

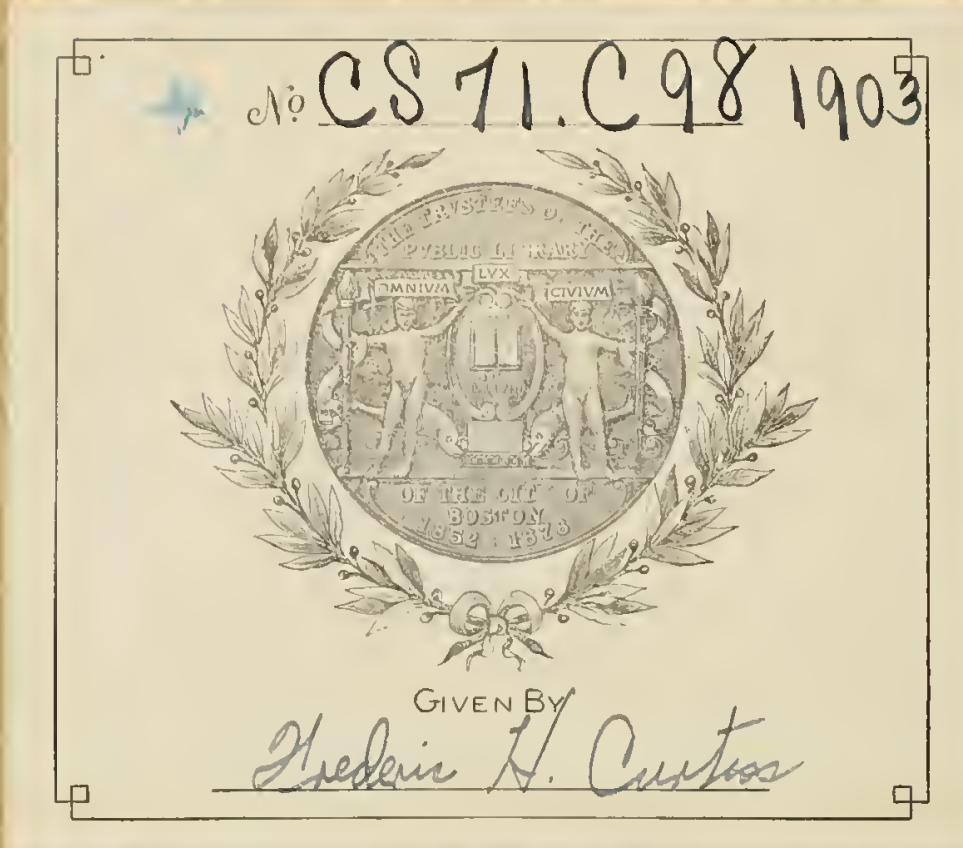






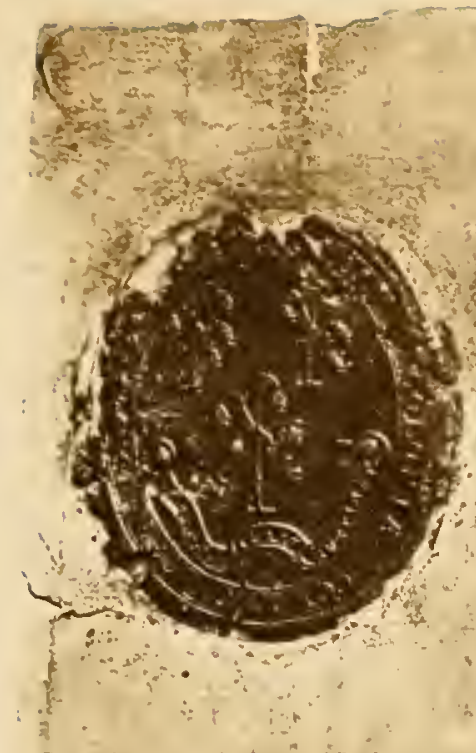

articreasthe Gener at courto Dotend of

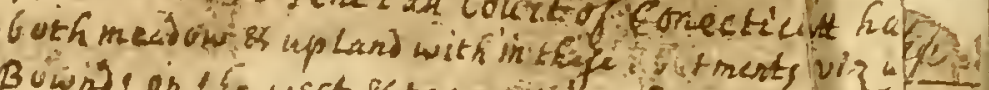

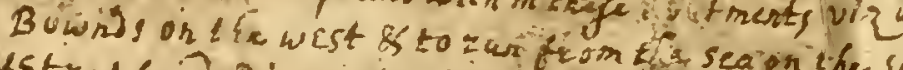

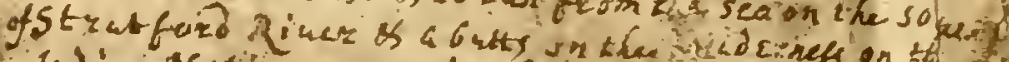

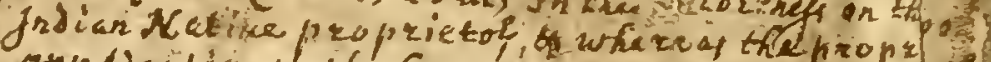

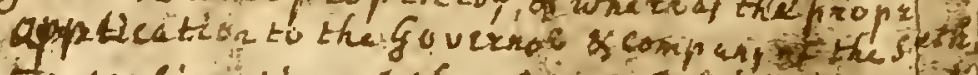

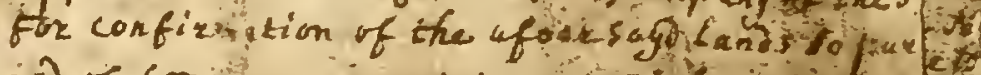

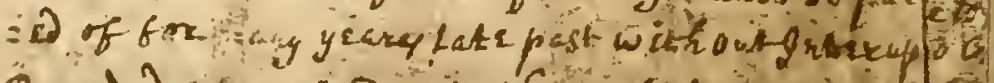
Bownd id a a sing unto the prefett proprictof

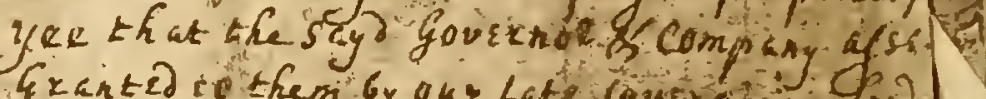
Granted to them Gy okz Late vouragin 20 2

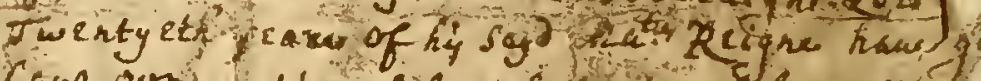

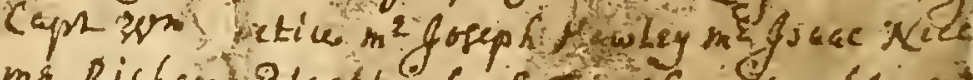

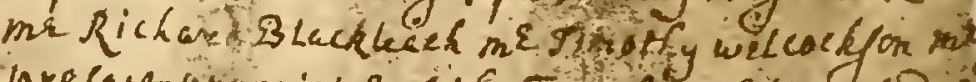

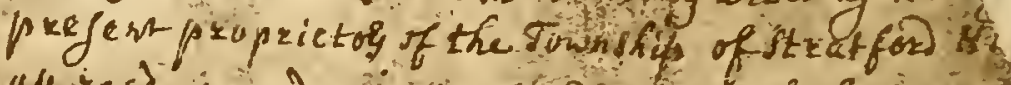

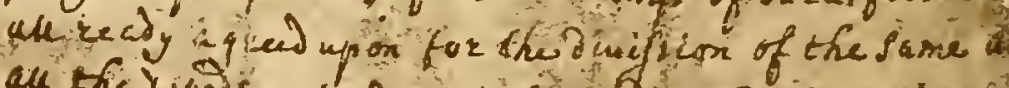

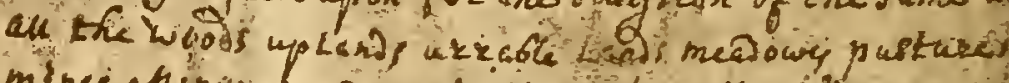

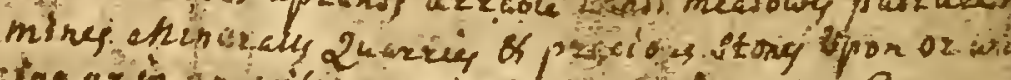

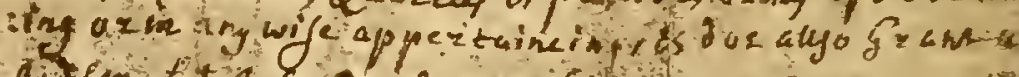

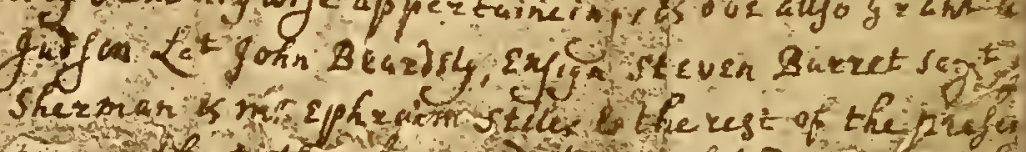

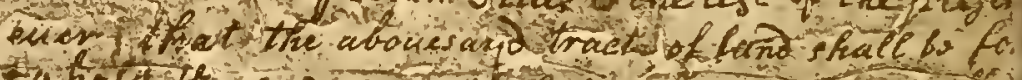

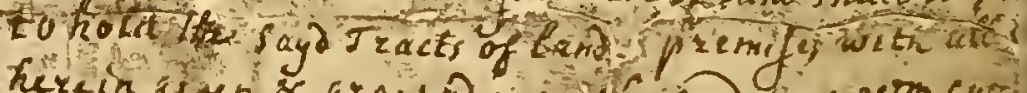

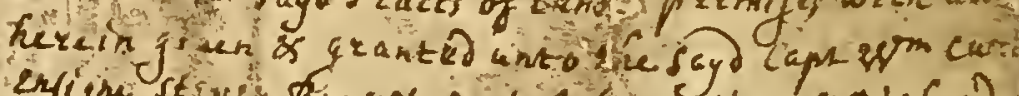

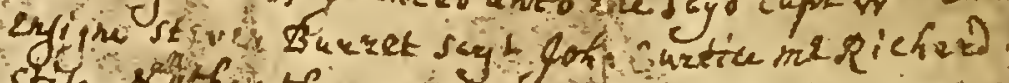

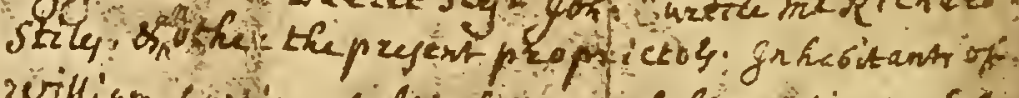

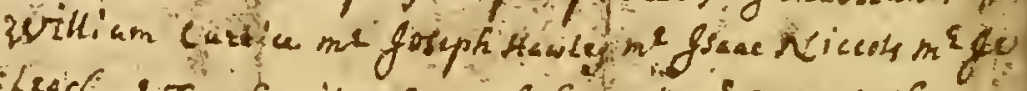

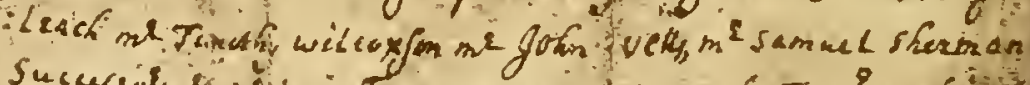

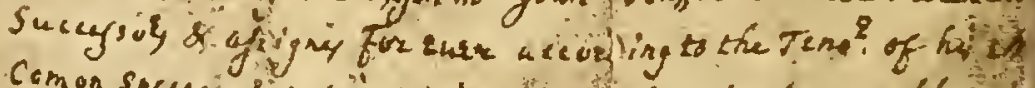

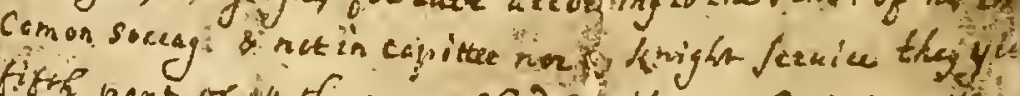

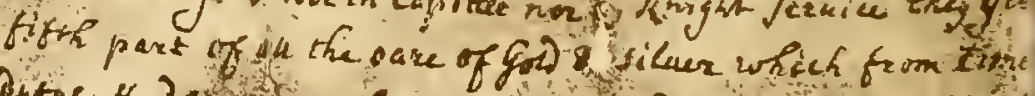

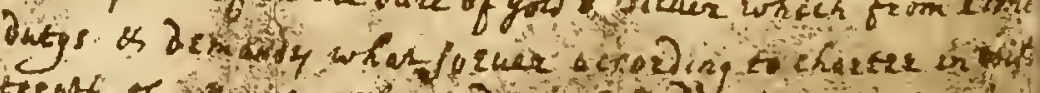

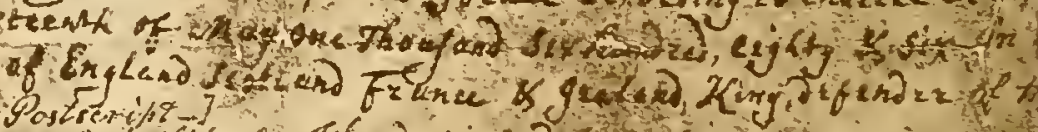

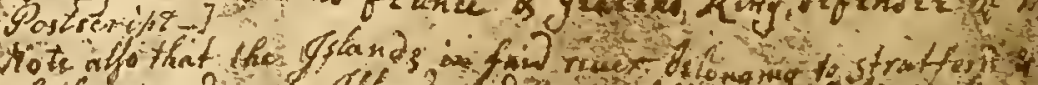

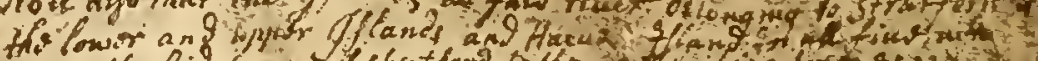

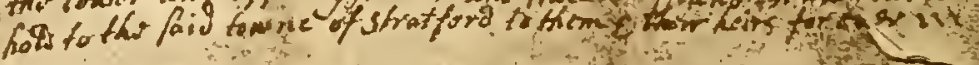

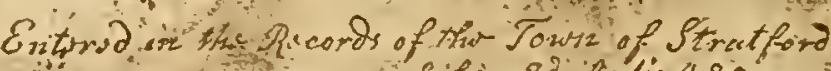

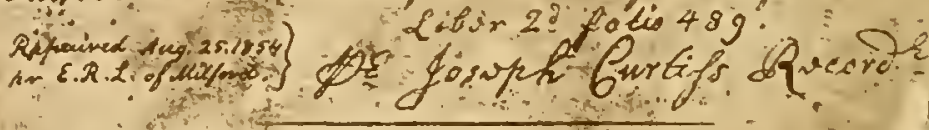

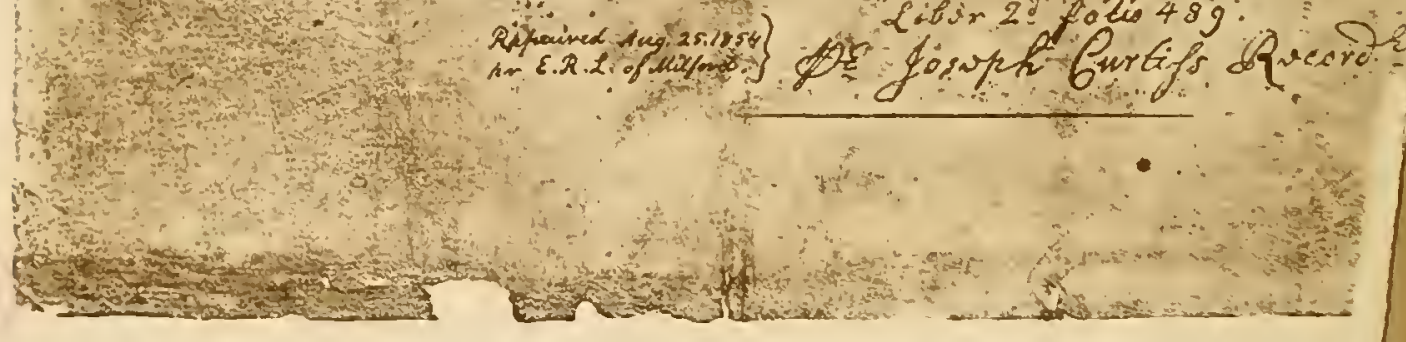




\section{Town of etritfore}

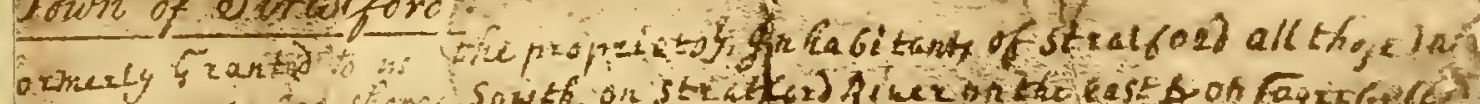

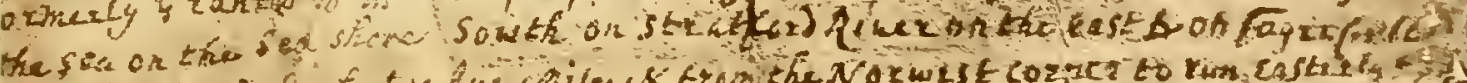

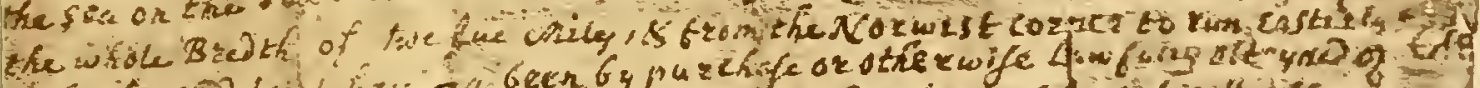

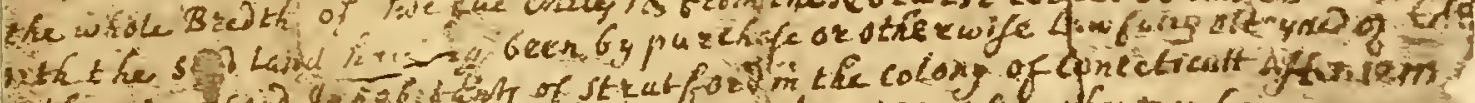

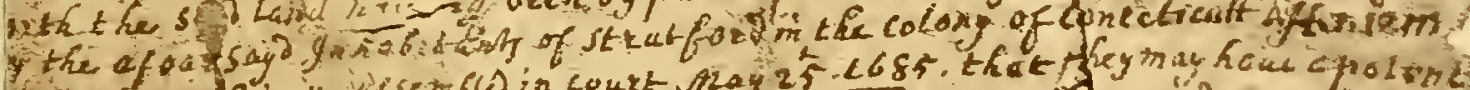

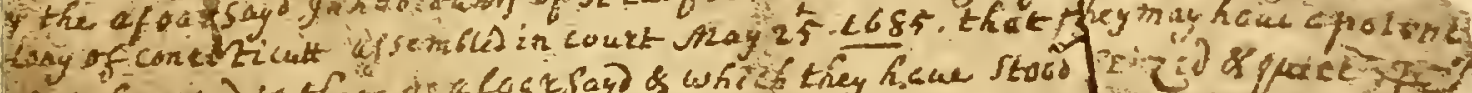

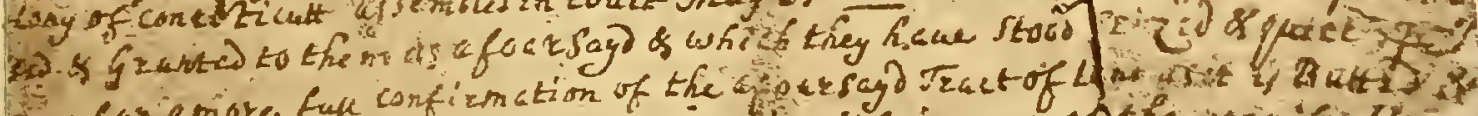

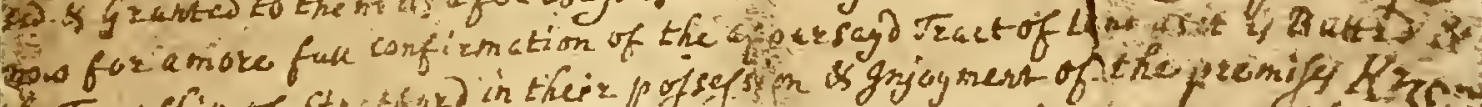

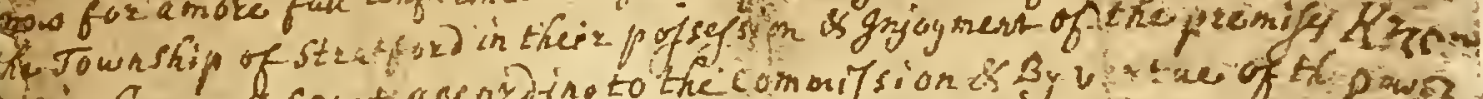

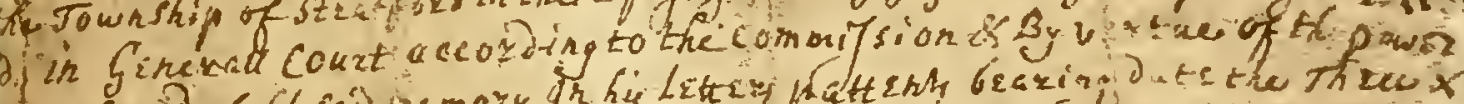

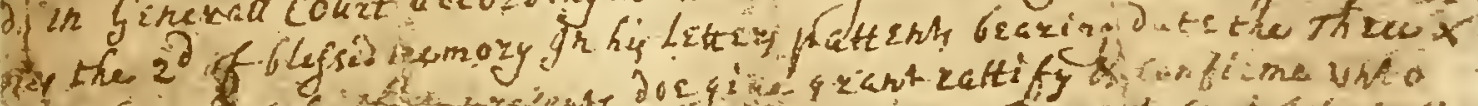

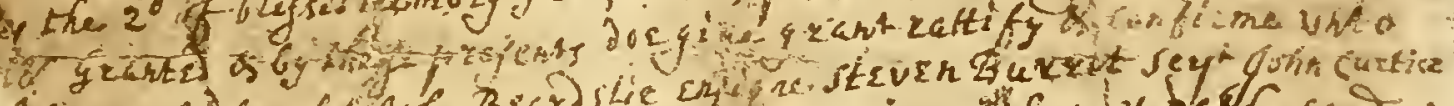

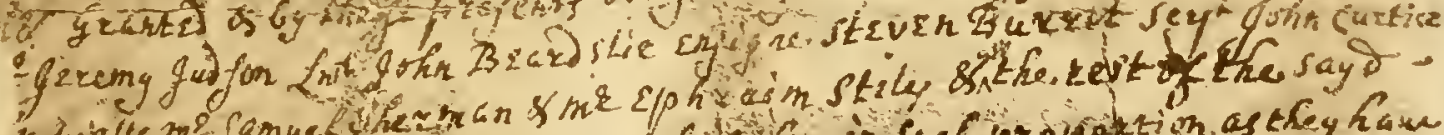

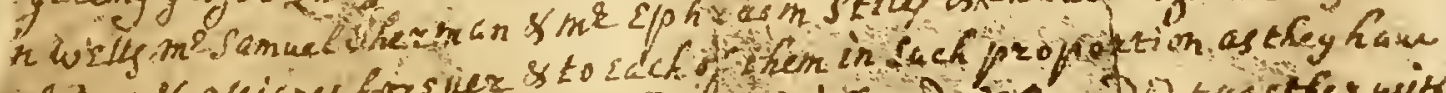

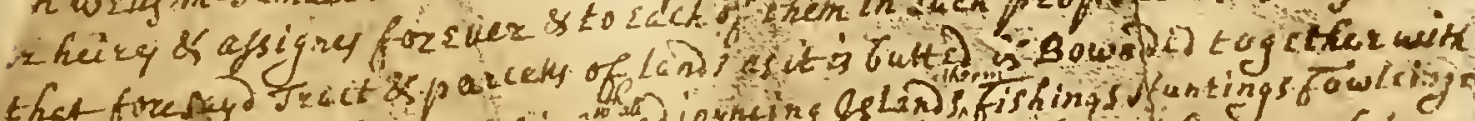

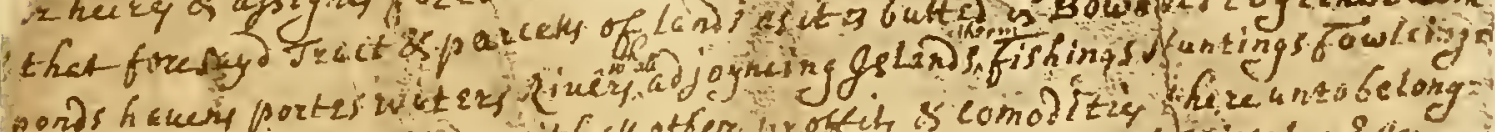

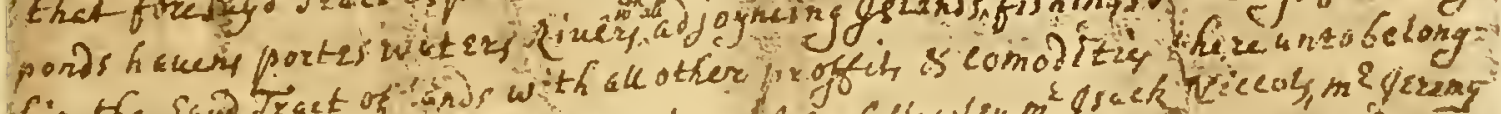

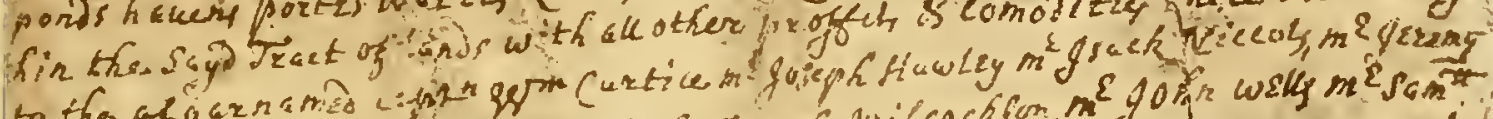

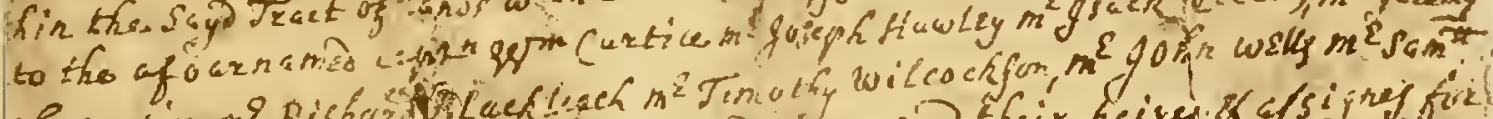

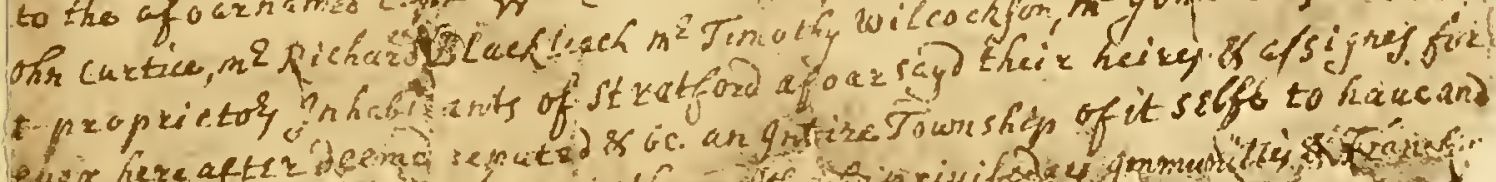

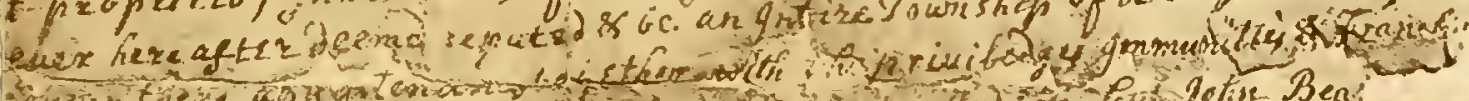

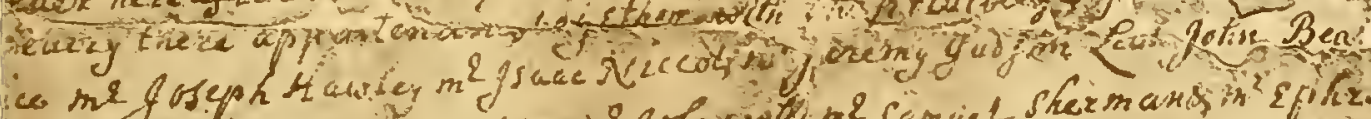

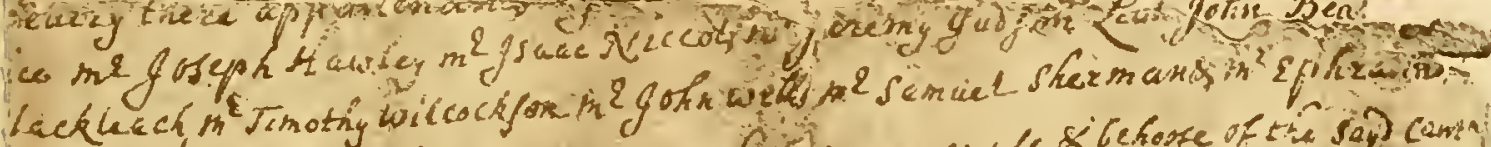

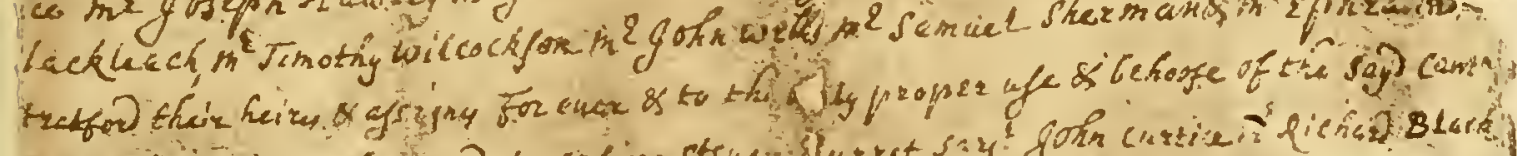

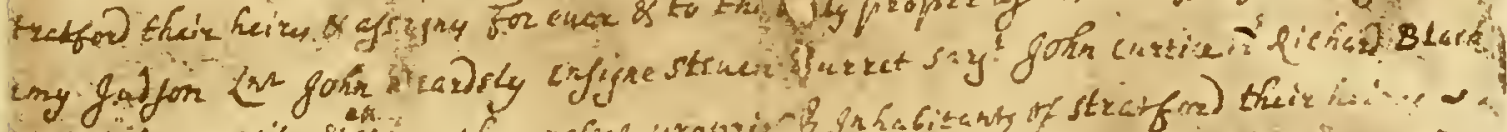

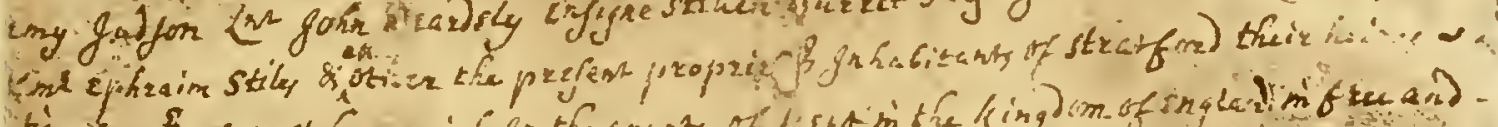

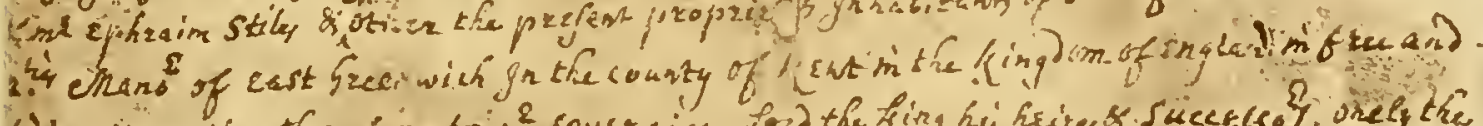

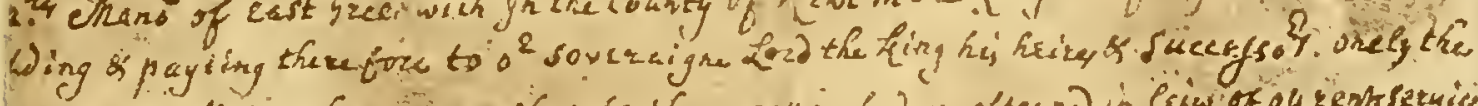

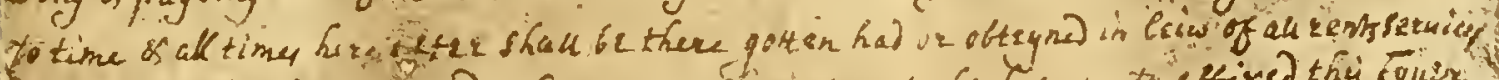

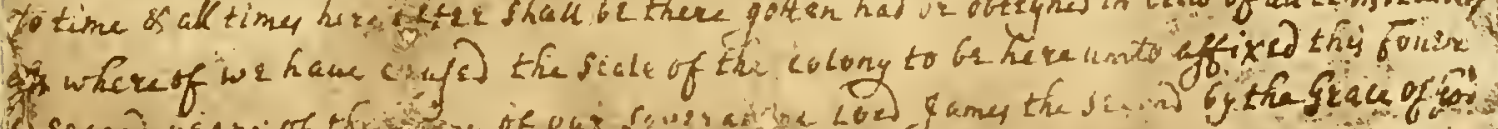

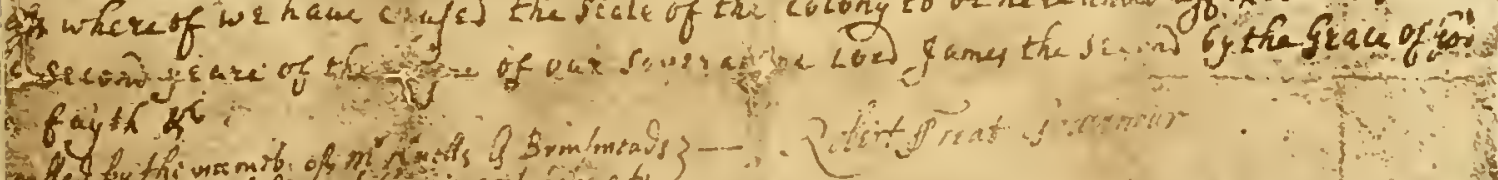

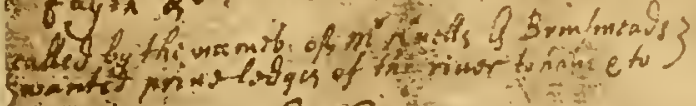

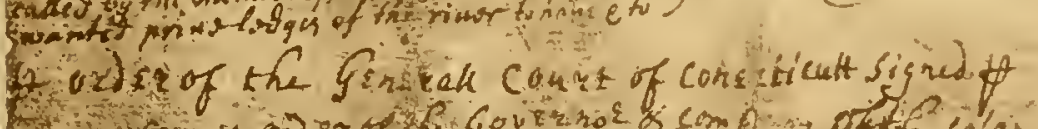

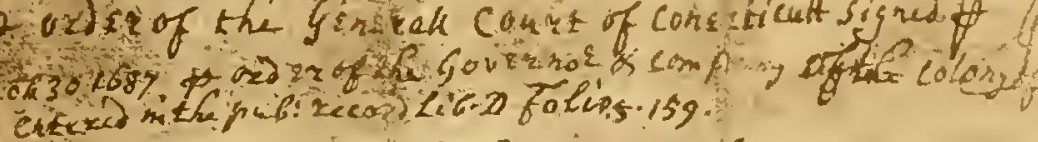

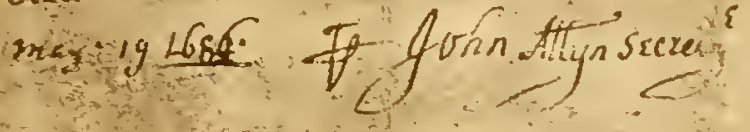

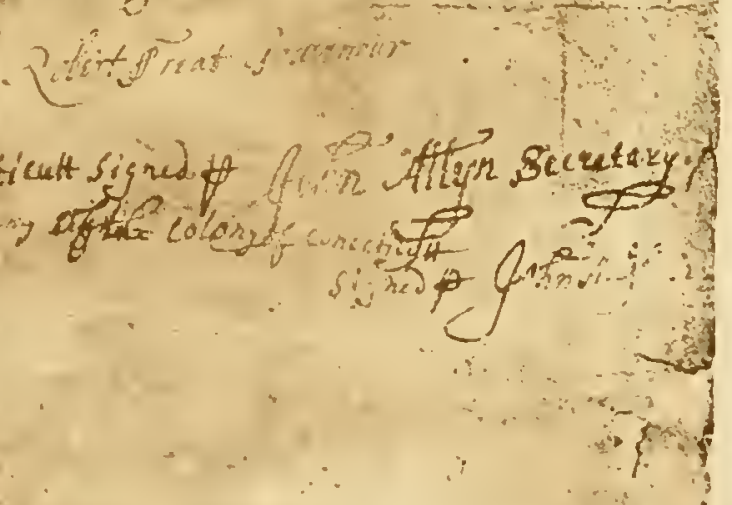





\section{A GENEALOGY}

OF THE

CURTISS FAMILY 



\title{
A GENEALOGY
}

OF THE

\section{CURTISS FAMILY}

\author{
BEING A RECORD OF THE
}

DESCENDANTS OF WIDOW ELIZABETH CURTISS

WHO SETTLED IN STRATFORD, CONN., I639-40

BY

FREDERIC HAINES CURTISS

Member of the New England Historic-Genealogical Society; Berkshire Historical Society; Colonial Society; Bostonian Society, etc.

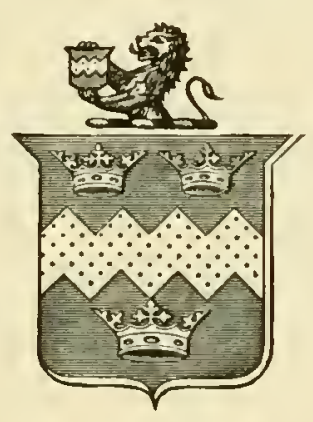

BOSTON

ROCKWELL AND CHURCHILL PRESS

c 1903 
*CS

C. 78

$190 ?$

$$
\begin{aligned}
& -2+4+4 x=113 \\
& \text { th }
\end{aligned}
$$




\section{PREFACE}

IN giving this book to the public the compiler realizes how incomplete it is in many respects, but as it is the first attempt to publish a genealogy of this family, he trusts the reader will pardon its shortcomings. The data contained herein have been gathered almost entirely from original sources such as town, church, land, and private records.

The compiler wishes to take this opportunity to thank the many persons who have assisted him in preparing this work. His thanks are especially due to Mr. George M. Curtis of Meriden, Conn., who has placed in his hands a mass of data, comprising the work of many years, and to whom he is indebted for almost all of the data relating to the Wallingford, Conn., branch of this family.

The compiler of this book will be glad to receive corrections or additional data from any nember of this family, and if another edition of this genealogy is published at a later date, such corrections will be made and all additional material will be used.

Frederic Haines Curtiss.

Boston, Mass.

(v) 



\section{THE PROBABLE ENGLISH ANCESTRY OF THE CURTISS FAMILY OF STRATFORD, CONN.}

There came to New England in the ship "Lyon," which sailed from London, June 22, I 632, William Curtiss and Thomas Ufford, both natives of Nazing, Essex County, England. Landing at Boston, N.E., on Sunday, Sept. 16,1632 , they proceeded to Roxbury, where John Eliot, the "Apostle to the Indians," who was the brother-in-law of William Curtiss, had settled the previous year. In the ship "Lyon," so Mr. Winthrop tells us, there came one hundred and twenty-three passengers, of whom fifty were children. These persons were all of the better class, were well educated and possessed a certain degree of wealth that enabled them to live in the enjoyment of comforts.

On the records of Nazing, England, ${ }^{2}$ we find that William Curtiss was baptized Nov. 12, 1592, and married Sarah, daughter of Bennett Eliot, of Nazing, Aug. 6, 16 18. The baptisms of his several children also appear on these records. William Curtiss died in Roxbury, N. E., Dec. 8, 1672, aged 8I years, and Sarah his widow, March 20, I673, aged 73 years. ${ }^{3}$ Most of the historians have supposed this IVilliam Curtiss to have been the husband of widow Elizabeth Curtiss, of Stratford, Conn., but my researches have led me to believe he was brother of her husband, who was John Curtiss. The Nazing records also show the following entries:

John Curtis, baptized Sept. I 5, I 577.

John Curtyce married Elizabeth Hutchins, April 19, I610; and the following baptisms, supposedly those of their children:

John Curtis, Feb. 26, I614-15; William Curtis, June 21, 16 18; and Thomas Curtis, March 12, $1619-20$.

John Curtis, aged 2I, sailed with Thomas Heath, aged 23, in the ship "Safety," Aug. 10, 1635. This may have been the above son, as the name of Heath is found both in Nazing and in Roxbury.

1 "The Curtiss Family," by Stiles H. Curtiss, A. M. N. E. Genealogical and Historical Register,

2 "Memorials of the Pilgrim Fathers of Nazing," by W. Winter. Waltham Abbey, 1882.

3 Page xxx. 
John Curtis ' of London, England, had his coat of arms confirmed May 9, 1632 , just forty-two days before William of Roxbury sailed for New England. In the visitation of London, in 1633 , his name does not appear. In " A Note of ye Estates and Persons of the Inhabitants of Rocksbury," I $638-40$, appears the name of John Corteis, having fifteen acres of land and with five persons in his family. No further mention of this family appears on the Roxbury records, but in 1639 a John Curtis ${ }^{3}$ was a resident of Wethersfield, Conn., and owned land in that town on what was then called "The North Road to the Great Meadow." Thomas Ufford, who left Roxbury in 1635, with William Pynchon, we also find in Wethersfield at this time. Rev. Thomas Hooker, the famous divine of Hartford, was a native of Morfield, Leicestershire, England. He was a Fellow of Emmanuel College about the same time as John Eliot, and Eliot assisted him in his school at Little Baddow, Essex. Through persecution Hooker left England and sought refuge in Holland. After a while he heard that some of his Essex friends were going to New England, and he speedily united with them and sailed in the "Griffin" from Downs in 1633. It is therefore probable that when he settled in Hartford many of the Roxbury people, natives of Essex, joined him there.

John Curtis left Wethersfield in I 640 for Cuphag (Stratford), and his name disappears, but we find the name of widow Elizabeth Curtiss and her two sons on the earliest records of Stratford. It is therefore probable that the father died soon after arriving at Stratford, or on the journey. Thomas Ufford left Wethersfield at about the same time and went to Milford, but a few years later we find him in Stratford where he, with his family, spent the remainder of his life. There has been a tradition in Stratford that the Ufford and Booth families had intermarried with the Curtiss before they left England, but this I have been unable to substantiate. Mr. Trumbull ${ }^{4}$ in his " History of Connecticut," in writing of the settlement of Stratford, says that "Mr. John and Mr. William Curtiss and Mr. Joseph Hawley were from Roxbury, and Mr. William Judson, and Mr. William Wilcoxson from Concord, in Massachusetts." "These were the first principal gentlemen in the town and church of Stratford." " A few years after the settlement commenced, Mr. John Birdseye removed from Milford and became a man of eminence both in the town and church." "Mr. Adam Blackman who had been episcopally ordained in England, and a preacher of some note, first at Leicester and afterwards in Derbyshire, was their minister and one of the first planters. It is said that he was followed by a

1 Page xii.

${ }^{2}$ Town Records of Roxbury, N. E.

3 " Memorial History of Hartford County, Conn.," page 439.

4 "History of Connecticut," by Benjamin Trumbull. New Haven, Conn., 1789.

(viii) 


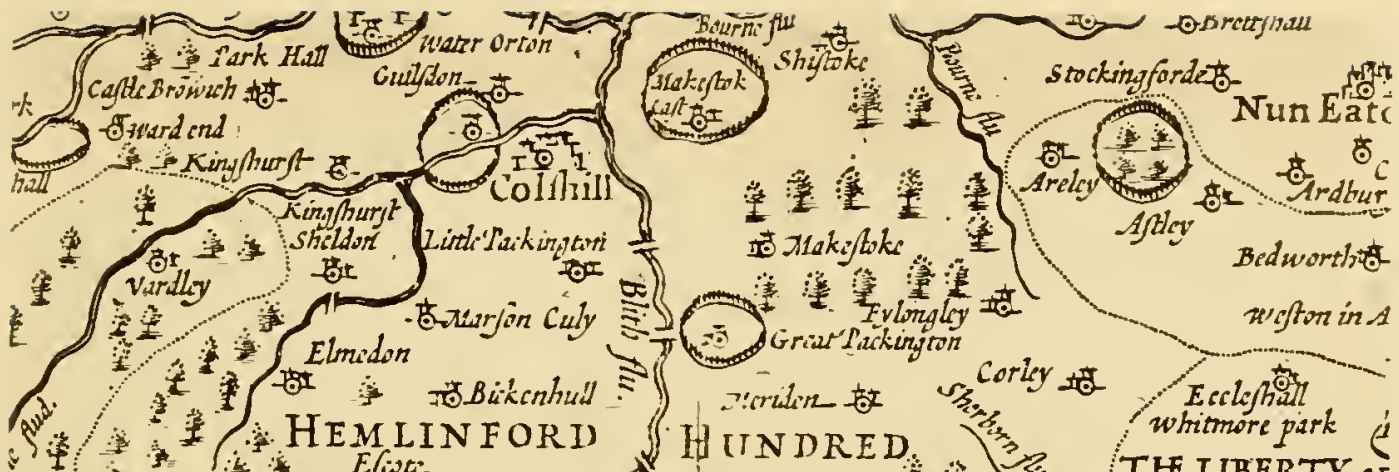

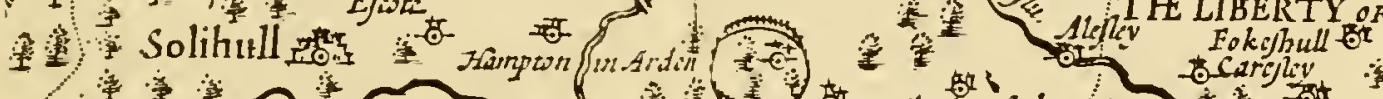

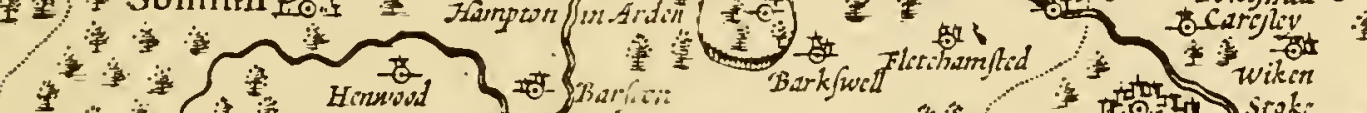

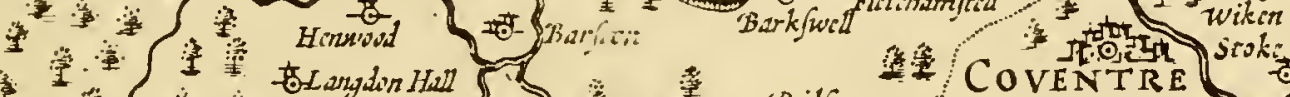

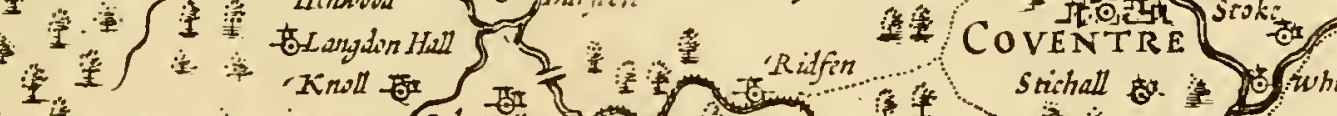

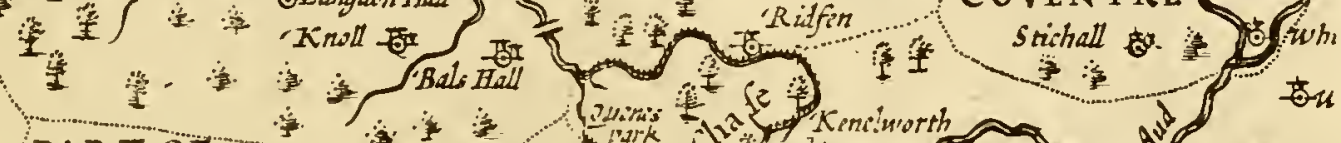
PAR I OF

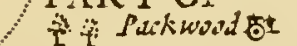
KINETON HLNDD

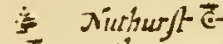
Intwith Tiungli Ford

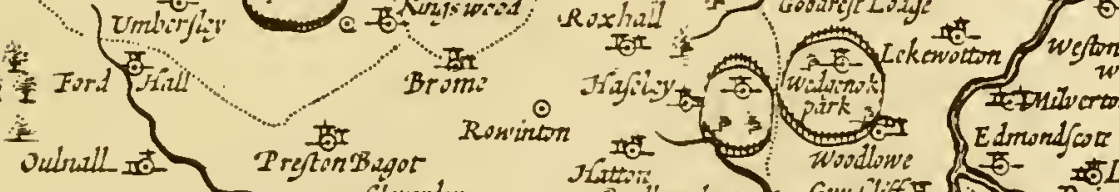

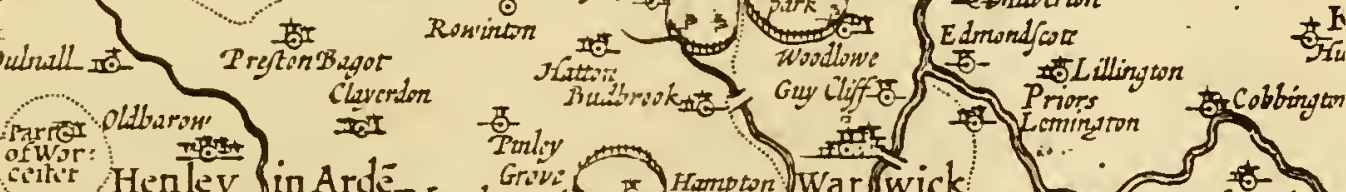

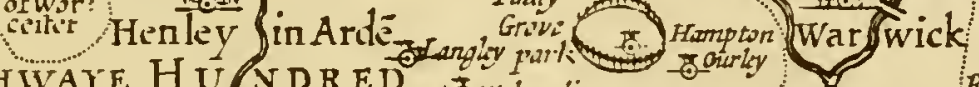
CHWAIE HU (NDRED 㙂Woluritur: Alini Lody wawis: Ed Evon

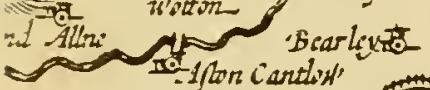

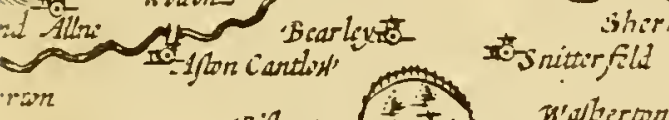

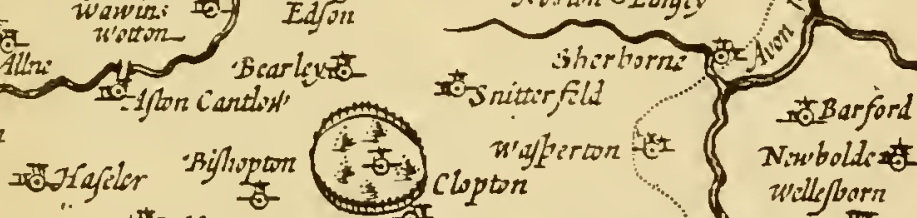

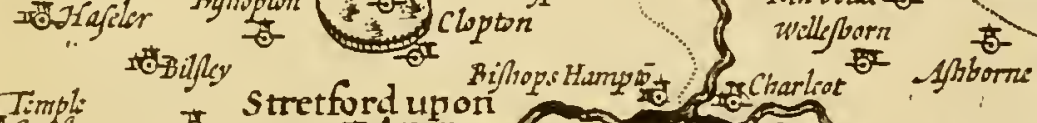
Timpli Stretford upon

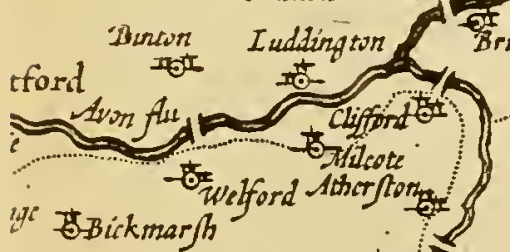

Prgfor tor Aulton Loxy

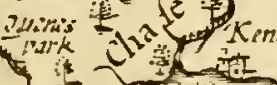

\section{,}



number of the faithful into this country." "These doubtless collected about him in the infant settlement." Mr. Blakeman is supposed to have come to Stratford from Wethersfield, and as the house lot of widow Elizabeth Curtiss was near or adjoining that of Mr. Blakeman's, this family may have come to Stratford to be under his ministry. In the will of Elizabeth Curtiss," John Birdseye, Henry Wakelyn, and Joseph Hawley were appointed overseers. Of these Joseph Hawley came from Roxbury, N. E., and also came to this country in the ship "Planter," in I635, with Adam Blakeman, William Wilcoxson, and William Beardsley. William Curtiss's ${ }^{2}$ family furnishes us with further proof of his having come from Wethersfield, Conn., and Nazing. He married for his second wife Sarah, daughter of Matthew Morris, ${ }^{3}$ of Hartford, Conn., and widow of William Goodrich, of Wethersfield, Conn., and two of his daughters married into Wethersfield families those of Rose and Welles. The latter perhaps was John Welles, son of Gov. Thomas Wells, as on the records John Welles calls John Curtiss uncle.

The Curtiss family was from all accounts one of the most prominent among the first settlers of Stratford, and it has been claimed that they suggested naming the town Stratford after Stratford-onAvon, ${ }^{4}$ England. If this be true it adds another proof towards the acceptance of the pedigree with the coat-of-arms, ${ }^{5}$ for Makestoke and Hatton were but small hamlets and Stratford-on-Avon was the nearest town of any size. That the name of Curtiss was well known in Shakespeare"s town is shown by the fact that that poet used it as one of the characters in "The Taming of the Shrew." William Beardsley, Thomas Alsop, and Richard Booth are also supposed to have been natives of Stratford-on-Avon, England, and as the Earl of Warwick had letters patent ${ }^{6}$ to that part of Connecticut, it is more than probable that many of the early settlers throughout that colony were from Warwickshire.

1 Page 1.

2 Page 3.

3 The name of Morris is found in Nazing, England.

4 There is also a town near Nazing, and now a part of London, called Stratford-on-Bow, and 1 have found the name of Curtis on tombstones in the parish churchyard of that town.

5 Page xii.

6 See Patent of Connecticut. 



\section{THE CURTISS COAT OF ARMS}

BEFORE going into the subject of the Curtiss coat of arms I feel that it will not be out of place to quote a portion of the report of the Committee on Heraldry of the New England Historic Genealogical Society, regarding the bearing of arms, -

"As there is no person and no institution in the United States with authority to regulate the use of coats of arms, your Committee discourages their display in any way or form.

"Prior to the Revolution, as subjects of a government recognizing heraldry, certain of the inhabitants were entitled to wear coats of arms; but only such as were grantees of arms, or who could prove descent in the male line from an ancestor to whom arms were granted or confirmed by the Heralds.

" Females did not regularly bear arms, but the daughter of an arms-bearing father could use the paternal coat in a lozenge. When she married, such arms did not descend to her children (except by special authority), unless she were an heiress marrying an armiger, and then only as a quartering of her husband's arms.

"The mere fact that an individual possessed a painting of a coat of arms, used it upon plate, or as a bookplate or seal, or had it put upon his gravestone, is not proof that he had a right to it.

"Proof of right must either be found in the Herald's records or be established by authenticated pedigree direct from an armiger.

"A coat of arms did not belong with a family name, but only to the particular family, bearing the name, to whose progenitor it had been granted or confirmed; and it was as purely individual a piece of property as a homestead. Hence it was as ridiculous to assume arms without being able to prove the right, as it would now be to make use of a representation of the Washington mansion at Mt. Vernon, and claim it as having been the original property of one's family, unless bearing the name of Washington and being of the line of those who owned it."

After the above, I hesitated to make any mention of coats of arms in this book, but as I have made a careful research on the subject, I feel that the results of this work may help some future genealogist to learn more of the early history of the Curtiss family.

The coat-of-arms on the title page of this book is claimed to be 
the one borne by the ancestor of the Stratford, Conn., branch of the Curtiss family. An ancient emblazonment on parchment has been in the possession of this branch of this family beyond any doubt for over one hundred and fifty years and is owned to-day by an elderly lady residing in Bridgeport, Conn. It is claimed this emblazonment was brought over to this country by the first ancestor, but this I have been unable to substantiate. On the reverse side of this parchment certain data relating to the Stratford and Roxbury members of the family have been written, supposedly in the handwriting of Joseph Curtiss, Town Clerk of Stratford, Conn., I 678-1728.

The records of the College of Heraldry, in London, Eng., show that this coat-of-arms was confirmed to "John Curtis, of London, Gent., son of William Curtis, of Hatton in the County of Warwick, Gent., son of Eustace Curtiss, of Malestock, in the said County, Gent., son of William who was son and heir of John Curtis, of Malestock aforesaid, Gent., by Sir Richard St. George Clarencieux, the 9th of May, 1632," in the 8th year of the reign of King Charles I., and that this crest was granted at the same time. In the visitation of London, in 1633 , the name of John Curtis does not appear, so it is probable that he left for New England soon after having these arms confirmed.

For further proof of the above see page viii.

The curious description of these arms on the records is as follows: "Azure a fesse dancette between three ducal crowns or,"

"Crest: A Lyon in his ppr. collers Issuing forth of his Collers Or and Azure supporting in his right pawe a Shield Azure \& thereon a fess dancette Or Mantled gules doubled argent."

A general search in the archives of the College of Heraldry, London, England, gives the following record of arms borne by persons of the Curtiss, Curtis, etc., name. The numbers and initials on the left are the reference numbers to those records.

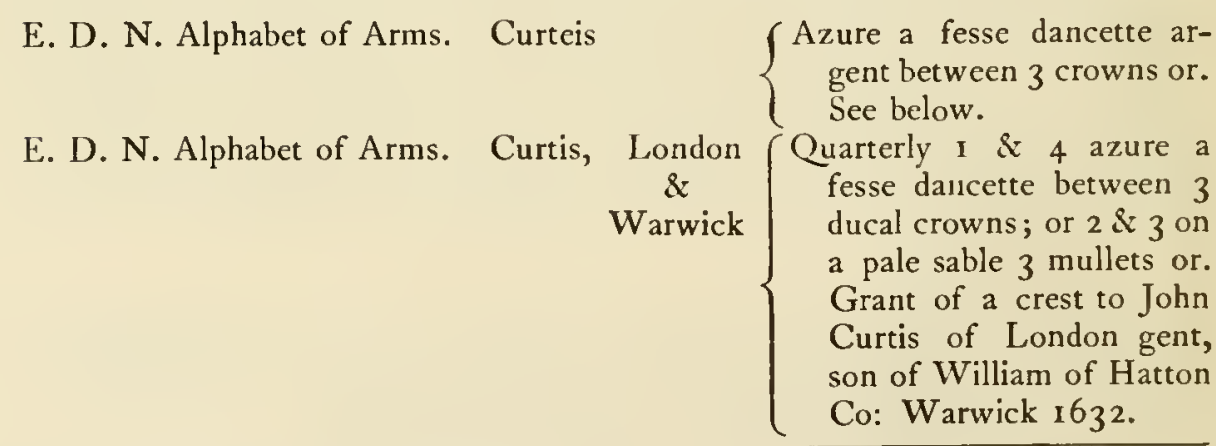

${ }^{1}$ William Curtiss, who settled in Roxbury, N. E., and who is claimed to have been a brother of John Curtiss, sailed for New England June 22, 1632. 

C I6,79 . . . . . Curtys, Kent.
C I6, I64 . . . . . Curtis, Kent.
C 33,9 - . . . . Curteys, Derby.
C 34,70 . . . . Curtys, Derby.
D I 8, I $19 . \quad . \quad . \quad$ Curtis, Kent.
D 18,167 . . . . Curteis, Kent.

E I 5, 8 I . . . . Curtes, Cornwall. 2nd E. 2,26 . . . . Curtis.

$6 \mathrm{E}, 25$. . . . Curtys, Lincoln.

G 2,76 . . . . Curteys, Cornwall.

H I 8, I20 • . . . . Curteys, Cornwall.

$\mathrm{K} 8$, i 10 . . . . . Curtis, London.

M I I, 8

Misc. Grants . . . . Curtis, London

\&

Warwick.

Misc. Grants 5, I9 . . Curteys, London.

Misc. Grants 5, 2 I . . Curtyes, Berkshire.

Misc. Grants 5, I24 . . Curtes, London.

Misc. Grants 5, 210 . . Curtes, London.

Misc. Grants 5, 124 . . Curtes, Lincoln.

Misc. Grants 5, 2 I I . Curteys, I,incoln.

Old Grants 39 . . Curties, London.

Grants I, I68 . . . Curtyes, London.

Grants I, I82 . . . Curteys, London.

Grants I 8, 396 Curtis, Southampton. Grant of Arms.

Grants 22, Curtis, Middlesex. Grant of Arms.

Grants 28, I32 - . . . Curtis, Middlesex. late Courtney.

Grants 28, 368 . . . Curtis, London.

Grant of Arms.
$\{4$ gens to I6I9 signed Will-

$\{$ iam Curtis. Arms.

(of Tenterden 2 gens. connected with Philips of same.

fof Somerlees. 6 gens. to I634 signed Francis Curteis: Arms.

fof Somerlees. 4 gens. to I 662, certified by Robert Curtys. Arms.

fof Tenterden. 4 gens. to I 663 certified by Edward Curtis.

(of Chart, near Sutton. 4 gens. to 1663 signed

George Curteis.

Match (early date).

Match Arms.

of Ely. Match.

of Pyll. 6 gens. Arms.

of Pyll. Match. Arms.

$\left\{\begin{array}{c}\& \text { Portsmouth. } 3 \text { gens to } \\ 1686 \text { signed Thos. Cur- }\end{array}\right.$ tis. Arms.

Arms.

Confirmation of arms to John Curtis of London. son of William C. of Hatton Co. Warwick giving a descent of 5 gens. Arms as No. I.

$\int$ Grant of arms. $4 \& 5$ Philip \& Mary.

Grant of arms. 2 Elizabeth.

Grant of Arms.

Grant of Arms.

Grant of Crest.

Grant of Arms.

Grant of Arms.

Grant of Arms.

$\{$ Sir Roger Bart. Grant of Supporters. 


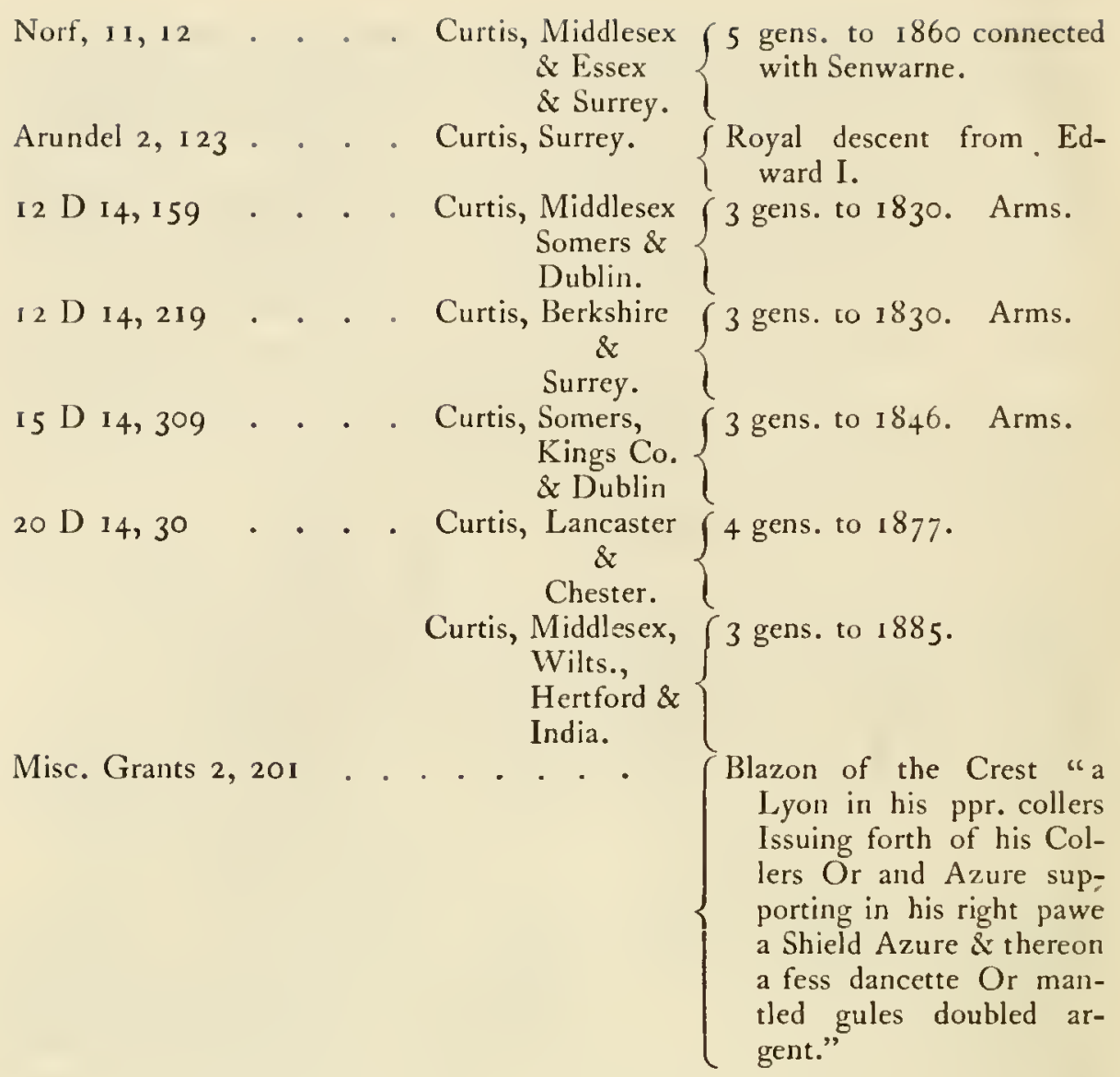

The following arms and crests are from the numerous heraldic publications :

Curteis of Appledore and Otterden Place, Kent, of Tenterden and Rye and of Windham Hill, Sussex: Argent a chevron between three bulls' heads cabossed sable. Crest : A unicorn passant or, between four trees proper.

CURTEIs: A wolf's head couped argent, collared and spiked sable, chained or.

Curteis, Curtess, Curteys, Curtois or Curtoys : A demi husbandman vested azure, holding over the shoulder an ox-yoke proper. (Another holding over the shoulder a plowshare.)

Curtis: An arm in mail garnished argent.

Curtss: Catscombe, County Hants. Per fesse wavy argent and sable in chief the rock of Gibraltar surrounded by fortifications and the sea proper in base, three fleur-de-lis of the first, on a canton gules. Crest: A sword erect proper, pommel and hilt or, entwined with a palm branch vert. 
Curtis : Sir Arthur Colin, Bart., of Gatscombe Hantz. Out of a naval coronet or, an arm unbound, vested azure, cuffed argent supporting a flagstaff proper, thereon a flag of the second charged with a wolf's head or, and a canton gyronné of four, gules and azure, thereon a cross of the third within a brodure of the first. Motto: Per ardua.

Curtis: Ireland. Crest : A hand holding four arrows, points downward.

Curtis of East Cliff, Devonshire. Crest: An arm in armor embowed, holding in the hand a scimitar argent, hilt and pommel or.

Curtis: Ermine a chevron argent between three fleur-de-lis or. Crest: An arm embowed in armor holding in the hand a sword proper.

CuRtis: Crest : An arm in armor erect, holding in the hand a sword, all proper, hilt and pommel or. Motto: Velle bene facere.

Curtis: Crest: An arm in mail proper holding in the hand a scimitar proper.

Curtis: Matthew, Esquire, of Thornfield, Lancashire. Crest: In front of a horse's head argent, holding in the mouth a fleur-de-lis azure, a fasces fessewise proper. Motto: Perseverando vinco.

Curtis : Sir William Michael, Bart., of Cullands Grove, Middlesex, Lord Mayor, 1796. Paly of six or and azure a fesse chequay argent and sable on a canton gules, a dragon wing erect of the third, in base a sword proper, pommel and hilt gold surmounting a silver key in saltire. Crest : A ram's head couped argent, surmounted by two branches of oak in saltire proper. Motto: Grandatione vincimus.

CuRT1S: Suffolk. Azure a chevron dancette between three mural coronets or. Crest: A lion sejant proper supporting with his dextra paw an escutcheon of the arms. Motto: Saepere aude.

Curtrs of Roscrea, County Tipperary, Ireland. Crest : A dolphin naiant azure pierced in the side with a feather, or.

Curtis: Shropshire. Crest: A leopard face or.

Curtis: Crest: A greyhound's head between two roses. 



\section{RECORDS IN ENGLAND}

The following entries occur in the Nazing and Waltham Abbey Parish registers: ${ }^{1}$

\section{NAZING.}

Baptisms.

Thomas Curtis, Aug. 25, 1560.

John Curtis, Oct. I I, I 562 .

Nicholas Curtis, January, i 576-7.

John Curtis, Sept. 15, 1577 .

Martha Curtis, October, 1587.

Mary Curtis, March, I589.

Elizabeth Curtis, March I4, I 590-I.

Philip Curtis, September, 1591.

William Curtis, Nov. I 2, I 592.

Mary Curtis, Jan. 20, 1593-4.

Thomas Curtis, July 21, 1594.

George Curtis, October, I 596.

Mary Curtis, daughter of Edward Curtis, Nov. 3, 1600.

John Curtis, Feb. I3, I602-3.

Susannah Curtis, Oct. 6, I605.

John Curtis, October, 1607.

John Curtis, Feb. 26, I6I4-15.

William Curtis, June 2I, I6I8.

Thomas Curtis, March I 2, 1619-20.

Mary Curtis, March II, I620-I.

Thomas Curtis, Jan. 19, I622-3.

Elizabeth Curtis, daughter of William Curtis, Feb. I 3, I624-5.

Margaret Curtis, daughter of George Curtis, March I 9, 1625-6.

Sarah Curtis, daughter of William Curtis, Aug. 5, I627.

Mary Curtis, daughter of George Curtis, Oct. 6, 1628.

John Curtis, July I 7, 1629.

Edward Curtis, son of George Curtis, April 20, 163 I.

Susan Curtis, daughter of George Curtis, May I6, I63I.

Philip Curtis, March 28, 1632 .

George Curtis, Oct. 20, 1633 .

1 "Memorials of the Pilgrim Fathers of Nazing," by W. Winters, Waltham Abbey, I 882.

(xvii) 
'Thomas Curtis, son to George Curtis, March 25, $1636-7$. John Curtis, July 17, 1643.

\section{Marriages.}

William Tomson and Marion Curtis, May 19, 1561.

John Read and Mary Curtis, Aug. I9, 1582.

Thomas Curtis and Mary Camp, Aug. 24, 1585.

Thomas Curtis and Mary Shelley (wid.), July 3, 1596.

John Curtyce and Ann Sansome, June 22, 1608.

John Curtyce and Elizabeth Hutchins, April 19, 16 ro.

William Curtis and Sarah Eliot, Aug. 6, 1618.

Thomas Ruggles and Marye Curtes, Nov. I, I 620.

John Beech and Marie Curtis, Nov. I7, 1627.

Gabriel Curtis and Anne Keyes, May 2, 1642.

\section{Burials.}

Mother Curtis, Nov. 20, 1561.

William Curtis, homo, May 1 7, I585.

Mary, wife of Thomas Curtis, Nov. 24, 1594.

Thomas Curtis, puer, July 3, I 594 .

George Curtis, senex, June 27, I602.

Thomas Curtis, homo, Jan. 6, 1605-6.

Wife of George Curtis, Aug. 14, 1606.

\section{Waltham Abrey (or Holy Cross).}

Baptisms.

Robert Corteys, the sonne of William Courteys, Aprill I 5, I 565. Jone Courtes, the dowghtie of George Courtes, July I 8, I 568.

Madlyng Cortes, the dowghter of George Cortes, Oct. 16, 1575.

Joane, daughter of Thomas Curtice, Nov. 22, 1603.

John Curttes, Son of George, March 3, $163^{8}-9$.

Robert Curtis, son of George, May I 2, I64 I.

Thomas Curtis, son of George, June 22, I645.

\section{Marriages.}

Thomas Curtes \& Alles Avnderson, wedow, April 22, 1577.

Willm Newman \& Catheryne Curtys, June 10, 1590.

John Curtis \& Phillip Brown, June 5, I6 I4.

Danicll Buckley \& Elizabeth Curtisse, Nov. 10, I6I6. 1623.

George Curtis, of Nazing, \& Margaret Wells, of this parish, July 3I,

John Curtis \& Mary Brown, Aug. I9, I66r.

John Curtis \& Mary Tailor, Dec. 7, 1662 .

\section{Burials.}

George Cortes, the sonne of Wm Cortes, of London, July 2 I, 1566.

Madlyng Cortes, the dowghter of George Cortes, Nov. 4, I 575.

$$
\text { (xviii) }
$$




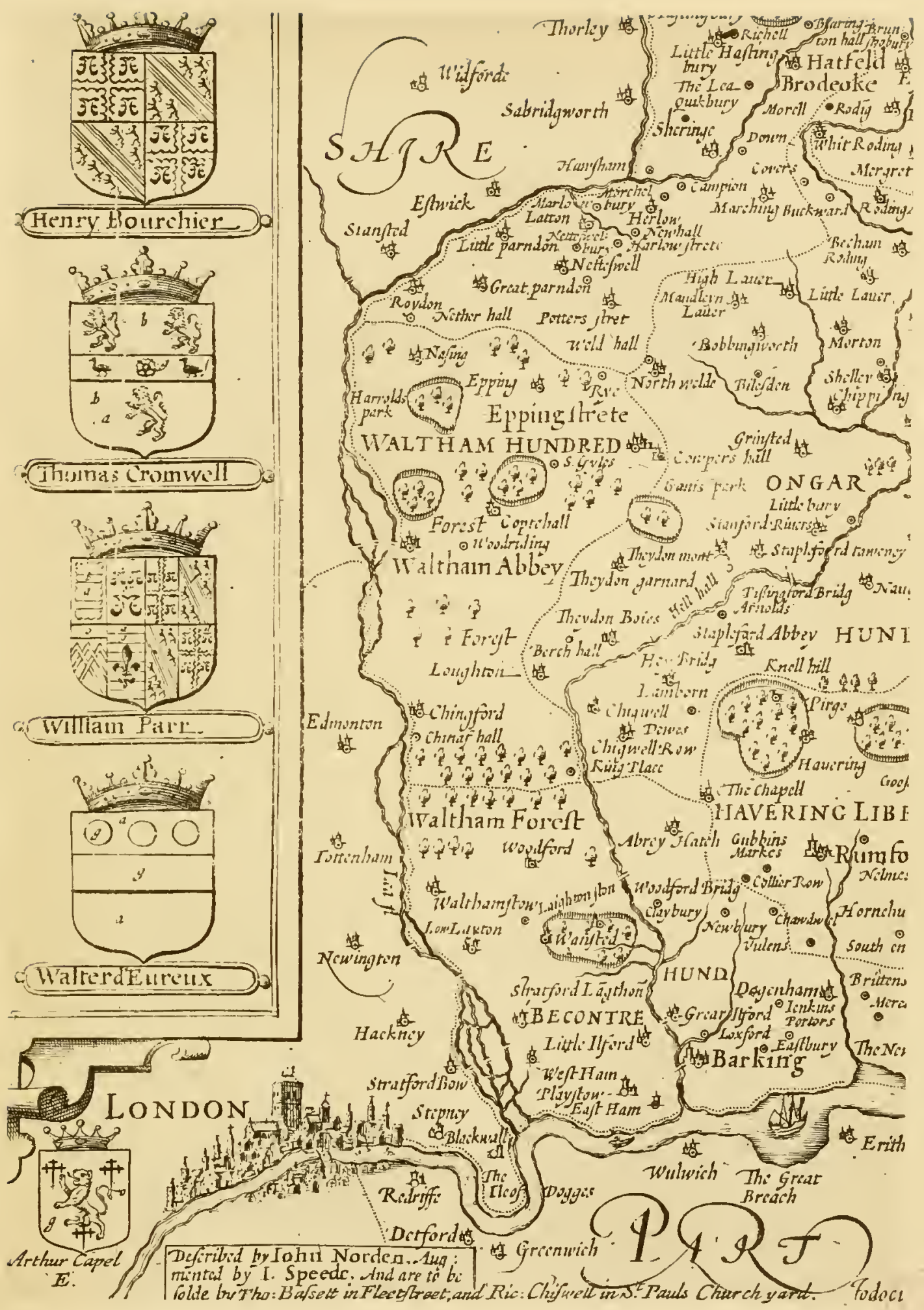



Mary, wyffe of Edw. Curtiss, May 25, 1612.

Curtise, daughter to Edward Curtise, Aug. 16, I612.

Sara Courteouse, daughter of Edward, Jan. 9, I6 I 5-16.

The wyfe of Ed. Courteouse, June 26, 1616.

Edward Courteouse, carpenter, Aug. I 3, i6 6 .

A nurse child of Goodman Curtess, of Copthall green, March I6, I6I6-17.

The widow Curtis, an old woman, Sept. II, I625.

Thomas Curtis, son of George, Sept. 22, 1638.

Mary Curttiss, a widow, Jan. 21, I638-9.

Mary Handford, a nurse child of George Curttises, Jan. 2, 1639-40.

A child of George Curtis, March 20, 1642-3.

George Curtisse, Sen., was buried Jan. 28, 1645-6.

Nathaniel, son of Christopher Curtis, Oct. I0, I66ı.

Mary, wife of John Curtis, Jan. 29, I66I-2.

Joseph, son of John and Margaret Curtis, Feb. 21, 1663-4.

\section{Cheshunt.}

Baptized.

Thomas and Anthony, twins of Henry Curtis, Feb. 14, 1562-3.

In the "Acts of the Court of High Commission," "under date June 26, 1634, appears the name of George Curtis, Esq., of Little Chart, Kent. He took oath to answer certain charges made against him. Two days previous Isaac Heath, of Ware, collar maker, appeared on his oath before the same court. Lyson mentions William Curtis, Commander in the East India Company, who died 1669 , and was buried in Stepney churchyard. On his tomb is inscribed the following lines :

\section{"Who in this life fifty years did stand, And to East India did bear command; Who in his lifetime kept not fast his door, And afterwards provided for the poor - Sixty pounds per annum for ever."}

This same person in 1669 bequeathed to the parish of Limehouse $£ 6$ per annum to apprentice two poor children, and $£ 6$ once in two years to be divided between twelve paupers of Limehouse, on the alternate years to be appropriated to the redemption of poor captives. ${ }^{2}$

Thomas Curtis, of London, was "fined xiij li. vis. viii. at the coming in of Queene Mary to the crowne, for not receiving the order of Knighthood," etc. 'Thomas Curtis was Sheriff of London 30 Henry VIII. In the Additional Manuscripts, British Museum, 
are several notices of the Curteis family. The name is variously spelt. Richard Curteis, of Tutbury, County Staffordshire, had a son William, who settled at Hanbury in the same county, temp. Ed. II. He had two sons and one daughter - (I) John, of Hanbury, living in the time of Ed. III., (2) Thomas, of the same place, and Matilda, who married (i.) John Hutton, of 'Tutbury, (ii.) William de Burton.'

A large family of this name resided for many years at Appledore, ${ }^{2}$ County Kent. Stephen Curteis had three sons, Richard, John, and Reginald. Richard settled in Newenden, County Kent, and had four daughters: Matilda married a Hamon, of Hurst, Jane married John LeSargent, Isabel married Robert Vincent, and Alice married Robert Scarlet. John (Stephen), the second son, was of Appledore, County Kent, married Petronill, and had issue: John, Stephen, and Joane. John (John, Stephen) had sons: William, John, and Thomas. William (John, John, Stephen), of Fairfield, temp. Henry VIII., married Ellen and had issue: Richard. Reginald (Stephen) married Margaret, daughter of Reginald, Lord Cobham, of Starborough Castle, and had issue: Reginald, of Westcliff. Thomas (John, John, Stephen), of Appledore, married Joane, daughter and co-heir of . . Twaight, and had son William and a daughter. William ('Thomas, John, John, Stephen), of Tenterden, County Kent, married first Joane Buntinge. Married second, about 1560 , Joane Pattenden, and died I 582. They had issue: William, Thomas, Dorothea, Peter, who married Susan Johnson of Canterbury, Robert, who married first an Everden, and second, Joane Mills, and had issue: George, John, and Mary, who married Michael Symons, and second, Richard Duke, brother of Sir Edward Duke, Stephen, George, and John. ${ }^{3}$ In Cole's MSS. ${ }^{4}$ is an able letter from Dr. Stukeley to Noah Curtis, lord of the manor of Woolstrop, respecting the escape of Charles I. from the Scotch army before Newark, 1646. In the same collection of MSS., ${ }^{5}$ dated Jan. 31, 1645, is an account of a fine of 100 marks for the discharge of the delinquency of Wm. Curtis, of Orvell, County Cambs. Cole states the said William Curtis "went to Oxford, and upon his return thence, being summoned, refused to come to his place of habitation. His estate is $£ 40$ per annum in lands, whereof part is copyhold, 6 cottages," etc. In another part of the MSS. he is called Wm. Curtis, of Basingbourn, County Cambs. Walter le Curteis de Wells confirmed a charter to the Abbey of West Dereham, County Norfolk, temp. Henry III. ${ }^{6}$

\footnotetext{
1 Additional Manuscripts, British Museum, 6046, f. 133 .

2 See coat of arms of Curteis, of Apledore, page vii.

${ }^{3}$ Berry's Pedigree of the Families of the County of Kent.

4 Add. MSS. Brit. Museum 5886, f. I ; 5846 , f. 372.

${ }^{5}$ Add. MSS. Brit. Museum 5819 , f. 14.

6 Add. MSS, Brit, Museum 5842, f. 2 I 7.
} 
Philip Curtis married Amy Washington, Aug. 8, I620. She was sister of John Washington, who emigrated to Virginia in 1657 . Walter Curteys, a blacksmith, I3 Ed. III. 1339, was charged with stealing in the "Ward of Chepe" several foreign articles, for which he was hanged."

Anthony Curtis and Anne Herryson were married Feb. 14, 1545-6, in St. Devine, Bockchurch, London. Willian Curtys and Eleanor Stone were married June 17, 1565 , at Allhallow, London Wall.

Oct. 16,1632 , William Searle and Elizabeth his wife surrendered land into the hand of the lord of the manor of Nazing, by the Rod, according to the custom of the said manor, by the hands of John Curtys and Richard Campe, customary tenants.

I 637, To be assigned for jury in Nazing, John Curtis, John Ruggles, John Hutchins, etc.

I638, Thomas Hutchin surrendered a tenement by the hands of Richard Campe and Samuel Curtis, two customary tenants of Nazing. 



\section{PATENT OF THE TOWN OF STRATFORD}

WhEREAs the Generall court of Conecticutt have formerly Granted to us, the proprietors, Inhabitants of Stratford all those lands both meadow \& upland with in these abutments viz upon the sea on the Sea shore South on Stratford River on the east $\&$ on Fayerfield Bownds on the west \& to run from the sea on the south the whole Bredth of twelve Miles \& from the Norwest corner to run easterly of Stratford River \& abutts on the Wilderness on the North the said Land having been by purchase or otherwise Lawfully obteyned of the Indian Native proprietors \& whereas the proprietors the afoar Sayd Inhabitants of Stratford in the colony of Conecticutt after making Application to the Governor \& company of the Sayd Colony of conecticutt assembled in court May $25^{\text {th }} 1685$ that they may have a patent for confirmation of the afoar Sayd Lands so purchassed \& Granted to them as afoar Sayd \& which they have Stood Seized \& quietly possessed of for many yeares late past without Interruption now for a more full confirmation of the afoar Sayd Tract of Land as it is Butted \& Bownded afoar Sayd unto the present proprietors of the Township of Stratford in their possession \& Injoyment of the premises Know yee that the sayd Governor \& Company assembled in Generall Court according to the commission \& By vertue of the power Granted to them by our Late Soveraigne Lord Charles the $\mathrm{II}^{\mathrm{d}}$ of blessed memory In his Letters pattents bearing date the three \& Twentyeth yeare of his Sayd Mates Reigne have given \& Granted \& by these presents doe give grant rattify \& confirme unto Capt $W^{m}$ Curtice $M^{r}$ Joseph Hawley $\mathrm{M}^{\mathrm{r}}$ Isaac Niccols $\mathrm{M}^{\mathrm{r}}$ Jercmy Judson Le ${ }^{t}$ John Beardslie cnsigne Steven Burrit Sergt John Curtice $M^{r}$ Richard Blackleach $M^{r}$ Timothy Wilcockson $M^{r}$ John Wells $M^{r}$ Samuel Sherman \& $M^{r}$ Ephraim Stiles \& all the rest of sayd present proprietors of the Township of Stratford \& their heires $\&$ assignes forever \& to each of them in Such proportion as they have all ready agreed upon for the divission of the Same all that fore Sayd Tract \& parcels of Land as it is butted \& Bownded together with all the woods uplands arrable lands meadowes pastures ponds havens portes waters Rivers $w^{\text {th }}$ all adjoyneing Islands fishings therein Huntings fowleings mines Mineralls Quarries \& precious Stones upon or within the Sayd Tract of lands with all other proffits $\&$ comodities there unto belonging or in any wise appertaineing $\mathcal{S}$ doe allso Grant unto the afoarnamed Capt ${ }^{n} W^{m}$ Curtice $M^{r}$ Joseph Hawley $M^{r}$ Isack Niccols $\mathrm{M}^{\mathrm{r}}$ Jeremy Judson Let John Beardsly Ensign Steven Burret Sergt John Curtice $M^{r}$ Richard Blackleach $M^{r}$ Timothy Wilcockson $M^{r}$ John Wells $M^{r}$ Sam ${ }^{11}$ Sherman \& $\mathrm{M}^{r}$ Ephraim Stiles \& the rest of the present proprietors Inhabitants of Stratford afoarsayd their heires \& assigns forever that the abovesayd (xxiii) 
tract of land shall be forever hereafter deemed reputed $\&$ be an Intire Township of it selfe to have and to hold the Sayd Tracts of land premises with all \& cvery there appurtenance together with the priviledges Immunities \& Franchises herein given \& granted unto the said Capt $W^{\mathrm{m}}$ Curtice $M^{\mathrm{r}}$ Joseph Hawley $M$ Isaac Niccols $M^{\mathrm{r}}$ Jeremy Judson Leut John Beardsly Ensign Steven Burret Sargt John Curtice $M^{r}$ Richard Blackleach $M^{r}$ Timothy Wilcockson $M^{r}$ John Wells $M^{\mathrm{r}}$ Samuel Sherman \& $M^{\mathrm{r}}$ Ephraim Stiles \& all others the present proprietors Inhabitants of Stratford their heires \& assignes forever \& to the only proper use $\&$ behoofe of the said Capt ${ }^{\mathrm{n}}$ William Curtice $\mathrm{M}^{\mathrm{r}}$ Joseph Hawley $\mathrm{M}^{\mathrm{r}}$ Isaac Niccols $M^{r}$ Jeremy Judson Leu John Beardsly Ensign Steven Burret Sargt John Curtice $M^{r}$ Richard Blackleach $M^{r}$ Timothy Wilcoxson $M^{r}$ John Wells $M^{r}$ Samuel Sherman \& $M^{r}$ Ephraim Stiles \& all others the present proprietors Inhabitants of Stratford their heires \& successors \& assignes forever according to the Tenor of his Maties Manor of east Greenwich In the county of Kent in the Kingdom of England in free and common sociag \& not in Capitee nor by Knight Service they yielding and payeing therefore to or Soveraigne Lord the King his heires and successors onely the fifth part of all the oare of Gold \& Silver which from time to time and all times hereafter shall be there gotten had or obteyned in leiv of all rents services dutys and demands whatsoever according to charter in Witness whereof we have caused the Seale of the colony to be here unto affixed This Fourteenth of May One Thousand Six hundred eighty \& Six in the second yeare of the Reigne of our Soveraigne Lord James the Second by the Grace of God of England Scotland France \& Ireland, King, defender of the fayth $\mathbb{E}^{\mathrm{r}}$.

Robert Treat Governour

Postscript] Note also that the Islands in said river belonging to Stratford and called by the mouth of Messrs Knells \& Brinsmeads the lower and uppor Islands and Harnd Island In all five $\mathrm{w}$. . . . . wanted priveledges of the river to have \& to hold to the said towne of Stratford to them \& their heirs forever.

Pr order of the Generall Court of Conecticutt. Signed Pr John Allyn Secretary March $30^{\text {th }} 1687 \mathrm{Pr}$ order of the Governor \& Company of the Colony of Conecticutt, Signed Pr John Allyn Secy Entered in the pub record Lib D. Folio 159.

May I $9^{\text {th }} 1686 \mathrm{Pr}^{\mathrm{J}}$ John Allyn Secrety $y^{\mathrm{r}}$ Entered in the Records of the Town of Stratford Lib $2^{\mathrm{d}}$ folio 489 Pr Joseph Curtiss Recordr ${ }^{3}$

See frontispieces. 


\title{
A LIST OF THOSE FAMILIES
}

\author{
BEARING
}

\section{THE CURTIS, CURTISS, CURTICE NAMES}

\author{
That Settled in New England Prior to i 700
}

DEODATE CURTIS ${ }^{1}$ settled in Braintree, Mass., about $1643 ; \mathrm{m}$. , and had son Solomon; m. 2d, Rebecca

and had Ruth, b. Braintree, Mass., Jan. 8, I648.

ELIZABETH CURTISS, mother of John and William Curtiss, of Stratford, Conn. See page I.

FRANCIS CURTIS settled in Plymouth, Mass., in r67 I ; m. Hannah, d. of John Snith, of Plymouth, Mass., Dec. 28, I67 I.

They had children:-

i. John, b. Plymouth, Mass., July I6, I673.

ii. Benjamin, b. Plymouth, Mass., Aug. 11, 1675; m. Mary, d. of Nehemiah Besse, of Sandwich, 1700.

iii. Francis, b. Plymouth, Mass., April , I679; m. Hannah Bosworth, of Plymouth, Mass, in 1700.

iv. Elizabeth, h. Plymouth, Mass., June 15, 1681.

v. Elisha, b. Plymouth, Mass., March , 1683; m. Amy West.

vi. Ebenezer, b. Plymouth, Mass; m. Mary, d. of Hezekiah Pinkham, of Plymouth, Mass., $1710 ;$ m. 2d, Martha, d. of John Doty, of Plymouth, Mass., $\quad 17$ I 8.

GEORGE CURTIS settled in Boston, Mass., where he was made a freeman May 13, I640. He was a servant to Mr. John Cotton. Dec. 30 , 1640 , he was granted land for two heads.

' As far as possible the compiler has adopted that spelling of the name in each instance which appears on the earliest records. 
HENRY CURTIS, b. in England, I 608. He sailed to New England in the ship "Elizabeth and Ann," May 6, 1635, and settled in Watertown, Mass., in 1636 ; m. Mary, d. of Nicholas Guy, of Watertown, Mass. In I64I he removed to Sudbury, Mass., where he died May 8, 1678. The Topsfield and Worcester families are descended from him.

They had children :-

i. Ephraim, b. Sudbury, Mass., March 31, 1642 ; he was made a freeman in Topsfield, Mass., in 1686, and was prominent in King Philip's War.

ii. John, b. Sudbury, Mass., I642 ; m. Sarah Locke, of Topsfield, Mass., Dec. 4, 1.672. He d. in 1679.

iii. Joseph, b. Sudbury, 1647 ; m. Abigail Grout. He d. Nov. 26, 1 700. She d. April 28, 1745 .

HENRY CURTIS, b. (Stratford-on-Avon or Coventry ?) England, I621. A graduate of Oxford? He settled in Windsor, Conn., in I645; m. Elizabeth Abel, of Windsor, Conn., May I3, I645; he removed to Northampton, Mass., in 1653 , where he d. Nov. 30, 1661. His widow 11. Robert Weller, of Farmington, Conn., June 22, I662. Descendants in Coventry, Conn., and Northampton, Mass.

They had children :-

i. Rowland, b. Windsor, Conn., I647; d. y.

ii. Samuel, b. Windsor, Conn., April 26, I649; m. Sarah, d. of George Alexander, of Northfield, Mass., July 23, 1678. He d. in (Coventry, Conn.)? July 28, I 721 .

iii. Nathaniel, b. Windsor, Conn., July 1 5, 1651. He was killed by the Indians at Northfield, Mass., Sept. 2, 1675.

HENRY CURTIS, of Boston, Mass. ; m. Jane. His descendants are found in Braintree, Quincy, and Stoughton, Mass.

They had children :-

John, b. Boston, Mass., July 2, 1657 .

Theophilus, b.

Jane, b.

JOHN CURTISS, b. England, 16I1. He settled in Stratford, Conn. See page 2.

JOHN CORTEIS owned fifteen acres of land in Roxbury, Mass., in $1638-40$, and had five persons in his family. No further 
record is found of him on the records of that town. He is supposed to have been the husband of Elizabeth Curtiss, of Stratford, Conn., and father of John and William.

JOHN CURTIS owned land in Wethersfield, Conn., in 1639-40, but disappears, leaving no trace. In the "Memorial History" of Hartford County it says that he left in 1639-40 for Cupheag (Stratford), Conn. He is supposed to be the same person as the above.

JOHN CURTIS, "ex-representative," was accepted into the town of Dover, N.H., in 1657 .

JOHN CURTIS settled in Scituate, Mass., in I640; probably brother of Thomas, Richard, and William, of Scituate.

RICHARD CURTIS settled in Scituate, Mass., in I640, probably with his brothers John, Thomas, and William. He removed to Marblehead, Mass., in I 648 , but returned to Scituate the following year; m. Ann, d. of John Hallet, in 1649; m. 2d, Lydia. He d. in Scituate, Mass., in 1693 . His descendants are found in Scituate and Hanover, Mass.

They had children :-
i. Ann, b. Scituate, Mass.,
ii. Elizabeth, b. Scituate, Mass.,
1649 . Scituate, Mass., in 1678 .
I651; m. Nathaniel Brooks, of
iii John, b. Scituate, Mass., Dec. I, 1653; m. Miriam, d. of Willian Brooks, of Scituate, Mass., I678.
iv. Mary, b. Scituate, Mass., I655; m. Mr. Badcocke.
v. Martha, b. Scituate, Mass., I657; m. Thomas Clark, of Scituate, Mass., in 1676 .
vi. Thomas, b. Scituate, Mass., March 18, 1659; m. Mary, d. of William Cooke, of Scituate, in 1694.
vii. Deborah, b. Scituate, Mass.,
viii. Sarah, b. Scituate, Mass., 1663.

RICHARD CURTICE, of Salem, Mass.; m. Sarah. Descendants in Southold, L.I., Hebron, and Sharon, Conn.

They had children :-

i. Caleb, b. Salem, Mass., July 24, 1646; m. Elizabeth, d. of Thomas and Abigail Terry Rider, of Southold, L.I., Dec. 1, I670. He d. Southold, L.I., March 18, 1730.

ii. Samuel, b. Salem, Mass., Feb. 1, 1651 .

(xxvii) 
iii. Richard, b. Salem, Mass., Dec. 14, 1652.

iv. Sarah, b. Salem, Mass., Jan. 19, 1654.

v. Hannah, b. Salem, Mass., July 16, 1656.

vi. John, b. Salem, Mass., Dec. 2, I658; d. y.

vii. John, b. Salem, Mass., April 4, r66o; d.y.

viii. Mary, b. Salem, Mass., Dec. I I, 1662.

RICHARD CURTIS, b. in England, I6I0. Settled in Dorchester, Mass., in I642; m. Elizabeth . She d. May 28, I657 ; m. 2d, Sarah $\quad$ Sept. 25,1657 . He was a shoemaker. In 1678 he removed to Wallingford, Conn., where he d. Sept. 17, I68 I. His descendants are found in Wallingford, New Haven, Watertown, and Farmington, Conn.

They had children :-

i. Elizabeth, b. Dorchester, Mass., July $17,1643$.

ii. Isaac, b. Dorchester, Mass., June 1 7, I658; m. Sarah, d. of Robert and Sarah Foote, of Branford, Conn., Aug. 13, 1682. He d. in Wallingford, Conn., July I 5, I 712.

iii. Joseph, b. Dorchester, Mass., Sept. 4, 166 I.

SAMUEL CURTIS, b. Sheffield, Eng. Emigrated to this country in 1690 , and settled in Windsor, Conn.; m. Lois Wentworth.

They had a child : -

Elnathan, b. Windsor, Conn., April ro, I7I2; m. Rose Weller, of Guilford, Conn., March Io, I 737. He d. in Stockbridge, Mass., Aug. 20, I 78 I. She d. June I, I 808 , aet. 94 years.

THOMAS CURTIS, b. England, 1598 ; m. Elizabeth

He settled in Wethersfield, Conn., in 1639 , where he d. Nov. I 3 , I 68I, leaving an estate of $\{717-13 \mathrm{~s}-9 \mathrm{~d}$, a large estate for that time. His descendants are found in Hartford, Farmington, and through Litchfield County, Conu.

They had children :-

i. John, b. Wethersfield, Conn., Jan. I, 1639; m. Lydia, Nov. 20, I666. He d. in Wethersfield, Conn., Nov. 8, I 7 I 2.

ii. James, b. Wethersficld, Conn., Sept. I 5, I64 I ; m. Abigail, July 8, I686. He d. in Wethersfield, Conn., Sept. 5, I 690.

iii. Joseph, b. Wethersfield, Conn., March 31, 1644; m. Mercy, Feb. 8, 1674. He d. in Wethersfield, Dec. 31, 1683.

iv Samuel, b. Wethersfield, Conn., April , $1645 ; \mathrm{m}$. Sarah Feb. 20, I683. He d. in Wethersfield, Conn., Nov. 26, I688.

v. Isaac, b. Wethersfield, Conn., 1647. Settled in Wallingford. (?)

$$
\text { (xxviii) }
$$


vi. Ruth, b. Wethersfield, Conn. Hartford, Conn.

vii. Elizabeth, b. Wethersfield, Conn. May 26, 1674.
; m. Eleazer Kimberly, of

; m. John Stoddar, jr.,

THOMAS CURTIS, settled in York, Maine, and in 1648 removed to Scituate, Mass., but in 1663 returned to York, Maine; probably brother of John, William, and Richard, of Scituate; $\mathrm{m}$.

They had children :-

i. Elizabeth, b. Scituate, Mass., I649.

ii. Samuel, b. Scituate, Mass., 1659; m. Elizabeth.

iii. Benjamin, b. York, Maine. He owned land in Portsmouth and in Newcastle, 168 r.

iv. Joseph, b.

; m. Sarah, d. of Richard Foxwell, 1678.

WILLIAM CURTIS, b. in England, r6r8. He settled in Stratford, Conn. See page 3 .

WILLIAM CURTIS settled in Scituate in 1643 ; probably brother of Richard, John, and Thomas; $m$. His descendants are found in Scituate, Hanover, and Boston, Mass.

They had children :-

i. Joseph, b. Scituate, Mass., May , I664; m. Rebecca 1692.

ii. Benjamin, b. Scituate, Mass., Jan. , 1667 ; m. Mary, d. of Capt.

Joseph Sylvester, of Scituate, Mass., I689.

iii. William, b. Scituate, Mass., Jan. , 1669.

iv. John, b. Scituate, Mass., I674; m. Experience, d. of John Palmer, of Scituate, Mass., March 4, 1708.

v. Miriam, b. Scituate, Mass., April , 1673.

vi. Mehitabel, b. Scituate, Mass., Dec. , 1675.

vii. Stephen, b. Scituate, Mass., Sept. , 1677.

viii. Sarah, b. Scituate, Mass., Aug. , 1679; m. William Cook, Aug. 30,1705 .

ix. Samuel, b. Scituate, Mass., June , I68 I; m. Anne Barstow, Sept. 11,1707 .

WILLIAM CURTIS, of Salem, Mass., m. Alice

They had children :-

i. Ann, b. Salem, Mass., Aug. 30, 1658.

ii. Sarah, b. Salem, Mass., Oct. 13, 1660. She d. Aug. 25, 1860.

iii. William, b. Salem, Mass., Oct. 26, 1662.

iv. Abigail, b. Salem, Mass., Aug. 15, 1664 .

(xxix) 
v. John, b. Salem, Mass., May I4, I666.

vi. Elizabeth, b. Salem, Mass., Jan. , 1668.

vii. Hannah, b. Salem, Mass., Aug. , 1670.

WILLIAM CURTISS (so spelt on the Roxbury records), b. Nazing, England, Nov. 13, 1592; m. Sarah, d. of Bennett Eliot, of Nazing, England, Aug. 6, I6I8. He sailed for this country in the ship "Lyon" on June 22, I632, and landed at Boston, Mass., on Sunday, Sept. I6, I632. He settled in Roxbury, Mass., where he was made a freeman, March 4, I633. He d. in Roxbury, Mass., Dec. 8, 1672. She d. in Roxbury, Mass., March 26, I672. He was probably the brother of John Curtiss, of Wethersfield, who was supposed to have been the husband of widow Elizabeth Curtiss, of Stratford, Conn. His descendants are found in Boston and Sharon, Mass.

They had children :-

i. William, b. Nazing, England, I6I8; he came to New England in 1631 with Rev. John Eliot, and settled in Roxbury. "William was a hopeful scholar, but God took him in the end of the year 1634." - Roxbury Church Records.

ii. Mary, bapt. Nazing, England, March I I, I620-I.

iii. Thomas, bapt. Nazing, England, Jan. I9, 1622-3. He d. probably June 26, 1650.

iv. Elizabeth, bapt. Nazing, England, Feb. I 3, I624-5; m. Isaac Newhall, of Roxbury, Mass., Dec. I4, I659.

v. John, bapt. Nazing, England, July 17, 1629; m. Rebecca, d. of Thomas Wheeler, of Boston, Mass., Dec. 26, I66I; m. 2d, Dorcas, d. of Charles and Dorcas Peak, of Roxbury, Mass. He d. 1654 .

vi. Philip, bapt. Nazing, England, March 28, 1632 ; m. Obedience, d. of John Holland, of Dorchester, Mass., Oct. 20, 1658 . He was killed by the Indians in King Philip's War, at Grafton, Mass., Nov. 9, 1675 .

vii. Sarah, bapt. Nazing, England, Aug. 5, 1627.

viii. Hannah, b. Roxbury, Mass.; m. William Geary, Aug. 25, I651.

ix. Isaac, b. Roxbury, Mass., July 22, I64I-2; m. Hannah, d. of John Puse. Palley, of Roxbury, Mass., May I0, I670. He d. in Roxbury, Mass., May 31, I695. She d. Feb. 6, I 720.

ZACHEUS CURTIS, of Salem, Mass. He came in the ship "James," from Southampton, Eng., in I653, and his native town was Downton, in Wiltonshire, Eng. 
Hatton in his list of persons who sailed from Great Britain to the American Plantations, I600-1700, gives the following:-

ELIZABETH CURTIS, aged 22 years, sailed to Barbadoes in the ship "Faulcon," from London, April xiij-1 635 .

HENRY CURTIS, aged 27 years, sailed in the ship "Elizabeth," May 6, 1635 .

JAMES CURTIS, aged 18 years, to St. Christopher in the ship "Amatie," Oct. 13, 1635 .

JOHN CURTIS, aged $2 \mathrm{I}$ years, sailed to Virginia in the ship "Safety," Aug. 10, 1635 .

JOHN CURTIS, aged 22 years, sailed in the "Flying Hart," I62I, and appears on the muster rolls of Elizabeth Cittie, Virginia.

JOHN CURTIS was living in Virginia, Feb. I6, 1623.

THOMAS CURTIS, aged 24 years, a servant, came in the "Flying Harte," in I621, and settled at Newport Newes, Virginia.

THOMAS CURTIS was living in Virginia in Feb. $16,1623$.

WILLIAM CURTIS, aged 19 years, sailed to Virginia in the ship "Georges," Aug. 2, 1635 .

WILLIAM CURTIS sailed from London, Eng., to New England, June 22,1632 . 

ELIZABETH CURTISS, or as she is generally called on the Stratford, Conn., Town Records, "Widow Curtiss," first appears on these records, in a list of property owners (about I650), with her two sons, John and William Curtiss. Her lot was near or adjoining that of Rev. Adams Blakeman. She died June, I6 58 , and her will was proved Nov. 4, 1658, at Fairfield, Conn., and reads as follows:-

Fairfield Probate Records, Vol. I648-56, Page I 7 .

The Last Will and Testament of Elizabeth Curtiss, ... being weak yeat perfect in her minde as is . .

Inp ${ }^{\mathrm{r}}$ is Unto her two Sonns John Curtiss and William doe give my Mare and Coalt. ...

It. Unto John Curtiss \& Jonathan Curtiss $y^{\oplus}$ Sonnes of my Sonnes John \& William my Grandchildren I doe give the coalt that my young Mare hath: And after the sayd young mare hath up her first coalt, Then I doe give $y^{\theta}$ sayd young mare to the Rest of my Grandchildren, Sonns and Daughters of my aforefd Sonns John \& William :

It. I doe give one houfe and Lott to my Grandchildren, John Curtiss \& Ifrael \& Jonathan \& Sarah Curtiss $y^{e}$ children of $y^{e}$ said John \& William: And they to enjoy it when they come to $y^{\theta}$ age of twenty:

It. I doe give unto my Grandchilde Mary Curtiss $y^{e}$ daughter of Thomas Curtiss forty shillings \& to bee paid unto her by my sonns John \& William Wthin a yeare after my defesase :

It. I doe give unto my Sonns John \& William Curtiss my two cowss, one hiefer, one bullock and a calf after my decsas: provided $y^{t}$ if my say ${ }^{\mathrm{d}}$ young mare before given to my Grandchildren should miscarry. Then my Will is if my two sonns John and William Curtiss should give foure pounds each of them to there Children $y^{t}$ lave no share in my house and Lott to be paid att $y^{e}$ Age of twentie:-

It. I doe give my Bible to John Curtiss my Grandchilde the Sonn of John Curtiss allso my desire is $y^{t}$ there be so much of my Corn sould as may buy a bible for Jonathan Curtiss $y^{\circ}$ Sonn of my Sonn William and given to him :-

The Rest of my goods IVthin $y^{e}$ house that are moveable goods I doe give to me Sonns John \& William Curtiss to be equally divided betwixt then,

My two Sonns John Curtiss \& William Curtiss I doe make executors : my will is $y^{t}$ John Burdseye Henry Wakelyn and Joseph hawly shall bee overseers of this my Last Will and Testament.

In ye prsence of

$$
\left.\begin{array}{ll}
\text { John } & \text { Brimsmaid } \\
\text { John } & \text { Washborn. }
\end{array}\right\}
$$

Elizabeth Curtiss.

This will is proved by John Curtiss and William Curtiss to bee $y^{e}$ Last Will and Teftant of Elizabeth Curtiss of Stratford: And $y^{\ominus}$ Court Approvs of it : it being attested to : $4^{\text {th }}: 4^{\text {th }}:\left(5^{8}\right)$ 
She had children : -

(2) i. John, b. in England, I6II ; m. Elizabeth Welles, and d. in Stratford, Conn., Dec. 6, 1707 .

(3) ii. William, b. in England, 1618; m. (i.) Mary, m. (ii.) Sarah, d. of Matthew Morris, of Hartford, Conn., and widow of William Goodrich, of Wethersfield, Conn., about I680. She d. I 702. He died in Stratford, Conn., Dec. 2 I, I 702.

iii. Thomas, b. in England. His name does not appear on the records of Stratford, Conn., and no further record has been found of him except that he had a daughter Mary, mentioned in his mother's will.

\section{2}

JOHN CURTISS (Elizabeth), b. in England in r6ir. His name appears among the original patentees of Stratford, Conn. (see frontispiece), and also on the list of property owners of 1650 . He married Elizabeth Welles (supposed to have been the sister of Gov. Thomas Welles). He was made a freeman in May, 1658. He was a prominent citizen, and took an active part in the work of settling the town, but was not as much of a public man as his brother William. In 1678 he and his brother William were on a committee to build a new meetinghouse. Sergeant John Curtiss was elected Town Treasurer, Dec. 29, I675. He served as a soldier in King Philip's War, and attained the rank of Ensign (second lieutenant). He died in Stratford, Comn., Dec. 6, 1707, æt. 96 years. She died in Stratford, Conn., March 9, I68 I-2.

They had children :-

i. John, b. in Stratford, Conn., Oct. 14, 1642; m. Hannah, widow of Abraham Kimberly, of Stratford, Conn. In 1667 he became a proprictor of Newark, N. J., and held a prominent position, not only in that town, but also in the county, until his death, in 1704 . He represented Newark at the General Court from 1683-8. A commissioner to lay out highways and assessor of Essex County, $1683_{3}-8$, Justice of the Court, etc. He also appears on the Newark records with the rank of Captain. They had no children, as far as the records of Newark show. He d. in Newark, N. J., Sept. 17, 1704 .

(4) ii. Israel, b. Stratford, Conn., April 3, 1644; m. Rebecca, He died in Woodbury, Conn., Oct. 28, 1704.

iii. Elizabeth, b. Stratford, Conn., May 2, 1647.

(5) iv. Thomas, b. Stratford, Conn., Jan. I4, I648; m. Mary, d. of Nathaniel and Abigail Merriman, of Wallingford, Conn., June 9, 1674. He d. about 1736 , in Wallingford, Conn.

(6) v. Joseph, b. Stratford, Conn., Nov. 12, 1650; m. Bethia, d. of Richard and Elizabeth Booth, of Stratford, Conn., Nov. 9, 1676. He d. 1742. 
(7) vi. Benjamin, b. Stratford, Conn., Sept. 30, 1652 ; m. (i.) Esther, d. of Joseph and Sarah Judson, of Stratford, Conn., March 23, I680-1. She d. Aug. 27, 17i3. He m. (ii.) Bathsheba, d. of Henry Tomlinson, of Derby, Conn., and widow of Ephraim Stiles, Dec. 1, 17I4. She d. I735. He d. 1733, in Stratford, Conn.

vii. Hannah, b. Stratford, Conn., Feb. 2, I654-5; m. Benjamin Lewis, of Stratford, Conn., about $167 \mathrm{I}$, and settled in Wallingford, Conn. She d. in 1728 .

WILLIAM CURTISS (Elizabeth), b. England, I6I 8 ; m. (i.) Mary, m. (ii.) Sarah, d. of Matthew Morris, of Hartford, Conn., and widow of William Goodrich, of Wethersfield, Conn., about I680. He came to Stratford, Conn., with his mother and brother John, and his name appears among the list of property owners of 1650 . He was an original proprietor, of Stratford, Conn. (see frontispiece), and was a most prominent man. As early as $165^{\circ}$ he had attained the rank of sergeant, and represented Stratford at the General Court, almost continuously from 1667 to I686. May 9, I672, liberty was granted by the General Court to Mr. Samuel Sherman, Lt. William Curtiss, Joseph Judson, and John Minor, themselves and their associates, to erect a plantation at Pomperaug (Woodbury). In June, I672, he was appointed captain of the "Train Band" of Stratford, and at the same time the General Court declared that " until further order be taken, Capt. Nathan Gold shall be deemed Chief Military Officer of Fairfield County, and Capt. William Curtiss his second." In August, I672, Capt. William Curtiss was appointed by the General Court one of six commissioners, with the Governor, Deputy Governor, and Assistants as a War Council against the Dutch at New York, "to act as the Grand Committee of the Colony in establishing and commissionating Military Officers, in pressing men, horses, ships, barques or other vessels, arms, ammunition, provisions, carriages, or whatever they judge needful for our defense, and to manage, order and dispose of the Militia of the Colony in the best way and manner they can for our defense and safety." He distinguished himself for bravery in King Philip's War. October, 1675, upon the report of the Indians being ready at Narragansett to attack Connecticut, Capt. William Curtiss was appointed to command those forces raised in Fairfield County, with power to appoint his inferior officers. In 1676 he was a commissioner of both Stratford and Woodbury. In May, 1676, he was chosen an Assistant, or member of the Governor's Council. He d. in Stratford, Conn., Dec. 21, I702. His wife d. I70r. 
His will was entered for Probate Dec. 31, 1702, at Fairfield, Conn., and reads as follows:-

Fairfield Probate Records, Vol. 1702-1750, Page 9:-

\section{Wili of William Curtiss.}

In the name of God, Amen, I William Curtiss of Stratford in the County of Fairfield in $y^{\theta}$ Colony of Connecticut being aged \& weak of body yet through $y^{\ominus}$ goodness of God of perfect memory \& understanding do make this my Last Will \& Testament as followeth Imprs I Commit my Soule to God hoping for Salvation in \& through $y^{\ominus}$ righteousness \& Merits of Jesus Christ my Only Saviour \& Redeemer \& my body after this Life to be decently buried at $y^{*}$ discretion of my surviving Relations. And having disposed of all my Children in Marriage \& gave them such portions as I thought fitt according to my ability \& having Now but Little to dispose of ny Will is that after Funerall Expences \& Just Debts being paid my Estate be disposed of as followeth :

I Give and bequeath to my Loving Son Daniell Curtiss my Comman Meadow Lyeing In the great Neck \& my meadow in $y^{\ominus}$ Salt Pond in said Neck \& one third part of all my Land Lyeing in Paquanock field \& four acres in the New Pasture and a fourth part of all my wood land laid out and to be laid out.

It. I give \& bequeath to my Loveing Son Ebenezer Curtiss three acres of Meadow Lying at Nesumpaws being the equall half of my Lot out there and one third part of all my Land Lyeing In Paquanock field \& hallf my farme in the woods on Turky hill \& one fourth part of all my Wood Land Layd out or to be laid out.

It. I give and freely bequeath to my Loveing Son Zachariah Curtiss one acre of Meadow at Nesumpaws and one acre of Land at $y^{\mathbf{e}}$ North Ende of the Towne next adjoining to his owne land \& one third part of all my Land lyeing in Paquanocke field \& half my farme at Turkey Hill \& one forth part of all my Wood land laid out and to be laid out.

It. I give \& freely bequeath to my Loveing Sonn Josiah Curtiss one acre of Land at the North End of the Towne \& four acres of land Lyeing next to Samuell French his land \& three acres of Land In the Intack \& three acres of Land near harveys Farme in the Ox pasture \& two acres \& half of Land in the farfield \& two acres of Land of the two miles division near Nesumpaws Creek and one quarter part of all my wood land laid out $\&$ to be laid out.

It. I give to my Daughter, Sarah Wells five pounds; and to my daughter Elizabeth Rose five pounds and to my Son Joshua Curtiss five pounds; to be paid by my Executors hereafter named: I Also give to Jonathan Curtiss the Son of Jonathan Curtiss the summ of twenty shillings, all the Rest of my Estate within doors and without Goods \& Chattells I freely Give \& bequeath to my four sons Daniell, Ebenezar, Zachariah \& Josiah to be Equally divided amongst them: and I do hereby ordaine and make my said Sons Daniell Curtiss, Ebenezar Curtiss, Zachariah Curtiss and Josiah Curtiss sole Executors of this my Last will \& testanent allways during Life Reserving for my Comfort to dispose of any of my owne Estate that this is my Last Will \& Testament Revoking all other wills this fifteenth day of December in $y^{e}$ year of $o^{r}$ Lord God 1702.

William Curtiss. 
Signed and declared by the said William Curtiss to be his Last Will and testament in presents of us

Joseph CURtiss JOHN BEACH

Mr. Joseph Curtiss \& Mr. John Beach appeared ate $y^{e}$ Prorogative Court held in Fairfield on the 3 Ist of Decemb 1702 and made oth that they saw Capt. William Curtiss signe $y^{e}$ above Instrument and heard him declare it to be his Last Will and testament and that they Judged him to be Competent.

Captaine IVilliam Curtiss of Stratford being deceased his Last Will \& Testament being Exhibited to $y^{\mathrm{e}}$ Prorogative Court Siting in Fairfield December 3 Ist $1702 \mathrm{v}^{\mathrm{e}} \mathrm{s}^{\mathrm{d}}$ WVill being proved is by the Court approved and is ordered to be Recorded.

Nathan Gold, Clerk.

They had children : -

i. Sarah, b. Stratford, Conn., Oct. Io, I642; m.

Wells.

(8) ii. Jonathan, b. Stratford, Conn., Feb. 14,1644 ; m. Abigail, d. of John and Mirabel Thompson, of Stratford, Conn., I669, and d. I68I.

(9) iii. Joshua, b. Stratford, Conn., Oct. J, 1646; m. and died I706, in Woodbury.

iv. Abigail, b. Stratford, Conn., April 21, 1650.

(Io) v. Daniel, b. Stratford, Conn., Nov. 16,1652 ; m. Mary, and d. Feb. 6, 17 I8-19.

vi. Elizabeth, b. Stratford, Conn., Sept. 13, 1654; m. Rose,

(II) vii. Ebenezer, b. Stratford, Conn., July 6, I657; m. Ruth, d. of Nathaniel and Elizabeth Porter, of Stratford, Conn. She d. May 20, 1739, aet. 70 years. He d. in Stratford, Conn., in $175 \mathrm{~J}$.

(I2) viii. Zachariah, b. Stratford, Conn., Nov. 14, 1659; m. Hannah, d. of Nathaniel and Elizabeth Porter, of Stratford, Conn. She d. in 1738 , aet. 73 years. He d. in Stratford, Conn., June I 2, 1748. (13) ix. Josiah, b. Stratford, Conn., Aug. 30, 1662; m. (i.) Abigail, d. of Lt. Joseph and Sarah Judson, of Stratford, Comn., July, 1692. She d. r697. He m. (ii.) Mary, d. of Benjamin and Mary Beach, of Stratford, Conn., 1698. He d. in Stratford, Conn., I 745.

ISRAEL CURTISS ( $(7 o h n$, Elizabeth), b. Stratford, Conn., April 3,1644 ; m. Rebecca,

He, with his cousin Joshua, son of William Curtiss, was an original proprietor, of Woodbury, Conn., in 1672 . He attained the rank of lieutenant in the "Train Band" of Woodbury, and in 1689 was chosen a deputy to the General Court. He was also a commissioner of Woodbury. He d. in Woodbury, Conn., Oct. 28, 1704. The settlement of his estate was filed in Fairfield, Conn., Nov. 10, I70.4. 
They had children :-

i. Israel, b. Woodbury, Conn., March 18, 1666-7; d. y.

(14) ii. Israel, b. Woodbury, Conn., May I2, I668; m. Mary Mallory, of Woodbury, and d. in Danbury, Conn.

(15) iii. John, b. Woodbury, Conn., Oct. 7, I670; m. Johannah, She d. Sept. I, 1 749. He d. April 14, 1754.

(I6) iv. Stephen, b. Woodbury, Conn., Aug. 24, 1673; m. Sarah Miner, of IVoodbury, Conn., Nov. 2, I699. He d. in Woodbury, Conn., June I, 1723 .

v. Peter, b. Woodbury, Conn.; d. y.

vi. Hannah, b. Woodbury, Conn.

; m. Thomas Miner.

vii. Rebecca, b. Woodbury, Conn., November, 1677; m. Ephraim Minor, Aug. 21, 1701, in Woodbury, Conn.

viii. Ruth, bapt. Woodbury, Conn., I680-I; d. y.

ix. Ruth, bapt. Woodbury, Conn., Sept., I686-7; d. y.

x. Josiah, bapt. Woodbury, Conn., Nov., 1682-3.

THOMAS CURTISS (Fohn, Elizabeth), b. Stratford, Conn., Jan. 14, 1648; m. Mary, d. of Capt. Nathaniel and Abigail Merriman, of Wallingford, Conn., June 9, 1674 . In October, 1670, he was made a freeman. He attained the rank of captain of the "Train Band" of Wallingford, Conn. He was an original proprietor in Wallingford, Conn., in October, 1669, and a signer of the covenant or original agreement. He was a surveyor. His name appears as acting in a church meeting in 1670 , the earliest record of the town. He was deputy to the Colonial Legislature in 1689,1714 , and 1717 , and was a very prominent man in town affairs until 1720 . He was elected Constable, Dec. 17, 1681; Town Treasurer in 1686. His will was filed May 5, 1736, in the Probate Records of New Haven, Conn., Vol. 6, page 184 .

\section{Will of Thomas Curtiss.}

In the Name of God, Amen, this Ninth Day of Augt in $y^{\mathrm{e}}$ year of our Lord A. D. one Thousand Seven hundred \& thirty-three, I Thomas Curtiss Sen? of Wallingford in $y^{\theta}$ County of New Haven and Collony of Connecticut in New England aged about 85 years yet of perfect mind and memory, thanks be to God and Calling to mind $y^{\mathrm{e}}$ Mortallity of the Body, do make \& ordain this my Last Will \& Testament that to Say principally \& first of all \& Recommend my Soul into $y^{\mathbf{e}}$ hands of God $y^{t}$ gave it in hopes of his mercy through Jesus Christ and my Body I Commit to $y^{\mathbf{e}}$ Earth to be decently buried at $y^{\mathrm{e}}$ Discretion of my Executors hereafter named in hopes of a Glorious Resurrection and as Touching Worldly Estate wherewith it has pleased God to Bless me in this Life after my just Debts \& Funerall expenses are paid, I Give, Divise \& Dispose of $y^{e}$ Same in $y^{e}$ Manor \& form following (viz)

To my beloved Wife Mary, I Give \& Bequeath one half of $y^{\ominus}$ House \& Home Lott and Six acres of Land in the Plain near Capt. Yales and $y^{e} \mathrm{Im}-$ provement of all my meadow on $y^{e}$ West Side of $y^{e}$ River during her Nattural 
Life, my Mare, a cow \& heifer and all my Movables in $y^{e}$ house, these Movables Estate I Give unto my Wife to use \& Improve \& finally to dispose of at her pleasure.

Item. I Give to my Son, Nathaniel Curtiss my Chestnut Ground Lott, four acres near Jeremiah Hulls Home Lott joining to Land of John Ives also four acres at $y^{\bullet}$ Plain field each piece as thev are butted \& bounded upon Record.

Item. To my Son Samuel Curtiss I Give \& Bequeath $y^{\mathbb{e}}$ one half of my fourth Division and each end of $s_{-}^{\mathrm{d}}$ Lott.

Item. To my Son Thomas Curtiss I Give \& Bequeath $y^{\ominus}$ other half of my fourth Division which is at West end of $s^{\mathrm{d}}$ Lott.

Item. To my Son Joseph Curtiss I Give \& Bequeath three acres of Land upon $y^{e}$ Clear Plain near Capt Yales House at his Mothers Disease.

Item. To my Son John Curtiss I Give \& Bequeath $y^{e}$ other half of my Home Lott at our Disease with $y^{\ominus}$ Buildings \& orchard upon it also $y^{e} \mathrm{Re}-$ mainder of my Plain Lott near Capt. Yales at his Mothers Disease

Item. To my Daughters, namely Mary ${ }^{\ominus}$ Wife of John Crain, Elizabeth the Wife of Nathaniel Hall, Sarah the Wife of Joseph Parker to my Grandchildren borne of $y^{e}$ Body of my Daughter Abigail Dead, Late Wife to Joseph Holt and Jemima, Wife of Nathan Beech and Rebeckah $y^{\ominus}$ Wife of William Munson to those above mentioned I Give \& Bequeath four acres of Meadow \& Plain Land on $y^{e}$ West Side of $y^{e}$ River at a place Call ${ }^{d} y^{\ominus}$ horse pasture also my Sixth Division near $y^{\ominus}$ West Rocks as they are Butted \& Bounded upon Record all this I Give \& Bequeath unto my Daughters \& Grandchildren above named to be Divided to each of them in proportion with what I have already given them to make them equal Shares of my estate.

Finally I make Conscitute \& ordain my Son Nathaniel Curtiss and my Son in Law Joseph Parker Execut ${ }^{\text {rg }}$ of this my Last IVill \& Testament hereby revoking \& Disannulling \& making void all other Wills \& Testaments Bequeaths \& Executors by me made. Establishing \& Confirming this \& no other to be my Last Will \& Testament in Wittness I have hereunto Sett my hand \& Seal signed sealed Published \& Declared by this I Thomas Curtis to be his Last Will \& Testament in wittnes signed

JOHN PECK

Thomas Curtis (seal)

Daniel Hall $\}$ Wittnesess

Benjamin Hall $\}$ Wallingford May $y^{e} 4$ th, day $173^{6}$.

John Peck, Daniel \& Benjamin Hall all of Wallingford personally appeared and made Solomı Oath that they saw Capt Thomas Curtiss Late of Wallingford Deas dign, Seal Publish \& declare this Instrument to be his Last Will \& Testament and $y^{t}$ they signed in $y^{e}$ presence of $y^{\theta}$ Testat ${ }^{r}$ as wittnesess and $y^{\theta}$ Tastat ${ }^{r}$ was of sound mind \& memory at $y^{\theta}$ time thereof.

Certified

\section{Theophilus Yale}

\section{They had children :-}

Justice of Peace.

i. Mary, b. Wallingford, Conn., Oct. 13, r675; m. John Crain.

(I7) ii. Nathaniel, b. Wallingford, Conn., May 14, 1677; d. March 4, I 763. m. (i.) Sarah Hall, of Wallingford, Conn., A pril 6, 1697; she d. Dec. 13, 1700 . m. (ii.) Sarah How, of Wallingford, Conn., July 9, 1702 ; she d. Jan. 4, 1740. m. (iii.) Mrs. Phebe Palmer, of Wallingford, Conn., Oct. I1, I74I ; she d. Jan. 5,1763 . 
$(I S)$ iii. Samuel, h. Wallingford, Conn., Feb. 3, I678; m. Elizabeth Fredericks, Jan. 3, 1704-5.

iv. Elizabeth, b. Wallingford, Conn., Sept. II, I680; m. Nathaniel Hall, of Wallingford, Conn., May II, I699.

v. Hamnah, b. Wallingford, Conn., Dec. 3, I682; d. Oct. 12, I 703.

(I9) vi. Thomas, b. Wallingford, Conn., Aug. 26, 1685; m. Mary,

vii. Sarah, b. Wallingford, Conn., Oct. I, I687; m. Joseph Parker, of Wallingford, Conn., June 17, 1705 .

viii. Abigail, b. Wallingford, Conn., Nov. 3, 1689; m. Joseph Holt, of Wallingford, Conn., June 8, I 709. She d. Jan. 1 2, 1730.

(20) ix. Joseph, b. Wallingford, Conn., Oct. I, I691 ; m. Martha Collins, March 14, I7 I2. He d. January, 1756.

x. Jemina, b. Wallingford, Conn., Jan. I5, 1694; m. Nathaniel Beech, of Wallingford, Conn., Sept. 29, 1713 .

xi. Rebecca, b. Wallingford, Conn., Aug. 21, 1697 ; m. (i.) Lambert Johnson, of Wallingford, Conn., March I, 1716; m. (ii.) William Munson, of Wallingford, Conn.

(2I) xii. John, b. Wallingford, Conn., Sept. 18, I699; m. Jemima, d. of William Abernathy, of Wallingford, June 17,1723 . He d. April 4, 1775 .

\section{6}

JOSEPH CURTISS (Fohn, Elizabeth), b. Stratford, Conn., Nov. 12, 1650; m. Bethiah, d. of Richard and Elizabeth Hawley Booth, of Stratford, Conn., Nov. 9, 1676 . He was one of the most prominent citizens of Stratford for many years. He held the position of town clerk for fifty successive years, 1678-1 728 . In all the records during his service he always spelled the name "Curtiss." In 1698 he was elected an Assistant or member of the Governor's Council, and served as such for twenty-three years. He was several years Judge of the County Court, and was appointed on several State committees of importance. In October, I 709, Capt. Joseph Curtiss, with the Deputy Governor and three others, was appointed as a Committee of War for the County of Fairfield. In I710, with Hon. Nathan Gold and Peter Burr, he was appointed, with a committee from the Colony of New York, to locate the boundary line between that Colony and Connecticut as settled by the authorities in 1700 . He was lieutenant in the town band, and was a representative from Stratford for a number of years. He was known as "the Worshipful Joseph Curtiss."

They had children : -

i. Elizabeth, b. Stratford, Conn., Jan. I 7, s677; m. Samuel Uffoot, of Stratford, Conn., Dec. 5, 1694 .

ii. Anna, b. Stratford, Conn., Sept 1, 1679.

iii. Mary, b. Stratford, Conn., I68I ; m. Joseph Nichols, Dec. 26, 1704. 
(22) iv. Ephraim, b. Stratford, Conn., Dec. 31, r684; m. Elizabeth, d. of Ephraim Stiles, of Stratford, Conn., June 26, 1707. She d. Oct. 5, 1775. He d. May 9, 1776.

(23) v. Joseph, b. Stratford, Conn., Nov. r, I687; m. Elizabeth, d. of John Welles, Jr., of Stratford, Conn., July I 5, I 7 I I.

(24) vi. Nathan, b. Stratford, Conn., Feb. 21, I689-90; m. Eunice, d. of Jeremiah Judson, Jr., of Stratford, Conn., June 23, 1713 .

vii. Josiah, b. Stratford, Conn., March 31, I69 I; d. April 26, I 709.

viii. Bethia, b. Stratford, Conn., May $17,1695-6$; m. Benjamin Burton, of Stratford, Conn., Dec. 7, 1714.

ix. (Eliazur, b. Stratford, Conn., July 30, 1699 ; d. Oct. 21, 1699 .

x. Ebenezer, b. Stratford, Conn., Aug. I, I699; d. 1699.

(25) xi. $\left.\begin{array}{c}\text { Eliphalet, b. Stratford, Conn., Aug. 1, I699; m. Hannah, } \\ \text { d. of John Burroughs, of Stratford, Conn., Dec. 7, 1720. }\end{array}\right\}$

Triplets.

BENJAMIN CURTISS (Fohn, Elizabeth), b. Stratford, Conn., Sept. 30, 1652; m. Esther, d. of Joseph and Sarah Judson, of Stratford, Conn., March 23, 1680-I. She d. Aug. 27, I713. m. (ii.) Bathsheba, widow of Ephraim Stiles, of Stratford, Conn., and d. of Henry Tomlinson, of Derby, Conn., Dec. I, 1714. She d. in 1735, aet. 74 years. He was an original settler in Huntington. He was a prominent man in the society of the place, and held many positions of trust. He was a representative from Stratford for many years. In May, I 7 I 4, he was appointed "Naval Officer" of the port of Fairfield. The inventory of his estate was filed at Fairfield, Conn., Oct. 4, 1733 .

They had children :-

(26) i. John, b. Stratford, Conn., Feb. 18, 1680; m. (i.) Margaret, d. of David Pickett, of Stratford, Conn., Feb. 19, I 706-7; she d. Oct. 8, I 74. m. (ii.) Hanuah, d. of Col. Ebenezer Johnson, of Derby, Conn., Nov. 3, 1715 .

(27) ii. David, b. Stratford, Conn., $\quad 1682$; m. (i.) Dinah Weakley, Nov. 26, 1719; she d. June, 1725. m. (ii.) Esther, d. of John Hurd, 2nd, of Stratford, Nov. 10, 1725; m. (iii.) Mehitabel, d. of Richard Hubbell, of Stratford, Conn., about I 735.

iii. Ruth, b. Stratford, Conn., Jan. I I, I683; m. John Thompson, of Stratford, Nov. 15, 1705. She d. April 23, 1721.

iv. Hester, b. Stratford, Conn., 1691; d. Aug. 26, I701.

(28) v. James, b. Stratford, Conn., May 14, 1686; nı. Hannah, d. of John Coe, of Stratford, Conn. He d. in Durham, Conn., 1766.

vi. Hester, bapt.
ford, Conn., Dec. 3, 1724 . 
JONATHAN CURTISS (William, Elizabeth), b. Feb. 14, 1644, at Stratford, Conn.; m. Abigail, d. of John and Mirabel Thompson, 1669. He was made a freeman October, 1670. He was lieutenant of the "Train Band" of Stratford. Inventory of his estate was filed at Fairfield, Conn., Oct. I0, I681. His widow married Nicholas Huse, of Stratford, Conn., in 1692. He died and she married Samuel Sherman, Jr., of Stratford, Conn., Aug. I, 1695, and d. March 2, 173 I.

They had childrer: -

i. Abigail, b. Stratford, Conn., Oct. 17, r671; m. Ebenezer Blakeman, of Stratford, Conn., Nov. 3, 1692 .

ii. Sarah, b. Stratford, Conn., Sept. 12, 1673; m. Francis Griffin, of Stratford, Conn., Nov. 25, 1697. She d. 1707.

iii. William, b. Stratford, Conn., 1675 .

(29) iv. Jonathan, b. Stratford, Conn., June 28, 1679 ; m. (i.) Hannah, m. (ii.) Mary Summers, Dec. 24, 1718.

JOSHUA CURTISS (William, Elizabeth), b. Stratford, Conn., Oct. I, 1646. He, with his cousin, Israel Curtiss, was one of the original proprietors of Woodbury in $1672 ; \mathrm{m}$.

$\mathrm{He}$ d. at Cohanzy, 1706. Administration was granted on his estate at Fairfield, Conn., May 22, 1706.

They had children :-

i. William, b. Woodbury, Conn. m. Rebecca Blakeman, Dec. 1, 1708 . ii. Mary, bapt. "“ "Sept., I687; m. John Marcy, of Salem, N. J. iii. Anna, bapt. " " " 6880 ; m. Thomas Rowell, of Salem, N. J. iv. Bethia, bapt. " " February, $1685-6 ; \mathrm{m}$. Dayton.

10

DANIEL CURTISS (William, Elizabeth), b. Stratford, Conn., Nov. 16,$1652 ;$ m. Mary,

He d. Feb. 6, 1718. Inventory of his estate was filed in Fairfield, Conn., Feb. 19, 1718-19.

They had children :-

(3o) i. Daniel, b. Stratford, Conn., 1695; m. Mary, d. of Joseph Curtiss, May I $1, \mathbf{1} 73^{6}$. He d. October, 1763 . She d. Octoher, 1782 , aet. 68 years.

(3I) ii. Hezekiah, b. Stratford, Conn., r711; m. Mehitabel, She d. I 790, act. 77 years.

iii. A bel, b. Stratford, Conn. He d. 1730 .

iv. Elizabeth, b. Stratford, Conn.; m. Elisha Blagg, of Stratford, Conn., Dec. $16,1723$. 
v. Esther, b. Stratford, Conn.

vi. Abigail, b. Stratford, Conn.

vii. Rebecca, b. Stratford, Conn.

EBENEZER CURTISS (William, Elizabeth), b. Stratford, Conn., July 6, I657; m. Ruth, d. of Nathaniel Porter. She d. May 20 , 1739 , aet. 70 years. He represented Stratford at the General Court in May, 1724. January 26, 1702-3, the town of Stratford granted liberty to Ebenezer Curtiss, James Lewis and Edmund Lewis to erect a sawmill near Misha hill. His will was filed at Fairfield, Conn., Oct. I, I75I.

They had children : -

(32) i. Ebenezer, b. Stratford, Conn. I $73^{\circ} ; \mathrm{m}$. (ii.) Mary,

m. (i.) Abigail Clarke, Nov. 25, $\mathrm{He}$ d. in 1749 .

ii. Hannah, b. Stratford, Conn., April 30, I691; d. y.

iii. Samuel, b. Stratford, Conn., Sept. 27, I693; d. March 18, I715-16.

iv. Ruth, b. Stratford, Conn., Jan. 7, r695-6; d. y.

v. Deborah, b. Stratford, Conn., Sept. 28, I699; m. Nathan Bennet, Feb. 25, 1724. She d. 1785 .

vi. Sarah, b. Stratford, Conn., April 7, r702; m. Theophilus Nichols, of Stratford, Conn., January 27, $1723-4$. She d. Sept. 26, I 769.

ZACHARIAH CURTISS (William, Elizabeth), b. Stratford, Conn., Nov. I4, I659; m. Hannah, d. of Nathaniel Porter. She d. Feb. I 4, I 738 , aet. 73 years. He d. June I 2,1748 , aet. 89 years.

They had children : -

(33) i. Nathaniel, b. Stratford, Conn.,

m. Hannah, d. of

Samuel Welles, of Hartford, Conn., Nov. 27, I 7 I 2. He d. I 760.

ii. Hannah, b. Stratford, Conn.,

d. unm., I 749 .

iii. Elizabeth, b. Stratford, Conn., r692; m. Zachariah Beardsley, of Stratford, Conn., June 4, I7 13. She d. in Ripton, Conn., I 776.

(3.) iv. Isaac, b. Stratford, Conn., m. (i.) Elizabeth, d. of Zachariah and Elizabeth Bostwick, of Stratford, Conn., Sept. 8, I726. m. (ii.) Sarah, He d. 1746.

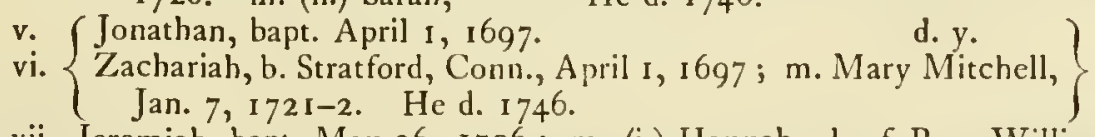

(36) vii. Jeremiah, bapt. May 26, I706; m. (i.) Hannah, d. of Rev. William and Hannah Burnham, of Kensington, Conn., Jan. 7, 1730; she d. April IO, 1772. m. (ii.) Rachel, d. of William Guernsey, of Waterbury, Conn., May 4, 1744; she d. Oct. 2I, I794, aet. 88 years. He d. March 31, 1795 . 
JOSIAH CURTISS (William, Elizabeth), b. Stratford, Conn., Aug. 30,1662 ; m. (i.) Abigail, d. of Lieut. Joseph and Sarah Judson, of Stratford, Conn., in July, I692. She d. I697. m. (ii.) Mary, d. of Benjamin and Mary Beach, of Stratford, Conn. In May, I 7 I4, the General Court appointed him captain of the "Train Band" of Stratford. In I 76 he was a deputy to the General Court. Dec. 29, 1725, liberty was granted to him and John Wilcoxson, $\mathrm{J} r$., to erect a sawmill on the halfway river. He d. 1745. She d. 1759. Will probated Nov. 20, 1745, at Fairfield, Conn.

They had children :-

(37) i. William, b. Stratford, Conn., Sept. 22, 1693; m. Prudence, d. of Samuel Judson, of Stratford, Conn., March 24, 1733.

ii. Abigail, b. Stratford, Conn., I695; m. Capt. Hezekiah Hooker, of Woodbury, Conn., Dec. 18, 1716; d. 1733.

iii. Anna, b. Stratford, Conn., I697; d. m. Zachariah Booth, June 12, 1718 , and d. 1733 .

iv. Eunice, b. Stratford, Conn., Aug. 1, 1699; m. Lieut. Robert Wells, of Stratford, Conn., Oct. 24, 1720.

(38) v. Abraham, b. Stratford, Conn., May 10, r 701 ; m. Elizabeth Webb; Feb. 25, 1724-5. He d. Sept. 7, 1779 .

(39) vi. Josiah, b. Stratford, Conn., Jan. 6, 1702-3; m. Mary Judson, of Woodbury, Conn., Nov. 28, 1727.

(70) vii. Benjamin, b. Stratford, Conn., Dec. 25, 1704; m. Elizabeth, d. of Abel and Comfort Birdscy, of Stratford, Conn., Aug. 31, 1727, and d. July 2, 1773, in Newtown, Conn.

(4I) viii. Peter, b. Stratford, Conn., April I, 1707 ; m. (i.) Hester, d. of Ephraim and Hester Clarke, of Stratford, Conn., Nov. 4, 1;28; she d. July 6, 1744. m. (ii.) Mary, d. of Henry and Mary Hawley, of Stratford, Conn.

ix. Matthew, b. Stratford, Conn., Dec. 16, 1708. d. y.

x. Mary, b. Stratford, Conn., July 25, 1711; m. John Patterson, of Stratford, Conn., June 4, 1730 .

(42) xi. Matthew, b. Stratford, Conn., Dcc. I, 17 I 2 ; m. (i.) Phebe, d. of Capt. David and Phebe Judson, of Stratford, Conn., June 2, 1737. She d. Sept. 18, 1758. m. (ii.) Abigail Thompson, of Fairfield, Conn. He d. Nov. 29, 1796.

(43) xii. Charles, b. Stratford, Conn., Jan. 1, 1715-16; m. Desire, xiii. Mehitabel, b. Stratford, Conn., Jan. 1, 17 15-16.

\section{4}

ISRAEL CURTISS (Israel, Fohn, Elizabeth), b. Woodbury, Conn., May 12, 1668; m. Mary Mallory, of Woodbury. He settled in Danbury, Conn. In 1719 he represented that town at the General Court. He was town clerk. 
They had children : -

i. Samuel, bapt. Woodbury, Conn., January, I694-5.

(44) ii. Josiah, bapt. Woodbury, Conn., April, 1697 ; m. Hannah, He d. I 740, in Danbury, Conn.

(45) iii. Stephen, bapt. Woodbury, Conn., April 22, I699; m. Abigail Knap, and d. in 1740 , in Danbury, Conn.

iv. Rebecca, b. Woodbury, Conn., July 22, I70I.

\section{5}

JOHN CURTISS (Israel, Fohn, Elizabeth), b. Woodbury, Conn., Oct. 7, I670; m. Johannah about I696. He was ensign of the "Train Band." In I 7 I 7 he was sent as a deputy from Woodbury to the General Court. He d. April I4, I754. She d. Sept. I, I 749.

They had children : -

i. Elizabeth, bapt. Woodbury, Conn., September, I697; m. John Mitchell, of Stratford, Conn., Jan. I7, 16 I 7 .

ii. Harriet, b. Woodbury, Conn., Nov. I, 1699.

(46) iii. Nathan (Elnathan), b. Woodbury, Conn., Feb. 2, 1 70I-2; d. Oct. I 7, 1761 ; m. Martha Preston, of Woodbury, Conn., May, 1732. She d. June 23,1764 , aet. 53 years. He d. Oct. 17, $176 \mathrm{r}$.

iv. Abigail, b. Woodbury, Conn., Feb. 29, 1703-4; m. David Hurd, of Woodbury, Conn., Jan. 6, $1723-4$.

v. Esther, b. Woodbury, Conn., April 5, 1706; m. Joseph Judson, of Woodbury, Conn., Dec. 20, I734.

vi. Joanna, b. Woodbury, Conn., Sept. 5, I708; m. Eliakim Stoddard, of Woodbury, Conn., Dec. 4, 1729.

vii. Hannah, b. Woodbury, Conn., Nov. 1, 1698-9.

(47) viii. John, b. Woodbury, Conn., Feb. 3, I71 ; m. Abiah, He was killed in 1743 .

ix. Olive, b. Woodbury, Conn., May 6, I7 I3; m. Gideon Stoddard, of Woodbury, Conn., Jan. 24, 1733 .

(48) x. Peter, b. Woodbury, Conn., Jan. I, I 7 I5-I6; m. Mrs. Mary Chittenden, Nov. 27, $1746-7$.

(79) xi. David (Daniel), b. Woodbury, Conn., Jan. 21, I 7 18; m. Eunice, He d. Sept. 1 5, I 782 . She d. March 5, 1783 , aet. 65 years.

xii. Eunice, b. Woodbury, Conn., March 20, I720; m. Abijah Stoddard, of Woodbury, Conn., Aug. I, 1739.

\section{6}

STEPHEN CURTISS (Israel, Fohn, Elizabeth), b. Woodbury, Conn., Aug. 24, I673; m. Sarah, d. of John Minor of Stratford, Conn., Nov. 2, I699. He was deputy from Woodbury to the General Court in I7 I8. He d. June I, I723, in Woodbury. 
They had children : -

(50) i. Joseph, b. Woodbury, Conn., Oct. 20, r 700 ; m. Mary, d. of Rev. A. Stoddard, of Woodbury, Conn. He d. June 5, 1727.

(5I) ii. Daniel, b. Woodbury, Conn., Jan. 12, 1702-3. He d. Aug. I 4 , 1784. m. (i.) Phebe Martin, June 3,1730; m. (ii.) Sarah Hinman, Sept. 12, 1760 . She d. Jan. I 2, 1807 , aet. 78 years.

iii. Sarah, b. Woodbury, Conn., July 28, 1705; m. Richard Peet, of Woodbury, Conn., March I I, $1724-5$.

iv. Rebecca, b. Woodbury, Conn., July 28, 1705; m. Elisha Stoddard.

v. Ruth, b. Woodbury, Conn., Jan. I 4, 1707; m. Samuel Galpin.

vi. Stephen, b. Woodbury, Conn., Feb. 23, 1710; m. (i.) Lois Hicock, Dec. 20, 1733 ; she d. June 29, 1776 , aet. 68 years. m. (ii.) widow Huldah Hicock, July 16, 1777 . He d. I 781 , childless.

vii. Mary, b. Woodbury, Conn., Sept. 15, 1713; m. Stephen Caulkins, of Woodbury, Conn., June 15, 1733.

(52) viii. Israel, b. Woodbury, Conn., July 23, I 7 16; m. Martha Towner, Jan. 31, I738; m. (ii.) Eunice Towner, Feb. 1, 1739. He d. 1796.

ix. Elizabeth, b. Woodbury, Conn., Sept. 19, 1 7 18; d. y.

x. Grace, b. Woodbury, Conn., July II, I720; m. Eldad King, of Stratford, Conn., March 3, 1743.

NATHANIEL CURTISS (Thomas, Fohn, Elizabeth), b. Wallingford, Conn., May 14, 1677; m. (i.) Sarah Hall, of Wallingford, Conn., April 6, 1697; she d. Dec. 17, 1700. m. (ii.) Sarah, d. of Zachariah and Sarah Gilbert How, of Wallingford, Conn., July 9, I 702 ; she d. Jan. 4, I 740, aet. 65 years. m. (iii.) Phebe Bartholomew, widow of Micah Palmer, of Branford, Conn., Oct. II, I741; she d. Jan. 5,1763 , aet. 69 years. He was a farmer, and lived in that part of Meriden, Conn., called Falls Plains, now Hanover. He was constable in 1729 , selectman, I7 I 7 , and he also held many other town offices. He d. March 4, 1763. Will filed, New Haven Probate Records, June, 1763 .

They had children :-

(53) i. Benjamin, b. Wallingford, Conn., April 27, 1703; m. Merriam, d. of Samuel and Hope Cook, of Wallingford, Conn., Dec. 12, 1727. He d. Oct. 29, 1754 .

ii. Hannah, b. Wallingford, Conn., Feb. 19, 1705; m. Joseph Matthews, Oct. 4, 1726.

(54) iii. Moses, b. Wallingford, Conn., Aug. 9, 1706; m. Eunice Cole, Nov. 9, 1731. He d. March 3, 1737, and his widow m. Jedediah Norton, of Farmington, Conn., Nov. 3, 1737 .

(55) iv. Enos, b. Wallingford, Conn., March 19, 1708; m. Eunice Yale, of Wallingford, Conı., June I $1,1732$. 
(56) v. Nathan, b. Wallingford, Conn., May 12, I 709 ; m. Esther Bishop, Oct. 7, 1740. He d. before 1759 .

(57) vi. Jacob, b. Wallingford, Conn., Aug. 23, I710; m. Abigail, d. of Joseph Foot, Jr., of Branford, Conn. He d. $173^{8 .}$

vii. Sarah, b. Wallingford, Conn., March 30, I7 12; m. Eliada Parker, of Wallingford, Conn., Dec. $2 \mathrm{I}, \mathrm{I} 73^{2}$.

viii. Abigail, b. Wallingford, Conn., April 9, I 7 I 3 ; d. y.

ix. Eliada, b. Wallingford, Conn., March 30, I714; m. Thomas Hall, of Wallingford, Conn., A pril 24, 1734.

x. Comfort, b. Wallingford, Conn., Oct. 30, I 7 16; m. Ephraim Munson, of Wallingford, Conn., Feb. 6, 1739 .

(58) xi. Nathaniel, b. Wallingford, Conn., Jan. I, 1718; m. Lois, d. of Joseph and Anne Curtis, of Wallingford, Conn., April 19, 1749.

xii. Rhoda, b. Wallingford, Conn., of Wallingford, Conn., June 28, 1737.

m. Waitstill John Plum,

\section{8}

SAMUEL CURTISS (Thomas, Fohn, Elizabeth), b. Wallingford, Conn., Feb. 3, I678; m. Elizabeth, d. of William and Mary Tuttle Fredericks, of New Haven, Conn., Jan. 3, I704-5. He was surveyor of highways, 1732 ; constable in 1745 . He bought land in Waterbury, Conn., in 1743, and d. there in 1769 . His will was filed Dec. 5, 1769 .

They had children :-

i. Mary, b. Wallingford, Conn., March 18, 1706; m. Sergt. Joseph Benham, of Wallingford, Conn., April 5, 1732.

ii. Elizabeth, b. Wallingford, Conn., Jan. I I, 1707 ; m. - Andruss.

(59) iii. Samuel, b. Wallingford, Conn., Sept. 28, I 709 ; m. Eunice Atwater, of Wallingford, Conn., Nov. 6, I734. She d. July 23, 1756.

(6o) iv. Enoch, b. Wallingford, Conn., Jan. 21, I 7 I ; m. Rachel Plum, June 6, I 739 .

v. Emma, b. Wallingford, Conn., July 29, I714; m. Joseph Benham, of Wallingford, Conn., Jan. 7, 1735.

vi. Abbie, b. Wallingford, Conn., Sept. 29, I7 16 ; m. Hezekiah Clark, Jan. 8, 1735 .

(6I) vii. Azor, b. Wallingford, Conn., Feb. 9, 1718; m. Margery, d. of Daniel Andruss, of New Haven, Conn.

viii. Ada, b. Wallingford, Conn., Jan. 26, I721 ; m. Samuel Leavitt, in Meriden, Conn., Sept. I 3, I 739.

ix. Desire, b. Wallingford, Conn., Jan. 23, 1723; d. Oct. 12, I $74 \mathrm{I}$.

x. Mindwell, b. Wallingford, Conn., Jan. 8, 1727; m. Ebenezer Roice, April 20, 1746.

xi. Alice, b. Wallingford, Conn., June 3, I 728.

xii. Olive, b. Wallingford, Conn.,

xiii. Content, b. Wallingford, Conn.

m. Andruss. 
THOMAS CURTISS ('Thomas, Fohn, Elizabeth), b. Wallingford, Conn., Aug. 26, I685; m. Mary,

He was a farmer, and lived in New Cheshire, Conn. He was town surveyor in I 728 and 1740 . Will filed February, 1759, New Haven Probate Records.

They had children : -

i. Rachel, b. Wallingford, Conn., Oct. 14, 1715; m. Jeronimus Bunnell, Sept. 20, I 739 .

ii. Phebe, b. Wallingford, Conn., Dec. 16, I 718 ; m. Samuel Thompson, of Wallingford, Conn., Nov. 5, 1741 .

iii. Margaret, b. Wallingford, Conn., May 8, I721 ; m. Timothy Smith, of Wallingford, Conn., Sept. 3, 1740.

(62) iv. Gideon, b. Wallingford, Conn., Jan. 25, 1726; m. Merriam Hotchkiss, June 16,1747 .

(63) v. Thomas, b. Wallingford, Conn., Aug. 4, 1729; m. Esther, d. of Abijah Benham, of Wallingford, Conn., July 11, I751. He d. 1776 .

vi. Mary, b. Wallingford, Conn.; m. John Benham, of Wallingford, Conn., Sept. 23, I 747 .

JOSEPH CURTISS (Thomas, Fohn, Elizabeth), b. Wallingford, Conn., Oct. I, I69I ; m. Martha, d. of William Collins, of New Haven, Conn., March 14, 17 I2. She d. July 29, 1769, aet. 86 years. He d. I756. His will was entered, New Haven Probate Records, Feb. I, I 756. He was a farmer, and owned farms in Wallingford and Farmington.

They had children : -

(67) i. $\left\{\begin{array}{l}\text { Peter, b. Wallingford, Conn., Jan. 5, 1713; m. Chestina Parker, } \\ \text { Nov. 22, } 1732 .\end{array}\right\}$

ii. Joseph, b. Wallingford, Conn., Jan. 5, I 7 I 3 ; d. Jan. I I, I I I 3.

(65) iii. Jonathan, b. Wallingford, Conn., April 1 3 , 1715; m. (i.) Deborah, d. of Thomas and Deborah Mix, of Wallingford, Conn., Feb. 4, 1736; m. (ii.) Hannah Maple, of Wethersfield, Conn.

(66) iv. James, b. Wallingford, Conn., Jan. 10, 1717; m. Thankful,

(67) v. Abner, b. Wallingford, Conn., Jan. 27, 1719; m. (i.) Mary Chapman, of Unity, Dec. 7, 1738 . m. (ii.) Mrs. Mary Whitıng, of Farmington, Conn., Nov. 28, 1782 .

(68) vi. Ethan, b. Wallingford, Conn., Dec. 27, 1720; m. Lowly Holt.

(69) vii. Joseph, b. Wallingford, Conn., Feb. 2, 1723; m. Rebecca Salter, June 5,1749 . 
viii. Martha, b. Wallingford, Conn., Jan. 29, 1725; m. John Miles, Nov. 14, 1743 .

(7o) ix. Caleb, b. Wallingford, Conn., Feb. 3, 1727; m. (i.) Charity Combs. m. (ii.) Lucy Putney, May 21, 1796. He d. March 21, I 802.

(7I) X. Joshua, b. Wallingford, Conn., March 7, I73I ; m. (i.) Sarah Salter, Feb. 27, 1753; she d. June, 1757. m. (ii.) Lydia Martin, May 23, 1760, in Southington, Conn. He d. March 21, I8 14.

2 I

JOHN CURTISS (Thomas, Fohn, Elizabeth), b. Wallingford, Conn., Sept. I 8, I699; m. Jemima, d. of William Abernathy, of Wallingford, Conn., June 17,1723 . He was surveyor of highways in 1736 , fenceviewer in 1728 , hayward in $1733 . \mathrm{He}$ d. in Wallingford, Conn., April 4, 1775. She d. March IO, I 77 I, aet. 69 years.

They had children : -

i. Mary, b. Wallingford, Conn., Nov. 20, 1724; m. (i.) Nathaniel Mattoon. m. (ii.) Philip Curtis, of Wallingford, Conn., Feb. I7, $1745-6$.

ii. Hannah, b. Wallingford, Conn., June 5, 1726; m. Edward Dunbar, of Waterbury, Conn.

iii. Ann, b. Wallingford, Conn., May 26, 1728 ; m. Ambrose London, Nov. 9, 1752.

iv. Abigail, b. Wallingford, Conn., June 8, $173^{\circ}$

v. Lois, b. Wallingford, Conn., Aug. 20, I 732.

(72) vi. John, b. Wallingford, Conn., Feb. 3, I735; m. Anne, d. of Abel Ives.

(73) vii. Giles, b. Wallingford, Conn., June I I, I737; d. Jan. 24, 1754.

viii. Jemima, b. Wallingford, Conn., March 18, I739; m. Waitstill Yale in 1763 . She d. Sept. 12, I 772.

ix. Elizabeth, b. Wallingford, Conn., April I I, I 74 I.

x. Sarah, b. Wallingford, Conn., June 28, I 744 ; d. Sept. 19, 1752.

22

EPHRAIM CURTISS (Foseph, Fohn, Elizabeth), b. Stratford, Conn., Dec. 3I, I684; m. Elizabeth, d. of Ephraim Stiles, June 26, 1707. He represented Stratford at the General Court for a number of years. He was also a Justice of the Peace of Fairfield County. She d. Oct. 5, 1775, aet. 9 I years. He d. May 9, 1776.

They had children :-

(74) i. Stiles, b. Stratford, Conn., March 18, 1708; m. Rebecca Judson, of Stratford, Conn., Nov. $7,173^{\circ}$.

(75) ii. Henry, b. Stratford, Conn., Oct. I 2, I 709 ; m. Anne, d. of Ambrose Thompson, of Stratford, Conn., April 12, 1739. 
iii. Anna, b. Stratford, Conn., Aug. 31, 1713; m. Robert Fairchild, of Durham, Conn., May i 8, I 730.

iv. Phebe, b. Stratford, Conn., Aug. 31, I7I3; m. David Lewis, of Stratford, Conn.

v. Elizabeth, b. Stratford, Conn., Aug. 9, I7 15; d. Oct. I1, I 7 I6.

vi. Ephraim, b. Stratford, Conn., Aug. 30, 1717 ; d. Dec. 2, 1737.

vii. Elizabeth, b. Stratford, Conn., Oct. 2, I 7 I9; d. July 3, I 728.

viii. Martha, b. Stratford, Conn., Nov. 26, 1721 ; m. Joseph Wells, Jr., Nov. 26, I741 ; d. Oct. I6, I 760.

ix. Ruth, b. Stratford, Conn., Oct. 27, 1723; m. Rev. James Beebee, of Stratford, Conn., July I 3, 1749.

$(76)$ x. Edmund, bapt. Stratford, Conn., August, $1725 ; \mathrm{m}$. Sarah Welles, of Stratford, Conn., April 10, 1746.

xi. Bathsheba, b. Stratford, Conn., November 7, 1728.

(77) xii. Elnathan, b. Stratford, Conn., January 4, 1726-7; m. Sarah Ufford in 1748 . He died Jan. 3, I812. She d. May 19, 1804.

\section{3}

JOSEPH CURTISS (Foseph, Fohn, Elizabeth), b. Stratford, Conn., Nov. 6, I687; m. Elizabeth, d. of John Wells, Jr., July 5, I7I I. His will was filed at Fairfield, March 6, 1738-9.

They had children : -

i. Robert, b. Stratford, Conn., June 7, 1713; d. Nov. 22, I 7 I3.

ii. Mary, b. Stratford, Conn., Sept. 2, 1714; m. Daniel Curtiss, of Stratford, Conn., May I 1, 1736; d. October, 1782.

(78) iii. (James, b. Stratford, Conn., Oct. 24, I 7 I6; m. (i.) Elizabeth, d. of

Edward Burroughs, of Stratford, Conn., Feb. 2, I 743-4; m.

(ii.) Keziah, 1747 .

iv. Joseph, b. Stratford, Conn., Oct. 24, 1716; d. y.

v. Elizabeth, b. Stratford, Conn., Jan. I6, 17 18-19; m. James Judson, of Stratford, Conn.; d. July 22, 1750.

(79) vi. Joseph, b. Stratford, Conn., March 28, 1721 ; m. (i.) Martha, d. of James Judson, of Stratford, Conn., Dec. 24, 1740; m. (ii.) Esther Blakeman, 1797 .

vii. Gideon, b. Stratford, Conn., Jan. 28, I722-3; d. unm., I 754.

viii. Tabitha, b. Stratford, Conn., Nov. 2, 1724; m. Richardson Minor, Jr., Jan. I 3, I 764 ; d. December, 1789.

(8o) ix. Robert, b. Stratford, Conn., Aug. 10, 1727 ; m. (i.) Amy Judson, of Stratford, Conn., Jan. 3, 1762; m. (ii.) Phebe Curtiss, of Stratford, Conn., Nov. 9, I 780.

x. Bethia, b. Stratford, Conn., January, I730-1 ; m. Abraham Beardsley, of Stratford, Conn., Oct. 21, 1753. She d. Aug. 4, I801.

NATHAN CURTISS (Foseph, Fohn, Elizabeth), b. Stratford, Conn., Feb. 21, I689-9o; m. Eunice, d. of Jeremiah Judson, Jr., June 23, 1713 . 
They had children : -

(8I) i. Jeremiah Judson, b. Stratford, Conn., April 4, 1716; m. Mary Edwards, of Stratford, Conn., July, 1745-6.

ii. Mary, b. Stratford, Conn., Aug. 28, I717; d. April 19, 1720.

iii. Eunice, b. Stratford, Conn., Nov. I I, 1719. She d. unm. in 1803 .

iv. Mary, b. Stratford, Conn., Sept. 6, I 72 I.

v. Judson, b. Stratford, Conn., May 22, I 723 ; m. Eunice,

vi. Hannah, bapt. Stratford, Conn., August, I 725 .

vii. Nathan, b. Stratford, Conn., Nov. 14, I727. He d. unm. Jan. 1776. viii. Phebe, b. Stratford, Conn., Aug. I3, I $73^{\circ}$.

ix. Charity, b. Stratford, Conn., Nov. I3, 1732; m. William Southworth, of Stratford, Conn., July, I 754 .

ELIPHALET CURTISS (Foseph, Fohn, Elizabeth), b. Stratford, Conn., Aug. I, I699; m. Hannah, d. of John and Patience Burroughs, of Straṭford, Conn., Dec. 7, i720. He d. 177 I.

They had children :-

i. Bethia, b. Stratford, Conn., Nov. I3, I72I ; m. Dr. William Russell, of Stratford, Conn., July 2, I74I. She d. March, I 749 .

ii. Hannah, b. Stratford, Conn., Feb. 26, 1730-1; m. Agur Judson, of Stratford, Conn., Dec. 23, 1746. She d. Nov. 14, 1747.

iii. Martha, b. Stratford, Conn., Nov. 26, I 722.

JOHN CURTISS (Benjamin, Fohn, Elizabeth), b. Stratford, Conn., Feb. I 8,.168I-2; m. (i.) Margaret, d. of Daniel and Mary Pickitt, Feb. 19, 1706-7. She d. Oct. 8, I714. m. (ii.) Hannah, d. of Col. Ebenezer Johnson, of Derby, Conn., Nov. 3, 1715. m. (iii.) Rebecca, He d. I745.

They had children :-

i. Mary, b. Stratford, Conn., June 28, I709; m. Samuel Fairchild, of Stratford, Conn., April 5, I 745. She d. 1783 .

ii. Sarah, b. Stratford, Conn., Sept. 2, 1710; m. James Beach, of Stratford, Conn., Dec. 23, 1730.

iii. Abigail, b. Stratford, Conn., Sept. 28, I7 I9; d. y.

iv. John, b. Stratford, Conn., Oct. I72I; d. y.

27

DAVID CURTISS (Benjamin, Fohn, Elizabeth), b. Stratford, Conn., $\quad 1682$; m. (i.) Dinah Weakley, Nov. 26, I7I9; she d. June, 1725. m. (ii.) Esther, d. of John and Abigail Hurd, Nov. 10, 1725. Will filed in Fairfield, Conn., June 30, 1761 . 
They had children : -

$\left(S_{2}\right)$ i. $\{$ Benjamin, b. Stratford, Conı., Dec. 10, 1720; m. Phebe,

ii. $\{$ David, b. Dec. 10, 1720.

(S3) iii. Samuel, b. Stratford, Conn., Nov. 25, r 722-3; m. Hannah, She d. July 19, 1746. He m. (ii.) Tabatha, and d. I 768.

iv. Esther, b. Stratford, Conn., March 30, 1725.

v. Dinah, b. Stratford, Conn., Nov. 28, I726; m. Ebenezer Fairchild, of Stratford, Conn., April, 1752.

vi. Anna, b. Stratford, Conn., June 23, 1729; m. Edmund Curtiss, of Trumbull, Conn., Aug. 20, 1750.

vii. Abigail, b. Stratford, Conn., May, I 732; m. William Wainwright.

viii. Ruth, b. Stratford, Conn., September, 1734; d. y.

\section{8}

JAMES CURTISS (Benjamin, Fohn, Elizabeth), b. Stratford, Conn., May 4, 1686; m. Hannah, d. of Lieut. John Coe, of Stratford, Conn. He was an original proprietor in Durham, 1708. His will was filed at Middletown, in 1766 , and dated at Durham.

They had children :-

(84) i. James, b. Durham, Conn.,

m. Hannah Buel, Sept. 12, I 734 .

ii. Ruth, b. Durham, Conn., Conn., Dec. I 728 .

m. Ezra Baldwin, of Milford,

iii. Sarah, b. Durham, Conn., March I 5, I 7 I2-13; m. Ithamar Parsons.

iv. Esther, b. Durham, Conn., Aug. 22, 1715; m. Parsons.

v. Mary, b. Durham, Conn., July 4, 1717 .

vi. Phebe, b. Durham, Conn.. Oct. 4, $1719 ; \mathrm{m}$. Mattoon.

(85) vii. John, b. Durham, Conn., Nov. 21,1721 ; m. Dinah, d. of Samuel and Dinah Norton, of Durham, Conn., Nov. $18,1747$.

(S6) viii. David, b. Durham, Conn., July 5, I 724 ; m. Thankful Thompson, of Durham, Conn., Jan. 22, 1747 .

ix. Hannah, b. Durham, Conn., Oct. 8, I727; m. Moses Austin.

x. Abigail, b. Durham, Conn., Sept. 17, 1732; m. Robinson.

xi. Eunice, b. Durham, Conn., June 23, I734; m. Samuel Sutliff, of Haddam, Conn., March 2I, 1755.

\section{9}

JONATHAN CURTISS (Fonathan, William, Elizabeth), b. Stratford, Conn., June 28, 1679; m. (i.) Hannah, m. (ii.) Mary Summers, Dec. 24,1718 ; m. (iii.)

He died in I $77 \mathrm{I}$, and his will was entered at Fairfield, Conn., April I 5, 177 I. 
They had children : -

$(87)$ i. Jonas, b. Stratford, Conn., I I00-I ; m. Eunice, d. of John Burroughs, Sept. II, I727. He d. I792.

(88) ii. Edmund, b. Stratford, Conn., June 26, 1702; m. Obedient Mallory, Dec. I2, 1723. He d. Oct. 20, 1726.

iii. Josiah (Jonah), b. Stratford, Conn., Oct. 26, I 703.

iv. Hannah, b. Stratford, Conn., Aug. 24, 1705; m. Benjamin Bennett, of Stratford, Conn., Jan. 28, I725.

(89) v. Jonathan, b. Stratford, Conn., She d. in 1796 , aet. 77 years.

vi. Sarah, b. Stratford, Conn., I 7 I2; m. Adams.

vii. Mary, b. Stratford, Conn.; m. John Haines, of Stratford, Conn., Sept. 6, 1739 .

viii. Israel, b. Stratford, Conn.

ix. Stephen, b. Stratford, Conn.

x. Timothy, b. Stratford, Conn.

DANIEL CURTISS (Daniel, William, Elizabeth), b. Stratford, Conn., I695; m. Mary, d. of Joseph and Elizabeth Curtiss, of Stratford, Conn., May i I, I736. She d. October, 1782, aet. 68 years. He d. October, I 763.

They had children :-

(go) i. Daniel, b. Stratford, Conn., May 20, 1737; m. Abigail Osborn. He d. September, 1795.

ii. Mary, b. Stratford, Conn., Oct. 1739 .

iii. Anna, b. Stratford, Conn., Oct. I 741 ; m. (i.) Smith; m. (ii.) Thomas Stratton, of Stratford, Conn., May 3, I771, and d. Jan. I I, $18 \mathrm{I}$.

$(g I)$ iv. Abijah, b. Stratford, Conn., July 1743; m. Phebe Clark, Sept. 4, I77.

v. Eunice, b. Stratford, Conn., Nov. 8, I 744 .

vi. Elihu, b. Stratford, Conn., May 1747. He d. Sept. 23, 1820.

vii. Hannah, b. Stratford, Conn., Sept. $\quad$ I 749 .

viii. Elizabeth, b. Stratford, Conn., Sept. I75 I m. Samuel Edwards, November, 5770 , in Trumbull, Conn.

HEZEKIAH CURTISS (Daniel, William, Elizabeth), b. Stratford, Conn., I7II ; m. Mehitabel, She d. I 790, aet. 77 years. His will was filed at Fairfield, Conn., October, I 77 I. He d. Oct. 9, I77I. 
They had children : -

i. Urania, b. Stratford, Conn., Sept. 26, 1743; m. Enoch Buckingham, of Huntington, and d. March i 8, 1823 .

ii. Abel, b. Stratford, Conn., May 29, I 745. He d. Nov. 18, 1830.

(g2) iii. Ezra, b. Stratford, Conn., Oct. 9, 1746; m. Ruth Ann. He d. May 4, 1809 .

(93) iv. Hezekiah, b. Stratford, Conn., Dec. 23, I 747 ; d. October, 1799; m. (i.) d. March 5, 1822 . m. (ii.) Sarah Thompson, February, I 771 ;

v. Mehitabel, b. Stratford, Conn., Aug. 4, I75I; m. Benjamin De Forest, of Ripton, Conn.

vi. Beulah, b. Stratford, Conn., I $754 ; \mathrm{m}$. —Curtiss. She d. Sept. I6, 1771 .

EBENEZER CURTISS (Ebenezer, William, Elizabeth), b. Stratford, Conn., she d. Nov. 29, I 746, aet. 32. m. (ii.) Mary, His will was filed at Fairfield, Conn., Dec. 5, 1749.

They had children :-

i. Ruth, b. Stratford, Conn., Dec. 7 .

ii. Phebe, b. Stratford, Conn., Nov. 27, 173 r.

iii. Anna, b. Stratford, Conn., Feb. 4, I $733-4$.

iv. Samuel, b. Stratford, Conn., Jan. 8, I 735-6; d. y.

NATHANIEL CURTISS (Zachariah, William, Elizabeth), b. Stratford, Conn.; m. Hannah, d. of Samuel Wells, of Hartford, Conn., Nov. 27, I712. He was a constable in 1720 . She d. 1761. His will was filed in Fairfield, Conn., 176r.

They had children :-

i. Thomas, b. Stratford, Conn.

ii. Nathaniel, b. Stratford, Conn.

iii. Sarah, b.

iv. Comfort, b.

v. Hannah, b.

m. Matthew Seymour, of Redfield, Conn.

vi. Oliver, bapt. Stratford, Conn., May, I73I; m. Hannah Clark.

(94) vii. Jeremiah, b. Stratford, Conn., December, I 732 ; m. Elizabeth, d. of Rev. Richardson Minor, of Stratford, Conn., Oct. 3, I 754.

(95) viii. Samuel, b. Stratford, Conn., June, I 735 ; m. Huldah, d. of Rev. Hezekiah Gold, of Stratford, Conn., Dec. 20, 1759.

ix. Mercy, b. Stratford, Conn., April, I737; m. Jacob Ressique, of Redfield, Conn. 
x. Elizabeth, bapt. Stratford, Conn., August, I742; m. Samuel Birdscy, of Goshen, Conn.

xi. Joshua, bapt. Stratford, Conn., September, 1746. He d. unm. in 176 I.

ISAAC CURTISS (Zachariah, William, Elizabeth), b. Stratford, Conn.; m. (i.) Elizabeth, d. of Zachariah and Elizabeth Bostwick, of Stratford, Conn., Sept. 8, 1726. m. (ii.) Sarah

His estate was divided Feb. 28, I746-7, in Fairfield, Conn. His widow m. Abraham Blakeman, of Stratford, Conn.

They had children :-

i. Jerusha, b. Stratford, Conn., July 4, I727; m. Nathan Peck, Jr., of Greenwich, Conn.

ii. Elizabeth, b. Stratford, Conn., Dec. 2, I 729.

iii. Sarah, b. Stratford, Conn., May 9, I 73 I.

iv. Benjamin, b. Stratford, Conn., Feb. 20, 1732 . He d. 1738.

v. Naomi, b. Stratford, Conn., Feb. 2, 1735-6.

ZACHARIAH CURTISS (Zachariah, William, Elizabeth), b. Stratford, Conn., I697; m. Mary Mitchell, of Stratford, Conn., Jan. 7, 1721-2. She d. Jan. 3, 1745, aet. 43. He d. in Unity, Conn., Feb. 18, 1746, aet. 49 years. His will was filed at Fairfield, Conn., March 4, 1746.

They had children : -

i. Mitchell, b. Stratford, Conn., Jan. I 72 I-2; d. y.

ii. Eunice, b. Stratford, Conn., Oct. 3, I722; m. John Hinman, of Unity, Conn.

iii. Mary, b. Stratford, Conn., Nov. 6, I 724 ; m. Richard Salmon, of Stratford, Conn.

iv. Rhoda, b. Stratford, Conn., Jan. 19, 1726; d. unm. in I 749.

v. Beulah, b. Stratford, Conn., Feb. $5,1727-8$; d. unm. in 1749 .

(g6) vi. Mitchell, b. Stratford, Conn., Sept. 21, I 729 ; d. Aug. I 5, I808; m. Phebe, d. of Deacon Thomas Peet, of Stratford, Conn., Feb. I1, I 753 .

vii. Susannah, b. Stratford, Conn., Oct. 6, 1736; m. David Seeley, of Stratford, Conn., Dec. $17,1755$.

\section{6}

JEREMIAH CURTISS (Zachariah, William, Elizabeth), bapt. Stratford, Conn., May 26, 1706 ; m. (i.) Hannah, d. of Rev. William and Hannah Wolcott Burnham, of Kensington, Conn., Jan. 7, I730; she d. April I0, 1772. m. (ii.) Rachel, widow 
of Joseph Guernsey, of Westbury, Conn., May 4, 1774. She d. Oct. 21, 1794, aet. 88 years; he d. March 31, 1795. From the time he was four years old until fourteen he was under the pastoral supervision of that distinguished scholar, Timothy Cutler, D. D., who was the second President of Yale College. In 1725 he graduated from Yale College, and at the age of twenty-one appears at Southington as a candidate for the pulpit of that society. He probably was a licentiate of the Fairfield County Association. He was ordained the first pastor of the Congregational Church in Southington, Conn., Nov. 27, 1728, which position he held until dismissed in November, 1754 .

They had children :-

i. Sarah, b. Southington, Conn., April 12, I733; m. Noah Gridley, of Southington, Conn., Aug. 15, 1751. She d. Aug. 6, 1806.

ii. Hannah, b. Southington, Conn., Sept. I, I735; m. Job Lewis, of Southington, Conn., Nov. 13, 1755. She d. May 4, 18 10.

(97) iii. Samuel, b. Southington, Conn., Nov. 15, 1737; m. (i.) Margaret, d. of Jonathan and Ruth Root, of Southington, Conn., May I4, 1766; she d. Dec. 20, 1769. m. (iii.) Mary Ann, d. of Aaron and Susannah Stanley Day. He d. May 15, I801. She d. March 18,1824 , act. 77 years.

(98) iv. John, b. Southington, Conn., Jan. 20, 1739-40; m. Mary, d. of Nathan and Mary Lewis, of Southington, Conn., Dec. 2, 1762. He d. March 25, I80I. She d. Sept. 9, I815, aet. 73 years.

v. Mary, b. Southington, Conn., Aug. 20, 1742; m. Dr. Phineas Clark, of Southington, Conn., Aug. 7, 1769. She d. October, $177 \mathrm{r}$.

vi. Lucy, b. Southington, Conn., Aug. 5, 1745; m. Elisha Root, of Southington, Conn., Feb. 17, 1764. She d. May 6, 1773 .

vii. Jeremiah, b. Southington, Conn., Nov. 7, 1752. He d. Dec. 23, 1758.

viii. Abigail, b. Southington, Conn.

37

WILLIAM CURTISS (Fosich, William; Elizabeth), b. Stratford, Conn., Sept. 22, 1693; m. Prudence, d. of Samuel and Mary Judson, of Stratford, Conn., March 24, $1725-6$. His will was filed at Fairfield, Conn., July 7, i 767.

They had children:-

(99) i. William, b. Stratford, Conn., July, r727; d. y.

ji. Anne, b. Stratford, Conn., Oct. 24, I $73^{\circ}$.

iii. Abigail, b. Stratford, Conn., Oct. IO, 1731; m. Treat Mills, of Stratford, Conn. 
ABRAHAM CURTISS (Fosiah, William, Elizabeth), b. Stratford, Conn., May 16, 1701; m. Elizabeth, d. of John and Mary Welles, of Stratford, Conn., Feb. 25, 1724-5. She d. in Stratford, Aug. 31, I770, aet. 68 years. He d. in Stratford, Sept. 7, I 779.

They had children :-

$(I O 0)$ i. Stephen, b. Stratford, Conn., June 3,$1727 ;$ m. (i.) Tahatha Beardslee, July 28, 1746 ; m. (iii.) Sarah, d. of Capt. David and Phebe Judson, of Stratford, Conn., Oct. 17, 1750. He d. May 8, 1806.

ii. Elizabeth, b. Stratford, Conn., Nov. 25, 1729 .

JOSIAH CURTISS (Fosiah, William, Elizabeth), b. Stratford, Conn., Jan. 6, 1702-3; m. Mary, d. of John and Elizabeth Judson, of Woodbury, Conn., Nov. 28, 1727. He d. May 26, 1773.

They had children :-

i. Mary, b. Stratford, Conn., Oct. 26, 1728.

(IOI) ii. Agur, b. Stratford, Conn., Sept. I1, 1730; m. Mercy Hinman, Jan. 30, I 755. He d. Feb. 8, 1784. She d. June 28, 1785, aet. 50 years.

(IO2) iii. Abner, b. Stratford, Conn., Oct. I I, 1732; m. Dec. 19, 1779 .

$\left(\mathrm{IO}_{3}\right)$ iv. Josiah, b. Stratford, Conn., Jan. 2I, I734-5; m. Mary

He d. Feb. 6, 1804 . She d. May 20, 1817 , aet. 80 years.

(107) v. Samuel, b. Stratford, Conn., March 24, $1736-7$.

(IO5) vi. David, b. Stratford, Conn., March 10, 1 739-40.

vii. Ann, b. Stratford, Conn., Jan. 27, 1742-3; m. Ephraim Curtiss, of Stratford, Conn., Dec. 23, 1764. She d. Jan. 15, 1802.

40

BENJAMIN CURTISS (Fosiah, William, Elizabeth), b. Stratford, Conn., Dec. 15, I704; m. (i.) Elizabeth, d. of Abel and Comfort Welles Birdsey, of Stratford, Conn., Aug. 27, 1727-8. She d. Feb. 24, 1773. He m. (ii.) Bathsheba Ford, of Stratford, Conn., June 17,1773 . He d. July 28, 1782-3. Will filed in Danbury, Conn., Aug. 6, 1782 . He and his brothers, Matthew and Josiah, settled in Newtown about 1728 , where he became a most prominent man. He represented his town at the General Court.

They had children:-

(Io6) i. Nehemiah, b. Newtown, Conn., Feb. 2, 1727-8; m. Martha Clark, of Newtown, Conn., Feb. 8, 1756 . He d. March 26, 1804.

(25) 
ii. Phebe, b. Newtown, Conn., Oct. 5, 1729; m. Daniel Morehouse, of Fairfield, Conn., Dec. 25, 1751.

iii. Eunice, b. Newtown, Conn., Jan. 13, 1731 ; m. Amos Hurd, of Zoar, Con11., Dec. 25, 1753.

iv. Elizabeth, b. Newtown, Conn., Oct. 26, I733; m. Capt. John Glover, of Hanover, Conn.

(107) v. Benjamin, b. Newtown, Conn., Feb. 13, 1735; m. (i.) Phedina, d. of Nathaniel Nichols, of Newtown, Conn., Nov. 23, 1758; she d. Feb. I5, I773, aet. 35 years. m. (ii.) Mary Devine, 1774; she d. Aug. 23, I795. m. (iii.) Phebe Town, May 6, I80I. She d. 1831. He d. Feb. 20, 18 I 7 .

vi. Abel, b. Newtown, Conn., Feb. 7, 1738. He d. July 8, 1767 .

$(I O S)$ vii. Abijah, b. Newtown, Conn., Jan. 3I, I740; m. (i.) Sarah Birdsey, Oct. 28,1762 ; m. (ii.) Mary, He d. Nov. 20, I817.

viii. Salmon, b. Newtown, Conn., He d. May 9, 1759.

ix. Sarah, b. Newtown, Conn., March 25, I 744; m. Nirom Hurd, July I7, I764. She d. Jan. I I, 1828 .

\section{$4 \mathrm{I}$}

PETER CURTISS (Fosiah, William, Elizabeth), b. Stratford, Conn., April I, I 707 ; m. (i.) Hester, d. of Ephraim and Hester Clarke, of Stratford, Conn., Nov. 4, I 728 ; she d. July 6, 1744. m. (ii.) Mary, d. of Henry and Mary Hawley, of Stratford, Conn. His will was probated Aug. 5, I766, at Fairfield, Conn.

They had children : -

i. Bethiah, b. Stratford, Conn., March 30,1730 ; m. Abel Hawley, of Woodbury, Conn.

ii. Mehitabel, b. Stratford, Conu., Aug. 5, 1732; m. Samuel Blakeman, of Stratford, Conn.

iii. Elihu, b. Stratford, Conn., February, 1733-4; d. y.

iv. Martha, b. Stratford, Conn., June 30,1735 ; m. Samuel Adams, of Stratford, Conn.

(109) v. Peter, b. Stratford, Conn., Oct. 31, 1736.

( $I I O)$ vi. Silas, b. Stratford, Conn., Aug. 6, 1741 ; m. Ruth, d. of Abel and Phebe Birdsey, of Stratford, Conn., March 25, 1763. He d. July 27,1818 . She d. Sept. II, 1818 , aet. 71 years.

(III) vii. Ezra, b. Stratford, Conn., June 24, 744 ; m. Ruth Ann, d. of Dr. Joseph Perry, Oct. 3, 1773. He d. March 29, 18 10. She d. Jan. 22, 1813 , aet. 55 years.

(112) viii. Henry, b. Stratford, Conn., June 8, 1754, m. Anne, d. of Zachariah and Amy Tomlinson, of Stratford, Conn., Aug. 14, 1774. He d. Jan. I I, I 806 .

(1I3) ix. Elijah, b. Stratford, Conn., $\quad$ I $747 ; \mathrm{m}$. Jane, d. of John Moss, of Stratford, Conn. She d. $183^{1}$, aet. 81 years. He d. $\quad 1817$.

(IIf) X. Elihu, b. Stratford, Conn., I749; m. (i.) Sarah, d. of Zachariah and Amy Tomlinson, of Stratford, Conn., March 14, I 769 ; m. (ii.) Sarah Lewis. He d. Nov. 2, 1804. She d. Dec。 I 3,1840 , aet. 98 years. 
MATTHEW CURTISS (Fosiah, William, Elizabeth), b. Stratford, Conn., Dec. I, I7 12 ; m. (i.) Phebe, d. of Capt. David and Phebe Judson, of Stratford, Conn., June 2, I730. She d. Sept. I 8, I758. m. (ii.) Abigail Thompson, of Fairfield, Conn. He settled in Newtown, Conn., with his brother Benjamin, and d. there Nov. 29, $179^{6}$. Administration was granted on his estate to Gould Curtiss, at Danbury, Conn., Dec. 15, 1796.

They had children : -

i. Phebe, b. Newtown, Conn., Feb. 20, I737-38; m. Rev. John Beach, of Newtown, Conn., Aug. 3, 1756.

(115) ii. David, b. Newtown, Conn., Jan. 4, 1741. He d. I 783.

(II6) iii. Niram, b. Newtown, Conn., April 5, I744; m. Mabel,

$\mathrm{He}$ d. 1794 .

(II7) iv. Matthew, b. Newtown, Conn., Feb. 4, I746; m. Hannah Ford, March 25, 1773 .

(II 8$)$ v. Stiles, b. Newtown, Conn., Nov. 4, I748; m. Hannah Bishop, Nov. 26, 1769.

(II9) vi. John, b. Newtown, Conn., Nov. I I, 1750.

(I20) vii. Josiah, b. Newtown, Conn., Jan. 7, I752; m. Ann Ford, Jan. 27, 1774 .

viii. Abner, b. Newtown, Conn., May 27, I754. He d. March 7, 1772.

(121) ix. Reuben, b. Newtown, Conn., May 13, 1757. He d. I816.

(I22) x. Gould, b. Newtown, Conı., Dec. 17, 1763; m. Elizabeth Gold, of Fairfield, Conn., Dec, 178 r. He died 1840.

43

CHARLES CURTISS (Fosiah, William, Elizabeth), b. Stratford, Conn., Jan., I7 I5-16; m. Desire,

They had children :-

(I23) i. Daniel Brewster, b. Stratford, Conn., Aug. 6, 1737. He was a soldier in the war of the Revolution.

ii. Mary, b. Stratford, Conn., March 15, $1739-40$.

iii. Ann, b. Stratford, Conn., Aug. 28, I 744.

(124) iv. John, b. Stratford, Conn., Feb. 1753.

44

JOSIAH CURTISS (Israel, Israel, Fohn, Elizabeth), bapt. Woodbury, Conn., April, 1697; m. Hannah, He d. in Danbury, Conn., about 1740.

They had children

(125) i. Seth, b. Danbury, Conn.

ii. Hannah, b. Danbury, Conn. 
STEPHEN CURTISS (Israel, Israel, Fohn, Elizabeth), bapt. Woodbury, Conn., April 22, 1699; m. Abigail Knapp. He d. in Danbury, Conn., about 1740 . Will filed at Fairfield, Conn., March 2, 1740-4I.

They had children :-

i. Rebecca, b. Danbury, Conn.

( 260$)$ ii. Stephen, b. Danbury, Conn.; m. Ruth, She d. 1798. He d. 1799 .

iii. Abigail, b. Danbury, Conn.

NATHAN CURTISS (Fohn, Israel, Fohn, Elizabeth), b. Woodbury, Conn., Feb. 2, 170I-2; m. Martha, d. of William and Martha Preston, May, 1732. He d. Oct. 19, 176r. She d. June 23, 1764 , aet. 53 years.

They had children :-

i. Elizabeth, bapt. Woodbury, Conn., Oct. 28, 1733; m. Thomas Bull, of Woodbury, Conn., Oct. 19, 1754.

ii. Martha, bapt. Woodbury, Conn., March 13, 1737; m. Dr. Andrew Graham, of Woodbury, Conn., June 4, 1753 .

iii. Ann, bapt. Woodbury, Conn., March 30, 1740; m. Edward Hinman, of Woodbury, Conn., July 18,1764 .

(127) iv. Nathan, bapt. Woodbury, Conn., April 3, 1748; m. (i.) Esther, She d. April 5, 1803 , act. 53 years. m. (ii.) Martha, d. of Jehiel Preston. She d. Feb. 16,1845 , aet. 86 years. He d. in Southbury, Conn., Nov. 21, 1845 .

JOHN CURTISS (Fohn, Israel, Fohn, Elizabeth), b. Woodbury, Conn., Feb. 3, I7II; m. Abiah, He was killed by lightning, I 743 .

They had children : -

i. Joanna, b. Woodbury, Conn., June 26, 1730; d. y.

(I28) ii. Abraham, b. Woodbury, Conn., Feb. 1732.

(I29) iii. Amos, b. Woodbury, Conn., Oct. 1733.

(130) iv. Gideon, b. Woodbury, Conn., June 8, 1735; m. Mary Hicock, Sept. 28, 1756. He d. 1793 .

v. Joanna, b. Woodbury, Conn., May 15, i737; m. Henry Cramer, of Woodbury, Conn., Dec. 5, 1753.

vi. Abiah, bapt. Woodbury, Conn., Jan. 21, 1739; m. Stephen Galpin.

(I.3I) vii. John, bapt. Woodbury, Conn., Oct. 12, 1740; m.

He d. 1803 .

viii. Hannah, b. Woodbury, Conn. 
ix. Eve, bapt. Woodbury, Conn., May I6, 1742.

x. Hester, b. Woodbury, Conn., Dec. II, 1743; m. Elijah Hinman, of Stratford, Conn., Jan. 3, 177 , and settled in Vermont.

48

PETER CURTISS (Fohn, Israel, Fohn, Elizabeth), b. Woodbury, Conn., Jan. I, I7I6; m. Mrs. Mary Chittendon, of Woodbury, Conn., Nov. 27, 1746-7. He d. in Lanesboro, Mass., March I 2, I775. She d. July 4, i 8 ro. After graduating from Yale College in 174I, he returned to Woodbury, Conn., where he lived till about $\mathbf{1 7 5 4}$, when he removed to Kent, Conn., and in 1759 to Lanesboro, Mass., where he became a most prominent citizen. In 176 i he was chosen moderator, and in 1772 selectman. He represented Lanesboro at the General Court in 1772 , 1773, and July 6, 1774 , he was chosen to be one of the three delegates from Lanesboro to a conference held at Stockbridge, Mass., regarding the rights of the American Colonies, and at a convention held soon afterward was one of the committee that brought in resolutions to abstain from drinking tea while it should be taxed. Aug. 3I, I774, a town meeting was called "to see if town will send agent to meet Dr. Ward of Worcester representing Committee of Correspondence in that town and to raise money for powder and shot and choose committee to consider the present alarming situation that the people of this Province is brought into by the British ministry, and to meet with other committees to consult on measures most prudent and effectual to guard against the slavery that is manifestly intended to involve us in, that the unanimity of the people of this County and Province may take place relative to this important affair." Mr. Curtiss was sent to represent the town before Dr. Ward and was appointed a member of the Committee of Safety, Dec. 27, I774. He was sent as delegate to the Provincial Congress held in Cambridge.

They had children :-

i. Mabel, b. Woodbury, Conn., March I6, r747-S.

ii. Mary, b. Woodbury, Conn., Aug. 30, 1752 ; m. John Young.

(132) iii. Peter Burr, b. Kent, Conn., A pril 30, 1755; m. Phebe, d. of Job Sherman of New Haven, Conn., Nov. 1 2, I775. He d. March 25, 1837. She d. Dec. 12, 1830.

DAVID CURTISS (Fohn, Israel, Fohn, Elizabeth), b. Woodbury, Conn., Jan. 2 I, I7 I 8 ; m. Eunice, d. of Lieut. Francis and Mary Stiles, of Southbury, Conn. He d. Sept. I 5, I 782 ; she d. March 5, 1783 , aet. 65 years. 
They had children :-

(133) i. David, bapt. Woodbury, Conn., Jan. 30, 1743; m. Sarah, d. of Captain Matthew Minor, of Woodbury, Conn., Sept. 27, 1764. She d. Feb. I4, 1820 . He d. Nov. I I, 1819.

(Ij7) ii. Asa, bapt. Woodbury, Conn., Aug. 3, I746; m. Eunice Woodward, Oct. 9, I765. He d. Sept. I, I 794.

iii. Eunice, bapt. Woodbury, Conn., Feb. 26, 1750; m. Ithiel Hicock, Woodbury, Conn., I 770 .

iv. Olive, bapt. Woodbury, Conn., April 5, 1752.

v. Love, bapt. Woodbury, Conn., Aug. 31, 1755 ; m. Graham Hurd, June IO, I 782 .

$$
50
$$

JOSEPH CURTISS (Stephen, Israel, Fohn, Elizabeth), b. Woodbury, Conn., Oct. 20, I 700 ; m. Mary, d. of Rev. Anthony Stoddard, of Woodbury, Conn. He d. June 5, 1727; she d. May 27, 1727 .

They had children :-

i. Prudence, b. Woodbury, Conn., June, r 724 ; m. William Martin, of Bethlehem, Conn.

(135) ii. Thaddeus, b. Woodbury, Conn., April 24, I726; m. Stoddard. He d. in $18 \mathrm{I} I$.

\section{1}

DANIEL CURTISS (Stephen, Israel, Fohn, Elizabeth), b. Woodbury, Conn., Jan. 12, 1703 ; m. (i.) Phebe, d. of William and Abigail Martin, of Woodbury, Conn., June 3, 1730; m. (ii.) Sarah, d. of Titus Hinman, of Southbury, Conn., and widow of David Hinman, Sept. 2, I760. He was a deputy to the General Court in May, 1742. He d. Aug. 14, 1784. She d. Jan. I 2, 1807 , aet. 78 years.

They had children :-

i. Phebe, b. Woodbury, Conn., Oct. 8, i 736; m. Amos Hicock, Jan. I 5,1759 .

ii. Elizabeth, bapt. Woodbury, Conn. June, I $73^{8}$; d. y.

iii. Sarah, bapt. Woodbury, Conn., Aug. 6, I762; m. Col. Joel Hinman, of Horse Neck, Conn., April Io, 1778 .

iv. Mary, bapt. Woodbury, Conn., June 7,$1767 ; \mathrm{m}$. Park Brown, of Stratford, Conn., May 2, I785. She d. June 30, 1825.

\section{2}

ISRAEL CURTISS (Stephen, Israel, Fohn, Elizabeth), b. Woodbury, Conn., June 23, I716; m. (i.) Martha Towner, Jan. 3I, $173^{8}$; m. (ii.) Eunice Towner, Jan. 31, 1739. He d. $179^{6 .}$ 
They had children :-

(136) i. Joseph, b. Woodbury, Conn., March 22, 1740; m. Keturah Hatch, of Woodbury, Conn., July I I, I763. He d. June 9, I798. She d. April 19, I 797, aet. 52 years.

(I37) ii. Israel, b. Woodbury, Conn., April 24, I742; m. Abigail Mallory, May 9,1769 .

.$(138)$ iii. Stephen, b. Woodbury, Conn., Jan. 8, I744; m. Mary Mallory. He d. Feb. 15, I8 I 3 .

(139) iv. Daniel, b. Woodbury, Conn., May $18,1746$.

v. Eunice, b. Woodbury, Conn., July 8,1748 ; m. Stephen Bateman, of Stratford, Conn., Sept. 26, 1768. She d. July 14, 1804.

vi. Sarah, b. Woodbury, Conn., April 3, 1749; m. Thomas Strong, Dec. 16,1767 .

(140) vii. Benjamin, b. Woodbury, Conn., May 18, I 751 ; m. Esther Benham, of Southbury, Conn., June I, I 778 . He d. July 28, I 798.

viii. Ruth, b. Woodbury, Conn., Aug. 24, I 753 ; m. Luther Bateman, Nov. 18, 1773 .

(I41) ix. Reuben, b. Woodbury, Conn., Feb. 25, 1755; m. Hannah Peck, of Southbury, Conn., March I6, 178 I. She d. July 28, I798, aet. 75 years. He d. June 21 , 1804 .

(142) x. Abijah, b. Woodbury, Conn., Sept. 26, 1756; m. Olive Hicock, May 5, I 778 . He d. Oct. I 2, I So5.

xi. Olive, b. Woodbury, Conn., Nov. 24, I759; m. Solomon Johnson, of Woodbury, Conn., Aug. 22, 1782. She d. Oct. 18, 1797.

BENJAMIN CURTISS (Nathaniel, Thomas, Fohn, Elizabeth), b. Wallingford, Conn., April 27, 1703; m. Miriam, d. of Samuel and Hope Cook, of Wallingford, Conn., Dec. 12, 1727. He d. Oct. 30, 1754. She d. May 29, 1776, aet. 74 years. He was made a member of the Meriden church, May IO, I73I. In 1744 and 1753 he held the position of tithing man, and in 1751 was appointed surveyor of highways.

They had children :-

i. Esther, b. Wallingford, Conn., Oct. 2, I 728 ; m. Timothy Thompson, of East Haven, Conn., July 20, 1749.

(143) ii. Abel, b. Wallingford, Conn., Dec. 22, 1729; m. (i.) Hannah Foster, of Wallingford, Conn., March 1, I759; m. (ii.) Patience, widow of Isaac IVickham. He d. Nov. I, 1797. She d. Dec. 22, 1828, aet. 86 years.

iii. Susannah, b. Wallingford, Conn., Nov. 9, 1732 ; m. Stephen Perkins, May 12, 1756. She d. Oct. 19, 1809.

iv. Lois, b. Wallingford, Conn., Sept. 30, 1733. She d. Oct. 19, 1749 .

(144) v. Benjamin, b. Wallingford, Conn., Oct. 27, 1735; m. Mindwell Hough, March 31, 1763. He d. Jan. 6, 1822. She d. June 8, I807. 
vi. Miriam, b. Wallingford, Conn., Aug. 30, I737; m. Daniel Parker, Nov. I8, 1762 .

vii. Sarah, b. Wallingford, Conn., May 29, I 739. She d. April 7, 1746. viii. Ruth, b. Wallingford, Conn., Sept. 2I, I74I ; m. John Woodward, of East Haven, Conn.

ix. Aaron, b. Wallingford, Conn., Nov. 8, 1744. He was thrown from a horse and killed, Dec. 18,1763 .

\section{4}

MOSES CURTISS (Nathaniel, Thomas, Fohn, Elizabeth), b. Wallingford, Conn., Aug. 4, I706; m. Eunice, d. of Joseph and A bigail Cole, of Meriden, Conn., Nov. 9, 1731. He d. March 3, 1737 , and his widow married Jediah Norton, of Farmington, Conn., Nov. 3, 1737. He was a member of the Meriden church, March 29, 1730.

They had children :-

i. Eldad, b. Wallingford, Conn., Nov. I3, 1732; m. (i.) Tabatha Perkins, Feb. 6, I 754. She d. Nov. I2, I754; m. (ii.) Clotilda Meeks, of Woodbury, Conn., May 20, 1756.

They had a child:

Eunice, b. Meriden, Conn., Nov. 12, 1754.

ii. Abigail, b. Meriden, Conn., Sept. 7, 1735.

\section{5}

ENOS CURTISS (Nathaniel, Thomas, fohn, Elizabeth), b. Wallingford, Conn., March 15, 1708 ; m. (i.) Mary, d. of Thomas Yale, of Wallingford, Conn., June II, I732. He was county tax collector from 1737 to 1739 . In 1740 he was constable. In 1762 he sold his farm in Meriden, Conn., and removed to Stockbridge, Mass., where he had bought land the previous year. He and his wife joined the Stockbridge church in 1764. His wife d. in Stockbridge, Mass., Feb. 27, I775, aet. 67 years, and he m. (ii.) Sarah, who survived him. His will was filed March 4, 1788 .

They had children : -

i. Titus, b. Meriden, Conn., June 28, 1732. He d. May I, I 733 .

(175) ii. Titus, b. Mcriden, Conn., April I3, 1734.

iii. Mary, b. Meriden, Conn., Nov. 8, 1736; m. Mr. Andrews.

iv. Anna, b. Meriden, Conn., July 29, I74I ; m. Mr. Martingale.

v. Comfort, b. Meriden, Conn., June 25, $1744^{\circ}$; m. Mr. Richards.

(I76) vi. Enos, b. Meriden, Conn., June 27, I746; m. Abigail,

vii. Lois, b. Meriden, Conn., March I, I 752; m. Mr. Hollister.

\section{6}

NATHAN CURTISS (Nathaniel, Thomas, Fohn, Elizabeth), b. Wallingford, Conn., May 12, I709; m. Esther Bishop, of Wallingford, Conn., Oct. 7, I740. In 1735 he was appointed 
fence viewer, and in 1745 , town surveyor. He sold his land in Wallingford in 1766 , and removed to Lenox.

They had children :-

(I47) i. Moses, b. Wallingford, Conn., Aug. 8, I74I.

(148) ii. Amos, b. Wallingford, Conn., March 24, 1742-3.

iii. Esther, b. Wallingford, Conn., March 7, 1744-5.

\section{7}

JACOB CURTISS (Nathaniel, Thomas, John, Elizabeth), b. Wallingford, Conn., Aug. 23, I710; m. Abigail, d. of Joseph Foot, Jr., of Branford, Conn., June 23, I736-7. He settled in Branford, Conn., in 1736, and died there in $1737-8$. His widow $m$. Solomon Palmer, Feb. 9, 1737-8.

They had children :-

(149) i. Jacob, b. Branford, Conn., Oct. I, I 737-8.

$$
5^{8}
$$

NATHANIEL CURTISS (Nathaniel, Thomas, Fohn, Elizabeth), b. Wallingford, Conn., Jan. I, I7 I 8 ; m. Lois, d. of Joseph and Ann Curtis, of Wallingford, Conn., April 19, 1749. He signed the oath of fidelity the second Thursday of May, 1777. He was a farmer, and lived in that part of Meriden now called Hanover. He d. about 1784 .

They had children :-

i. Eunice, b. Meriden, Conn., April I 2, 1750.

(150) ii. Nathaniel, b. Meriden, Conn., June I3, I 756; m. Esther Parker.

(ISI) iii. Jacob, b. Meriden, Conn., Sept. I4, I 758; m. Catherine,

SAMUEL CURTISS (Samuel, Thomas, Fohn, Elizabeth), b. Wallingford, Conn., Sept. 28, I709; m. (i.) Eunice Atwater, Nov. 6, I 734 ; she d. July 23, I 750 . He was a farmer. He owned land in Wallingford, Farmington, New Cheshire, and Northbury.

They had children : -

i. Samuel Edmond, b. Wallingford, Conn., Oct. IO, I739. He d. June 4,1740 .

(152) ii. Solomon, b. Wallingford, Conn., June 2, 174I ; m. Abigail, d. of Zachariah Gillett, of Southington, Conn., Feb. 13, 1766. He d. Aug. I, I 820 .

iii. Eunice, b. IVallingford, Conn., Aug. 30, 1743; m. Robert Patten, May I 0,1764 .

iv. Samuel, b. Wallingford, Conn., Sept. I6, I 745 ; d. Dec. 23 , I 745.

v. Thankful, b. Wallingford, Conn., March 16, 1746-7.

vi. Elizabeth, b. Wallingford, Conn., March 26, I 749. She d. July $15,1750$. 
ENOCH CURTISS (Samuel, Thomas, Fohn, Elizabeth), b. Wallingford, Conn., Jan. 2I, I7 I I ; m. Rachel Plum, June 6, I739. He lived in New Cheshire in 1743 , and removed to Farmington, Conn., where he died in 1760 . He was a farmer.

They had children : -

(153) ii. Enoch, b. Waterbury, Conn., Jan. 26, I 745-6.

iii. Elizabeth, b. Waterbury, Conn., March 5, I 748 ; d. y.

iv. Elizabeth, b. Farmington, Conn., Oct. 16, 175 I.

v. Ada, b. Farmington, Conn., March I 2, I 753 .

\section{I}

AZOR CURTISS (Samuel, Thomas, Fohn, Elizabeth), b. Wallingford, Conn., Feb. 9, I7 I 8 ; m. Margery, d. of Daniel Andruss, of New Haven, Conn. In 1749 he bought land in Farmington, Conn., which he sold in 1765 . June 17,1767 , he bought land in Lanesboro, Mass., where he removed. His will was entered at Stockbridge, Mass., May 2, I 784.

They had children : -

(154) i. Azor, b. Farmington, Conn., Oct. 22, 1750. He was a soldier in the Revolutionary army.

ii. Deliverance, b. Farmington, Conn., Jan. 7, I752; m. Mr. Beebee.

iii. Lois, b. Farmington, Conn., April 3, 1754 ; m. Mr. Powell.

iv. Margery, b. Farmington, Conn., Feb. 6, 1756.

(155) v. Samuel, b. Farmington, Conn., Feb. 6, 1758. He enlisted as a private in Benjamin Simond's' regiment, Sept. 5, 1777 .

vi. Elidia, b. Farmington, Conn., Oct. I7, I762.

vii. Vasti, b. Farmington, Conn., Jan. 1, 1765.

(156) viii. Ury, b. Lanesboro, Mass., July 29, I 767 .

(157) ix. Ephraim, b. Lanesboro, Mass., July I4, I 770.

$\left({ }_{15} 8\right)$ x. Silas, b. Lanesboro, Mass., Oct. 9, 1772.

(159) xi. Solomon, b. Lanesboro, Mass.

\section{2}

GIDEON CURTISS (Thomas, Thomas, Fohn, Elizabeth), b. Wallingford, Conn., Jan. 25, 1726; m. Mirriam Hotchkiss, June 16,1747 . He was a farmer, and lived in Cheshire, Conn. In 1777 he signed the oath of fidelity. Jan. I 4 , I778, he was a member of the committee of inspection. He sold his house in Cheshire in 1776.

They had children :

i. Lucy, b. Wallingford, Conn., March 29, 1 747-8.

ii. Phebe, b. Wallingford, Conn., Nov. $18,1750$. 
iii. Mirriam, b. Wallingford, Conn., June 28, I753; m. Ephraim Parker.

iv. Lue, b. Wallingford, Conn., Sept. 9, 1755 .

v. Sarah, b. Wallingford, Conn., July 10,1758 .

vi. Merib, b. Wallingford, Conn., Nov. 20, i 760.

( 160$)$ vii. Simeon, b. Wallingford, Conn., July 5, I 766.

$(I 6 I)$ viii. Gideon, b. Wallingford, Conn., July I $3,1767$.

(I62) ix. Alanson, b. Wallingford, Conn., Oct. I 2, I 768.

x. Decidamia, b. Wallingford, Conn., Feb. 25, I 771 .

\section{3}

THOMAS CURTISS (Thomas, Thomas, Fohn, Elizabeth), b. Wallingford, Conn., Aug. 4, I729; m. Esther, d. of Abijah Benham, of Wallingford, Conn., July I I, 175 . He d. in Cheshire, where he lived, Oct. 13,1776 .

They had children :-

i. Lydia, b. Cheshire, Conn., Nov. 24, 1754 .

ii. Esther, b. Cheshire, Conn., Jan. 10, I757; m. Asahel Wilmot, Jan. Io, 1776 .

(163) iii. Gilbert, b. Cheshire, Conn., April 30, 176I; m. Lucy Smith, in Cheshire, Conn., Dec. 8, 1784 .

$(167)$ iv. Thomas, b. Cheshire, Conn., Aug. I, 1764.

v. Mary, b. Cheshire, Conn.

vi. Hannah, b. Cheshire, Conn., July I I, I767; d. y.

vii. Hannah, b. Cheshire, Conn., April 22, I 769 .

$$
64
$$

PETER CURTISS (Foseph, Thomas, Fohn, Elizabeth), b. Wallingford, Conn., Jan. 5, 1713; m. Chestina, d. of Eliphalet and Hannah Parker, of Wallingford, Conn., Nov. 22, 1732 . He was a farmer, and lived in Farmington, Conn., until 1744, when he removed to West Simsbury, Conn. He d. in Simsbury, Conn., Dec. 20, 1756. His widow m. Sylvanus Woodruff, of Farmington, Conn., Feb. 18, 1767. She d. Feb. 15, 1777 .

They had children : -

( $\left.6_{5}\right)$ i. Israel, b. Meriden, Conn.; m. Elizabeth Andrews, of Simsbury, Conn., March 10, 1756 .

(I66) ii. Eliphalet, bapt. Meriden, Conn., February, $1735-6$; m. Margaret Dyer, of Simsbury, Conn., March 24, 1757. He d. 1806. She d. I 8 I 2 .

iii. Lucy, bapt. Meriden, Conn., April 2, I738; m. Col. Amasa Mills, of Simsbury, Conn., May 7, 1756.

$\left({ }^{6} 67\right)$ iv. Peter, bapt. Meriden, Conn., June 8,$1740 ; \mathrm{m}$. Ruth, She d. Dec. 28, 1815. He d. May 19, 1797 . 
v. Asenath, bapt. Southington, Conn., June 24, 1744; m. Bigelow Lawrence, and removed to Birkhampstead.

vi. Atheldred, bapt. Southington, Conn., Jan. I I, I 746-7; m. Eli Case.

vii. Chestina, b. to Nine Partners, N. Y.

viii. Prudence, bapt. Southington, Conn., April 30, 1749; m. Joshua Woodruff, Jr., Oct. Io, 1768 .

(I6S) ix. Gideon, b. Simsbury, Conn., 1752; m. Elizabeth, d. of Rev. Gideon Mills. He d. June 8, I789. She d. I 825 .

(I69) x. Solomon, b. Simsbury, Conn., 1753. He d. June I9, 1777, of small-pox. He cnlisted May 8, I775, as a private, at the Lexington Alarm, and served until Dec. 18, 1775. He served at the Siege of Boston as corporal, March 26, 1777, May 5, 1777, and also in New York.

xi. Charles, b. Simsbury, Conn., 1756. He d. Jan. 19, 1777.

\section{5}

JONATHAN CURTISS (Foseph, Thomas, Fohn, Elizabeth), b. Wallingford, Conn., April I3, I7 15; m. (i.) Deborah, d. of Thomas and Deborah Mix, of Wallingford, Conn., Feb. 4, 1736 .

They had children : -

i. Lois, b. Wallingford, Conn., June 23, 1737 .

ii. Deborah, b. Wallingford, Conn., Jan. 20, 1739.

iii. Thankful, b. Wallingford, Conn., April 21, 174I ; m. John Smith, of Saybrook, Conn., June 28, 1759.

( 170$)$ iv. Amasa, b. Wallingford, Conn., July 7, 1743; m. Lucy Garfield, of Monterey, Mass. He d. I822.

v. Esther, b. Wallingford, Conn., Sept. 5, 1745.

( $I 7 I)$ vi. Jonathan, b. Wallingford, Conn., Oct. 28, I747; m. (i.) Mary, d. of Richard Jacobs, of Sheffield, Mass., i 768. m. (ii.) Prudence, He d. in Sheffield, Mass., April 7, 1829.

vii. Abijah, b. Wallingford, Conn., Dec. 18, I 749 .

(172) viii. Elisha, b. Wallingford, Conn., Feb. 26, 1752; m. Sarah,

JAMES CURTISS (Foseph, Thomas, Fohn, Elizabeth), b. Wallingford, Conn., Jan. Io, I7I7; m. Thankful Clark, Nov. I I, I $73^{8}$. He settled in Cheshire, and from there removed to Pownall, Albany Co., N. Y., about 1763 . 
They had children : -

(I75) i. David, b. Wallingford, Conn., Feb. I, I740; m. (i.) Eunice Perkins, of Wallingford, Conn., July $30,176 \mathrm{r}$. m. (ii.) Huldah Andrews, May 3, 1783. He d. April 4, 1821. She d. Nov. 23,1827 .

ii. Jonah, b. Wallingford, Conn., Sept. 6, 1742. He d. Aug. 26, I754.

iii. Hannah, b. Wallingford, Conn., April 5, 1745. She d. May 9, I 746 .

iv. Thankful, b. Wallingford, Conn., May 4, 1748. She d. Feb. 9, I $750-1$.

v. Thankful, b. Wallingford, Conn., Nov. I6, I750.

$\left({ }_{17} 6\right)$ vi. James, b. Wallingford, Conn., July 9, 1753.

\section{7}

ABNER CURTISS (Foseph, Thomas, Fohn, Elizabeth), b. Wallingford, Conn., Jan. 27, 1719; m. (i.) Mary Chapman, of Unity, Conn., Dec. 7, 1738 ; she d. May 24, 1778 , aet. 66 years. m. (ii.) Mrs. Mary Whiting, of Farmington, Conn., Nov. 28, 1782. In 1764 he sold his farm in Wallingford, and removed to Farmington, where he died in 1799 .

They had children :-

(177) i. Abner, b. Wallingford, Conn., Aug. 8, 1738; m. Miriam Clark, Jan. 8, $176 \mathrm{r}$.

ii. Achseh, b. Wallingford, Conn., Oct. 4, 1739; m. (i.) Asa Bronson, Jan. 20, 1757. m. (ii.) William Churchill.

iii. Mary, b. Wallingford, Conn., June 6, 1741; m. William Andrews, Dec. 27,1759 .

iv. Silas, b. Wallingford, Conn., Jan. 21, 1744. He d. May 25, 1762.

v. Eunice, b. Wallingford, Conn., April 2, 1746; m. Robert Patten, in Meriden, Conn., May ro, 1764.

$(I 78)$ vi. Jesse, b. Wallingford, Conn., April 2, 1748; m. Martha Root, of Farmington, Conn., Dec. 2, 1772.

(I79) vii. Daniel, b. Wallingford, Conn., Feb. 21, 1750; m. Mary Andruss, of Farmington, Conn., Feb. 4, 1773.

( 180 ) viii. Amos, b. Wallingford, Conn., April 4, I752.

ix. Huldah, b. Wallingford, Conn., April 30, 1755. She d. Oct. 15, 1756.

x. Phineas, b. Wallingford, Conn., March 26, 1758. He d. Dec. 14, 1759 .

xi. Ruth, b. Farmington, Conn., April 29, 1784 .

JOSEPH CURTISS (Foseph, Thomas, Fohn, Elizabeth), b. Wallingford, Conn., Feb. 2, 1723; m. Rebecca Salter, of Cordlandt Manor, N. Y., June 5, 1749. He was a farmer, and about 1760 sold his lands in Meriden, and removed to Hanover, N. J. 
They had children : -

i. Sybil, b. Meriden, Conn., A pril 12, 1750.

ii. Jeptha, b. Meriden, Conn., March 21, 1752.

iii. Jerusha, bapt. Meriden, Conn., May 3, 1752 .

70

CALEB CURTISS (Foseph, Thomas, Fohn, Elizabeth), b. Wallingford, Conn., Feb. 3, 1727; m. (i.) Charity Combs. m. (ii.) Lucy Putney, May 21, 1796. He was a licentiate in Litchfield South Association in 1758 , and became the first settled minister of the church in Charlton, Worcester Co., Mass., in 1761, where he continued until 1776 , when he left to take charge of the Church of Christ at Dudley, Mass. In 1775 Rev. Caleb Curtiss represented Charlton at the Provincial Congress at Watertown, Mass. He died March 21, I 802, and is buried at Charlton, Mass.

They had children:-

i. Damaris, b. Charton, Mass., July 1 9, 1762 .

ii. Caleb, b. Charlton, Mass., March 17, 1766 . He d. March 5, 1769 .

$\left(\right.$ I $\left.S_{I}\right)$ iii. Caleb, b. Charlton, Mass., March 12, 1770; m. Polly Davis, of Charlton, Mass., Feb. I2, I794.

iv. Charity, b. Charlton, Mass., Sept. 4, I773; m. Marshall Mower, July 1,1790 .

\section{1}

JOSHUA CURTISS (Foseph, Thomas, Fohn, Elizabeth), b. Wallingford, Conn., March 7, 1731 ; m. (i.) Sarah Salter, of Wallingford, Conn., Feb. 27, 1753. She d. June, 1757. m. (ii.) Lydia Martin, in Southington, Conn., May 23, 1760. He d. in Burlington, Conn., March 21, 1814. She d. Sept. 14, 1811, aet. 7 I years.

They had children:-

(182) i. Ethan, b. Wallingford, Conn., Dec. 3, 1753.

ii. Martha, b. Wallingford, Conn., March 3, 1755 ; m. Joel Parks. She d. April 20, 1835 .

iii. Sarah, b. Wallingford, Conn., March 19, 1757; m. John Kidder, and settled in Hebron, Batavia Co., N. Y.

(183) iv. Simeon, b. Wallingford, Conn., Nov. 19, 1760; m. Elizabeth Sledge, of Middletown, Conn. He was killed Dec. I3, I809.

(I84) v. Joshua, b. Wallingford, Conn., May 6, 1762.

vi. Lydia, b. Wallingford, Conn., May 10, 1764; m. Mr. Bacon.

$(185)$ vii. Robert, b. Wallingford, Conn., May 20, 1766.

(186) viii. Aaron, b. Wallingford, Conn., Jan. $1767 ; \mathrm{m}$. Comfort Foot.

$(187)$ ix. Asa, b. Wallingford, Conn., March I, 1768. 
JOHN CURTISS (Foln, Thomas, Fohn, Elizabeth), b. Wallingford, Conn., Feb. 3, 1735; m. (i.) Anne Ives, d. of Abel Ives, of Wallingford, Conn. m. (ii.) Louisa, In I77 I he bought land in Canaan, Conn., and removed there with his family. $\mathrm{H}$ is will, dated Oct. 17, 1805, at Berkhamstead, was filed Dec. 3,1805 .

They had children

i. Sarah, b. Wallingford, Conn., May 8, I 762.

ii. Esther, b. Wallingford, Conn., April 30, I 763 .

(188) iii. Giles, b. Wallingford, Conn., March 22, 1764; m. Hannah Westover, of Sheffield, Mass.

iv. Elizabeth, b. Wallingford, Conn., Feb. 28, 1767.

v. Rhoda, b.,

vi. Francis, b.,

(189) vii. David, b.,

74

STILES CURTISS (Ephraim, Foseph, Fohn, Elizabeth), b. Stratford, Conn., March 8, I708; m. Rebecca Judson, d. of David and Phebe Judson, of Stratford, Conn., Nov. 7, 1730. He d. April 22 , 1785 . She d. July I, I 798 , aet. 89 years. His will was filed May 23, 1785. He was captain of the militia for many years.

They had children :-

i. Sarah, b. Stratford, Conn., May 17, I731; m. Daniel Judson, of Stratford, Conn., Jan. I, I751-2. She d. May 30, 1808 .

ii. Elizabeth, b. Stratford, Conn., Dec. 28, 1732 ; m. Benjamin Judson.

iii. Elihu, b. Stratford, Conn., Dec. 17, 1734. d. April 11, I $73^{8}$.

iv. Hannah, b. Stratford, Conn., Dec. 15, 1736; m. Abner Judson, of Stratford, Conn., Dec. I 9, 1756.

(IgO) v. Ephraim, b. Stratford, Conn., March 27, 1739; m. Anne, d. of Josiah and Mary Curtiss, of Stratford, Conn., Dec. 23, I 764. He d. Aug. 30, 1794. She d. Jan. I 5, I802.

(IgI) vi. Elihu, b. Stratford, Conn., May I O, I74I; m. Phebe, d. of John and Phebe Burritt, of Stratford, Conn., Dec. 13, 1769. He d. Aug. 20, I 820 .

(192) vii. Silas, b. Stratford, Conn., June 14, I743; m. Hannah, d. of Rev. Nathan and Hannah Birdsey, of Stratford, Conn., Feb. 17, 1765. He d. Jan. 1 5, I 816 . She d. Nov. 25, I 8 I I, aet. 65 years.

(193) viii. John, b. Stratford, Conn., Sept. 29, 1745; m. Mary, d. of Daniel and Mary Shelton, of Stratford, Conn., Aug. 17, 1769. He d. Aug. 31, I 825. She d. Aug.,

ix. Rebecca, h. Stratford, Conn., Feb. I 4, 1747-8; m. John Thompson, of Waterbury. Conn.

x. Kate, b. Stratford, Conn., June I752; m. Abijah Hull of Derby, Conn. 
HENRY CURTISS (Ephraim, Foseph, Fohn, Elizabeth), b. Stratford, Conn., Oct. 12, 1709; m. Anna, d. of Ambrose and Anne Thompson, of Stratford, Conn., April 12, 1739. He d. May 23, 1804 . She d. Sept. 14, I $78_{3}$, aet. 68 years. Will filed at Bridgeport, Conn.

They had children :-

i. Jabez, b. Stratford, Conn., Oct. 26, 1739. He d. in I 829. unm.

ii. Lucy, b. Stratford, Conn., March 4, I74I; m. John Booth, of Stratford, Conn., Dec. 23, 1762. d. Sept, 17, I 817 .

(I97) iii. Elijah, b. Stratford, Conn., May 22, I 742 . d. Sept. $23,1776$.

(I95) iv. Andrew, b. Stratford, Conn., Jan. 20, I744; m. Sarah, d. of Job Peck, of Stratford, Conn., Sept. 22, 1768 . He d. in 1830.

(196) v. Lewis, b. Stratford, Conn., March 12, 1745; m. Hepzibah, d. of James and Jerusha Walker, of Stratford, Conn., Jan. 28, I773. He d. March 5, 18 84 . She d. April I6, I 8 I 9 , aet. 7 I years.

(197) vi. Solomon, b. Stratford, Conn., July 24, $1747 ; \mathrm{m}$. Jerusha Wheeler, of Stratford, Conn., June 1786. He d. July I3, I824. She d. Aug. 29, I 834 , aet. 76 years.

vii. Anne, b. Stratford, Conn., March 29, 1749. d. Aug. 8, 1749 .

(IgS) viii. Henry Thompson, b. Stratford, Conn., March 22, 1751 ; m. Phebe, d. of Ebenezer Sherman, of Stratford, Conn., Dec. 1778. He d. April 18, 1814. She d. Feb. 5, 1826, aet. 67 years.

ix. Anne, b. Stratford, Conn., Dec. I2, 1752; m. James Welles, of Stratford, Conn., Sept. 13, 1778.

(Ig9) x. David, b. Stratford, Conn., Sept. 3, 1754; m. Anna Gorham.

(200) xi. Samuel, b. Stratford, Conn., June 27, I 757; m. Dorothy Tibbals, of Milford, Conn., Oct. 9, 1783. He d. May 19, 1826. She d. Sept. I 9, I 829 , aet. 66 years.

xii. Huldah, b. Stratford, Conn., Oct. 24, I759; m. Curtiss Judson, of Stratford, Conn., Sept. 9, I782.

\section{6}

EDMUND CURTISS (Ephraim, Foseph, Fohn, Elizabeth), b. Stratford, Conn., Aug. $1725 ; \mathrm{m}$. Sarah, d. of Robert Welles, of Stratford, Conn., March 9, I 746.

They had children:-

i. Thaddeus, b. Stratford, Conn., Nov. I 9, I 746 . d. Sept. 23, I 776.

(20I) ii. Samuel, b. Stratford, Conn., June 28, 1748.

(202) iii. (Edmund, b. Stratford, Conn., May I, I 751 ; m. Sarah Fairchild, of Stratford, Conn., March 17,1775 .

iv. Sarah, b. Stratford, Conn., May I, I 75I; m. Capt. David 
(203) v. Ehenezer, b. Stratford, Conn., Sept. I1, I 753. He was a soldier in the war of the Revolution; $d$.

vi. Elizabeth, b. Stratford, Conn., Jan. 9, 1755; d. unm. in 1823.

(204) vii. Robert Welles, b. Stratford, Conn., March 12, 1757.

ELNATHAN CURTISS (Ephraim, Foseph, Fohn, Elizabeth), b. Stratford, Conn., Jan. 4, 1726-7; m. Sarah, d. of Thomas and Phebe Ufford, of Stratford, Conn., in 1745. He d. in Huntington, Conn., Jan. 3, I812. She d. May I9, 1804. His will was filed in Bridgeport, Conn., April 23, I 812.

They had children : -

i. Charity, b. Stratford, Conn., Nov. 7, 1745. She d.

I 753 .

ii. Ruth, b. Huntington, Conn., May 27, 1748; m. Luke Summers, of Ripton, Conn., Jan. 5, 1769 .

(205) iii. William, b. Huntington, Conn., Aug. 24, 1750.

iv. Phebe, b. Huntington, Conn., Aug. 20, $175^{2}$; m. Ezra Birdseye, of Stratford, Conn.

v. Charity, b. Huntington, Conn., Sept. 9, I754; m. Joseph Wooster, of Stratford, Conn.

vi. Sarah, b. Huntington, Conn., May 3, $1757 ; \mathrm{m}$. Agur Tomlinson, of Huntington, Conn., May 20, 1781 .

vii. Anne, b. Huntington, Conn., Jan. 27, 1759.

(206) viii. Stiles, b. Huntington, Conn., Feb. 3, 1761; m. (i.) Clara Adams, June 178I. She d. May 4, 1796. m.(ii.) Betsey Hitchcock, of New Milford, Conn. She d. April 29, 1816. He d. Nov. 8, 1827 .

(207) ix. Elle, b. Huntington, Conn., March 1 2, 1763.

x. Betsey, b. Huntington, Coun., Nov. 10, 1765. She d. 1793 .

\section{8}

JAMES CURTISS (Foseph, Foseph, Fohn, Elizabeth), b. Stratford, Conn., Oct. 24, I7 I6 ; m. (i.) Elizabeth, d. of Edward Burroughs, Feb. 2, 1743-4. She d. Jan. I 4, 1745. m. (ii.) Keziah,

1747. James Curtiss enlisted as a private, May 10, 1775, in Capt. Samuel Whiting's Company, Col. Waterbury's Regiment.

They had children :-

i. Ann, b. Stratford, Conn., Oct. 28, 1744.

ii. Elizabeth, b. Stratford, Conn., Jan. 1745-6.

(208) iii. John, b. Stratford, Conn., April 14, 1748.

iv. Sarah, b. Stratford, Conn., Sept. 1751. 
JOSEPH CURTISS (Foseph, Foseph, Foln, Elizabeth), b. Stratford, Conn., March 28, 172I ; m. (i.) Martha, d. of James and Martha Judson, of Stratford, Conn., Dec. 24, 1740. She d. 1796. m. (ii.) Esther Blakeman, Nov. 4, 1797. He d. March 1 5, 1801. Will filed at Bridgeport, Conn., 1801 .

They had children :-

(209) i. Nehemiah, b. Stratford, Conn., $\quad$ 1740; m. (i.) Phebe, d. of Joseph and Martha Welles, of Stratford, Conn., April 4, 1763. She d. July 24,1776 , aet. 32 years. m. (ii.) Sarah, d. of William Lewis, and widow of Josiah Booth, Nov. 26, 1778 . She d. Feb. 2, 1812 , aet. 79 years. He d. May I3, i8 10.

ii. Joseph, b. Stratford, Conn., June 1741; d. y.

(210) iii. Phineas, b. Stratford, Conn., May I $742-3$; m. Huldah, d. of Samuel and Bethia Judson, of Stratford, Conn., and widow of Josiah Gilbert, Feb. 23, I 784 .

(2II) iv. Joseph, bapt. Stratford, Conn., Aug. I745; m. (i.) Mary, d. of John and Mehitabel Thompson, of Stratford, Conn., Nov. 13, 1768.

v. Abigail, b. Stratford, Conn., Nov. 1747; m. Abel Booth.

(212) vi. Silas, b. Stratford, Conn., May I 750 ; m. Mary, d. of Nathan and Tabitha Peck, of Stratford, Conn., Oct. 1770. He d. Aug. 5, I 822. She d. April I I, I805, aet. 50 years.

vii. Martha, b. Stratford, Conn.,

I753; m. Isaac Brooks.

viii. Judson, bapt. Stratford, Conn., Jan. 1755 .

(213) ix. Agur, b. Stratford, Conn., April 1757; m. Mercy, d. of Zechariah Blakeman. He d. Nov. Io, 1838 . She d. Dec. 17,1850 , aet. 90 years.

x. Charity, b. Stratford, Conn., May 1762; m. Philo Lewis, of Stratford, Conn., May 24, I 78 I.

xi. Phebe, b. Stratford, Conn. Stratford, Conn., Nov. 9, 1780.

m. Robert Curtiss, of

\section{0}

ROBERT CURTISS (Foseph, Foseph, Fohn, Elizabeth), b. Stratford, Conn., Aug. IO, $1727 ;$ m. Amy Judson, Jan. 3, 1762. She d. $\quad 1769$.

They had children :-

i. Amy, b. Stratford, Conn., July 3, 1766; m. Hilkiah Booth, Jr., Feb. 29, 1792 , and settled in Waterloo, N. Y.

(214) ii. Everett, b. Stratford, Conn., Nov. 6, 1762; m. Lucy, d. of Samuel and Esther Patterson, of Weston, Conn. He d. in 1826.

iii. Ruth, b. Stratford, Conn., Aug. 3, I 764 ; m. (i.) Isaac Jones, of Stratford, Conn., Nov. 22, I 789 . m. (ii.) Silas Judson, of Stratford, Conn., May 24, I 795.

iv. Robert, b. Stratford, Conn., March 24, I769. d. I 775. 
JEREMIAH JUDSON CURTISS (Nathan, Foseph, Fohn, Elizabeth), b. Stratford, Conn., April 4, 1716; m. Mary, d. of Gershom Edwards, of Stratford, Conn., July I745-6. He d. Sept. 4, 1782. His will was filed at Bridgeport, Conn., Sept. 23, 1782.

They had children :-

i. Edwards, b. Stratford, Conn., July 1746; d. y.

ii. Eunice, b. Stratford, Conn., July 1747; m. Peter Mallett, of Stratford, Conn., May 7, 1765.

iii. Elizabeth, bapt. Stratford, Conn., Feb. 1751; m. Benjamin Welles, of Stratford, Conn., March 19, 1796.

iv. Ann, b. Stratford, Conn., m. John McEwen, of Stratford, Conn., Jan. 3, 1774 .

v. Jerusha, b. Stratford, Conn., Feb. I761; m. Joseph Whitney.

82

BENJAMIN CURTISS (David, Benjamin, Fohn, Elizabeth), b. Stratford, Conn., Dec. 10, 1720; m. Phebe,

They had children :-

(215) i. Solomon, b. Stratford, Conn., May 6, 1748.

ii. Phebe, b. Stratford, Conn., Oct. 9, 1749.

(216) iii. Abel, bapt. Stratford, Conn., Feb. 23, 1752.

SAMUEL CURTISS (David, Benjamin, Fohn, Elizabeth), b. Stratford, Conn., Nov. 25, I722-3; m. (i.) Hannah, She d. July 19, 1746. m. (ii.) Tabatha, His will was filed at Fairfield, Conn., Nov. $31,1768$.

They had children :-

i. Hannah, b. Stratford, Conn., July 1 3, I 746; m. Judson

(2I7) ii. David, b. Stratford, Conn., Aug. $21,1748$.

(2I8) iii. Isaac, b. Stratford, Conn., Aug. 20, I 750.

(219) iv. Samuel, b. Stratford, Conn., Sept. 17, 1754.

v. Esther, b. Stratford, Conn., Sept. 1, 1756; d. y.

vi. Nehemiah, bapt. Stratford, Conn., Nov. 12, I 765 ; m. Ann Beardsley, of North Stratford, Conn., April 1787. He d. May 11, 1816. She d. Nov. I6, I836, aet. 77 years.

They had a child :-

Lydia, b. Trumbull, Conn., March 20, I 848 .

I787. She d. 
JAMES CURTISS (Fames, Benjamin, Fohn, Elizabeth), b. Durham, Conn.; m. Hannah Buel, Sept. I2, I734. He represented Durham in the General Assembly. His will was filed at

Middletown, Feb. 8, 1790.

They had children:-

(220). i Nathan, b. Durham, Conn., June 23, I735; m. Anna Booth, of Durham, Conn., May I 3,176 I.

(221) ii. Aaron, b. Durham, Conn., Sept. 9, 1737; m. Hannah Griswald, of Durham, Conn., May I1, 1763 .

\section{5}

JOHN CURTISS (Fames, Benjamin, Fohn, Elizabeth), b. Durham, Conn., Nov. 2I, I72I; m. Dinah, d. of Samuel and Dinah Norton, of Durham, Conn., Nov. 18, 1747. He represented Durham at the General Court. She d. Sept. 6, I 800 , aet. 77 years. He d. July I, I 800 .

They had children:-

(222) i. Abijah, b. Durham, Conn., March 2, 1750; m. Ann Bishop, of Durham, Conn., Aug. 13, 1777. He d. Sept. 8, 1825. She d. April $3, \mathrm{I}_{1} \mathrm{I}_{3}$, aet. 63 vears.

ii. Phebe, b. Durham, Conn., June I8, I752; m. David Scranton, of Durham, Conn.

iii. Hannah, b. Durham, Conn., Jan. 8, I755; m. John Hall, of Durham, Conn.

(223) iv. John, b. Durham, Conn., May 5, 1757 ; m. (i.) Lydia Hall, Dec. 29, I794. She d. July 6, I799; m. (ii.) Ruth Parmalee, of Durham, Conn., June 2, I80I. He d. in I 802.

v. Lois, b. Durham, Conn., July 15, I760; m. Deacon John Johnson.

vi. Sarah, b. Durham, Conn., Oct. I I, I 762 ; m. (i.) Elnathan Camp, and $\mathrm{m}$. (ii) Deacon Abraham Pierson.

vii. Dinah, b. Durham, Conn., Jan. 21, 1766; d. 1786.

DAVID CURTISS (Fames, Benjamin, Fohn, Elizabeth), b. Durham, Conn., July 5, 1724; m. Thankful Thompson, Jan. 22, 1747 .

They had children:-

(224) i. Ebenezer, b. Durham, Conn., Jan. 17, I749.

(225) ii. David, b. Durham, Conn., May I9, 1750.

iii. Esther, b. Durham, Conn., Nov. 15, 1751; m. Phineas Bates, of Durham, Conn., Dec. 5, $177 \mathrm{I}$.

iv. Helen, b. Durham, Conn., Nov, 15,175 I.

(226) v. Daniel, b. Durham, Conn., Oct. $7,1753$.

vi. Sarah, b. Durham, Conn., May 7, 1755 . 
JONAS CURTISS (Fonathan, Fonathan, William, Elizabeth), b. Stratford, Conn., I700-I; m. Eunice, d. of Johnl and Patience Burroughs, of Stratford, Conn., Sept. I I, I727. His will was filed at Bridgeport, Conn., May 27, 1792. Jonas Curtiss enlisted, May 8, I775, in Capt. Samuel Whiting's Regiment, of Stratford, Conn., Fifth Regiment, Col. Waterbury.

They had children :-

i. Eunice, b. Stratford, Conn., April I 8, 1728 ; m. Ichabod Hawley, of Trumbull, Conn., Jan. 3, I 75 I.

(227) ii. Edmund, b. Stratford, Conn., March 22, 1729-30; m. Anna, d. of David and Esther Curtiss, of North Stratford, Conn., Aug. 20, 1750 .

(228) iii. William, b. Stratford, Conn., May 23, 1732.

iv. Abigail, b. Stratford, Conn., Nov. I6, 1734 ; m. John Fayerweather, of Stratford, Conn., Feb. I4, I 760.

v. Hannah, b. Stratford, Conn., April 23, 1739.

vi. Sarah, b. Stratford, Conn., June 2I, r74I.

\section{8}

EDMUND CURTISS (Fonathan, Fonathan, William, Elizabeth), b. Stratford, Conn., June 26, 1702; m. Obedience Mallory, of Stratford, Conn., Dec. 12, 1723. He died Oct. 20, 1726. His widow m. Nathan Fairchild, of Stratford, Conn., Feb. 14, 1727-8, and then Benjamin Sherman, of Stratford, Conn., in 1740.

They had a child :-

i. Hannah, b. Stratford, Conn., June 18,1725 ; m. Washburn.

89

JONATHAN CURTISS (Fonathan, Fonathan, William, Elizabeth), b. Stratford, Con11., 1710; m. Eunice Summers. She d. in 1796, aged 77 years. His will was filed at Bridgeport in 1789 . Jonathan Curtiss enlisted May I I, I775, in Capt. Samuel Whiting's Company.

They had children :

i. Mary, b. Stratford, Conn., March 16, I748; m. Ephraim Judson, of New Stratford, Conn.

(229) ii. Timothy, b. Stratford, Conn., Aug. 17, 1749; m. (i.) Jerusha Wilcox, July 1,1773 ; m. (ii.) Hepzibah,

iii. Sarah, b. Stratford, Conn., Feb. 25, I75 I m. Gideon Hawley, of New Stratford, Conn.

(230) iv. Jonathan, b. Stratford, Conn.,

(23I) v. Andrew, b. Stratford, Conn., July 18, 1756. 
DANIEL CURTISS (Daniel, Daniel, William, Elizabeth), b. Stratford, Conn., May 20, 1737 ; m. Abigail Osborn. He d. Sept., 1795; she died 1796. He served as Coast Guard in Stratford, Conn., in 1778.

They had children :-

(232) i. Daniel, b. Stratford, Conn., 1775.

ii. Phebe, b. Stratford, Conn.,

(233) iii. Ebenezer, b. Stratford, Conn.,

(23f) iv. Samuel, b. Stratford, Conn.,

(235) v. Charles, b. Stratford, Conn.,

(236) vi. William, b. Stratford, Conn.,

(237) vii. James, b. Stratford, Conn.,

(238) viii. Aaron, b. Stratford, Conn.,

ABIJAH CURTISS (Daniel, Daniel, William, Elizabeth), b. Stratford, Conn., July, 1743; m. Phebe Clarke, Sept. 4, 1771. He served as Coast Guard in Stratford, Conn., in 1778.

They had children :-

i. Ann, b. Stratford, Conn., Sept. 4, 1772.

ii. Betsey, b. Stratford, Conn., Sept. 25, 1774 .

(239) iii. Abijah, b. Stratford, Conn., Sept. I0, 1776.

$(270)$ iv. Joseph Merchant, b. Stratford, Conn., March 10, 1779.

v. Sally, b. Stratford, Conn., July 26, I 78 I.

vi. Alice, b. Stratford, Conn., Feb. 17, 1783 .

vii. Ruth, b. Stratford, Conn., May I 8, 1786 ; m. Ephraim B. DeForrest, of Stratford, Conn., Feb. , 1812.

(24I) viiii. Philo, b. Stratford, Conn., Jan. 4, I 789.

ix. David, b. Stratford, Conn., July , I792.

\section{3}

HEZEKIAH CURTISS (Hezekiah, Daniel, William, Elizabeth), b. Stratford, Conn., Dec. 23, 1747; m. (i.) _ ; m. (ii.) Sarah, d. of Hezekiah and Sarah Thompson, in Feb. I77I. He died in Huntington, Conn., March 5, 1822 ; she d. in Huntington, Dec. 7,1805 , aet. 58. His will was filed at Bridgeport, Conn., April 2, 1822 . He served as a private in the Revolutionary War in Capt. Birdseye's Co., in 1777 .

They had children :-

i. Kate, b. Stratford, Conn., Dec. 2 I, I768 ; m. Philo Nichols.

ii. Hannah, b. Stratford, Conn., Feb 15, 1771 ; m. Asa Blackman, I $79 \mathrm{I}$, and d. in Huntington, Conn., 1799.

(46) 
iii. Sarah Jane, b. Stratford, Conn., June 22, I773; m. Isaac Bennet.

(242) iv. Hezekiah, b. Stratford, Conn., April 2, I775; m. Elizabeth Ann, He d. Dec. 3I, I 807.

v. Eli, b. Stratford, Conn., March 6, I781. d. Mar. 16, I783.

\section{4}

JEREMIAH CURTISS (Nathaniel, Zachariah, William, Elizabeth), b. Stratford, Conn., Dec. , I732; m. Elizabeth, d. of Rev. Richardson and Elizabeth Minor, Oct. 3, 1754.

They had children :-

i. Anna, b. Stratford, Conn., Sept. 7, I 755.

ii. Hannah, b. Stratford, Conn., Dec. 6, 1757 .

(243) iii. Joshua, b. Stratford, Conn., May 4, I 76 I.

iv. Wells, b. Stratford, Conn., May 2, I 764 ; m. Betsey Clark, March 1789 .

They had a child : -

Alice, b. Sept. 9, I 789 .

\section{5}

SAMUEL CURTISS (Nathaniel, Zachariah, William, Elizabeth), b. Stratford, Conn., June 1735 ; m. (i.) Huldah, d. of Rev. Hezekiah Gold, of Stratford, Conn., Dec. 20, 1759. She d. April 28, I 765 , aet. 27 years. m. (ii.) Hannah Gorham, He d. in Stratford, Conn., Sept. 8, 1802. His will was filed at Bridgeport, Conn., Oct. 4, 1802 . He was a farmer and resided in Stratford, Conn.

They had children : -

(244) i. Agur, b. Stratford, Conn., Nov. I7, I 760 ; m. Hulda, d. of Benjamin and Elizabeth Lewis, of Stratford, Conn., Feb. 22, 1788. He d. April 22, I84 I. She d. June 6, 1858 , aet. 92 years.

ii. Elizabeth, b. Stratford, Conn., June Io, I 763 ; m. Peter Pixley, of Stratford, Conn., May i 8, 1786.

iii. Huldah, b. Stratford, Conn., March 16, I 765 ; d. July 1765.

(245) iv. Samuel, b. Stratford, Conn., June I 4, I769; m. (i.) Temperance Ann, d. of Abel and Amy Patterson, of Stratford, Conn., Feb. I 793. She d. Aug. 30, I 800 , aet. 30 years. m. (ii.) Alice, d. of Elihu and Sarah De Forrest, of Stratford, Conn. She d. Dec. 1 3, I 859 , aet. 75 years. He d. Feb. 15,1833 .

v. Isaac, b. Stratford, Conn., April I 3 , I 775 ; m. Elizabeth, and d. Jan. 17, 1796. She d. July 1, 1 797, aet. 26. 


\section{6}

MITCHELL CURTISS (Zachariah, Zachariah, William, Elizabeth), b. Stratford, Conn., Sept. 21, I729; m. Phebe, d. of Thomas and Phebe Pect, of North Stratford, Conn., Jan. IO, 1753. He d. in Trumbull, Conn., Aug. 15, 1808. His will was filed Feb. 3, t809, in Bridgeport.

They had children : -

(246) i. Zachariah, b. Stratford, Conn., Sépt. I 7, I753; m. Naomi, d. of John and Huldah Edwards, of Stratford, Conn., Dec. 28, I 775. He d. Feb. 4, 1809; she d. Dec. I 7, r 808.

(2+7) ii. Daniel Mitchell, b. Stratford, Conn., Jan. 5, I 755 ; m. (i.) Hepzibah Burr, June 24, I 779. She d., and he m. (ii.) Huldah

He d. April 27, I83 I ; she d. May 7, 1832.

iii. Mary Ann, b. Stratford, Conn., Nov. 7, 1756; d. y.

iv. Phebe, b. Stratford, Conn., July , I 764.

(248) v. Isaac, b. Stratford, Conn., Dec. , 1766.

vi. Eunice, b. Stratford, Conn.

SAMUEL CURTISS (Feremiah, Zachariah, William, Elizabeth), b. Southington, Conn., Nov. 15, 1737 ; m. (i.) Margaret, d. of Jonathan and Ruth Root, of Southington, Conn., May I 4, 1766. She d. Dec. 20, 1769; m. (ii.) Mary Ann, d. of Aaron and Susannah Stanley Day. He d. in Southington, Conn., May I 5, 1801. She d. March I 8, I 824, aet. 77 years. He kept a tavern at the south end of Southington, Conn. In the war of the Revolution he served a few months as a private.

They had children : -

i. Infant, b. Southington, Conn., Nov. 4 , I 768 ; d. y.

(249) ii. Samuel, b. Southington, Conn., Dec. 8, I 769 ; m. Peggy Dyer, Feb. 24, I 799 .

iii. Day, b. Southington, Conn., Dec. 20, 1772. He d. Nov. 23, 1794 .

iv. Carlos, b. Southington, Conn., Dec. 20, I 774 . He d. Oct. 8, I 792.

(250) v. Leverett, b. Southington, Conn., 1776 ; m. Ruth, d. of Asa and Sarah Barnes, of Southington, Conn., Jan. 25, I 796. He d. Oct. I 2, I 826 . She d. April 9, 1850 , aet. 78 years.

vi. Margaret, bapt. Southington, Conn., Feb. I 782 ; m. Harvey Hough, March 25, I804.

(25I) vii. Leonard, bapt. Southington, Conn., Feb. $\quad$ I 782.

viii. Infant, b. Southington, Conn., Jan. 29, I 786 ; d. y.

ix. Mary Ann, b. Southington, Conn., m. Moses Rice. 
JOHN CURTISS (Feremiah, Zachariah, William, Elizabeth), b. Southington, Conn., Jan. 20, 1739-40; m. Mary, d. of Nathan and Mary Lewis, of Southington, Conn., Dec. 2, 1762. He d. March 25, I801. She d. Sept. 9, I815, aet. 73 years. He was a most prominent man in Southington, having been its town clerk for years, and also a Justice of the Peace. He was a member of the State Convention called to ratify the Federal Constitution, and voted in the affirmative.

They had children :-

i. Lovina, b. Southington, Conn., Sept. I I, I 763 ; m. Selah Deming, of Southington, Conn., Jan. 1 3, I 788 , and removed to Ohio.

(252) ii. Lucas, b. Southington, Conn., June 17, $1766 ; \mathrm{m}$. Laurinda, d. of Abel and Rhoda Lewis Carter, of Southington, Conn., Nov. 25, 1799. He d. Aug. 22, 1847. She d. March 7, 1857.

(253) iii. Jeremiah, b. Southington, Conn., Sept. 6, 1770; m. Rachel, d. of Abel and Rhoda Lewis Carter, of Southington, Conn. He d. June 2I, I 8 I 3 . She d. March 6, I 858 , aet. 86 years.

iv. Polly, b. Southington, Conn., June 5, 1773 .

v. Lucy, b. Southington, Conn., July 25, 1776; m. James Coach Carter, of Southington, Conn., Oct. 29, 1797. She d. Sept. 27, 1855 .

(254) vi. Marcus, b. Southington, Conn., Sept. 19, I780; m. Catherine, d. of Isaac Newell, of Southington, Conn., Nov. 30, 1804. He d. Dec. 1, 1868 ; she d. Sept. 10, 1870.

(255) vii. Erastus, b. Southington, Conn., May 30, I 784 ; m. Mary, d. of Timothy Lee, of Southington, Conn., Nov. 24, 1808 . He d. April 4, 1825; she d. August 31, 1863.

100

STEPHEN CURTISS (Abraham, Fosiah, William, Elizabeth), b. Stratford, Conn., June 3, 1727 ; m. (i.) Tabatha Beardslee, July 28, I745; m. (ii.) Sarah Judson, Oct. 17, 1749. She d. Nov. 2, 1794 , aged 64. He d. May 8, 1806 . His will was filed in Bridgeport, Conn., June 2, 1806.

They had children : -

i. Abraham, b. Stratferd, Conn., Nov. I747; d. y.

$V$ ii. James, b. Stratford, Conn., Aug. I 7, I 748.

iii. Sarah, b. Stratford, Conn., Oct. I0, $175^{\circ}$.

iv. Jerusha, b. Stratford, Conn., Aug. I, 175 I ; d. y.

v. Jerusha, b. Stratford, Conn., March 21, 1753; m. Stephen Lewis, of Stratford, Conn., Sept. 12, 1768 . She d. in $183^{8}$.

vi. Abraham, b. Stratford, Conn., Feb. 6, I 754 ; m. Anna,

He d. Sept. 4, 1776.

They had a child : -

Sally, b. Stratford, Conn.

m. Oliver S. Wilcox. 
vii. Hannah, b. Stratford, Conn., July 14, 1755.

viii. Stephen Judson, b. Stratford, Conn., July 5, I 757. He d. June I 7, I 760 .

ix. William, b. Stratford, Conn., Aug. $\quad 1758$.

x. Stephen Judson, b. Stratford, Conn., I76r. He d. April i 8, I 766 .

xi. Sarah, b. Stratford, Conn., Aug. 4, I765. She d. April 9, I 766.

xii. Belle, b. Stratford, Conn., Feb. 6, I767. She d. Oct. 9, 1777.

xiii. Phebe, b. Stratford, Conn., Sept. 6, 1769; m. Lewis Beers, of Oswego, N. Y.

(256) xiv. Levi, b. Stratford, Conn., March 26, i 772 ; m. Sarah

\section{1}

AGUR CURTISS (Fosiah, Fosiah, William, Elizabeth), b. Stratford, Conn., Sept. II, I730; m. Mercy, d. of Capt. Wait Hinman, of Woodbury, Conn., Jan. 30, I 755. He d. in Southbury, Conn., Feb. 8, 1784. She d. in Southbury, Conn., June 28, I 785 , aged 50 years. Sept. 19, 1775, he was chosen a member of the Committee of Inspection or observation of the town of Woodbury, Conn. He served as a captain in the war of the Revolution.

They had children : -

(257) i. Wait, bapt. Woodbury, Conn., Jan. I 8, I 756; m. Olive, d. of Selah and Esther Strong, of Woodbury, Conn., June 1 8, I 789. He d. Oct. I 7, I 798 .

ii. Anna, b. Woodbury, Conn., July 20, 1759 ; m. Dr. Anthony Burritt, of Newtown, Conn., April 7, I 782, and d. Sept. 30, 1808.

(258) iii. Josiah, bapt. Woodbury, Conn., July 25, I 762 ; m. Olive, d. of Increase and Patience Hinman Moseley, of Woodbury, Conn., Dec. I 3,1787 .

102

ABNER CURTISS (Fosiah, Fosiah, William, Elizabeth), b. Stratford, Conn., Oct. II, I732; m. He d. Dec. I 9,1779 .

They had children :-

i. Mary, bapt. Stratford, Conn., July , 1763.

(259) ii. Isaac Judson, bapt. Stratford, Conn., June, I 767 ; m. Charity Booth, Jan. I, I791. He d. July 17, 1845. She d. Dec. I6, I 846 .

(260) iii. Samuel, bapt. Stratford, Conn., March, I 769.

106

NEHEMIAH CURTISS (Benjamin, Fosiah, William, Elizabeth), b. Newtown, Conn., Feb. 20, 1727-8; m. Martha Clark, of Newtown, Conn., Feb. 8, 1756. He d. in Newtown, Conn., March 26, 1804 . 
They had children :-

i. Phebe, b. Newtown, Conn., Feb. 15, 1757.

(26r) ii. Salmon, b. Newtown, Conn., Aug. I7, 1759; m. Esther Holbrook, of Newtown, Conn., July 4, 1782. He d. Jan. 2, I814. She d. 1818 .

iii. Apame (Appime), b. Newtown, Conn., March 8, 1762.

iv. Parthenia, b. Newtown, Conn., April I6, 1764.

(262) v. Abel, b. Newtown, Conn., Nov. 2, 1766; m. Abiah Hurd, of Newtown, Conn., Nov. 24, I79I.

vi. $\{$ Sarah, b. Newtown, Con1., Sept. 5, I 77 I.

vii. $\{$ Eunice, b. Newtown, Conn., Sept. 5, I 77 I.

107

BENJAMIN CURTISS (Benjamin, Fosiah, William, Elizabeth), b. Newtown, Conn., Feb. 14, 1736; m. (i.) Phedenia, d. of Nathaniel Nichols, of Newtown, Conn., Nov. 23, 1758. She d. Feb. 15, 1773, aged 35; m. (ii.) Mary Devine, 1774. She d. Aug. 23, 1795; m. (iii.) Phebe Town, May 6, 1801. She d. in 1831. He d. Feb. 20, 1817 , in Newtown, Conn. His will was filed Dec. 16, 1817, in Danbury, Conn. Benjamin Curtiss, Jr., was a private in Capt. David Smith's Company, April 21, 1776.

They had children:-

(263) i. Philo, b. Newtown, Conn., June 27, 1760; m. Huldah Hubbard. He d. in Newtown, Conn., March , I818.

ii. Elizabeth Birdsey, b. Newtown, Conn., Sept. 2I, I766; m. Ephraim Peck.

iii. Anna, b. Newtown, Conn., Dec. 20, I769; m. Silas French, of Southbury, Conn., Dec. 22, I794.

(264) iv. Benjamin, b. Newtown, Conn., Feb. I 5, I 772.

v. Sarah, b. Newtown, Conn., Nov. 24, I775. d. Jan. , 1802.

vi. Artemesia, b. Newtown, Conn., May 5, $1778 ; \mathrm{m}$. Linus Sherman.

vii. Aurilla, b. Newtown, Conn., Nov. 30, I780. d. April 23, 1796.

(265) viii. Alfred Devine, b. Newtown, Conn., Jan. 24, I783; m. Sarah, d. of Miron and Sarah Hurd, of Newtown, Conn. He d. Sept. 30, I850. She d. April 6,1858 , aet. 75 years.

(266) ix. Epenetus, b. Newtown, Conn., Oct. 4, 1786; m. Sabrina, d. of Amos Hurd, of Newtown, Conn. He d. in Bridgeport, Conn., Dec. 15,1828 .

x. Phedina, b. Newtown, Conn., Southbury, Conn., Nov. 9, I79I.

m. Daniel Clarke, of

\section{8}

ABIJAH CURTISS (Benjamin, Fosiah, William, Elizabeth), b. Newtown, Conn., Jan. 31, 1740; m. (i.) Sarah Birdsey, of Newtown, Conn., Oct. 28, 1762. She d. in 1796 ; m. (ii.) Mary,

He d. in Newtown, Conn., Nov. 20, 1817 . He was a farmer, 
and owned land in Newtown and Sandy Hook, Conn., and was also interested in mills in Huntington, Conn. He was a Justice of the Peace and Tax Collector in Newtown.

They had children :-

(267) i. John, b. Newtown, Conn., 1764; m. Hannah, d. of John and Phebe Beech, of Huntington, Conn. He d. Oct. 19, 1820-1.

(26S) ii. Benjamin, b. Newtown, Conn., I766; m. Mehitable - He d. in Newtown, Conn., July 2, 1825 . No issue. He was a prominent physician, and one of the first to use inoculation.

(269) iii. Abijah Birdsey, b. Newtown, Conn., 1772; m. Anna, d. of James and Eunice Glover, of Newtown, Conn., Sept. 22, 1793. She d. Oct. I3, 1829, aet. 56 years. He d. May I7, 1857.

\section{IO}

SILAS CURTISS (Peter, Fosiah, William, Elizabeth), b. Stratford, Conn., Aug. 6, I74r; m. Ruth, d. of Abel Birdsey, of Stratford, Conn., March 21, 1763. She d. Sept. I I, I 818 , aet. 7 I years. He d. in Stratford, Conn., July 27, I 8 I 8.

They had children : -

i. Esther, b. Stratford, Conn., Oct. I 5,1763 ; d.y.

ii. Phebe, b. Stratford, Conn., May 15, 1765; m. Samuel Beardsley, of Huntington, Conn., Jan. 28, 1783. She d. Jan. 27, 1857.

iii. Ruth, b. Stratford, Conn., Jan. I 9, 1766.

iv. Esther, b. Stratford, Conn., Jan. 7, 1767; m. Philo Beardsley, of Kent, Conn.

v. Sally, M., b. Stratford, Conn., June 5, 1769; m. James Beach, of Stratford, Conn., Nov. 23, 1786.

(270) vi. Agur, b. Stratford, Conn., Oct. 27, I771; m. Polly Buckingham. He d. April 2, 1866 . She d. July 2, I 843 , aet. 67 years.

(27I) vii. Eben Birdsey, b. Stratford, Conn., April 22, 1773 ; m. Patty Morse. viii. Marcia R., b. Huntington, Conn., April 9, 1776; m. Elnathan Camp, of Huntington, Conn.

(272) ix. Ezekiel, b. Stratford, Conn., Feb. 3, I779; m. Anna Clark.

x. Lucy, b. Stratford, Conn., Feb. 3, 1782 .

xi. Aurilla, b. Stratford, Conn., May 22, $1784 ; \mathrm{m}$. Thomas G. Northrop, of Kent, Conn.

xii. Annis, b. Stratford, Conn., July 15, I 786 ; m. Ezra C. Wheeler, of Huntington, Conn.

I I I

EZRA CURTISS (Peter, Fosiah, William, Elizabeth), b. Stratford, Conn., June 6, I744; m. Ruth Ann, d. of Dr. Joseph Perry, Oct. 3, 1773. He d. in Huntington, Conn., March 29, I810. She d. Jan. 22, I8I3, aet. 55 years. He was a physician. 
They had children :-

(273) i. Harmon, b. Huntington, Conn., May 1 3, 1774.

ii. Ruth Ann, b. Huntington, Conn., May 3, I776; m. Atwell.

iii. Betsey, b. Huntington, Conn., Feb. 25,1778 ; m. Lewis.

iv. Sylvia, b. Huntington, Conn., Aug. I9, I 780 ; m. Agur Beardsley, of Monroe, Conn., 1779.

(274) v. George Preston, b. Huntington, Conn., July 10, 1782 ; m. Sarah, He d. March 22, I 856 . She d. Oct. 2, I 869 , aet. 83 years.

vi. Maria, b. Huntington, Conn., Feb. 12, I784; m. Sherwood.

(275) vii. Abel, b. Huntington, Conn., Dec. ro, 1785.

viii. Esther, b. Huntington, Conn., Nov. 30, 1 787 . She d. unm. Jan I 7 , 1825 .

(276) ix. Henry, b. Huntington, Conn., Jan. I, I 790.

x. Delia, b. Huntington, Conn., Oct. 30, 1791 ; m. Botsford.

xi. Sally, b. Huntington, Conn., Oct. Io, 1793.

xii. Julia, b. Huntington, Conn., Sept. 4, I 795 .

xiii. Eliza, b. Huntington, Conn., April r 2, I 797 ; m. Ezra Abel Beardsley, of Monroe, Conn. ; m. Jan. I 3, I 822.

I I 2

HENRY CURTISS (Peter, Fosiah, William, Elizabeth), b. Stratford, Conn., June 8, I754; m. Anne, d. of Zachariah and Amy Tomlinson, of Huntington, Conn., Aug. 14, I774. He d. Jan. II, I796, and she m. Rev. David Ely, of Huntington, Conn., She d. Jan. 3I, I849, aet. 9I years. He had the title of Captain (probably in the militia).

They had children : -

(277) i. William Pixlee, b. Huntington, Conn., May 29, 1778.

ii. Sarah Ann, b. Huntington, Conn., May 27, I780; m. Gideon Beardslcy, M. D.

iii. Lydia, b. Huntington, Conn., May 20, 1785; m. Elias Youngs Hawley, of Ballston, N. Y.

(278) iv. Lucius, b. Huntington, Conn., April 6, I 787 .

v. $\left\{\begin{array}{l}\text { Maria, b. Huntington, Conn., Dec. 29, I } 790 . \\ \text { Eloisa Ann, b. Huntington, Conn., Dec. 29, I } 790 ; \text { m. Elisha } \\ \text { Mills Ely, of }\end{array}\right\}$

(279) vii. Henry Tomlinson, b. Huntington, Conn., Sept. 2 I, I 796 ; d. July, 1876.

(280) viii. Hiram, b. Huntington, Conn., June 6, I 793.

113

ELIJAH CURTISS (Peter, Fosiah, William, Elizabeth), b. Stratford, Conn., $\quad 1747$; m. Jane, d. of John Moss. He d. 
in Huntington, Conn., 1817 . She d. 1813 , aged 81 . Will filed in Bridgeport, Conn., Oct., i 8 I7. Elijah Curtiss appears as an ensign in Capt. Samuel Beardslee's Company, Col. Samuel Whiting's Regiment, at Fishkill-on-Hudson, N. Y., Oct. 1777 .

They had children :-

(2SI) i. Charles, b. Monroe, Conn., $\quad$ I 758 ; m. Sarah Catherine, d. of Judson Lewis.

ii. Mary, b. Monroe, Conn., Feb. I, I770; m. (i.) Capt. Cyrus Hawley, of Monroe, Conn., Sept. I 2, I79I. m. (ii.) Hall Beardsley, She d. March 20, I 840.

iii. Sally M., b. Monroe, Conn.;

m. Sylvester Hinman.

iv. Nancy M., b. Monroe, Conn., June 19, I781; m. Gen. Eli L. Hawley, of Monroe, Conn., April 23, I800. She d. Feb. I 7, I 814.

v. Rebecca, b. Monroe, Conn.,

m.

Curtiss.

\section{4}

ELIHU CURTISS (Peter, Fosiah, William, Elizabelh), b. Stratford, Conn., I749; m. (i.) Sarah, d. of Zachariah and Amy Tomlinson, of Stratford, Conn., March 14, 1769; m. (ii.) Sarah, w. of Robert Lewis, and d. of John Moss. She d. Dec. I3, I 840 , aged 98. He d. in Huntington, Conn., Nov. 2, 1804. Will filed at Bridgeport, Jan. 7, 1805 . An Elihu Curtiss served as a private in the Revolutionary War.

They had children : -

i. Amy, b. Huntington, Conn., Feb. I9, I77I ; m. Lewis Nichols, of New York City, N. Y.

(282) ii. Zachariah Tomlinson, b. Huntington, Conn., May I0, 1774.

(283) iii. Isaac, b. Huntington, Conn., Oct. I 2, 1777.

iv. Rosillee, b. Huntington, Conn., Aug. 16, I 781 ; d. Oct. I 7, 1791.

v. Polly, b. Huntington, Conn., Jan. I, I 786 ; m. Agur Lewis.

vi. William, b. Huntington, Conn., July I 2, I 779 ; d. Dec. 30, I 79 I.

I 17

MATTHEW CURTISS (Matthew, Fosiah, William, Elizabeth), b. Newtown, Conn., Feb. 4, I746 ; m. Hannah Ford, of Newtown, Conn., March 25, 1773. He d. in Newtown, Conn., Nov. 29, 1796.

They had children : -

(284) i. Judson, b. Newtown, Conn., Jan. 8, I 774.

ii. Polly, b. Newtown, Conn., I 775.

iii. Richard, b. Newtown, Conn., Nov. 8, I 777 . He d. unm. I 799.

iv. Sarah Ann, b. Stratford, Conn., Feb. 26, 1780. 
REUBEN CURTISS (Matther, Fosiah, William, Elizabeth), b. Newtown, Conn., May I3, 1757; m. Silence, d. of Micah and Mary (White) Allen, of New Haven, Conn., Oct. 4, 1775. He d. in Danbury, Conn., I 8 I 6.

They had children : -

(285) i. Abner, b. Danbury, Conn., Dec. I 5, I 776; m. Mary, d. of Moses and Mary (White) Osborn, of Danbury, Conn., Nov. I, I 796. He d. May I 4, I 827 . She d. Aug. I 852, aet. 76 years.

ii. Nabby, b. Danbury, Conn., I778; m. Thomas Addison.

iii. Joseph, b. Danbury, Conn., I 78 I. d. y.

(286) iv. Joseph, b. Danbury, Conn., Sept. I 9, I782; m. Dorothy, d. of Stephen Hopkins, of Hempstead Harbor, L. I., in I 803 . He d. in New York City, N. Y., April, I 856.

(287) v. Matthew, b. Danbury, Conn., Jan. 5, I783; m. (i.) Polly Lacey, of Stratford, Conn., June 7, I 809 . She d. Nov. I 4, I 8 I 9 , aet. 33 years. m. (ii.) Rebecca - She d. Aug. IO, 1864, aet. 7 I years. He d. Aug. 25, I 864.

(288) vi. \{ David, b. Danbury, Conn., 1786.

vii. Phebe, b. Danbury, Conn., I 786.

(289) viii. Thomas, b. Danbury, Conn., I 788 ; m. Esther

ix. Mary, b. Danbury, Conn., I 790 ; m. Solomon Lyman, of Easthampton, Mass.

x. Hannah, b. Danbury, Conn., I 792 ; m. John L. Moffatt.

xi. Alben, b. Danbury, Conn., I 794 ; d. I 795 .

xii. Reuben, b. Danbury, Conn., I 796 ; d. 1797.

xiii. $\{$ Silence, b. Danbury, Conn., I 797 .

(29o) xiv. \{Reuben Judson, b. Danbury, Conn., I 797.

xv. Betsey, b. Danbury, Conn., I 799; m. Thomas Addison.

xvi. Adeline, b. Danbury, Conn., I800; m. William Allen. She d. 1832 .

xvii. Angeline, b. Danbury, Conn., I 800 .

122

GOLD CURTISS (Matthew, Fosiah, William, Elizabeth), b. Dec. 17, I763; m. Elizabeth Gould, of Fairfield, Conn., Dec. 2, I781. He was a farmer, and lived and died in Newtown, Conn. He d. March 22, 1840 . She d. Nov. 26, 1839 , aet. 80 years.

They had children :-

i. Abigail, b. Newtown, Conn., Oct. 14, r782; m. Cyrus Hurd, of Newtown, Conn., Dec. 5, 1802.

ii. Betsey, b. Newtown, Conn., March I 5, I 784 ; d. July 6, I 807 .

iii. Deborah, b. Newtown, Conn., March I 9, 1786 ; m. Hermon Warner, of Newtown, Conn. 
(29I) iv. Gould, b. Newtown, Conn., Dec. 27, I787; m. Joanna Peck, of Newtown, Conn.

v. Marilla, b. Newtown, Conn., March I I, I 790; d. unm.

(292) vi. John Gould, b. Newtown, Conn., March 3, 1792; m. Lucy Blackman, of Newtown, Conn.

vii. Phebe, b. Newtown, Conn., Oct. 25, 1793; d. July 18, I810.

(293) viii. Hezekiah, b. Newtown, Conn., March 25, 1796 ; m. Marcia Glover, of Newtown, Conn.

(297) ix. David, b. Newtown, Conn., July 28, I798; m. Jeannette Shelton, of Monroe, Conn.

(295) x. Daniel, b. Newtown, Conn., Jan. 26, r801 ; m. Elizabeth Beach, of Trumbull, Conn., March 4, I829. He d. June 21, 1835. She d. Oct. 27,1869 , aet. 64 years.

127

NATHAN CURTISS (Nathan, Fohn, Israel, Fohn, Elizabeth), b. Woodbury, Conn., April 3, 1748; m. (i.) Esther,

She d. April 5, I803, aged 53 years; m. (ii.) Martha, d. of Jehiel and Betterus Preston. She d. in Southbury, Conn., Feb. I 6, I 845 , aged 86 years. He d. in Southbury, Conn., Nov. 2 I, 1845 .

They had children :-

i. Elizabeth, bapt. Southbury, Conn., July 5, 1772.

ii. Martha, bapt. Southbury, Conn., March I3, 1774.

iii. Betty, bapt. Southbury, Conn., June 9, 1776; m. Truman Hinman, of Stratford, Conn., Nov. 22, 1798.

130

GIDEON CURTISS (Fohn, Fohn, Israel, Fohn, Elizabeth), b. Woodbury, Conn., June 8, I 735; m. Mary, d. of Stephen and Bethiah Hicock, of Southbury, Conn., Sept. 28, I756. He d. in Woodbury, Conn., I793. In August, 1757, he marched from Woodbury, Conn., as a private in Capt. Ebenezer Down's Company to the relief of Fort William Henry.

They had children : -

i. Beatrice, bapt. Woodbury, Conn., June 19, 1757.

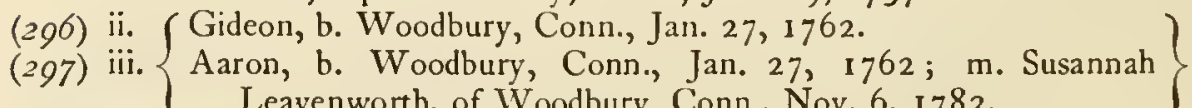
Leavenworth, of Woodbury, Conn., Nov. 6, 1783 .

iv. Susanna, bapt. Woodbury, Conn.

(29S) v. Eldad King, bapt. Woodbury, Conn., Aug. 7, 1773.

vi. Mary Eve, bapt. Woodbury, Conn., Sept. 24, 1775. 
JOHN CURTISS (Fohn, Fohn, Israel, Fohn, Elizabeth), bapt. Woodbury, Conn., Oct. 12, 1740; m.

Conn., $\quad 1803$.

They had children: -

(299) i. Sherman, b. Woodbury, Conn., Stratford, Conn., June 2, I 796.

m. Sarah Binham, of

(300) ii. Levi, b. Woodbury, Conn.

(30r) iii. John, b. Woodbury, Conn.

\section{2}

PETER BURR CURTISS (Peter, Fohn, Israel, Fohn, Elizabeth), b. Kent, Conn., April 30, 1755 ; m. Phebe, d. of Job Sherman, of New Haven, Conn., Nov. 12, 1775. He d. in Lanesboro, Mass., March 25, 1837. She d. Dec. I2, 1830. He was taken to Lanesboro by his father in 1759 , where he became a prominent citizen. At the Lexington Alarm, in April, 1775, he enlisted in the Revolutionary Army as a private, and served until 1780. In March, 1782 , he was chosen town treasurer, and served in that position a number of years. After leaving the army, he opened a hotel in Lanesboro, which he kept until his death. His will was filed in Pittsfield, Mass., July 1 I, I 837.

They had children:-

i. Mary, b. Lanesboro, Mass., Oct. 2, I 777; m. Amos Foot.

(302) ii. Peter Burr, b. Lanesboro, Mass., Sept. 27, 1779; m. Tammah Mead. She d. Nov. 29, 1860 . He d. March 9, 1837.

iii. Electa, b. Lanesboro, Mass., April 22, 1781; m. John Rockwell Wildman, Sept. 19, I 800.

iv. Eunice, b. Lanesboro, Mass., Oct. 10, 1782; m. David Babbitt, Feb. 27,1808 .

(303) v. Burr, b. Lanesboro, Mass., March 19, 1784; m. Lucy Brown, of Lanesboro, Mass., March 17, 1808. He d. June 6, 1825.

(304) vi. Almond, b. Lanesboro, Mass., April 22, 1786 ; m. Lucy Sprague, of Lanesboro, Mass., Dec. 24, 1806. He d. Nov. 19, 1864. She d. Jan. 26, $186 \mathrm{I}$.

vii. Roxa, b. Lanesboro, Mass., Dec. 8, I787; m. William Smith, Feb. 6, 1806 . She d. Nov., 1855.

(305) viii. David, b. Lanesboro, Mass., June 21,$1789 ;$ m. Rachel Cowan in I 8 I0. He d. Jan. 8, 184 I. She d. May 25, 1884.

(306) ix. Henry, b. Lanesboro, Mass., April I, I 791 ; m. Hannah Lyman, of Hinsdale, Mass. He d. July 26, 1867.

x. Sally, b. Lanesboro, Mass., Nov. 25, 1792. She d. unm. July 6, 1843 . 
xi. Abel Sherman, b. Lanesboro, Mass., July 3, I 794. He d. March 27, I 796.

xii. Lucy, b. Lanesboro, Mass., Dec. 21, I 796. She d. unm. Feb. 28, 1877 .

(307) xiii. Sherman, b. Lanesboro, Mass., April I3, I799; m. Maria, d. of Silas Butler, of Lanesboro, Mass., April 25, I 820. He d. Jan. 23, I 877 . She d. Sept. 20, I 888 , aet. 89 years.

\section{33}

DAVID CURTISS (David, Fohn, Israel, Fohn, Elizabeth), bapt. Woodbury, Conn., Jan. 30, I743; m. Sarah, d. of Capt. Matthew Minor, of Woodbury, Conn., Sept. 27, I764. He d. in Woodbury, Conn., Nov. I I, I819. She d. Feb. I4, I820, aet. 77 years.

\section{They had children :-}

i. Phebe, b. Woodbury, Conn., Feb. I4, I765; m. Anthony Strong, of Woodbury, Conn., Nov. 13, 1791. She d. March 25, 1836.

(308) ii. David Stiles, b. Woodbury, Conn., Jan. 14, 1 767 ; m. Sybilla, d. of Daniel and Sybilla Huntington, of Woodbury, Conn. She d. Dec. 30,1837 , aet. 68 years. He d. Jan. 22, 1846.

(309) iii. William Preston, b. Woodbury, Conn., May I I, I 769.

iv. Anna, b. Woodbury, Conn., Oct. 26, 177 ; m. Aaron Sherman, of Woodbury, Conn., Feb. 19, I 795. She d. Feb. I8, 1847 .

v. Sarah, b. Woodbury, Conn., Jan. 23, 1774; m. Curtiss Hicock, of Washington, Conn., July 2I, 1796.

vi. Elizabeth, b. Woodbury, Conn., Oct. 26, 1776; m. Bennet French. vii. Currence, bapt. Woodbury, Conn., Oct. 5, I $78_{3} ; \mathrm{m}$. Rev. Philo Judson.

\section{34}

ASA CURTISS (David, Fohn, Israel, Fohn, Elizabeth), bapt. Woodbury, Conn., Aug. 3, I 746 ; m. Eunice Woodward, of Woodbury, Conn., Oct. 9, I765. He d. Sept. I, I794. He served in the Army of the Revolution under Capt. Thomas Ball, and was with Washington during his retreat through New Jersey in December, I 776 .

They had children : -

i. Leonia, b. Woodbury, Conn., March 4, I767; m. Gideon Munn, Jan. 6, 1785 .

(3Io) ii. Russell, b. Woodbury, Conn., March I, I769.

(3II) iii. Truman, b. Woodbury, Conn.

iv. Olive, b. Woodbury, Conn.

v. Mary, b. Woodbury, Conn.,

vi. Eunice, b. Woodbury, Conn.,

m. James Hinman.

m. David Hawley. 
THADDEUS CURTISS (Foseph, Stephen, Israel, Fohn, Elizabeth), bapt. Woodbury, Conn., A pril 24, I 726; m. (Mary, d. of Elisha?) Stoddard, of Woodbury, Conn. In August, 1757, he marched as a private in Capt. Ebenezer Down's Company, which marched from Woodbury, Conn., to the relief of Fort William Henry. In 1765 he removed to Lanesboro, Mass., and in 1780 to Rutland, $\mathrm{Vt}$.

They had children :-

i. Mary, b. Woodbury, Conn., April 8, 1750; d. y.

ii. Sarah, b. Woodbury, Conn., Sept. 24, 1752.

(312) iii. Thaddeus, b. Woodbury, Conn., Sept. 29, 1754.

iv. Mary, b. Woodbury, Conn., July i I, 1756; m. Wolcott Hubbell, of Woodbury, Conn., August , 1775. She d. April 19, 1836.

v. Elizabeth, b. Woodbury, Conn., Feb. I $9,1758$.

vi. Prudence, b. Woodbury, Conn., May 25, I 760.

\section{36}

JOSEPH CURTISS (Israel, Stephen, Israel, fohn, Elizabeth), b. Woodbury, Conn., March 22, 1740; m. Keturah Hatch, of Woodbury, Conn., July II, 1763. She d. in Southbury, Conn., April I9, I797, aet. 52 years. He d. in Southbury, Conn., Jan. 9, 1798 .

They had children :-

i. Hannah, b. Southbury, Conn., Aug. 29, I764; m. David Mallory, of Southbury, Conn., Sept. 30, 1783 .

(313) ii. Simeon, b. Southbury, Conn., Jan. 2, 1768 ; m. Mary Bradley, of Southbury, Conn., Aug. I2, 1790. He d. April 2, 1852. She d. Jan. 19,1850 .

(314) iii. Amos, b. Southbury, Conn., March 29, 1771. He d. Nov. 9, 1834.

(315) iv. Abel, b. Southbury, Conn., March 2, 1776; m. Hannah Atwater, of Southbury, Conn., Feb. I, I 802 . He d. Nov. 9, 1834 .

v. Eunice, b. Southbury, Conn., June 30, 1781 ; m. Jared Munson, of Southbury, Conn., Jan. 3, 1805 . She d. Sept. 20, 1838 .

137

ISRAEL CURTISS (Israel, Stephen, Israel, Fohn, Elizabeth), b. Woodbury, Conn., April 24, 1742; m. Abigail, d. of Gideon and Olive Mallory, of Woodbury, Conn., May 9, I769. He was a soldier in the War of the Revolution

They had children :-

(316) i. Truman, b. Woodbury, Conn., April 8, $177^{\circ}$

ii. Polly, b. Woodbury, Conn., Aug. 4, I77I. 
iii. Charlotte, b. Woodbury, Conn., Oct. 30, 1773 .

iv. Mary, b. Woodbury, Conn., Feb. 24, I 776.

(3I7) v. Martin Luther, b. Woodbury, Conn., Oct. 19, 1778.

vi. Alathea, b. Woodbury, Conn., Aug. 23, 1785 .

\section{$13^{8}$}

STEPHEN CURTISS (Israel, Stephen, Israel, Fohn, Elizabeth), b. Woodbury, Conn., Jan. 8, 1744; m. Mary, d. of John and Mary Mallory, of Woodbury, Conn., in 1772 . H d. in Woodbury, Conn., Feb. 15,18 I 3. He was a farmer.

They had children : -

(31S) i. Abner, b. Southbury, Conn., Aug. , 1772.

ii. Amy, b. Woodbury, Conn., Feb. , I $774 ; \mathrm{m}$. Mr. Tomlinson.

(319) iii. Sheldon, b. Woodbury, Conn., Jan. , 1776.

iv. Mabel, b. Woodbury, Conn., July , I 778 .

v. Nabby, b. Woodbury, Conn., Aug. , i 780 ; m. Mr. Hinman.

vi. Betsey, b. Woodbury, Conn., April , I 783 .

(320) vii. Stephen, b. Woodbury, Conn., July , I 786.

(321) viii. Charles Ransom, b. Southbury, Conn., May 7, I 788 ; m. Jennet, d. of Benjamin and Esther Preston Stiles, of Southbury, Conn., March 25, I 8 I 7 . She d. I 875 , aet. 85 years. He d. I 844.

ix. Leony, b. Southbury, Conn., Aug. , 1790.

(322) x. Louis, b. Southbury, Conn., May , 1792.

140

BENJAMIN CURTISS (Israel, Stephen, Israel, John, Elizabeth), b. Woodbury, Conn., May 18, 175 I; m. Esther Bennham, of Southbury, Conn., June 1, I778. He d. July 28, 1798.

They had children :-

(323) i. Japhet, b. Southbury, Conn., Dec. 1 2, I 779 ; m. Lucy Strong, of Southbury, Conn., June 5, I 805 .

(324) ii. Sherman, b. Southbury, Conn., Janl. 26, I 78 I ; m. Jerusha, d. of Thaddeus Bronson, Jan. 25, i 78 I. He d. I 848 .

(325) iii. Cyrus, b. Southbury, Conn., Dec. I 2, I 786 ; m. Julia, d. of Benjamin Strong.

iv. Anne, b. Southbury, Conn., Oct. 7, I789; m. David Porter, of Middlebury, Conn.

ABEL CURTISS (Benjamin, Nathaniel, Thomas, Folm, Elizabeth), b. Wallingford, Conn., Dec. 22, 1729; m. (i.) Hannah Foster, of Wallingford, Conn., March 7, 1759; m. (ii.) Patience, widow of Isaac Wickham. He d. Nov. I, 1797. She d. Dec. 22, 1828, aet. 86 years. He was tax collector for Meriden in 1775, and took the oath of fidelity April 2, 1779. 
They had children :-

i. Sarah, b. Wallingford, Conn., Oct. I0, I 759 ; m. Thomas Tryon.

(326) ii. Jauncey, b. Meriden, Conn., I 768 ; m. Jerusha Hall, of Wallingford, Conn., Dec. 3, I 793. He d. Oct. 7, I845.

(327) iii. Harvey, b. Meriden, Conn., Middletown, Conn., Jan. 30, 1825 .

(328) iv. Andrew, b. Meriden, Conn., m. Huldah Curtiss, of Harwinton, Conn., Nov. I3, I 792. He d. Sept. 23, I80 I.

v. Polly, b. Meriden, Conn.,

m. Amos Curtiss, of Wallingford, Conn., March I 8, I802. She d. April 30, 1818.

(329) vi. Levi, b. Meriden, Conn.,

m. (i.) Lucy Hall, March 28, I 790 ; m. (ii.) Sarah Francis, Dec. 6, I 792.

vii. William, b. Meriden, Conn., I781. He d. Oct. I4, I80o.

(330) viii. Chauncey, b. Meriden, Conn., Dec. 20, I 786 ; m. Anna Hall, Sept. 9, 1804 . He d. Dec. 6, 1824. She d. Nov. 5, 1826.

ix. Hannah, b. Meriden, Conn., m. Oliver Andrews, Dec. $27,1796$.

\section{4}

BENJAMIN CURTISS (Benjamin, Nathaniel, Thomas, Fohn, Elizabeth), b. Wallingford, Conn., Oct. 27, I735; m. Mindwell, d. of Daniel Hough, of Wallingford, Conn., March 31, I763. He d. Jan. 16, I 822. She d. June 8, I 807. He was town surveyor in 1775 and 1776 , and signed the oath of fidelity in 1777 . He was one of the largest landowners in Wallingford, and became a most prominent citizen. He was a man of strong individuality, shrewd and successful. He served in the French and Indian wars during the campaign of $175^{8}$, in the same company of which his future father-in-law was ensign.

They had children :-

i. Lucy, b. Wallingford, Conn., Feb. I4, I 764; m. Whiting Andrews, and moved to Claremont, N. H.

(33I) ii. Aaron, b. Wallingford, Conn., Feb. 9, I 765; m. Susanna Butler, Oct. I 3, 1793 .

(332) iii. Benjamin, b. Wallingford, Conn., May I9, I766; m. Molly, d. of Capt. Daniel Collins, Jan. 22, I 796. He d. Oct. 23, 1843. She d. Feb. 20, I 83 , aet. 60 years.

iv. Eunice, b. Wallingford, Conn., Jan. I 3, I 768 ; m. Judge Nathaniel Merriam, of Wallingford, Conn., Dec. 2, 1792, and removed to Leydon, Lewis Co., N. Y., in 1800.

v. Amasa, b. Wallingford, Conn., June I9, I770; m. Mehitabel Hall, of Wallingford, Conn., Aug. 4, I796, and d. Dec. 2, I808. He was a merchant in Meriden, and did a large business. In 1803 he was a representative from Wallingford, and from Meriden in I 807 . He was a member of the Compass Lodge of Wallingford previous to 1822 . 
They had a child :-

i. Cornelia, b. Wallingford, Conn., Sept. 28, 1798; m. Dr. Benjamin Potwin, of Tolland, Conn., and settled in Ellington, N. Y.

(333) vi. Ivah, b. Wallingford, Conn., Oct. I9, I77 I m. Hannah Ives, Aug. 4; 1796 . He d. May 27, 1852 . She d. Oct. 8, 1844 , aet. 66 years.

(337) vii. Elisha, b. Wallingford, Conn., June I 9 , I 773 ; m. Abigail, d. of Capt. Enos Hall, of Walling ford, Conn., Feb. I8, 1796. He d. July 28,1847 . She d. Feb. 28,1826 , aet. 47 years.

viii. Lydia, b. Wallingford, Conn., May 4, 1775. She d. June 1777 .

ix. Ruth, b. Wallingford, Conn., March 18,1777 ; m. Silas Rice, Aug. $4,1796$.

(335) x. Amos, b. Wallingford, Conn., April 14, I779; m. (i.) Polly, d. of Abel Curtiss, of Wallingford, Conn., March 18, I802. She d. April 30, 1818 , aet. 34 years. m. (ii.) Louisa Johnson, Dec. 6, I8 18. He d. Sept. 25, I857. She d. in Williamsport, Pa., April I6, 1872, aet. 8 I years.

(336) xi. Samuel, b. Wallingford, Conn., June 30, I78 i ; m. Polly Andrews, Nov. 20, 1805. She d. Nov. 5, 1846, aet. 61 years. He d. Nov. 8,1838 .

xii. Lydia, b. Wallingford, Conn., Feb. 5, I 785 ; m. Col. Elijah Smith, of Tolland, Conn., July I6, I 8 i 1 .

(337) xiii. Asahel, b. Wallingford, Conn., July 2, I 786 ; m. Mehitable, d. of Augustus Redfield, of Clinton, Conn., Nov. 8, $18 \mathrm{I} 2$.

(338) xiv. Roswell, b. Wallingford, Conn., Dec. 24, i788; m. Eunice Scott, July 30 , I 809 .

\section{46}

ENOS CURTISS (Enos, Nathaniel, Thomas, Fohn, Elizabeth), m. Abigail, He was taken to Stockbridge by his father in 1761 . In 1769 his father gave him land in Lenox. In I801 he sold this land and removed to Queensborough, Washington Co., N. Y. He was a soldier in the Revolutionary War, and appears with rank of sergeant in Capt. Jos. Woodbridge's Company, Col. Brown's Regiment, which marched from Stockbridge on the evacuation of Ticonderoga.

They had children :-

(339) i. Thomas Yale, b. Stockbridge, Mass., Oct. I 2, I 773.

(340) ii. Justus, b. Stockbridge, Mass., April 27, 1775.

(37I) iii. Levi, b. Stockbridge, Mass., June 6, 1777.

I 50

NATHANIEL CURTISS (Nathaniel, Nathaniel, Thomas, Fohn, Elizabeth), b. Wallingford, Conn., June 13, 1756; m. Esther 
Parker. He was a farmer, and lived in Falls Plains, a part of Wallingford now called Hanover.

They had children : -

(342) i. Charles, b. Wallingford, Conn.,

(3+3) ii. Horace, b. Wallingford, Conn.,

He d. Oct. Io, I 826.

$27, \quad 1832$.

m. Julia Bradley, Jan.

151

JACOB CURTISS (Nathaniel, Nathaniel, Thomas, Fohn, Elizabeth), b. Meriden, Conn., Sept. 14, 1758; m. Catherine,

They had children :-

(374) i. Amasa, b. Wallingford, Conn., Feb. 18, 178g.

ii. Nancy, b. Wallingford, Conn., March 27, 1792. She d. April 25, I 795 .

(345) iii. Ira, b. Wallingford, Conn., Jan. 21, I 794.

(346) iv. Asahel, b. Wallingford, Conn., May i I, I 796.

v. Lois, b. Wallingford, Conn., Dec. 24, 1797. She d. June 22, 1800.

(377) vi. Jacob, b. Wallingford, Conn., Aug. 4, 1800.

vii. Nancy, b. Wallingford, Conn., Feb. I I, 1802 .

I 52

SOLOMON CURTISS (Samuel, Samuel, Thomas, Fohn, Elizabeth), b. Wallingford, Conn., June 2, 1741; m. Abigail, d. of Zachariah Gillett, of Southington, Conn., Feb. 13, 1766. She d. June 8,1820 , aet. 78 years. He d. Aug. 1, 1820 . He was a farmer, and lived in Southington, Conn.

They had children : -

(343) i. Levi, b. Southington, Conn., Jan. 10, I772; m. Nancy, d. of Cornelius and Jemima Andrews Dunham. He d. May $3 \mathrm{I}$, 1840. She d. June 12, 1831 , aet. 60 years.

ii. Sarah, b. Southington, Conn., Nov. 6, I 776 .

iii. Solomon, bapt. Southington, Conn., Dec. 24, r78o. He d. Dec. I 9, I 793 .

iv. Eunice, b. Southington, Conn., April 16, 1778.

v. Abigail, b. Southington, Conn., Dec. Io, I 779 ; m. Levi Barnes, Oct. 29, 1797 .

vi. Lydia, bapt. Southington, Conn., Dec. 24, I780; m. Samuel Barnes, Jan. 19, 1803 .

vii. Ruth, bapt. Southington, Conn., Sept. 1 2, 1784.

(349) viii. Samuel, bapt. Southington, Conn., May 27, 1787; m. Polly Phelps Nov. 20, 1808.

I 56

URIE CURTISS (Azor, Samuel, Thomas, Fohn, Elizabeth), b. Lanesboro, Mass., July 29, 1767 ; m. Huldah, d. of David and Martha 
(Chatfield) Le Baron. He d. in Rutland Co., Vt. She d. in Le Roy, N. Y.

They had children :-

(350) i. Azor, b. Rutland Co., Vt., July 23, I792; m. (i.) Catherine Fowler, Dec. 20, I8I 2 ; m. (ii.) Eliza Ann Standing, Oct. 28, I840. He d. in Le Roy, N. Y., Aug. 5, I880.

ii. Sally, b. Rutland Co., Vt.; m. Simeon Clark.

iii. Laura, b. Rutland Co., Vt. She d. unm. in the West.

iv. Huldah, b. Rutland Co., Vt. She d. unm. in the West.

\section{3}

GILBERT CURTISS (Thomas, Thomas, Thomas, Fohn, Elizabeth), b. Cheshire, Conn., April 30, I76I ; m. Lucy Smith, of Cheshire, Conn., Dec. 8, 1784. Administration on his estate granted Sept. 24,1808 .

They had children :-

i. Silas, b. Cheshire, Conn., I 805 .

ii. Patience, b. Cheshire, Conn.,

iii. Betsey, b. Cheshire, Conn.,

iv. Esther, b. Cheshire, Conn.,

m. Lumaud Cook, Dec. 22,

m. Bunnell.

\section{5}

ISRAEL CURTISS (Peter, Joseph, Thomas, Fohn, Elizabeth), b. Meriden, Conn., m. Elizabeth Andrews, of Simsbury, Conn., March 10, 1756 . He removed to Windsor, Cumberland Co., N. Y., previous to the Revolutionary War. He was a soldier in the Northern Continental Army, and attained the rank of major. Hed. in the army May $19,1776$.

They had children :-

(35I) i. Zebina, bapt. Farmington, Conn., Sept. 2 I, 1760. He settled in Windsor, Cumberland Co., N. Y., where he became most prominent.

ii. Elizabeth, bapt. Farmington, Conn., d. y.

166

ELIPHALET CURTISS (Peter, Foseph, Thomas, Fohn, Elizabeth), bapt. Meriden, Conn., Feb. I735-6; m. Margaret, d. of Benjamin Dyar, of Simsbury, Conn., March 24, 1757. He was a representative from Simsbury in 1780 . In 1800 he sold his farm in West Simsbury, Conn., and removed to Western New York, where he died in 1806. She died in I8I2. He enlisted in the army at the Lexington Alarm, and served from 
May 4, I775, to Dec. I 8, I775, as a sergeant in Gen. Spencer's Regiment, Seventh Company, Capt. Abel Pettebone. He served during the Siege of Boston, as second lieutenant under Gen. Gates, Col. Mott's Fifth Company, and also at Fort Ticonderoga and vicinity. Captain at the Danbury raid, April 25, 1772. In New Haven Alarm, July 5, I777, as captain. Captain Eighteenth Militia Regiment, 1778 .

They had children :-

(352) i. Eliphalet, b. Simsbury, Conn., Nov. 9, 1758 ; m. Deborah, and $\mathrm{d}$. $\quad$ I $8 \mathrm{I} 6$.

ii. Margaret, b. Simsbury, Conn., June I4, I76I. d. I 798, unm.

iii. Agnes, b. Simsbury, Conn., Feb. 26, 1763; m. Solomon Dill, Jr.

iv. Chestina, b. Simsbury, Conn., Sept. 29, I 764 ; m. Solomon Woodruff.

v. Theodosia, b. Simsbury, Conn., m. (ii.) Banajah Holcomb, Jr.

vi. Narcissa, b. Simsbury, Conn., m. (i.) Zebina Moses;

(353) vii. Horace, b. Simsbury, Conn., I77 ; m. Chloe Poison. He settled in Whitestone, Oneida Co., N. Y.

(354) viii. Gad, b. Simsbury, Conn., I774; m. Cherissa, d. of Amos Wilcox, and d. I 853 .

(355) ix. Israel, b. Simsbury, Conn.,

x. Sarah, b. Simsbury, Conn., I780. d. $\quad$ I 785 .

\section{7}

PETER CURTISS (Peter, Foseph, Thomas, Fohn, Elizabeth), bapt. Meriden, Comn., June 8, I740; m. Ruth, Dec. 28,1815 , aet. 80 years. He settled in Farmington, Conn., and died there, May 19, 1797. Dec. 12, 1774, he was elected one of Committee of Inspection. At the Lexington Alarm he enlisted and served as lieutenant under Capt. Hooker, Sixth Company, Gen. Spencer, May 1, 1775. Dec. 18, 1775, this Company took post at Roxbury, and served during the siege of Boston. November, 1776, he was appointed captain Third Battalion, Col. Ely, under Gen. Spencer. He was at Peekskill under Col. Whiting in October, 1777. At the New Haven Alarm, July 5, 1777. After the Revolutionary War, he was made a brigade major, and also a major of the line. He was the first keeper of the Newgate Prison in Granby (1790), and left it in 1796 in declining health. He was admitted to the Society of the Cincinnati, July 7,1784 .

They had children :-

(356) i. Adna, bapt. Farmington, Conn., 1766; m. Almira

He d. May 25, I8 I 5. She d. Sept. I 8, I 848, aet. 8 I years.

ii. Jeptha, bapt. Farmington, Conn., Sept. 4, 1768. 
iii. Rhoda, bapt. Farmington, Conn., June 26, 1774 .

(357) iv. Charles, bapt. Farmington, Conn., March 29, I 778.

v. Nancy, bapt. Farmington, Conn., m. Danie! Newell.

vi. Betsey, bapt. Farmington, Conn., m. David Lash.

vii. Chloe, bapt. Farmington, Conn., m. Appleton Robbins.

\section{68}

GIDEON CURTISS (Peter, Foseph, Thomas, Fohn, Elizabeth), b. Simsbury, Conn., I752; m. Elizabeth, d. of Rev. Gideon Mills. Hed. on June 8, I789. His widow m. Rev. Rufus Hawley, of Avon, and died in 1825 . He was a blacksmith. At the call for troops at the Lexington Alarm, he enlisted as a private in Capt. Amos Wilcox's Company.

They had children : -

i. Child, b. Simsbury, Conn., I774. d. $\quad$ I774.

ii. Gideon, b. Simsbury, Conn., Nov. $18,1776$.

(358) iii. Solomon, b. Simsbury, Conn., March 2, 1778; m. a d. of Samuel Eggleston.

(359) iv. Drayton Mills, b. Simsbury, Conn., Oct. 7, 1783.

(360) v. Norman, b. Simsbury, Conn., Feb. 20, 1785.

vi. Elizabeth, b. Simsbury, Conn., Feb. 9, 1788 ; m. Thomas Gleason, of Avon.

\section{0}

AMASA CURTISS (Fonathan, Foseph, Thomas, Fohn, Elizabeth), b. July 7, I 743; m. Lucy, d. of Isaac Garfield, of Monterey, Mass. (Tyringham). In the War of the Revolution he served as sergeant in Capt. Solomon Jackson's Company, Col. John Ashley's Regiment. He d. in Tyringham, Mass., I 822.

They had children :-

i. Diantha, b. Tyringham, Mass., Aug. 9, I 770 . She d. April I o, I 77 I.

ii. Lucy, b. Tyringham, Mass., Feb. 26, 1772.

iii. Diantha, b. Tyringham, Mass., Dec. 27, 1773.

(361) iv. Amasa, b. Tyringham, Mass., Aug. 17, 1776; m. Electra,

v. Mary (Mercy), b. Tyringham, Mass., Sept. 23, 1778. She d. Sept. 29,1783 .

vi. Sarah, b. Tyringham, Mass., Sept. 26, 1 780 . She d. Sept. 30, I 780.

(362) vii. Zebinah, b. Tyringham, Mass., Nov. 25, 1781 ; m. Julia Buel, of Monterey, Mass.

(363) viii. Caleb, b. Tyringham, Mass., Feb. 3,$1784 ; \mathrm{m}$. Hines.

ix. Sally, b. Tyringham, Mass., Nov. 28,1785 .

x. Rhoda, b. Tyringham, Mass., Feb. 28, 1788 ; m. John Langdon, of Monterey, Mass.

xi. Mercy, b. Tyringham, Mass., Jan. 14, I790; m. John Langdon, of Monterey, Mass.

xii. Electa, b. Tyringham, Mass., March 30, I 792. 
JONATHAN CURTISS (Fonathan, Foseph, Thomas, Fohn, Elizabeth), b. Wallingford, Conn., Oct. 28, 1747 ; m. (i.) Mary, d. of Richard and Thankful Kellogg Jacobs, of Sheffeld, Mass., in I768. She d. Aug. I 7, I 813, aet. 64 years. m. (ii.) Prudence, She d. July I I, I 827 , aet. 76 years. He d. April 7 , 1829. He was taken when a boy by his father to Wethersfield, Conn., and then removed to Sheffield about 1760-5. He was a farmer.

They had children :-

(364) i. Joseph, b. Sheffield, Mass., June 25, I768; m. Ruth, d. of Abner Roys, of Sheffield, in 1790.

ii. Josiah, b. Sheffield, Mass., April 29, 1770 . He d.

iii. Mary, b. Sheffield, Mass., I 1772 . She d. 1775 . 1775 .

(365) iv. Abijah, b. Sheffield, Mass., June 7, I773; m. Betsey, d. of Lieut. Thomas and Hannah Stevenson, of Middletown, Conn., Sept. 1 793. He d. Feb. I7, I834. She d. Nov. I6, I 858 .

(366) v. Josiah, b. Sheffield, Mass., Nov. 3, I775; m. Mary, d. of Timothy Kellogg, of South Egremont, Mass., Nov. 23, 1800. He d. Oct. 18, 1819. She d. March 4, 1860, aet. 80 years.

vi. Amanda, b. Sheffield, Mass., $\quad$ 1778; m. Isaac Roys, 1798. vii. Ira, b. Sheffield, Mass., d. $y$.

viii. Thankful, b. Sheffield, Mass., May 7, 1787 ; m. Capt. Levi Roys, I 804. She d. I 84 I.

ix. Aurora, b. Sheffield, Mass., March 1789; m. Donald Kellogg.

x. Sabra, b. Sheffield, Mass., May 14, I791; m. Capt. Asa Haskins. She d. Jan. 2, I 869 .

xi. Pamelia, b. Sheffield, Mass., Dec. 23, I793; m. Samuel Newman, Jr., 18 r 3 .

xii. Laura L., b. Sheffield, Mass., June 13, 1797. She d. May 25, I859, unm.

ELISHA CURTISS (Fonathan, Foseph, Thomas, Fohn, Elizabeth), b. Wallingford, Conn., Feb. 26, I752; m. Sarah,

They had children :-

i. Lucy, b. Wallingford, Conn., Oct. 9, 1776.

ii. Sarah, b. Wallingford, Conn., July 4, 1779 .

(367) iii. Caleb, b. Wallingford, Conn., June 9, I 782 . 
DAVID CURTISS (Fames, Foseph, Thomas, Fohn, Elizabeth), b. Wallingford, Conn., Feb. I, I740; m. (i.) Eunice, d. of Elisha Perkins, of New Haven, Conn., July 30, I 76 I ; m. (ii.) Huldah Andrews, March I3, I783. He d. April 4, I 82 I. She d. Nov. 23,1827 , aet. 76 years.

They had children :-

i. Eunice, b. Cheshire, Conn., April 7, 1762.

ii. Damaris, b. Cheshire, Conn.,

(37I) iii. David, b. Cheshire, Conn.,

iv. Hannah, b. Cheshire, Conn.,

v. Loly, b. Cheshire, Conn.,

vi. Nancy, b. Cheshire, Conn.,

m. Eben Bunnell, July 2, I 788 .

vii. Sally, b. Cheshire, Conn.,

viii. Miriam, b. Cheshire, Conn.,

ix. Lucy, b. Cheshire, Conn.

x. Hila Andrews, bapt. Cheshire, Conn., Sept. I, I 793 .

xi. Huldah, bapt. Cheshire, Conn., Aug. 17, 1794.

(372) xii. Leonard Chester, b. Cheshire, Conn., Hitchcock, July 23, I809.

(373) xiii. James Herod, b. Cheshire, Conn., Aug. 20, 1809.

$1787 ; \mathrm{m}$. Abigail

1790 ; m. Ruth Brooks,

177

ABNER CURTISS (Abner, Foseph, Thomas, Fohn, Elizabeth), b. Wallingford, Conn., Aug. 8, I738; m. Mirriam Clark, Jan. 8, $176 \mathrm{I}$. He was given land by his father in Farmington, Conn. Farmington Town Records, Dec. 25, I 780 : "Voted on Memorial of Abner Curtiss, Jr., respecting his sons in Continental Army to grant them the sum of ten pounds state money same as to those that enlisted June last. Also voted to abate tax to Abner Curtiss on List I779." In September, I 765, he was living in Cambridge, Conn.

They had children :-

i. Phineas, b. Wallingford, Conn., Oct. 25, 176r. He d. Nov. I, I 761 .

(374) ii. Chauncy, b. Wallingford, Conn., Oct. 31, 1762. He was a soldier in the Revolutionary War.

(375) iii. Clark, b. Wallingford, Conn., April 29, 1764. He was a soldier in the Revolutionary War. 


\section{8}

JESSE CURTISS (Abner, Foseph, Thomas, Fohn, Elizabeth), b. Farmington, Conn., April 2, I 748; m. Martha, d. of Josiah and Keziah Root, of Farmington, Conn., Dec. 2, I772. She d. in Meriden, Conn., Nov. 7, I 830 , aet. 80 years. He d. July 4 , 1823. He lived and died in Farmington, Conn. Jesse Curtiss enlisted at Waterbury in the Revolutionary Army, and attained the rank of major.

They had children :-

i. Lucy, b. Farmington, Conn., March 22, 1774. She d. unm. July I 2,1833 .

ii. Azubah Smith, b. Farmington, Conn., April 6, 1776. d. Nov. 24, 1777.

(376) iii. Azubah Smith, b. Farmington, Conn., Oct. 27, 1778.

(377) iv. Marcus, b. Farmington, Conn., Nov. 13, 1781. He d. Dec. 1 1, I 8 I 2.

(378) v. (Jesse, b. Farmington, Conn., July I 3,1785 ; m. Rhoda Pantlin, ) Oct. 11, I 8 10. He d. Sept. I1, I855. She d. Aug. 10,

vi. $\begin{gathered}1844, \text { aet. } 76 \text { years. } \\ \text { Benoni, b. Farmington, Conn., July } 13,1785 \text {; d. Aug. } 7,1785 \text {. }\end{gathered}$

vii. Phebe, b. Farmington, Conn., Aug. 4,1787 .

viii. Julia, b. Farmington, Conn., March 9, 1790.

ix. \{ Catherine, b. Farmington, Conn., Aug. I9, 1792.

x. $\{$ Nancy, b. Farmington, Conn., Aug. I 9, 1 792. $\}$

xi. Susan, b. Farmington, Conn., Oct. 26, 1794.

xii. Martha, b. Farmington, Conn., July 24, 1797 .

I 80

AMOS CURTISS (Abner, Foseph, Thomas, Fohn, Elizabeth), b. Wallingford, Conn., April 4, I752; m. His will was dated at New Hartford, Conn., May 27, i821. Filed April 21, I 821 .

They had children : -

(379) i. Amos, b.

(380) ii. Phineas, b.,

iii. Abigail, b.,

iv. Huldah, b.,

v. Shuvall, b.,

$(38 I)$ vi. Alvan, b.,

I 8 I

CALEB CURTISS (Caleb, Foseph, Thomas, Fohn, Elizabeth), b. Charlton, Mass., March I2, I770; m. (i.) Polly Davis, of Charlton, Mass., Feb. I2, 1794. She d. Jan. 4, 1801, aet. 25 years; m. (ii.) Anna, d. of Samuel Robinson. She d. April 27, 
I 8 I 4, aet. 37 years; m. (iii.) a widow, Mrs. Daggett. He removed to Calais, Vt., in 1795 , and became a prominent man in that town. He was colonel in the militia. He d. in Calais, Vt., Jan. 4, 1836 .

They had children :-

(382) i. Pliny, b. Charleton, Mass., Nov. 14, I 795; m. Relief, d. of Darius Boydon, of East Montpelier, Vt., Dec. I 7, I8 18.

ii. Ruth, b. Calais, Vt., Jan. I I, I 799 ; m. John Gilman, M. D., May 20, 1819. She d. July 30, 1865 .

iii. Polly, b. Calais, Vt., July 6, 1804 ; m. Ira Kent, of Calais, Vt., Aug. 3 I, I83 I. She d. Jan. 24, I88 I.

iv. Stillman H., b. Calais, Vt., Dec. 20, 1808 . He d. unm. in Plainfield, March , I 844 .

v. Amanda, b. Calais, Vt., July 9, I810; m. Libeus H. Chase. She d. March 23, I837.

vi. Minerva, b. Calais, Vt., April 18,1813 ; m. Ezekiel Kent, of Calais, Vt., Nov. I 3, 1836. She d. 1871 .

vii. Dauphna, b. Calais, Vt., Aug. I 3, I 8 I6; m. Rev. L. H. Tabor. She d. Jan. I I, 1880.

viii. Laura A., b. Calais, Vt., Aug. 28, I819; m. J. V. R. Kent, of Calais, Vt., Nov. I844. She d. Aug. 3I, I85I.

ix. Fanny H., b. Calais, Vt., July 24, 1822 ; m. Abdiel Kent, of Calais, Vt., June 7, 1845. She d. Dec. 24, 1854 .

183

SIMEON CUR'TISS (Foshua, Foseph, Thomas, Fohn, Elizabeth), b. Wallingford, Conn., Nov. I 9, I760; m. Elizabeth Sledge, of Middletown, Conn., He was killed in Burlington, Conn., where he lived, by falling into a well, Dec. I3, I 809 . His widow m. Jacob Catlin, of Harwinton, Conn., and d. March I3, I 856 , aet. 76 years and 6 months.

They had children :-

i. Cornelia, b. Burlington, Conn.,

(384) ii. Joshua, b. Burlington, Conn., Gillett, of Milford, Conn.

$17^{8} 3 ;$ m. Polly, d. of John

iii. Betsey, b. Burlington, Conn., d. Nov. 17, 1873 .

I $789 ; \mathrm{m}$. John Beach. She

iv. Polly, b. Burlington, Conn., Feb. I2, I793; m. Charles H. Todd, Sept. 17, 1815 .

v. Chester, b. Burlington, Conn.,

(385) vi. Major, b. Burlington, Conn., July 4, r797; m. Clarissa Dowd in 1822 .

vii. Amanda, b. Burlington, Conn., 1802; m. Samuel Harrison, of Cheshire. 
AARON CURTISS (Foshua, Foseph, Thomas, Fohn, Elizabeth), b. Wallingford, Conn., Jan. I767; m. Comfort Foot,

He was in Delhi, Delaware Co., N. Y., in $1815 . \mathrm{He}$ d. in Burlington, Conn., July 9, 1853 . She d. March 4, 1850.

They had children : -

(386) i. Miles, b.

ii. Charlotte M., b. Burlington, Conn., Feb. 21, 1810; m. Philo Curtiss, of Burlington, Conn., Sept. 3, 1829.

$(387)$ iii. Merritt, b. Burlington, Conn., 1804. d. Oct. 27, 1804.

iv. Martin, b. Burlington, Conn.,

\section{88}

GILES CURTISS (Fohn, Fohn, Thomas, Fohn, Elizabeth), b. Wallingford, Conn., March 22, I764; m. Hannah Westover, of Sheffield, Mass. He came with his father to Ashley Falls, Mass. He was a soldier in the Revolutionary Army, and served three years and four months. Hed. I 832 .

They had children :-

i. Mary, b. Sheffield, Mass., April 20, 1785.

ii. Elizabeth, b. Sheffield, Mass, Nov. 3, 1786.

(.388) iii. Elias, b. Sheffield, Mass., Aug. 28, 1788.

iv. Roswell, b. Sheffield, Mass., Feb. 25, 1790. He kept a hotel in Sheffield, Mass., and d. without issue 1829 .

(389) v. James, b. Sheffield, Mass., Feb. 26, 1792.

vi. Huldah, b. Sheffield, Mass., May 28, 1797 ; m. Stephen March, of Goshen, Conn., April 3, 1817 .

(390) vii. Edmund, b. Sheffield, Mass., May 28, 1777.

viii. Roxey, b. Sheffield, Mass., April 28, 1799 ; m. Stephen Cahoon, of Great Barrington, Mass., 1814 .

ix. Edna, b. Sheffield, Mass., March 1 I, I80I ; m. Augustus Bartholomew, of Goshen, Conn., Dec. 4, 1817 .

x. Jane, b. Sheffield, Mass., Jan. 30, 1803 .

xi. Hannah, b. Sheffield, Mass., June 3, 1805.

xii. Cornelia, b. Sheffield, Mass., May 3, 1807 .

xiii. Electa, b. Sheffield, Mass., March 20, 1809.

xiv. Clinton, b. Sheffield, Mass., Jan. 21, 1811. He was killed by being run over by horses.

xv. De Witt, b. Sheffield, Mass., Jan. 14, 1814. He was a farmer, and d. unm. in Sheffield, Mass., Jan. 1881.

\section{90}

EPHRAIM CURTISS (Stiles, Ephraim, Foseph, Fohn, Elizabeth), b. Stratford, Conn., March 27, I739; m. Ann, d. of Josiah and Mary Curtiss, of Stratford, Conn., Dec. 23, 1764. He d. in 
Huntington, Conn., Aug. 30, I 794. She d. Jan. I 5, I 802 , aet. 60 years. Ephraim Curtiss was a lieutenant in the Revolution in Capt. Beach Tomlinson's Co., 1777.

They had children :-

(391) i. Levi, b. Huntington, Conn., Dec. 29, 1765; m. (i.) Abigail, She d. Jan. 9, 1809; m. (ii.) Ann, She d. Sept. 8, 1826. He d. April 8, 185 I.

ii. Mary Ann, b. Huntington, Conn., Sept. 14, 1767; m. Eli Blakeman. (392) iii. Philo, b. Huntington, Conn., Oct. 25, 1772; m. Sarah, She d. Dec. 26, I823, aet. 45 years. He d. April 2I, I8 32 .

(393) iv. Ephraim, b. Huntington, Conn., July I 2, 1780.

v. Rebecca, b. Huntington, Conn., Sept. I7, I 782.

vi. Agur, b. Huntington, Conn., Feb. I9, I 786. He d. Aug. 30, 180 r.

\section{9 I}

ELIHU CURTISS (Stiles, Ephraim, Joseph, Fohn, Elizabeth), b. Stratford, Conn., May Io, I74I ; m. Phebe, d. of John and Mercy Burritt, of Stratford, Conn., Dec. 13, 1769. He d. Aug. 20, 1820 , in Ripton, Conn. Will was filed in Bridgeport. She d. I 840 .

They had children :-

i. Catherine, b. Stratford, Conn., Dec. 20, 1770; m. Benjamin Ufford. She d. I83 I.

ii. Phebe, b. Stratford, Conn., Feb. 5, 1773; m. Benjamin Ufford, of Stratford, Conn., Dec. 31, I798. She d.

(397) iii. Elihu, b. Stratford, Conn., Dec. 20, I774; m. Clemence, d. of Samuel Peck, of Milford, Conn., April I829. He d. ${ }_{1} 858$.

(395) iv. Burritt, b. Stratford, Conn., Nov. I0, 1776.

v. Molly, b. Stratford, Conn., Nov. 30, I 779 ; m. Josiah Booth.

(396) vi. Stephen Welles, b. Stratford, Conn., Sept. 24, I 782.

vii. Nancy, b. Stratford, Conn., Dec. 16,1784 ; m. Charles Hawley.

viii. Betsey, b. Stratford, Conn., Nov. 21, I787; m. Philo Birdsey, of Stratford, Conn. She d. 1813 .

\section{2}

SILAS CURTISS (Stiles, Ephraim, Foseph, Fohn, Elizabeth), b. Stratford, Conn., June I 4, I 743 ; m. Hannah, d. of Rev. Nathan and Dorothy Birdsey, of Stratford, Conn., Feb. I7, I765. She d. Nov. 25, I 8 I I, aet. 65 years. He lived in Oronoke, Conn. He d. Jan. I 5,18 I 6.

They had children :-

(397) i. Ezra, b. Stratford, Conn., Aug. 26, i 765; m. Anna, d. of Samuel and Abigail Ufford, of Stratford, Conn. He was a physician. He d. in Litchfield, Conn., Nov. 17, 1797.

ii. Hannah, b. Stratford, Conm., June 26,$1767 ; \mathrm{m}$. Seymour Conway Whiting, of Stratford, Conn., Nov. 24, 179 J. 
iii. Helen, b. Stratford, Conn., July 7, I 769 ; m. (i.) Joseph Thompson, of Stratford, Conn.; m. (ii.) Aaron Judson, of Stratford, Conn.

iv. Rebecca, b. Stratford, Conn., Aug. 7, $177 \mathrm{I}$; m. Eli Walker Lewis, of Stratford, Conn., Dec. 24, I80I. She d. June 30, 1823.

v. Susannah, b. Stratford, Conn., Aug. I3, I 773 ; m. Asa Curtis, of Stratford, Conn., Nov. 2, 1797. She d. Feb. 2, 1852.

(39S) vi. Silas, b. Stratford, Conn., Feb. 27, I776; m. (i.) Helen, d. of Stiles and Naomi Judson, of Stratford, Conn., m. (ii.) Avis Newton, d. of Daniel and Mehitable Shelton, of Stratford, Conn., He d. Sept. I 4,1845 .

(399) vii. William, b. Stratford, Conn., Aug. 14, 1778; m. (i.) Rebecca, d. of Stiles and Naomi Judson, of Stratford, Conn., Feb. 9, 1803. She d. Dec. 23, 1822; m. (ii.) Helen, d. of Ezra and Phebe Birdsey, of Stratford, Conn., March 5, 1825. She d. Feb. 13, 1875. He d. Jan. I7, 1855 .

viii. Thaddeus, b. Stratford, Conn., Sept. 24, I78o. d. Dec. 25, I80 r.

ix. Abigail Betsey, b. Stratford, Conn., Feb. 17, I $78_{3}$; m. Abijah Booth, of Stratford, Conn., i 803 .

(400) x. Eli, b. Stratford, Conn., March I I, I785; m. Sarah, d. of Zachariah and Anna Blakeman, of Stratford, Conn.

(401) xi. Lucius, b. Stratford, Conn., Oct. 20, 1785-6; m. Harriet, d. of John and Mary Curtiss, of Stratford, Conn.

\section{3}

JOHN CURTISS (Stiles, Ephraim, Foseph, Fohn, Elizabeth), b. Stratford, Conn., Sept. I745; m. Mary, d. of Daniel and Mary Sheldon, of Stratford, Conn., April 17, 1769. He d. Aug. 31, 1825. She d. Aug. 31, 1825 , aet. 78 years. He served in the War of the Revolution, and attained the rank of lieutenant.

They had children :-

i. Sarah, b. Stratford, Conn., Sept. 29, 1769; m. Joseph Tomlinson, of Stratford, Conn., March 20, 1790.

(402) ii. Stiles, b. Stratford, Conn., April I 3,1771 ; m. Sarah, d. of Samuel Beard, of Huntington, Conn., Oct. 5, 1794. He d. Jan. 20, 1850. She d. July 26, 1862.

(703) iii. Judson, b. Stratford, Conn., Feb. 15, 1774.

iv. John, b. Stratford, Conn., Feb. 8, 1778 . He d. unm.

(704) v. Ezekiel, b. Stratford, Conn., June 6, 1779; m. (i.) Anna Wooster, of Stratford, Conn.; m. (ii.) Electa Lord.

(405) vi. Daniel, b. Stratford, Conn., April 8, 1781; n. Elizabeth, d. of Peter and Elizabeth Pixlee, of Stratford, Conn., Jan. 10, 18 ro. He d. $\quad 1852$.

vii. Julia, b. Stratford, Conn., Nov. 27, 1784; m. Burritt Curtiss, of Stratford, Conn.

viii. Harriet, bapt. Stratford, Conn., Feb. I791 ; m. Lucius Curtiss, of Stratford, Conn. 
ANDREW CURTISS (Henry, Ephraim, Foseph, Fohn, Elizabeth), b. Stratford, Conn., Jan. 20, 1744; m. Sarah, d. of Deacon Job and Betty Peck, of Stratford, Conn., Sept. 22, 1768. Will filed in Bridgeport, Conn., April 30, 1830.

They had children:-

i. Sarah, b. Stratford, Conn., April 23, 1769; m. Matthew Beers, of Stratford, Conn., Feb. I4, 1785.

ii. Mary, b. Stratford, Conn., Nov. 4, 1771. She d. Sept. 20, 1776.

iii. Anne, b. Stratford, Conn., Jan. 8, I775. She d. Jan. 24, I775.

iv. Job, b. Stratford, Conn., Jan. 6, 1776 . He d. Sept. 22, 1776 .

(406) v. Job, b. Stratford, Conn., July I I, I777; m. Polly,

$\mathrm{He}$ d. in Stratford, Dec. 4, 1859 .

vi. Hannah, bapt. Stratford, Conn., 1783 .

vii. Polly, b. Stratford, Conn., March Io, I 783.

\section{6}

LEWIS CURTISS (Henry, Ephraim, Foseph, Fohn, Elizabeth), b. Stratford, Conn., March 12, 1745; m. Hepzibah, d. of James and Jerusha Walker, of Stratford, Conn., Jan. 28, 1773. He d. March 5, I 834. She d. April 16, I819, aet. 71 years. His will was filed in Bridgeport, Conn., April $\quad$ I 834 .

They had children :-

(407) i. Philo, b. Stratford, Conn., March 4, I774.

ii. Naomi, b. Stratford, Conn., Jan. 2, 1776.

iii. Hepzibah, b. Stratford, Conn., Jan. I9, 1778. She d. unm. in 1832 .

iv. Huldah, b. Stratford, Conn., Feb. 10, I780; m. Peet.

(408) v. David, b. Stratford, Conn., Dec. 17, I781.

(409) vi. Ira, b. Stratford, Conn., Dec. 30, 1783.

vii. Alice, b. Stratford, Conn., Nov. 5,$1787 ; \mathrm{m}$. Staples.

\section{7}

SOLOMON CURTISS (Henry, Ephraim, Foseph, Fohn, Elizabeth), b. Stratford, Conn., July 24, 1747 ; m. Jerusha, d. of Nathaniel and Rachel Wheeler, of Stratford, Conn., June i786. She d. in Monroe, Aug. 29, 1834 , aet. 76 years. He d. July I3, I 824 .

They had children :-

i. Rejoice, b. Stratford, Conn., Sept. I787. He d. Oct. II, 186I, unm.

ii. Jabez, b. Stratford, Conn., Oct. r789. d. Feb. 8, I797.

iii. Jerusha, b. Stratford, Conn., July I791; m. James Prince, of Stratford, Conn. She d. Dec. 29, 1873. 
(4Io) iv. Solomon, b. Stratford, Conn., Feb. 1794.

(4II) v. Nathaniel Wheeler, b. Stratford, Conn., Sept. 16, I795.

vi. Charlotte, b. Stratford, Conn., Sept. I 7, 1798. She d. March 13, I 866, unm.

$$
198
$$

HENRY THOMPSON CURTISS (Henry, Ephraim, Joseph, Fohn, Elizabeth), b. Stratford, Conn., March 22, I 75 I ; m. Phebe, d. of Ebenezer Sherman, Dec. 1778. He d. April i 8, 1814. She d. Feb. 5, I 826 , aet. 67 years.

They had children :-

(412) ii. Elijah, b. Stratford, Conn., Oct. I6, 1779; m. Anne, d. of Thomas and Anne Curtis, of Stratford, Conn. He d. Jan. II, 1873. She d. March 4, I862.

ii. Abiah, b. Stratford, Conn., June 26, 1782; m. Elijah Blakeman, of Stratford, Conn., Aug. 26, i 8 I 0 .

iii. Anna, b. Stratford, Conn., Dec. 26, $1784 ; \mathrm{m}$.

iv. Phebe, b. Stratford, Conn., June I, $1787 ; \mathrm{m}$. Blakeman. She d. Jan. 2, I864.

v. Betsey, bapt. Stratford, Conn., July 1790. She d. March 2I, 1843.

vi. Harriett, bapt. Stratford, Conn., June 1793. She d. June 20, I 793.

(413) vii. Henry, b. Stratford, Conn., April I 795.

(4I4) viii. George, b. Stratford, Conn., Jan. 1798. He d. in Illinois, Sept. I8, I 822 .

ix. Eliza, b. Stratford, Conn., $\quad$ 1800. d. March 7, 1800.

I 99

DAVID CURTISS (Henry, Ephraim, Foseph, Fohn, Elizabeth), b. Stratford, Conn., Sept. 3, I754 ; m. Annis Gorham, of Stratford, Conn., Jan. 23, I777. He served as a private in Samuel Whiting's Company, Col. Waterbury's Regiment, in the War of the Revolution.

They had children:-

i. Annis, b. Stratford, Conn., Feb. 22, 1778 . d. y.

ii. Nabby, b. Stratford, Conn., Oct. 29, I 780 . d. y.

(715) iii. David, b. Stratford, Conn., April 7, i788. d. y.

iv. Polly, b. Stratford, Conn.,

200

SAMUEL CURTISS (Henry, Ephraim, Foseph, Fohn, Elizabeth), b. Stratford, Conn., June 27, I757; m. Dorothy Tibbals, of Milford, Conn., Oct. 9, 1783. He d. May 19, 1826. She d. Sept. 19, I 829, aet. 66 years. 
They had children :-

i. Mira, b. Stratford, Conn., Sept. 16, 1784. d. Nov. 18, 1786.

ii. Charles, b. Stratford, Conn., Jan. 16, 1786. He d. Jan. 8, I893.

( 716$)$ iii. Marcus, bapt. Stratford, Conn., March 1786.

(4I7) v. Charles, b. Stratford, Conn., Oct. I795.

\section{2}

EDMUND CURTISS (Edmund, Ephraim, Foseph, Fohn, Elizabeth), b. Stratford, Conn., May I, I751 ; m. Sarah Fairchild, of Stratford, Conn., March I7, 1775 .

They had children :-

$\left(4 I^{\mathcal{S})}\right.$ i. Ezra, b. Stratford, Conn., May r2, 1776; m. Anna Welles, of Stratford, Conn.

ii. Catherine, b. Stratford, Conn., Oct. 6, 1777 ; m. David Lewis, of Stratford, Conn.

iii. Mary, b. Stratford, Conn., Aug. I2, I779; m. Middlebrook.

(4I9) iv. Beech, b. Stratford, Conn., Jan. I, I78I ; m. Laura Hawley, of Hawleyville, Conn.

v. Benjamin, b. Stratford, Conn., Nov. 21, 1783. He d. unm.

(420) vi. William, b. Stratford, Conn., July 20, 1785 ; m. Annie Fayerweather, of Trumbull, Conn. He d. March 3I, I867. She d. July I8, I 860, aet. 72 years.

(42I) vii. Jabez, b. Stratford, Conn., Sept. 15, 1787; m. Sarah, d. of Stephen Burritt, of Stratford, Conn., Jan. 1810. He d. I867.

viii. Sarah, b. Stratford, Conn., April I4, I 787 ; m. Warren Bonney, of Cornwall, Conn.

\section{6}

STILES CURTISS (Elnathan, Ephraim, Foseph, Fohn, Elizabeth), b. Huntington, Conn., Feb. 3, 176I ; m. (i.) Clara, d. of Elijah Adams, of No. Guilford, Conn., June I781. She d. May 4, 1796, aet. 34 years. m. (ii.) Betsey Hitchcock, of New Milford, Conn. She d. April 29, I8 6 . He d. Nov. 8, 1827.

They had children :-

i. Hannah, b. Huntington, Conn., Sept. 18, 1782.

ii. Susan, b. Huntington, Conn., Jan. 3,1786 .

iii. Lepy, b. Huntington, Conn., July I4, 1789 .

(422) iv. Joseph, b. Huntington, Conn.,

v. Betsey, b. Huntington, Conn.,

(123) vi. Aaron, b. Huntington, Conn., July 26, 1798.

vii. Sally, b. Huntington, Conn., Jan. I I, I 800 ; m. Albert Hotchkiss.

(424) viii. Morgan, b. Huntington, Conn., Jan. I I, I802; m. Margaret Owen, d. of Daniel and Elizabeth Curtiss, of Stratford, Conn., Feb. I3, I833. He d. Sept. 30, I88 i. She d. March 20, I868.

ix. Polly, b. Huntington, Conn., Dec. 7, 1803 ; m. John Brush

(425) x. Stiles, b. Huntington, Conn., Sept. 3, 1806. 
NEHEMIAH CURTISS (Foseph, Foseph, Foseph, Fohn, Elizabeth), b. Stratford, Conn., I $\quad$ I 740 ; m. (i.) Phebe, d. of Joseph and Martha Welles, of Stratford, Conn., April 4, 1763. She d. July 24,1776 , aet. 32 years. m. (ii.) Sarah, d. of William Lewis, and widow of Josiah Booth, Nov. 26, 1778. She d. Feb. 2, I812, aet. 79 years. He d. May 13, 1810. He served as a private in the War of the Revolution in Capt. Joseph Birdsey's Company, Col. Whiting's Regiment.

They had children :-

i. Abigail, b. Stratford, Conn., Feb. 11, 1767 .

(426) ii. Judson, b. Stratford, Conn., Aug. 18,1768; m. (i.) Mary. d. of Benjamin and Elizabeth Lewis, of Stratford, Conn。 ; m. (ii.) Ellen. He d. May 13, 1828 .

iii. Martha, b. Stratford, Conn., Nov. 12, $177^{\circ}$.

iv. Gideon, b. Stratford, Conn., Dec. 3, 1773. d. y.

(427) v. Nehemiah, b. Stratford, Conn., June I $3,1775$.

vi. Enoch, b. Stratford, Conn., Nov. 2, 1779. He d. unm. 1813.

(428) vii. Freeman, b. Stratford, Conn., Dec. 6, 1782; m. (i.) Anna, d. of Judson Peck, of Stratford, Conn., Aug. 6, I803; m. (ii.) Polly E. McEwen, Dec. 28, 1828 . He d. Jan. 5, I862.

viii. Sarah, b. Stratford, Conn., July 2, I784. She d. unm. 1827.

ix. Phebe, b. Stratford, Conn., March 26, 1787 . d. Nov. 1827, unm.

x. Lucy, bapt. Stratford, Conn., Dec. 12, 1792; m. Thaddeus Peck, of Stratford, Conn., Jan. 12, 1817. She d. April 5, 1874.

\section{0}

PHINEAS CURTISS (Foseph, Foseph, Foseph, Fohn, Elizabeth), b. Stratford, Conn., May I $742-3 ; \mathrm{m}$. Huldah, d. of Samuel and Bethia Judson, of Stratford, Conn., and widow of Josiah Gilbert, Feb. 23, 1784 .

They had children :-

i. Hepsey, b. Stratford, Conn., Feb. 6, 1785; m. Mr. Barteau, of Long Island.

ii. Martha, b. Stratford, Conn., Jan. 7, 1787 ; m. David Trubee, of Fairfield, Conn.

iii. Betsey, b. Stratford, Conn., Dec. 25, 1788; m. Samuel Comfort Trubee, of Fairfield, Conn., Oct. 22, 1809. She d. Sept. 17, 1879.

(429) iv. Andrew, b. Stratford, Conn.,

v. Huldah, b. Stratford, Conn.,

Fairfield, Conn.

m. Aaron Turney, of 
JOSEPH CURTISS (Foseph, Foseph, Foseph, Fohn, Elizabeth), bapt. Stratford, Conn., Aug. 1745; m. Mary, d. of John and Ruth Thompson, of Stratford, Conn., Nov. I 3, 1768.

They had children :-

(430) i. John, b. Stratford, Conn., Nov. 10, 1769; m. Anne Curtiss.

( 731 ) ii. Abel, b. Stratford, Conn., July 20, I 77 I.

(\$32) iii. Joseph, b. Stratford, Conn., June 16, I773.

iv. Mary, b. Stratford, Conn., March 29, I 775

(433) v. William Thompson, b. Stratford, Conn., April 9, 1777.

(434) vi. Zechariah, b. Stratford, Conn., Feb. 8, 1779.

(435) vii. Eli, b. Stratford, Conn., Dec. 9, 178 1.

viii. Alice, b. Stratford, Conn., Feb. I5, 1784.

ix. Sarah, b. Stratford, Conn., June 20, 1787. She d. Sept. 1787 .

(436) x. Victory, b. Stratford, Conn., Jan. 4, i 789.

xi. Nancy, b. Stratford, Conn., July 179 I.

212

SILAS CURTISS (Foseph, Foseph, Foseph, Fohn, Elizabeth), bapt. Stratford, Conn., May 1750; m. Mary, d. of Nathan and Tabatha Peck, of Stratford, Conn., Oct. 1770. He d. in Stratford, Aug. 5, I 822 . She d. April I I, I 805 , aet. 50 years. His will was filed in Bridgeport, Conn., Aug. 6, 1822 .

They had children : -

(437) i. Nathan, b. Stratford, Conn., Oct. 7, I 770.

$(738)$ ii. Silas, b. Stratford, Conn., July 7, 1773.

iii. Ann, b. Stratford, Conn., June 15,1776 ; m. Francis Percival.

(439) iv. Thaddeus, b. Stratford, Conn., June 5, 1779.

(440) v. Josiah, b. Stratford, Conn., Nov. 5, I 784.

vi. Theodosia, b. Stratford, Conn., Jan. 2 I, I786; m. Broadwell.

(471) vii. Joel, b. Stratford, Conn., Dec. I791; m. Harriet, d. of Abner Judson, of Stratford, Conn.

(442) viii. Birdseye, b. Stratford, Conn., Nov. I 2, I 789.

\section{3}

AGUR CURTISS (Foseph, Foseph, Foseph, Fohn, Elizabeth), b. Stratford, Conn., April 1757 ; m. Mercy, d. of Zechariah Blakeman. He d. in Stratford, Conn., Nov. 10, I 838. She d. Dec. 17, 1850, aet. 90 years. Agur Curtiss appears as a pensioner of the Revolutionary War in Fairfield Co., in 1832 .

They had children :-

(713) i. Ezekiel, b. Stratford, Conn.,

$(\not+7)$ ii. James, b. Stratford, Conn.,

He d. unm. I824. 
(445) iii. Agur, b. Stratford, Conn., I $785 ; \mathrm{m}$. Alice, d. of Josiah and Helen Birdseye, of Stratford, Conn. He d. March 12, 1868. She d. $\quad 1878$, aet. 89 years.

iv. Dolly, b. Stratford, Conn.,

m. Welles Beach, of Trumbull, Conn.,

v. Ellen (Helen), b. Stratford, Conn.,

Baldwin, of Milford, Conn.,

m. 'Thaddeus

\section{I 4}

EVERETT CURTISS (Robert, Foseph, Foseph, Fohn, Elizabeth), b. Stratford, Conn., Nov. 6, r762; m. Lucy, d. of Samuel and Esther Patterson, of Weston, Conn. He d. in Stratford, Conn., I826. Will filed in Bridgeport, Nov. $\quad$ I 826.

They had children :-

(476) i. Everett, b. Stratford, Conn.,

(47) ii. John, b. Stratford, Conn.,

iii. Rodney, b. Stratford, Conn., 1810 ; m. (i.) Abby Ann Brooks, of Bridgeport, Conn., Dec. 14, 1836 . She d. Sept. 9, I 842 , aet. 33 years. m. (ii.) Lucy Burr, of Bridgeport, Conn., Dec. 28 , I 845 ; m. (iii.) Esther L., He d. in Bridgeport, Conn., May 29, 1891.

iv. Abby, b. Stratford, Conn., d. unm.

v. Helen, b. Stratford, Conn., Aug. 2, 1794; m. Samuel Peet, of Bridgeport, March 4, I8I3. She d. Jan. I, I86I.

vi. Clarissa, b. Stratford, Conn., m. Benjamin Plumb.

vii. Sarah J., b. Stratford, Conn., m. Benjamin.

220

NATHAN CURTISS (Fames, Fames, Benjamin, Fohn, Elizabeth), b. Durham, Conn., June 29, I735; m. Anna Booth, of Durham, Conn., May 13, 176 . He d. before 1782 .

They had children :-

i. James, bapt. Durham, Conn., Feb. 14, I 762. d. y.

(448) ii. James, bapt. Durham, Conn., May 22, 1763 .

iii. Phebe Ann, bapt. Durham, Conn., Sept. 9, 1764; m. Bridgeman

Guernsey, Feb. 5, 1786.

iv. Augustus, bapt. Durham, Conn., Oct. 12, 1766. d. y.

v. Clarissa, bapt. Durham, Conn., May i 768 .

vi. Anna, bapt. Durham, Conn., May 13, 1770.

vii. Hannah, bapt. Durham, Conn., May I, I 772.

(4+9) viii. Augustus, bapt. Durham, Conn., Oct. 23, 1774.

AARON CURTISS (Fames, Fames, Benjamin, Fohn, Elizabeth), b. Durham, Conn., Sept. II, I737; m. Hannah Griswold, of Granville, Mass., May I3, 1763 . 
They had children :-

(450) i. Thaddeus, b. Granville, Mass., April 24, 1764.

$(75 I)$ ii. Levi, b. Granville, Mass., Nov. I 3, 1767; m. Rachel,

(752) iii. Natlan, b. Granville, Mass., Aug. 22, I776; m. Nancy Wadsworth, of Granville, Mass., Sept. 9, 1804 .

iv. Statirah, b. Granville, Mass., June 6, I78I ; m. (i.) David B. Curtiss, of Sandisfield, Mass., Aug. 25, I809; m. (ii.) Bevil Seymour, of Otis, Mass., Nov. I0, 1821.

(753) v. Samuel, b. Granville, Mass., July 20, 1783; m. Sally Fairchild.

ABIJAH CURTISS (Fohn, Fames, Benjamin, Fohn, Elizabeth), b. Durham, Conn., March 2, I750; m. Ann Bishop, of Durham, Conn., Aug. 13, 1777. He d. in Durham, Conn., Sept. 8, 1825. At the Lexington Alarm he enlisted as a private in Capt. Stephen Norton's Company.

They had children :-

i. Olive, b. Durham, Conn., April 24, 1778; m.

Fairchild. She d. Nov. 28, 1822 .

ii. Anne, b. Durham, Conn., Dec. 6, 1779; m. Scranton. She d. Dec. 1815 .

iii. Phebe, b. Durham, Conn., Feb. 27, 1783; m. Thomas Robinson Bray, of Guilford, Conn., Oct. I0, I804.

iv. Dinah, b. Durham, Conn., July 23, 1785 .

(454) v. Samuel, b. Durham, Conn., July 13, 1787; m. Lucretia Brooks, of Durham, Conn., Oct. 3, 1810. He d. in Medina, Ohio, in November, 1846 .

vi. Ichabod, b. Durham, Conn., May 17, I790. He d. May IO, I791. (455) vii. Ichabod, b. Durham, Conn., April 3, 1792; m. Selina Camp, of Durham, Conn., in I8I5. He d. Jan. 17, 1867.

(456) viii. David, b. Durham, Conn., Jan. 31, 1795. He d. Nov. 19, I816.

JOHN CURTISS (Fohn, Fames, Benjamin, Fohn, Elizabeth), b. Durham, Conn., May 5, I757; m. (i.) Lydia Hall, She d. July 6, r799. m. (ii.) Ruth Parmalee, of Durham, Conn., June 2, 1801 . He d. in 1802 .

They had children :-

i. Lydia, b. Durham, Conn.,

m. Benjamin Coe, Oct. 13,1823 .

ii. Anne, b. Durham, Conn.,

iii. Ruth, b. Durham, Conn., Oct. 7, 1802. 
DAVID CURTISS (David, Fames, Benjamin, Fohn, Elizabeth), b. Durham, Conn., May 19, 1750; m. Prudence Bishop, of Durham, Conn.

They had children :-

(457) i. Charles, bapt. Durham, Conn., Feb. 16, 1772.

$\left(45^{8}\right)$ ii. Lucius, bapt. Durham, Conn., Nov. 21, 1773 .

EDMUND CURTISS (Fonas, Fonathan, Fonathan, William, Elizabeth), b. Stratford, Conn., March 22, 1729-30; m. Anna, d. of David and Esther Curtiss, of North Stratford, Conn., Aug. 20, 1750.

They had children :-

i. Ruth, b. North Stratford, Conn., March 4, 1750.

(459) ii. Jonas, b. North Stratford, Conn., March 14, 1753.

(460) iii. John, b. North Stratford, Conn., Oct. I4, 1758.

iv. Anna, bapt. North Stratford, Conn., Aug. 26, 1764 .

v. Beula, b. North Stratford, Conn., July 1767 .

(46I) vi. William, b. North Stratford, Conn., Jan. 1770.

(462) vii. Daniel, b. North Stratford, Conn., Nov. 1772.

(463) viii. Elijah, b. North Stratford, Conn., April I9, 1775.

TIMOTHY CURTISS (Fonathan, Fonathan, Fonathan, William, Elizabeth), b. Stratford, Conn., Aug. 17, I749; m. (i.) Jerusha Wilcox, of Stratford, Comn., July I, I773; m. (ii.) Hepzibah

He d. in Huntington, Conn., 1813 .

They had children :-

i. Sarah Ann, b.,

(464) ii. Philo, b.,

iii. Lydia, b.,

$(465)$ iv. David, b.,

v. Hepzibah, b.,

vi. Anna, b.,

(466) vii. Josiah, b.,

(467) viii. Ezra, b.,

ix. Mary, b.,

(468) x. William, b.,

m. George L. Sherman.

HEZEKIAH CURTISS (Hezekiah, Hezekiah, Daniel, William, Elizabeth), b. Stratford, Conn., April 2, 1775; m. Elizabeth Ann

He d. in Huntington, Conn., Dec. 31 , 1807.

(81) 
His widow m. Isaac Cummings, - She d. March 9, I857, aet. 77 years.

They had children :-

$\left(f^{6} 9\right)$ i. Eli, b.,

$(470)$ ii. Charles, b.,

\section{4}

AGUR CURTISS (Samuel, Nathaniel, Zachariah, William, Elizabeth), b. Stratford, Conn., Nov. 17, I760; m. Hulda, d. of Benjamin and Elizabeth Lewis, of Stratford, Conn., Feb. 22, I 786. She d. June 6,1858, aet. 92 years. He d. in Stratford, Conn., April 22, I 844. He was a farmer, and always lived in Stratford, Conn. He was a deacon in the Congregational Church of that town.

They had children :-

(47I) i. Lewis, b. Stratford, Conn., April 30, 1787; m. (i.) Mary Elizabeth, d. of Joseph D. and Mary C. Beers, of New York City, N. Y., Feb. 4, 1824. She d. Dec. 15, 1835, aet. 31 years. m. (ii.) Elizabeth Overing, of New York City, N. Y., Oct. 7, 1840. She d. Nov. 26, 184 I. He d. New York City, N. Y., Jan. 30, 1883 .

(472) ii. Benjamin, b. Stratford, Conn., Nov. II, I790; m. (i.) Rebecca Nappan Edwards, Sept. 2, I830, in Paris, France. She d. July 22, $1844 ;$ m. (ii.) Laura, d. of David and Ann A. Hadden, of New York City, N. Y., Jan. 29, 1856. She d. Nov. 7, I894. He d. Feb. 19, 188I.

\section{5}

SAMUEL CURTISS (Samuel, Nathaniel, Zachariah, William, Elizabeth), b. Stratford, Conn., June I4, I769; m. (i.) Temperance Anna, d. of Abel and Amy Patterson, of Stratford, Conn., Feb. 1793. She d. Aug. 30, i 800 , aet. 30 years; m. (ii.) Alice, d. of Elisha and Sarah Hubbell De Forest, of Stratford, Conn. She d. Dec. I 3, I 859 , aet. 75 years. He d. Feb. 15, I833. He was a farmer, and lived and died in Stratford, Conn.

They had children :-

i. Mary Ann, b. Stratford, Conn., I 8 II.

ii. Henrietta, b. Stratford, Conn., 1803 .

iii. Samuel, b. Stratford, Conn.,

1793. She d. April II,

1796. She d. Aug. 26,

iv. Henrietta, b. Stratford, Conn., Jan, 25, I8 I I. She d. unm. April 30, 1874 .

v. Mary Ann, b. Stratford, Conn., Jan. I8I4. She d. June 14, I8I4. 
vi. Mary, b. Stratford, Conn., Oct. 15, I815; m. Asa S. Curtis, of Stratford, Conn., Feb. 4, I841, She d. Bec. 1 I, I 876.

vii. Elizabeth, b. Stratford, Conn., Jan. 14, I819; m. Robert Miller, May 17,1858 .

viii. Emily, b. Stratford, Conn., April 3, 1822; m. Ezra Wheeler, of New York City, N. Y., Dec. 23, 1849. She d. Feb. 3, I 896.

ix. Samuel Elisha, b. Stratford, Conn., Dec. 9, I825. He is a farmer, and lives in Stratford, Conn., unm.

x. Albertina C., b. Stratford, Conn,, Dec. 21, I830. She d. unm. April 25, I 887 .

\section{6}

ZACHARIAH CUR'TISS (Mitchell, Zachariah, Zachariah, William, Elizabeth), b. Stratford, Conn., Sept. 17, 1753; m. Naomi, d. of John and Huldah Edwards, of Stratford, Conn., Dec. 28, I775. He d. in Trumbull, Conn., Feb. 4, 1809 . Will entered for probate at Bridgeport, Conn., Feb. 13, I 809 . She d. Dec. 17, I 808 , aet. 53 years.

They had children :-

(473) i. Elijah Edwards, b. Stratford, Conn., Jan. 21, I779; m. Elizabeth J. He d. Dec. I 5, I 847 . She d. Dec. 6, I 835 , aet. 56 years.

ii. Mary Ann, b. Stratford, Conn., Jan. I6, I 78 I.

(474) iii. James Rose Edwards, b. Stratford, Conn., April I, 1788.

247

DANIEL MITCHELL CURTISS (Mitchell, Zachariah, Zachariah, William, Elizabeth), b. Stratford, Conn., Jan. 5, I755; m. (i.) Hepzibah Burr, June 24, I779; 111. (ii.) Huldah

She d. May 7, 1832 , aet. 74 years. He d. in Trumbull, Conn., April 27, 1831. Will filed in Bridgeport, Conn., May 25, 183 $\mathrm{J}$. In 1777 he was a drummer in the Revolutionary Army.

They had children :-

(475) i. Justus Burr, b. Trumbull, Conn., Jan. 27, I780; m. Huldah Edwards, He d. Aug. 10, I832. She d. Dec. 3, 1855.

(476) ii. Eli, b. Trumbull, Conn., Sept. I6, I781 ; m. (i.) Sarah, d. of Zachariah and Anna H. Blakeman, of Trumbull, Conn.; m. (ii.) Hepzibah, . She d. Oct. 29, I 866, aet. 82 years. He d. April 23, I8 19.

iii. Mary, b. Trumbull, Conn., Aug. 2, 1783 ; m. Andrew Beardsley, of Trumbull, Conn. She d. May I, I864.

(477) iv. David, b. 'Trumbull, Conn., Feb. I9, I 786.

v. Daniel, b. Trumbull, Conn., March 8, I 788 . He d. unm. May 6, 1842. 
vi. Hepzibah, b. Trumbull, Conn., Sept. 29, 1790; m. Andrew S. Nichols, of Stratford, Conn.

vii. Huldah, b. Trumbull; Conn., ley, of Stratford, Conn.

viii. Lucy, b. Trumbull, Conn.; m. $(47 \mathcal{S})$ ix. Alvin, b. Trumbull, Conn.

(179) x. Ira, b. Trumbull, Conn.,
1795; m. Abijah R. HawShe d. March 18, i 846. Nichols.

1801. He d. June 21, 1849 .

\section{0}

LEVERETT CURTISS (Samuel, Feremiah, Zachariah, William, Elizabeth), b. Southington, Conn., I776; m. Ruth, d. of Asa and Sarah (Atkins) Barnes, of Southington, Conn., Jan. 25, I 796. He d. in Southington, Conn., Oct. I2, I826. She d. April 9, I 850 , aet. 78 years.

They had children : -

$\left({ }_{f}\right.$ So) i. Carlos, b. Southington, Conn., Feb. 22, 1798; m. Jerusha Brooks, of Cheshire, Conn., Oct. 29, 1820.

ii. Laura, b. Southington, Conn., June 22, I800; m. Lemuel Andrews, of Southington, Conn., Jan. 1, 1821. She d. Dec. 22, 1822.

iii. Rodney, b. Southington, Conn., Sept. 26, 1803 . He graduated from Yale College in 1828 , and then entered the Divinity School of Yale College. He d. Aug. I 8, I 829.

$\left(f_{1}\right)$ iv. Samuel Ellery, b. Southington, Conn., March 6, 1808; m. Mary B. Andrews, of New Britain, Conn., April 21, 1830. He d. 1830.

v. Allen Barnes, b. Southington, Conn., Nov. 6, I8 12 ; m. Laura He d. in Southington, Conn., Feb. 14, 1856.

They had a child : -

Isaac Leverett, b. Southington, Conn., $18{ }_{3} 8$. He d. Feb. 4,1854 .

252

LUCAS CURTISS (Fohn, Feremiah, Zachariah, William, Elizabeth), b. Southington, Conn., June I 7, I 766 ; m. Laurinda, d. of Abel and Rhoda (Lewis) Carter, of Southington, Conn., Nov. 25, 1799. She d. March 7, 1857 . He d. in Southington, Conn., Aug. 22, I 847. He occupied the homestead of his father, and for many years was active in the public affairs of the town.

They had children :-

i. Mary, b. Southington, Conn., Aug. 6, I80 I ; m. Bennet Bishop, April 29, 1822. She d. Aug. 7,1827 .

ii. Rachel Jane, b. Southington, Conn., June 17, 1805; m. Dr. Wyllys Woodruff, April, 1825 . 
JEREMIAH CURTISS (Fohn, Feremiah, Zachariah, William, Elizabeth), b. Southington, Conn., Sept. 6, 1770; m. Rachel, d. of Abel and Rhoda (Lewis) Carter, of Southington, Conn. She d. March 6, I858, aet. 86 years. He d. June $2 \mathrm{I}, 18 \mathrm{I} 3$.

They had children :-

i. Polly, b. Southington, Conn., Feb. 24, 1790; m. William Hall, Jan., 1815. She d. Sept. 17, I848.

(482) ii. John, b. Southington, Conn., Feb. 17, 1795; m. Sarah Carter, Dec. 25, I817. She d. Sept. 22, 1869, aet. 71 years. He d. May $27, \mathrm{I} 866$.

iii. Lucy, b. Southington, Conn., I807. She d. July 9, I815.

iv. Charles, b. Southington, Conn., May, I8 14. He d. Sept. 6, I8 15.

\section{4}

MARCUS CUR'TISS (Fohn, Feremiah, Zachariah, William, Elizabeth), b. Southington, Conn., Sept. 19, I780; m. Catherine, d. of Isaac and Rachel Pomeroy Newell, of Southington, Conn., Nov. 30, 1804 . He removed to Geneva, N. Y., in September, 1808, and d. there Dec. 1, 1868.

They had children :-

$(483)$ i. Augustus, b. Southington, Conn., June 2, 1806 ; m. Jane Wheatley, June 22, 1842 . He d. Dec. 28, I 863.

(484) ii. Leonard, b. Genoa, Ohio, April 4, I8I I m. Mary A. Hough, May $4,1836$.

(485) iii. Henry, b. Genoa, Ohio, March I 2, I8I4; m. Jane Monroe.

( $\$ 86)$ iv. Charles, b. Genoa, Ohio, Jan. 30, 1816 ; m. Martha Higley.

v. Mary, b. Genoa, Ohio, Sept. 2I, 18 I 8 ; m. Ransom Keeler She d. Dec. Io, 1852 .

$(487)$ vi. Oliver, b. Genoa, Ohio, July 30,1821 ; m. Jane Badger He d. Oct. 20, I 866.

viii. Rachel, b. Genoa, Ohio, Aug. 25, 1825 ; m. Charles Rugg.

\section{5}

ERASTUS CURTISS (Fohn, Feremiah, Zachariah, William, Elizabeth), h. Southington, Conn., May 30 , I $784 ; \mathrm{m}$. Mary, d. of Timothy and Lucy Camp Lee, of Southington, Conn., Nov. 24, 1808. He d. in Southington, Conn., April 4, r825. She d. Aug. 31, 1863 .

They had children :-

(488) i. John, b. Southington, Conn., March I2, 1812; m. Hannah M. Gridley, Sept. I 2, 1838 . 
ii. Susan, b. Southington, Conn., May 2I, I 8 I4; m. Russell Peck, of Southington, Conn., Sept. 26, 1839.

iii. Mary Emeline, b. Southington, Conn., June 8, I8 I7; m. Ezra Loveland.

\section{6}

LEVI CURTISS (Stephen, Abraham, fosiah, William, Elizabeth), b. Stratford, Conn., March 26, 1772; m. (i.) Sarah ; m. (ii.) Elizabeth. He d. in Stratford, Conn., Feb. 2 I, I 854.

They had children :-

i. Phebe, b. Stratford, Conn., m. Welles.

ii. Betsey, b. Stratford, Conn., m. Lovejoy.

iii. Cornelia, b. Stratford, Conn., m. Skelton.

iv. Sarah, b. Stratford, Conn., m. Isaac Welles, of Stratford, Conn., Oct. 2 I, 1827.

(489) v. Elbert, b. Stratford, Conn.

(490) vi. Stephen, b. Stratford, Conn.

(49I) vii. Willis, b. Stratford, Conn., He d. 1867.

(492) viii. Levi, b. Stratford, Conn.

WAIT CURTISS (Agur, Fosiah, Fosiah, William, Elizabeth), bapt. Woodbury, Conn., Jan. 18, 1756; m. Olive, d. of Selah and Esther Mead Strong, of Woodbury, Conn., June 8, $1789 . \mathrm{He}$ d. in Southbury, Conn., Oct. 17, I798. She m. Adonijah St. John, of Norwalk, Conn. Dec. I 799. She d. Aug. I 847 , aet. 84 years. He served as a private in the Revolutionary War. $\mathrm{He}$ was a farmer, and resided in Southbury, Conn.

They had children :-

i. Fanny, b. Southbury, Conn., Nov. 1, I790; m. Alanson Beecher, of Southbury, Conn. She d. June, I851.

ii. Phebe, b. Southbury, Conn., Dec. 27, 1792; m. Willian Stebbins, of Clinton, N. Y.

iii. William, b. Southbury, Conn., Sept. 13, 1794. He d. July 4, I 801 .

(493) iv. Cyrus, b. Southbury, Conn., Dec. 8, I797; m. Lydia Van der Burgh, of Hudson, N. Y., March I2, 1823.

ISAAC JUDSON CURTISS (Abner, Fosiah, Fosiah, William, Elizabeth), b. Stratford, Conn., April 12, I $767 ; \mathrm{m}$. Charity, d. of James and Abigail Booth, of Stratford, Conn., Jan. I, I79I. He d. in Stratford, Conn., July 17, 1845. She d. Dec. I6, 1846, aet. 75 years. 
They had children :-

i. Mary, b. Stratford, Conn., April 1 3, I 792 ; m. Joel Jones, of Stratford, Conn., Oct. 16, 1811. She d. Jan. 15, 1817 .

ii. Catharine, b. Stratford, Conn., Aug. 7, 1794; m. Marcus Curtiss, of Stratford, Conn., Oct. 8, 1817. She d. June 17, 1855; no children.

iii. Dillisenia, b. Stratford, Conn., Dec. 10, 1796. She d. Jan. I 4, 1817 .

iv. Harriet, b. Stratford, Conn., March 31, 1799; m. Samuel Barnum, of Stratford, Conn., Nov. 11, I824. She d. Feb. 28, 1856.

v. Rebecca, b. Stratford, Conn., Dec. 14, 1801; m. Lewis Beers, of Stratford, Conn., April 19, 1826 . She d. Nov. $21,1835$.

(494) vi. Isaac, b. Stratford, Conn., Jan. 10, 1803 ; m. Sarah Lucinda Beers, of Stratford, Conn., June I, 1827. She d. Nov. 7, I844; m. 2d, Caroline Lewis, of Stratford, Conn., March 4, 1845. He d. June 2, 1862.

vii. Charity, b. Stratford, Conn., May 2, I 805. She d. Aug. I 9, 1805. (495) viii. Charles Dillisenea, b. Stratford, Conn., July 6, I 806 ; m. Elizabeth A. Porter, of Stratford, Conn., Nov. 11, 1830. She d. Nov. 27, 1860 , aet. 47 years. He d. April 2, 1880.

They had a child : -

i. Alma, b. Stratford, Conn. She is unm. and lives in Stratford, Conn.

(496) ix. Sidney I., b. Stratford, Conn., June 23, I 808; m. Christina Demarest, of Stratford, Conn., Sept. 19, 1833 .

x. Mary Caroline, b. Stratford, Conn., Sept. 25, 18 I I ; m. IVillian Brooks, of Stratford, Conn., Oct. 15, 1835. She d. Aug. 20, I 852 .

(497) xi. Lucius, b. Stratford, Conn., Feb. 22, 18 14 ; m. Frances Ufford, of Stratford, Conn., March 18, 1839. He d. Oct. 18, 1848. She d. Jan. $7,188_{3}$.

\section{1}

SALMON CURTISS (Nehemiah, Benjamin, Fosiah, William, Elizabeth), b. Newtown, Conn., Aug. 16, 1759; m. Esther Holbrook, of Derby, Conn., July 4, 1782. She d. I818. He died Jan. 2, I 8 I4. Will filed in Danbury, Conn., Feb. 8, I8 I4.

They had children:-

i. Polly Ann, b. Newtown, Conn., April 2, 1783; m. Capt. Isaac Tomlinson, of Newtown, Conn., Oct. 28, 1799.

(498) ii. Holbrook, b. Newtown, Conn., July 14, 1 787 ; m. Elizabeth P., d. of Hon. William Edmond, Oct. 7,1832 . He d. Feb. 21, 1858 . 
ABEL CURTISS (Nehemiah, Benjamin, Fosiah, William, Elizabeth), b. Newtown, Conn., Nov. 2, I766; m. Abiah Hurd, of Newtown, Conn., Nov. 24, I791.

They had children :-

i. Aurilla, b. Newtown, Conn., June 6, 1792.

(799) ii. John, b. Newtown, Conn., May 2, 1796.

(500) iii. Charles, b. Newtown, Conn., July I 2, I80 I.

263

PHILO CURTISS (Benjamin, Benjamin, Fosiah, William, Elizabeth), b. Newtown, Conn., June 27, I $760 ; \mathrm{m}$. Huldah Hubbell, of Weston, Conn. He d. in Newtown, Conn., March 7, I8 I8. Will filed in March, I 8 I 8 . She d. Jan. 25, I 853 , aet. 96 years. He was selectman of Newtown, Conn., i 802-1806, i 808, i 809.

They had children :-

(5or) i. Nichols, b. Newtown, Conn., Sept. 27, 1784; m. Sarah Ann, d. of Amos Bennett, of Newtown, Conn., Nov. 3, 1803 . He d. April 20, 1852 . She d. Oct. 29, I 858 , aet. 7 I years.

(502) ii. Carlos, b. Newtown, Conn., Sept. 29, 1786; m. Polly Sample, of Newtown, Conn., Sept. 20, 1807. He d. Jan. 8, 1844. She d. April 26, I 858 .

iii. Phedenia, b. Newtown, Conn., Sept. 27, 1787; m. Thomas Beers, of Newtown, Conn.

(503) iv. Philo, b. Newtown, Conn., Sept. 20, I791 ; m. Sally Maria Birch, of Newtown, Conn., Jan, 16, 1825. He d. Jan. 15, I872. She d. March 2, 1893 , aet. 90 years.

v. Polly, b. Newtown, Conn., m. John Glover, of Newtown, Conn. She d. I 8 rg.

vi. Betsey, b. Newtown, Conn., Nov. 14, 1795 ; m. Amasiah Beers, of Newtown, Conn. She d. April 25, 1858 .

vii. Huldah, b. Newtown, Conn.,

m. Chauncy Hatch, of Oxford, Conn. She d. 1876 .

viii. Julia, b. Newtown, Conn. She d. unm. Jan. 3, I835.

265

ALFRED DEVINE CURTISS (Benjamin, Benjamin, Fosiah, William, Elizabeth), b. Newtown, Conn., Jan. 24, I783; m. Sarah, d. of Nirom and Sarah Hurd, of Newtown, Conn. She died April 6, I855, aet. 75 years. He died Sept. 30, I850.

They had children :-

i. Sophia, b. Newtown, Conn., Sept. 7, 1802; died

1881 . 
(504) ii. Nirom, b. Newtown, Conn., March 28, I805; m. Matilda Rogers. He d. Feb. 24, I 846.

iii. Phebe, b. Newtown, Conn., May 24, 1807; m. Joseph Nettleton. She d. I 893-4.

iv. Mary, b. Newtown, Conn., May 3, I809; m. John Clark. She d. Sept. I 5, I 839 .

(505) v. William, b. Newtown, Conn., Sept. I4, 18 I I m. Fannie Moore. He d. April I I, I 857.

(500) vi. Cyrenius, b. Newtown, Conn., Dec. 5, 1814; m. Christa Ann Beardsley, May 15, I838. He d. May 27, 1890. She d. May I I, I 89 I.

(507) vii. Alfred, b. Newtown, Conn., June 8, 1817; m. Matilda Rogers, wid. of Nirom Curtiss. He d. Aug. 26, 1850.

viii. Sarah, b. Newtown, Conn., Sept. 5, I8 I g. She d. May 23, I 834.

$(508)$ (ix. Edwin Augustus, b. Newtown, Conn., Jan. 25, 1825 ; m. Matilda

Rogers, wid. of Nirom Curtiss and Alfred Curtiss.

x. Edward Augustus, b. Newtown, Conn., Jan. 25, 1825. He d. Sept. I 2, I 825 .

\section{6}

EPENETUS CURTISS (Benjamin, Benjamin, Fosiah, William, Elizabeth), b. Newtown, Conn., Oct. 4, I786; m. Salina, d. of Amos Hurd, of Newtown, Conn. He d. in Bridgeport, Conn., Dec. I 5,1828 . He was a merchant and shipowner.

They had children :-

i. Susan, b. Bridgeport, Conn., I8I8; n. Anson Smith, of Brookfield, Conn.

ii. Charles, b. Bridgeport, Conn., I821. He d. in Georgia unm. in 1857 .

(509) iii. Henry Francis, b. Bridgeport, Conn., May 4, 1823; m. Lany, d. of Hon. Risdon McDaniel, of Jones Co., N. C., Aug. I 2, 1847. He d. in Knox Co., Tenn., Oct. 23, 1893.

JOHN CURTISS (Abijah, Benjamin, Fosiah, William, Elizabeth), b. Newtown, Conn., I764; m. Hannah, d. of John and Phebe Curtiss Beach, of Huntington, Conn. He d. in Huntington, Oct. 19, 1820. She d. May 11, 1816. He lived in Huntington, Conn., where he owned large tanning and dyeing mills, and was a Justice of the Peace.

They had children : -

i. Betsey, b. Huntington, Conn., I79I. She d. I837.

ii. Carlos, b. Huntington, Conn., I794. He d. Oct. I6, 18 I 7 .

iii. Sarah, b. Huntington, Conn., I 796. She d. Jan. I I, I815.

iv. Charles, b. Huntington, Conn., 1799. He d. July 27, 1820. 
v. Russell, b. Huntington, Conn., I 80 I. He d. Oct. I 2, I 820.

vi. Lucy, b. Huntington, Conn., I 803 . She d. Oct. 31, I 820.

vii. David B., b. Huntington, Conn., I 806 . He d. Oct. I I, I 820.

viii. John, b. Huntington, Conn., I 809 . He d. Sept. 29, I820.

(5I0) ix. Abijah Beach, b. Huntington, Conn., I 8I I-I 2; m. Abigail Allen, of Rochester, N. Y. He d. March 26, 184I-2.

\section{9}

ABIJAH BIRDSEY CURTISS (Abijah, Benjamin, Fosiah, William, Elizabeth), b. Newtown, Conn., 1772 ; m. (i.) Anna, d. of James and Eunice Glover, of Newtown, Conn., Sept. 22, 1793. She d. Oct. I3, I 829 , aet. 56 years; m. (ii.) Mary Judson, of Newtown, Conn., Oct. I 8, I831. She d. Dec. 21, 1859. He d. in Newtown, Conn., May 17, 1857 . He was a captain in the 2d Company, 6th Regiment State troops in the War of 1812, and was afterwards made a major.

They had children :-

$(5 I I)$ i. Elihu Starr, b. Newtown, Conn., July 8, I794; m. Mary He d. Jan. I, I 850.

ii. Marcia, b. Newtown, Conn., July 18, 1796; m. John Beach, of Newtown, Conn., May I0, 1818 . She d. Aug. 6, I861.

(512) iii. Horatio Nelson, b. Newtown, Conn., July 27, I798; m. Marcia Nefus, of Rochester, N. Y., . He d. Jan. 3, I 87 I. She d. April I 3,1876 , aet. 68 years.

They had a child : -

Sarah, b. Rochester, N. Y., I 836 ; m. Carlos G. Curtiss, of Rochester, N. Y.

iv. Charlotte, b. Newtown, Conn., July 22, 1800 ; m. Nichols Booth Lake, Nov. 24, 1818 . She d. 1883.

v. Ann, b. Newtown, Conn., Aug. I I, I 802 ; m. Simeon Nichols. She d. Sept. 9, I 852 .

vi. Joseph Beebe, b. Newtown, Conn,, Jan. 18, 1805 ; m. Elizabeth. He d. July 25, 1834 , and his widow m. David S. Baldwin. No issue.

(513)vii. Birdscy Glover, b. Newtown, Conn., Jan. I8, 1807; m. Louisa Ketcham, of Beloit, Wis. He d. July 25, 1834 .

viii. Caroline, b. Newtown, Conn., July I I, I 8 I, I ; m. Simeon B. Peck. She d. May 27, I 858 .

(514)ix. Ira Lawrence, b. Newtown, Conn., Nov. 9, 1813; m. Marietta, d. of John and Polly Curtiss Glover, of Newtown, Conn., Jan. I 8 , I 835. He d. Jan. I, I843. She d. Aug. 30, 1887.

\section{0}

AGUR CURTISS (Silas, Peter, Fosiah, William, Elizabeth), b. Stratford, Conn., Oct. 27, 1771; m. Polly Buckingham. He d. in 
Winfield, N. Y., April 2, i 866 . She d. July I 8, I843, aet. 68 years. He removed to Exeter, N. Y., about 1794, driving through from Connecticut with ox teams. He was a farmer.

They had children :-

(515) i. Roswell, b. Exeter, N. Y., Aug. I, 1795; m. Jerusha Bennet, Oct. I3, 1816. He d. Oct. 25, I 869. She d. July 24, I869, aet. 75 years.

ii. Betsey, b. Exeter, N. Y., March 28, 1796.

(516) iii. David, b. Exeter, N. Y., July 26, I 799 ; m. (i.) Sophia May, March I, I827; m. (ii.) Betsey Rose, of Exeter, N. Y., Nov. 28, I 844. He d. Oct. 13, 1876. She d. May 7, I888, aet. 75 years.

iv. Esther, b. Exeter, N. Y., June 5, I802; m. Sherman Williams, March 4, 1828 . She d. April 15, I 888.

v. Laura, b. Exeter, N. Y., June 7, 1804; m. Jerre Shepherd, of Exeter, N. Y., March 16, 1824.

(517) vi. Lester, b. Exeter, N. Y., May 31, I806; m. (i.) Caroline Bartlett; m. (ii.) Sarah Holmes. He d. Aug. 1 3, I 834.

$\left({ }^{\prime} I^{8}\right)$ vii. Lee, b. Exeter, N. Y., May 30,$1808 ; \mathrm{m}$. Phebe Ann Higbee, of New York City, N. Y., Oct. 1832. He d. Aug. I3, 1834 .

(519) viii. Shelden, b. Exeter, N. Y., Oct. 19, 1810. He d. Aug. 17, I813.

ix. Lucy, b. Exeter, N. Y., Aug. 21, 1813; m. (i.) William Wilbur, of New York City, N. Y., Jan. 31 , I8 33 ; m. (ii.) Daniel Wilcox, of Litchfield, N. Y., Feb. 9, 1859. She d. Sept. 18, 1882 . He d. Feb. 6, 1875 .

(520) x. Charles S., b. Exeter, N. Y., Aug. 18, 1815; m. Abigail M. Robinson, of Exeter, N. Y., Jan. 6, 1836 . He d. Aug. I8, 1852.

xi. Birdseye, b. Exeter, N. Y., June 19, 1818. He d. unm. Feb. 13, 1839 .

xii. Marcia Maria, b. Exeter, N. Y., May 3, I820; m. Samuel McLaughlin, of Winfield, N. Y., Sept. 23, 1846. She d. Jan. 2, I 883. He d. June 22, I 889 .

EBEN BIRDSEYE CURTISS (Silas, Peter, Fosiah, William, Elizabeth), b. Stratford, Conn., April 22, 1773; m. Patty Morse.

They had children :-

(521) ii. John, b.

iii. Matilda, b.,

iv. Celestia, b.,

(522) v. Morse, b., 
LUCIUS CURTISS (Henry, Peter, Fosiah, William, Elizabeth), b. Huntington, Conn., April 6, 1787; m. Polly Ann, d. of Elnathan Bostwick, Oct. 12, 1809. He d. Nov. 2, 1870. She d. Dec. 26, I 83 I.

They had children :-

i. Ann Eloisa, b. Huntington, Conn., Aug. 19, 1810; m. Henry Banks, of North Salem.

ii. Oliver, b. Huntington, Conn., Oct. I7, I8 I 2. He d. Newbern, N. C., Dec. I 2, I 835 .

iii. Eliza Maria, b. Huntington, Conn., Aug. 30, I818; m. Stephen Stillman.

iv. Henry, b. Huntington, Conn., July I 8, 1825.

285

ABNER CURTISS (Reuben, Mattherw, Fosiah, William, Elizabeth), b. Danbury, Conn., Dec. 15, 1776; m. Mary, d. of Moses Osborn, of Danbury, Conn., Nov. 20, 1796. They removed to New York City, N. Y., in 1801 , and he became Clerk of the Court and Chief of Police in that city. He d. in New York City, N. Y., May 14, 1827. She d. in Brooklyn, N. Y., Aug. 1852 , aet. 76 years.

They had children :-

i. Julia, b. Danbury, Conn., Dec. I3, I797; m. Anthony Moffatt, of New York City, N. Y., June 19, I820. She d. March I865.

(523) ii. Edwin C., b. Danbury, Conn., Dec. I 8, I 798 ; m. (i.) Henrietta, d. of John Pitts, of Milledgeville, Ga., May I4, I 823. She d. Feb. 25,1836 ; m. (ii.) Mary, d. of William and Phebe Van de Water, of New York City, N. Y., June 7, 1836. He d. Nov. I I, I864.

(524) iii. Paul Allen, b. Danbury, Conn., Jan. 26, 1800 ; m. Mary Cecilia, d. of Jonas and Catherine Stansbury, of New York City, N. Y., Sept. 30, 1823 .

(525) iv. Moses Osborn, b. New York City, N. Y., Nov. 23, 1801; m. Rebecca, d. of Thomas Whitfield, of New York City, N. Y., July I824. He d. New York City, N. Y., April 1850. She d. Fort Lee, N. J., Nov. 5, 1873, aet. 67 years. No issue. He lived in New York City, N. Y., and was a shoe dealer.

(526) v. Joseph, b. New York City, N. Y., Nov. 23, r803; m. Sarah Anne, d. of William and Phebe Van de Water, of New York City, N. Y., May I3, I826. He d. in New York City, N. Y., July 9, 1867 .

vi. Mary, b. New York City, N. Y., Oct. 19, 1805; m. William Halsey, of New York City, N. Y., Nov. 5, 1828. 
vii. Emma, b. New York City, Sept. 15, 1807. She d. unm. in Brooklyn, N. Y., Sept., I 868.

viii. Harriett, b. New York City, N. Y., Feb. 3, 1809 ; m. John Halsey, of New York City, N. Y., April 28, 1830.

ix. Susan, b. New York City, N. Y., Oct. 28, 1810; m. (i.) Robert Riddel McKay, of New York City, N. Y., Oct. I 4, 1831; m. (ii.) Henry E. Kummel, of New York City, N. Y., May I 2, 1836 .

(527) x. Abner, b. New York City, N. Y., July I 2, I 8 I 2. He d. Feb. 22, I 813 .

(528) xi. Abner, b. New York City, N. Y., June I I, I 8 1 4. He d. unm. Nov., 1835 .

xii. Caroline, b. New York City, N. Y., Feb. 3, 1816; m. Charles Halsey, of New York City, N. Y., IShe d. Jan. 30, 1872.

MAT'THEW CURTISS (Reuben, Matthew, Fosiah, William, Elizabeth), b. Danbury, Conn., Jan. 5, I783; m. (i.) Polly Lacey, of Stratford, Conn., June 7, I 809 . She d. Nov. I 4, I 819 , aet. 33 years; m. (ii.) Rebecca . She d. Aug. Io, I 864, aet. 7 I years. He d. in Bridgeport, Conn., Aug. 25, I864. He served as a lieutenant in the war of I 8 I 2 .

They had children :-

i. Sarah Ann, b. Stratford, Conn., Nov. 16, 1809; m. Jarvis Wilson.

ii. Mary, b. Stratford, Conn., June 7, I8 I i m m. Beardslee.

(529) iii. Frederick Lacey, b. Bridgeport, Conn., Nov. 20, 1813; m. Elizabeth Knapp, of Bridgeport, Conn., Sept. 26, I832.

iv. Harriet, $m$. Noble.

(530) v. Russell T., b. $\quad$ Conn., 822 ; m. Louisa M. Hubbell, of Bridgeport, Conn., Nov. 2, 1847 . He d. Nov. 24, I 877.

vi. Caroline, $b$.

Conn.,

m.

Thompson.

\section{I}

GOULD CURTISS (Gold, Matthew, Fosiah, William, Elizabeth), b. Newtown, Conn., Dec. 27, I787; m. Joanna, d. of Samuel Peck, of Newtown, Conn., April 9, I 8 I 5. He d. in Newtown, Conn., Feb. 3, 1858. She d. in Newtown, Conn., Nov. 7, 1860, aet. 72 years. He was a farmer, and in early life manufactured whips.

They had children :-

i. Mary, b. Newtown, Conn., Jan. 19, 1817; m. Cyrenius Beers, of Newtown, Conn., Oct. 7, 1837 . 
(531) ii. Samuel, b. Newtown, Conn., April 27, 1818; m. Mary Nichols, of Newtown, Conn., Jan. 5, 1848 . He resides in Sandy Hook, Conn.

iii. Elizabeth, b. Newtown, Conn., Sept. 25, 1824; m. Simeon B. Peck, of Newtown, Conn., March 2, I 859.

iv. Sarah, b. Newtown, Conn., Oct. 4, 1834 ; m. Monroe Judson, M.D., of Newtown, Conn., Feb. 3, 1869. He d. March 28, I898. She resides in Newtown, Conn.

292

JOHN GOULD CURTISS (Gold, Matthew, Fosiah, William, Elizabeth), b. Newtown, Conn., March 3, I792; m. Lucy Blakman He was a tanner, and lived in Newtown, Conn., all his life.

They had children :-

(532) i. Daniel Blakman, b. Newtown, Conn., m. Mary A. Brown, of Bath, Steuben Co., N. Y., March 6, I863. He d. Aug. 6, i 883.

(533) ii. Simeon, b. Newtown, Conn.

(534) iii. John, b. Newtown, Conn.

(535) iv. David, b. Newtown, Conn.

293

HEZEKIAH CURTISS (Gold, Mattbew, Fosiah, William, Elizabeth), b. Newtown, Conn., March 25, I796; m. Marcia Glover, of Newtown, Conn. He was a farmer, and lived in Newtown, Conn.

They had children :-

i. Betsey, b. Newtown, Conn., Conn.

$\left(53^{6}\right)$ ii. Benjamin, b. Newtown, Conn., Conn.

m. David Hinman, of Southbury, m. Laura Lewis, of Monroe,

(537) iii. Henry, b. Newtown, Conn., Iowa.

m. Fannie Parker, of Dubuque,

294

DAVID CURTISS (Gold, Matthew, Fosiah, William, Elizabeth), b. Newtown, Conn., July 28, I 798 ; m. Jennet Shelton, of Monroe, Conn. He was a comb manufacturer in early life. He removed to Steuben Co., N. Y., and engaged in the lumber business, converting the pines into lumber, and rafted them down the river to Port Deposit, Maryland.

They had children :-

i. Antoinette, b., 
ii. Phebe, b.,

DANIEL CURTISS (Gold, Matthew, Fosiah, William, Elizabeth), b. Newtown, Conn., Jan. 26, I801; m. Elizabeth Beach, of Trumbull, Conn., March 4, I829. He d. in Bridgeport, Conn., June 21, 1835 . She d. Oct. 27, I868, aet. 64 years.

They had children :-

i. John Beach, b. Bridgeport, Conn., Dec. 15, 1830. He d. Feb. 26, 1859 .

ii. Cornelia Mary, b. Bridgeport, Conn., Aug. 20, 1832; m. Edwin Banks, of Bridgeport, Conn., Dec. 2, 1863. She d. Jan. I, 1885 .

302

PETER BURR CURTISS (Peter B., Peter, Fohn, Israel, Fohn, Elizabeth), b. Lanesboro, Mass., Sept. 27, I779; m. Tammah Mead. He d. in West Martinsburgh, N. Y., March 9, 1837. She d. in West Martinsburgh, N. Y., Nov. 29, I 860.

They had children :-

i. Samantha, b. West Martinsburgh, N. Y., . She d. unm. Aug. 13, 1831 .

(539) ii. Peter Burr, b. West Martinsburgh, N. Y., m. He d. Aug. 23, 1871 .

iii. Maria, b. West Martinsburgh, N. Y., Shipman, $\quad$ She d. in Genoa, N. Y.

(540) iv. Uriah Bradley, b. West Martinsburgh, N. Y., March 17, 1808; m. Sophia Shumwag, of West Martinsburgh, N. Y. . He d. in West Martinsburgh, N. Y., Oct. 22, 1876. She d. Dec. 4, 1872 , aet. 58 years.

v. Veletta, b. West Martinsburgh, N. Y., Hazen, . She d. in Illinois.

vi. Orman, b. West Martinsburgh, N. Y.

m. Charles

BURR CURTISS (Peter B., Peter, Fohn, Israel, Fohn, Elizabeth), b. Lanesboro, Mass., March 19, 1784; m. Lucy, d. of Dexter Brown, of Lanesboro, Mass., March I 7, I 808. He d. June 6, 1825 .

They had children :-

i. Mary, b. Lanesboro, Mass., Dec. 19, 1808; m. Stephen Van Renselear Hoxey, of Lanesboro, Mass., Feb. 9, 1830. 
(541) (ii. Henry, b. Lanesboro, Mass., Dec. 18, 18 13; m. Sarah, d. of $\{$ Charles Buckley, of Granville, N. Y., May 24, I 843.

iii. Harriet, b. Lanesboro, Mass., Dec. I8, I813. She d. March I7, I 814 .

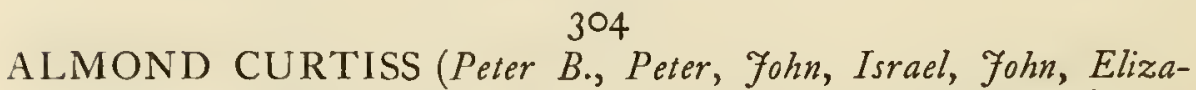
beth), b. Lanesboro, Mass., April 22, 1786; m. Lucy, d. of John Sprague, of Lanesboro, Mass., Dec. 24, 1806. He d. in Lanesboro, Mass., Nov. 19, I 864. She d. Jan. 26, I861. He was a farmer, and most of his life was spent in Lanesboro, Mass., but he also lived in Martinsburgh, N. Y., and Dorset, Vt. In the latter place he kept a hotel.

They had children :-

i. Fanny Maria, b. Lanesboro, Mass., Sept. I4, I807; m. Collins Morse, Jan. 5, 1826 . She d. Oct. $30,1838$.

ii. Hannah Eliza, b. Martinsburgh, N. Y., Feb. 19, 1810; m. David Deming, of Dorset, Vt. She d. Nov. 26, 1872.

iii. Martha Ann, b. Dorset, Vt., Dec. I 4, I82I ; m. William T. Filley, of Lanesboro, Mass. She d. Feb. IO, 1883 .

305

DAVID CURTISS (Peter B., Peter, Fohn, Israel, Fohn, Elizabeth), b. Lanesboro, Mass., June 21, 1789; m. Rachel Cowan in I 8 Io. He was a farmer and lived in Lanesboro, Mass., where he d. Jan. 8, 1841. She d. May 25, 1884.

They had children:-

i. Sophronia, b. Lanesboro, Mass., Feb. 2I, I8I I ; m. Curtiss Tillotson, of Lanesboro, Mass., April 25, 1833 . She lives in Lanesboro, Mass.

ii. Clarissa Jane, b. Lanesboro, Mass., July 5, 18 I 2 ; m. Alonzo Tyler, - She d. July 27,1864 .

iii. Hannah Maria, b. Lanesboro, Mass., Feb. I4, 1814.

iv. David R., b. Lanesboro, Mass., Feb. 2, 181 7. He is a farmer, and resides in Lanesboro, Mass., unm.

v. Almira C., b. Lanesboro, Mass., Nov. 9, 1819. She d. unm. June I0, 1849 .

vi. Abel S., b. Lanesboro, Mass., Dec. I822; d. y.

$$
306
$$

HENRY CURTISS (Peter B., Peter, Fohn, Israel, Fohn, Elizabeth), b. Lanesboro, Mass., April I, I791 ; m. Hannah, d. of Samuel Lyman, of Hinsdale, Mass. He d. in West Martinsburgh, N. Y., July 26, 1867. She d. Jan. 5, I 862 , aet. 69 years. 
They had children :-

(542) i. Charles Henry, b. West Martinsburgh, N. Y., March 9, I815; m. (i.) Mary Lyman, . She d. May 6, I853, aet. 48 years. m. (ii.) Julia Abbe, $\quad$ He d. Sept. 7, I89 I.

ii. John Nelson, b. West Martinsburgh, N. Y., July 5, I819. He was a soldier in the Union Army in the War of the Rebellion and died in the South, Sept. 7, I863, unmarried.

iii. George Dwight, b. West Martinsburgh, N. Y., Aug. 9, 1821; m. Rozella Lyman, of Trenton, N. Y. He d. Oct. 22, 1878.

iv. Lyman Sylvester, b. West Martinsburgh, N. Y., Feb. 23, 1823; m. Sarah Maria Lyman, of West Martinsburgh, N. Y. She d. March 8, 1871, aet. 44 years.

v. Phebe Amanda, b. West Martinsburgh, N. Y., Feb. 8, 1829; m. Edward Bayard Livingston, Sept. 6, 1849, and lives in Brooklyn, N. Y.

vi. Alvira Thresa, b. West Martinsburgh, N. Y., Nov. 29, 1830; m. George Sawyer. She d. in Fond du Lac, Wis.

vii. Caroline Maria, b. West Martinsburgh, N. Y., April 31, 1832; m. William Antisdel, and lives in Topeka, Kan.

viii. Sarah Melvina, b. West Martinsburgh, N. Y., Jan. 3I, I834; m. Frank Buck, and lives in Sedgwick, Kan.

\section{7}

SHERMAN CURTISS (Peter B., Peter, Fohn, Israel, Fohn, Elizabeth), b. Lanesboro, Mass., April I3, I799; m. Maria, d. of Silas Butler, of Lanesboro, Mass., April 25, 1820 . He d. in Pittsfield, Mass., Jan. 22, 1878. She d. Sept. 20, I 888, aet. 89 years.

They had children :-

i. Louisa Amelia, b. Lanesboro, Mass., Dec. II, 182I ; m. Simon Wood, of Lanesboro, Mass. She d. Oct. 25, I867.

(543) ii. Charles Butler, b. Lanesboro, Mass., June 5, 1824; m. (i.) Maria Wright ; m. (ii.) Rebecca Lamphere. They reside in Elgin, Ill.

iii. Henry Burr, b. Lanesboro, Mass., July 17, 1826. He d. unm. Sept. 2, 1844 .

iv. Sarah Maria, b. Lanesboro, Mass., July 8, г 829 ; m. Charles Henry Read, of No. Adams, Mass., Nov. 28, 1850 . They live in No. Adams, Mass.

v. Phebe Augusta, b. Lanesboro, Mass., Feb. 3, 1832 ; m. James Sy!vester Rathburn, Dec. 25, i 850 . She d. March 18, I889.

vi. Margaret Hoxey, b. Lanesboro, Mass., Aug. I 7, 1838; m. John R. Bartlett. She d. July 12, 1893 .

vii. $\{$ Martha Evaline, b. Lanesboro, Mass., May 30, 184I.

viii. \{ Mary Emaline, b. Lanesboro, Mass., May 30, I 841 ; m. Simeon Morton Dean, Dec. 25, I860. 
DAVID STILES CURTISS (David, David, Fohn, Israel, Fohn, Elizabeth), b. Woodbury, Conn., Jan. I4, I767; m. Sybilla, d. of Daniel and Sybilla Huntington, of Woodbury, Conn. She d. Dec. 31 , I 837 , aet. 68 years. He was a farmer and resided in Woodbury, Conn., where he d. Jan. 2 I, I 846.

They had children :-

i. Sybilla Cleora, bapt. Woodbury, Conn., Nov. 23, I 794 ; m. Roderick Stiles, $\quad$ She d. Nov. $27,1852$.

(547) ii. David Huntington, bapt. Woodbury, Conn., April 3, 1796; m. (i.) Maria Summers, of Woodbury, Conn., Dec. 20, I825. She d. Oct. 5, 1828, aet. 24 years; m. (ii.) Anna Guernsey. He d. Jan. 25, I862. She d. Aug. 31, I 866, aet. 59 years.

iii. Sarah, bapt. Woodbury, Conn., Feb. I I, I 798 ; m. Rufus Stiles, of Woodbury, Conn, Nov. 9, 1822.

iv. Nathan, bapt. Woodbury, Conn., March I 9, I 799 ; d. y.

(545) v. Daniel, bapt. Woodbury, Conn., Nov. 8, I80 I ; m. Julia Flora, d. of John and Flora Strong, of Woodbury, Conn., Jan. 27, 1835. He d. May i6, I 878 .

vi. Elvira, bapt. Woodbury, Conn., Nov. I3, I803. She d. unm. Dec. 24,1837 .

vii. Mary Ann, bapt. Woodbury, Conn., June 6, I805; m. Oliver S. Weller, of Stratford, Conn., Jan. 22, 1838.

viii. (Maria, bapt. Woodbury, Conn., Aug. I 3, 1808 .

ix. Caroline, bapt. Woodbury, Conn., Aug. I 3, I 808.

x. Harriet, bapt. Woodbury, Conn., Aug. I 3, I 808 .

xi. (Eliza, bapt. Woodbury, Conn., Aug. I 3, I 808 .

xii. William, bapt. Woodbury, Conn., Sept. 24, I809.

xiii. Charles, bapt. Woodbury, Conn., July 5, I8 I 2.

SIMEON CURTISS (Foseph, Israel, Stephen, Israel, Fobn, Elizabeth), b. Southbury, Conn., Jan. 2, 1768; m. Mary Bradley, of Southbury, Conn., Aug. I2, I 790. He died in Southbury, Conn., April 2, 1852 . She d. Jan. 19, I 850 , aet. 85 years. He was a farmer.

They had children :-

$(546)$ i. Erastus, b. Southbury, Conn., Nov. 26, 1792. He was a physician.

(547) ii. Jason, b. Southbury, Conn., July I I, I794; m. Maria E. ; no issue. He was a farmer.

(548) iii. Reuben Bradley, b. Southbury, Conn., Dec. I 9, I 796; m. Minerva, d. of David and Sally Booth McEwen, of Oxford, Conn., March I I, I 820. He d. Aug. 1 5, I882. She d. Oct. 20, 1867 . 
(549) iv. William, b. Southbury, Conn., April 25, 1798.

v. Mary Ann, b. Southbury, Conn., Jan. 29, I804; m. Josiah Hines.

(550) vi. Simeon, b. Southbury, Conn., Nov. I3, I805; m. Hannah Bronson.

3 I 5

ABEL CURTISS (Foseph, Israel, Stephen, Israel, Fobn, Elizabeth, bapt. Southbury, Conn., May 10, I776; m. Hannah Atwater, of Southbury, Conn., Feb. I, I802. He d. in Southbury, Conn., Nov. 9, 1834 .

They had children :-

i. Alford, bapt. Southbury, Conn., April 24, 1803 .

(55I) ii. Charles, bapt. Southbury, Conn., Jan. 30, 1805 .

CHARLES RANSOM CURTISS (Stephen, Israel, Stephen, Israel, Fohn, Elizabeth), b. Southbury, Conn., May 7, I788; m. Jeannette, d. of Benjamin and Esther Preston Stiles, of Southbury, Conn., March 24, I817. He was a farmer and lived in Southbury, Conn., where he d. June 24, I 844 . She d. in Southbury, Conn., Aug. 8, I 875 , aet. 84 years.

They had children:-

i. Harriet Stiles, b. Southbury, Conn., Aug. 7, 1818; m. Jasper Osborn, of Southbury, Conn. She d. in East Oakland, Cal., March 20, 1890.

ii. Mary Mallory, b. Southbury, Conn., May 24, 1820. She d. unm. Oct. I0, 1878 .

(552) iii. Benjamin Stiles, b. Ellisville, N. Y., July 23, I822; m. Martha Jennette, d. of Stoddard and Jennette Mallory Strong, of Woodbury, Conn., Jan. 27, I850. They reside in Woodbury, Conn.

iv. Charles Burke, b. Ellisville, N. Y., Sept. 5, I824. He d. unm. in East Oakland, Cal., Dec. 2, I 888.

v. Walter Henry, b. Ellisville, N. Y., April 2, I827; m. Margeth Hinman, of Southford, Conn. No issue.

(553) vi. Horace Horatio, b. Ellisville, N. Y., March 16, I829; m. Mary, d. of Stephen Sypert, of Jackson, Tenn., Aug. 3I, i 853. They live in Jackson, Tenn.

vii. Theron Skeele, b. Ellisville, N. Y., Aug. 5, I831. He d. unm. Aug. 5, 1852 .

JAPHET CURTISS (Benjamin, Israel, Stephen, Israel, Fohn, Elizabeth), b. Southbury, Conn., Dec. I 2, I 779 ; m. Lucy Strong, of Southbury, Conn., June 5, 1805. 
They had children :-

i. Lucy, b. Southbury, Conn.,

ii. Louisa, b. Southbury, Conn.

iii. Maria, b. Southbury, Conn.,

(554) iv. Benjamin, b. Southbury, Conn.,

v. Julia, b. Southbury, Conn.,

(555) vi. Japhet, b. Southbury, Conn.,

(556) vii. Theron E., b. Southbury, Conn.

I813. She d. Dec. 12, I814.

557) viii. Carlos B., b. Southbury, Conn., June, I 824 ; m. Rachei

d. in Bridgeport, Conn., March 30, I893.

They had a child :-

Charles T., b. . He lives in Bridgeport, Conn.

324

SHERMAN CURTISS (Benjamin, Israel, Stephen, Israel, Fohn, Elizabeth), b. Southbury, Conn., Jan. 26, I78 I ; m. Jerusha, d. of Thaddeus Bronson, of Woodbury, Conn.,

He d. in Southbury, Conn., I 848.

They had children :-

$\left(55^{8}\right)$ i. Chester, b. Southbury, Conn.

ii. Esther, b. Southbury, Conn.

iii. Mary Ann, b. Southbury, Conn.

iv. Olive, b. Southbury, Conn.

325

CYRUS CURTISS (Benjamin, Israel, Stephen, Israel, Fohn, Elizabeth), b. Southbury, Conn., Dec. I 2, I 786 ; m. Julia, d. of Benjamin Strong. He d. in Southbury, Conn.,

I 875 .

They had children :-

(559) i. Henry, b. Southbury, Conn.

ii. Annis, b. Southbury, Conn.

(560) iii. Benjamin Strong, b. Southbury, Conn.

iv. Julia, b. Southbury, Conn.

$$
326
$$

JAUNCEY CURTISS (Abel, Benjamin, Nathaniel, Thomas, Fohn, Elizabeth), b. Meriden, Conn., I 768 ; m. Jerusha Hall, Dec. 3, 1793. He d. in Wallingford, Conn., Oct. 7, I 845 .

They had children :-

$(563)$ i. Jauncey, b. Wallingford, Conn.

$(567)$ ii. Orrin, b. Wallingford, Conn., in 1823 .

I800; m. Sally Bristol 
iii. Mary, b. Wallingford, Conn.

iv. Sarah, b. Wallingford, Conn., $\quad 1803$.

(565) v. William, b. Wallingford, Conn.

(567) vi. Harvey, b. Wallingford, Conn., April 3, 18 15; m. Julantia E. Terrell, of Cheshire, Conn.

\section{8}

ANDREW CURTISS (Abel, Benjamin, Nathaniel, Thomas, Fohn, Elizabeth), b. Meriden, Conn., m. Huldah Curtiss, of Harwinton, Conn., Nov. 13, 1792. He d. Sept. 23, I801. His widow m. Elnathan Corner.

They had children : -

(568) i. Bradley, b. Wallingford, Conn., Oct. 3, r 793.

(569) ii. Chandler, b. Wallingford, Conn., May 7,1795 . He taught school in N. W. district of Meriden, and then became a Methodist minister and went South.

iii. Elizabeth, b. Wallingford, Conn., May 22, 1797.

iv. Amanda, b. Wallingford, Conn., July 25, 1799.

(570) v. Andrew, b. Wallingford, Conn., Sept. 8, I80I; went to New York City, N. Y.

\section{9}

LEVI CURTISS (Abel, Benjamin, Nathaniel, Thomas, Fohn, Elizabeth), b. Meriden, Conn.; m. (i.) Lucy Hall, March 28, I790; m. (ii.) Sarah, d. of Jacob Francis, Dec. 6, 1792. In 1807 he removed to Orange, New Haven Co., Conn.

They had children : -

(57I) i. Levi, b. Wallingford, Conn., May 12, 179 I.

ii. Lucy, b. Wallingford, Conn., May 24, 1794.

(572) iii. Jerry, b. Wallingford, Conn., Feb. 24, 1796.

(573) iv. Alfred, b. Wallingford, Conn., Jan. 1, 1798.

v. Patty, b. Wallingford, Conn., Oct. 24, I 800.

(574) vi. Hawley, b. Wallingford, Conn., March 2, I80x.

(575) vii. Leonard, b. Wallingford, Conn., June 15, 1805.

CHAUNCEY CURTISS (Abel, Benjamin, Natbaniel, Thomas, Fohn, Elizabeth), b. Meriden, Conn., Dec. 20, I786; m. Anna Hall, Sept. 9, 1804. She d. Nov. 5, 1826. He lived in Meriden, Conn., where he d. Dec. 6, I 824 .

They had children : -

i. Louisa, b. Meriden, Conn., Aug. 22, I805; m. Eli Elisha Curtiss, of Meriden, Conn., Feb. 27, 1825 . 
ii. Caroline, b. Meriden, Conn., Nov. 25, 1807. She d. April 7, I8I I.

iii. Julia A., b. Meriden, Conn., April 7, I810; m. Eli Elisha Curtiss, of Meriden, Conn., Aug. 31, 1830.

(576) iv. Isaac W., b. Meriden, Conn., Oct. 27, 1812; m. Amy Ann Webster, of Southington, Conn., July I 4,1834 .

v. Clarissa, b. Meriden, Conn., Jan. 22, I 8 I 4 ; m. Ezra Rutley, Sept. 16,1832 .

(577) vi. Harrison, b. Meriden, Conn., Aug. 20, 1817 ; m. (i.) Eliza Bradley, Oct. I 7, 1838 ; m. (ii.) Martha L. , Sept. 29, I 850. He d. Jan. I 5,1869 .

$(578)$ vii. Edmund B., b. Meriden, Conn., Oct. 22, I819; m. Hannah L. Lane, Feb. 15, I 852 . He d. Nov. 27, 1880.

viii. Caroline, b. Meriden, Conn., Nov. 30, I82 I ; m. Edward N. Hall, Jan. $\quad$ I 843 .

\section{$33^{\mathrm{I}}$}

AARON CURTISS (Benjamin, Benjamin, Nathaniel, Thomas, Fohn, Elizabeth), b. Wallingford, Conn., Feb. 9, I765; m. Susanna Butler, Oct. 13, 1793. He was a farmer, and lived in Meriden, Conn. He d. Dec. 24, I 84 I.

They had children :-

(579) i. Oliver Bronson, b. Meriden, Conn., Aug. 9, I 794. He d. unm.

(580) ii. Asahel Benham, b. Meriden, Conn., Jan. I0, I797; m. Emily M. Hubbard, of Durham, Conn., I 820 . He d. July 7, 1864. She d. Aug. 25, I886.

iii. Daniel Reed, b. Meriden, Conn., April 24, I800. He lived in Whitestone, L. I., and d. unm.

BENJAMIN CURTISS (Benjamin, Benjamin, Nathaniel, Thomas, Fohn, Elizabeth), b. Wallingford, Conn., May 19, I766; m. Molly, d. of Capt. Dan Collins, Jan. 22, I796. He d. in Meriden, Conn., Oct. 23, 1843. She d. Feb. 20, 1835, aet. 60 years.

They had children :

i. Laura, b. Meriden, Conn., Nov. 2 I, I797. She d. unm. Aug. 5, I 872 .

$(58 I)$ ii. Homer, b. Meriden, Conn., Aug. 4, 1800; m. Julia Ann Upson, of New Britain, Conn., Nov. 26, I835. He d. May 30, I880.

iii. Julia, b. Meriden, Conn., I80 I. She d. unm. Dec. I 3, I 856 .

iv. Henry, b. Meriden, Conn., 1802 ; m. Henrietta Malone.

They had a child : -

i. Henry, b. Meriden, Conn., Oct. 1844. He died July, I 852 .

v. Mary, b. Meriden, Conn.,

m. Samuel Roberts, May 5, 1829, and removed to Kentucky.

( 102 ) 
(582) vi. Dan Collins, b. Meriden, Conn., Oct. 23, 1807; m. (i.) Harriet Atwater, April 12, 1842; m. (ii.) Frances Ann French, May 9, I 847 .

vii. Louisa, b. Meriden, Conn., and moved to Brownsville, $\mathrm{Pa}$.

viii. Elizabeth, b. Meriden, Conn., of Baltimore, Md., Aug. 13, 1832.

m. William Seeright,

m. Edwin L. Lines,

\section{3}

IVAH CURTISS (Benjamin, Benjamin, Nathaniel, Thomas, Fohn, Elizabetb), b. Wallingford, Conn., Oct. 19, 177 i ; m. Hannah Ives, of Wallingford, Conn., Aug. 4, 1796. He d. Aug. 27, 1852 . She d. Oct. 8,1844 , aet. 66 years.

They had children :-

i. Lucretia Ives, b. Wallingford, Conn., June 22, 1798; m. Geo. Wilcox, of Saratoga, N. Y., in 1816.

ii. Samuel Ives, b. Wallingford, Conn., Sept. 8, I80I. He d. April 25,1802 .

$(583)$ iii. Samuel Ives, b. Wallingford, Conn., March 5, I803; m. (i.) Rebecca Tuttle Hough, of Wallingford, Conn., Oct. 3, I8 32 . She was killed March 25, $1842 ; \mathrm{m}$. (ii.) Eliza, d. of Jesse Ives, of Stafford Springs, Conn., Jan. 30, I843. She d. May 31, I 865 ; m. (iii.) Mrs. Deborah N. G. Curtiss, widow of Erastus Ives, June I 5, I866. He d. March 26, I880. She d. I 88 I.

(584) iv. Erastus Ives, b. Meriden, Conn., May I9, I805; m. Deborah N. G., d. of Latkin Newton, of Petersham, Mass., May, I833. He d. March I9, 186I.

$(585)$ v. Charles Augustus, b. Meriden, Conn., Aug. 17, 1807; m. Laura Paulk, of Tolland, Conn. He d. Sept. 5, 1853.

vi. Emily Melissa, b. Meriden, Conn., Dec. 25, 1809; m. Kirtland Hall, of Wallingford, Conn., Oct. 22, 1833 .

vii. Mary Rice, b. Meriden, Conn., April 9, 18 12. She d. July I, 1832 , in Otis, Mass.

viii. Hannah, b. Meriden, Conn., March 30, I822; m. Rev. John T. Cushing, Sept. I 5, I 847 .

\section{4}

ELISHA CURTISS (Benjamin, Benjamin, Nathaniel, Thomas, Fohn, Elizabeth), b. Wallingford, Conn., June 19, 1773; m. (i.) Abigail, d. of Capt. Enos Hall, Feb. 18, I796. She d. Feb. 28, I826, aet. 47 ; m. (ii.) Mary, d. of Capt. Enos Hall, April 3, I 826. She d. Aug. 29, 1857 , aet. 72. He d. July 28, I 847 . He was a representative from Meriden, Conn, in 1818 , and was senior warden of St. Andrew's Church for many years. 
They had children :-

$(586)$ i. Enos Hall, b. Meriden, Conn., Sept. I 2, I 796 ; m. Lois K. Bailey. He d. April 30, 1862. She d. May 10, 1887, aet. 86.

ii. Lucy, b. Meriden, Conn., April I 4, I800; m. Horace Redfield, Dec. 25,1817 .

$\left(55_{7}\right)$ iii. Eli Elisha, b. Meriden, Conn., Feb. 27, 1804 ; m. (i.) Louisa, d. of Chauncey Curtiss, of Meriden, Conn., Feb. 27, I825. She d. May 18, $1830 ;$ m. (ii.) Julia A., d. of Chauncey Curtiss, of Meriden, Conn., Aug. 31, I830. He d. Feb. I 1, 1861.

iv. Edwin Erastus, b. Meriden, Conn., Sept. 1, 1805; m. Aurelia Benham, Dec. 24, I826. He d. April 29, 1885. She d. Sept. 4, 1879. For many years he was a manufacturer in Meriden, and president of the Meriden Savings Bank. He held many positions of trust. At his death he bequeathed most of his property to St. Andrew's Church, to which during his lifetime he had given many gifts.

(588) v. Lemuel Johnson, b. Meriden, Conn., Jan. 15, 1814; m. Bedotha Pierpont Button, of North Haven, Conn., Dec. 24, I835. He d. Jan. I0, I 888 .

vi. James Harrison, b. Meriden, Conn., March 5, 1817. He d. March 2, 18 I9.

(589) vii. Alanson, b. Meriden, Conn., Oct. 27, 1818; m. Harriet Eliza Sullivan, Feb. I, I 840 . He d. Sept. $27,1856$.

AMOS CURTISS (Benjamin, Benjamin, Nathaniel, Thomas, Fohn, Elizabeth), b. Wallingford, Conn., April 14, 1779; m. (i.) Polly, d. of Abel Curtiss, of Wallingford, Conn., March 18, I802. She d. April 30, 1818, aet. 34 years; m. (ii.) Louisa Johnson, Dec. 6, 1818. She d. in Williamsport, Penn., April 16, 1872, aet. $8 \mathrm{I}$ years. He d. Sept. 25,1857 . He held many positions of trust, and was a prominent man in Meriden. He was town clerk of that town from $1826-1830$, and a representative in 1824 .

They had children :-

i. Amelia, b. Meriden, Conn., Sept. 7, 1804 ; m. David Borbul Gilbert, of Tolland, Conn., Nov. 30, 1824 . She d. July 1 5, 1827 .

ii. Maria, b. Meriden, Conn., July 16, 1806. She d. April 13, I818.

iii. Ruth, b. Meriden, Conn., March I 4, I 809 . She d. Dec. 3, I 8 I 2.

iv. Polly, b. Meriden, Conn., March I6, I8 I 2. She d. April I0, I826.

v. William Amos, b. Meriden, Conn., May 26, 1816; m. (i.) Sarah Higley, of Canton, Conn., Sept. 12, 1838 . She d. March 24, 1847 ; m. (ii.) Adeline Higley, of Canton, Conn., Aug. 22, 1849. He d. April 19, 1893.

They had a child: -

i. William Munson, b. Meriden, Conn., July 24, 1848 .

He d. Aug. 15, 1856. 
vi. Lucy Maria, b. Meriden, Conn., Jan. 15, I820; m. Edgar Munson, of Bradford, N. Y., June 15, I 852 . They live in Williamsport, $\mathrm{Pa}$.

(59o) vii. Sylvester Johnson, b. Meriden, Conn., Sept. 30, I8 26 ; m. Harriet A., d. of Thomas Hulse, of New York City, N. Y., April 23, 1851 .

$$
336
$$

SAMUEL CURTISS (Benjamin, Benjamin, Nathaniel, Thomas, Fohn, Elizabeth), b. Wallingford, Conn., June 30,1781 ; m. Polly Andrews, Nov. 20, 1805 . She d. Nov. 5, 1846, aet. 61 years. His will was filed Dec. I 9,1838 .

They had children : -

i. Eliza Ann, b. Wallingford, Conn., March 4, 1807 ; m. Henry D. Booth, of Bridgeport, Conn., Aug. 29, 1831. She d. April 29, I 884 .

ii. Emeline, b. Wallingford, Conn., Sept. I2, r808; m. Lewis S. Green, April 19, 1827. She d. Feb. 5, I881.

(59I) iii. Alfred Pierpont, b. Wallingford, Conn., July 4, I817; m. (i.) Emeline Amelia, d. of Levi and Abigail Ann Bradley, Oct. 19, 1838. She d. July 19, I 847 , aet. 27 years; m. (ii.) Sarah M. Hitchcock, of Ashtabula, Ohio. She d. Dec. 5, I 857 , aet. 24 years ; m. (iii.) Julia Hannah Phelps, of Ashtabula, Ohio, Jan. I 4, 1868 . He d. July 21, I 870 .

iv. Polly Samantha, b. Wallingford, Conn., Jan. I 8, I8 I 2; m. Wm. H. Green, Nov. 4, I830, of New Haven.

\section{7}

ASAHEL. CURTISS (Benjamin, Benjamin, Nathaniel, Thomas, Fohn, Elizabeth), b. Wallingford, Conn., July 2, 1786; m. Mehitabel, d. of Augustus and Ann Redfield, of Clinton, Conn., Nov. 8, 18 1 2. He was a member of the Compass Lodge of Wallingford, Conn., and a charter member of the Meriden Lodge in 1851 and the first Senior Warden of the latter. He was appointed Ensign by Gov. Oliver Walcott, May 20, 1820; Tax Collector, June 6, 1825; Town Treasurer, June I 8, I 834; and a representative from Meriden, Conn., in 1836 . Asahel Curtiss was a private in war of 1812 .

They had children : -

i. Jennett, b. Meriden, Conn., March I 4, I81 4; m. Charles H. Clark, of Meriden, Conn., Dec. 25, 1836.

ii. Phebe Ann, b. Meriden, Conn., June 2I, I $815 ; \mathrm{m}$. Rev. Robert A. Hallam, of New London, Conn., Nov. 4, 1834 . She d. Jan. 27, I891. 
iii. Benjamin Upson, b. Meriden, Conn., July 20, 1817. He d. Sept. 2, I 835 .

(592) iv. Asahel, b. Meriden, Conn., Feb. 25, 1821 ; m. Annie E., d. of Silas and Abigail Wilcox, of Clinton, Conn., Feb. 29, 1844. He d. Dec. 2, 1889 .

(593) v. George Redfield, b. Meriden, Conn., Dec. 25, I 825 ; m. Augusta, d. of Jesse and Sophia Munson, "of Bradford, N. Y., May 22, 1855. He d. May 20, 1893.

\section{$33^{8}$}

ROSWELL CURTISS (Bemjamin, Benjamin, Nathaniel, Thomas, Fohn, Elizabeth), b. Wallingford, Conn., Dec. 24, 1788; m. Eunice Scott, July 30, 1809. He d. and his widow m. Darius Benham, May 4, 1836 .

They had children :-

i. Amasa Horatio Nelson, b. Wallingford, Conn., Jan. 30, t810. d. y.

ii. Lauretta, b. Wallingford, Conn., Sept. 3, I81 2 ; m. William Matthews, of Southington, Conn., Dec. $23,1832$.

343

HORACE CURTISS (Nathaniel, Nathaniel, Nathaniel, Thomas, Fohn, Elizabeth), b. Wallingford, Conn., m. Julia Bradley, Jan. 27, 1832. He d. in Wallingford, Conn., 1840. Horace Curtiss was a private in the war of 1812 .

They had children :-

i. Horace, b. Wallingford, Conn., $\quad 1840$.

\section{8}

LEVI CURTISS (Solonion, Samuel, Samuel, Thomas, Fohn, Elizabeth), b. Southington, Conn., Jan. 10, 1772; m. Nancy, d. of Cornelius and Jemima Andrews Dunham. He d. May 31, 1840 . She d. June 12, 1831 , aet. 60 years. He lived on Redstone Hill.

They had children :-

(594) i. Chester, b. Southington, Conn., March 3 I, I 793; m. Elizabeth Hitchcock, of Cheshire. He d. July 6, 1826 . She d. Oct. 8, I 826 , aet. 25 .

ii. Emily, b. Southington, Conn., Nov. 12, 1795. She d. July 27, I 8 I 8.

(595) iii. George, b. Southington, Conn., March 18, 1797; m. Ann E. Leyden, of North Carolina.

iv. Nancy, b. Southington, Conn., Aug. 1 0, I798; m. Andrew Langdon.

(596) v. Levi, b. Southington, Conn., Feb. 19, I800; m. Julia Brooks, of Cheshire. 
(597) vi. Solomon, b. Southington, Conn., Sept. 3, I80 I ; m. Sarah Cooke.

vii. Lydia, b. Southington, Conn., April 25, 1803. She d. Oct. I 8, I 826 .

$\left\{\begin{array}{l}\text { viii. Triplets, b. Southington, Conn. } \\ \text { ix. } \\ \text { x. }\end{array}\right\}^{\text {d. y. }}$

(598) xi. Charles, b. Southington, Conn., Sept. I3, I807.

xii. Sarah, b. Southington, Conn., April I 7, 1809; m. Henry A. Clark, Nov. 15,1832 .

xiii. Jeanette, b. Southington, Conn., July 27, 18 I I ; m. Asahel Carter. xiv. Abigail, b. Southington, Conn., Nov. $\quad 18$ I 3 .

\section{0}

AZOR CURTISS (Urie, Azor, Samuel, Thomas, Fohn, Elizabeth), b. Rutland Co., Vt., July 23, 1792 ; m. (i) Catherine Fowler, Dec. 20, I8 1 2. She d. Dec. 23, I839, aet. 46 years; $m$. (ii) Eliza Ann Standing, Oct. 28, I 840 . She d. A pril, i 898 , aet. 84 years. He d. in Le Roy, N. Y., Aug. 5, 1880.

They had children :-

i. Happalorna Katherine, b. Le Roy, N. Y., Oct. I I, I8 I3. She d. Jan. 29,$1838 ; \mathrm{m}$. John Longworthy in 1834 .

ii. Betsey Lorage, b. Le Roy, N. Y., Aug. 3, I8 I 5 ; m. Asa Currier, Dec. I 2, I833. She d. Oct. 22, I860.

iii. Sally Ann, b. Le Roy, N. Y., March I, 1817 ; m. B. Evans Ensign, of Perry, N. Y., Nov. 14, I840. She d. Sept., I 843.

iv. Cynthia, b. Le Roy, N. Y., Dec. 10, I8 18 ; m. B. Evans Ensign, of Perry, N. Y.

v. Alfred Azor, b. Le Roy, N. Y., Sept. I 2, I 820 ; m. Diana Farnum, of Le Roy, N. Y., Dec. I, 1841. He d. Dec., 1847.

vi. Harriet, b. Le Roy, N. Y., Aug. 9, 1822. She is unm. and resides in Le Roy, N. Y.

(599) vii. Stephen Fowler, b. Le Roy, N. Y., Sept. 6, I 824 ; m. Sally L. Stevens, of Bergen, N. Y., April 23, I 846. They reside in Le Roy, N. Y.

viii. Trephena Emily, b. Le Roy, N. Y., Oct. 22, I826; m. William E. Palmer, of Auburn, N. Y., Oct., 1845. She d. Sept. 24, 1855 .

ix. Elsie Berry, b. Le Roy, N. Y., March 28, I829; m. Le Roy Hart Kimberly, of Auburn, N. Y., Jan. I I, I855. They reside in Auburn, N. Y.

(60o) x. Solomon, b. Le Roy, N. Y., Dec. I 8, 1831 ; m. Mary Pugeley, of Rochester, N. Y. He d. in Le Roy, N. Y., in 1894.

xi. Urie Carlton, b. Le Roy, N. Y., March 3, 1842. He d. June 4, 1842.

xii. Jane, b. Le Roy, N. Y., Jan., I843; m. John Walker. They reside in Le Roy, N. Y. 
xiii. John B., b. Le Roy, N. Y.,

xiv. Phcbe, b. Le Roy, N. Y.,

xv. William, b. Le Roy, N. Y., Sept. 25, I848. He is a resident of Berlin, Mich.

xvi. Horace, b. Le Roy, N. Y., Jan. 5, 1851 . He is a resident of Le Roy, N. Y.

xvii. Mary Eliza, b. Le Roy, N. Y., June 6, I853.

xviii. James, b. Le Roy, N. Y., March 24,1858 . He is a resident of Detroit, Mich.

\section{2}

ELIPHALET CURTISS (Eliphalet, Peter, Foseph, Thomas, Fohn, Elizabeth), b. Simsbury, Conn., Nov. 9, 1758 ; m. Deborah He removed to Granby, but returned to Simsbury, where he d. in 8 I 7. He enlisted in the Revolutionary army at the Lexington alarm, and was made a sergeant. In June, I776, he was made a $2 \mathrm{~d}$ lieutenant in Captain Robinson Co. under Colonel Mott, and served at Fort Ticonderoga. At the Danbury Raid, April, 1777, he appears as a captain.

They had children :-

i. Harriet, b. Simsbury, Conn.,

ii. Herman, b. Simsbury Conn.,

iii. Jabez, b. Simsbury, Conn.,

iv. Marian, b. Simsbury, Conn.,

v. Edmund, b. Simsbury, Conn.,
1800.

1801 .

1805 .

1808.

I 8 Io.

356

ADNA CURTISS (Peter, Peter, Foseph, Thomas, Fohn, Elizabeth), bapt. Farmington, Conn., $1766 ; \mathrm{m}$. Almira

He d. May 25, I 8 1 5. She d. in Buffalo, N. Y., Sept. I 8, I 848, aet. 8 I years.

They had children :-

i. Sylvia, b. Farmington, Comn,, I 789 .

ii. Peter, b. Farmington, Conn., N. Y., in 1823.

iii. Ruth A., b. Farmington, Conn., 13,1802 .

iv. Sylvia, b. Farmington, Conn.,

1787. She d. Nov. 7,

He removed to Buffalo,

1794. She d. March

m. Abner Bidwell.

357

CHARLES CURTISS (Peter, Peter, Foseph, Thomas, Fohn, Elizabeth), b. Farmington, Conn., March 22, $1778 ; \mathrm{m}$. Fanny, d. of Gen. Solomon Cowles, of Farmington, Conn., about i 803 . They 
removed to Madison, Ohio, in 1811 , making the journey through the wilderness in ox teams. He d. Dec. 27, 1833. She d. Nov. 26,1859 , aet. 76 years.

They had children:-

i. Henry A., b.

He d.

They had a child:
Feb. 10, 1804 ; m. Zilpha Henries, 1873 .

He resides in New York State.

ii. Fanny C., b. Sept. I6, 1805; m. Ethan A. Judson,

iii. Polly I., b. She d. Dec. 27,1848 .

iv. Louisa A., b. - She d. Aug. 27,$1807 ; \mathrm{m}$. Walter Crocker, - She d. 1893 .

Aug. 29, 1809; m. Warren Maltbie, 1893 .

v. Charles S., b. Madison, Ohio, March 3, 1812; m. Emeline Baker. They had a child :-

i. Stephen C., b.,

He is living in Elgin, Ill.

vi. Mary A. R., b. Madison, Ohio, Oct. 26, I8 14; m. Darius Crombie, of Chicago, Ill. She d. Aug. 26, 1896.

vii. Chloe R., b. Madison, Ohio, Jan. 26, 1817 ; m. Ambrose Branch.

viii. Harriet N., b. Madison, Ohio, Aug. 8, 1819; m. Alva Ross. She d. May 28, I 893 .

ix. Martha C., b. Madison, Ohio, Oct. 17, 1821. She d. unm.

x. Sarah E., b. Madison, Ohio, July 23, 1824 ; m. S. J. Wadsworth. She resides in San Francisco, Cal.

(602) xi. George Cowles, b. Madison, Ohio, Feb. 21, 1827; m. Mary E. Pancost. They reside in Painsville, Ohio.

$$
361
$$

AMASA CURTISS (Amasa, Fonatban, Foseph, Thomas, Fohn, Elizabeth), b. Tyringham, Mass., Aug. 17, 1776 ; m. (i.) Electra m. (ii.) Fanny

They had children :-

i. Betsey, b. Monterey, Mass., July 23, 1797 ; m. Barnabas Bidwell.

ii. Orlando, b. Monterey, Mass.

iii. Edwin, b. Monterey, Mass. d. y.

\section{2}

ZEBINAH CURTISS (Amasa, Fonathan, Foseph, Thomas, Fobn, Elizabeth), b. Tyringham, Mass., Nov. 25, 1785 ; m. Julia H. Buel, of Monterey, Mass. He d. in Tyringham, Mass., Oct. 21,1818 .

They had children :-

i. Lambert B., b. Monterey, Mass., Aug. 6, I809. 
ii. Julia Ann, b. Monterey, Mass., Aug. 23, 1812; m. (i.) Henry Underwood; m. (ii.) Oliver Baird.

$(607)$ iii. Orlando F., b. Monterey, Mass., Oct. 30, 1815. He went out West.

363

CALEB CURTISS (Amasa, Fonathan, Foseph, Thomas, Fohn, Elizabeth), b. Tyringham, Mass., Feb. 3, 1784; m. Harriet Hines.

They had children : -

(605) i. Zebinia, b. Monterey, Mass.

(606) ii. Thomas H., b. Monterey, Mass.,

m. Martha Hyde, of Monterey, Mass.

iii. Mary, b. Monterey, Mass.,

Great Barrington, Mass.

m. Gilbert Granger, of

364

JOSEPH CURTISS (Fonathan, Fonathan, Foseph, Thomas, Fohn, Elizabeth), b. Sheffield, Mass., June 25, I768; m. Ruth, d. of Abner Roys, of Sheffield, Mass.,

1790.

They had children :-

(607) i. Milton, b. Sheffield, Mass., Aug. I9, 1793; m. Harriet Doolittle, of Bloomfield, N. Y.

$$
365
$$

ABIJAH CURTISS (Fonathan, Fonathan, Foseph, Thomas, Fohn, Elizabeth), b. Sheffield, Mass., June 7, I773; m. Betsey, d. of Lieut. Thomas and Hannah Stevenson, of Middletown, Conn., Sept. 1793. She d. Nov. 16, I 858 . He d. Feb. 17, 1834 . He was a farmer, and lived and died on the farm which he inherited from his father. He was a Colonel of the Militia. In I 826 he was a member of the State Legislature.

They had children :-

(608) i. Ira, b. Sheffield, Mass., March 1 5, 1794; m. Artimesia, d. of Salmon Willoughby, of Cornwall, Conn., Jan. 19, 1820 . He d. March 20, 1870 .

ii. Almira, b. Sheffield, Mass., Nov. 28, 1 795. She d. Jan. 28, I834.

(609) iii. Orren, b. Sheffield, Mass., May 17, 1797; m. (i.) Carolina, d. of Col. Ira and Sophia Standish Owen, of Sheffield, Mass., March 24, I825. She d. May I4, I870; m. (ii.) Maria Hagerman. He d. Dec. 20, I 883 .

$(610)$ iv. Julius, b. Sheffield, Mass., March 18, 1799; m. Lucinda Tuttle, of Salisbury. She d. Nov. I 892 . He d. April 12, I 845 .

v. Eliza, b. Sheffield, Mass., Feb. 11, 1801. She d. Oct. 13, 1802. 
vi. Julia, b. Sheffield, Mass., Feb. I 8, I803; m. David B. Litchfield, of South Egremont, Mass., in 1829 . He d. April I 7, 1842.

$\left(G_{I I}\right)$ vii. Charles Abbey, b. Sheffield, Mass., March 14, 1805; m. (i.) Sabrina, d. of Timothy Robinson, of Sheffield, Mass., June 30, 1825 . She d. Oct. 2, I 877 ; m. (ii.) Sarah A. Green, Aug. 26, 1879. He d. Nov. 30, i 880 .

viii. Abijah, b. Sheffield, Mass., May 20, 1807. He d. Feb. 7, 1830, unm.

ix. Betsey, b. Sheffield, Mass., June I 2, I 809 ; m. Nicholas Race, of Great Barrington, Mass., Aug. 30, 184 I, and she d. June 19, 1873 .

x. Mary Ann, b. Sheffield, Mass., July 23, I 8 i 2 ; m. Newton Jayner, of South Egremont, Mass., Dec. 2, I $83^{\circ}$, and she d. May 10, I 854 .

xi. Emily, b. Sheffield, Mass., Oct. 4, I8 84 ; m. Almiron Dowd, of Florence, Ky., Aug. 20, 1846.

xii. Hannah, b. Sheffield, Mass., Aug. I4, I816; m. Harvey Roys, of Sheffield, Mass. She d. Feb. 1894.

\section{6}

JOSIAH CURTISS (Fonathan, Fonathan, Foseph, Thomas, Fohn, Elizabeth), b. Sheffield, Mass., Nov. 3, 1775; m. Mary, d. of Timothy Kellogg, of South Egremont, Mass., Nov. 23, I 800. He d. Oct. 18, 1819. She m. (ii.) Onias Tuller, of Penfield, N. Y., July 2 I, I 829. She d. March 4, I 860, aet. 80 years.

They had children :-

i. Harriet B., b. Sheffield, Mass., Sept. 5, I80 I ; m. (i.) Alonson Brazee, of Renfield, N. Y., Dec. I, I830; m. (ii.) Clarendon Mayo. She d. Dec. 23, I869.

(6I2) ii. James J., b. Sheffield, Mass., March 20, 1803; m. Nancy Steele, of Westmoreland, N. Y., Sept. 20, 1828. He d. April I 7, I 840 .

iii. Sarah, b. Sheffield, Mass., June I, I 805 ; m. Philo Upson, of South Egremont, May I, I 828 . She d. May I 9, I 877 .

$(613)$ iv. Trueman, b. Sheffield, Mass., Jan. 28, 1808; m. Charlotte, d. of Lemuel Lombard, of Brimfield, Mass., Sept. 23, i 840 . He d. March 28, 1852 .

v. Fanny W., b. Sheffield, Mass., Aug. I I, I 8 Iо. She d. Aug. 7, I 83 , unm.

vi. Mary Electra, b. Sheffield, Mass., July 22, I814; m. Phineas Mansfield, of South Egremont, March 26, i 839. She d. Nov. $25,184 \mathrm{I}$.

(6r4) vii. Jonathan G., b. South Egremont, Mass., Oct. 22, I 8 I 7 ; m. Charlotte Sophia, d. of Abel Hull, of South Egremont, Mass., March 4, 1847. He d. Feb. 3, I869. 
JAMES H. CURTISS (David, Fames, Foseph, Thomas, Fohn, Elizabeth), b. Cheshire, Conn., 1790 ; m. (i.) Ruth M. She d. June 28, I 859, aet. $7 \mathrm{I}$ years; $\mathrm{m}$. (ii.) Laura A. Brooks, Feb. 9, г 860. She d. May 6, I 882, aet. 76 years. He was a farmer, and lived in Cheshire, Conn., where he became quite prominent in town affairs, holding many town offices. He d. in Cheshire, Conn., Sept. I86 I.

They had children :-

$(6 I 8)$ i. James H., b. Cheshire, Conn., He removed to Shelbyville, Tenn., where he d., leaving a large family. In dry goods business.

(6rg) ii. John Lucky, b. Cheshire, Conn.,

I826. Went to Tenn. with his brother, and from there to Kankakee, Ill., where he d., leaving two children, one daughter and one son, James Anson Curtiss, b. I846, now living in Meriden, Conn.

\section{8}

JESSE CURTISS (Fesse, Abner, Foseph, Thomas, fohn, Elizabeth), b. Farmington, Conn., July 13, 1785; m. Rhoda Pantlin, Oct. 11, 1810 . He d. Sept. 11, 1855. She d. Aug. 10, 1844, aet. 65 years. He was a farmer and lived in Meriden, Conn.

They had children :-

(620) i. Jeremiah, b. Meriden, Conn., Jan. 25, 1812; m. Mary Ann Stephens.

ii. Marienina, b. Meriden, Conn., April I3, 1818.

iii. Martha Root, b. Meriden, Conn., Jan. I, 1825.

iv. Mary E., b. Meriden, Conn.,

m. Loomis Lamb.

\section{2}

PLINY CURTISS (Caleb, Caleb, Foseph, Thomas, Fohn, Elizabeth), b. Charleton, Mass., Nov. I4, 1795 ; m. Relief, dau. of Darius Boyden, of East Montpelier, Vt., Dec. 17, 1818. He was taken by his father to Calais, Vt., in 1795 . They removed to Columbus, Ohio, in 1840 . He d. Feb., I 853 . She d. Aug. I3, I 862.

They had children : -

(62I) i. Nathaniel Bancroft, b. Calais, Vt., Sept. I I, I8I9; m. Jane Warren, of Warrenville, Ill., in 1853 . He d. in 1872.

ii. Darius Boyden, b. Calais, Vt., Sept. 17, 1821. He d. unm. Nov. 7, 1844 .

iii. Caroline Amanda, b. Calais, Vt., Sept. 23, 1823; m. Jonas K. Hall, of Calais, Vt. 
(62.2) iv. Pliny, b. Calais, Vt., March 29, 1826; m. Cornelia Baldwin, of Peoria, 111.

v. Maria, b. Calais, Vt., 1846 .

vi. Lucinda, b. Calais, Vt., vii. Mary, b. Calais, Vt., viii. Levon, b. Calais, Vt., m. Dr. E. S. Deming, of Calais, Vt., Sanger, of Peoria, Ill. Sanger, of Peoria, Ill.

d. $y$.

\section{4}

JOSHUA CURTISS (Simeon, Foshua, Foseph, Thomas, Fohn, Elizabeth), b. Burlington, Conn., I783; m. Polly, d. of John Gillett, of Milford, Conn. He d. in Burlington, Conn., Sept. 22, 1843. She d. April 10, 1854 , aet. 74 years. He was a farmer.

They had children :-

(623) i. Philo, b. Burlington, Conn., July 14, I808; m. Charlotte M., d. of Aaron and Comfort Curtiss, of Burlington, Conn., Sept. 3, 1829 . She d. Oct. 27, I883. He d. June $10,1875$.

(624) ii. Henry, b. Burlington, Conn., Aug. I3, I810; m. Elizabeth Dowd, Jan. 2, 1833. She d. Jan. 3, I 884 . He d. Dec. 30, 1883.

iii. Amy, b. Burlington, Conn., Nov. 27, 18 I 2 ; m. Trumball Beach, of Harwinton, Conn., Oct. 7, 1832. She d. Oct. 24, 1891 .

(625) iv. Simeon, b. Burlington, Conn., $1816 ; \mathrm{m}$. Maria Hoskins, of Burlington, Conn. He d. April 3, 1882. She d. Jan. 30, 1878 .

(626) v. (Sylvester, b. Burlington, Conn., March 5, I819; m. Eveline Horton, Aug. 25, 1840 .

Celestia, b. Burlington, Conn., March 5, 1819; m. Loderick Shurrell

vi. Lewis, of New Hartford, Conn., July 4, 1842. She d. in Forrestville, Conn., Nov. 12, 1893 .

vii. Laura, b. Burlington, Conn., May 2, 1822; m. Daniel Burnes, of Forrestville, Conn., January, 1843 .

(627) viii. Coydon, b. Burlington, Conı., March 30, I823; m. Mary Ann Dayton, of Hamdon, Conn., Sept. 3, 1843 . He d. Feb. 16, 1885 .

$$
3^{85}
$$

MAJOR CURTISS (Simeon, Foshua, Foseph, Thomas, Fohn, Elizabeth), b. Burlington, Conn., July 4, $1797 ; \mathrm{m}$. Clarissa Dowd in 1822 .

She d. Jan. 16, I 890 , aet. 87 years. He d. Dec. 3 I, I 875 .

They had children :

i. Orrin, b. Burlington, Conn., Dec. 29, I823. He d. unm. July 26, I 863.

ii. Nancy, b. Burlington, Conn., Oct. 3, $1825 ; \mathrm{m}$. Crane.

iii. Lyman, b. Burlington, Conn., Jan. 16, 1827. He d. unm. March 30, I $85 \mathrm{I}$. 
iv. Polly, b. Burlington, Conn., Feb. 15, 1829.

$(62 S)$ v. Hiram, b. Burlington, Conn., July I 2, I831 ; m. Susan E. Alcott, Sept. 6, I 856 .

vi. Elizabeth, b. Burlington, Conn., Aug. 8, I833; m. Andrew O. Norton, of New Hartford, Conn., May 9, 1852.

vii. Maria, b. Burlington, Conn., July 20, 1836; m. Orrin Woodon, Jan. $31,185^{8}$.

(629) viii. Thomas, b. Burlington, Conn., Nov. 10, 1839.

$(6 ; 0)$ ix. Sanuel, b. Burlington, Conn., March 20, 1841 .

x. Mary, b. Burlington, Conn., April 28, 1843 .

xi. James, b. Burlington, Conn., Aug. 15, 1845. He d. Aug. I 2, I 846 .

(631) xii. James, b. Burlington, Conn., Sept. I3, I 847. He was a soldier in the Union Army and d. in Andersonville Prison, Sept. 20, 1864.

\section{8}

ELlAS CURTISS (Giles, Fohn, Fohn, Thomas, Fohn, Elizabeth), b. Sheffield, Mass., Aug. 28, 1788 ; m. He went West, but returned to his farm in Sheffield, Mass., where he d. in 1886.

They had children : -
i. Bird, b.
ii. Jerome, b.
iii. Benjamin, b.
iv. Diana, b. Jan. I 7, 1839 .
v. Marcia, b.
vi. Adeline, b.

m. Catherine.

m. Amelia.

m. Watson Brown, of Chester, Mass.,

m. Dr. Edwin Lucas, of Springfield, Mass.

JAMES CURTISS (Giles, Fohn, Fohn, Thomas, Fohn, Elizabeth), b. Sheffield, Mass., Fieb. 26, I 792 ; m. Sarah farmer, and lived in Sheffield, Mass., where he d. in 1832.

They had children :-

$(6,32)$ i. John J., b. Sheffield, Mass.,

$(6,3)$ ii. James, b. Sheffield, Mass.,

iii. Nancy, b. Sheffield, Mass.,

iv. Huldah, b. Sheffield, Mass.

He was a

v. Betsey, b. Sheffield, Mass.

vi. Sarah, b. Sheffield, Mass.

(6,34) vii. Elbridge, b. Sheffield, Mass.

m. Ellen Babcock.

m. Helen Upton.

m. Giles C. Calhoun, of Col-

391

LEVI CURTISS (Ephraim, Stiles, Ephraim, Foseph, Fohn, Elizabeth), b. Huntington, Conn., Dec. 29, 1765; m. Abigail

She d. 
Jan. 9, 1809 , aet. 42 years ; m. ii, Abbie

She d. Sept. 9, 1836 , aet. 59 years. He lived in Huntington, Conn., where he d. April 8, 1851 .

They had children :-

(635) i. Ephraim, b. Huntington, Conn.

$(636)$ ii. Samuel C., b. Huntington, Conn., He d. May 8, 1867. She d. Nov. I, 1867.

iii. Sally, b. Huntington, Conn., m. Cyrus Wheeler.

iv. Anna, b. Huntington, Conn.,

v. Melissa, b. Huntington, Conn.,

vi. Kate, b. Huntington, Conn.,

m. David Welles. m. A. Blockman.

m. Roswell Welles.

\section{2.}

PHILO CURTISS (Ephraim, Stiles, Ephraim, Foseph, Fohn, Elizabeth), b. Stratford, Conn., Oct. 25, I772; m. Sarah

She d. Dec. 26, 1823 , aet. 45 years. He d. April 21, I832.

They had children :-

i. Sarah H., b. Huntington, Conn., 24,1821 .

ii. Ann Rebecca, b. Huntington, Conn., 1803. She d. unm. Oct. 4, 1811 .

\section{4}

ELIHU CURTISS (Elihu, Stiles, Ephraim, Foseph, Fohn, Elizabeth), b. Stratford, Conn., Dec. 20, I774; m. Clemence, d. of Samuel Peck, of Milford, Conn., April, 1829. He d. I 858.

(637) Stephen Edwin, b. Stratford, Conn., May, 1831 . Emily Peck, b. Stratford, Conn., Dec., 1832.

Catherine Ufford, b. Stratford, Conn., July, i 835.

$(638) \quad$ Charles Elihu, b. Stratford, Conn., Aug., 1839.

\section{8}

SILAS CURTISS (Silas, Stiles, Ephraim, Foseph, Fohn, Elizabeth), b. Stratford, Conn., Feb. 27, 1776; m. Helen, d. of Stiles and Naomi Judson, of Stratford, Conn. She d. April 2, I801, aet. $2 \mathrm{I}$ years; $\mathrm{m}$. ii, Avis Newton, d. of Daniel and Mehitabel Shelton, of Stratford, Conn., April 18, 1802. He d. in Stratford, Conn., Sept. I 4, I 845 .

They had children :-

i. Helen Marietta, b. Stratford, Conn., Jan. 2, 1804; m. Ambrose Shelton. She d. March 14, 1877 .

ii. Mehitabel Jane, b. Stratford, Conn., Sept. 15, 1805; m. George Welles. 
iii. Ezra Eli, b. Stratford, Conn., July 26, 1807. He d. Oct. 21, 1830 .

iv. Hannah Susan, b. Stratford, Conn., Dec. 31, 1810; m. Edwin Shelton, of Stratford, Conn.

v. Nancy Maria, b. Stratford, Conn., Dec. 28, I8ı3; m. James Scribner.

vi. Abigail Avis, b. Stratford, Conn., July 2I, I8I6; m. Silas H. Marks.

vii. Elizabeth Sophia, b. Stratford, Conn., July 30, 1818; m. Nathan Birdsey Booth, of Stratford, Conn.

WILLIAM CURTISS (Silas, Stiles, Ephraim, Foseph, Fohn, Elizabeth), b. Stratford, Conn., Aug. I 4, I778; m. Rebecca, d. of Stiles and Naomi Judson, of Stratford, Conn., Feb. 9, I 803 . She d. Dec. 23, 1822; m. 2d, Helen, d. of Ezra and Phebe Curtiss Birdsey, of Stratford, Conn., March 5, 1825. She d. Feb. I3, I 875. He was a farmer and resided in Stratford, Conn., where he d. Jan. I 7, I 855 .

They had children :-

(639) i. Stiles, b. Stratford, Conn., Feb. 17, 1805; m. Hannah Whiting, of Stratford, Conn., Oct. 3, I830. She d. Feb. 8, 1838; m. ii, Jane I. Whiting, of Bridgeport, Conn., Sept. 23, I8 38 . She d. March $3, \mathrm{~s} 866 ; \mathrm{m}$. iii, Jane Hanson Bartow, of New Haven, Conn., April I6, I867. She d. April I 5, I 868; m. iv, Martha Abbott Hull, of New Haven, Conn., May i 9, I869. He d. Nov. I I, 1882 .

ii. Myra, b. Stratford, Conn.,

iii. William, b. Stratford, Conn.,

She d. unm. Oct. 27,1843 .

He d. unm. April I 4,1837 .

$(6 \neq 0)$ iv. Lewis Judson, b. Stratford, Conn., m. Fannie Olmstead, of Norwalk, Conn., Aug. 19, 1843.

(6.7I) v. Thaddeus Birdsey, b. Stratford, Conn., July I 2, I 808; m. Jane, d. of Noah and Anne Belden Olmstead, of Norwalk, Conn., Nov. 19, I 846. She d. Feb. I, 187 I. He d. May 5, I 864.

$(6,2)$ vi. Elbert, b.Stratford, Conn., m. Mary F., d. of Anson and Polly Quintord, of Norwalk, Conn., Sept. 29, I846. She d. Dec. 26, I $855 ; \mathrm{m}$. ii, Harriet Ann, d. of Samuel C. and Matilda Curtiss, of Huntington, Conn., Oct. 7, 1857. She d. May 20, I 868. He resides in Norwalk, Conn.

EII CURTISS (Silas, Stiles, Ephraim, Foseph, Fohn, Elizabeth), b. Stratford, Conn., March I I, I785; m. Sarah, d. of Zachariah and Anna Blakeman, of Stratford, Conn. 
They had children :-

i. Eliza Ann, b. Stratford, Conn., Feb. I6, I 809.

$(673)$ ii. Birdsey, b. Stratford, Conn., Dec. 25, 18 10. He d. July 1 3, 1882.

iii. Myra, b. Stratford, Conn., Dec. I I, I8 I 2. She d. July I, I 844.

iv. Harriet, b. Stratford, Conn., Oct. 15, I814. She d. July I, 1847.

(644) v. Cornelius, b. Stratford, Conn., Jan. 28, i 8 I 7 .

$(675)$ vi. Eli, b. Stratford, Conn., May 6, 18 19; m. Sarah E. Booth. vii. Sarah, b. Stratford, Conn., July I I, I 82 I.

(646) viii. Lucius Treat, b. Stratford, Conn., July I 8, I 824.

$(677)$ ix. Charles Henry, b. Stratford, Conn., June I4, 1827.

\section{2}

STILES CURTISS (Fohn, Stiles, Ephraim, Foseph, Fohn, Elizabeth), b. Stratford, Conn., April 13, 177 I ; m. Sarah, d. of Samuel Beard, of Huntington, Conn., Oct. 5, 1794. He d. in Woodbury, Conn., Jan. 20, 1850 . She d. July 26, I 862.

They had children :-

i. Amzi, b. Woodbury, Conn., Sept. 14, I 795. Hed.unm. Nov. I 7, I 869 .

(65I) ii. John, b. Woodbury, Conn., July I3, I 797; m. Maria Gilbert, of Woodbury, Conn., Oct. 18, 1818. She d. in 1879.

iii. Maria, b. Woodbury, Conn., Nov. 13, 1800; m. William G. Moseley, of Southbury, Conn., Nov. 12, 1821. She d. in Roxbury, Mass., in 1895.

iv. Caroline, b. Woodbury, Conn., May 29, r802; m. Samuel Smith, of South Britain, Conn.

v. Harriet, b. Woodbury, Conn., March 29, I804; m. Truman Minor, of Woodbury, Conn., Dec. I8, I8 27.

vi. Eliza, b. Woodbury, Conn., Dec. 29, i 806 . She d. unm. Sept. 4, 1840 .

(652) vii. Charles, b. Woodbury, Conn., May 23, 18 I 2; m. Emily A. Lambert, of Woodbury, Conn., March 14, I8 38 . She d. Oct. 20, $184 \mathrm{I}$; m. 2d, Mary Gleeson Goodwin, Aug. 3, I842. He d. Dec. 27,1872 .

viii. Henry Stiles, b. Woodbury, Conn., Feb. 24, 18 I4; m. Elizabeth L. Benham, of South Britain, Conn. He d. Oct. 23, 1869.

They had a child : -

i. Henry, b. Woodbury, Conn.,

Oct. 20, 1853 .

JUDSON CURTISS (Fohn, Stiles, Ephraim, Foseph, Fohn, Elizabeth), b. Stratford, Conn., Feb. I 5, I774; m.

1841. Hed.

( 117 ) 
They had children :-

(65f) i. Judson, b.

$(655)$ ii. George, b.

iii. Eliza, b.

EZEKIEL CURTISS (Fohn, Stiles, Ephraim, Foseph, Fohn, Elizabeth), b. Stratford, Conn., June 6, 1779; m. Anna Wooster, of Stratford, Conn.; m. 2d, Electa Lord, of Oneida, N.Y. He was a ship-owner and captain out of Bridgeport, Conn., for many years, and during one of his cruises was captured by the French. About 1826 he settled in Oneida Co., N.Y., where he had inherited land from his father, and became a farmer. He d. in Utica, N.Y., 1840.

They had children:-

i. Son, b. Bridgeport, Conn.

ii. Daughter, b. Bridgeport, Conn.

iii. Charles Gould, b. Oneida Co., N.Y., d. y.

iv. Daughter, b. Oneida Co., N.Y., m. Foster, of Utica, N.Y.

v. Emily, b. Oneida Co., N.Y., m.

m. Mitchell.

(656) vi. Thomas Emmons, b. Oneida Co., N.Y., m. Mary Southworth.

vii. Juliette, b. Oneida Co., N.Y.,

$\mathrm{m}$.

Williams, of

viii. Ann, b. Oneida Co., N.Y.

(657) ix. Lyman Lord, b. Oneida Co., N.Y.,

$(658)$ x. Charles Gould, b. Oneida Co., N.Y.,

m. Ellen

m. Amelia Lent Main, of Binghamton, N.Y., April, 1852 . He d. Sept. 27, 1893. She d. Nov. 30, 1881 .

\section{5}

DANIEL CURTISS (Fohn, Stiles, Ephraim, Foseph, Fohn, Elizabeth), b. Stratford, Conn., April 8, I781 ; m. Elizabeth, d. of Peter and Elizabeth Pixlee, of Stratford, Conn., Jan. 10, 1810 . His will was filed in Stratford, Conn., April 17, 1852.

They had children :-

i. Mary Elizabeth, b. Stratford, Conn., Nov. 2, 1810; m. Nathan Birdsey, of Stratford, Conn., April, 1833. She d. Oct. 3, I86I.

(659) ii. Sheldon Pixlee, b. Stratford, Conn., May 26, 18 I 2 ; m. Sarah McEwen, of Stratford, Conn., Sept. 23, 1835. He d. Jan. 9, 1875 .

iii. Margaret Owen, b. Stratford, Conn., Oct. 19, 1813; m. Morgan Curtiss, of Huntington, Conn., Feb. 13, 1833. She d. March 20,1868 . 
(660) iv. Charles Henry, b. Stratford, Conn., Jan. 23, 1815; m. Electa A. Woolsey. He d. Feb. 5, 1888.

v. Peter Pixlee, b. Stratford, Conn., Feb. Io, I817. He d. May Io, I 8 i 7 .

$(66 I)$ vi. Peter Pixlee, b. Stratford, Conn., Oct. 26, I 820; m. Mary Esther Fitch, of Stratford, Conn., Nov. 30, I843. He d. June 16, 1882. She $d$. in 1890 .

vii. Elizabeth Ann, b. Stratford, Conn., Feb. 26, 1824; m. Edward D. Fitch, of Stratford, Conn., July 18, 1853 .

PHILO CURTISS (Lewis, Henry, Ephraim, Foseph, Fohn, Elizabeth), b. Stratford, Conn., March 4,1774; n. Elizabeth Frost, of Stratford, Conn., March 1797. He d. in Stratford, Conn., May 5, I 852 . She d. Feb. I I, I 844 , aet. 68 years.

They had children :-

i. Elvira, bapt. Stratford, Conn., I799; m. Eli Welles.

(662) ii. Lewis Walker, bapt. Stratford, Conn., I802.

iii. Julia, bapt. Stratford, Conn., 1806 ; m. Judson Gorham, of Stratford, Conn., May 23, 1827 .

409

IRA CURTISS (Lewis, Henry, Ephraim, Foseph, Fohn, Elizabeth), b. Stratford, Conn., Dec. 30, I783; m. Lucy . He d. in Bridgeport, Conn., March 12, 1834. She d. April I, I872, aet. 83 years.

They had children :-

i. Isaac B., b. Stratford, Conn., I8I2. He d. Sept. I8, I812.

ii. Elisha, b. Stratford, Conn., 1825. He d. Sept. I 2, I 826.

$(663)$ iii. Isaac, b.

(667) iv. Lewis, b.

v. Ruth Ann, b.

EZRA CURTISS (Edmund, Edmund, Ephraim, Foseph, Fohn, Elizabeth), b. Stratford, Conn., May 12, 1776; m. Anna Welles.

They had children : -

i. Emma.

ii. Catherine m. Osborn Chambers.

419

BEECH CURTISS (Edmund, Edmund, Ephraim, Foseph, Fohn, Elizabeth), b. Stratford, Conn., Jan. I, I78I ; m. Laura Hawley, of Hawleyville, Conn. 
They had children :-

i. Maria, b.

ii. Harriet, $b$.

iii. Catherine, $b$.

iv. Charles, b.

m. Moses Secor, of New York City, N.Y. m. Charles Guyon.

\section{0}

WILLIAM CURTISS (Edmund, Edmund, Ephraim, Foseph, Fohn, Elizabeth), b. Stratford, Conn., July 20, 1785 ; m. Anna Fayerweather, of Trumbull, Conn. He d. in Trumbull, Conn., March 3I, 1867 . She d. July 18,1860 , aet. 72 years.

They had children :-

i. Almira, b. Trumbull, Conn., m. Alonzo Sherman.

ii. Carlos, b. Trumbull, Conn., June I, I 8 I I; m. Jane B. Smith,

She d. Nov. 2, 1895 . He was a member of the firm of Hubbell \& Curtis, of Bridgeport, Conn., cabinet-makers, and also a director in the Bridgeport National Bank. In 1875 he was elected a representative from Bridgeport, and in $\mathrm{I} 882$ was mayor of that city. He d. in Bridgeport, Conn., May 30, 1896.

They had a child : -

Mary B., b. Bridgeport, Conn., Oct. 18, I856; m. Eugene B. Peck, of Bridgeport, Conn., Oct. I I, I 876.

iii. Spencer, b. Trumbull, Conn.

$42 \mathrm{I}$

JABEZ CURTISS (Edmund, Edmund, Ephraim, Foseph, Fohn, Elizabeth), b. Stratford, Conn., Sept. 15, 1787 ; m. Sarah, d. of Stephen and Anne Burritt, of Stratford, Conn., January, 18 ro. He d. in Stratford, Conn., March 18, 1867.

They had children :-

i. Mary Elizabeth, b. Stratford, Conn, Oct. I 2, 1810; m. Theodosius

F. Sicor, of New York City, N.Y., April 13, 1829.

ii. Burritt, b. Stratford, Conn., March 28, 1814 ; d. y.

iii. Susan A., b. Stratford, Conn., Oct. 26, 1817; m. Rufus Brooks, of

New York City, N.Y., April I 8, I 836.

iv. Edward Beach, b. Stratford, Conn., Oct. 27, 1823; m. Margaret, d. of Agur Curtiss, of Stratford, Conn.

They had a child : -

Edward Grant, b.

v. John, b. Stratford, Conn., Aug. I 0, 1828 .

MORGAN CURTISS (Stiles, Elnathan, Ephraim, Joseph, John, Elizabeth), b. Huntington, Conn., Jan. II, 1802 ; m. Margaret 
Owen, d. of Daniel and Elizabeth Pixlee Curtiss, of Stratford, Conn., Feb. 13, I833. He d. in Huntington, Conn., Sept. 30, I 88 r. She d. March 20, I 868.

They had children :-

i. Jane Pixlee, b. Huntington, Conn., Dec. 31, I833; m. Robert Comstock Naramore, Sept. 24, 1854.

(666) ii. Franklin, b. Huntington, Conn., Dec. 6, 1835 ; m. Margaret, d. of Peter and Susan Schmitz, of Germany, Oct. 28, 1860. They reside in Nevada, Ia.

(667) iii. Charles Henry, b. Huntington, Conn., Nov. I I, I837; m. Frances Catherine, d. of Charles Spencer, of Saybrook, Conn., July 16, 1868. They reside in Detroit, Mich.

iv. Frederick William, b. Huntington, Conn., May I 8, 1840; m. Sarah Matilda, d. of Cyrenius and Christe Amn Curtiss, of Newtown, Conn., May 10, I868. She d. Nov. 28, 1883 . He served as a private in the I 7 th Connecticut during the War of the Rebellion. He lives in Shelton, Conn., and is street commissioner of that town. No issue.

v. Margaret, b. Huntington, Conn., April 9, 1850.

$$
426
$$

JUDSON CURTISS (Nehemiah, Foseph, Foseph, Foseph, Fohn, Elizabeth), b. Stratford, Conn., Aug. I 8, I768; m. Mary, d. of Benjamin and Elizabeth Lewis, of Stratford, Conn. He was killed while felling a tree, May 13, 1828. She d. Sept. I7, 1817, aet. 45 years. He was a farmer and a tanner, and lived in Stratford, Conn.

They had children :-

(668) i. Ashbel, b. Stratford, Conn., 1794; m. (i.) Deborah Sniffen, Conn., Nov. 18, 1840. He d. May 14, 1880.

ii. Louisa, b. Stratford, Conn., $\quad 1796$; m. Lewis Fairchild, She d. Oct. 16, 1867.

(669) iii. Roswell, b. Stratford, Conn., Junc 29, 1799; m. Sarah, d. of Thaddeus and Hannah Peck, of Stratford, Conn., Nov. 8, 1836. He d. Oct. 18, 1880 . She d. June 8, 1851, aet. 38 years.

iv. Elizabeth, b. Stratford, Conn., I802; m. Nathan Peck, of Stratford, Conn., March 24, I824. She d. 1829.

v. Maria, b. Stratford, Conn., 1803. She d. 1865

$(670)$ vi. Judson, b. Stratford, Conn., Oct. 2, 1806; m. Martha, d. of Thaddeus and Hannah Peck, of Stratford, Conn., March 24, I 833 . She d. June 5,1854 , act. 43 years.

vii. Mary, b. Stratford, Conn., $\quad$ i808; m. Thaddeus Peck, Jr., of Stratford, Conn., May 6, 1829 . 
$(671)$ viii. Agur, b. Stratford, Conn.,

I $811 ; \mathrm{m}$. Mary Hard, of Woodbury, Conn. He d. June 2, 1889. She d. Jan. 17, I 886.

ix. Ann, b. Stratford, Conn.,

$1812 ; \mathrm{m}$. Lewis Curtiss, of Stratford, Conn., April 7, 1834 . She d. Feb. 28, 1846.

$(672) x$. Enoch, b. Stratford, Comn., Dec. 30, I813; m. Susan, d. of Silas and Hannah Bcers, of Stratford, Conn., June 16, 1839 . He d. May 20, 1876 . She d. May 9, 1896 , aet. 81 years.

\section{8}

FREEMAN CURTISS (Nehemiah, Foseph, 7oseph, Foseph, Fohn, Elizabeth), b. Stratford, Conn., Dec. 6, 1782 ; m. Anne, d. of Judson and Mary (Blakeman) Peck, of Stratford, Conn., Aug. 6, I 803 . She d. July I 4 , I 828 , aet. 42 years. m. (ii.) Polly E. McEwen, of Stratford, Conn., Dec. 28, i 828. She d. March i6, I 879 , aet. 84 years. He d. Jan. 5,1862 .

They had children :-

i. Stilcs, b. Stratford, Conn., Jan. 3I, I 804. He d. Oct. 2, 1804.

ii. Dolly, b. Stratford, Conn., Oct. I 3 , I 805 ; m. John M. Andrews, 1822. She d. April 24, I891.

(673) iii. Stiles, b. Stratford, Conn., June 7, r808; m. Catherine Mumford, d. of John Robertson, of New York City, N. Y. He d. May 4, I 876 . She d. Oct. 7, 1893.

(674) iv. Fnoch Olds, b. Stratford, Conn., Nov. I7, 1810. He d. in Darius, Ia., April 30, 1831 .

v. Lucy Ann, b. Stratford, Conn., Oct. I 4, I8I2; m. George Thompson, of Stratford, Conn., April i 8, i 833. She d. Dec. 6,1888 .

vi. Angeline, b. Stratford, Conn., Oct. 7, 18 16; m. John Stevenson, of Bridgeport, Conn., Feb. 4, 1836 . She d. Dec. 22, 1885 .

(675) vii. Elbert F., b. Stratford, Conn., April 30, 1819; m. Ann Jennett Gorham, of Westport, Conn., Sept. 26, i 840. He d. May I3, I 883 .

$(676)$ viii. William L., b. Stratford, Conn., June 22, 1821 ; m. Jane M. Lcwis, Oct. 22, I 844. He d. June 15, 1849. She d. Jan. 4, I 858 .

ix. Freeman L., b. Stratford, Conn., June 27, 1824; d. Sept. . 29, I 825 .

$(677)$ x. Frecman L., b. Stratford, Conn., Jan. 9, I 828; m. Georgie Howard, of New York, Oct. 2, 1856.

xi. Edwin, b. Stratford, Conn., Dec. 9, 1829. He d. Sept. 10, I 830.

\section{0}

JOHN CURTISS (Foseph, Foseph, Foseph, Foseph, Fobn, Elizabeth), b. Stratford, Conn., Nov. 10, $1769 ; \mathrm{m}$. Anne Curtiss. 
They had children :-

i. Adelia, b. Stratford, Conn., Nov. 24, $1794 ; \mathrm{m}$. Conway Lewis, in Madison, N. Y., Oct. 13, 1814. She d. Sept. 22, I848.

ii. Julia, b. Stratford, Conn., m. Curtiss Woodhull.

iii. Susan M., b. Stratford, Conn.,

iv. Charles, b. Stratford, Conn., m. Mr. Alvord.

d. $y$.

NATHAN CURTISS (Silas, Foseph, Foseph, Foseph, Fohn, Elizabeth), b. Stratford, Conn., Oct. 7, I770; m. Sally Curtiss,

She d. Jan. I859, aet. 79. He d. in Stratford, Conn., in I 824 .

They had children :-

i. Polly, b. Stratford, Conn.

ii. Anna, b. Stratford, Conn.

iii. Julia, b. Stratford, Conn.

iv. Caroline, b. Stratford, Conn.

v. Silas, b. Stratford, Conn., Conn., Sept. IO, I828. He d. in I 837 .

They had a child :-

i. Marie, b.

$(678)$ vi. Ira, b. $\mathrm{m}$.

(679) vii. Joseph, b. Stratford, Conn., 1802; m. Helen Peck, of Stratford, Conn., Oct. I 8, 1825 .

\section{$44^{1}$}

JOEL CURTISS (Silas, Foseph, Foseph, Foseph, Fohn, Elizabeth), b. Stratford, Conn., Dec. 1791 ; m. Harriet, d. of Abner Judson, She d. in Stratford, Conn., Sept. 11, 1885 , aet. 92 years.

They had children :-

$(680)$ i. Lewis, b. Stratford, Conn., m. Ann, d. of Judson and Mary Lewis Curtiss, of Stratford, Conn., April 7, 1834 . She d. Feb. 28,1846 , aet. 34 years.

ii. Fanny, b. Stratford, Conn.

iii. Harriet, b. Stratford, Conn., m. Levi Lewis, of Stratford, Conn., March 7, 1847 .

EZEKIEL CURTISS (Agur, Foseph, Foseph, Foseph, Fohn, Elizabeth), b. Stratford, Conn. m. Sarah She d. I 843 .

They had children:

i. Sophia, b. Stratford, Conn.,

ii. Abigail, b. Stratford, Conn.

m.

Stratton. 
iii. Phoebe, b. Stratford, Conn.,

m.

Peck.

iv. Betsey, b. Stratford, Conn.,

v. Ezekiel, b. Stratford, Conn.,

He had a child : -

i. Ezekiel, b. Stratford, Conn.,

I813. He d. Sept. I 9, I 893 .

445

AGUR CURTISS (Agur, Foseph, Foseph, Foseph, Fo.'n, Elizabeth), b. Stratford, Conn., $\quad 1785$; m. Alice, d. of Josiah and Helen Birdseye Peck, of Stratford, Conn. She d. in I878, aet. 89 years. He was a farmer, and in winter taught school. He d. in Stratford, Conn., March 12, I 868.

They had children :-

$(68 x)$ i. Victory, b. Stratford, Conn., Feb. 28, I806; m. Susan J. Miles, He d. Nov. 20, I89 I. She d. Jan. 3I, I87 I, aet. 63 years.

$(682)$ ii. Cornelius Agur, b. Stratford, Conn., June 28, 1808; m. Phebe Lewis, of Stratford, Conn., Sept. 23, 1833 .

iii. Sarah Peck, b. Stratford, Conn., Jan. 24, I8I I ; m. Elbert Welles, of Stratford, Conn., Sept. 22, 1830.

iv. Helen Birdseye, b. Stratford, Conn., March 21, I813; m. Joseph H. Stagg, of Stratford, Conn., Sept. I7, I833.

v. Louisa, b. Stratford, Conn., Oct. 22, I8I6; m. Benjamin Welles.

vi. Abigail, b. Stratford, Conn., June 26, 18 19; m. Frederick Brooks.

$(683)$ vii. Alfred, b. Stratford, Conn., Jan. 22, I 822 ; m. Sarah A. Wheeler, of Stratford, Conn., May 2, I 844.

(687) viii. Norris Newton, b. Stratford, Conn., Sept. 2, I 824 ; m. Sarah Buched.

ix. Margaret Ann, b. Stratford, Conn., Jan. I 4, I 828 ; m. Edward Beach Curtiss, of Stratford, Conn.

447

JOHN CURTISS (Everett, Robert, Joseph, Joseph, John, Elizabeth), b. Stratford, Conn., m.

They had children :-

i. Jennie, b.

ii. Susan M., b.

m. Frederick B. Hawley, of Bridgeport, Conn. N. Y.

m. Geo. L. Holmes, of New York City,

LEVI CURTISS (Aaron, Fames, Fames, Benjamin, Fohn, Elizabeth), b. Granville, Mass., Nov. 13, 1767; m. Rachel. 
They had children : -

(685) i. Talcott, b. Granville, Mass., June 17, I795; m. Mercy J. Squires, Sept. 14, I 8 I 8 .

ii. Eliza, b. Granville, Mass., Aug. 1 I, I 797. She d. April 9, I8I 2.

iii. Lavinia, b. Granville, Mass., April 26, I 799.

iv. Sarah, b. Granville, Mass., April I 7, 1801. She d. June 18, 1802.

v. Aaron Lester, b. Granville, Mass., May 6, I 809 .

NATHAN CURTISS (Aaron, Fames, Fames, Benjamin, Fohn, Elizabeth), b. Granville, Mass., Aug. 22, I776 ; m. Nancy Wadsworth, of Granville, Mass.

They had children

i. Marcia, b. Granville, Mass., July 10,1805 ; m. Israel M. Parsons, of Granville, Mass., April 29, 1824.

ii. Frederick Griswold, b. Granville, Mass., May I I, I807.

iii. Elisha Mix, b. Granville, Mass., April 7, I8 го.

iv. Charles Wadsworth, b. Granville, Mass., Dec. Io, I8 I I.

\section{4}

SAMUEL, CURTISS (Abijah, Fohn, Fames, Benjamin, Fohn, Elizabeth), b. Durham, Conn., July 13, 1787; m. Lucretia Brooks, of Durham, Conn., Oct. 3, i 8 10. He d. in Waterloo, Wis., Nov. 2 I, 1846 . She d. in Medford, Minn., March 29, 1869, aet. 83 years.

They had children :-

$(68 S)$ i. John, b. Durham, Conn., Nov. 21, I8I I ; m. Emily Sophia Lindslay, of Medina, Ohio, Jan. 23, I834. He d. June 6, 1893.

$(689)$ ii. Samuel Brooks, b. Durham, Conn., July 13, I8 I3; m. Sarah M. Chapman, of Medina, Ohio, April 26, 1836. He d. Oct. 2, I 896. She d. May 7, 1889.

iii. Anna Elizabeth, b. Durham, Conn., May I I, I8I5; m. Sylvester Hawkins, of Medina, Ohio, Aug. I4, I833. She d. 1891.

iv. Phebe, b. Durham, Conn., April I 2, I8I7; m. (i.) Samuel C. Smith, of Medina, Ohio, Sept. 1, I 834. He d. April 25, I 843 ; m. (ii.) Newton Reed, of Medina, Ohio, May I, I 845. She d. June 25,1847 .

v. Sarah, b. Durham, Conn., May 31, I819. She d. July I 2, I 825 .

vi. Hannah Hall, b. Durham, Conn., Aug. 29, I823; m. Samuel S. Logan, of Medina, Ohio, Aug. 29, 1843.

vii. David, b. Durham, Conn., July 25, 1825. He d. Nov. 4, 1837.

viii. Ira Brooks, b. New York State, Jan. 15, 1827. He d. unm. in Sacramento, Cal., June 4, 1858. 
(690) ix. James Francis, b. Medina, Ohio, Feb. 24, 1829; m. Nancy I. Kimball, Feb. 22, 1858. They live in Mantorville, Minn.

They had a child : -

i. Walter, b.

x. Sarah, b. Medina, Ohio, Jan. 3, 1832; m. Walter D. Smith, of Medina, Ohio, May 21, I852. She d. Dec. 6, I863.

$(691)$ xi. Ichabod Francis, b. Medina, Ohio, April I, I834; m. Martha Matilda, d. of John and Geraldine Goss, of Turner, Ill., Nor. I 4, I86I. He resides in Paonia, Col.

\section{5}

ICHABOD CURTISS (Abijah, Fohn, Fames, Benjamin, Fohn, Elizabeth), b. Durham, Conn., April 3, I792; m. Selina Camp, of Durham, Conn., 1815 . In 1820 he removed to Riga, N. Y., and then to Bergen, N. Y., and in 1833 to Kingsville, Ohio. He d. Jan. 17, 1867. He served as drum-major in the war of 18 I 2 .

They had children : -

i. Phebe, b. Durham, Conn.,

ii. Theodore, b. Durham, Conn., d. in Oquawka, Ill., I 845 .

They had a child : -

$$
\text { i. Theodore, } b \text {. }
$$

iii. Mary, b. Bergen, N. Y.,

I 8 I 6 ; d. $y$.

I 8 I 7 ; m. Julia Bailey. He

He lives in Gladstone, Ill.

m. John Danforth, of New York City, N. Y. She d. in Owego, N. Y.

$(692)$ iv. Carlos E., b. Bergen, N. Y., m. Julia E. Niles, of Kingsville, Ohio, in I 859. He d., Kingsville, Ohio, May 3, I 886.

v. Elmira, b. Bergen, N.Y., They live in Chicago.

vi. Clara, b. Bergen, N. Y., m. Elihu Hosford, of Vermont. Owego, N. Y., 1857 ; I $831 ; m$. W. E. Bouta, of Bowen, Ill.

vii. Susan, b. Kingsville, Ohio, I 833 ; d. y.

viii. Elihu, b. Kingsville, Ohio, I 834 ; d. y.

ix. Elihu, b. Kingsville, Ohio, June, I 835 ; m. Helen Gerrish, 1862. He d. Feb. I I, I 892.

x. James Francis, b. Kingsville, Ohio, 1837 ; m. Lucile Sherwood, of Elkhart, Ind., I866. He d. at Petoskey, Mich., I875. They live in Marysville, Wash.

457

CHARLES CURTISS (David, David, Fames, Benjamin, Fohn, Elizabeth), b. Durham, Conn., Feb. 16, 1772; m. Lucy . His father settled in Granville, Mass., about 1780. 
They had children :-

i. Polly, b. Granville, Mass., Oct. 14, 1795.

ii. Chauncey, b. Granville, Mass., Sept. 4, 1797.

iii. Lucretia, b. Granville, Mass., May 20, 1799.

(693) iv. Charles Lewis, b. Granville, Mass., July 31, 1801.

(697) v. Barnes, b. Granville, Mass., July 31, 1803.

vi. Harriet, b. Granville, Mass., April I 5, 1805.

vii. Anna Maria, b. Granville, Mass., July i I, 1807.

\section{1}

LEWIS CURTISS (Agur, Samuel, Nathaniel, Zachariah, William, Elizabetb), b. Stratford, Conn., April 30, 1787 ; m. Mary Elizabeth, dau. of Joseph Davis and Mary Chapman Beers, of New York City, N. Y., Feb. 4, 1824 . She d. Dec. 1 5, I 835 ; m. (ii.) Elizabeth Overing, of New York City, N. Y., Oct. 7, I 840 . She d. Nov. 26, 184 I. He died in New York City, N. Y., Jan. 30, 1883. He removed to New York City, N. Y., in March, I807, and became a large and wealthy importer of silk, with his brother Benjamin, under the firm name of L. \& B. Curtis. He was president of the Farmers' Loan and Trust Co., a member of the Board of Foreign Missions of the Protestant Episcopal Church, from its formation until his death, a director of the Union Bank and of the Atlantic Mutual Insurance Company of New York, etc.

They had children :-

(695) i. Joseph Davis Beers, b. New York City, N. Y., Jan. 5, 1825; m. He d. May 22, 1870 . His son, Geo. Washington, lives in Catskill, N. Y.

ii. Mary Elizabeth, b. New York City, N.Y., Nov. 4, 1827. She d. Feb. 9, 1828 .

iii. Lewis Benjamin, b. New York City, N. Y., Jan. 3, 1829. He d. Jan. $29,1832$.

iv. Mary Beers, b. New York City, N. Y., May 13, 183I ; m. William Ogden Giles.

v. Benjamin Lewis, b. New York City, N. Y., March 27, 1833. He is unm. and is living in New York City, N. Y.

(696) vi. Lewis Agur, b. New York City, N. Y., Dec. 15, I835; m. Emma Sophia, d. of Roderick Cadwell and Delia Augusta (Perry) Steele, of Auburn, N.Y., June 9, 1859 . She d. May 27, I893, aet. 57 years. He resides in Southport, Conn.

472

BENJAMIN CURTISS (Agur, Samuel, Nathaniel, Zachariah, William, Elizabeth), b. Stratford, Conn., Nov. II, I790; m. 
Rebecca Tappan Edwards, Sept. 2, 1830, in Paris, France. She d. at sea, July 22, 1844 ; m. (ii.) Laura, d. of David and Ann Aspinwall Hadden, of New York City, N. Y., Jan. 29, I 856. She d. Nov. 7, 1894. He d. in New York City, N. Y., Feb. I9, I881. After graduating from the school in Stratford, Conn., he removed to New York City, N. Y., and entered into partnership with his brother Lewis Curtis, under the firm name of L. \& B. Curtis, silk importers. He represented the above firm in Paris for upwards of twenty years, returning to New York City in 1844 , where he resided until his death. He retired from business in 1868 .

They had children:-

i. Benjamin Farquhar, b. Philadelphia, Penn., Aug. 5, 1857; m. Eva H., dau. of Edward C. Bogert, of New York City, N. Y., Sept. 28, 1882. She d. Nov. I8, 1883; m. (ii.) Anabel, d. of Wm. B. Clarke, of New York City, N. Y., April 26, 1897. He graduated from Columbia College in 1878 , and from the College of Physicians and Surgeons of Columbia College in $188 \mathrm{I}$. He is a practising physician in New York City, N. Y.; attending surgeon to St. Luke's Hospital since I 888; Cancer Hospital since 1890; Professor of Surgery at the Woman's Medical School of the New York Infirmary since 1892 ; Professor of Surgery in the New York Post-graduate Medical School. In I 887 he wrote the Cartwright Prize Essay, College of Physicians and Surgeons, Columbia College. In 1885 he translated into English Professor Von Esmarch”s “ Handbuch der Kriegschirurgischen Ischuik." In 1898 he wrote with Dr. H. R. Wharton, of Philadelphia, "A Practice of Surgery."

ii. Anne Aspinwall, b. New York City, N. Y., Jan. 26, 1859. She is unm. and resides in New York City, N. Y.

iii. William Hadden, b. New York City, N. Y., Dec. 27, I86r. He is unm. and resides in New York City, N. Y.

ELIJAH EDWARDS CURTISS (Zachariah, Mitchell, Zachariah, Zachariah, William, Elizabeth), b. Stratford, Conn., Jan. 21, I779; m. Elizabeth Jane . He d. in Trumbull, Conn., Dec. I 5,1847 . She d. Dec. 6, I 835 , aet. 56 years.

They had a child : -

i. Zachariah P., b. Trumbull, Conn., m. Eliza Jane

He d. in Bridgeport, Conn., in 1842.

They had a child:-

i. George, b. Bridgeport, Conn., I 828 . He d. Jan. 2, 1846 . 
JAMES ROSE EDWARDS CURTISS (Zachariah, Mitchell, Zachariab, Zachariah, William, Elizabeth), b. Stratford, Conn., April I, $1788 ; \mathrm{m}$.

They had children :-

(697) i. Lewis B., b.

ii. James E., b.

iii. Ellen, b. m. Wakeley.

iv. Anna, b. m. Turney.

$(698)$ vi. Albert, b.

vii. Maria, b.

JUSTUS BURR CURTISS (Daniel M., Mitchell, Zachariah, Zachariah, William, Elizabeth), b. Trumbull, Conn., Jan. 27, 1780; m. Huldah Edwards She d. Dec. 3, 1855 . He d. in Nichols, Conn., Aug. 10, 1832 . He served as a private in the War of 1812 .

They had children :-

i. Matilda, b. Trumbull, Conn., Feb. 21, I801; m. Stevens. She d. July 25, 1852 .

ii. Emeline, b. Trumbull, Conn., Sept. I, I802. She d. unm. Dec. I4, 1855 .

iii. Susan, b. Trumbull, Conn., ; m. David Shelton.

iv. Monson Edwards, b. Trumbull, Conn., Oct. I5, I8I I ; m. Julia Beach He d. Dec. 15, I841. No issue.

(699) v. Elliot Plumb, b. Nichols, Conn., April 14, 1814; m. Clarissa Bulkley, of Greenwich, Conn., . He d. March 10, I 896. She d. Dec. 31, I888, aet. 70 years.

vi. Sylva, b. Nichols, Conn., ; m. Edwin Barlow She d. March 28, I 842 .

vii. Elizabeth, b. Nichols, Conn., viii. Henry B., b. Nichols, Conn., 1839 .

$$
476
$$

ELI CURTISS (Daniel M., Mitchell, Zachariah, Zachariah, William, Elizabeth), b. Trumbull, Conn., Sept. 16, 1781 ; m. Sarah, d. of Zachariah and Anna Hawley Blakeman, of Trumbull, Conn., ; m. (ii.) Hepzibah . She d. Oct. 29, 1886, aet. 88 years. He d. April 23, 1819. 
They had children :-

i. Caroline, b. Trumbull, Conn.

ii. Lucy, b. Trumbull, Conn.,

I 8 i $2 ; \mathrm{m}$. John Burr, of Trumbull, Conn., March 16,$1836 ; \mathrm{m}$. (ii.) Rodney Curtiss, of Bridgeport, Conn., Dec. 28, 1845. She d. Dec. 21 , I882.

iii. Alanson, b. Trumbull, Conn., I814. He d. Aug. 26, I842.

(70o) iv. Isaac, b. Trumbull, Conn.

\section{8}

ALVIN CURTISS (Daniel M., Mitchell, Zachariah, Zachariah, William, Elizabeth), b. Trumbull, Conn., r798; m. Dolly

He d. in Trumbull, Conn., Sept. 18, 1879. She d. Oct. 21 , 1885 , aet. 86 years.

They had children : -

i. Delia, b. Trumbull, Conn.,

I $829 ; \mathrm{m}$. George W. Peck,

(70I) ii. David, b. Trumbull, Conn.

(702) iii. Miles L., b. Trumbull, Conn.

\section{9}

I RA CURTISS (Daniel M., Mitchell, Zachariah, Zachariah, William, Elizabeth), b. Trumbull, Conn., 1801 ; m. Sarah Burritt, of Stratford, Conn., Dec. 26, 1833. He d. in Trumbull, Conn., June 24, 1849 .

They had children :-

i. Caroline, b. Trumbull, Conn.,

ii. Lewis M., b. Trumbull, Conn.,

(703) iii. Henry B., b. Trumbull, Conn.,

I 835 ; d. $y$.

I 837 . Hed. June 5,1855 . I $84 \mathrm{I} ; \mathrm{m}$. Ellen $\mathrm{T}$. Curtiss, of Trumbull, Conn., Oct. 16, I866. She d. Jan. I9, I 870 ; m. (ii.) Anna A. Marshall, of Trumbull, Conn., Nov. I 2, 1871 . She d. Aug. 7, 1878, aet. 37 years.

$$
480
$$

CARLOS CURTISS (Leveret,, Samuel, Feremiah, Zachariah, William, Elizabeth), b. Southington, Conn., Feb. 22, 1798 ; m. Jerusha Brooks, of Cheshire, Conn., Oct. 29, 1820.

They had children :-

(704) i. Harry Brooks, b. Southington, Conn., Nov. 26, I82 I ; m. Frances E. Doolittle, Nov. I, 1843 .

ii. William Day, b. Southington, Conn., Feb. 24, i 825 . He d. Sept. 22,1825 .

iii. Laura, b. Southington, Conn., Feb. 7, I 828 . She d. Jan. $21,183^{8}$. ( 130 ) 


\section{$48 \mathrm{I}$}

SAMUEL ELLERY CURTISS (Leveret,, Samuel, Feremiah, Zachariah, William, Elizabeth), b. Southington, Conn., March 8, I 808 ; m. Mary Bidwell, d. of Ezekiel and Roxana Andrews, of New Britain, Conn., April 2r, I830. He was a shoemaker, but later in life became interested in photography, and took that for his profession. In 1833 he settled in New Britain, Conn., and a few years later removed to Broadalbin, N. Y., and thence to Madison, Wis., where he d. during the summer of I883. His wife d. in 1880 , aet. 73 years.

They had children :-

i. Frances Marian, b. New Britain, Conn., May 30, 1834. She d. unm. April 27, 1860 .

(705) ii. Edwin Rodney, b. Southington, Conn., May 6, 1836; m. Eva A. Lingenfelter, of Fonda, N. Y., May I I, 1859 . Thev live at Madison, Wis.

(706) iii. Nathan Selah, b. New Britain, Conn., Oct. 19, I838; m. Fannie Bouta, August, 1859 . They reside in Syracuse, N. Y.

iv. George Frederick, b. New Britain, Conn., Oct. I I, 1849. He d. June I 2, I 854 .

WILLIS CURTISS (Levi, Stephen, Abrabam, Fosiah, William, Elizabeth), b. Stratford, Conn., I801; m. Catherine Jane She d. Nov. 3, I 864, aet. 66 years; m. (ii.) Mary R. Welles, of Stratford, Conn., Nov. 5, 1865. He d. in Stratford, Conn., April I 3, I 867 .

They had children :-

i. Mary Cordelia, b. Stratford, Conn.

(707) ii. Elbert O., b. Stratford, Conn., of Stratford, Conn., Dec. 4, 1860.

I $831 ;$ m. Emma L. Reeve,

493

CYRUS CURTISS (Wait, Agur, Fosiah, Fosiah, William, Elizabeth), b. Southbury, Conn., Dec. 8, I797; m. Lydia Van der Burgh, of Hudson, N. Y., March 12, I823. He settled in Hudson, N. Y., in 18I7, and entered the dry goods business, in which he continued until 1830 , when he changed to an importer of sperm oil, whale oil, and whale bone. In 1846 he removed to New York City, N. Y., where for twelve years he carried on the same business. In 1860 he organized the Washington Life Insurance Company, of New York, and was elected its first president, which position he held for a number of years. 
They had children :-

i. William Lafayette, b. Hudson, N. Y., Dec. 25, 1823. He d. Dec. 7, 1824 .

$(708)$ ii. Henry Hobart, b. Hudson, N. Y., May 29, 1826. He was a health officer in New York, and d. April 26, 1851.

(709) iii. Cyrus Stebbins, b. Hudson, N. Y., Sept. 18, 1827 ; m. Mary Helena, d. of Henry F. Peck, of New Haven, Conn., Oct. 15, 1850. He d. Oct. 8,1853 .

(710) iv. Edward Middleton, b. Hudson, N. Y., April 30, I830.

\section{4}

ISAAC CURTISS (Isaac F., Abner, Fosiah, Fosiah, William, Elizabeth), b. Stratford, Conn., Jan. Io, I 803 ; m. Sarah Lucinda Beers, of Stratford, Conn., June 1, I 827 . She d. Nov. 7, I844; m. (ii.) Caroline Lewis, of Stratford, Conn., March 4, I845. He d. in Stratford, Conn., June 2, 1862.

They had children :-

i. Elbert, b. Stratford, Conn., Feb. 18, 1828 . He d. March 8, I828.

ii. Sarah Jane, b. Stratford, Conn., Sept. 6, 1829; m. Lewis B. Carpenter, Sept. 6, 1870. She lives in Chicago, Ill.

iii. Caroline Elizabeth, b. Stratford, Conn., Sept. 29, I831 ; m. Edward Benjamin, Oct. 23, $185 \mathrm{I}$.

iv. Charles Dillissenea, b. Stratford, Conn., Feb. 18, 1834; m. Ellen Stebbins, Oct. 15, 1867. He d. Feb. 2, 1870.

They had a child : -

i. Kittie, b. June 3, 1869. She d. Oct. 3, 1869 .

v. Lewis Frederick, b. Stratford, Conn., June 10,1836 ; m. Mary E. Hamilton, Nov. IO, J858. She d. June I, I869; m. (ii.) Mary C. Baldwin, Oct. 25, 1871. He lives in New Milford, Conn. They had a child:

i. Frederick, b. New Milford, Conn., June 8, J875.

vi. Frances Ann, b. Stratford, Conn., March 25, 1839. She is unm.

vii. John Beers, b. Stratford, Conn., April I I, I841 ; m. Lucretia Smith, June 2I, I866. He lives in San Francisco, Cal.

They had a child :-

i. Agnes D., b. April 22, I867 ; m. Frederick L. Wright, Aug. 31, 1893 .

viii. Susan Rebecca, b. Stratford, Conn., April ı 8, I843; m. C. Cady.

\section{6}

SIDNEY F. CURTISS (Isaac F., Abner, Fosiah, Fosiah, William, Elizabeth), b. Stratford, Conn., June 23, I808; m. Christina Demarest, of Stratford, Conn., Sept. I9, I 833 . 
They had children :-

i. Helen Maria, b. Stratford, Conn., Dec. 21, 1836 .

ii. Cornelius D., b. Stratford, Conn., April 5, 1839.

$(7 I I)$ iv. William Henry, b. Stratford, Conn., April II, I841 ; m. Helen A. Bertine, of Danbury, Conn., . He d. Jan. 8, 1887.

v. Christina, b. Stratford, Conn.

LUCIUS CURTISS (Isaac $\mathcal{F}_{.,}$Abner, Fosiah, Fosiah, William, Elizabeth), b. Stratford, Conn, Feb. 22, I814; m. Frances A. Ufford, of Stratford, Conn., March i 8, i 839. He d. in Stratford, Conn., Oct. 18, 1848. She d. Jan. 17, 1883.

They had children :-

i. Sidney Ufford, b. Stratford, Conn., Sept. 29, I84I ; m. Carrie A. Pratt, of Northampton, Mass., July 5, I864. He is cashier of the Western Union Telegraph Co., at Hartford, Conn., where he lives.

They had a child : -

i. Robert William, b. Bridgeport, Conn., July 4, s 869.

ii. Francis Eugene, b. Stratford, Conn., Jan. 14, 1845. He d. unm. Feb. 11, 1874 .

$$
498
$$

HOLBROOK CURTISS (Salmon, Nehemiah, Benjamin, Fosiah, William, Elizabeth), b. Newtown, Conn., July 14, I787; m. Elizabeth P., d. of Hon. William and Elizabeth Edmond, of Watertown, Conn., Oct. 7, 1822. He d. Feb. 21, 1858.

They had a child :-

(7I2) i. William Edmund, b. Litchfield, Conn., I824; m. Mary Anne, d. of William and Eunice Scovill, of Waterbury, Conn., Sept. 2, 1851. He d. in Watertown, Conn., July 6, I880.

501

NICHOLS CURTISS (Philo, Benjamin, Benjamin, Fosiah, William, Elizabeth), b. Newtown, Conn., Sept. 27, 1784; m. Sarah Ann, d. of Amos Bennett, of Newtown, Conn., Nov. 3, I803. He d. in Newtown, Conn., April 20, I852. She d. Oct. 29, I 858 , aet. 71 years.

They had children :-

i. Charlotte Nichols, b. Newtown, Conn., June 13, 1821; m. Monroe Downs, of Southbury, Conn., in 1843. She d. in Newtown, Conn., April 6, 1881. 
(7I3) ii. Julius Bolěvar, b. Newtown, Conn., Dec. Io, I825; m. Mary, d. of Peter and Mary Acker, of Greenwich, Conn., Oct. 30, 1854. She d. Jan. 23, I 864, aet. 56 years; m. (ii.) Alice, d. of Henry and Margaret Kneeland, of New York City, N. Y.

\section{2}

CARLOS CURTISS (Philo, Benjamin, Benjamin, Fosiah, William, Elizabeth), b. Newtown, Conn., Sept. 29, 1786 ; m. Polly Sample, of Newtown, Conn., Sept. 20, 1807 . She d. in Taylorville, Ill., April 26, I 858 , aet. 71 years. He removed from Newtown, Conn., to Berkshire, Delaware County, Ohio, in $1810-1812$, and then in I 836 to Coles County, Ill., where he d. Jan. 8, I 844 . He was a farmer and a musician. During the younger days of his life he taught the violin and was a writer and composer of some note.

They had children :-

(714) i. William Hubbell, b. Newtown, Conn., Oct. 17, I808; m. Hannah, d. of Jacob Rosecrans, of Coles County, Ill, Oct. 29, I 829 . He d. Tuscalo, Ill., Feb. 28, I 884 . She d. Jan. 5, I 857 , aet. 47 years.

ii. Charlotte, b. Newtown, Conn., Sept. 20, I 8 Io; m. Kerby Benedict, of Delaware County, Ohio, in 1837 . She d. at Decatur, Ill., Nov. 1 5, I876. He d. at Santa Fe, N. M., Feb. 26, I 874 .

(715) iii. Nichols, b. Delaware County, Ohio, Jan. I3, I8 18. He d. Jan. I 2, 1844 .

$(716)$ iv. Derwin, b. Delaware County, Ohio, Jan. 21, I820. He d. Nov. $22, \mathrm{I} 847$.

(717) v. Ira B., b. Delaware County, Ohio, Oct. I 8, I 823 ; m. Jane Butler,

He d. in Decatur, Ill., Dec. I6, I89I.

$(718)$ vi. Philander, b. Delaware County, Ohio, Oct. 28,$1825 ; \mathrm{m}$. Ann Elizabeth, d. of Robert and Elizabeth Modrul, Jan. 16, I844. He d. Nov. 15,1876 .

503

PHILO CURTISS (Philo, Benjamin, Benjamin, Fosiah, William, Elizabeth), b. Newtown, Conn., Sept. 20, I791 ; m. Sally Maria Birch, of Newtown, Conn., Jan. 16, I 825. He d. in Newtown, Conn., Jan. I 5, I 872 . She d. March 2, I 893 , aet. 90 years.

They had children :-

i. Cornelia, b. Newtown, Conn., Sept. 24, 1827. She resides in Newtown, Conn.

ii. Jane, b. Newtown, Conn., July 9, I 829. She d. Oct. I 5, I 862. 
CYRENIUS CURTISS (Alfred D., Benjamin, Benjamin, Fosiah, William, Elizabeth,) b. Newtown, Conn., Dec. 5, i814; m. Christa Ann Beardsley, of Monroe, Conn., May 1 5, I838. She d. May I I, I 89 I, aet. 76 years. He d. in Bridgeport, Conn., May 27, 1890.

They had children :-

i. Alfred, b. Newtown, Conn., Dec. 3, 1839 . He d. Nov. 24, I850.

ii. William, b. Newtown, Conn., Aug. 20, 1842 . He d. Nov. 5 , 1850 .

(719) iii. Joseph, b. Newtown, Conn., April 5, 1844; m. Jane Eliza Tyler, of East Haddam, Conn., Feb. I4, I869. She d. Jan. 19, I890, aet. 43 years. He lives in New Haven, Conn.

iv. Sarah Matilda, b. Newtown, Conn., Nov. 7, I846; m. Frederick William Curtiss, of Huntington, Conn., May I0, r868. She d. Nov. 28, 1883 .

v. Phebe Ann, b. Newtown, Conn., Sept. 15, 1850; m. James A. Wilson, of Bridgeport, Conn., Nov., 1867. She resides in Bridgeport, Conn.

vi. Bertha Celia, b. Newtown, Conn., May 20, I853. She is unm. and lives in New Haven, Conn.

vii. Eva Adelaide, b. Newtown, Conn., May I7, 1856. She d. July 19, I 868 .

509

HENRY FRANCIS CURTISS (Epenetus, Benjamin, Benjamin, Fosiah, William, Elizabeth), b. Bridgeport, Conn., May 4, 1823 ; m. Lany, d. of Hon. Risdon McDaniel, of Jones County, N. C., Aug. I 2, I 847 . He d. in Knox County, Tenn., Oct. 23, I 893 . He went to sea when a boy and travelled nearly all over the world, finally settling in North Carolina in 1846.

They had children :-

i. Joseph, b. Jones County, N. C., July 25, I848. He removed with his parents to Connecticut, before the Civil War. He enlisted in the Federal army, at the age of fifteen years, and served until the end of the war. He d. in New Orleans, La., June 3, 1895 .

ii. Susan, b. Jones County, N. C., Nov. I, 1850. She resides in Knoxville, Tenn.

(720) iii. Henry William, b. Jones County, N. C., May I r, I852; m. Ida, d. of Josiah Whitlow, of Bedford County, Va., Sept. 4, 1877 . He resides in Knoxville, Tenn.

(721) iv. Charles, b. Jones County, N. C., Jan. 2, 1854; m. Rachel Barnum. He resides in Danbury, Conn.

v. Salena, b. Jones County, N. C., Feb. 3, 1856; m. Richard Randall, . She resides in Shelton, Conn. 
vi. Lany, b. Jones County, N. C., July 25, 1857. She resides in Shelton, Conn., unm.

vii. Sarah, b. Jones County, N. C., Nov. 7, 1865 ; m. H. B. Branner, of Knoxville, Tenn. She d. Oct. I I, I 894.

viii. Frances, b. Jones County, N. C., May 2I, 1869 . He is a schoolteacher at Grand Junction, Col.

\section{0}

ABIJAH BEACH CURTISS (Fobn, Abijah, Benjamin, Fosiah, William, Elizabeth), b. Huntington, Conn., I80I-2; m. A bigail Allen, of Rochester, N. Y.

They had children : -

(722) i. Carlos G., b. Rochester, N. Y.; m. Sarah, d. of Horatio Nelson and Marcia Curtiss, of Rochester, N. Y.

They had children :

i. Elizabeth, b. Rochester, N. Y.; m. Andre Meymeis, of Paris, France.

ii. Edward, b. Rochester, N. Y. d. y.

(723) ii. John Beach, b. Rochester, N. Y.

(724) iii. Jacob, b. Rochester, N. Y. He d. in Brooklyn, N. Y., I 876.

iv. Elizabeth, b. Rochester, N. Y.

v. Louisa, b. Rochester, N. Y.

\section{I 4}

IRA LAWRENCE CURTISS (Abijah B., Abijah, Benjamin, Fosiah,.William, Elizabeth), b. Newtown, Conn., Nov. 9, I8 I3; m. Marietta, d. of John and Polly Curtiss Glover, of Newtown, Conn., Jan. I 8, I 835 . He d. Newtown, Conn., Jan. I, I 843 . She d. Bedford, Ind., Aug. 30, 1887.

They had children :-

i. Elizabeth, b. Newtown, Conn., Oct. 19, 1835; m. Daniel Webster Parker, of New Haven, Conn., Sept. 19, 1860.

ii. Juliette, b. Newtown, Conn., July 9, 1837; m. Winthrop Alvin Foote, of Newtown, Conn., Sept. 16, 1858.

\section{5}

ROSWELL CURTISS (Agur, Silas, Peter, Fosiah, William, Elizabeth), b. Exeter, N. Y., Aug. I, I795; m. Jerusha Bennett, of Exeter, N. Y., Oct. I3, I8 I6. He was a farmer and lived in Starkey, N. Y., where he died Oct. 29, I 869. She d. July 24, I 869 , aet. 75 years. 
They had children :-

(725) i. Thomas Bennett, b. Starkey, N. Y., July 28, I817; m. Alvira, d. of William B. and Martha Briggs, Sept. I0, 1842 .

ii. Esther, b. Starkey, N. Y., June 28, 1820 ; m. Rev. Manderville Tuttle, May 14, 1839; m. (ii.) Dr. J. Esack Moshier, of Anı Arbor, Mich. She d. April 5, 1866.

(726) iii. Charles, b. Starkey, N. Y., May 22, 1823; m. Roxy Doty, July 5,1869 . He lives in Howell, Mich.

iv. Caroline M., b. Starkey, N. Y., March 7, 1825; m. John T. Williams, Aug. 29, I845. She d. July 13, 1854.

v. Eliza Jane, b. Starkey, N. Y., Aug. 29, 1827 ; m. William Doty, Feb. 21, 1850. She lives in South Lyons, Mich.

(727) vi. Lester, b. Starkey, N. Y., Jan. 29, 1829; m. Amanda White, April 25,1850 . She d. March, 1851 ; m. (ii.) Sarah Bennett, June 6, I853. She d. April, 1896. He d. July I 2, 1892.

(728) vii. Samuel Bennett, b. Starkey, N. Y., May 5, I834; m. Augusta Pebbles, Dec. 10, 1863. She lives in Bath, Ill.

viii. Isabella May, b. Starkey, N. Y., Feb. 24, 1843; m. John Jay Hooper, March 26, 1862. She lives in South Lyons, Mich.

\section{6}

DAVID CURTISS (Agur, Silas, Peter, Fosiah, William, Elizabeth), b. Exeter, N. Y., July 26, I799; m. Sophia May, Nov., 1825. She d. Feb. 5, I 844 , aet. 4I years; m. (ii.) Betsey Rose, of Exeter, N. Y., Nov. 28, 1844 . She d. May 7, 1888 . He was a farmer and lived in Exeter, N. Y., where he died Oct. I3, I 876.

They had children : -

(729) i. Walter Shelden, b. Exeter, N. Y., Feb. 18, 1828 ; m. Amelia Bishop, of Cortland, N. Y., Aug. I1, I 859 . No issuc.

ii. Marinda, b. Exeter, N. Y., Aug. 17, 1829; m. Thomas White, of Gravelrun, Mich., March 30, 1854.

(73o) iii. Delos, b. Exeter, N. Y., April 17, 1831 ; m. Lovina May, of Cortland, N. Y., Nov., 1857 . No issue.

iv. Irene Morse, b. Exeter, N. Y., June 15, 1834 ; m. Lafayette Gates, June 15,1856 .

(731) v. Harmon May, b. Exeter, N. Y., Oct. 23, 1842; m. Matilda A. Howard, Aug. 27, 1867. He d. Feb. 17, 1875 .

(732) vi. George Thomas, b. Exeter, N. Y., July 3, 1847 ; m. Eliza Hagabone, Feb. 5, 1874 . No issue.

(733) vii. Lester Lee, b. Exeter, N. Y., Jan. 30, I849; m. Amelia J. Dingee, April 6, 1879 .

They had a child :-

i. Charles, b. Exeter, N. Y., March 2I, 188 I.

(734) viii. Dorre Rose, b. Exeter, N. Y., March 14, 1851; m. Mary, d. of (137) 
Almon and Cynthia Henry, of Exeter, N. Y., Dec. 22, I881. $\mathrm{He}$ is a farmer and lives in Excter, N. Y.

They had a child:

$$
\text { i. William Ray, b. Exeter, N. Y., Nov. } 2 \text { I, } 1888 .
$$

(7j5) ix. Charles Birdsey, b. Exeter, N. Y., Nov. 10, 1853; m. Anna Cass, of Exeter, N. Y., Jan. 2, I884.

$\left(73^{6}\right)$ x. Sherman William, b. Exeter, N. Y., Feb. 14, 1857.

\section{$5 \mathrm{I} 7$}

LESTER CURTISS (Agur, Silas, Peter, Fosiah, William, Elizabeth),

b. Exeter, N. Y., May 31, 1806; m. Caroline Bartlett; m. (ii.) Sarah Holmes. He was a merchant and resided in New York City, N. Y., where he d. Aug. I 3, I 834 .

They had children :-

i. Mary, b. Exeter, N. Y.,

(737) ii. Lester, b. Exeter, N. Y.,

m. William Burgess.

He lives in Chicago, Ill.

\section{I 8}

LEE CURTISS (Agur, Silas, Peter, Fosiah, William, Elizabeth), b. Exeter, N. Y., May 30, 1808 ; m. Phebe Ann Higbee, of New York City, N. Y., Oct., I 832. She d. Jan. 2 I, I 880, aet. 74 years. He was a physician and resided in Exeter, N. Y., where he d. Aug. I 3,1834 .

They had children :-

i. Helen, b. Exeter, N. Y., July 4, s833; m. Alvin Morelle Coleman, April 8, 1854 .

$\left(73^{8}\right)$ ii. Oscar Lee, b. Exeter, N. Y., ～; m. Hannah Stone.

\section{0}

CHARLES S. CURTISS (Agur, Silas, Peter, Fosiah, William, Elizabeth), b. Exeter, N. Y., Aug. I 8, I 8 I 5 ; m. Abigail M. Robinson, of Exeter, N. Y., Jan. 6, I836. He d. Aug. I8, 1852. She m. George May, Jan. 14, I 854, and d. April 13, I 882.

They had a child :-

i. Damon C., b. Exeter, N. Y., Oct. I I, I 838 ; m. B. Pliana Caunrad, of Exeter, N. Y., Dec. 23, 1863. He d. in Exeter, N. Y., Dec. 4, 1892. No issue. He was an undertaker and resided in Excter, N. Y.

\section{3}

EDWIN C. CURTISS (Abner, Reuben, Matthew, Fosiah, William, Elizabeth), b. Danbury, Conn., Dec. I 8, I798; m. Henrietta, d. of John Pitts, of Milledgeville, Ga., May I4, I823. She d. 
Feb. 25,1836 , aet. 30 years ; m. (ii.) Mary, d. of William and Phebe Van de Water, of New York City, N. Y., June 7, 1836. $\mathrm{He}$ was a book dealer in Milledgeville, Ga., for several years, but removed to Cahaba, Ala., where he lived on a cotton plantation until 1835 , when he settled in New York City, N. Y. In 1836 he removed to Campo, Conn., where he engaged in farming until I 840 , when he returned to New York City and entered the crockery business. He d. in New York City, N. Y., Nov. I I, 1864 .

They had children :-

(739) i. Edwin Pitts, b. Cahaba, Ala., Dec. 2, 1824. He enlisted as a member of an Illinois cavalry troop at the outbreak of the war of the Rebellion and d. of typhoid fever in a hospital at Trenton, Tenn., Aug. I 3, I 862.

(740) ii. John Abner, b. Cahaba, Ala., April I 5, r 826; m. Frances Angeline, d. of William and Adeline Curtiss Allen, of New York City, N. Y., Nov., I 848 . He was a member of the firm of Haggerty \& Co., auctioners. He d. in New York City, N. Y., Dec. 30, 1865 ; no issue.

iii. Julia Emma, b. Cahaba, Ala., Feb. 6, I829. She d. in New York City, Dec., I846; unm.

iv. Mary Henrietta, b. Cahaba, Ala., Jan. 25, I831; m. John Hunt Fowle, of Northampton, Mass., Sept. I8, 1850.

v. William Osborn, b. Cahaba, Ala., Oct. 14, 1833. He d. New York, Feb. 6, I836.

vi. Susan Caroline, b. New York City, N. Y., Feb. 10, 1836. She d. at Campo, Conn., April 29, I836.

vii. Phœebe Eliza, b. Campo, Conn., Aug. I 3, I837.

viii. Rebecca, b. Campo, Conn.

ix. Sarah Ann, b. Campo, Conn., July I0, I840. She d. in New York, Jan. 5, I 849 .

(741) x. William Osborn, b. Campo, Conn., Feb. 4, I842.

xi. Caroline Halsey, b. New York City, N. Y., Sept. 26, I844. She d. Aug. I, I 849 .

\section{$5^{24}$}

PAUL ALLEN CURTISS (Abner, Reuben, Matthew, Fosiah, William, Elizabeth), b. Danbury, Conn., Jan. 26, 1800 ; m. Mary Cecilia, d. of Jonas and Catherine Stansbury, of New York City, N. Y., Sept. 30, I823. He was a silk merchant and resided in New York City, N. Y.

They had children :-

(742) i. Allen Stansbury, b. New York City, N. Y., Aug. 3, I824; m. Mary Elizabeth, d. of William Lyons, of New York City, N. Y., 
Jan. 6, I 846. He d. in New York City, N. Y., July 22, I 868 .

ii. Catherine Stansbury, b. New York City, N. Y., Sept. I, I826; m. Henry Osmond Pinneo, of Milford, Conn., Nov. 23, I 846.

iii. Mary Cecilia, b. New York City, N. Y., Dec. 29, I828; m. Theodore Wallace Todd, of New York City, N. Y., June 24, I 85 I. She d. in New York City, N. Y., Feb. 23, I869.

iv. John Leonard, b. New York City, N. Y., Sept. 5, I831. He d. New York City, N. Y., Oct., I835.

(743) v. William Henry, b. New York City, N. Y., April I, I834; m. Annie Martin, of New York City, N. Y., Nov. I7, 1858.

vi. John Lconard, b. Westport, Conn., Feb. 2, I 836 . Hc d., New York City, N. Y., Feb. 22, 1844.

vii. Charles Halsey, b. New York City, N. Y, March 23, I838. He d. April I6, I840.

viii. Pauline, b. New York City, N. Y., June I2, I840. She d. New York City, N. Y., March 23, 187 I ; unm.

ix. Irene Augusta, b. New York City, N. Y., Nov. 7, 1842; m. Cornelius Brinkman Outcault, of New York City, N. Y., June 6, I 867 .

x. Clemence, b. New York City, N. Y., June 8, I846.

xi. Floyd Ferris, b. New York City, N. Y., Oct. 28, 1849. He d. unm.

\section{6}

JOSEPH CURTISS (Abner, Reuben, Matthew, Fosiah, William, Elizabeth), b. New York City, N. Y., Nov. 23, 1803; m. Sarah Anne, d. of William and Phebe Van de Water, of New York City, N. Y., May ı 3, i 826. He d. in New York City, N. Y., July 9,1867 . He was an importer of silks and ribbons, and resided in New York City, N. Y.

They had children :-

(744) i. William Van de Water, b. New York City, N. Y., March 6, 1827 ; m. Amy Cornell McIntire, d. of William and Ruth Lovett McIntire, of New York City, N. Y., May I I, I848. He d. 1873 .

ii. Harriet Amelia, b. New York City, N. Y., June 29, I829.

(745) iii. Alfred Leonard, h. New York City, N. Y., May 3, 1832; m. Mary Elizabeth, d. of Thomas and Eleanor Johnson Hilson, of New Orleans, La., April I, I854. She d. in New York City, N. Y., June 8, r869, aet. 35 years; m. (ii.) Elizabeth McDonald, d. of Oliver and Elizabeth McDonald (McIntire) Strong and widow of Frank Jaudon, of New York City, N. Y., Aug. 4, I872. He d. I 897 .

iv. Adeline Allen, b. New York City, N. Y., Sept. 9, I834; m. Alfred Gardner Perry, of R. I., Sept. 7, I854. He d. in New York City, N. Y., May 3, I862, aet. 32 years. She d. Feb. 4, I88I. 
v. Sarah Isabel, b. New York City, N. Y., Jan. 1, 1837.

vi. Mary Caroline, b. New York City, N. Y., March 2, 1839.

vii. Julia Emma, b. New York City, N. Y., July IO, I841; m. Myron Winslow, Aug., I 88 I.

(746) viii. John Halsey, b. New York City, N. Y., Oct. 4, I843; m. Mary Harriet Rider.

They had a child :-

i. Sarah Theodora, b. 1881 .

$53 \mathrm{I}$

SAMUEL CURTISS (Gould, Gold, Matthew, Fosiah, William, Elizabeth), b. Newtown, Conn., April 27, I818; m. Mary Nichols, of Newtown, Conn., Jan. 5, 1848. He is a manufacturer of combs and buttons and resides in Sandy Hook, Conn.

They had children :-

(750) i. Henry Gould, b. Newtown, Conn., Dec. 8, 1848; m. Anna E., d. of George and Sarah Peck Beers, of Newtown, Conn., Sept. 23,1874 .

ii. Julia, b. Newtown, Conn., July 3 I, 1855 ; m. Henry S. Hawley, of Newtown, Conn., Nov. 17, 1879.

\section{$53^{6}$}

BENJAMIN CURTISS (Hezekiah, Gold, Matthew, Fosiah, William, Elizabeth), b. Newtown, Conn., Monroe, Conn., Oct. I, I 857 .

They had children :-

i. Hobart Henry, b. Newtown, Conn., ; m. Ruth A. Nichols, of Newtown, Conn.

ii. Jennie, b. Newtown, Conn.

iii. Frederick L., b. Newtown, Conn.

iv. Newton, b. Newtown, Conn.

HENRY CURTISS (Hezekiah, Gold, Mattherw, Fosiah, William, Elizabeth), b. Newtown, Conn., ; m. Fannie Parker, of Dubuque, Iowa, . He settled in Dubuque, Iowa, and was killed there by the explosion of a boiler on a steamer.

They had children:-

i. Florence, b. Dubuque, Iowa.

(75I) ii. Charles, b. Dubuque, Iowa, . He lives in Tacoma, Wash. 
PETER BURR CURTISS (Peter B., Peter B., Peter, Fohn, Israel, Fohn, Elizabeth), b. West Martinsburgh, N. Y., ; m.

He d. Aug. 23, 187 I.

They had children :-

$(752)$ i. Benjamin, b.,

(753) ii. Alonzo, b.

- He was killed on the railroad in Illinois.

$54^{\circ}$

URIAH BRADLEY CURTISS (Peter B., Peter B., Peter, Fohn, Israel, Fohi, Elizabeth), b. West Martinsburgh, N. Y., March 17, $1808 ; \mathrm{m}$. Sophia Shumway, of West Martinsburgh, N. Y., He died in West Martinsburgh, N. Y., Oct. 22, 1876.

She d. in West Martinsburgh, N. Y., Dec. 4,1872 , aet. $5^{8}$ years. They had children :-

i. Augusta, b. West Martinsburgh, N. Y., Dec. I, 1835; m. Anson Cummings, . She lives in Watertown, N. Y.

ii. Anna, b. West Martinsburgh, N. Y., May 31, I837; m. A. J. Gordon, - She d. in West Martinsburgh, N. Y., Feb. I I, 1864 .

(754) iii. Byron, b. West Martinsburgh, N. Y., Sept. 5, I840; m. Mary Philleo, He d. in Lowville, N. Y.

(755) iv Beri, b. West Martinsburgh, N. Y., July I, I 842 ; m. Emily Philleo, . They live in West Martinsburgh, N. Y.

They had a child : -

i. Grant, b. West Martinsburgh, N. Y.

v. Benjamin, b. West Martinsburgh, N. Y., March 10,$1845 ; \mathrm{m}$ Helen Stevens, . They live in West Martinsburgh, N. Y. No issue.

vi. Adelia, b. West Martinsburgh, N. Y., March 23, I847; m. A. J. Gordon, They live in West Martinsburgh, N. Y.

vii. Emory, b. West Martinsburgh, N. Y., July 5, I848; m. Maria Stevens, . They live in West Martinsburgh, N. Y. They had a child:

i. Anna, b. West Martinsburgh, N. Y., Oct. $5,1878$. She d. March 6, I889.

viii. Ella, b. West Martinsburgh, N. Y., July 2I, I850; m. Ira Sharp, They live in Lowville, N. Y.

ix. Bradley, b. West Martinsburgh, N. Y., Oct. 3, 1855. He d. March 7, 1860 .

542

CHARLES HENRY CURTISS (Henry, Peter B., Peter, Fohn, Israel, Fohn, Elizabeth), b. West Martinsburgh, N. Y., March 9, 
$1815 ; \mathrm{m}$. Mary Lyman, She d. May 6, 1853 , aet. 48 years; m. (ii.) Julia Abbe, - He d. in Lowville, N. Y., Sept. 7, 1891. His widow lives in Lowville, N. Y.

They had children :-

i. Charles Merrick, b. West Martinsburgh, N. Y., Sept. 29, $18{ }_{3} 8$. He d. unm. in Lowville, N. Y., Feb. 10, I872.

ii. Mary Cornelia, b. West Martinsburgh, N. Y., July 2 I, I 84 I ; m. Ferris Newman, . They live in Low ville, N. Y.

(756) iii. George Henry, b. West Martinsburgh, N. Y., June I 7, I $845 ; \mathrm{m}$. Martha Curtiss,

- They live in Rosendale, Wis.

\section{3}

CHARLES BUTLER CURTISS (Sherman, Peter B., Peter, Fohn, Israel, John, Elizabeth), b. Lanesborough, Mass., June 15, 1824; m. Maria Wight, . She d. in 1863 ; m. (ii.) Rebecca Lamphere. He was a farmer until 1854 , when he went in to the stockraising business. He resides with his wife in Elgin, Ill.

They had children :

(757) i. George S., b.

$\left(75^{\circ}\right)$ ii. Fred D., b.

iii. Margaret, b.

iv. Effie, b.

(759) v. Herman, b.

Feb. $4,1848$.

Feb. 25,1855 .

Nov. 20, 1857 .

Aug. 17, 1859 .

Feb. 23,1862 .

\section{4}

DAVID HUNTINGTON CURTISS (David $S_{.}$, David, David, Fohn, Israel, Fohn, Elizabeth), bapt. Woodbury, Conn., April 3, I 796 ; m. Maria Summers, of Woodbury, Conn., Dec. 20, 1825.

She d. Oct. 5, 1828 , aet. 24 years ; m. (ii.) Anna Guernsey,

She d. Aug. 31, I 866, aet. 59 years. He d. in Woodbury, Conn., Jan. 25, I 862 . He was a justice of the peace.

They had children:-

i. Maria, b. Woodbury, Conn.,

1827. She d. April i I, 1836 .

(760) ii. James Guernsey, b. Woodbury, Conn., Aug. 8, 1835; m. Mary Jennette, d. of Henry B. and Frances Stiles, of Southbury, Conn., May I 7, I 860. She d. Sept. 20, I 893.

$(761)$ iii. David, b. Woodbury, Conn., Sept. 25, 1837.

\section{5}

DANIEL CURTISS (David S., David, David, Fohn, Israel, Fohn, Elizabeth), bapt. Woodbury, Conn., Nov. 8, 1801 ; m. Julia F., d. of John and Flora Preston Strong, of Woodbury, Conn., Jan. 
27,1835 . He was a manufacturer of woolen goods and a prominent citizen of Woodbury, Conn. After his death, May I6, 1878, his business was carried on by his three sons under the name of Daniel Curtiss' Sons. He was a representative and senator from Woodbury, Conn. His widow resides in Woodbury, Conn.

They had children :-

(762) i. Walter Stiles, b. Woodbury, Conn., Feb. 9, 1836; m. Eunice, d. of Phineas Averill, of South Britain, Conn., Nov. 29, 1865. They reside in Woodbury, Conn.

ii. Emily Amelia, b. Woodbury, Conn., Oct. 30, 1837; m. Homer S. Tomlinson, of Woodbury, Conn., June I, 1872.

iii. Ellen C., b. Woodbury, Conn., Nov. 8, 1839; m. Perry Averill, of Woodbury, Conn., Sept. 6, I876.

$(763)$ iv. Horace Daniel, b. Woodbury, Conn., July 24, I84 I ; m. Harriet F. Atwater, of New Haven, Conn., Oct. 6, 1868 . They reside in Woodbury, Conn.

v. Cordelia, b. Woodbury, Conn., March I 3, I $843 ; \mathrm{m}$. Geo. C. White, of Brooklyn, N. Y., Sept. 19, 1867. She d. Nov. 9, 1887.

$(764)$ vi. Edward John, b. Woodbury, Conn., Jan. 24, I845; m. Elizabeth Sanford, of Roxbury, Conn., May 8, 1872 . She d. Oct. I2, 1878 ; m. (ii.) Ella L. Abrams, in Woodbury, Conn., June 7, 1882. She d. April, 1884. He resides in Woodbury, Conn.

S vii. Anna, b. Woodbury, Conn., July 7,1848 .

\{viii. Frances J., b. Woodbury, Conn., July 7, 1848. She resides in Woodbury, Conn., unm.

ix. Elizabeth Stiles, b. Woodbury, Conn., Sept. 7, 1850; m. Edward F. Cole, of Waterbury, Conn., Jan. 3, 1878 . She d. Jan. II, 1882 .

\section{8}

REUBEN BRADLEY CURTISS (Simeon, Foseph, Israel, Stephen, Israel, Fohn, Elizabeth), b. Southbury, Conn., Dec. 19, 1796; m. Minerva, d. of David and Sally Booth McEwen, of Oxford, Conn., March I I, I 820 . She d. Oct. 20, I 867 , aet. 65 years. He d. Southford, Conn., Aug. I 5, I882. He was a farmer.

They had children :-

i. James McEwen, b. Oxford, Conn., Nov. 25, 1822. He d. unm. Aug. 27, 1854 .

(765) ii. Wales Hatch, b. Southford, Conn., July 29, 1826; m. Mary Frances, d. of William and Esther Sanford Hammill, of Williamsburg, L. I., Dec. 24, 1850 . He lives in Southford, Conn.

iii. Mary Ann, b. Southford, Conn., Dec., 1827. She d. unm. Nov. $2 \mathrm{I}, 1849$. 
SIMEON CURTISS (Simeon, Foseph, Israel, Stephen, Israel, Fohn, Elizabeth), b. Southbury, Conn., Nov. I3, 1805; m. Hannah Bronson.

They had children :-

i. Israel Josiah, b. Southbury, Conn., I833. He d. July 7, 1868.

(766) ii. Franklin Luther, b. Southbury, Conn., Jan. 19, 1835; m. Mary Louise, d. of Richard Hines, of Waterbury, Conn., Sept. 28, I 858 . He resides in Waterbury, Conn.

iii. Hannah, b. Southbury, Conn., Sept. 20, 1836. She d. Sept. I I, I 892 .

\section{2}

BENJAMIN STILES CURTISS (Charles R., Stephen, Israel, - Stephen, Israel, Fohn, Elizabeth), b. Ellisville, N. Y., July 23, $1822 ; \mathrm{m}$. Martha Jennette, d. of Stoddard and Jennette (Mallory) Strong, of Woodbury, Conn., Jan. 27, I 850 . He came to Southbury, Conn., in 1838 , and has lived in Woodbury, Conn., since his marriage. $\mathrm{He}$ is engaged in mechanical pursuits. They reside in Woodbury, Conn.

They had children:-

(777) i. Charles Strong, b. Woodbury, Conn., March 23, 1856 ; m. Ellen Mallory, d. of George B. French, of Watertown, Conn., Nov. 8,1883 . They reside in Woodbury, Conn.

ii. Jennette Waterbury, b. Woodbury, Conn., Aug. 12, 186 I. She d. Nov. 24,1877 .

iii. Benjamin Lincoln, b. Woodbury, Conn., March 8, 1867 ; m. Mary Curtiss, d. of Francis Atwood, of Woodbury, Conn., in 1893. He d. May, I893; no issue.

HORACE HORATIO CURTISS (Charles R., Stephen, Israel, Stephen, Israel, Fohn, Elizabeth), b. Ellisville, N. Y., March I6, $1829 ;$ m. Mary, d. of Stephen Sypert, of Jackson, Tenn., Aug. 3I, I 853. He settled in Jackson, Tenn., where he wás a manufacturer until $189 \mathrm{I}$, when he removed to Texas, returning from there in 1897 . He resides in Jackson, Tenn. During the Civil War he served two years on detail service in the Confederate army.

They had children:-

i. Mary Jennette, b. Jackson, Tenn., Oct. I0, 1854; m. Robert Mann, of Jackson, Tenn., Jan. 26, 1875.

ii. Charles Sypert, b. Jackson, Tenn., Feb. 22, 1857. He d. Jackson, Tenn., March 20, 1885. 
$(77 \mathcal{S})$ iii. Edward Benjamin, b. Jackson, Tenn.; m. Corinna B., d. of Robert J. Ruffin, of Jackson, Tenn., Dec. 2I, I887. He lives in Jackson, Tenn.

iv. Ellen Elizabeth, b. Jackson, Tenn., Aug. 27, 1863; m. Charles H. Hughes, of Jackson, Tenn., Dec. 20, 1882. She d. Feb. 3, 1884.

v. Minnie, b. Jackson, Tenn., Feb. 26, 1868 ; m. W. Thomas Anderson, Dec. 22, 1887. She d. April 21, 1890.

ORRIN CURTISS (Fauncey, Abel, Benjamin, Nathaniel, 'Thomas, Fohn, Elizabeth), b. Wallingford, Conn., I $800 ; \mathrm{m}$. Sally Bristol, of Waterbury, Conn., I823. He d. Sept. 24, I88 I. She d. Feb. 23, I 879 , aet. 74 years.

They had children :-

i. Sarah Jerusha, b. Wallingford, Conn.,

ii. Ann Elizabeth, b. Wallingford, Conn.,

$1825 ; \mathrm{m}$. Parker. 6,1853 .

iii. Mary Cornelia, b. Wallingford, Conn., $\quad 1830$.

iv. Julia M., b. Wallingford, Conn., I833; m.

(779) v: James J., b. Wallingford, Conn., 1835 .

(7So) vi. William O., b. Wallingford, Conn., 1839.

vii. Levi, b. Wallingford, Conn., I843. He d. Sept. I 8, I 844.

$\left(7 S_{I}\right)$ viii. Henry B., b. Wallingford, Conn.,

(782) ix. Edgar A., b. Wallingford, Conn.,

x. Jane A., b. Wallingford, Conn., I 845 .

1847 .

I $849 ; \mathrm{m}$.

Johnson.

\section{7}

HARVEY CUR'TISS (Fauncey, Abel, Benjamin, Nathaniel, Thomas, Fohn, Elizabeth), b. Wallingford, Conn., April 3, I 8 I 5 ; m. Juliante E. Terrell, of Cheshire, Conn. She d. Jan. I5, I888, aet. 60 years. He d. Jan. I7, I 892.

They had children :-

$(783)$ i. George H., b. Wallingford, Conn., Nov. 24, 1850.

ii. Andrew, b. Wallingford, Conn., April 29, I852. He d. Aug. 27, I 852 .

iii. Mary E., b. Wallingford, Conn., Oct. I 8, I853. She d. unm. Sept. 28,1894 .

iv. Ira, b. Wallingford, Conn., Sept. I , I 857 . He d. June 26, I 858.

v. William B., b. Wallingford, Conn., March 7, 1863. He d. July, $19,1863$.

vi. Willis B., b. Wallingford, Conn., March 7, r863. He d. Oct. 6, 1863 .

vii. Wilber B., b., Wallingford, Conn., March 7, 1863. He d. Dec. 14,1863 . 


\section{6}

ISAAC W. CURTISS (Chauncey, Abel, Benjamin, Nathaniel, Thomas, Fohn, Elizabeth), b. Meriden, Conn., Oct. 27, $1812 ;$ m. Amy Ann Webster, of Southington, Conn., July I4, I 834. He d. May 7, 1884. She d. June 23, I86I.

They had children :-

i. Sarah A., b. Meriden, Conn., I 835. She d. Oct. 15, 1872 .

ii. Isaac R., b. Meriden, Conn., Sept. 30, I838; m. Emma L. Tryon, of Newark, N. J., May 27, 1865.

iii. Watson W., b. Meriden, Conn., Sept. 22, I843. He d. April 22, I 897 ; m. Agnes Dempater, of Naugatuck, Conn., Sept. 27, 1865 .

HARRISON W. CURTISS (Chauncey, Abel, Benjamin, Nathaniel, Thomas, Fohn, Elizabeth), b. Meriden, Conn., Aug. 20, $1817 ; \mathrm{m}$. Eliza Bradley, Oct. I 7, I 838 . She d. ; m. (ii.) Martha L. Sept. 29, I 850 , in Cincinnati, Ohio. He d. Jan. I 5 , 1869. She d. Sept. 9, 1890. He was a manufacturer and lived all his life in Meriden, Conn.

They had children :-

i. Rollin William, b. Meriden, Conn., Aug. 31, I84I; m. Emily Adelaide Francis, of Meriden, Conn., May 7, I862.

They had a child :-

i. Carlotta May, b. Meriden, Conn., Aug. 29, I 864.

(784) ii. Louis K., b. Meriden, Conn., June 29, 1851 ; m. Sarah Elizabeth, d. of Edward Walker and Nancy Boris Hatch, of Meriden, Conn., Oct. I, I 879 .

(785) iii. Floyd, b. Meriden, Conn., Dec. 25, I854; m. Clara, d. of Edward Furniss, of Meriden, Conn., Dec. 2, I 879 .

iv. Mattie Louise, b. Meriden, Conn., Oct. 10, 1859 ; m. H. E. Partridge, Jan. 16, 1883, and lives in St. Paul, Minn.

v. Zella, b. Meriden, Conn., June 3, I86I; m. F. A. Wallace, of Wallingford, Conn., Jan. I6, I884.

vi. Rosa, b. Meriden, Conn., April, I866. She d. June Io, I 866.

\section{8}

EDMUND B. CURTISS (Chauncey, Abel, Benjamin, Näthaniel, Thomas, Fohn, Elizabeth), b. Meriden, Conn., Oct. 22, I $819 ; 11$. Hannah L. Lane, Feb. I 5, I 852 . He d. Nov. 27, 1880. His widow is living in South Meriden, Conn. 
They had children:-

i. Ellen L., b. Meriden, Conn., Sept. 7, 1855; m. Philip Mills, May 16,1878 .

$(\neg S \circ)$ ii. Frederick, b. Meriden, Conn., July 23, 1858; m. Alice Stebbins, Oct. 2 I, 1887 .

iii. Enma A., b. Meriden, Conn., Dec. 6, I860; m. Harry Heaton, April 27, I880.

$\left(7 S_{7}\right)$ iv. John E., b. Meriden, Conn., April 2, 1862; m. Lizzie Seymour, Oct. 20, 1888.

v. Edwin R., b. Meriden, Conn., March 23, 1870.

ASAHEL BENHAM CURTISS (Aaron, Benjamin, Benjamin, Nathaniel, Thomas, Foln, Elizabeth), b. Meriden, Conn., Jan. Io, 1797 ; m. Emily M. Hubbard, of Durham, Conn., in 1820 . He d. July 7, I 864 . She d. Aug. $25,1886$.

They had children :-

i. Susan M., b. Meriden, Conn., $1821 ; \mathrm{m}$. Origen Utley in I 840 .

ii. Emily M., b. Meriden, Conn., March 2, 1825; m. Waldo Corbin, I 852 .

iii. Sarah H., b. Meriden, Conn., July 2, 1830 ; m. A. J. Edgerton, Oct. 8, 1850 .

$5^{8 \mathrm{I}}$

HOMER CURTISS (Benjamin, Benjamin, Benjamin, Nathaniel, Thomas, Fohn, Elizabeth), b. Meriden, Conn., Aug. 4, 1800; m. Julia Ann Upson of New Britain, Conn., Nov. 26, 1835 . He was one of Meriden's earliest manufacturers, a member of the firm of Isbell, Curtiss \& Co., and later of Curtiss, Morgan \& Co.; both firms were manufacturers of locks. He was one of the active conductors of the underground railway during slavery times. He d. in Meriden, Conn., May 30, I880.

They had children:-

i. Elizabeth Collins, b. Meriden, Conn., Sept. I3, I836; m. Sherman Cogswell, of Meriden, Conn., Oct. I 2, I 862.

ii. Augusta Louisa, b. Meriden, Conn., June 28, 184I ; m. Zeno K. Pease, of Hartford, Conn.

iii. Helen M., b. Meriden, Conn., Feb. 22, I 843 .

(790) iv. Homer Augustus, b. Meriden, Conn., June 14, 1846; m. Mary A. Hussey, of New York City, N. Y., Aug. 28, 1873.

$5^{82}$

DAN COLLINS CURTISS (Benjamin, Benjamin, Benjamin, Nathaniel, Thomas, Fohn, Elizabeth), b. Meriden, Conn., Oct. 23, I 807 ; 
m. Harriet, d. of James and Nancy Atwater, of New Haven, Conn., April 12, 1842. She d. in Brookfield, Conn., Oct. I I, I 844 ; m. (ii.) Frances Ann, d. of James and Melissa French, of Monroe, Conn., May 9, I 847 . She d. in Detroit, Mich., Aug. I 1, I 894. He d. in Fort Howard, Wis., Aug. 9, I883. He graduated from Yale College, in the Class of 1836 , and from the Yale Theological Seminary in 1839 . On June 4, 1840, he was installed pastor of the Congregational church in Greens Farms, Westport, Conn., which pulpit he held until January, 1843. In the autumn of that year he accepted a call to the church in Brookfield, Conn., where he preached for 12 years. In October, 1855 , he was installed minister of the church at Fort Atkinson, Wis., where he labored for 8 years. November, 1863 , he went to Fort Howard, Wis., where he was pastor of a church until a few months before his death. He contributed much to the papers, and many of his sermons were published.

They had children :-

i. Harriet Louisa, b. New Haven, Conn., May 3, 1843 .

ii. Julia Atwater, b. Brookfield, Conn., Oct. 3, I844; m. Daniel Davidson, in Fort Howard, WVis., Oct. I 4, 1879.

iii. Edward Collins, b. Brookfield, Conn., Jan. 30, I 849; m. Alice M., d. of Rev. Zackary and Malvinia R. Eddy, April 26, I886. She d. Aug. 25, 1889. No issue. He lives in Detroit, Mich.

iv. Mary Frances, b. Brookfield, Conn., April 25, 1851 ; d. Sept. 26, 1857 .

v. Laura Jane, b. Brookfield, Conn,, Aug. 7, 1853.

\section{$5^{8} 3$}

SAMUEL IVES CURTISS (Ivah Benjamin, Benjamin, Nathaniel, Thomas, John, Elizabeth), b. Meriden, Conn., March 5, I 803 ; m. (i.) Rebecca Tuttle Hough, of Wallingford, Conn., Oct. 3, I 832 . She was struck by lightning March 25, I 842 . He m. (ii.) Eliza, d. of Jesse Ives, of Stafford Springs, Conn., Jan. 30, 1843. She d. May 31, 1865; m. (iii.) Mrs. Deborah H. G. Curtiss, widow of his brother Erastus Curtiss and d. of Larkin Newton, of Petersham, Mass., June 15, I 866. She d. in 1881, aet. 76 years. He d. at Union, Conn., March 26, I 880 . He studied for the ministry at Bangor and New Haven Seminaries and was ordained and installed pastor of the Congregational Church at East Hampton, Conn., November, I 832 , where he continued for about five years. He then went to West Woodstock, Conn., and in April, 1843, was installed in the church at Union, Conn. 
They had children:-

i. Ann, b. East Hampton, Conn., March 21, 1834. She was killed with her mother, March 25, I842.

(79I) ii. George, b. East Hampton, Conn., Jan. 5, 1837 ; m. (i.) Elvira, d. of Healy Corbin, Jan. 1 3, 1864. She d. April I7, I875; m. (ii.) Mrs. Phebe M. Bishop, of New Haven, Conn., March 8, 1877 . She d. June 27,1877 ; m. (iii.) Martha A. Blakely, of Bristol, Conn., Sept. 15, 1880. She d. Dec. 24, 1888. He lives in Mayville, North Dakota.

(792) iii. Samuel Ives, b. Union, Conn., Feb. 5, I844; m. Mrs. Laura W., widow of Jared D. Sessions, May 10, 1870 . He resides in Chicago, Ill.

\section{$5^{84}$}

ERASTUS IVES CURTISS (Ivah, Benjamin, Benjamin, Nathaniel, Thomas, Jobn, Elizabeth), b. Meriden, Conn., May 19, I805; m. Deborah Hall Grosvenor, d. of Larkin Newton, of Petersham, Mass., May, I 833. He d. at Greenwich, Mass., March I9, I 86I. His widow $\mathrm{m}$. his brother Samuel Ives Curtiss, of Meriden, Conn., June I 5, 1866, and d. in 188 I. He graduated from Union College in 1829 and from the Yale Theological Seminary in I 832. He was ordained a minister of the Gospel at Marlboro, N. H., in 1833 . He served as pastor at the Western Reserve, Ohio, Marlboro, N. H., North New Salem, Mass., Milton and Salem, Me., and closed his ninisterial labors at New Salem, Mass., in 1860 .

They had children :-

i. Francis, b. Marlboro, N. H., March, I834; d. young.

ii. Francis Ivah, b. North New Salem, Mass., Oct. I, I833. He d. July I, 1855 .

(iii. Emma Hall, b. North New Salem, Mass., June 5, I839; m. Rev.

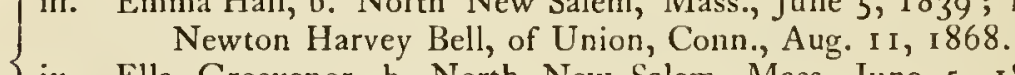

iv. Ella Grosvenor, b. North New Salem, Mass., June 5, I839; m. John Adams Spelman, of Union, Conn., Nov. 27, 1873 .

CHARLES AUGUSTUS CURTISS (Ivah, Benjamin, Benjamin, Nathaniel, Thomas, Fohn, Elizabeth), b. Meriden, Conn., Aug. I7, I807; m. Laura, d. of Eliakim and Sally Craw Paulk, of Tolland, Conn. He d. Sept. 5, I853. She m. Joseph Dresser, and d. Aug. 1 2, 1888.

They had children :-

i. Sarah Jane B., b. Meriden, Conn., Feb. 1849. She d. June 8, I 849 . 
(793) ii. George A., b. Meriden, Conn., May 4, I852; m. Frances M. Smith, Sept. 3, 1884. She d. He m. (ii.) Frances E. Slocum, September, 1888. He removed to Troy, N. Y., in 1868 , and to Lansingburgh, N. Y., in 1878 , where he now lives.

\section{6}

ENOS HALL CURTISS (Elisha, Benjamin, Benjamin, Nathaniel, Thomas, Fohn, Elizabeth), b. Meriden, Conn., Sept. 12, I796; m. Mrs. Lois K. Bailey. He d. April 30, 1862. She d. May 10, 1887 , aet. 86 years. He represented Meriden, Conn., in 1833. For many years he was a prominent manufacturer in Meriden. He was the first president of the Meriden Savings Bank.

They had children :-

i. Asahel Harrison, b. Meriden, Conn., Jan. I, 1819; m. Juliette Yale, Sept. I 2, I84I. He d. July 23, I877, without issue. He was postmaster of Meriden for many years; a representative in 1858,1875 , and 1876 . Treasurer of the Meriden Savings Bank. His widow lives in Meriden, Conn.

ii. Elisha Stevens, b. Meriden, Conn., June 18, 1826 ; m. Harriet R., d. of Silas and Abigail Wilcox, of Clinton, Conn. He d. Sept. I 9, I893. She d. Dec. 23,1883 , aet. 57 years.

They had a child :-

i. Edgar Wilcox, b. Meriden, Conn., Dec. 7, 1848 . $\mathrm{He}$ is living unm. in Meriden.

ELI ELISHA CURTISS (Elisha, Benjamin, Benjamin, Nathaniel, Thomas, Fohn, Elizabeth), b. Meriden, Conn., Feb. 27, I804; m. Louisa, d. of Chauncey and Anna Hall Curtiss, of Meriden, Conn., Feb. 27, 1825. She d. May 18, 1830 , aet. 25 years; m. (ii.) Julia A., d. of Chauncey and Anna Hall Curtiss, of Meriden, Conn., Aug. 31, 1830. She d. April 27, 1859, aet. 49 years. He d. Feb. I I, I861.

They had children :

i. Lemuel, b. Meriden, Conn., Aug. 17, 1827; m. Hannah Maria. He d. Dec. 6, I 853 . She d. Oct. 4, I853, aet. 2 I years. They had a child :-

i. Daughter, b. Meriden, Conn., Sept. 8, 1853 ; d. September, 1853 .

LEMUEL JOHNSON CURTISS (Elisha, Benjamin, Benjamin, Nathaniel, Thomas, Fohn, Elizabeth), b. Meriden, Conn., Jan. I5, 
I $814 ; \mathrm{m}$. Bedotha Pierpont, d. of Newbury and Bedotha Pierpont Burton, of North Haven, Conn., Dec. 24, 1835. He d. Jan. 10, 1888. His widow lives in Meriden, Conn. He began business as a manufacturer of britannia ware in partnership with I. C. Lewis and was one of the founders of the Meriden Britannia Co., in 1852 , in which he was a director until his death. He was also director and officer in numerous other companies. For years he was a warden of St. Andrew's Church and gave munificently to its support. In 1883 he founded an orphans' and old woman's home in Meriden, known as "The Curtiss Home," and at his death in 1888 left over $\$ 700$,000 as an endowment for this Home.

They had children : -

i. Celia Jennette, b. Meriden, Conn., Oct. 4, $183^{8}$. She d. unm. Oct. 23, 1893 .

ii. Adelaide Asenath, b. Meriden, Conn., July 30, I842; m. John E. Parker, Dec. 14, 1865. She d. March 9, 1869.

\section{$5^{89}$}

ALANSON CURTISS (Elisha, Benjamin, Benjamin, Nathaniel, Thomas, Fohn, Elizabeth), b. Meriden, Conn., Oct. 27, 1818; m. Harriet Eliza Sullivan, Feb. 1, 1840. He d. Sept. 27, 1856. His widow lives in Meriden, Conn.

They had a child : -

i. John William, b. Meriden, Conn., Feb. 17, 1843; m. Sarah L. Gridley, Dec. 18,187 ı.

They have a child :-

i. Cordie Aurelia, b. Meriden, Conn., Feb. I4, 1874 .

\section{0}

SYLVESTER JOHNSON CURTISS (Amos, Benjamin, Benjamin, Nathaniel, Thomas, Fohn, Elizabeth), b. Meriden, Conn., Sept. 30, 1826 ; m. Harriet A., d. of Thomas Hulse, of New York City, N. Y., April 23, 1851 . At the age of twenty-one years he in company with some other young men in New York chartered a ship, fitted it out and started for California by way of Cape Horn. He returned by the Isthmus of Panama, having been quite successful. He then started in business in New York City, and was for many years a broker and a member of the New York Stock Exchange. $\mathrm{He}$ has retired from active business and resides in New York City. 
They had children :-

(795) i. Thomas E., b. New York City, N. Y., Feb. 13, I852; m. Ada H. Hulse, New York City, N. Y., June 8, I88r. She d. Feb. I8, 1896.

ii. Harriet Louise, b. New York City, N. Y., Aug. 13, 1855. She is unm.

591

ALFRED PIERPONT CURTISS (Samuel, Benjamin, Benjamin, Nathaniel, Thomas, Fohn, Elizabeth), b. Wallingford, Conn., July 4, I8 17 ; m. Emeline Amelia, d. of Levi and Abigail Ann Bradley, of Meriden, Conn., Oct. 19, I838. She d. July 19, 1847, aet. 27 years; m. (ii.) Sarah Maria, d. of Augustus and Lois Atwater Hitchcock, Oct. I 5, I 85 I. She d. Dec. 3, I 857 , aet. 24 years; m. (iii.) Julia Hannah, d. of Asaph and Sophia Pearl Phelps, of Ashtabula, Ohio, Jan. I4, I868. He d. July 21, 1870 .

They had children :-

i. Jane Maria, b. Meriden, Conn., Jan. 4, I842; m. Geo. A. Fay, of Meriden, Conn., Sept. II, I865.

(796) ii. Levi Bradley, b. Meriden, Conn., Aug. 10, 1843 ; m. Alice Maria, dau. of Henry Francis and Abby Bicknell Cushing, Dec. 23, 1868.

(797) iii. Alfred William, b. Meriden, Conn., June 2, 1847; m. Clara Augusta, d. of IVillian and Augusta Savage Horner, Feb. I7, 1870 .

iv. Ellen Eliza, b. Meriden, Conn., June 9, 1869; m. Charles Edward Homan, Dec. 3I, I89I.

592

ASAHEL CURTISS (Asabel, Benjamin, Benjamin, Nathaniel, Thomas, Fohn, Elizabeth), b. Meriden, Conn., Feb. 25, 1821 ; m. Ann E., d. of Silas and Abigail Wilcox, of Clinton, Conn., Feb. 29, I 844. He d. Dec. 2, 1889. His widow lives in Bradford, N. Y. He was a merchant and lived in Meriden, and the latter part of his life devoted himself to farming.

They had children :-

(798) i. Robert Hallam, b. Meriden, Conn., Dec. 4, 1845; m. Laura Sophia Hotchkiss, of Gouverneur, N. Y. She d. March 5, 1891 .

ii. Anna Wilcox, b. Meriden, Conn., March 7, s848; m. Jesse Munson, Jr., of Bradford, N. Y., April 20, 1870.

iii. Hetty Redfield, b. Meriden, Conn., April 24, 1853 . She d. June 22, I86r.

iv. Alice Antoinette, b. Meriden, Conn., July 28, 1856. She d. Sept. I5, 1862 . 
GEORGE REDFIELD CURTISS (Asahel, Benjamin, Benjamin, Nathaniel, Thomas, Fohn, Elizabeth), b. Meriden, Conn., Dec. 25, $1825 ; \mathrm{m}$. Augusta, d. of Jesse and Sophia Talmadge Munson, of Bradford, Steuben Co., N. Y., May 22, I 855 . He d. May 20, 1893. He started in business in Middletown, Conn., as a clerk in a dry goods store. After a few years he began to teach school near Rochester, N. Y., and a year or two later in Meriden, Conn. A bout 1850 he became teller in the Meriden Bank, and in 1852 when the Meriden Britannia Co. was organized he accepted the position of treasurer of that company, which position he retained until his death. He was also president of the Meriden Silver Plate Co., Meriden Gas Light Co., Meriden Electric R. R. Co., and a director in numerous other corporations. He was mayor of Meriden 1879-1881, and filled many other positions of trust and influence. He was always deeply interested in St. Andrew's Church, and was its junior and senior warden for many years, and many times its delegate to Diocesan Conventions, and in 1892 he was a State delegate to the general convention of the Protestant Episcopal Church held in Baltimore, Md. He gave largely to the Memorial Church in Meriden, which his sister, Mrs. R. A. Hallam, has caused to be built, and the day before his death he presented the new church with a rectory.

They had children :-

(799) i. George Munson, b. Meriden, Conn., May 27, 1857; m. Sophia Phillips, d. of Thomas Trowbridge and Catherine Hurlburt Mansfield, of New Haven, Conn., Nov. 30, 1886.

ii. Frederick Edgar, b. Meriden, Conn., Aug. 12, 1861. He d. Sept. 10, 1869 .

iii. Agnes Deshon, b. Meriden, Conn., April ro, 1863; m. Allan B. Squire, May 22, 1890.

\section{6}

LEVI CURTISS (Levi, Solomon, Samuel, Samuel, Thomas, Fohn, Elizabeth), b. Southington, Conn., Feb. 19, 1800; m. Julia Brooks, of Cheshire. He was a farmer and lived most of his life in Southington, Conn., although later he removed to Plainville.

They had children :-

i. Eliza, b. Southington, Conn., April r6, 1837 ; m. Silah Lewis, Jan. I, 1863. She d. Oct. 7, 1873 .

$(800)$ ii. George Brooks, b. Southington, Conn., Aug. 2, 1842 ; m. (i.) Frances M., d. of Edwin and Maria Bristol Barnes. She d. Nov. 4, 1866 ; m. (ii.) Laura M. Clarke. 
SOLOMON CURTISS (Levi, Solomon, Samuel, Samuel, Thomas, Fohn, Elizabeth), b. Southington, Conn., Sept. 3, 180 I ; m. Sally Leavenworth, d. of Joseph and Anna Bronson Cook, of Plymouth, Conn., Jan. I, 1827 .

They had children :-

i. Sarah Emily, b. Southington, Conn., Feb. I 5, I 828 ; m. Frank W. Bristol.

$(8 O I)$ ii. Charles W., b. Southington, Conn., June 25, I83I; m. Mary Decker, . He graduated from Yale in 1852.

iii. Anna E., b. Southington, Conn., April I I, I838; m. Josiah H. Merriman.

STEPHEN FOWLER CURTISS (Azor, Urie, Azor, Samuel, Thomas, Fohn, Elizabeth), b. Le Roy, N. Y., Sept. 6, 1824; m. Sally Stevens, of Bergen, N. Y., April 23, I846. They reside in Le Roy, N. Y.

They had children :-

i. Marian Eleanor, b. Le Roy, N. Y., March 12, 1847. She d. Dec. 12,1858 .

ii. L. Josephine, b. Le Roy, N. Y., Nov. 8, 1849. She is unm. and lives in Le Roy, N. Y.

iii. Elsie Catherine, b. Bergen, N. Y., May 22, I 854 ; m. Alva Lewis, of Batavia, N. Y., June 28, I88 I. She d. March 23, I 889.

iv. Earl Stevens, b. Bergen, N. Y., Oct. 7, I 856 . He d. in Le Roy, N. Y., April 17, I871.

v. Le Roy Gaines, b. Bergen, N. Y., June 19, 1861. He d. Aug. 9, I86I.

600

SOLOMON CURTISS (Azor, Urie, Azor, Samuel, Thomas, Fohn, Elizabeth), b. Le Roy, N. Y., ; m. Mary Pugeley, of Rochester, N. Y. He d. in Le Roy, N. Y., $\quad$ I 894.

They had children :-

i. Charles, b. Le Roy, N. Y.

ii. Marion, b. Le Roy, N. Y.

iii. Adaline, b. Le Roy, N. Y., of Le Roy, N. Y. She resides in Le Roy, N. Y.

602

GEORGE COWLES CURTISS (Charles, Peter, Peter, Foseph, Thomas, Fohn, Elizabeth), b. Madison, Ohio, Feb. 21, 1827; $\mathrm{m}$. Mary E. Pancost. He is a merchant and has resided in Painesville, Ohio, since I 852 . 
They had children :-
i. Frank C., b.
ii. Charles F., b.
iii. George WV., b.
iv. John H., b.

\section{6}

THOMAS H. CURTISS (Caleb, Amasa, Fonathan, Foseph, Thomas, Fohn, Elizabeth), b. Monterey, Mass., ; m. Martha Hyde, of Monterey, Mass.

They had children :-

(Soz) i. John, b. Monterey, Mass., in New York.

ii. Clara, b. Monterey, Mass.,

; m. Sarah Bosworth and lives ; m. Frank Joyner.

\section{8}

IRA CURTISS (Abijah, Fonathan, Fonathan, Foseph, Thomas, Fobn, Elizabeth), b. Sheffield, Mass., March 15, 1794 ; m. Artemesia, d. of Salmon and Salome Jeffers Willoughby, of Cornwall, Conn., in Lenox, Mass., Jan. 19, 1820. He was a farmer, merchant, and distiller and lived the greater part of his life on the "Old Tuttle Farm " under the mountain in Sheffield, Mass. He represented the district in the General Court and served as State Senator. He always took a leading part in the town affairs. He died March 20, i 870 , and his wife, Jan. 4, I 879 , aet. So years.

They had children :-

(803) i. Albert Willoughby, b. Sheffield, Mass., Nov. 10, 1820; m. Susan, d. of John Campbell and Grace Knott Whitehead, of Stockbridge, Mass., Dec. I 5, I 85 I. He d. Nov. 21, I 893 .

ii. Eliza M., b. Sheffield, Mass., Jan. 3I, I 822 ; m. Dr. Joel Bacon Merriman, of Sheffield, Mass., Jan. I, I 843. She d. April I, I 894 .

(Sof) iii. George Graham, b. Sheffield, Mass., Sept. I, I 825 ; m. Mary, d. of Joseph H. and Hannah Lewis, of Fallston, Md., June, I 86I.

iv. Frederick Alonzo, b. Sheffield, Mass., Oct. 2I, I828. He is unm. and lives in Bradford, Pa., and is a producer of petroleum.

v. Infant son, b. Sheffield, Mass., Dec. I 3, I 832 ; d. young.

vi. Artemesia, b. Sheffield, Mass., Dec. 2, I833; unm.

vii. Mary Ellen, b. Sheffield, Mass., Nov. 26, I $840 ; \mathrm{m}$. Rev. Charles E. Becker, of Philadelphia, Pa., June, I 870.

viii. Infant son, b. Sheffield, Mass., Nov. 26, I 842 ; d. young.

609

ORRE.N CURTISS (Abijah, Fonathan, Fonathan, Foseph, Thomas, Gohn, Elizabeth), b. Sheffield, Mass., May 17, 1797 ; m. Caroline, 
d. of Col. Ira and Sophia Standish Owen in Sheffield, Mass., March 24, I825. She d. May 14, 1870; m. (ii.) Maria Hagerman. He was a farmer, and lived at the old homestead which belonged to his grandfather, and which is now owned by his son, Frank Curtiss. In 1840 he represented his town in the State Legislature. He d. in Sheffield, Mass., Dec. 20, I 883.

They had children:-

i. Sophia, b. Sheffield, Mass., Nov. 24, 1825 ; m. George Hoffman, of Claverack, N. Y., May 15, 1845. She resides in New York City, N. Y.

ii. Emma, b. Sheffield, Mass., April 20, 1828; m. John Bascom, of Genoa, N. Y., Jan. 8, I 856. She resides at Williamstown, Mass.

(805) iii. Abijah, b. Sheffield, Mass., March 1 2, 1831 ; m. Mary Elizabeth, d. of Rev. Seldon and Catherine Butler Haines, at Skaneateles, N. Y., June 5, 1855 . He d. Aug. 2, 1888.

iv. Orren, b. Sheffield, Mass., Aug. 25, I 833 ; m. Elizabeth Joyner. He d. Sheffield, Mass., Dec. I 3, 1876. She d. March 25, I860, aged 20 years.

They had a child :-

i. Lizzie, b. Sheffield, Mass., March 24, 1860; d. Sheffield, Mass., Dec. 4, 1860.

(So6) v. Franklin, b. Sheffield, Mass., Dec. 15, 1838; m. Susan, d. of Simon Sage, of Seekonk, Mass., Jan. I 5, 1862; m. (ii.) Ella, d. of Abner and Jane Spurr Roys, of Sheffield, Mass., June 5, 1878. He resides in New York City, N. Y.

\section{0}

JULIUS CURTISS (Abijah, Fonathan, Fonathan, Foseph, Thomas, Fohn, Elizabeth), b. Sheffield, Mass., March 18, I799; m. Lucinda Tuttle, of Salisbury, Conn. She d. November, I 892 . He d. in Utica, N. Y., April 12, I 845 .

They had children :-

i. Jane A., b. Egremont, Mass., October, 1834. She d. unm. June I 2,1855 .

ii. Julia, b. Egremont, Mass.

iii. Alden J., b. Egremont, Mass., June, 1836 . He d. unm. in Utica, N. Y., 1895 .

\section{1}

CHARLES ABBEY CURTISS (Abijah, Fonathan, Fonathan, Foseph, Thomas, Fohn, Elizabeth), b. Sheffield, Mass., March I 4, I 805 ; m. Sabrina, d. of Timothy Robinson, of Sheffield, Mass., June 30, 1825 . He removed to Freedom, Ohio, in 1835 , and d. there Nov. 30, I880. His wife d. Oct. 2, I 877 . He was a farmer. 
They had children :-

i. Lorinda, b. Sheffield, Mass., May 28, 1826; m. Darius Freeman, of Freedom, Ohio, June I, 1846.

ii. Susan, b. Sheffield, Mass., Sept. 6, 1828 ; m. William B. Harmon, of Freedom, Ohio, Jan. 19, 1860. She d. Jan. 20, 1894.

(So7) iii. Charles Henry, b. Sheffield, Mass., Oct. 23, I833; m. (i.) Jannette, d. of Charles Wilson, of Shalerville, April 15, 1855; m. (ii.) Martha, d. of Dexter and Hannah Abbey, of Chardon, Ohio, April I6, I865.

$(S \circ S)$ iv. Lewis Emerson, b. Freedom, Ohio, Oct. 17, 1838; m. Harriet Monervia, d. of George and Harriet Williams, of Joliet, Ill., Dec. I 9, I87 .

v. Albert, b. Freedom, Ohio, July 26, 184 I. He was a soldier in the Union army and was killed Dec. I 5, 1862, at Cairo, Tenn.

vi. Emma Elizabeth, b. Freedom, Ohio, Jan. 13, 1847. She d. April $5, \mathrm{I} 85 \mathrm{I}$.

\section{I 2}

JAMES J. CURTISS (Fosiah, Fonathan, Fonathan, Foseph, Thomas, Fohn, Elizabeth), b. South Egremont, Mass., March 20, I 803 ; m. Nancy, d. of Michael and Deborah Lacey Steele, of Westmoreland, N. Y., Sept. 20, I 828 . He d. in Albany, N. Y., April I 7, 1840.

They had children :-

(809) i. Jason Parker, b. Westmoreland, N. Y., Feb. 4, I830; m.

He d. in Pioche, Nev., May 5, I879.

ii. Josiah A., b. Westmoreland, N. Y., Dec. 2 I, 1834 ; m. Juliette Bradley, d. of Dr. Alonzo and Janette Phelps. She d. in Kirkland, N. Y., June 1 5, 1894. He lived in Pioche, Nev., for a number of years, and was county assessor of Lincoln County.

They had a child : -

i. Alonzo Parker, b. Westmoreland, N. Y., Jan. 2, 1862.

He was ordained a priest in the Episcopal Church at St. Paul's Cathedral, Fond-du-Lac, Wis., Nov. 20, 1893, and is now in charge of the Church of St. Agnes by the Lake, in Ohnapee, Wis.

iii. Francis E., b. Westmoreland, N. Y., Dec. 21, 1834.

iv. Sarah G., b. Westmoreland, N. Y., Sept. 28, 1843 .

\section{I 3}

TRUMAN CURTISS (Fosiah, Fonathan, Fonathan, Foseph, Thomas, Fohn, Elizabeth), b. South Egremont, Mass., Jan. 28, i 808 ; m. Charlotte, d. of Lemuel and Lucinda Lombard, of Greenfield, Mass., Sept. 23, I 840 . He was a farmer and lived in Westmoreland, N. Y., where he d. March 28, i 852. 
They had children :-

i. Philo Upson, b. South Egremont, Mass., Dec. 30, 1842.

ii. Fanny, b. Westmoreland, N. Y., Oct. 12, 1850 ; m. James Bridges, of Warren, Mass. She d. Feb. 20, I889.

\section{4}

JONATHAN G. CURTISS (Fosiah, Fonathan, Fonathan, Foseph, Thomas, Fohn, Elizabeth), b. Sheffield, Mass., Oct. 22, I 8 I 7 ; 11. Charlotte Sophia, d. of Abel and Electa Hull, of Egremont, Mass., March 4, 1847 . In 1847 he removed to Jersey City, N. J., when he began to practise dentistry and became a most successful dentist. He d. in Jersey City, N. J., Feb. 3, I 869.

They had children :-

i. Jessie Louise, b. Jersey City, N. J., Dec. 5, I847; m. Edward P. de Mott, of Jersey City, N. J., Oct. 4, I876; m. (ii.) Henry C. Littlefield, of Great Barrington, Mass., Jan. 28, I89 I.

ii. Fred Hull, b. Jersey City, N. J., Nov. 21, I853. He d. at Great Barrington, Mass., Dec. 5, I 892 . He was a poet and writer, and contributed to the principal magazines and weeklies.

\section{0}

JEREMIAH CURTISS (Fesse, Fesse, Abner, Foseph, Thomas, Fohn, Elizabeth), b. Meriden, Conn., Jan. 25, I 8 I2; m. Mary Ann Stephens.

They had children :-

$(S r o)$ i. Winfield Scott, b. Meriden, Conn., July I4, 1848; m. Elizabeth Grumley, Oct. 27, I874.

\section{3}

PHILO CURTISS (Foshua, Simeon, Foshua, Foseph, Thomas, Fohn, Elizabeth), b. Burlington, Conn., July I4, 1808 ; m. Charlotte Marietta, d. of Aaron and Comfort Curtiss, of Burlington, Conn., Sept. 3, I 829. He d. in Bristol, Conn., June 10, I 875. She d. in Waterbury, Conn., Oct. 27, 1883 .

They had children :-

$\left(\delta_{I I}\right)$ i. Lucius Wellington, b. Bristol, Conn., Oct. 5, I $83^{\circ} ; \mathrm{m}$. Harriett A. Barker, of South Meriden, Conn., May 15, I853. She d. Dec. I I, 1854; m. (ii.) Olive Webb Hotchkiss, of Meriden, Conn., Dec. I 5, 1857. He d. April 27, 1885.

(812) ii. Jonas Platt, b. Bristol, Conn., May 29, 1832 ; m. Laura Ann Stevens, of Killingsworth, Conn., Jan. 15, 1852. 
(SI 3) iii. George Henry, b. Bristol, Conn., Sept. 2, I 834 ; m. Frances R. Casterline, of Derby, Conn., Sept. 29, 1856 ; m. (ii.) Mary Frister, of Galena, Ill., June 4, I 868.

$\left(\mathcal{S}_{1 . f}\right)$ iv. Edwin Mills, b. Bristol, Conn., June I, I 837 ; m. Emeline Sheldon, of Bristol, Conn., Jan. 2, 1860.

v. Ellen Louise, b. Bristol, Conn., Sept. 8, I839; m. Gilbert Wetmore, of Middletown, Conn., July 3, I 865 .

vi. Laura Maria, b. Bristol, Conn., Jan. 6, I842; m. Orlando Sheldon, of Derby, Conn., April 5, I870.

vii. Andrew Jackson, b. Bristol, Conn., Oct. 26, I844; m. Sarah Elizabeth Ayres, of Troy, Bradford County, Pa., Jan. I, 1873.

They a child :-

i. Miriam Blanche, b. Bristol, Conn., Oct. 25, I 873.

viii. Ann Amelia, b. Bristol, Conn., Dec. 25, I 846 . She d. Jan. 10, 1848 .

ix. Ann Eliza, b. Bristol, Conn., July 4, 185 I ; m. Lucius Sidney Beldon, of Bristol, Conn., Sept. 27, 1868.

x. Emma Jane, b. Bristol, Conn., Sept. I4, I853; m. George Newton Downs, of Bristol, Conn., May I $4,1872$.

\section{4}

HENRY CURTISS (Foshua, Simeon, Foshua, Foseph, Thomas, Fohn, Elizabeth), b. Burlington, Conn., Aug. I3, I810; m. Elizabeth Dowd, Jan. 2, 1833. She d. Jan. 3, I884, aet. 79 years. He d. Dec. 30,1883 . He was a farmer and lived in Burlington, Conn.

They had children :-

$(815)$ i. Royal, b. Burlington, Conn., Dec. 21, 1833; m. Almira Fulmer, Aug. 31, I865. He died Aug. I, I 880 .

ii. Son, b. Burlington, Conn., June Io, I 835 ; d. I 835 .

$(S I 6)$ iii. Austin, b. Burlington, Conn., Aug. 7, I836; m. Lucy J. Steele, Sept. 18,186 I.

iv. Evaline, b. Burlington, Conn., Sept. $30,183^{8}$; m. J. T. Root. She d. April 8, I $88 \mathrm{I}$.

$\left(8_{I 7}\right)$ v. Cornelius, b. Burlington, Comn., July 6, I844; m. Effie J. Healey, Dec. 3I, 1870 .

\section{5}

SIMEON CURTISS (Foshua, Simeon, Foshua, Foseph, Thomas, Fohn, Elizabeth), b. Burlington, Conn., i 8 I 6 ; m. Maria Hoskins, of Burlington, Conn. He d. April 3, I882. She d. in Bristol, Conn., Jan. 30, 1878 .

They had children :-

i. Adeline F., b. Burlington, Conn., I845. She d. Sept. $26,1866$. 
ii. Esther Alvira, b. Burlington, Conn., Feb. I 5 , I 848 ; m. Julius B. Smith, of Burlington, Conn., Nov. 21, 1865. She d. in Burlington, Conn., July 15,1877 .

\section{6}

SYLVESTER CURTISS (Foshua, Simeon, Foshua, Foseph, Thomas, Fohn, Elizabeth), b. Burlington, Conn., March 5, 1819; m. Eveline, d. of Jared and Nancy Houghton, of Burlington, Comn., Aug. 25, 1840. She d. June 15, i 888 , aet. 59 years; m. (ii.) a Brainard. He d. March 7, 1889, in Burlington, Conn., where he had always lived.

They had children :-

i. Josephine, b. Burl ngton, Conn., Nov. I3, $1842 ; \mathrm{m}$. Willis A. Hamlin, of Plainville, Conn., Nov. 14, I 863.

ii. Eugenia, b. Burlington, Conn., Oct. 20, I844; m. Washburn G. Mills, of Canton, Conn.

(SIS) iii. Waldo Loren, b. Burlington, Conn., Dec. I, I846; m. Mary, d. of Samuel Camp, of Plainville, Conn. She d. Feb. 5, 1875 , aet. 27 years. He m. (ii.) Nettie Root, of Berlin, Conn., Dec. 25, 1878 .

iv. Truman, b. Burlington, Conn., May I I, I849. He d. March 24, 1849 .

v. Grovenor William, b. Burlington, Conn., Feb. 22, $185 \mathrm{I}$; m. Ella C., d. of Linus and Vincy Ann Birdsey, of Meriden, Conn., Feb. 24,1875 . He is a merchant and resides in Hartford, Conn. No issue.

\section{7}

COYDEN CURTISS (Foshua, Simeon, Foshua, Foseph, Thomas, Foln, Elizabeth), b. Burlington, Conn., March 30, I823; m. Mary Ann Dayton, of Hansden, Conn., Sept. 3, 1843. He d. Feb. 16, 1885 .

They had children :-

i. Lanette, b. Bristol, Conn., Sept. I 8, I 860 . She d. Feb. 24, I 864.

ii. Carrie M., b. Bristol, Conn., May 6, 1865 ; m. Henry A. Calkins, in Plainville, Conn., Aug. 10, 1885.

$$
630
$$

SAMUEL CURTISS (Major, Simeon, Foshua, Foseph, Thomas, Fohn, Elizabeth), b. Burlington, Conn., March 20, I841; m. Hannah Maria Meserah, Oct. 6, 1864. He left Burlington when young and became a resident of Meriden, where he now lives. 
They had children :-

$\left(\mathcal{S}_{19}\right)$ i. Burton Samuel, b. Meriden, Conn., May 10, 1867 ; m. Anna Eliza Howell, May 4, I 895 .

$(\$ 20)$ ii. Clinton James, b. Meriden, Conn., Oct. 24, I87 I ; m. Blanche Eliza Kelly, Oct. 3I, I 895 .

\section{3}

JAMES CURTISS (Fames, Giles, Fohn, Fohn, Thomas, Fohn, Elizabeth), b. Sheffield, Mass.; m. Helen Upton.

They had children :-

i. Charles, b. Sheffield, Mass., Aug. 2, I860.

ii. Hattie, b. Sheffield, Mass., Oct. ro, I 865.

iii. Emma, b. Sheffield, Mass., Aug. 5, I 867.

iv. Irving G., b. Sheffield, Mass., July 28, 1869.

v. Daisy, b. Sheffield, Mass., Oct. I I, 1872.

vi. Ada May, b. Sheffield, Mass., Aug. 28, 1877 .

$$
636
$$

SAMUEL C. CURTISS (Levi, Ephraim, Stiles, Ephraim, Foseph, John, Elizabeth), b. Huntington, Conn., $\quad$ I789; m. Melissa $\quad$ She d. Nov. I, I 867 , aet. 78 years. He d. in Huntington, Conn., May 8, I 867.

They had children :-

i. Harriet, b.

ii. Maria, b.

Oct. 22, I 850 .

; m. Abram C. Lewis, of Stratford, Conn.,

iii. Levi, b. Huntington, Conn., I819. He d. Sept. 6, 1820.

iv. David W., b. 1826. He d. June 22, I867.

639

STILES CURTISS (William, Silas, Stiles, Ephraim, Foseph, Fohn, Elizabetb), b. Stratford, Conn., Feb. I 7, i 805 ; m. Hannah, d. of Seymour Conway and Hannah Curtiss Whiting, of Stratford, Conn., Oct. 3, I830. She d. in Norwalk, Conn., Feb. 8, I8 38 , age 35 years; m. (ii.) Jane Isabella, d. of Nathan Whiting, of Bridgeport, Conn., Sept. 23, I 838 . She d. in Norwalk, Conn., March 3, I866; m. (iii.) Jane Hanson, d. of Rodney and Cornelia Barton, of New Haven, Conn., April 16, I867. She d. in Norwalk, Conn., April I 5, I 868 ; m. (iv.) Martha Abbott Hull, of New Haven, Conn., May I9, I 869. He has been warden of borough for many years. He was vice president of the Fairfield 
County Bank of Norwalk, president of the Bank of Norwalk, and president of the Union Mfg. Company, of Norwalk, Conn. He d. in Norwalk, Conn., Nov. I I, I 882 .

They had children :-

(821) i. Stiles Whiting, b. Norwalk, Conn., Sept. I4, I831; m. Harriet, d. of Eli Barnum and Anah Eversley, of Norwalk, Conn., Sept. 5, 1853 .

ii. Helen Birdseye, b. Norwalk, Conn., of Norwalk, Conn., Oct. 27, 1852.

(822) iii. William Conway, b. Norwalk, Conn., ; m. Fanny M. Norton, of Eastport, Me., Aug. 26, 1868.

(823) iv. David Seymour, b. Norwalk, Conn., ; m. Cornelia I. Raymond, of Norwalk, Conn., Dec. 18, 1860.

v. Catherine Sophia, b. Norwalk, Conn., leck, of Troy, N. Y.

vi. Henry Elbert, b. Norwalk, Conn., ; unm.

vii. Mary Jane, b. Norwalk, Conn., ; m. Frederick Stanton Perrin, of New York City, N. Y.

\section{0}

LEWIS JUDSON CURTISS (William, Silas, Stiles, Ephraim, Foseph, Fohn, Elizabeth), b. Stratford, Conn.; m. Fannie, d. of Noah and Anna Belden Olmstead, of Norwalk, Conn., Aug. 19, 1843 .

They had children :-

i. Charles Lewis, b. Norwalk, Conn., March 19, I847. He d. March $23,1862$.

ii. Maria Louise, b. Norwalk, Conn., May 18, 1850. She d. July 17,1864 .

iii. Anna Belden, b. Norwalk, Conn., March 27, 1853 ; m. Charles Eversley Curtiss, of Norwalk, Conn., Oct. 20, I885. She d. Aug. 29, I 890.

$(824)$ iv. Franklin Judson, b. Norwalk, Conn.; m. Julia Boquet, of New York City, N. Y.

THADDEUS BIRDSEY CURTISS (William, Silas, Stiles, Ephraim, Foseph, Fohn, Elizabeth), b. Stratford, Conn., July I2, I 808 ; m. Jane, d. of Noah and Anna Belden Olmstead, of Norwalk, Conn., Nov. 19, 1846. He was a farmer and lived in Stratford, Conn., where he d. May 5, I884. She d. Feb. I, i 87 I, aet. 59 years. 
They had children :-

i. Myra, b. Stratford, Conn., Sept. 5, 1847.

ii. Emma, b. Stratford, Conn., Feb. 18, 1851. She d. Sept. 27, 1876.

iii. Louise Wakely, b. Stratford, Conn., Feb. 16, 1857.

\section{2}

ELBERT CURTISS (William, Silas, Stiles, Ephraim, Foseph, Fohn, Elizabeth), b. Stratford, Conn.; m. (i.) Mary Frances, d. of Anson and Polly Quintow, of Norwalk, Conn. She d. Dec. 26, I 855 ; m. (ii.) Harriet Ann, d. of Samuel and Matilda Curtiss, of Huntington, Comn., Oct. 7, 1857. She d. May 20, 1868. He is a tailor and resides in Norwalk, Conn.

They had children : -

i. Rebecca Judson, b. Norwalk, Conn., Sept. 7, 1847 .

$(\$ 25)$ ii. William Anson, b. Norwalk, Conn., Dec. 7, 1849.

iii. Francis Elbert, b. Norwalk, Conn., Nov. 9, 1855 . He d. Aug. 9, 1856.

$\left.\begin{array}{l}\text { jv. Harriet Ann, } \\ \text { v. Minnie, }\end{array}\right\}$ b. Norwalk, Conn., Feb. 27, 186 I.

\section{5}

ELI CURTISS (Eli, Silas, Stiles, Ephraim, Foseph, Fohn, Elizabeth), b. Stratford, Conn., May 6, I8I 9 ; m. Sarah E. Booth, July 12, 1853. He settled in Port Chester, N. Y.

They had children :-

j. Charles Henry, b. Sept. 2, I854; d. Oct. I4, 1858.

ii. Caroline Booth, b. Jan. 28, I8 56 ; m. Charles A. Stridwell, Oct. I6, 1878 .

iij. Mary Ada, b. Oct. 21, 1857 ; d. Jan. I6, 1873 .

iv. Howard, b. Nov. I 4, I 858 ; d. April I I, I 86 I.

v. Francis Ella, b. Jan. 25, 1861 ; d. Dec. 21 , 1865.

vi. Laura, b. Dec. I9, 1868.

\section{$65 \mathrm{I}$}

JOHN CURTISS (Stiles, Fohn, Stiles, Ephraim, Foseph, Fohn, Elizabeth), b. Woodbury, Conn., July I 3, I797 ; m. Maria Gilbert, of Woodbury, Conn., Oct. I 8, I 8 I 8 . She d. 1879 , aet. 82 years. They lived in Woodbury, Conn.

They had children :-

i. Sarah H., b. Woodbury, Conn., ; m. Benjamin Russell, of IVoodbury, Conn.

ii. Harriet M., b. Woodbury, Conn., 1829; m. James H. Lindsley, of Woodbury, Conn., Oct. 9, 1849 . She d. June 22, 1879. 
CHARLES CURTISS (Stiles, Fohn, Stiles, Ephraim, Foseph, Fohn, Elizabeth), b. Woodbury, Conn., May 23, 1812; m. Emily A. Lambert, of Woodbury, Conn., March I4, I838. She d. Oct. 20, I 841 ; m. (ii.) Mrs. Mary Gleeson Goodwin, d. of Moses and Polly (Richardson) Gleeson, of Saratoga County, N. Y., Aug. 3, 1 84 2. She d. May 6, 1896 , aet. 83 years. He d. in Cleveland, Ohio, Dec. 27, 1872. He removed to Ohio in 1839 and took up his residence there in Summit County, where he became a man of prominence. In 1860 he removed to Cleveland, where he engaged in business. He was a director in the Euclid National Bank until his death.

They had children : -

i. Charles Edmund, b. Summit County, Ohio, June 9, I844. He enlisted at the outbreak of the war of the Rebellion and was severely wounded at the Battle of Ivy Mountain and was disabled for several years. He removed to Arizona and engaged in business there. He was adjutant-general of that territory at the time of his death. He d. unm. Jan. 22, I879.

(826) ii. Stiles Henry, b. Summit County, Ohio, May 27, I 846 ; m. Lucia Marcia, d. of Edwin and Marcia Lucia (Cobb) Stair, Sept. 30, 1875. They reside in Cleveland, Ohio.

iii. Anna Maria, b. Summit County, Ohio, Aug. 28, i 849 ; n. Thomas H. Brooks, of Cleveland, Ohio, Sept. 9, 1873 .

\section{6}

THOMAS EMMONS CURTISS (Ezekiel, Fohn, Stiles, Ephraim, Foseph, Fohn, Elizabeth), b. Oneida County, N. Y.; m. Mary Southworth. He resides in Buffalo, N. Y.

They had children :-

i. Nettie, b. ; m. Frederick Post, of Buffalo, N. Y.

ii. Clarence, $b$.

$(\delta 27)$ iii. Frederick Ernest, b.

iv. Georgia, b.

v. Charlotte, b.

LYMAN LORD CURTISS (Ezekiel, Fohn, Stiles, Ephraim, Foseph, John, Elizabeth), b. Oneida County, N. Y.; m. Ellen

They had children :-

$(\mathcal{2} 2)$ i. Edwin, b. $\quad$; m. Eliza Dorchester, of Buffalo, N. Y.

(\$29) ii. Charles Main, b. ; m. Rosa Puiner.

They had a child : -

i. Henry Puiner, b.

(830) iii. Walter Lord, b. ; m. Grace Griffiths, of Buffalo, N. Y. 
CHARLES GOULD CURTISS (Ezekiel, Fohn, Stiles, Ephraim, Foseph, Fohn, Elizabeth), b. Oneida Co., N. Y.; m. Amelia Lent Main, of Binghamton, N. Y., April, 1852. She d. Nov. 30, 188I. He was a grain merchant and lived in Buffalo, N. Y. In 1870 he was elected president of the Buffalo Board of Trade. He was a trustee of the New York State Hospital for Insane, a director of the Third National Bank of Buffalo, trustee of the Buffalo Savings Bank. In 1884 he was the Democratic presidential elector of the $32 \mathrm{~d}$ District, New York State. He d. in Buffalo, N. Y., Sept. 17, I 893 .

They had children:-

i. Charlotte Electa, b. Buffalo, N. Y., Dec. 30, 1853. She d. Jan. $16,1866$.

$\left(\mathcal{S}_{31}\right)$ ii. Alexander Main, b. Buffalo, N. Y., Oct. 6, I856; m. Sophia Jane Colman, of Dunkirk, N. Y., June 9, 1880 . He resides in Buffalo, N. Y.

iii. Harlow Clarke, b. Buffalo, N. Y., Nov. 6, 1858; m. Ethel Mann, of Buffalo, N. Y., June I8, I896. He graduated from Trinity College, Hartford, Conn., in 1881, and received the degree of M. A. from that college in 1888 . He was admitted to the bar of New York State in 1883. He is a director of the Niagara Falls and Suspension Bridge Railway Co., Professor of Medical Jurisprudence in Niagara University, 1893-1896. In October, $\mathbf{1 8 9 5}$, he was a deputy from diocese of Western New York to General Convention of the Protestant Episcopal Church at Minneapolis, Minn. He resides in Buffalo, N. Y., where he practises law.

iv. Mary, b. Buffalo, N. Y., Oct. 13, 1861; m. Charles McClellan Clarke, of Buffalo, N. Y., June 2, I892. She d. Sept. I4, 1893.

SHELDON PIXLEE CURTISS (Daniel, Fohn, Stiles, Ephraim, Foseph, Fohn, Elizabeth), b. Stratford, Conn., May 26, 1812 ; m. Sarah, d. of Joel and Tryphena Gorham McEwen, of Stratford, Conn., Sept. 23, 1835. He was a farmer and lived in Trumbull, Conn., where he d. Jan. 9, I 875 .

They had children :-

$(832)$ i. Robert W., b. Stratford, Conn.

$\left(\$_{33}\right)$ ii. Alfred H., b. Stratford, Conn.

$(834)$ iii. Charles B., b. Stratford, Conn.

$(835)$ iv. Frederick, b. Stratford, Conn. 
PETER PIXLEE CURTISS (Daniel, Fohn, Stiles, Ephraim, Foseph, Fohn, Elizabeth), b. Stratford, Conn., Oct. 26, I820; m. Mary Esther Fitch, of Stratford, Conn., Nov. 30, i 843. He d. in Stratford, Conn., June I6, I882. She d. in 1890.

They had children : -

i. Sarah Fitch, b. Stratford, Conn., June 20, 1850; m. Wilmot C. Wheeler, of Trumbull, Conn., March 31,1873 .

ii. Elizabeth F., b. Stratford, Conn., Nov. 23, i 855 .

iii. Mary Pixlee, b. Stratford, Conn., Aug. 10,1858 ; m. Geo. H. Booth, of Trumbull, Conn., April 24, 1872.

iv. Esther Louisa, b. Stratford, Conn., April 25, 1865.

v. Martha B., b. Stratford, Conn., ; m. Charles H. Weeks.

\section{6}

FRANKLIN CURTISS (Morgan, Stiles, Elnathan, Ephraim, Foseph, Fohn, Elizabeth), b. Huntington, Conn., Dec. 6, I835; m. Margaret, d. of Peter and Susan Schmitz, of Germany, Oct. 28, 1860. He is a farmer and lives in Nevada, Iowa, where he is engaged in cattle raising.

They had children :-

$(8,6)$ i. Charles Franklin, b. Nora, Ill., Dec. 12, I863; m. Olive Wilson, Feb. 16, 1893.

ii. Fannie Edith, b. Nevada, Iowa, May 25, 1867.

iii. Effie Jane, b. Nevada, Iowa, Jan. 26, 1870.

iv. Frederick Morgan, b. Nevada, Iowa, May 25, 1872 .

v. Guy Cook, b. Nevada, Iowa, June 7, 1876.

vi. Gertrude May, b. Nevada, Iowa, Jan. 7, 1879.

CHARLES HENRY CURTISS (Morgan, Stiles, Elnathan, Ephraim, Foseph, Fohn, Elizabeth), b. Huntington, Conn., Nov. II, $1837 ; \mathrm{m}$. Frances Catherine, d. of Charles Chauncey and Lucretia Marsylva Spencer, of Saybrook, Conn., July I6, 1868. On June I9, I86I, he enlisted as a soldier in the Union Army at Detroit, and served through the War of the Rebellion. Aug. 22, I86 I, he was appointed quartermaster in the 7 th Michigan Infantry, and each year was promoted until March I 2, I 864, when he was appointed captain of this same regiment. On June 12,1864 , he resigned on account of disability. He is a merchant and resides in Detroit, Mich. 
They had children :-

i. Edith Maria, b. Saybrook, Conn., Sept. 5, 1869. She d. March IO, 1877 .

ii. Bessic Margaret, b. Detroit, Mich., Oct. 13, 1870.

iii. Frances Eddy Spencer, b. Detroit, Mich., Oct. 2, 1876.

668

ASHBEL. CURTISS (Fudson, Nehemiah, Foseph, Foseph, Foseph, Fohn, Elizabeth), b. Stratford, Conn.

Sniffin, of Stratford, Conn.
40 years ; m. (ii.) Elizabeth Lewis, of Derby, Conn., Nov. I 8 ,

1794; m. Deborah I 840 . She d. March I0, 1892 , aet. 80 years. He served as a private in the War of $18 \mathrm{I} 2$. He was a shoemaker and resided in Stratford, Conn., where he d. May i4, i 880.

They had children :-

$\left(S_{37}\right)$ i. William, b. Stratford, Conn. Derby, Conn.

He d.

; m. Jennette Gilbert, of I 866.

ii. Susannah, b. Stratford, Conn.,

I 823 ; m. Otis Beers, of Stratford, Conn., Feb. II, 1847. She d. June 28, 1854.

\section{9}

ROSWELL CURTISS (Fudson, Nehemiah, Foseph, Foseph, Foseph, Fohn, Elizabeth), b. Stratford, Conn., June 29, 1799 ; m. Sarah, d. of Thaddeus and Hannah Peck, of Stratford, Conn., Nov. 8, I 836 . He d. Oct. I 8 , I 880 . She d. June 8, I 85 I, aet. 38 years. He was a merchant, and resided in Stratford, Conn.

They had children :-

i. Lucy, b. Stratford, Conn., Nov. 12, 1837; m. John V. Huiell, of New York, June 17, 1857 .

ii. Albertina, b. Stratford, Conn., April 30, 1840. She d. March 15, 1874 .

iii. Anna Augusta, b. Stratford, Conn., July 1 3,$1843 ; \mathrm{m}$. John V. Huiell, of New York City, N. Y., April 17, 1878. She d. Oct. 8,1887 .

iv. Mary Elizabeth, b. Stratford, Conn., June 28, I 845. She d. Nov. 10, 1876 .

v. Jane Rosilla, b. Stratford, Conn., Jan. II, I848; m. Samuel Edward Seaman, of Bay Ridge, L. I., Aug. 18, 1875 .

\section{0}

JUDSON CURTISS (Fudson, Nehemiah, Foseph, Foseph, Foseph, Fohn, Elizabeth), b. Stratford, Conn., Oct. 2, i 806 ; m. Martha, d. 
of 'Thaddeus and Hannah Peck, of Stratford, Conn., March 24, 1833. She d. June 5, 1854 . $\mathrm{He}$ is a farmer and resides in Stratford, Conn.

They had children : -

i. Sarah Peck, b. Stratford, Conn., March 23, I 834 ; m. Rev. Joseph Smith, of Birmingham, England, March 31, I 879 .

ii. Judson, b. Stratford, Conn., June 3, I 836 . He d. June $13,1836$.

iii. Rodney Valentine, b. Stratford, Conn., Sept. 22, I8 37 ; m. Harriet N. Atwood, of New York City, N. Y., October, 1863. He d. in Marquette, Mich., Sept. 7, I870. He was a bank cashier.

They had a child:

i. Ella Hewitt, b. Marquette, Mich., Sept. I I, I 870. She d. February, 1873 .

iv. Helen Frances, b. Stratford, Conn., Jan. I 3, I 840.

$\left(83^{8}\right)$ v. Hamilton Burton, b. Stratford, Conn., Aug. 4, 1842 ; m. Emma W. Curtiss, October, 1874 .

vi. Emily Jane, b. Stratford, Conn., Sept. II, I845. She d. June I 7 , I 875 .

vii. Theresa Ann, b. Stratford, Conn., May 6, I 848 .

\section{1}

AGUR CURTISS (Fudson, Nehemiah, Foseph, Foseph, Foseph, Fohn, Elizabeth), b. Stratford, Conn., I8 I ; m. Mary Hurd, of Woodbury, Conn. She d. Jan. 17, 1886, aet. 80 years. He d. June 2, 1889. He was a comb-maker.

They had children :-

$(839)$ i. Franklin A., b. Stratford, Conn.

ii. Hannah Olive, b. Stratford, Conn.

iii. Ella, b. Stratford, Conn., April 4, I 849. She d. July I 9, I 884.

iv. David, b. Stratford, Conn., 1854. He d. March 26, 1877.

$$
672
$$

ENOCH CURTISS (Fudson, Nehemiah, Foseph, Foseph, Foseph, Folın, Elizabeth), b. Stratford, Conn., Dec. 30, 18 I 3 ; m. Susan, d. of Silas and Hannah Beers, of Stratford, Conn., June 16,1839 . He d. May 20, I876. She d. May 9, 1896, aet. 8 I years. He was a comb-maker, and lived in Stratford, Conn.

They had children :-

$(8 \neq 0)$ i. Melville Judson, b. Stratford, Conn., April 5, I 840 ; m. Clarissa F., d. of John and Mary Baldwin, of Milford, Conn., July 14, I 863 .

$(841)$ ii. Otis Beers, b. Stratford, Conn., Feb. 2, I 843 ; m. Harriett, d. of Andrew and Harriett Clark, of Nichols, Conn., Oct. I I, I 870.

(169) 
iii. Irene, b. Stratford, Conn., May 31, I846; m. William Thompson, of Stratford, Conn., June 23, I88 I.

iv. Mary Edwards, b. Stratford, Conn., March 18, I849.

\section{3}

STILES CURTISS (Freeman, Nehemiah, Foseph, Foseph, Foseph, John, Elizabeth), b. Stratford, Conn., June 7, 1808 ; m. Catherine Mumford, d. of John Robertson, of New York City, N. Y. He d. in New York City, N. Y., May 4, 1876. She d. in Plainfield, N. J., Oct. 7, 1893 .

They had children :-

$(842)$ i. John Robertson, b. New York City, N. Y., I 838.

(843) ii. Albert Arthur, b. New York City, N. Y., I 841 .

iii. Mary Robertson, b. New York City, N. Y., I845; m. J. Augustus Rogers, of Newburg, N. Y., $\quad$ I876.

iv. Henrietta Swords, b. New York City, N. Y., 1849; m. Brion Foulke, of New York, 1884 .

\section{5}

ELBERT F. CURTISS (Freeman, Nehemiah, Foseph, Foseph, Foseph, Fohn, Elizabeth), b. Stratford, Conn., April 20, 18 19; m. Ann Jewett Gorham, of Westport, Conn., Sept. 26, I840. He d. in Bridgeport, Conn., May I3, I883. She d. April 8, I893, aet. 72 years.

They had children :-

i. Margett, b. Bridgeport, Conn., May 2, I 842 ; d. May 29, I 842 .

ii. William Douglass, b. Bridgeport, Conn., Oct. I 5,1857 ; d. Oct. 6,1859 .

(844) iii. Freeman G., b. Bridgeport, Conn., Oct. 4, 1860.

$$
676
$$

WILIIAM I. CURTISS (Freeman, Nehemiah, Foseph, Foseph, Foseph, Fohn, Elizabeth), b. Stratford, Conn., June 22, I82 I ; m. Jane M. Lewis, Oct. 22, I 844. He d. in Corning, N. Y., June I 5, I 849 . She d. Jan. 4, I 858 .

They had children :-

(845) i. Frank Thompson, b. Burdett, N. Y., July I2, I 846.

(846) ii. William Lewis, b. Corning, N. Y., March 2 I, I 848.

$$
\text { ( I } 70)
$$


FREEMAN L. CURTISS (Freeman, Nehemiah, Foseph, Foseph, Foseph, Fohn, Elizabeth), b. Stratford, Conn., Jan. 9, 1828; m. Georgia Howard, of New York City, N. Y., Oct. 2, 1856 . He lives in the old homestead, built about I 713, at Stratford, Conn.

They had children :-

(847)i. Howard J., b. Stratford, Conn., June 29, 1857 ; m. Ellen V. Talbot, June 5,1888 . He resides in Bridgeport, Conn.

ii. Alice E., b. Stratford, Conn., Feb. I 4, 1860. She is unm. and lives in Stratford, Conn.

$(\delta \not \delta)$ iii. Lee Le Count, b. Stratford, Conn., Nov. I4, I 865.

iv. Emma Louisa, b. Stratford, Conn., Oct. 3, 1868; m. Elmer H. Havens, of Bridgeport, Conn., June Io, I89 I.

\section{8}

IRA CURTISS (Nathan, Silas, Foseph, Foseph, Foseph, Fohn, Elizabeth), b.

They had children:-

i. Ira Elnathan, b.

ii. Jane Augusta, b.

\section{9}

JOSEPH CURTISS (Nathan, Silas, Foseph, Foseph, Foseph, Fohn, Elizabeth), b. Stratford, Conn., I802; m. Helen Peck, Oct. I 8, I 825. He d. in Stratford, Conn., April I6, I 859. He was a cooper.

They had children :-
i. Julius A., b.
ii. John P., b.
iii. Hannah C., b.
iv. Julia A., b.

680

LEWIS CURTISS (Foel, Silas, Foseph, Foseph, Foseph, Fohn, Elizabeth), b. Stratford, Conn.; m. Ann, d. of Judson and Mary Lewis Curtiss, of Stratford, Conn., April 7, 1834. She d. Feb. 28, I 846 , aet. 34 years.

They had children :-

i. Louise, b. Stratford, Conn., Derby, Conn., June 9, 1858 .

$\left(8_{49}\right)$ ii. Lewis Francis, b. Stratford, Conn., $1835 ;$ m. I. Mix Gilbert, of He d. Sept. 1 3, I 86 r.

iii. Fanny, b. Stratford, Conn., Conn., Nov. 30, 1882 .

$$
183^{8} ; \mathrm{m} \text {. Eliza }
$$

1841 ; m. Job Peck of Stratford, 
VICTORY CURTISS (Agur, Agur, Foseph, Foseph, Foseph, Fohn, Elizabeth), b. Stratford, Conn., Feb. 28, 1806; m. Susan J., d. of Captain Miles. She d. Jan. 31, 1871, aet. 63 years. He d. in Bridgeport, Conn., Nov. 20, 1891.

They had children :-

i. Josiah Miles, b. Bridgeport, Conn., June 7, 1828 .

ii. Susan J., b. Bridgeport, Conn., Jan. I6, I 830 ; m. Philo Beach, of Trumbull, Conn.

$\left(S_{50}\right)$ iii. Elliot Miles, b. Bridgeport, Conn., Oct. 192 I831; m. Ellen Stone, of New Milford, Conn.

iv. Louise, b. Bridgeport, Conn., March 15, I834; m. David W. Clarke, of Tidioute, Penn.

$\left(\delta_{51}\right)$ v. Cornelius, b. Bridgeport, Conn., Feb. 5, 1837. He settled in Chicago, Ill.

vi. Sarah E., b. Bridgeport, Conn., April 16, 1839; m. H. B. Glover, of Dubuque, Iowa.

$\left(\delta_{52}\right)$ vii. Frederick, b. Bridgeport, Conn., Aug. 26, I84I ; m. Elizabeth Beck, of Bridgeport, Conn., May 3, 1870 .

(853) viii. John Calvin, b. Bridgeport, Conn., April 17, 1845; m. Adela Stuart, of New Haven, Conn., Nov. 8, 1870.

$\left(S_{54}\right)$ ix. Victory B., b. Bridgeport, Conn., Dec. 25, 1848. He settled in Dallas, Texas.

682

CORNELIUS AgUR CURTISS (Agur, Agur, Foseph, Foseph, Foseph, Fohn, Elizabeth), b. Stratford, Conn., June 28, i808; m. (i.) Phebe, d. of Stephen Curtiss and Hannah Wheeler Lewis, of Stratford, Conn., Sept. 23, 1833 . She d. May 15, I866, aet. 55 years; m. (ii.) Sarah Jane Wentworth, of Winsted, Conn., in 1869. He was a manufacturer of shell combs and resided in North Stratford, Conn., until 1852, when he removed to Derby, Conn., where he d. Feb. 21, I883.

They had children :-

i. Catherine Lewis, b. Stratford, Conn., July 27, 1834; m. George Curtiss, Dec. 8, 1859 .

$\left(8_{55}\right)$ ii. Augustine Beers, b. Stratford, Conn., April 18,1836 ; m. (i.) ; m. (ii.) Alice Beal, of Peru, Ind. He d. April 25, $188 \mathrm{I}$. His widow m. Leopold Kraus.

iii. Elizabeth Ann, b. Stratford, Conn., Aug. 10, 1838 ; m. Samuel Hitchcock Brush, of Derby, Conn., Nov. 4, 1857. She d. June 8,1891 . 
iv. Mary Jerusha, b. Stratford, Conn., March 29, I841; m. Ahijah Gilbert, of Derby, Conn., Oct. 26, 1859.

v. Cornelius Agur, b. Stratford, Conn., May 29, 1844. He d. Aug. 14,1845 .

vi. Norris Beach, b. Stratford, Conn., Sept. 27, 1847; m. Phebe Woodruff, of New Haven, Conn., in I 869. He was a merchant in East Brooklyn, N. Y., for twenty years, but is now manager of the firm of Harmon Bros., of New Haven, Conn., where he now resides. No issue.

683

ALFRED CURTISS (Agur, Agur, Foseph, Foseph, Foseph, Fohn, Elizabeth), b. Stratford, Conn., Jan. 22, I822; m. (i.) Sarah A. Wheeler, of Stratford, Conn., May 2, I844; m. (ii.) Althea J. Stratton, of Stratford, Conn., Oct. 5, I 858 . He is a carpenter and resides in Stratford, Conn.

They had children :-

i. Alice Louise, b. Stratford, Conn., June 29, 1870 ; m. Mr. Silliman, June 23,1890 .

ii. Helen Birdseye, b. Stratford, Conn., March 6, 1878 ; m. Mr. Beach.

\section{4}

NORRIS NEWTON CURTISS (Agur, Agur, Foseph, Foseph, Foseph, Fohn, Elizabeth), b. Stratford, Conn., Sept. 2, 1824; m. (i.) Sarah Belcher; m. (ii.) Martha Melbourne.

They had children :-

i. Lizzie, b. Stratford, Conn.

$(856)$ ii. Walter, b. Statford, Conn.

iii. Alice, b. Stratford, Conn.

\section{5}

TALCOT'T CURTISS (Levi, Aaron, Fames, Fames, Benjamin, Fohn, Elizabeth), b. Granville, Mass., June I7, I795; m. Mercy J. Squires, of Granville, Mass., Sept. I 4 , I 818 .

They had children : -

i. Abigail Bates, b. Granville, Mass., Sept. 2, 1820 .

ii. Jane Eliza, b. Granville, Mass., Sept. 5, 1825.

iii. Levi Johnson, b. Granville, Mass., Nov. I 2, $183^{6}$. 
JOHN CURTISS (Samuel, Abijah, Fohn, Fames, Benjamin, Fohn, Elizabeth), b. Durham, Conn., Nov. 2, 1811 ; m. Emily Sophia Lindslay, of Medina, Ohio, Jan. 23, 1834. He d. in Owatonna, Minn., July 6, 1893. He was educated at West Point Academy.

They had children :-

i. Benjamin Lindslay, b. Medina, Ohio, Dec. 2i, i 834 . He d. Aug. 7,1844 .

ii. David, b. Medina, Ohio, May 2, I837; m. Mary A. Sanborn, of Medford, Minn., July 16, 1867. No issue. He is a farmer and fruit grower and lives at Owatonna, Minn.

(\$57) iii. Thomas Jefferson Barnes, b. Medina, Ohio, Nov. I9, I838; m. Nellie E. Hubbard, of Owatonna, Minn., Aug. 24, 1867. She d. May, 1897, aet. 49 years. He resides in Lincoln, Neb.

iv. Hannah Lucretia, b. Medisia, Ohio, Oct. ro, $1840 ;$ m. W. N. Abbott, of Medford, Minn., Oct. 5, 1863 .

v. Benjamin Lindslay, b. Geneseo, Ill., Dec. 15, 1844. He is unmar. and resides in Owatonna, Minn. He is connected with the Estey Companies of Chicago, Ill.

$(858)$ vi. Samuel, b. Geneseo, Ill., May 18, 1847.

(\$59) vii. Henry James, b. Geneseo, Ill., Dec. 20, I849; m. Phebe Gillet, of Owatonna, Minn., Jan. 19, 1872.

viii. John Francis, b. Geneseo, Ill., Jan. 7, r 854 ; m. Florence A. Barlow, of Medford, Minn., Oct. 9, 1882.

They had a child :

i. Lindslay Byron, b. Medford, Minn., Jan. 31, 1885 .

689

SAMUEL BROOKS CURTISS (Samuel, Abijah, Fohn, Fames, Benjamin, Fohn, Elizabeth), b. Durham, Conn., July 13, I813; m. Sarah M. Chapman, of Medina, Ohio, April 26, 1836. He d. in Medina, Ohio, Oct. 2, 1896 . She d. May 7, 1889. He was taken by his father to Ohio in 1828 , and became a prominent citizen of Medina, Ohio, holding many positions of trust in the town and county.

They had children:-

(860) i. Roswell Chapman, b. Medina, Ohio, April 19, 1837; m. Frances E. . He was educated at Hillsdale College, and is an attorney-at-law, practising in Medina, Ohio, where he resides.

They had a child: -

i. Carrie Ball, b. Medina, Ohio, June 26, 1872 . She d. Dec. 3, $189 \mathrm{I}$. 
ii. Samuel Henry, b. Medina, Ohio, Jan. I6, I 839. He d. Sept. I2, I 845 .

iii. Emily Sophia, b. Medina, Ohio, Nov. 30, I 840 ; m. Orville B. Dealing, of Medina, Ohio, Jan. I, I 867.

$(S 6 I)$ iv. John Stokes, b. Medina, Ohio, July I 5, I 843 ; m. Sarah H. He resides in Lodi, Ohio.

v. Mary Almira, b. Medina, Ohio, March 20, 1846 . She studied at Oberlin College and lives unm. in Medina, Ohio.

(\$6z) vi. Frederic Henry, b. Medina, Ohio, Dec. 15, 1849; m. (i.) Medora C. ; m. (ii.) Cora T. . They live in Medina, Ohio.

vii. Clara Samantha, b. Medina, Ohio, March 26, 1852; m. O. F. White, at Medina, Ohio, Aug. I7, I88 I.

$(863)$ viii. Edward Shurtleff, b. Medina, Ohio, April r9, I856; m. (i.) Emma D., d. of Richard E. Jones, of Sparta, Wis., May 5, I885. She d. April 11, I 894; m. (ii.) Anna M. Miller, of Toledo, Ohio, Nov. 28, 1895. He resides in Toledo, Ohio.

\section{$69 \mathrm{I}$}

ICHABOD FRANCIS CURTISS (Samuel, Abijah, fohn, fames, Benjamin, Fohn, Elizabeth), b. Medina, Ohio, April 1, 1834; m. Martha Matilda, d. of John and Geraldine Goss, of Geneseo, I11., Nov. 14, 1861. In the Civil War he served as ist lieutenant of an Illinois cavalry company, and as captain in Company $\mathrm{H}$, 8th Kansas Infantry. He served two terms as county commissioner of Delta Co., Col. $\mathrm{He}$ is a fruit grower and resides in Paonia, Col.

They had children : -

i. Horace Ludington, b. Genesco, Ill., April 9, 1865. He is unm. and resides in Paonia, Col.

ii. John Goss, b. Geneseo, Ill., Oct. 12, I869; m. Lillian Oregon Parks, of Pitkin, Col., Dec. 24, 1894, where he now resides. They had a child :-

i. Jennie Frances, b. Pitkin, Col., March I 8, 1896.

692

CARLOS E. CURTISS (Ichabod, Abijah, Fohn, Fames, Benjamin, Fohn, Elizabeth), b. Bergen, N. Y., ; m. Julia E. Niles, of Kingsville, Ohio, in 1859 . He was a farmer and in 1852 went to California, where he spent five years in the gold mines, acquiring quite a fortune. He d. in Kingsville, Ohio, May 3, 1886.

They had children :-

i. Mary, b. Kingsville, Ohio, Sept. 5, 1860 ; m. James A. Fickinger, of Kingsville, Ohio, March 29, 1884. She d. Feb. 15, 1889. 
ii. Halden, b. Kingsville, Ohio, Nov. 2, 1869; m. Frank P. Blair, of Kingsville, Ohio, Dec. I I, I895. They live in Cleveland, Ohio.

(S6 f) iii. Albert, b. Kingsville, Ohio, March 12, 1871 ; m. Elizabeth $\mathrm{K}$. Luikart, of Collinwood, Ohio, Sept. 25, 1895 . He is station agent of the Lake Shore and Michigan Southern Ry., at Nottingham, Ohio.

\section{5}

JOSEPH DAVIS BEERS CURTISS (Lewis, Agur, Samuel, Nathaniel, Zachariah, William, Elizabeth), b. New York City, N. Y., Jan. 5, $1825 ; \mathrm{m}$.

They had children : -

i. Bessic, b. New York City, N. Y., I 849 ; m. Marquis Maurice de Talleyrand-Perigord, at Nice, France, March 18, 1867.

(865) ii. George Washington, b. New York City, N. Y. He resides at Catskill, N. Y.

iii. Josephine May, b. New York City, N. Y., 1865; m. Emanuel, Prince of Ruspoli and Prince of Poggiosuasa, Mayor of Rome.

696

LEWIS AGUR CURTISS (Lewis, Agur, Samuel, Nathaniel, Zachariah, William, Elizabeth), b. New York City, N. Y., Dec. I 5, 1835 ; m. Emma Sophia, d. of Roderick Cadwell and Delia Augusta Perry Stub, of Auburn, N. Y., June 9, I859. She d. May 27, 1893, aet. 57 years. He graduated from Columbia College in 1858 , and lived in New York City until May 4, 1868 , when he removed to Southport, Conn., where be now lives.

They had children :-

(866) i. Roderick Perry, b. New York City, N. Y., May 4, 1860; m. Louisa Wells.

(867) ii. Lewis Beers, b. New York City, N. Y., June 30, 1863; m. Alice Minerva, d. of Samuel I. and Mary B. Beardsley, of Long Hill, Conn., Oct. 17, 1888.

699

ELLIOTT PLUMB CURTISS (Fustus B., Daniel M., Mitchell, Zachariah, Zachariah, William, Elizabeth), b. Nichols, Conn., April s 4, I 8 I 4; m. Clarissa Bulkley, of Greenwich, Conn. He d. in Nichols, Conn., March 10, I 896. She d. Dec. 31, I 888, aet. 70 years. He was captain of the militia in 1850 . 
They had children: -

i. Hamilton, b. Nichols, Conn., June 8, 1845. He d. May io, I 848 .

ii. Lizzie, b. Nichols, Conn., March 1 5, 1849; m. Isaac W. Birdsey. She d. September, I 864 .

iii. Isabelle, b. Nichols, Conn., Jan. I6, I851.

(868) iv. Elliott Plumb, b. Nichols, Conn., July 26, 1853 ; m. Annabelle, d. of Ormiel Hall, of Bridgeport, Conn., June 14, I883. He d. July I 3, I 893 .

(869) v. Nathan Bulkley, b. Nichols, Conn., May I4, I857; m. Mary Ann, d. of William T. and Harriett L. Gilbert, of West Granby, Conn., Oct. 20, I881.

\section{5}

EDWIN RODNEY CURTISS (Samuel E., Leveret, Samuel, Feremiah, Zachariah, William, Elizabeth), b. Southington, Conn., May 6 , I 836 ; m. Eva A. Lingenfetler, of Fonda, N. Y., May 11, 1859. He is a photographer and resides in Madison, Wis.

They had children: -

$(870)$ i. Frederick Milard, b. Madison, Wis., Feb. I I, I 860 ; m. Catherine W. Ford, of Madison, Wis., Oct. 3, 1882. They reside in Madison, Wis.

ii. May Evelyn, b. Madison, Wis., Sept. I 8, I 869 ; m. Henry C. Geiger, of Madison, Wis., Oct. I 2, I 892. They live in Madison, WVis.

iii. Gertrude Helen, b. Madison, Wis., Jan. IO, I874. She is unm.

\section{1}

WILLIAM HENRY CURTISS (Sydney $F$., Isaac $F$., Abner, Fosiah, Fosiah, William, Elizabeth), b. Stratford, Conn., April 11, I $841 ; \mathrm{m}$. Helen A. Bertine, of Danbury, Conn. He d. in Stratford, Conn., Jan. 8, I 887 .

They had children:-

$(871)$ i. Frank L., b. $\quad$ 1867; m. Emma A. Atwood, of Stratford, Conn., May 28, I 88 .

ii. Cora May, b. Stratford, Conn., I868; m. Frank B. Beers, of Stratford, Conn., Nov. 9, I887.

iii. William H., b. Stratford, Conn., July 21, 1876 .

iv. Marion B., b. Stratford, Conn., July 20, 1880.

v. Nellic Iona, b. Stratford, Conn. 
WILLIAM EDMUND CURTISS (Holbrook, Salmon, Nehemiah, Benjamin, Fosiah, William, Elizabeth), b. Watertown, Conn., Sept. 23, 1823; m. Mary Ann, d. of William Henry and Eunice Scovill, of Waterbury, Conn., Sept. 2, 1851. He graduated from Trinity College in 1843 , and was admitted to the bar in 1847. He began practising law in New York City, where he rapidly rose in his profession. In $187 \mathrm{r}$ he was elected judge of the Superior Court of New York, and at the time of his death was chief justice of the Superior Court. He was a member of the Committee of the Board of Education of New York, and for a number of years its president. In 5862 Trinity College conferred on him the degree of LL.D. He was vice-president of the New York Geographical Society. He died in Watertown, Conn., July 6, 1880 .

They had children:-

i. William Edmond, b. New York City, N. Y., June 2, I 855 . He was educated at the Columbia Grammar School and at home in the country. In 1875 he was graduated at Trinity College, Hartford, Conn., with the degree A.B., and received that of M.A. in 1878 . He studied law at the Columbia College Law School, and graduated from that school in 1877 , and in June of that year he formed the law partnership of Stearns \& Curtis. He took an active part in the affairs of Trinity College, and was elected a trustee thereof by the alumni. At the conclusion of this term he was made a life trustee by the board. As a Democrat he was actively interested in politics, and in the interest of good government. He was secretary of the Democratic Club of New York City for seven years. He was a delegate to the Albany Conference of Anti-Snappers in February, I892, and also to their State Convention of Syracuse, and was a member of the Democratic Provisional State Committee. He took an active part in the Presidential Campaign of $\mathrm{I} 892$ in behalf of Mr. Cleveland, and was appointed by President Cleveland Assistant Secretary of the Treasury, and was confirmed by the Senate in April, J893. His duties in this office were mainly connected with the finances, the divisions employed on them being under his personal supervision. He resides in New York City, N. Y., and is a member of the law firm of Curtis, MalletPrévost, \& Colt. Unm.

$(872)$ ii. Henry Holbrook, b. New York City, N. Y., Dec. 15, I856; m. Josephine, d. of Hugh and Josephine Hall Allen, of Brooklyn, N. Y., June 19, 1884 . He lives in New York City, N. Y.

iii. Francis Randolph, b. New York City, N. Y., Oct. I I, I 858 . He was educated at St. Paul's School, Concord, N. H., and gradu- 
ated from Trinity College, Hartford, Conn., in 1880. He went to North Carolina to live and became interested in various enterprises in the South. He d. June 10, 1892, unm.

iv. Fustace Sanford, b. New York City, N. Y., June 13, 1860. He d. July 14, 188 I.

$(873)$ v. Frederick Kinsbury, b. New York City, N. Y., Feb. 3, I863; m. Marian S., d. of J. Montgomery Hale, of New York City, N. Y., Oct. 28, 1890. He resides in New York City, N. Y.

vi. Mary Alanthea, b. New York City, N. Y., Oct. 2, 1867.

vii. Elizabeth; b. New York City, N. Y., April 21, I 873.

JULIUS BOLĚVAR CURTISS (Nichols, Philo, Benjamin, Benjamin, Fosiah, William, Elizabeth), b. Newtown, Conn., Dec. 10, 1825 ; m. (i.) Mary, d. of Peter and Mary Acker, of Greenwich, Conn., Oct. 30, 1854 . She d. Jan. 23, 1884 , aet. 56 years; m. (ii.) Alice, d. of Henry and Margaret Kneeland, of New York City, N. Y., and widow of Francis H. Grain, May I I, 1886. He was educated at the public schools of his native town, finishing with the academy and at a private school. He then began the study of law and in December, I 850, was admitted to the bar of the State of Connecticut. He began the practice of law in Greenwich, Conn., in 1851 , but removed to Stamford, Conn., in 1864. From $1855-1865$ he was one of the burgesses of the borough of Greenwich and borough attorney during the same period. In $185^{8}$ and in 1860 he was a member of the State Senate and during the latter service senior member and ex-officio member of the corporation of Yale College. During the Civil War he served from 1861-1864 as a member of the Military Committee of the town of Greenwich. In I 867 he was elected judge of probate for the district of Stamford and served until I870. From 1887-1893 judge of the borough court of Stamford. Mr. Curtis has been a member of the American Bar Association since 1879, and was one of its vice-presidents from i $885^{-}$ 1889. He is also a director of the Stamford Street Railway. He resides in Stamford, Conn., and with his son forms the law partnership of Curtis \& Curtis.

They had children :-

i. Sarah Lena, b. Stamford, Conn., March 9, r866; m. T. J. Mackay, of New York City, N. Y., 1891 .

ii. Louis Julius, b. Stamford, Conn., March I 1, 1869. He graduated from Yale College in 1889 . In 1892 he was admitted to the bar of Connecticut. He served for four years as clerk of the 
City Court, of Stamford, Conn. He is a member of the law firm of Curtis \& Curtis, of Stamford, Conn., where he now resides.

William HUBBell CURTISS (Carlos, Philo, Benjamin, Benjamin, Fosiah, William, Elizabeth), b. Newtown, Conn., Oct. I7, i 808 ; m. Hannah, d. of Jacob Rosecrans, of Columbus, Ohio, Oct. 29, I 829 . She d. at Oakland, Ill., Jan: 5, I 857 , aet. 47 years. He was a farmer and lived in Illinois. He d. at Tuscola, Ill., Feb. 28, I 884 .

They had children : -

i. Charlotte Levina, b. Sunbery, Ohio, Sept. I I, I83 I ; m. (i.) John McClure, of Oakland, Ill., Jan. 25, 1852; m. (ii.) Albert Garbrant, of Oakland, Ill., Oct. 25, 1853 . She resides in Napa City, Cal.

(874) ii. George Sample, b. Sunbery, Ohio, Aug. 3, I833; m. (i.) Ann Eliza Jester, at Grandview, Ill., March 2, I856. She d. I 866 ; m. (ii.) Jerusha, d. of Joseph Walsh, of Monticello, Ill. He resides in Decatur, Ill.

(875) iii. Don Carlos, b. Sunbery, Ohio, Sept. 23, I835; m. Mary Hawkins McClure, widow of William McClure, at Arcola, Ill., Oct. 8, 1863. She d. Aug. 24, I869. He resides in Tuscola, IIl.

$(876)$ iv. Edwin, b. Oakland, IIl., Jan. 22, I 838 ; m. Emma, d. of Joseph Walsh, of Monticello, Ill., Sept. I, 1870.

v. Mary, b. Coles Co., Ill., May 19, 1840 ; m. Edwin Corwin, of Mt. Pulaski, Ill., July 23, I 857 . She d. May 5, I 858 .

vi. Cordelia, b. Coles Co., Ill., Dec. 24, I 843. Shed.unm. at Tuscola, Ill., Jan. I 2, I 876 .

vii. Henry Clay, b. Coles Co., Ill., Feb. I I, 1845. He died unm. at Tuscola, Ill., Jan. 12, 1876 .

( 877$)$ viii. Derrel, b. Oakland, Ill., March 20, I 848 ; m. Francis Stork, of Westfield, Ill., in 1875 . He resides in Paris, Ill.

IRA B. CURTISS (Carlos, Philo, Benjamin, Benjamin, Fosiah, William, Elizabeth), b. Delaware Co., Ohio, Oct. 18, 1823; m. Jane Butler. He was a physician and lived in Decatur, Ill., where he d. Dec. I6, I 89 I.

They had children :-

$(878)$ i. Otto E., b.

$(879)$ ii. Frank, b. 
PHILANDER C. CURTISS (Carlos, Philo, Benjamin, Benjamin, Fosiah, William, Elizabeth), b. Delaware Co., Ohio, Oct. 28, I825; m. Ann Elizabeth, d. of Robert and Elizabeth Modrell, Jan. I6, 1844. She d. July 28, 1879 , aet. 54 years. He d. Jan. 26, 1879 .

They had children :-

(880) i. Carlos C., b. Oakland, Ill., Dec. I6, 1845; m. Eliza Abbie Ficket, Oct. 27, 1872.

ii. Ira Lamar, b. Oakland, Ill., Feb. 17, I848. He d. March 13, 1848 .

iii. Isabelle Jane, b. Oakland, Ill., March 8, I 849 ; m. Alfred N. Chapman, Dec. 21,1871 .

iv. Charlotte Catherine, b. Oakland, Ill., Sept. I, I852; m. (i.) E. C. Brown, March 13, 1873; m. (ii.) E. A. Pierce, Oct. 26, I 88 I.

v. Arcelia Lacella, b. Oakland, Ill., May 16, I855; m. James Glassburner, Sept. 13, 1874. She d. June 16, 1893 .

(881) vi. Sterling Pemberton, b. Oakland, Ill., June I9, I858; m. Susan A. Taber, Aug. 18, 1879 .

vii. Walter A., b. Oakland, Ill., March I8, I86I ; m. Estella Strange, Oct. I9, I88 I. He d. April 30, I896.

They had a child : -

i. Nora, b. Oakland, Ill., July 31, I 884 .

719

JOSEPH CURTISS (Cyrenius, Alfred D., Benjamin, Benjamin, Fosiah, William, Elizabeth), b. Newtown, Conn., April 5, i 844 ; m. Jane Eliza Tyler, of East Haddam, Conn., Feb. 14, I 869. She d. in Branford, Conn., Jan. 19, 1890, aged 43 years.

They had children :-

i. Alfred Tyler, b. Branford, Conn., July I9, I870.

ii. William Perry, b. Branford, Conn., Scpt. I I, I 87 I.

iii. Hiram Foster, b. Branford, Conn., Sept. Io, 1872. He d. Nov. I 2, I 872 .

iv. Minnie, b. Branford, Conn., Sept. 1, 1873. She d. Oct. 7, 1873.

v. Julia Ann, b. Branford, Conn., Nov. 25, 1874 .

HENRY WILLIAM CURTISS (Henry $F$., Epenetus, Benjamin, Benjamin, Fosiah, William, Elizabeth), b. Jones County, N. C., May 1 1, 1852; m. Ida, d. of Josiah Whitlow, of Bedford County, 
Va., Sept. 4, I 877 . He was taken by his parents to Connecticut before the War of the Rebellion. In I87 I he removed to Knoxville, Tenn., where he now resides. $\mathrm{He}$ is a jeweller.

They had children :-

i. Lucy McDaniel, b. Knoxville, Tenn., Aug. 31, I878.

ji. Henry Willian, b. Knoxville, Tenn., Aug. I, I880.

\section{$72 \mathrm{I}$}

CHARLES CURTISS (Henry $F$, Epenetus, Benjamin, Benjamin, Fosiah, William, Elizabeth), b. Jones County, N. C., Jan. 2, I 854 ; m. Rachel Barnum. He resides in Danbury, Conn.

They had children:-

i. Henry Barnum, b.

ii. Anna, b.

\section{5}

THOMAS BENNETT CURTISS (Roswell, Agur, Silas, Peter, Fosiah, William, Elizabeth), b. Starkey, N. Y., July 28, 1817; m. Alvira, d. of William B. and Martha (Wilson) Briggs, Sept. I0, 1842. He is a farmer and lives in Starkey, N. Y. He is a justice of the peace, and has served as master of service and for several years as master of the poor.

They had children :-

i. Lydia Ann, b. Starkey, N. Y., Starkey, N. Y.

ii. Frances J., b. Starkey, N. Y., Rochester, N. Y.

; m. J. Elbridge Gano, of

; m. Myron Disbro, of

\section{6}

CHARLES CURTISS (Roswell, Agur, Silas, Peter, fosiah, William, Elizabeth,) b. Starkey, N. Y., May 22, I823; m. Roxy Maria Doty, July 5, I 869. He was a farmer.

They had a child :-

(882)i. Alanson Doty, b. , June 2i, I854; m. Belle Teasdale - He lives in Howell, Mich.

LESTER CURTISS (Roswell, Agur, Silas, Peter, Fosiah, William, Elizabeth), b. Starkey, N. Y., Jan. 29, 1829; m. (i.) Amanda White, April 25, I850; m. (ii.) Sarah Bennett, June 6, I 853. She d. April, 1896. He settled in Carlisle, Ark., where he d. July 12,1892 . 
They had children :-

$\left(88_{3}\right)$ i. William L., b.

(884) ii. Arthur Clayton, b.

; m. Helen Short.

- He resides in Carlisle, Ark.

728

SAMUEL BENNETT CURTISS (Roswell, Agur, Silas, Peter, Fosiah, William, Elizabeth), b. Starkey, N. Y., May 5, I $834 ; \mathrm{m}$. Augusta Pebbles, Dec. IO, I863. He lives in Bath, Ill.

They had children : -

i. Lydia, b.

ii. Lucella, b.

731

HARMON MAY CURTISS (David, Agur, Silas, Peter, Fosiah, William, Elizabeth), b. Exeter, N. Y., Oct. 23, I842; m. Matilda Howard, of Kansas, Aug. 27, 1867. He d. at Ann Harbor, Feb. I 7, I 875 .

They had children :-

$(885)$ i. William H., b. Kansas, May 23, 1868.

ii. Frank, b. Kansas, July I2, I87 I. He d. Sept. 2, I873.

iii. Charles H., b. Kansas, May II, 1874.

735

CHARLES BIRDSEY CURTISS (David, Agur, Silas, Peter, Fosiah, William, Elizabeth), b. Exeter, N. Y., Nov. Io, I853; m. Anna Carr, of Exeter, N. Y., Jan. 2, I 884.

They had children :-

i. Netta A., b. Exeter, N. Y., Dec. 28, 1886.

ii. Nellie, b. Exeter, N. Y., Feb. I6, I892.

\section{2}

ALLF, STANSBURY CURTISS (Paul A., Abner, Reuben, Matthew, Fosiah, William, Elizabeth), b. New York City, N. Y., Aug. 3, 1824; m. Mary Elizabeth, d. of William Lyons, of New York City, N. Y., Jan. 6, 1846. He d. in New York City, N. Y., July 22, 1868.

They had children :-

i. Ella, b. New York City, N. Y., Nov. I 3, I846; m. Angelo Godone, of New York City, N. Y., April 28, I87 I. He d. March I I, I 872 .

(886) ii. Paul Allen, b. New York City, N. Y., Jan. 6, I 850.

iii. Walter, b. New York City, N. Y., ; d. y. 
$\left(\mathcal{S} \mathcal{S}_{7}\right)$ iv. Alfred Halsey, b. New York City, N. Y.

v. Grace, b. New York City, N. Y.;

m. William Carroll.

743

WILLIAM HENRY CURTISS (Paul A., Abner, Reuben, Matthew, Fosiah, William, Elizabeth), b. New York, April I, I834; m. Annie Martin, of New York, Nov. 17, $185^{8}$.

They had children :-

$(S \& S)$ i. William Henry, b. Orange, N. J., Sept. 17, 1859.

ii. Mary Cecilia, b. Orange, N. J., Nov. 27, 1862.

iii. Frederick Martin, b. Orange, N. J., March 31, s864. He d. in Orange, N. J., March, 1868.

iv. Pauline, b. Orange, N. J., Aug. 20, 1867.

$(\$ \& 9)$ v. Frank, b. Orange, N. J., Aug. 22, I869.

vi. Annie Augusta, b. Orange, N. J., May 28, I873.

\section{4}

WILLIAM VAN DE WATER CURTISS (Foseph, Abner, Reuben, Matthew, Fosiah, William, Elizabeth), b. New York City, N. Y., March 6, 1827; m. Amy Cornell, d. of William and Ruth (Lovett) McIntire, of New York City, N. Y., May I I, I 848 . He d. in 1873 .

They had children :-

$(S 90)$ i. Joseph Osborn, b. New York City, N. Y., May 25, 1849; m. Ida F. Young, in 1876 . He resides in New York City, N. Y.

ii. Charles Henry, b. New York City, N. Y., Aug. 7, 1850. He d. April 2, 1853 .

iii. William McIntire, b. New York City, N. Y., July 3, 1852 . He d. in Rochester, N. Y., Feb. 23, 1869.

ALFRED LEONARD CURTISS (Foseph, Abner, Reuben, Matthew, Fosiah, William, Elizabeth), b. New York City, N. Y., May 3, 1832 ; m. Mary Elizabeth, d. of Thomas and Eleanor Johnson Hilson, of New Orleans, La., April I, I854. She d. June 8, 1869, aet. 35 years; m. (ii.) Elizabeth McDonald, d. of Oliver and Elizabeth McDonald (McIntire) Strong, and widow of Frank Jandon, of New York City, N. Y., Aug. 4, I872. He d. April i 8, I 897 .

They had children :-

i. Eleanor Maud, b. New York City, N. Y., March 1, 1857. She d. Sept. 18, 1860 .

ii. Georgina Pell, b. New York City, N. Y., Feb. 19, 1859. 
iii. Isabel, b. New York City, N. Y., Jan. 25, 186 I. She d. Feb. 25, I 862.

iv. Bertha, b. New York City, N. Y., Nov. 25, 1862; m. Rollin W. Larrabee.

v. Olive Strong, b. New York City, N. Y., 1875 ; m. Allen I. Minor.

\section{$75^{\circ}$}

HENRY GOULD CURTISS (samuel, Gould, Gold, Matthew, Fosiah, William, Elizabeth), b. Newtown, Conn., Dec. 8, I8+8; m. Anna E., d. of George and Sarah Peck Beers, of Newtown, Conn. He resides in Newtown, Conn., and is engaged in business at Sandy Hook, Conn., where he is a manufacturer of buttons and combs with his father.

They had children :-

i. Harry Beers, b. Newtown, Conn., May 30, 1879 .

ii. William Robert, b. Newtown, Conn., May I7, 1883 .

\section{4}

BYRON CURTISS (Uriah B., Peter B., Peter B., Peter, Fohn, Israel, Fohn, Elizabeth), b. West Martinsburgh, N. Y., Sept. 5, I 840 ; m. Mary Philles. He died in Lowville, N. Y.

They had children :-

i. Ray, b. West Martinsburgh, N. Y.

They live in West Martinsburgh, N. Y.

ii. Laura, b. West Martinsburgh, N. Y.

They live in Lowville, N. Y.

iii. Celia, b. West Martinshurgh, N. Y. ; m. Eugene Pitcher.

They live in Lowville, N. Y.

iv. Earl, b. West Martinsburgh, N. Y.

v. Willis, b. West Martinsburgh, N. Y.

760

JAMES GUERNSEY CURTISS (David H., David S., David, David, Fohn, Israel, Fohn, Elizabeth), b. Woodbury, Conn., Aug. 8, I835; m. Mary Jennette, d. of Henry B. and Frances Stiles, of Southbury, Conn., May I7, I860. She d. Sept. 20, I 893.

They had children :-

i. Henry Stiles, b. Woodbury, Conn., May 13, 1861; m. Frances

E. Eyre, of Woodbury, Conn., Aug. 28, 1884.

ii. Anna, b. Woodbury, Conn., May 2, 1862. 
iii. James Guernsey, b. Woodbury, Conn., Sept. I3, I863; m. Alma Maria Bossett, of Watertown, Conn., Feb. I3, 1890.

iv. Ellen E., b. Woodbury, Conn., Sept. 2I, I865.

v. Flora Preston, b. Woodbury, Conn., Aug. I3, I867; m. Lyman Wheeler Carrington, of Woodbury, Conn., May I4, 1890 .

vi. George Smith, b. Woodbury, Conn., Oct. 8, 1870. He was drowned June $27,1872$.

vii. Eva M., b. Woodbury, Conn., Aug. 26, 1875. She d. April I0, I 885 .

$$
762
$$

WALTER STILES CURTISS (Daniel, David S., David, David, Fohn, Israel, Fohn, Elizabeth), b. Woodbury, Conn., Feb. 9, I 842 ; m. Eunice, d. of Phenia Averill, of New Britain, Conn., Nov. 29, 1865 . He is a member of the firm of Daniel Curtiss Bros., woolen manufacturers of Woodbury, Conn., where he resides.

They had children :-

i. Daniel Averill, b. Woodbury, Conn., July 20, I867.

ii. Julia Eliza, b. Woodbury, Conn., Sept. 21, 1874 .

\section{3}

HORACE DANIEL CURTISS (Daniel, David S., David, David, Fohn, Israel, Fohn, Elizabeth), b. Woodbury, Conn., July 24, I $84 \mathrm{I}$; m. Harriet Atwater, of New Haven, Conn., Oct. 6, I 868. $\mathrm{He}$ is a member of the firm of Daniel Curtiss Bros., large woolen manufacturers in Woodbury, Conn., where he resides. He has served as representative and senator from Woodbury, Conn.

They had children :

i. Horace Atwater, b. Woodbury, Conn. I871 ; d. y.

ii. Charles Atwater, b. Woodbury, Conn., May 24, 1875. He graduated from the Sheffield Scientific School of Yale College in 1895 .

iii. Olivia Harriet, b. Woodbury, Conn., March 30, 1878 .

764
EDWARD JOHN CURTISS (Daniel, David S., David, David, Fohn, Israel, Fohn, Elizabeth), b. Woodbury, Conn., Jan. 24, I 845 ; m. Elizabeth Sanford, of Roxbury, Conn., May 8, i 872 . She d. Oct. I 2, I 878 ; m. (ii.) Ella L. Abrams, of Woodbury, Conn., June 7, I882. She d. April, I 884. He is a nember of the firm of Daniel Curtiss Bros., large woolen manufacturers in Woodbury, Conn., where he resides. 
They had children :-

i. Sarah Miranda, b. Woodbury, Conn., March 12, I 874.

ii. Eula Lambert, b. Woodbury, Conn., April 15, I 884.

\section{5}

WALES HATCH CURTISS (Reuben B., Simeon, Foseph, Israel, Stephen, Israel, Fohn, Elizabeth), b. Southford, Conn., July 29, $1826 ; \mathrm{m}$. Mary Frances, d. of William and Esther Sanford Hammill, of Williamsburg, L. I., Dec. $24,1850$. He is a farmer and lives in Southford, Conn.

They had children : -

i. William Reuben, b. Southford, Conn,, Feb. 19, 1852. He is unm. and lives in Southford, Conn.

ii. James McEwen, b. Southford, Conn., Jan. 30, 1854; m. Ida Hannah Crofton, of Woodbridge, Conn. He d. in Ansonia, Oct. 27, 1891. He was an architect and builder.

They had a child : -

i. Seta Ann, b. Ansonia, Conn., Feb. 20, 1879.

iii. Sarah Minerva, b. Southford, Conn1, Nov. 2, 1855; m. Cornelius Marcus Hard, of Seymour, Conn., Nov. 22, 1876.

iv. Charles Henry, b. Southford, Conn., March 6, I857; m. Ellen Hard, of Seymour, Conn. He lives in Beacon Falls, Conn.

$(89 r)$ v. Elnathan Atwater, b. Southbury, Conn., Feb. 19, 1859; m. Anna Isabel Hasard, of Ansonia, Conn., May 23, 1888 . He lives in Ansonia, Conn.

vi. Arthur Edward, h. Southford, Conn., Aug. 9, r86r ; m. Ida Isabel Hart, of Nangatuck, Conn., Nov. I $3,1890$.

vii. Jessie Hammill, b. Southford, Conn., April 24, 1864.

viii. $\{$ Isabel Sanford, b. Southford, Conn., Feb. 8, 1866.

ix. $\quad$ Estelle Sanford, b. Southford, Conn., Feb. 8, 1866.

x. Birdie May, b. Southford, Conn., Oct. I0, 1870; m. Charles Benjamin, of Stormiville, N. Y., Oct. 3, 1889.

xi. Nellie Elizabeth, b. Southford, Conn., Aug. 12, 1873; m. Charles Grant Ellison, of Arnot, Penn., Sept. I 2, 1894.

\section{6}

FRANKLIN LUTHER CURTISS (Simeon, Simeon, Foseph, Israel, Stephen, Israel, Fohn, Elizabeth), b. Southbury, Conn., Jan. I9, $1835 ; \mathrm{m}$. Mary Louise, d. of Richard Hines, of Waterbury, Conn., Sept. 28 , I 858 . In 1852 , after graduating from the district school in Southbury, he went to Waterbury for the purpose of attending high school. During a portion of the winter he taught school, and the same year became a clerk in the Water- 
bury post office. In 1853 he entered the Citizen's Bank of Waterbury as its teller. In I 864 he was made assistant cashier of that bank, and in 1868 its cashier, which position he now holds. $\mathrm{He}$ is also president of the Matthews and Willard Manufacturing Company.

They had children :-

i. Henry, b. Waterbury, Conn.

ii. Howard, b. Waterbury, Conn.

iii. Edith, b. Waterbury, Conn.

CHARLES STRONG CURTISS (Benjamin S., Charles R., Stephen, Israel, Stephen, Israel, Fohn, Elizabeth), b. Woodbury, Conn., March 23, I856; m. Ellen Mallory, d. of George B. French, of Watertown, Conn., Nov. 8, 1883. He is a farmer and resides in Woodbury, Conn.

They had children :-

i. George Benjamin, b. Woodbury, Conn., Sept. I3, 1884 .

ii. Louise French, b. Woodbury, Conn., April 23, 1886.

iii. Frederick Stoddard, b. Woodbury, Conn., Oct. 25, I889.

iv. Alice Mary, b. Woodbury, Conn., June 23, 1894.

\section{8}

EDWARD BENJAMIN CURTISS (Horace H., Charles R., Stephen, Israel, Stephen, Israel, Fohn, Elizabeth), b. Jackson, Tenn.; m. Corinna B., d. of Robert J. Ruffin, of Jackson, Tenn. He is a merchant and resides in Jackson, Tenn.

They had children:-

i. Charles Ruffin, b. Jackson, Tenn., Sept. 29, 1888.

ii. Minnie Merk, b. Jackson, Tenn., Sept. 6, i 890.

iii. Mary Melissa, b. Jackson, Tenn., Sept. I0, 1898 .

\section{4}

LOUIS K. CURTISS (Harrison $W$., Chauncey, Abel, Benjamin, Natbaniel, Thomas, Fohn, Elizabeth), b. Meriden, Conn., June 29, $1851 ; \mathrm{m}$. Sarah Elizabeth, d. of Dr. Edward Walker and Nancy C. Boies Hatch, of Meriden, Conn., Oct. I, I $879 . \quad$ He is cashier of the Southington National Bank of Southington, Conn.

They had children :-

i. Edward Hatch, b. Meriden, Conn., Nov. I3, I880.

ii. Nellie Thomson, b. Meriden, Conn., Aug. I8, 1882. 
FI.OYD CURTISS (Harrison $W$., Chauncey, Abel, Benjamin, Nathaniel, Thomas, Fohn, Elizabeth), b. Meriden, Conn., Dec. 25, $1854 ; \mathrm{m}$. Clara, d. of Edward Furniss, of Meriden, Conn., Dec. 2, I 879. He is teller of the First National Bank of Meriden, Conn., where he resides.

They had a child : -

i. Bessie Furniss, b. Meriden, Conn., Sept. 5, 1882.

\section{6}

FREDERICK CURTISS (Edmund B., Chauncey, Abel, Benjamin, Nathaniel, Thomas, Fohn, Elizabeth), b. Meriden, Conn., July 23, I $85^{8}$; m. Alice Stebbins, of Meriden, Conn., Oct. 2 I, I887. He resides in Meriden, Conn.

They had a child : -

i. Myrtle Almyra, b. Meriden, Conn., Oct. 22, I 896.

\section{7}

JOHN E. CURTISS (Edmund B., Chauncey, Abel, Benjamin, Nathaniel, Thomas, Fohn, Elizaheth), b. Meriden, Conn., April 2, I 862 ; m. Lizzie Seymour, Oct. 20, I 888 . He lives in Newark, N. J.

They had children :-
i. Ida, b.
ii. Frank, b.
April 2I, 1890.
, Nov. 24, 1892.

HOMER AUGUSTUS CURTISS (Homer, Benjamin, Benjamin, Benjamin, Nathaniel, Thomas, Fohn, Elizabeth), b. Meriden, Conn., June 14,1846 ; m. Mary A. Hussey, of New York City, N. Y., Aug. 28, 1873. He began his business career in New York, in the employ of the Meriden Cutting Company. In I 879 he returned to Meriden, Conn., and the following year was elected secretary of this company, and since that time has been very active in its management.

They had children :-

i. Adele L., b. New York City, N. Y., Sept. 2, 1875 .

ii. Caroline M., b. Meriden, Conn., July 2, 1879. 
GEORGE CURTISS (Samuel I., Ivah, Benjamin, Benjamin, Nathaniel, Thomas, John, Elizabeth), b. Easthampton, Conn., Jan. 5, 1837 ; m. Elvira, d. of Healy Corbin, Jan. 13, 1864. She d. at Harwinton, Conn., April 17, 1875. He m. (ii.) Mrs. Phebe M. Bishop, of New Haven, Conn., March 8, 1877. She d. at Amherst, Mass., June 27, 1877. He m. (iii.) Martha A. Blakesley, of Bristol, Conn., Sept. I 5, I880. She d. at Hartford, Conn., Dec. 24, 3888 . He graduated from Amherst College in 1860 , and studied in the Hartford Theological Seminary for two years, and graduated from the Andover Theological Seminary in 1863. He preached at Oxford and Pepperell, Mass., and Bristol, R. I., and took charge of the church in East Avon, Conn., November, 1864, where he was ordained Dec. 28,1865 , and installed pastor June 26,1866 . He was pastor of the church at Harwinton, Conn., eight years, having been installed there June $30,1869$. In 1872 he represented the town in Legislature. He was dismissed from the Harwinton Church, May 21, 1877, then ministered in Union, Comn., for three years. In May, I881, he received a call to the Wethersfield Avenue Congregational Church at Hartford, where he continued until 1889. Jan. 1, I 890 , he began work as pastor of the Congregational Church at Mayville, North Dakota, and was installed there in September of the same year, which position he is now filling.

They had children :-

i. Elsie Ann, b. East Avon, Conn., Sept. 29, 1865; m. Rev. Herbert K. Job, Sept. Io, I891.

ii. George Corbin, b. Harwinton, Conn., June 19, 1868. He d. Nov. 28,1876 .

iii. Mary Rebecca, b. Harwinton, Conn., June 9, 1873 .

iv. John Haugh, b. Harwinton, Conn., March 30, I874. He d. June 24,1877 .

v. Martha Eliza, b. Hartford, Conn., July 25, 188 I. She d. Jan. 26, I 882 .

SAMUEL IVES CURTISS (Samuel I., Ivah, Benjamin, Benjamin, Nathaniel, Thomas, Fohn, Elizabeth), b. Union, Con11., Feb. 5, I 844 ; m. Laura W., widow of Jared D. Sessions, May 10, 1870. He graduated from Monson Academy with highest honors, and entered Amherst College in 1862, but was obliged to leave college for a while on account of ill health. He graduated with honor in 1867. The same year he entered the Union Theological Sem- 
inary of New York. He was ordained by the New York Presbytery in 1874. After leaving the seminary he went to Europe, and studied at the University of Leipsic, where he received the degree of Doctor of Philosophy in 1876 . He was one of the founders and afterward pastor (1874-8) of the American chapel at Leipsic. The University of Berlin conferred on him the degree of Licentiate of Theology. In 1878 he received the degree of Doctor of Divinity from Iowa College. The same year he was called to the chair of Biblical Literature of the Chicago Theological Seminary. In 1879 he was transferred at this Seminary to the chair of OldTestament Literature and Interpretation, which he now holds. He is the author of numerous books on biblical subjects.

They had children :-

i. Paul, b. New York City, N. Y., Oct. 11, 187 I ; d. y.

ii. Pauline, b. Germany, Dec. 5, 1872. She d. March 7, 1890.

iii. John Christleib, b. Leipsic, Germany, Sept. 29, 1874 .

iv. Victor Delitzsch, b. Leipsic, Germany, Jan. 14, 1876. He d. Aug. 20,1876 .

THOMAS E. HULSE CURTISS (Sylvester F., Amos, Benjamin, Benjamin, Nathaniel, Thomas, fohn, Elizabeth), b. New York City, N. Y., Feb. 13, I 852 ; m. Ada H. Hulse, of New York City, N. Y., June 8, I88 I. She d. Feb. 18, 1 896.

They had children :-

i. Ada Florence, b. New York City, N. Y., July 26, 1882.

ii. Harriett Louisa, b. Cornwall-on-Hudson, N. Y., Aug. 22, 1884.

iii. Sylvia Johnson, b. New York City, N. Y.

\section{6}

LEVI BRADLEY CURTISS (Alfred P., Somuel, Benjamin, Benjamin, Nathaniel, Thomas, Fohn, Elizabeth), b. Meriden, Conn., Aug. 10, 1843 ; m. Alice Maria, d. of Henry Francis and Abby Bicknell Cushing, Dec. 23, 1868. He is connected with the Bradley Phosphate Company, of Boston, Mass. He served three years during the Civil War as a member of the Fifteenth Connecticut Regiment. Most of the time his regiment was connected with the Army of the Potomac.

They had children :-

i. Lillian Fay, b. Jan. 27, 1873 .

ii. Harold Marshall, b. April 25, 1878 . 
ALFRED WILLIAM CURTISS (Alfred P., Samuel, Benjamin, Benjamin, Nathaniel, Thomas, Fohn, Elizabeth), b. Meriden, Conn., June 2, I847; m. Clara Augusta, d. of William and Augusta Savage Homan, Feb. 17, 1870. He is a merchant and resides in Meriden, Conn.

They had children :-

i. Le Roy Homan, b. Meriden, Conn., Jan. 21, I872; m. Nettie, d. of Otis Benjamin and Emma Tinker Clark, Sept. 7, I892.

They had a child : -

i. Rachel, b. Meriden, Conn., Oct. 8, I893.

ii. Clarence Alfred, b. Meriden, Conn., Sept. 4, 1873 .

iii. Frederick Levi, b. Meriden, Conn., Nov. 29, I874. He d. Oct. 31, I890.

\section{8}

ROBERT HALL.AM CURTISS (Asahel, Asahel, Benjamin, Benjamin, Nathaniel, Thomas, Fohn, Elizabeth), b. Meriden, Conn., Dec. 4, I 845 ; m. Laura Sophia Hotchkiss, of Gouverneur, N. Y. She d. March 5, i891. He was educated at Cheshire Academy and Trinity College, Hartford, graduating in the class of i 868 . He taught school in Meriden a few years and then became secretary and treasurer of the Meriden Silver Plate Company and later its president. He resides in New York City, N. Y.

They had children : -

i. Howard Redfield, b. Meriden, Conn., Sept. 17, 1878.

ii. Jesse Monson, b. Meriden, Conn., Nov. 2, I 879 .

iii. Grace Hallam, b. Meriden, Conn., June I, I88 I. She d. Aug. 30; I 886.

GEORGE MUNSON CURTISS (George R., Asahel, Benjamin, Benjamin, Nathaniel, Thomas, Fohn, Elizabeth), b. Meriden, Conn., May 27, 1857 ; m. Sophia Phillips, d. of Thomas Trowbridge and Catharine Hurlburt Mansfield, of New Haven, Conn., Nov. 30, I 886. He is a graduate of Cheshire Academy, of Connecticut, and was at Trinity College, Hartford, Conn., three years, in the class of 1880 . Since 1880 he has been connected with the Meriden Britannia Company. In 1884 he was elected assistant treasurer of that corporation and in 1893 treasurer, which position he now holds. He resides in Meriden, Conn. 
They had a child :-

i. Agnes Mansfield, b. Meriden, Conn., Sept. 6, I887.

800

GEORGE BROOKS CURTISS (Levi, Levi, Solomon, Samuel, Samuel, Thomas, Fohn, Elizabeth), b. Southington, Conn., Aug. 2, I842; m. Frances M., d. of Edwin and Maria Bristol Barnes. She d. Nov. 4, 1866; m. (ii.) Laura M. Clarke. He graduated from Yale College in 1863 , and after teaching a short time started in business in New York City, N. Y., where he now resides.

They had children :-

i. George L., b. New York City, N. Y., March 3, 1871 .

ii. Agnes J., b. New York City, N. Y., Sept. 23, I873.

\section{ALBERT WILLOUGHBY CURTISS (Ira, Abijah, Fonathan,} Fonathan, Foseph, Thomas, Fohn, Elizabeth), b. Sheffield, Mass., Nov. 10, 820 ; m. Susan, d. of John Campbell and Grace Knapp Whitehead, of Stockbridge, Mass., Dec. 15, 185 r. He removed to Milwaukee, Wis., in 1852 , and to Whitewater, Wis., in 1853 , where he lived until 1868 . He then returned to Sheffield, Mass., where he died Nov. 21, 1893. He was a lumber, flour, and wheat merchant, and also a distiller. He served as a member of the State Legislature in 1876.

They had children :-

i. Ellenora, b. Whitewater, Wis., March I 8, I853; d. March II, I $88_{4}$; m. George F. Ranger, of Springfield, Mass., June I, I 876 .

(892)ii. Edward Whitehead, b. Whitewater, Wis., Sept. 7, I854; m. Grace, d. of Joel B. and Eliza Curtiss Merriman, of Sheffield, Mass., in Syracuse, N. Y., Jan. 7, I879. He resides in Sheffield, Mass.

iii. Della, b. Whitewater, Wis., April 27, $1856 ; \mathrm{m}$. Charles Stillman, of Sheffield, Mass., Oct. 6, I875.

iv. Elizabeth, b. Whitewater, Wis., Aug. 10, I859. She d. Jan. 2, 1872 .

v. Daniel Mason, b. Arena, Wis., Nov. 17, 1861. He is a mining engineer and is unm.

vi. Stella, b. Whitewater, Wis., Dec. 24, 1863; m. G. Burton Bacon, of Sheffield, Mass., May 2 I, I 887.

vii. Grace, b. Whitewater, Wis., Nov. I 2, I $865 ; \mathrm{m}$. Henry L. Wilcox, of South Egremont, Mass., Jan. I8, 1888.

viii. John, b. Sheffield, Mass., Jan. 24, I878. He d. Feb. I3, I879. 
GEORGE GRAHAM CURTISS (Ira, Abijah, Fonathan, Fonathan, Foseph, Thomas, Foln, Elizabeth), b. Sheffield, Mass., Sept. $\mathrm{I}, \mathrm{I} 825$; 11 . Mary, d. of Joseph $\mathrm{H}$. and Hannah Lewis, of Fallston, Md., June, I861. In I86 I he removed to Fallston, Md., where he now resides. $\mathrm{He}$ is a school-teacher and a civil engineer.

They had children :-

i. Ida, b. Sheffield, Mass., Aug. 5, I862. She is unm. and lives in Fallston, Md.

ii. Joseph, b. Sheffield, Mass., Jan. 23, I864. He is unm. and lives in Fallston, Md.

iii. Dora, b. Fallston, Md., Sept. 5, 1865. She is unm. and lives in Fallston, Md.

iv. Marion, b. Fallston, Md., March 8, 1867. She is unm. and lives in Fallston, Md.

v. Eva, b. Fallston, Md., April 23, 1868. She is unm. and lives in Fallston, Md.

vi. Ethel, b. Fallston, Md., March I2, I874. She is unm. and lives in Fallston, Md.

805

ABIJAH CURTISS (Orren, Abijah, Fonathan, Fonathan, Foseph, Thomas, Fohn, Elizabeth), b. Sheffield, Mass., March 12, I83 I ; m. Mary Elizabeth, d. of Rev. Selden and Catherine Butler Haines, at Skaneateles, N. Y., June 5, I 855 . He prepared for college at the district school in Sheffield, and entered the Troy Polytechnic School, from which he graduated in 1852 . In 1853 he removed to New York, where he became a prominent merchant. He was largely interested in the oil wells at Oil City, Penn. In 1873 he was offered the presidency of the Belt Line Surface Railroad, which position he accepted and filled for a number of years, resigning in favor of his brother. He then resigned from active business and retired to his estate at Yonkers-on-Hudson, N. Y. In I 884 he was elected president of the Sixth Avenue Surface Railroad, which position he accepted and held for a short period, resigning again in favor of his brother. He d. at Yonkerson-Hudson, N. Y., Aug. 2, 1888 . His widow resides in Yonkers.

They had children :-

(893) i. Frank Moody, b. New York City, N. Y., May 6, 1856; m. Florence Irene, d. of Ebenezer Franklin and Mary Robinson Tucke, of Exeter, N. H., Oct. I 2, I88 I ; m. (ii.) Josephine Thompson, of Yonkers-on Hudson, N. Y., 1894. He lives in Boston, Mass. 
ii. Kate Butler, b. New York City, N. Y., June 21, 1858 ; m. (i.) William B. Moore, of Yonkers-on-Hudson, N. Y., Oct. 25, I88 I ; m. (ii.) Dr. Isaac R. Sanford, of Sheffield, Mass., July 8, 1888 . She lives at Yonkers-on-Hudson, N. Y.

iii. Caroline, b. Yonkers-on-Hudson, N. Y., Sept. I3, I 862; m. John Quincy Adams Johnson, of Newburyport, Mass., Jan. 25, 1884. She lives at Yonkers-on-Hudson, N. Y.

(894) iv. Frederic Haines, b. Yonkers-on-Hudson, N. Y., Aug. I 4, I 869; $m$. Helen Lawrence, d. of Francis Orvis and Helen Lawrence Squire, of Arlington, Mass., Oct. I4, I891. He lives in Boston, Mass.

806

FRANKLIN CURTISS (Orren, Abijah, Fonathan, Fonathan, Foseph, Thomas, Fohn, Elizabeth), b. Sheffield, Mass., Dec. I5, I $838 ;$ m. Susan, d. of Simon Sage, of Seekonk, Mass., Jan. I 5 , r $862 ; \mathrm{m}$. (ii.) Ella, d. of Abner and Jane Spurr Roys, of Sheffield, Mass., June 5, 1878 . He removed to New York City, N. Y., about I 866 , and became a prominent merchant. In 1876 he was elected president of the Belt Line Surface Railroad, which position he held for a number of years. In I 884 he was elected president of the Sixth Avenue Surface Railroad, which position he still holds. He resides in New York City, N. Y.

They had children :-

i. Sophia M., b. Sheffield, Mass., April 15, 1863. She is unm. and lives in New York City, N. Y.

ii. William M., b. Sheffield, Mass., May 4, I868. He d. March Io, 1869.

iii. Roy Abijah, b. New York City, N. Y., Aug. 14, 188o. He lives in New York City, N. Y.

CHARLES HENRY CURTISS (Charles A., Abijah, Fonathan, Fonathan, Foseph, Thomas, Fohn, Elizabeth), b. Sheffield, Mass., Oct. 23,1833 ; m. Jannette, d. of Charles Wilson, of Shalerville, Ohio, April I 5, I 855 ; m. (ii.) Martha, d. of Dexter and Harriet Abbey, of Chardon, Ohio, April I6, 1865. He was taken by his father to Freedom, Ohio, in 1833 . During the Civil War he enlisted as a private in the 2 Ist Michigan Regiment, Company I, I 4th Army Corps, Ohio, July 31, I 862, and served thirty-five months. In 1883 he removed to Bassett, Neb., where he now lives, and is engaged in farming. 
They had children : -

(S95) i. Charles Albert, b. Freedom, Onio, Oct. I1, I 857 ; m. Lucinda, d. of George and Jane Vanderslip, of Bawn, Mich., Aug. 6, 1879. He lives in Freeport, Mich.

ii. Nellie Sabrina, b. Freedom, Ohio, Oct. 28, 1859. She d. Aug. $30,1863$.

iii. Maybel Edith, b. West Campbell, Mich., May 5, I866; m. William Moore, of Freedom, Ohio, March 21, I883.

iv. George Emerson, b. Freedom, Ohio, Sept. 4, I868.

v. Ida Castella, b. Freedom, Ohio, March, I, I 872; m. Fred C. Gale, of Bassett, Neb., March 10, 1888.

vi. Charles Dexter, b. Freedom, Ohio, Jan. 4, 1874. He d. Oct. 28, 1877 .

vii. Frederic Eugene, b. Freedom, Ohio, Nov. 21, 1875. He d. Aug. 9,1876 .

viii. Hadden Reuben, b. Freedom, Ohio, Jan. 28, 1878.

ix. Herbert Dewy, b. Bassett, Neb., Aug. 28, 1885 .

x. William Henry, b. Bassett, Neb., Feb. I 3, 189 I.

\section{8}

LOUIS EMERSON CURTISS (Charles A., Abijah, Fonathan, Fonathan, Foseph, Thomas, Fohn, Elizabeth), b. Freedom, Ohio, Oct. 17,1848 ; m. Harriet Minerva, d. of George and Harriet Williams, of Joliet, Ill., Dec. 19, I87I. He is a farmer and lives in Freedom, Ohio.

They had children :-

i. George William, b. Freedom, Ohio, Feb. 6, 1873 .

ii. Enma Lynn, b. Freedom, Ohio, March 14, 1876 .

iii. Albert Emerson, b. Freedom, Ohio, Aug. 8, i 877 . He d. July 8, 1878 .

iv. Ernest Morton, b. Freedom, Ohio, Nov. 14, 1880.

v. Charles Lewis, b. Freedom, Ohio, July 7, 1886.

vi. Lulu Rose, b. Freedom, Ohio, Feb. 28, 1889.

809

JASON PARKER CURTISS (Fames F., Fosiah, Fonathan, Fonathan, Foseph, Thomas, Fohn, Elizabeth), b. Westmoreland, N. Y., Feb. 4, 1830; m. He d. in Pioche, Nev., May 5, I879. He was a civil engineer and held the position of county surveyor of Lincoln County, Nev., and also those of deputy sheriff and United States marshal of that county.

They had children:-

(896) i. James Henri, b. Virginia City, Nev., Dec. 10, 1864. He lives in Ely, Nev. 
(897) ii. Francis Parker, b. Virginia City, Nev., Nov. 28, 1866. He lives in Sacramento, Cal.

8 I0

WINFIELD SCOTT CURTISS (Feremiah, Fesse, Fesse, Abner, Foseph, Thomas, Fohn, Elizabeth), b. Meriden, Conn., July 14, $1848 ; \mathrm{m}$. Elizabeth Grumley, Oct. 27, 1874. He lives in Meriden, Conn.

They had children :-

i. Wilfred Leroy, b. Meriden, Conn., Feb. 9, 1880. He d. Feb. 27, 1880.

ii. William Lester, b. Meriden, Conn., Aug. 25, 1882.

\section{1}

LUCIUS WELLINGTON CURTISS (Philo, Foshua, Simeon, Foshua, Foseph, Thomas, Fohn, Elizabeth), b. Bristol, Conn., Oct. 5, 1830 ; m. Hannah A. Barker, of South Meriden, Conn., May 15 , 1853. She d. Dec. I I, I 854 ; m. (ii.), Olive Webb Hotchkiss, of Meriden, Conn., Dec. 15, 1857. He lived in Meriden most of his life, but died in New Britain, Conn., April 27, 1885.

They had children :-

i. Nettie Barker, b. Meriden, Conn., Aug. 26, r860; m. George Horace Wilcox, of Meriden, Conn., Jan. 23, 1884.

ii. Bertha Olive, b. Meriden, Conn., April 33, 1865. She d.Sept. 1 1, 1865 .

JONAS PLATT CURTISS (Philo, Foshua, Simeon, Foshua, Foseph, Thomas, Fohn, Elizabeth), b. Bristol, Conn., March 29, 1832; m. Laura Ann Stevens, of Killingworth, Conn., Jan. 15, 1852 . He lives in New Britain, Conn.

They had children :-

i. Isadora Louise, b. New Britain, Conn., Nov. 14, 1852; m. Charles E. Andruss, in New Britain, Conn., Oct. 31, 1888.

ii. Hannah Amelia, b. New Britain, Conn., Jan. 14 , 1854 ; m. Roswell F. Perry, in New Britain, Conn., May 22, 1879.

814

EDWIN MILLS CURTISS (Philo, Foshua, Simeon, Foshua, Foseph, Thomas, Fohn, Elizabeth), b. Bristol, Conn., June I, $1837 ; \mathrm{m}$. Emeline Sheldon, of Bristol, Conn., Jan. 2, 1860. 
They had children :-

$\left(\mathcal{S}_{Q S} \mathcal{S}\right)$ i. Emerson Wardell, b. Meriden, Conn., Oct., 13, I86 I m. Emily Louise Sheldon, of Brewsters, N. Y., Nov. 26, 1883.

ii. Herbert Ellsworth, b. Meriden, Conn., Nov. 5, I 863. He d. May 7,1865 .

iii. Wallace Edwin, b. Bristol, Conn., June I 2, I866; m. Emily Clark, March 3, I885.

They had a child : -

i. Howard Everett, b. Bristol, Connn., Oct. 9, I887. He d. June IO, I889.

iv. Elbert Everett, b. Bristol, Conn., Oct. I I, I867. He was drowned Oct. I6, I890.

v. Frank b. Bristol, Conn., Dec. 10, I870. He d. Feb. I3, I871.

vi. Frederick \}b. Bristol, Conn., Dec. 10, 1870. He d. Feb. 14, 1871 .

vii. Ida May, b. Bristol, Conn., Nov. 23, 1872 .

viii. Franklin Burdette, b. Bristol, Conn., Aug. 22, 1875.

815

ROYAL CURTISS (Henry, Foshua, Simeon, Foshua, Foseph, Thomas, Fohn, Elizabeth), b. Burlington, Conn., Dec. 2 I, I 833 ; m. Almira Fulmer, Aug. 3I, I 865. He d. Aug. I, I 880, and his widow m. Mr. Traver and lives in Cooperstown, N. Y.

They had children : -

(S99) i. Roland Anthony, b. Burlington, Conn., Nov. 6, 1866.

(9oo) ii. Thomas Henry, b. Burlington, Conn., April Io, 1869 ; m. Agnes Group, Dec. I, I892. He lives in Cooperstown, N. Y.

\section{6}

AUSTIN CURTISS (Henry, Foshua, Simeon, Foshua, Foseph, Thomas, Fohn, Elizabeth), b. Burlington, Conn., Aug. 7, I836; m. Lucy J. Steele, Sept. I 8, I 86I. He is a merchant in Bristol, Conn.

They had children :-

(gor)i. Charles H., b. Bristol, Conn., Oct. I4, I864; m. Mary Smith, March 2, I887.

ii. George A., b. Bristol, Conn., July II, I875. He d. Aug. 3I, 1876 .

\section{I 7}

CORNELIUS CURTISS (Henry, Foshua, Simeon, Foshua, Foseph, Thomas, Fohn, Elizabeth), b. Burlington, Conn., July 6, I844; m. Effie J. Healey, Dec. 3I, I 870. 
They had children :-

(902)i. Isabelle E., b. Burlington, Conn., Feb. 21, 1872; m. William A. Andrews, Sept. 9, 1891.

ii. Burritt H., b. Burlington, Conn., May 4, 1873; m. Abbie O. Dorman, Dec. 24, 1895 .

iii. Wellington L., b. Burlington, Conn., April 4, 1875 .

8 I 8

WALDO LOREN CURTISS (Sylvester, Foshua, Simeon, Foshua, Foseph, Thomas, Fohn, Elizabeth), b. Burlington, Conn., Dec. 1, I 846 ; m. Mary, d. of Samuel Camp, of Plainville, Conn. She d. Feb. 5, 1875 , aet. 27 years. He m. (ii.) Nettie Root, of Berlin, Conn., Dec. 25, 1878 . He was educated in the public schools and finished at the Connecticut Literary Institute at Suffield, Conn., and the State Normal School, New Britain, Conn., For eighteen years after graduating he taught in several schools and then became interested in mining and later in manufacturing. He lives in New Britain, Conn.

They had children :-

i. Waldo Camp, b. Plainville, Conn., Jan. 25, 1875.

ii. Maud, b. Meriden, Conn., Jan. 23, 1880.

821

STILES WHITING CURTISS (Stiles, William, Silas, Stiles, Ephraim, Foseph, Fohn, Elizabeth), b. Norwalk, Conn., Sept. I 4, 1831 ; m. Harriet, d. of Eli Barnum and Anah Eversley Bennett, of Norwalk, Conn., Sept. 5, 1853. He resides in Norwalk, Conn., and has a steamship and pension agency. $\mathrm{He}$ is also a notary public.

They had children : -

i. Edwin Bennett, \} b. Norwalk, Conn., July 3, I 854.

(903) ii. Charles Eversley, b. Norwalk, Conn., July 3, I854; m. Anna Belden, d. of Lewis Judson and Fanny Curtiss, of Norwalk, Conn., Oct. 20, 1885 . She d. Aug. 29, 1890.

iii. Helen Birdseye, b. Norwalk, Conn., Dec. 17, 1858 .

822

WILLIAM CONWAY CURTISS (Stiles, William, Silas, Stiles, Ephraim, Foseph, Fohn, Elizabeth), b. Norwalk, Conn.; m. Fannie M. Norton, of Eastport, Me., Aug. 26, I 868 . He graduated from Williams College.

They had a child : -

i. Winterton Conway, b. Nov. 4, 1875 . 
DAVID SEYMOUR CURTISS (Stiles, William, Silas, Stiles, Ephraim, Foseph, Fohn, Elizabeth), b. Norwalk, Conn.; m. Cornelia 1. Raymond, of Norwalk, Conn., Dec. I 8, i 860 . He resides in Norwalk, Conn., and is commissioner of streets and sewers in that city.

They had children :-

(gof) i. Frederick Stiles, b. Norwalk, Conn., Oct. I I, I86 i m. Helen Purion, of Philadelphia, Pa.

ii. Walter Raymond, b. Norwalk, Conn., May 3, I863. He d. July 23,1864 .

iii. Fannie Isabella, b. Norwalk, Conn., Nov. 28, I867.

(905)iv. Raymond, b. Norwalk, Conn., Oct. I I, I869.

v. Marie, b. Norwalk, Conn., May I 2, 187 I.

vi. Seymour, b. Norwalk, Conn., May 29, I874.

vii. Keith, b. Norwalk, Conn., Dec. 29, I879; d. Aug. I I, I 88 I.

viii. Ruth, b.'Norwalk, Conn.; d. y.

\section{6}

STILES HENRY CURTISS (Charles, Stiles, Fohn, Stiles, Ephraim, Foseph, Fohn, Elizabeth), b. Summit County, O., May 27, $1846 ; \mathrm{m}$. Lucia Marcia, d. of Edwin and Marcia Lucia (Cobb) Stair, Sept. 30, I 875. He graduated from the Western Reserve College in 1867 with the degree of A.B., and received the degree of M.A. from the same college in 1870. After leaving college he practised law in Cleveland until the death of his father, when he succeeded him in business. He is a director in the Euclid Avenue National Bank, of Cleveland, Ohio, where he resides.

They had children :-

i. Charles Edwin, b. Cleveland, Ohio, Aug. i 8, 1876 .

ii. Henry Stiles, b. Cleveland, Ohio, Sept. 14, 1879 .

iii. Edwin Stair, b. Cleveland, Ohio, April 2, $188 \mathrm{I}$.

iv. Anna Marcia, b. Cleveland, Ohio, Nov. 24, I 885 .

\section{1}

ALEXANDER MAIN CURTISS (Cbarles G., Ezekiel, Fohn, Stiles, Ephraim, Foseph, Fohn, Elizabeth), b. Buffalo, N. Y., Oct. 6,$1856 ; \mathrm{m}$. Sophia Jane Colman, of Dunkirk, N. Y., June 9, 1880. He graduated from the New York Homœopathic Medical College in 1879 . In 1884 he was appointed surgeon for the West Shore R. R. at Buffalo. He is a director in the Third National Bank of Buffalo, N. Y., and also a trustee of the Fidelity Guaranty and Security Company of that city. He resides in Buffalo, N. Y. 
They had children :-

i. Colman, b. Buffalo, N. Y., Jan. 25, 188 r.

ii. Geoffrey, b. Buffalo, N. Y., Jan. 23, 1883 .

iii. Charles Gould, b. Buffalo, N. Y., July Io, 1884 .

$$
836
$$

CHARLES FRANKLIN CURTISS (Franklin, Morgan, Stiles, Elnathan, Ephraim, Foseph, Fohn, Elizabeth), b. Nora, Ill., Dec. 12, 1863; m. Olive Wilson, Feb. 16, 1893. He lives in Ames, Iowa.

They had children :-
i. Ruth, b.
ii. Edith, b.

\section{$83^{8}$}

HAMILTON BURTON CURTISS (Fudson, Fudson, Nehemiah, Foseph, Foseph, Foseph, Fohn, Elizabeth), b. Stratford, Conn., Aug. 4, I 842 ; m. Emma W., d. of David and Phœbe Curtiss, of Derby, Conn., October, 1874 . He is a merchant.

They had children:-

i. Walter Judson, b. Birmingham, Conn., May 10, I876. He d. in Redlands, Cal., "May I 2, I 896.

ii. David Raymond, b. Birmingham, Conn., Jan. I 2, I878.

iii. Ralph Hannilton, b. Birmingham, Conn., Feb. 8, 1880.

$$
840
$$

MELVILLE JUDSON CURTISS (Enoch, Fudson, Nehemiah, Foseph, Foseph, Foseph, Fohn, Elizabeth), b. Stratford, Conn., April 5, I 840 ; m. Clarissa F., d. of John and Mary Baldwin, of Milford, Conn., July I4, I 863. He lives in Stratford, Conn.

They had a child : -

(906)i. Robert Melville, b. Stratford, Conn., Jan. I, I865; m. Mary Stuart, d. of W. Alexander and Margaret Borden, of Partick, Scotland, Nov. 28, 1894. He d. Jan. 8, 1896.

\section{$84 \mathrm{I}$}

OTIS BEERS CURTISS (Enoch, Fudson, Nehemiah, Foseph, Foseph, Foseph, Fohn, Elizabeth), b. Stratford, Conn., Feb. 2, 1843; m. Harriett, d. of Andrew and Harriett Clark, of Nichols, Conn., Oct. 1 I, I 870. He is a dairyman, and resides in Stratford, Conn.

They had children :-

(907) i. Walter Stanley, b. Stratford, Comn., Dec. 27, 1871 ; m. Grace, d. of Frederick and Isabella Lillingston. 
ii. Clarissa May, b. Stratford, Conn., Sept. I 3, I 876.

iii. Clifford Clark, b. Stratford, Conn., April 3, i 882.

\section{7}

HOWARD J. CURTISS (Freeman L., Freeman, Nehemiah, Foseph, Foseph, Foseph, Fohn, Elizabeth), b. Stratford, Conn., June 29, I 857 ; m. Ellen V. Talcott, June 5, 1888 . He graduated from Yale College in $188 \mathrm{I}$, and from the Yale Law School in 1883. He was admitted to the bar of the State of Connecticut in 1883 . April 1, 1893, he was appointed Judge of the Court of Common Pleas of Fairfield County, and was reappointed April, I899. He resides in Stratford, Conn.

They had a child: -

i. Howard Whecler, b. Stratford, Conn., July 9, I89o.

\section{2}

FREDERICK CURTISS (Victory, Agur, Agur, Foseph, Foseph, Foseph, Fohn, Elizabeth), b. Stratford, Conn., 1842 ; m. Elizabeth E. Beck, of Bridgeport, Conn., May 3, 1870 . He served during the Civil War four years in the First Regiment Connecticut Cavalry as sergeant. He resides in Bridgeport, Conn.

They had children :-

i. Susan J., b. Bridgeport, Conn., July 8, I87 I.

ii. May L., b. Bridgeport, Conn., Nov. 2, 1873 .

iii. Ellen S., b. Bridgeport, Conn., Oct 9, I 877 .

iv. Frederick, b. Bridgeport, Conn., May 27, I 880.

853

JOHN CALVIN CURTISS (Victory, Agur, Agur, Foseph, Foseph, Foseph, Fohn, Elizabeth), b. Bridgeport, Conn., April 17, I845; m. Adela Stuart, of New Haven, Conn., Nov. 8, 1870. At the outbreak of the Civil War he enlisted as a private in Company I, Ninth Regiment Connecticut Volunteers, and served until mustered out at the close of the war. He rapidly rose through the various non-commission grades to the rank of sergeant-major, and at the time of his discharge was a first lieutenant. For especially brave service at the battle of Baton Rouge, La., in 1862, he received a medal of honor, voted to him by the United States Congress. He is general manager of the New England department of Adams Express Company, in whose employ he has been for thirty-six years. He resides in Boston, Mass. 
They had children :-

( $9 \circ 8)$ i. Victor Stuart, b. New Haven, Conn., Oct. $1871 ; \mathrm{m}$. Helen Louise, d. of Frederick Hawley, of Bridgeport, Conn., Oct. 25, 1893.

ii. Henry Sanford, b. New Haven, Conn., 1873 .

iii. Adeline Stuart, b. Bridgeport, Conn., Jan. 7, 1876 .

iv. John K., b. Stratford, Conn., Nov. 22, 1877.

v. Vera Cameron, b. Stratford, Conn., Oct. 5, 1879 .

vi. Gladys Hawthorne, b. Bridgeport, Conn., Aug. I 3, I 88 I .

vii. Clapp Spooner, b. Bridgeport Conn., 1884. He was drowned Aug. 14, 1889 .

viii. Sarah Glover, b. Bridgeport, Conn., May 3, 1886.

ix. Eliot Robertson, b. Bridgeport, Conn., May 13, 1890.

AUGUSTINE BEERS CURTISS (Cornelius A., Agur, Agur, Foseph, Foseph, Foseph, Fohn, Elizabeth), b. Stratford, Conn., April I 8,$1836 ; \mathrm{m}$. (i.) ; m. (ii.) Alice Beal, of Peru, Ind. He was a soldier in the War of Rebellion. He removed to Indiana in the early seventies, where he d. April 25, I $88 \mathrm{I}$.

They had children :-

i. Elmer, b. Derby, Conn., 1861 .

ii. William, b. Derby, Conn., 1863 .

iii. Lewis Charles, b. Peru, Ind., June 26, 1874. He d. June 24, 1894.

iv. Minnie Belle, b. Peru, Ind., Dec. 9, 1875. She d. April 23, 1882.

v. Frank Beal, b. Peru, Ind., March 14, 1877. He lives in New York City, N. Y.

\section{7}

THOMAS JEFFERSON BARNES CURTISS ( $\mathcal{F}_{0} \mathrm{~h} n$, Samuel, Abijah, Fohn, Fames, Benjamin, Fohn, Elizabeth), b. Medina, Ohio, Nov. 19, 1838 ; m. Nellie E. Hubbard, at Owatonna, Minn., Aug. 25, 1867. She d. in May, I 897 , aet. 49 years. He lived in Owatonna until i 883 , when he removed to Lincoln, Neb., where he now resides. He served three years in the late war. He is a member of the Lincoln Music Company.

They had children :-

i. Harriet, b. Owatonna, Minn., May 31, 1868; m. Fred Shepherd, of Lincoln, Neb. She d. May, 1897.

ii. Edna, b. Owatonna, Minn., March 12, 1877. 
SAMUEL CURTISS (Fohn, Samuel, Abijah, Fohn, Fames, Benjamin, Fohn, Elizabeth), b. Geneseo, Ill., May 18, I847; m.

They had children :-

i. Benjamin Richmond, b. Medford, Minn., June 8, 1879.

ii. Walter George, b. Medford, Minn., Sept. 30, 188I.

859

HENRY JAMES CURTISS (Fohn, Samuel, Abijah, Fohn, Fames, Benjamin, Fohn, Elizabeth), b. Geneseo, Ill., Dec. 20, I849; m. Phebe Gillett, at Owatonna, Minn., Jan. 19, 1872.

They had children :-

i. Myrtie, b. Northfield, Minn., Aug. 14, I874.

ii. Ella, b. Castle Rock, Minn., Jan. 17, 1878 .

\section{$86 \mathrm{I}$}

JOHN STOKES CURTISS (Samuel B., Samuel, Abijah, Fohn, Fames, Benjamin, Fohn, Elizabeth), b. Medina, Ohio, July 15, I $843 ; \mathrm{m}$. Sarah H. He was superintendent of the Home for Aged Women at Lodi, Ohio, where he now resides.

They had children :-

i. Clara Parthena, b. Medina, Ohio, Dec. 9, 1867; m. E. E. Harrington, of Chicago, Ill., May 27,1894 .

ii. Archy Ray, b. Medina, Ohio, Oct. 10, 1871 ; m. Jessie Stoddard, of Medina, Ohio, Oct. 5, 1898 .

862

FREDERIC HENRY CURTISS (Samuel B., Samuel, Abijah, Fohn, Fames, Benjamin, Fohn, Elizabeth), b. Medina, Ohio, Dec. I5, 1849 ; m. Medora C.; m. (ii.) Cora T. He is a mechanic and resides in Medina, Ohio.

They had children :-

i. Edna C., b. Medina, Ohio, Aug. 8, 1875 .

ii. Donald F., b. Medina, Ohio, June I, 1878 .

iii. Charlotte May, b. Medina, Ohio, April 20, 1884. She d. Nov. $4, \mathrm{I} 884$.

iv. Dorothy Lois, b. Medina, Ohio, Dec. 5, $189 \mathrm{I}$. 
EDWARD SHURTLEFF CURTISS (Samuel B., Samuel, Abijah, Fohn, Fames, Benjamin, Fohn, Elizabeth), b. Medina, Ohio, April 1 9, 1856 ; m. Emma D., d. of Richard E. Jones, of Sparta, Wis., May 5, 1885 . She d. April II, I 894 ; m. (ii.) Anna M. Miller, of Toledo, Ohio, Nov. 28, 1895 . He resides in Toledo, Ohio.

They had children :-

i. Arthur F., b. Wauseon, Ohio, March 8, 1886.

ii. Ray R., b. Wauseon, Ohio, July 31, I887.

iii. Harold Edward, b. Wauseon, Ohio, Dec. 19, 1893.

iv. Edward J., b. Toledo, Ohio, Oct. I I, 1898.

\section{7}

LEWIS BEERS CURTISS (Lewis A., Lewis, Agur, Samuel, Nathaniel, Zacbariah, William, Elizabeth), b. New York City, N. Y., June 30,$1863 ; \mathrm{m}$. Alice Minerva, d. of Samuel Gregory and Mary Beach Beardsley, of Long Hill, Conn., Oct. I 7, I 888. He was taken by his parents to Southport, Conn., in I 868 . In I 881 he graduated from the Hopkins Grammar School at New Haven, Conn., and studied law in Stanford, Conn., the two following years. From 1885 to 1887 he was business manager of the Church Record Association, resigning from that position to go into business with his brother Roderick P. Curtis, at Bridgeport, Conn. The firm of Curtis \& Curtis are prominent manufacturers of machinery and tools. His residence is at Southport, Conn.

They had a child :-

i. Alice Beardsley, b. Southport, Conn., Sept. 19, 1897.

\section{8}

ELLIOTT PLUMB CURTISS (Elliott P., Fustus B., Daniel M., Mitchell, Zachariah, Zachariah, William, Elizabeth), b. Nichols, Conn., July 26, I 853 ; m. Annabelle, d. of Ormel Hall, of Bridgeport, Conn., July I4, I 883 . He d. in Bridgeport, Conn., July i3, s 893 .

They had children :-

i. Clara Melville, b. Bridgeport, Conn., Dec. I I, 1884.

ii. Elliott Plumb, b. Bridgeport, Conn., May 7, 1886.

iii. Mildred Rebecca, b. Bridgeport, Conn., 1888.

iv. Louisa Hall, b. Bridgeport, Conn., I 890.

v. Elliott Plumb, b. Bridgeport, Conn., Jan. 15, 1892. 
NATHAN BULKLEY CURTISS (Elliott P., Fustus B., Daniel M., Mitchell, Zachariah, Zachariah, William, Elizabetb), b. Nichols, Conn., May I4, I 857 ; m. Mary Ann, d. of William Thatcher and Harriet Lavinia Gilbert, of West Granby, Conn., Oct. 20, I $88 \mathrm{I}$.

They had children :-

i. Clifford Gilbert, b. Bridgeport, Conn., Aug. 28, I883.

ii. Elizabeth Bulkley, b. Bridgeport, May 7, I885.

iii. John Burr, b. Bridgeport, Conn., Oct. 6, I887.

iv. Cornelia, b. Bridgeport, Conn., June 4, I89o. She d. Dec. 29, I891.

FREDERICK WILLARD CURTISS (Edwin R., Samuel B., Leverett, Samuel, Feremiah, Zachariah, William, Elizabeth), b. Madison, Wis., Feb. I1, 1860; m. Catherine W. Ford, of Madison, Wis., Oct. 3, 1882. He is a photographer and lives in Madison, Wis.

They had children :-

i. Mildred Harriet, b. Madison, Wis., Nov. 5, I883.

ii. Edwin Ford, b. Madison, Wis., Nov. 26, 1887.

iii. Frederick Willard, b. Madison, Wis., May 29, I895.

872

HENRY HOLBROOK CURTISS (William B., Holbrook, Salmon, Nehemiah, Benjamin, Fosiah, William, Elizabeth), b. New York City, N. Y., Dec. I 5,1856 ; m. Josephine, d. of Hugh and Josephine (Hall) Allen, of Brooklyn, N. Y., June I 9, 1884. He was educated at the Columbia Grammar School, the Gunnery at Washington, Conn., and the Cheshire, Conn., Military Academy, where he prepared for the Sheffield Scientific School of Yale College, from which institution he received the degree of $\mathrm{Ph} . \mathrm{B}$. in 1877. Three years later he received his degree of M.D. from the Yale Medical School. During one year of his medical studies he was in the office of Dr. Francis Bacon, of New Haven, as assistant. Dr. Curtis spent eighteen months in the Vienna and Paris hospitals. After his return from Europe he settled in New York City, N. Y., and began a general practice which he soon abandoned to make a specialty of the diseases of the respiratory tract. His mechanical genius led him to invent many instruments in nasal and throat surgery, which bear his name. $\mathrm{He}$ is a 
member of the British Laryngological Society and corresponding member of the Laryngological and Otological Society of France. $\mathrm{He}$ is also a member of the New York Academy of Medicine, the County Medical Society, and vice-president of the American Social Science Association. He is the author of many books and monographs on his specialty. Dr. Curtis resides in New York City.

They had children :-

i. Henry Holbrook, b. New York City, N. Y., April 6, i885. He d. Jan. $25, \mathrm{r} 888$.

ii. Marjorie Allen, b. New York City, N. Y., Nov. I I, 1888.

iii. William Edmond, b. New York City, N. Y., Jan. 4, 1890. He d. Aug. I 4, I 890.

FREDERICK KINGSBURY CURTISS (William E., Holbrook, Salmon, Nehemiah, Benjamin, Josiah, William, Elizabeth), b. New York City, N. Y., Feb. 3, I863; m. Marian Scott, d. of J. Montgomery Hare, of New York City, N. Y., Oct. 28, I 890. He was prepared for college at St. Paul's School, Concord, N. H., of which Rev. Henry A. C. Coit was then principal, and after six years of preparation, in 1880 entered Yale College, from which he graduated in 1884 , with the degree of $A . B$. In the autumn of the same year he entered the Columbia Law School, and obtained the degree of LL.B. in 1886 . This same year he was admitted to practice in New York State, and has ever since practised law with great success in the city of New York, and is at present a member of the firm of Curtis, Mallet-Prevost \& Colt, of that city.

They had a child:-

i. Mary Emlen, b. Dec. 20, I89 I ; she d. July I I, I 892.

$$
874
$$

GEORGE SAMPLE CURTISS (William H., Carlos, Philo, Benjamin, Benjamin, Fosiah, William, Elizabeth), b. Sunbury, Ohio, Aug. 3, 1833; m. Ann Eliza Jester, at Grandview, Ill., March 2, I 856 . She d. I 866 ; m. (ii.) Jerusha, d. of Joseph Walsh, of Monticello, Ill. He was a painting contractor, but retired from business in 1888 on account of ill health. He resides in Decatur, III.

They had children : -

(و०9) i. Cassius P., b. Indianapolis, Ind., Nov. 25, 1860; m. Lena, d. of Joseph Walsh, of Monticello, Ill., Nov. 25, I 883 . 
(rooo) ii. Ira Clifton, b. Oakland, Ill., Oct. 17, 1863; m. Flora, d. of Henry Bouta, at Indianapolis, Ind., Dec. 7, 1887 . He resides in Tuscola, Ill.

iii. Lowesa Jane, b. Newman, Ill., Oct. 7, r866; m. Mr. Hawkins, and lives at Camargo, Ill.

\section{5}

DON CARLOS CURTISS (William H., Carlos, Philo, Benjamin, Benjamin, Fosiah, William, Elizabeth), b. Sunbery, Ohio, Sept. 23 , 1835 ; m. Mary Hawkins McClure, widow of William McClure, at Arcola, Ill., Oct. 8, 1863. She d. Aug. 24, I 869. He is a painter, and resides in Tuscola, Ill.

They had children : -

i. Harry, b. Illinois, July 20, 1864. He d. Nov. 19, 1864.

ii. Elmer E., b. Illinois, Dec. I I, I865. He d. Oct 22, I884.

iii. $\int$ Milbern, b. Illinois, Nov. 20, r868. He d. Feb. 1, 1869.

iv. Wilber, b. Illinois, Nov. 20, 1868. He d. Feb. 19, 1869.

\section{7}

DERREL CURTISS (William H., Carlos, Philo, Benjamin, Benjamin, Fosiah, William, Elizabeth), b. Oakland, Ill., March 20, $1848 ; \mathrm{m}$. Frances Storks, of Westfield, Ill., in 1875 . He is a manufacturer of brooms, at Paris, Ill., where he resides.

They had children : -

i. Claude, b. Charleston, Ill., July 25, I $88 \mathrm{r}$.

ii. Harry, b. Charleston, Ill., July 25, I88 I ; m. Nora Bennett, of Paris, Ill., July I0, I897. He is in business with his father, at Paris, Ill.

They had a child

i. Ruth, b. Paris, Ill., June 24, 1898.

iii. S Maud, b. Garrett, Ill., Sept. 8, 1886. She d. March 5, I 892.

iv. \{Minnic, b. Garrett, Ill., Sept. 8, I 886. She d. March 6, 1892.

v. Grace, b. Tuscola, Ill., May I 8, 1890 .

vi. Blanch, b. Paris, Ill., Oct. 1 2, 1896.

\section{0}

CARLoS C. CURTISS (Philander C., Carlos, Philo, Benjamin, Benjanin, Fosiah, William, Elizabeth), b. Oakland, Ill., Dec. I6, 1845 ; m. Eliza Abbie Ficket, Oct. 27, I872. He resides in Wellington, Kan., and is connected with the Curtis Mercantile Company.

They had children :-

i. Fred Sterling, b. May 27, 1873 . 
ii. Mabelle Elizabeth, b. April 7, I875.

iii. Charles Hobart, b. April 8, 1878. He d. May 14, 1889.

iv. Lida Dot, b. Feb. 9, I 880 .

v. Son, b. May 5, 1882. He d. Aug. 20, i 882 .

vi. Bessie, b. Nov. 21, 1883. She d. June 19, 1884 .

vii. Gladys Cleo, b. Jan. I 7, I887.

STERLING PEMBERTON CURTISS (Philander C., Carlos, Philo, Benjamin, Benjamin, Josiah, William, Elizabeth), b. Oakland, Ill., June 19, 1858; m. Susan A. Taber, Aug. 18, 1879 . $\mathrm{He}$ resides in Oakland, Ill.

They had children :-

i. Herbert T., b. Oakland, Ill., March 23, I881. He d. Aug. I6, $188 \mathrm{I}$.

ii. Chase L., b. Oakland, Ill., Feb. 16, I896.

890

JOSEPH OSBORN CURTISS (William $V$. de W., Foseph, Abner, Reuben, Matthew, Fosiah, William, Elizabeth), b. New York City, N. Y., May 25, $1849 ; \mathrm{m}$. Ida F. Young. He resides in New York City, N. Y.

They had children:-

i. Martha Young, b. New York City, New York, 1877; m. George Thomas Stewart in 1898 .

ii. William Van der Water, b. New York City, N. Y., I88o.

\section{$89 \mathrm{I}$}

ELNATHAN ATWATER CURTISS (Wales $H$. , Reuben $B$, Simeon, Foseph, Israel, Stephen, Israel, Fohn, Elizabeth), b. Southbury, Conn., Feb. 19, 1859; m. Anna Isabel, d. of Thomas and Jane Hassard, of Ansonia, Conn., May 23, 1888 . He is a retail grocer and lives in Ansonia, Conn.

They had children :-

i. Mabel Hassard, b. Ansonia, Conn., April I 5, 1889.

ii. Raymond Hammill, b. Ansonia, Conn., Oct. 27, I 893.

\section{2}

EDWARD WHITEHEAD CURTISS (Albert $W$., Ira, Abijah, Fonathan, Fonathan, Foseph, Thomas, Fohn, Elizabeth), b. Whitewater, Wis., Sept. 7, I854; m. Grace, d. of Joel B. and Eliza Curtiss Merriman, of Sheffield, Mass., in Syracuse, N. Y., Jan. 
7, I 879. She d. in Chicago, Ill., March 2, 1893. He is a graduate of Williams College and is a mechanical engineer. $\mathrm{He}$ lives in Sheffield, Mass.

They had children :-

i. Howard M., b. Ithaca, N. Y., Sept. 23, 1879.

ii. Irving, b. Dixon, Ill., Sept. I I, I 882.

iii. Margaret, b. Chicago, Ill., Dec. 22, 1883 .

893

FRANK MOODY CURTISS (Abijah, Orren, Abijah, Fonathan, Fonathan, Foseph, Thomas, Fohn, Elizabeth), b. New York City, N. Y., May 6, 1856 ; m. (i.) Florence Irene, d. of Ebenezer F. and Mary Robinson 'Tucke, of Exeter, N. H., Oct. 12, 1881 ; m. (ii.) Josephine Thompson, of Farmington, Conn., in $1894 . \mathrm{He}$ entered Yale College in class of $\mathrm{I} 878$. He lives in Boston, Mass.

They had children :-

i. Sidney, b. New York City, N. Y., Sept. 23, I 882.

ii. Christine, b. Yonkers, N. Y., Dec. 6, 1884.

iii. Malcolm, b. Yonkers, N. Y., Feb. 28, 1889.

894

FREDERIC HAINES CURTISS (Abijah, Orren, Abijah, Fonathan, Fonathan, Foseph, Thomas, Fohn, Elizabeth), b. Yonkers-onHudson, N. Y., Aug. I 4, I869; m. Helen Lawrence, d. of Francis Orvis and Helen (Lawrence) Squire, of Arlington, Mass., Oct. 14, I 891. He entered Adams Academy, of Quincy, Mass., under Dr. William Everett, and graduated from there in 1887. The same year he entered Harvard College in the class of $189 \mathrm{I}$, but owing to ill health after two years he was forced to leave college, and in the summer of I 889 he went to Europe, where he passed the next year in study and travel. On his return he settled in Boston, Mass., and entered the Broadway National Bank of that city as clerk in January, 1890 . The following year he was elected assistant cashier of that bank and in I 899 cashier. In January, 1900, he accepted the position of cashier of the Massachusetts National Bank, which position he now holds. He is a director in that bank and a director in numerous other corporations and holds many positions of trust. He was the first-vicepresident of the Boston Bank Cashiers' Association. He resides in Boston, Mass.

They had children : -

i. Robert Lawrence, b. Boston, Mass., Jan. 2, 1898 . He d. Jan. I I, 1898 . 
ii. Eleanor, b. Boston, Mass., Aug. 2, 1899. She d. March 20, 1901.

\section{5}

CHARLES ALBERT CURTISS (Charles H., Charles A., Abijah, Fonathan, Fonathan, Foseph, Thomas, Fohn, Elizabeth), b. Freedom, Ohio, Oct. I I, I 857 ; m. Lucinda, d. of George and Jane Vanderslip, of Bawn, Mich., Aug. 6, 1879. He is a cabinet maker and resides in Freeport, Mich.

They had children :-

i. Jenny Belle, b. Campbell, Mich., Sept. I 9, 1880.

ii. Mattie May, b. Campbell, Mich., Jan. I 7, 1883.

iii. Susie, b. Freeport, Mich., June 8, i 885.

iv. Lewis Martin, b. Freeport, Mich., Sept. $10,1888$.

900

THOMAS HENRY CURTISS (Royal, Henry, Foshua, Simeon, Foshua, Foseph, Thomas, Fohn, Elizabeth), b. Burlington, Conn., April 10, 1869; m. Agnes Group, Dec. I, 1892. He lives in Cooperstown, N. Y.

They had children :-

i. Hazel May, b. Cooperstown, N. Y., Sept. 17, 1893.

ii. Royal, b. Cooperstown, N. Y., Feb. 28, 1896.

$9 \circ 9$

CASSIUS P. CURTISS (George S., William H., Carlos, Philo, Benjamin, Benjamin, Fosiah, IVilliam, Elizabeth), b. Indianapolis, Ind., Nov. 25, I860; m. Lena, d. of Joseph Walsh, of Monticello, Ill., Nov. 25, 1883 .

They had children : -

i. Eva May, b. Indianapolis, Ind., April 27, 1887.

ii. Kate, b. Indianapolis, Ind., Oct. I 8, 1888.

iii. Minnie, b. Indianapolis, Ind., April 24, 1891 .

iv. Jennie, b. Indianapolis, Ind., Oct. 17,1893 .

v. Freda, b. Indianapolis, Ind., Feb. 4, I895.

vi. James Herman, b. Indianapolis, Ind., Aug. 30, 1896.

\section{0}

IRA CLIFTON CURTISS (George S., William H., Carlos, Philo, Benjamin, Benjamin, Fosiah, William, Elizabeth), b. Oakland, Ill., Oct. I 7, I 863 ; m. Flora, d. of Henry Bouta, of Indianapolis, Ind., Dec. 7,1887 . He is a painting contractor-house, sign, and fresco - in Tuscola, Ill., where he lives. 
They had children : -

i. Montelle Clifton, b. Tuscola, Ill., Sept. 8, r889.

ii. Ida Fern, b. Tuscola, Ill., May 22, I 89 I.

iii. Cleona, b. Tuscola, Ill., Dec. 4, 1892 .

iv. L. Clyde, b. Tuscola, Ill., Sept. 2 I, 1894.

v. Susan Clarice, b. Tuscola, Ill., May II, 1897 .

THOMAS CUR'TISS (Nathaniel, Zachariah, William, Elizabeth), b. Stratford, Conn., ; m. Prudence, d. of Samuel and Abigail Welles, Jr., of Stratford, Conn. He died April 24, 1761.

They had children :-

i. Hannah, bapt. July 22, 1750 .

ii. Mehitable, bapt. April 8, I753; m. John Nichols, Jr. She d. April 9,1785 .

iii. Wells, bapt. Feb. 15, 1756; d. y. prob.

iv. Ruth, bapt. June II, 1758 .

v. Phoebe, bapt.

OLIVER CURTISS (Nathaniel, Zachariah, William, Elizabeth), b. Stratford, Conn., May, I73 I ; m. Hannah, d. of William Clark, of Derby, Conn., April 2, 1754. He d. in Derby, Conn., Jan. I 3, J794. She d. Oct. 3, I 803 , aet. 64 years.

They had children : -

i. Sheldon.

ii. Elizabeth, b. Derby, Conn., Feb. 28, r756; m. Gracey.

iii. Sarah, b. Derby, Conn., Aug. 26, 1757; d. y.

iv. (Sarah, b. Derby, Conn., May 12, I 765 .

v. Hannah, b. Derby, Conn.; m. Thompson.

vi. Eunice.

105

DAVID CURTISS (Fosiah, Fosiah, William, Elizabeth), b. Stratford, Conn., March IO, I739-40; m. Sally Burr. She d. March 6,180 I, aet. 60 years. He d. in Stratford, Conn., Nov. I, I819.

They had children :-

i. Comfort, b. Stratford, Comn.; m. Coe.

ii. David, b. Stratford, Conn.; m. Nancy Osborn, of Huntington, Conn., Oct. 3, 1833 .

iii. Sally, b. Stratford, Conn.; m. Phelps.

iv. Agur, b. Stratford, Conn., 177 1. He d. Oct. 9, 1776. 
MATTHEW CURTISS (Matthere, Fosiah, William, Elizabeth), b. Newtown, Conn., Feb. 4, 1746; m. Hannah Ford, March 25, 1773. He d. in Newtown, Conn., Nov. 29, 1796.

They had children:-

i. Judson, b. Newtown, Conn., Jan. 8, 1774.

ii. Polly, b. Newtown, Conn., I 775 .

iii. Richard, b. Newtown, Conn., Nov. 8, 1777 ; d. 1799.

iv. Sarah Ann, b. Newtown, Conn., Feb. 26, 1780.

204

ROBERT WELLES CURTISS (Edmund, Ephraim, Foseph, Fohn, Elizabeth), b. Stratford, Conn., March 12, I757; m. Phoebe, d. of Joseph and Martha Judson Curtiss, of Stratford, Conn., Nov.

9, 1780 . He d. in Monroe, Conn., I 854.

They had children:

i. Charity, b. Stratford, Conn., April I 4, I 78 I.

(IOOI) ii. Stiles, b. Stratford, Conn., Nov. 14, 1782; m. Nancy Thompson in 1804 . He died in Solsville, N. Y., Feb. I 8, I 858 . She d. in Solsville, N. Y., Nov. I 2, 1860 , aet. 74 years.

iii. Lewis, b. Stratford, Conn., March I 3, 1784.

iv. Asa, b. Stratford, Conn., Dec. 25, 1785 .

v. Welles, b. Stratford, Conn., Aug. 5, 1788.

vi. Isaac, bapt. Stratford, Conn., December, I 791.

vii. Hannah, bapt. Stratford, Conn., January, I 795 ; d. y.

viii. Charles, bapt. Stratford, Conn., August, 1796 ; d. y.

205

WILLIAM CURTISS (Elnathan, Ephraim, Foseph, Fohn, Elizabeth), b. Huntington, Conn., Aug. 24, 1750; m. Sarah.

They had children:-

i. Elizabeth Ann, b. Huntington, Conn., 1774 .

ii. William, b. Huntington, Conn., 1776.

iii. Eli, b. Huntington, Conn.

iv. Esther, b. Huntington, Conn.

v. Lucius, b. Huntington, Conn.

vi. Hepsa, b. Huntington, Conn.

vii. Lovie or Laura, b. Huntington, Conn.

viii. Phoebe, b. Huntington, Conn.

274

GEORGE PRESTON CURTISS (Ezra, Peter, fosiah, William, Elizabeth), b. Huntington, Conn., July 10, $1782 ; \mathrm{m}$. Sarah. He 
d. in Monroe, Conn., March 24, 1856. She d. Oct. 2, 1869, aet. 83 years.

They had children : -

i. Charlotte, b. Monroe, Conn., i8 I I ; m. Hill. She d. Oct. 3 r, 1894.

ii. John H., b. Monroe, Conn., 1819. He d. Sept. 27, I820.

iii. Henry, b. Monroe, Conn., I823. He d. Dec. 25, 1824.

\section{9}

HENRY TOMLINSON CURTISS (Henry, Peter, Fosiah, William, Elizabeth), b. Huntington, Conn., Sept. 21, 1796; m. Mary E. H. Wheeler. He d. in Fairfield, Conn., July I I, 1876. She d. Sept. I 5, I 886, aet. 64 years.

They had children :-
i. Julia A., b. 1842 . She d. Aug. 8,1856 .
ii. Julia W., b. Fairfield, Conn., Aug. 29, 1858.
iii. Julian.

286

JOSEPH CURTISS (Reuben, Matther, Fosiah, William, Elizabeth), b. Danbury, Conn., Sept. 19, 1782; m. Dorothy, d. of Stephen Hopkins, of Hampstead Harbor, L. I., in 1803 .

They had children :-

i. Alben, b. New York City, N. Y., I 807. He d. 1890.

ii. Anna, b. New York City, N. Y., 1809 .

iii. Alben, b. New York City, N. Y., 18I 2. He d. 1812.

iv. Joseph Reuben, b. New York City, N. Y., I8 13 ; m. Maria Robinson. He died February, I 886.

v. F.lla Maria, b. New York City, N. Y., 1816; m. Lewis G. Clark.

vi. Jane, b. New York City, N. Y., $1818 ; \mathrm{m}$. Telfair. She d. 1872 .

$$
388
$$

ELIAS CURTISS (Giles, Fohn, Fohn, Thomas, Fohn, Elizabeth), b. Sheffield, Mass., Aug. 28, I788; m. (i.) Mary Burrell, of Sheffield, Mass., June I 5 , I 8 I 2. She d. March 24, I 843 , aet. $5^{8}$ years; m. (ii.) Mrs. Desire Hewitt about I 850 ; m. (iii.) Mrs. Susan Aldrich, May, I857. She d. Feb. 27, I879. He was a farmer in Sheffield, Mass., where he d., Sept. 6, 1882.

They had children :-

i. Diana, b. Sheffield, Mass., Sept. 8, 1815 ; m. Watson Brown, Jan. 17,1843 . She d. in Michigan, Jan. Iо, 1886.

(214) 
ii. Bird, b. Sheffield, Mass., Aug. I 2, I 8 I7. He d. unın. in Indiana, Jan. 5, 1889 .

iii. Marcia A., b. Sheffield, Mass., Sept. 1, I819; m. John E. Lucas, of Springfield, Mass., May 17, 1843 . She d. in Springfield, Mass., Jan. 1 5, I 898.

(1002) iv. Jerome, b. Sheffield, Mass., Jan. 7, 1822; m. Catherine Johnson, of Sheffield, Mass., Oct. 31, 1844. She d. April 24, I872, aet. 46 years. He d. Feb. 19, 1888.

v. Adaline, b. Sheffield, Mass., March 20, 1824; m. Isaiah Thomas, Nov. I 3,1845 .

vi. Benjamin, b. Sheffield, Mass., Jan. I 5, 1826; m. Amelia Lapham, April 15,1851 .

1001

STILES CURTISS (Robert $W$. Edmund, Ephraim, Foseph, Fohn, Elizabeth), b. Stratford, Conn., Nov. 14, I 782 ; m. Nancy, d. of Nehemiah and Abigail Judson Thompson, of Stratford, Conn., 1 804. He was a farmer in Solsville, N. Y., where he d. Feb. I 8, 1858 . She d. in Solsville, N. Y., Nov. I 2, 1860 , aet. 74 years.

They had children :-

i. Charles, b. Solsville, N. Y., Sept. I 4, I805.

ii. Jane, b. Solsville, N. Y., April 24, 1807.

iii. Polly Ann, b. Solsville, N. Y., Nov. 27, I 810.

iv. Roswell, b. Solsville, N. Y., April I7, I8 82.

v. Lucetta, b. Solsville, N. Y., May I 5, I 8 I 5.

(1003)vi. Stiles, b. Solsville, N. Y., March 9, 1817; m. Priscilla, d. of Charles and Nancy Peckham, of Solsville, N. Y., Oct. I I, 1848 . She d. June 20, 1888 , aet. 61 years. He lives in Madison, N. Y.

vii. Nehemiah, b. Solsville, N. Y., Feb. 25, 1828.

\section{2}

JEROME CURTISS (Elics, Giles, Fohn, Fohn, Thomas, Fohn, Elizabeth), b. Sheffield, Mass., Jan. 7,1822 ; m. Catherine Johnson, of Sheffield, Mass., Oct. 31, 1844. She d. in New Marlboro, Mass., April 24, I872, aet. 46 years. He was a farmer and resided in New Marlboro, Mass., where he d., Feb. 19, 1888.

They had children :-

i. Mary E., b. New Marlboro, Mass., Aug. I3, 1846 .

ii. George A., b. New Marlboro, Mass., Oct. 22, 1847. He d. Jan. 26,1890 .

iii. Catherine, b. New Marlboro, Mass., Aug. I8, I85 I. She d. Oct. 6,1852 .

(Ioo4)iv. Joseph W., b. New Marlboro, Mass., Jan. 5, 1854; m. Ella L., d. of Sylvester and Philander (Jones) Barker, of Sandisfield, Mass., Feb. 20, 1878. 
v. William W., b. New Marlboro, Mass., April 6, 1860. He d. Oct. I4, I 86I.

vi. Frederic J., b. New Marlboro, Mass., April 29, 1863.

1003

STILES CURTISS (Stiles, Robert $W$., Edmund, Ephraim, Foseph, John, Elizabeth), b. Solsville, N. Y., March, 9, I817; m. Priscilla, d. of Charles and Nancy Peckham, of Solsville, N. Y., Oct. 11,1848 . She d. June 20,1888 , aet. 6 I years. He is a farmer and resides in Madison, N. Y.

They had children :- -

i. Minnie Jane, b. Solsville, N. Y., Oct. 3, I849. She is unm. and lives in Solsville, N. Y.

ii. Charles, b. Solsville, N. Y., Feb. 3, I854. He is unm. and lives in Solsville, N. Y.

\section{4}

JOSEPH W. CURTISS (Ferome, Elias, Giles, Fohn, Thomas, Fohn, Elizabeth), b. New Marlboro, Mass., Jan. 5, r 854 ; m. Ella L., d. of Sylvester and Philander (Jones) Barker, of Sandisfield, Mass., Feb. 20, I 878 . He is a civil engineer and surveyor, and lives in Great Barrington, Mass.

They had children :-

i. William E., b. New Marlboro, Mass., Nov. I, I 879.

ii. Fanny May, b. New Marlboro, Mass., Oct. 13, 1883. She d. Feb. 14,1889 . 
I should be glad to receive any additional data in regard to the Curtiss family which you may possess.

If, later, another edition of the work should be published, the additional data contributed will be included.

Be sure to send as complete data as possible.

\section{Address:}

Frederic H. Curtiss,

Box I 360 ,

Boston, Mass. 
v. William W., b. New Marlboro, Mass., April 6, I860. He d. Oct. I 4, 1861 .

vi. Frederic J., b. New Marlboro, Mass., April 29, 1863.

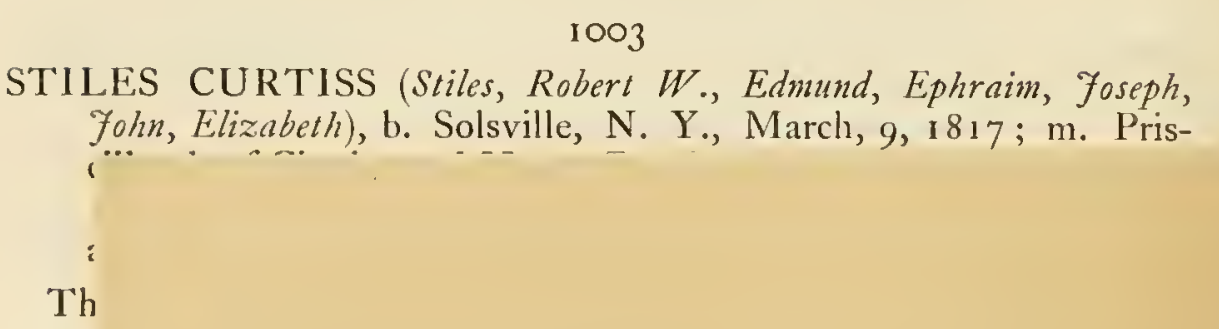

JOSE

Th 
I N D E X 



\section{N D E X}

Abbe, Julia, 97, r43.

Abbey, Dexter, 158, 195.

Abbey, Hannah, I 58, r 95.

Abbey, Martha, r 58 , 195 .

Abbott, W. N., I 74 .

Abel, Elizabeth, xxvi.

Abernathy, Jemima, 8, I 7 .

Abernathy, William, 8, 17 .

Abrams, Ella L., r44, I 86.

Acker, Mary, 134, 179.

Acker, Peter, r 34, r 79.

Adair, George, 126 .

Adams, - 2 r .

Adams, Clara, 4I, 76 .

Adams, Elijah, 76 .

Adams, Samuel, 26.

Addison, Thomas, 55 .

Alcott, Susan E., I 14 .

Aldrich, Susan, 2 I 4 .

Alexander, George, xxvi.

Alexander, Sarah, xxvi.

Allen, Abigail, 9o, r36.

Allen, Adeline Curtiss, 139.

Allen, Frances Angeline, 139.

Allen, Hugh, r $78,206$.

Allen, Josephine, r 78, 206.

Allen, Josephine Hall, I 78, 206.

Allen, Mary (White), 55 .

Allen, Micah, 55.

Allen, Silence, 55 .

Allen, William, 139 .

Allyn, John, xxiv.

Alsop, Thomas, ix.

Alvord, , 123.

Anderson, Alles, xviii.

Anderson, W. Thomas, r 46 .

Andrews, - 32 .

Andrews, Elizabeth, 35, 64 .

Andrews, Ezekiel, ${ }_{31}$.

Andrews, Huldah, 37, 68 .
Andrews, John M., 122.

Andrews, Lemuel, 84 .

Andrews, Mary Biclwell, 84, r 31 .

Andrews, Oliver, 6 I .

Andrews, Polly, 62, I05.

Andrews, Roxana, I $3 \mathbf{I}$.

Andrews, Whiting, $6 \mathrm{I}$.

Andrews, William, 37 .

Andrews, William E., 199.

Andrus(s), Daniel, I 5, 34 .

Andrus(s), Margery, r 5, 34 .

Andruss, - I 5 .

Andruss, Charles E., 197.

Andruss, Mary, 37 .

Antisdel, William, 97.

Atwater, Eunice, r 5, 33 .

Atwater, Hannah, 59, 99.

Atwater, Harriet, ro3, I 48 .

Atwater, Harriet F., I 44, r 86.

Atwater, James, 149.

Atwater, Mary, r 49.

Atwell, - 53 .

Atwood, Emma A., 177.

Atwood, Francis, I45.

Atwood, Harriet N., r 69 .

Atwood, Mary Curtiss, 145.

Austin, Moses, 20.

Averill, Eunice, r 44, 186.

Averill, Perry, r 44 .

Averill, Phineas, I 44, I 86.

Ayres, Sarah Elizabeth, 160.

Babbitt, David, 57.

Babcock, Ellen, I r 4 .

Bacon, - 38 .

Bacon, G. Burton, r 93 .

Badcocke, - xxvii.

Badger, Jane, 85 .

Bailey, Julia, I 26 .

Bailey, Lois K., I $04, \mathbf{I} 5$ r.

Baird, Oliver, I 10. 
Baker, Emeline, ro9.

Baldwin, Clarissa F., 169, 201.

Baldwin, Cornelia, 113 .

Baldwin, David S., 90 .

Baldwin, Ezra, 20.

Baldwin, John, 169, 201.

Baldwin, Mary, 169, 20 I.

Baldwin, Thaddeus, 79.

Bulwin, Mary C., 132.

Banks, Edwin, 95.

Banks, Ilenry, 92.

Barker, Ella L., 2 I 5, 2 I6.

Barker, Harriet A., I 59, 197.

Barker, l'hilander Jones, 215, 2 I 6.

Barker, Sylvester, 2 I 5,2 I 6.

Barlow, Edwin, I 29 .

Barlow, Florence A., I 74 .

Barnes, Asa, 48, 84 .

Barnes, Edwin, 154, 193.

Barnes, Frances M., 154, 193.

Barnes, Levi, 63.

Barnes, Maria Bristol, I 54, 193.

Barnes, Ruth, 48, 84 .

Barmes, Sarah, 4S, 84 .

Barnes, Simuel, 63 .

Barnum, Rachel, 135 .

Barnum, Samuel, 87. -

Barstow, Anne, xxix.

Barteau, — 77 .

Bartholomew, Augustus, 7 I.

Bartholomew, Phebe, I4.

Bartlett, Caroline, 91, 138.

Bartlett, John R., 97.

Barton, Cornelia, 162.

Barton, Rodney, 162.

Bartow, Jane Hanson, 116, 162.

Bascom, John, I 57.

Bateman, Luther, $3 \mathrm{I}$.

Bateman, Stephen, 3 I.

Bates, Phineas, 44 .

Beach, - 173 .

Beach, Benjamin, 5, 12.

Beach, Elizabeth, 56, 95 .

Beach, James, 19, 52 .

Beach, John, xviii, 5, 27, 70, 90 .

Beach, Mary, 5, 12.

Beach, Philo, I 72.

Beach, Trumball, 113 .

Beal, Alice, 1 $72,203$.

Beard, Samuel, 73, i 7 .

Beard, Sarah, 73,117 .

Beardslee, —, 93 .
Beardsley, Abraham, 18.

Beardsley, Agur, 53 .

Beardsley, Alice Minerva, 1 76, 205.

Beardsley, Andrew, $8_{3}$.

Beardsley, Ann, 43 .

Beardsley, Christa Ann, 89, I 35 .

Beardsley, Ezra Abel, 53.

Beardslcy, Gideon, 53.

Beardsley, Hall, 54.

Beardsley, John, xxiii, xxiv.

Beardsley, Mary 13., 1 76, 205.

Beardsley, Philo, 52.

Beardsley, Samuel, 52.

Beardsley, Samuel I., 176, 205.

Beardsley, Tabatha, 25, 49.

Beardsley, Zachariah, I I.

Beardsley, IVilliam, ix.

Beck, Elizabeth, I 72, 202.

Becker, Charles E., $15^{6}$.

Beebe, James, 18.

Beebee, - 34 .

Beech, Daniel, 9.

Beech, Hannah, 52, 89 .

Beech, Jemima, 7 .

Beech, John, 52, 89 .

Beech, Nathan, 7 .

Beech, Nathaniel, 8.

Beech, Phebe Curtiss, 52, 89.

Beech, Welles, 79.

Beecher, Alanson, 86.

Beers, Amasiah, 88.

Beers, Anna E., 141 , 185.

Beers, Cyrenius, 93.

Beers, Frank B., I 77 .

Beers, George, 141 , 185 .

Beers, Hannah, 1 22, 169 .

Beers, Joseph Davis, 82, 127.

Beers, Lewis, 50, 87 .

Beers, Mary Chapman, 82, 127 .

Beers, Mary Elizabeth, 82, I 27 .

Beers, Matthew, 74.

Beers, Otis, I 68.

Beers, Sarah Lucinda, 87, 132 .

Beers, Sarah Peck, I 4 I, 185 .

Beers, Silas, I 22, 169.

Beers, Susan, I 22, 169 .

Beers, Thomas, 88.

Belcher, Sarah, 173 .

Beldon, Lucius Sidney, I 60.

Bell, Newton Harvey, 150.

Benedict, Kerby, 134 .

Benham, Abijah, 16, 35 . 
Benham, Amelia, 104.

Benham, Darius, i o6.

Benham, Elizabeth L., I 7 .

Benham, Esther, 16, 31, 35, 60.

Benham, John, i 6.

Benham, Joseph, 15.

Benjamin, Charles, I87.

Benjamin, Edward, $\mathbf{3}^{2}$.

Bennet, Isaac, 47.

Bennet, Jernsha, 9 $1,136$.

Bennet, John F., $\mathbf{1} 63$.

Bennet, Nathan, I I.

Bennett, Amos, 88, 133.

Bennett, Benjamin, $2 \mathbf{x}$.

Bennett, Norah, 208.

Bennett, Sarah, I37, I 82 .

Bennett, Sarah Ann, 88, 133 .

Bertine, Helen A., I 33, 177 .

Besse, Mary, xxv.

Besse, Nehemiah, xxv.

Bidwell,. Abner, 108.

Bidwell, Barnabas, 109.

Binham, Sarah, 57.

Birch, Sally Maria, 88, I 34.

Birdsey, Comfort Welles, 25.

Birdsey, Dorothy, 72 .

Birdsey, Ella C., I 6 I.

Birdsey, Ezra, 73, I 16.

Birdsey, Hannah, 39, 72.

Birdsey, Helen, 73, I 6.

Birdsey, Isaac IV., 177 .

Birdsey, Linus, I6 I .

Birdsey, Nathan, 39, 72, I 18.

Birdsey, Phebe, 26, 73 .

Birdsey, Phebe Curtiss, I 6.

Birdsey, Philo, 72.

Birdsey, Ruth, 26, 52 .

Birdsey, Samnel, 23.

Birdsey, Saralı, 26, 5 r.

Birdsey, Vincy Ann, r6r.

Birdseye, Abel, 1 2, 25, 26, 52 .

Birdseye, Alice, 79.

Birdseye, Comfort, I 2.

Birdseye, Elizabeth, I2, 25 .

Birdseye, Ezra, $4 \mathbf{I}$.

Birdseye, Helen, 79.

Birdseye, Jolın, viii, ix.

Birdseye, Josiah, 79.

Bishop, Amelia, 137.

Bishop, Ann, 44, 80 .

Bishop, Bennet, 84 .

Bishop, Esther, 14, 32 .
Bishop, Hannah, 27.

Bishop, Phebe M., I 50, I9o.

Bishop, Prudence, 8 I.

Blackleach, Richard, xxiii, xxiv

Blackman, Asa, 46.

Blackman, Lucy, 56, 94 .

Blagg, Elisha, 10.

Blair, Frank P., 176.

Blakeman, —, 75 .

Blakeman, Abraham, 23.

Blakeman, Adams, viii, ix, ı .

Blakeman, Anna, 73, i 6 .

Blakeman, Anna H., 83, 129.

Blakeman, Ebenezer, 10.

Blakeman, Eli, 72.

Blakeman, Elijah, 75 .

Blakeman, Esther, 18, 42 .

Blakeman, Mercy, 42, 78 .

Blakeman, Rebecca, 10.

Blakeman, Samuel, 26.

Blakeman, Sarah, 73, 83, I I 6, 129.

Blakeman, Zachariah, 42, 73, 78, 83 , I 16 , I 29.

Blakesly, Martha A., 150, 190.

Blockman, A., I 5 .

Bogert, Edward C., r 28.

Bogert, Eva H., 128.

Bonney, Warren, 76 .

Booth family, viii.

Booth, Abel, 42.

Booth, Abigail, 86.

Booth, Abijah, 73 .

Booth, Anna, 44, 79 .

Booth, Bethia, 2, 8 .

Booth, Charity, 50, 86 .

Booth, Elizabeth, 2, 8.

Booth, George H., I67.

Booth, Henry D., I 05.

Booth, Hilkiah, Jr., 42 .

Booth, James, 86 .

Booth, John, 40.

Booth, Josiah, 42, 72, 77 .

Booth, Nathan Birdsey, 116.

Booth, Richard, ix, 2, 8 .

Booth, Sarah, 42, 77.

Booth, Sarah E., I $17,164$.

Booth, Zachariah, I 2.

Boquet, Julia, 163 .

Borden, Margaret, 201.

Borden, Mary Stuart, 20 .

Borden, W. Alexander, 201.

Bossett, Alena Maria, 186. 
Bostwick, Elizabeth, I $1,23$.

Bostwick, Elnathan, 92.

Bostwick, Polly Ann, 92.

Bostwick, Zachariah, r 1, 23.

Bosworth, Hannah, xxv.

Bosworth, Sarah, 156.

Botsford, - 53 .

Bouta, Fannie, I $3 \mathrm{r}$.

Bouta, Flora, 208,2 I r.

Bouta, Henry, 208, 2 r.

Bouta, W. E., 126.

Boydon, Darius, 70, 112.

Boydon, Relief, 70, 112.

Bradley, Abigail Ann, 105, I53.

Bradley, Eliza, 102, 147.

Bradley, Emeline Amelia, 105, 153.

Bradley, Julia, 62, Io6.

Bradley, Levi, ro5, 153.

Bradley, Mary, 59, 98.

Brainard, - I6 1 .

Branch, Ambrose, rog.

Branner, H. B., I 36 .

Bray, Thomas Robinson, 80.

Brazee, Alonson, $\mathrm{r}$ I I.

Bridges, James, 157 .

Briggs, Alvira, $137,182$.

Briggs, Martha (Wilson), I 37, 182.

Briggs, William B., 137,182 .

Brimsmaid, John, $x$.

Brinsmeads, - xxiv.

Bristol, Frank W., 155 .

Bristol, Sally, roo, r 46.

Broadwell, $\longrightarrow, 78$.

Bronson, Asa, 37.

Bronson, Hannah, 99, 145 .

Bronson, Jerusha, 60, 100.

Bronson, Thaddeus, 60 , 100.

Brooks, Abby Ann, 79 .

Brooks, Frederick, 124 .

Brooks, Isaac, 42.

Brooks, Jerusha, 84, r 30.

Brooks, Julia, ro6, 154 .

Brooks, Laura A., I I 2.

Brooks, Lucretia, 8o, 125.

Brooks, Miriam, xxvii.

Brooks, Nathaniel, xxvii.

Brooks, Rufus, 120.

Brooks, Ruth, 68, I 12.

Brooks, Thomas H., 165 .

Brooks, William, xxvii, 87 .

Brown, Dexter, 95.

Brown, E. C., 18 r.
Brown, Lucy, 57, 95 .

Brown, Mary, xviii.

Brown, Mary A., 94.

Brown, Park, 30.

Brown, IVatson, I I 4, 2 I 4 .

Brush, John, 76 .

Brush, Samuel Hitchcock, r 72.

Buched, Sarah, 124.

Buck, Frank, 97.

Buckingham, Enoch, 22.

Buckingham, Polly, 52, 90 .

Buckley, Charles, 96.

Buckley, Daniel, xviii.

Buckley, Sarah, 96.

Buel, Hannah, 20, 44 .

Buel, Julia, 66, rog.

Buel, Thomas, 28 .

Bulkley, Clarissa, I 29, 176.

Bunnell, $\longrightarrow, 64$.

Bunnell, Eben, 68.

Bunnell, Jeronimus, I 6 .

Buntinge, Joane, $x x$.

Burdseye, John, I.

Burgess, William, ${ }_{3} \delta$.

Burnes, Daniel, I 13 .

Burnham, Hannah, I r, 23.

Burnham, IVilliam, I r, 23 .

Burr, Hepzibah, 48,83 .

Burr, John, r 30.

Burr, Lucy, 79.

Burr, Peter, 8.

Burr, Sally, 2 I 2.

Burrell, Mary, 2 I 4 .

Burret, Steven, xxiii, xxiv.

Burritt, Anne, 120.

Burritt, Anthony, 50 .

Burritt, John, 39, 72.

Burritt, Mercy, 72.

Burritt, Phebe, 39, 72.

Burritt, Sarah, 76, I 20, I 30.

Burritt, Stephen, 76, 120.

Burroughs, Edward, r 8, 4 I.

Burroughs, Elizabeth, I 8, 41 .

Burroughs, Eunice, 21,45 .

Burroughs, Hannah, 9, 19.

Burroughs, John, 9, 19, 21, 45 .

Burroughs, Patience, 19,45 .

Burton, Benjamin, 9.

Burton, Newbury, ${ }^{2} 2$.

de Burton, William, xx.

Butler, Jane, r 34, I 80.

Butler, Maria, 58, 97 . 
Butler, Silas, 58, 97.

Butler, Susannah, 6I, 102.

Button, Bedotha Pierpont, 104, 152.

Cady, C., r 32 .

Cahoon, Stephen, 7 r.

Calhoun, Giles C., 1 I 4 .

Calkins, Henry A., I 61 .

Cam, Elnathan, 44, 52 .

Camp, Mary, xviii, 16r, 199.

Camp, Selina, 8o, 126 .

Camp, Samuel, r6r, rg9.

Campe, Richard, xxi.

Carpenter, Lewis B., 132 .

Carr, Anna, r83.

Carrington, Lyman Wheeler, 186.

Carroll, William, I 84 .

Carter, Abel, 49, 84, 85 .

Carter, Asabel, ro7.

Carter, James Coach, 49.

Carter, Laurinda, 49, 84 .

Carter, Rachel, 49, 85.

Carter, Rhoda Lewis, 49, 84, 85 .

Carter, Sarah, 85 .

Case, Eli, 36.

Cass, Anna, r 38 .

Casterling, Frances R., I60.

Catlin, Jacob, 70.

Caulkins, Stephen, I4.

Caunrad, B. Pliana, 138.

Chambers, Osborne, 1 I9.

Chapman, Alfred N., r8I.

Chapman, Mary, r6, 37 .

Chapman, Sarah M., I 25, I 74 .

Chase, Libens H.,70.

Chittenden, Mary, I 3, 29.

Clarencieux, Sir Richard St. George, xii.

Clark, Andrew, 169, 20 r.

Clark, Anna, 52.

Clark, Betsey, 47.

Clark, Charles H., ro5.

Clark, Emily, I98.

Clark, Emma Tinker, 192.

Clark, Haınah, 2 2, 2 I 2.

Clark, Harriett, $169,201$.

Clark, Henry A., 107.

Clark, John, 8 g.

Clark, Lewis G., 2 I 4 .

Clark, Miriam, 37, 68 .

Clark, Nettie, I92.

Clark, Otis Benjamin, r92.

Clark, Phebe, 2 r, 46.

Clark, Phineas, 24 .
Clark, Simeon, 64.

Clark, Thankful, 36 .

Clark, Thomas, xxvii.

Clark, William, 212.

Clarke, Abigail, I 1, 22.

Clarke, Anabel, 128.

Clarke, Charles McClellan, 166.

Clarke, Daniel, 5 .

Clarke, David IV., r 72 .

Clarke, Ephraim, I 2, 26 .

Clarke, Hester, 1 2, 26.

Clarke, Hezekiah, 15.

Clarke, Laura M., r 54, 193.

Clarke, Martha, 25, 50.

Clarke, IVilliam B., I 28.

Cobham, Richard, Lord, xx.

Coe, $\longrightarrow, 2$ I 2.

Coe, Benjamin, 80.

Coe, Hannah, 9, 20.

Coe, John, 9, 20.

Cogswell, Sherman, 148 .

Cole, Abigail, 32.

Cole, Edward F., 144 .

Cole, Eunice, I 4, 32 .

Cole, Joseph, 32 .

Colin, Sir Arthur, xii.

Collins, Daniel, 6r, 102.

Collins, Martha, 8, 16 .

Collins, Molly, 6r, roz.

Collins, William, I 6 .

Coleman, Alvin Morelle, $13^{8 .}$

Colnan, Sophia Jane, I 66, 200.

Combs, Charity, $17,3^{8}$.

Cook, Anna Bronson, 155.

Cook, Hope, 1 4, 3 .

Cook, Joseph, 155.

Cook, Lumand, 64.

Cook, Mirriam, I 4, 3 r .

Cook, Sally Leavenworth, 155 .

Cook, Sanuel, r 4, 31 .

Cooke, Sarah, 107.

Cooke, William, xxvii, xxix.

Corbin, Elvira, I 50, I 90.

Corbin, Healy, 150, I90.

Corbin, Waldo, I 48.

Corner, Elnathan, I 1 .

Corwin, Edwin, I 80.

Cotton, John, xxv.

Cowan, Rachel, 57, 96.

Cowles, Fanny, io8.

Cowles, Solomon, ro\$.

Crain, John, 7. 
Crain, Mary, 7 .

Cramer, Henry, 28.

Crane, - , 13 .

Crocker, Walter, ıog.

Crofton, Ida Hannah, 187 .

Crombie, Darius, 109.

Cummings, Anson, I 42.

Cummings, Isaac, 82 .

Currier, Asa, 107.

Curtis, Anne, r 5, 75 .

Curtis, Asa, 73.

Curtis, Asa S., 83 .

Curtis, Joseph, 15 .

Curtis, Lois, I 5 .

Curtis, Philip, $\mathbf{r} 7$.

Curtis, Thomas, 75 .

Curtiss, - 22.

Curtiss, Aaron, 32, 38, 44, 46, 56, 61, 71, 76,79 , 102, I 13,159 .

Curtiss, Aaron Lester, 125.

Curtiss, Abbie, $15, \times 15$.

Curtiss, Abby, 79.

Curtiss, Abel, ro, 22, 26, 3r, 43, 5 r, 53, $59,60,62,78,88,99,104$.

Curtiss, Abel S., 96 .

Curtiss, Abel Sherman, 58 .

Curtiss, Abiah, r $3,28,75$.

Curtiss, Abigail, xxviii, xxix, 5, 7, 8, ro, I 1, $\mathrm{r}_{2}, \mathrm{r}_{3}, \mathrm{r}_{5}, \mathrm{I}_{7}, \mathrm{r}_{9}, 20,24,28,32$, $42,45,55,62,63,69,72,77,107,114$, I $23, \mathrm{I} 24$.

Curtiss, Abigail.Avis, $x \times 6$.

Curtiss, Abigail Bates, r 73 .

Curtiss, Abigail Betsey, 73 .

Curtiss, Abijah, 2 r , 26, $31,36,44,46,51$, 67,80 , r хо, r 1 1, 157, r 94 .

Curtiss, Abijah Beach, 9o, I 36 .

Curtiss, Abijah Birdsey, 52, 90.

Curtiss, Abner, 16, 25, 27, 37, 50, 55, 6o, $68,92,93$.

Curtiss, Abraham, 12, 25, 28, 49 .

Curtiss, Achseh, 37 .

Curtiss, Ada, r 5, 34 .

Curtiss, Ada Florence, $19 r$.

Curtiss, Ada May, r62.

Curtiss, Adaline, I 55, 215 .

Curtiss, Adelaide Asenath, 152.

Curtiss, Adele L., r8g.

Curtiss, Adelia, 123,142 .

Curtiss, Adeline, 55, I 14 .

Curtiss, Adeline Allen, I 40.

Curtiss, Adeline F., 160 .
Curtiss, Adeline Stuart, 203.

Curtiss, Adna, 65, ro8.

Curtiss, Agnes, 65 .

Curtiss, Agnes D., 132.

Curtiss, Agnes Deshon, 154 .

Curtiss, Agnes J., I 93.

Curtiss, Agnes Mansfield, I 93.

Curtiss, Agur, 25, 42, 47, 50, 52, 72, 78 , $79,82,90,122,124,169,2$ 工 2.

Curtiss, Alanson, 35, 104, 130, 152.

Curtiss, Alanson Doty, I $8 z$.

Curtiss, Alathea, 60.

Curtiss, Alben, 55, 214 .

Curtiss, Albert, 129 , I 58, 1 76 .

Curtiss, Albert Arthur, r 7 o.

Curtiss, Albert Emerson, r 96.

Curtiss, Albert Willoughby, I 56, 193

Curtiss, Albertina, r 68.

Curtiss, Albertina C., 83 .

Curtiss, Alexander Main, I66, 200.

Curtiss, Alford, 99.

Curtiss, Alfred, 89, ror, r 24, r35, I 73.

Curtiss, Alfred Azor, ro 7 .

Curtiss, Alfred Devine, 5 r, 88.

Curtiss, Alfred H., I 66.

Curtiss, Alfred Halsey, 184 .

Curtiss, Alfred Leonard, r40, I 84 .

Curtiss, Alfred Pierpont, r05, I 53 .

Curtiss, Alfred Tyler, r $8 \mathrm{r}$.

Curtiss, Alfred William, r 53, r 92.

Curtiss, Alice, $\mathrm{xx}, \mathrm{x} 5,46,47,74,78$, 173.

Curtiss, Alice Antoinette, 153.

Curtiss, Alice Beardsley, 205 .

Curtiss, Alice E., I $7 \mathbf{r}$.

Curtiss, Alice Louise, r 73 .

Curtiss, Alice Mary, I 88.

Curtiss, Allen Barnes, 84.

Curtiss, Allen Stansbury, r 39, 133 .

Curtiss, Alma, 87 .

Curtiss, Almira, 65, 1 08, 1 1 0, I 20.

Curtiss, Almira C., 96 .

Curtiss, Almond, 57, 96 .

Curtiss, Alonzo, r 42.

Curtiss, Alonzo Parker, 158.

Curtiss, Alvan, 69.

Curtiss, Alvin, 84 , r 30 .

Curtiss, Alvira Thresa, 97.

Curtiss, Amanda, 67, 70, ror.

Curtiss, Amasa, 36, 6r, 63, 66, 109 .

Curtiss, Amasa Horatio Nelson, ro6.

Curtiss, Amelia, r 04 , 14. 
Curtiss, Amos, 28, 33, 37, 59, 61, 62, 69, I04.

Curtiss, Amy, 54, 60, 113 .

Curtiss, Amzi, 117.

Curtiss, Andrew, 40, 45, 6r, 74, 77, ror, I 46.

Curtiss, Andrew Jackson, r 60.

Curtiss, Angeline, 55, 122.

Curtiss, Ann, xxvii, xxix, 17, 25, 27, 28, $33,41,43,46,71,72,78,90$, I 18 , I 22, $123,150,17$ I.

Curtiss, Ann Amelia, I 60 .

Curtiss, Ann Elizabeth, ${ }_{4} 6$.

Curtiss, Ann Eloisa, 92.

Curtiss, Ann Rebecca, 155 .

Curtiss, Anna, $8,10,12,1 S, 20,21,22$, $32,45,47,49,50,5$ I, 58, 75, 79, 8г, I 5 , I $23, \mathbf{r}_{29}, \mathrm{I}_{42}, 144,182$, I $S_{5}, 2$ I 4 .

Curtiss, Anna Augusta, r 68 .

Curtiss, Anna Belden, 163, 199.

Curtiss, Anna E., I 55 .

Curtiss, Anna Elizabeth, 125.

Curtiss, Anna Hall, I 51.

Curtiss, Anna Marcia, 200.

Curtiss, Anna Maria, I 27,165 .

Curtiss, Anne, 24, 39, 40, 41, 60, 74, 78, So, I 22.

Curtiss, Anne Aspinwall, r 28 .

Curtiss, Annie Augusta, r 84 .

Curtiss, Annis, 52, 75, 9r, roo.

Curtiss, Anthony, xix, xxi.

Curtiss, Antoinette, 94 .

Curtiss, Apame (Appime), $5 \mathbf{r}$.

Curtiss, Arcelia Lacella, is r.

Curtiss, Archy Roy, 204.

Curtiss, Artensisia, 5 r, 156.

Curtiss, Arthur Clayton, $18_{3}$.

Curtiss, Arthur Edward, 187.

Curtiss, Arthur F., 205.

Curtiss, Asa, 3o, 38, 58, 2 I 3 .

Curtiss, Asahel, 63, ro5, ro6, 153 .

Curtiss, Asahel Benham, ro2, r 48 .

Curtiss, Asahel Harrison, 5 r.

Curtiss, Asenath, 36 .

Curtiss, Ashbel, $121,168$.

Curtiss, Atheldred, 36 .

Curtiss, Augusta, r 42.

Curtiss, Augusta I onisa, r 48.

Curtiss, Augustine Beers, I 72, 203.

Curtiss, Augustus, 79, 85 .

Curtiss, Aurilla, 5 r, 52, 88 .

Curtiss, Aurora, 67 .
Curtiss, Austin, I60, 198.

Curtiss, Azor, $15,34,64$, ro7.

Curtiss, Azubah Smith, 69.

Curtiss, Barnes, I 27.

Curtiss, Bathsheba, r 8 .

Curtiss, Beatrice, 56 .

Curtiss, Beech, 76, 119.

Curtiss, Belle, 5 o.

Curtiss, Benjamin, xxv, xxix, 3, 9, 12, $14,20,23,25,26,31,43,5 \mathrm{I}, 52,60$, $6 \mathrm{r}, 76,82,94,100,102,114,127,141$, 142,215 .

Curtiss, Benjamin Farquhar, r $2 \mathrm{~S}$.

Curtiss, Benjamin Lewis, 27 .

Curtiss, Benjamin Lincoln, I 45 .

Curtiss, Benjamin Lindslay, 174.

Curtiss, Benjamin Richmond, 204.

Curtiss, Benjamin Stiles, 99, I 45.

Curtiss, Benjamin Strong, roo.

Curtiss, Benjamin Upson, ro6.

Curtiss, Benoni, 69.

Curtiss, Beri, r 42.

Curtiss, Bertha, 185 .

Curtiss, Bertha Celia, r 35 .

Curtiss, Bertha Olive, 197.

Curtiss, Bessie, I 76, 209.

Curtiss, Bessie Margaret, r68.

Curtiss, Bethia, 18,19 .

Curtiss, Bethiah, 9, то, 26.

Curtiss, Betsey, 41, 46, 53, 55, 60, 64, $66,70,72,75,76,77,86,88,89,9$ I, 94, I O9, I I I, I I 4, 124.

Curtiss, Betsey Lorage, Io7.

Curtiss, Betty, 56 .

Curtiss, Beula, 8 I.

Curtiss, Beulah, 22, 23 .

Curtiss, Bird, r I 4 , 2 r 5 .

Curtiss, Birdie May, 187 .

Curtiss, Birdsey, г т.

Curtiss, Birdsey Glover, 90

Curtiss, Birdseye, 78,9 r.

Curtiss, Blanch, 208.

Curtiss, Bradley, ror, r 42 .

Curtiss, Burr, 57, 95.

Curtiss, Burritt, 72, 73, r 20.

Curtiss, Burritt H., I 99.

Curtiss, Burton Samuel, r 62.

Curtiss, Byron, $142,18_{5}$.

Curtiss, Caleb, xxvii, $17,38,66,67,69$, I IO.

Curtiss, Carlos, $48,84,88,89$, r 20,130 , I 34 . 
Curtiss, Carlos B., roo.

Curtiss, Carlos C., r8 8 , 208.

Curtiss, Carlos E., 126 , 175 .

Curtiss, Carlos G., 90, 136 .

Curtiss, Carlotta May, 147.

Curtiss, Caroline, 90, 93, 98, 102, 117 , $123,130,195$.

Curtiss, Caroline Amanda, r 12.

Curtiss, Caroline Booth, 164 .

Curtiss, Caroline Elizabeth, 132 .

Curtiss, Caroline Halsey, 139 .

Curtiss, Caroline M., 137, r89.

Curtiss, Caroline Maria, 97 .

Curtiss, Carrie Ball, r 74 .

Curtiss, Carrie M., r6r.

Curtiss, Cassius P., 207, 2 I 1 .

Curtiss, Catharine, 87.

Curtiss, Catherine, xviii, $33,62,69,72$, 76, 114, II9, 120, 215 .

Curtiss, Catherine Jane, 86, 131 .

Curtiss, Catherine Lewis, 172.

Curtiss, Catherine Sophia, I63.

Curtiss, Catherine Stansbury, 140.

Curtiss, Catherine Ufford, 1 I 5 .

Curtiss, Celestia, 9 I, II3.

Curtiss, Celia, 185 .

Curtiss, Celia Jennette, 152 .

Curtiss, Chandler, ror.

Curtiss, Charity, 19, 38, 41, 42, 87, 213.

Curtiss, Charles, I $2,27,36,46,54,63$, $66,76,8$ r, 82, 85, 88, 89, 98, 99, rо7, I 8 , I I 7,120 , I 23,126, I 35, I 37,14 I, $155,162,165,182,213,215,216$.

Curtiss, Charles Abbey, III.

Curtiss, Charles Albert, 1 96, 2 I I.

Curtiss, Charles Atwater, I 86.

Curtiss, Charles Augustus, $103,150$.

Curtiss, Charles B., I 66.

Curtiss, Charles Birdsey, $3_{3}^{8}$, I 83 .

Curtiss, Charles Burke, 99.

Curtiss, Charles Butler, 97, I 43.

Curtiss, Charles Dexter, 196.

Curtiss, Charles Dillisenea, 87, 132 .

Curtiss, Charles Edmund, 165 .

Curtiss, Charles Edwin, 200.

Curtiss, Charles Elihu, I I 5 .

Curtiss, Charles Eversley, I63, I 99.

Curtiss, Charles F., I 56 .

Curtiss, Charles Franklin, $167,201$.

Curtiss, Charles Gould, r18, 166, 201.

Curtiss, Charles H., I 83 , 198.

Curtiss, Charles Halsey, 140.
Curtiss, Charles Henry, 97, I I 7, I 9 , $121,142,158,164,167,184,187$, I 95 .

Curtiss, Charles Hobart, 209.

Curtiss, Charles Lewis, 1 $27,163,196$.

Curtiss, Charles Main, 165.

Curtiss, Charles Merrick, 143.

Curtiss, Charles Ransom, 60, 99.

Curtiss, Charles Ruffin, 188.

Curtiss, Charles S., 91, r 09, 138 .

Curtiss, Charles Strong, 145, 88.

Curtiss, Charles Sypert, 145 .

Curtiss, Charles T., roo.

Curtiss, Charles W., I 55 .

Curtiss, Charles Wadsworth, 125.

Curtiss, Charlotte, 60, 75, 90, $\mathrm{x}_{34}, \mathrm{r} 65$, 214.

Curtiss, Charlotte Catherine, $18 x$.

Curtiss, Charlotte Electa, 166.

Curtiss, Charlotte Levina, 180.

Curtiss, Charlotte M., 7 I, I I 3 .

Curtiss, Charlotte Marietta, I59.

Curtiss, Charlotte May, 204.

Curtiss, Charlotte Nichols, 133 .

Curtiss, Chase L., 209.

Curtiss, Chauncey, 6r, 68, ror, r04, r27, $15 \mathrm{I}$.

Curtiss, Chester, 70, roo, ro6.

Curtiss, Chestina, 36,65 .

Curtiss, Chloe, 66.

Curtiss, Chloe R., rog.

Curtiss, Christe Ann, 121.

Curtiss, Christina, I33.

Curtiss, Christine, 2 Io.

Curtiss, Christopher, xix.

Curtiss, Clapp Spooner, 203.

Curtiss, Clara, $126,156$.

Curtiss, Clara Melville, 205.

Curtiss, Clara Parthena, 204.

Curtiss, Clara Samantha, 175.

Curtiss, Clarence, 165.

Curtiss, Clarence Alfred, 192.

Curtiss, Clarissa, 79, 102.

Curtiss, Clarissa Jane, 96.

Curtiss, Clarissa May, 202.

Curtiss, Clark, 68.

Curtiss, Claude, 208.

Curtiss, Clemence, I 40.

Curtiss, Cleona, 2 I 2.

Curtiss, Clifford Clark, 202.

Curtiss, Clifford Gilbert, 206.

Curtiss, Clinton, 7 I. 
Curtiss, Cliuton James, 162.

Curtiss, Colman, 201.

Curtiss, Comfort, 1 5, 22, 32, 212.

Curtiss, Comfort (Foot), I I 3, I 59.

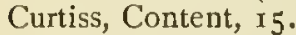

Curtiss, Cora May, 177 .

Curtiss, Cora T., I 75, 204.

Curtiss, Cordelia, ז 44, i 80.

Curtiss, Cordelia Aurelia, 152.

Curtiss, Cornelia, 62, 70, 71, 86, 1 34, 206.

Curtiss, Cornelia Mary, 95.

Curtiss, Cornelius, i 17, I $60,172,198$.

Curtiss, Cornelius Agur, I 24, I 72, 173.

Curtiss, Coydon, I 13,161 .

Curtiss, Currence, 58 .

Curtiss, Cynthia, 107.

Curtiss, Cyrenius, 89, 12 I , 135 .

Curtiss, Cyrus, 60, 86, г 00, I $3 \mathbf{1}$.

Curtiss, Cyrus Stebbins, 132 .

Curtiss, Daisy, I 62 .

Curtiss, Damaris, 38,68 .

Curtiss, Damon C., I 38 .

Curtiss, Dan Collins, I03, 148.

Curtiss, Daniel, 4, 5, 1 0, I 3, 14, I 8, 2 I, $30,31,37,44,46,56,73,76,81,83$, 95,98 , I I 8 , I 2 I, 143 .

Curtiss, Daniel Averill, 186.

Curtiss, Daniel Brewster, 27.

Curtiss, Daniel Mason, 193.

Curtiss, Daniel Mitchell, 48, 83 .

Curtiss, Daniel Reed, ro2.

Curtiss, Darius Boyden, I 12.

Curtiss, Dauphna, 70.

Curtiss, David, 9, 13, I9, 20, 25, 27, 29, $30,37,39,40,43,44,45,46,55,5^{6}$, $57,58,68,74,75,80,81,83,91,94$, $96,125,130,137,143,169,174,201$, 212 .

Curtiss, David B., 8o, 90.

Curtiss, David Huntington, 98, I 43.

Curtiss, David R., 96.

Curtiss, David Raymond, 2 ог.

Curtiss, David Seymour, I63, 200.

Curtiss, David Stiles, 58, 98.

Curtiss, David W., 162.

Curtiss, Day, 48.

Curtiss, Deborah, xxvii, I I, 36, 55, 65, I 08 .

Curtiss, Deborah H. G., $103,149$.

Curtiss, Decidamia, 35 .

Curtiss, Delia, 53, I 30.

Curtiss, Deliverance, 34 .
Curtiss, Della, I 93.

Curtiss, Delos, 137 .

Curtiss, Deodate, xxv.

Curtiss, Derrel, r 80, 208 .

Curtiss, Derwin, 134 .

Curtiss, Desire, г 2, т 5, 27,34 .

Curtiss, DelVitt, 7 г .

Curtiss, Diana, I I 4, 214 .

Curtiss, Diantha, 66.

Curtiss, Dillisenia, 87 .

Curtiss, Dinah, $20,44,80$.

Curtiss, Dolly, 79, 122 , 130 .

Curtiss, Donald F., 204.

Curtiss, Don Carlos, $180,208$.

Curtiss, Dora, I94.

Curtiss, Dorothea, xx.

Curtiss, Dorothy Lois, 204.

Curtiss, Dorre Rose, I 37.

Curtiss, Drayton Mills, 66 .

Curtiss, Earl, I 85 .

Curtiss, Earl Stevens, I 55.

Curtiss, Ebenezer, xxv, 4, 5, 9, 1 I, 22, 4 I, 44,46 .

Curtiss, Eben Birdsey, 52, 9 I.

Curtiss, Edgar A., 146.

Curtiss, Edgar Wilcox, 151 .

Curtiss, Edith, I 88, 2 0 . .

Curtiss, Edith Maria, I 68.

Curtiss, Edmund, $18,20,21,40,45,7$ I, $76,8 \mathrm{I}, \mathrm{I} 08$.

Curtiss, Edmund B., I02, 147.

Curtiss, Edna, 7 I, 203.

Curtiss, Edna C., 204.

Curtiss, Edward, xvii, xix, 136 .

Curtiss, Edward Augustus, 89 .

Curtiss, Edward Beach, I 20, I 24.

Curtiss, Edward Benjamin, I 46, I 88.

Curtiss, Edward Collins, 149.

Curtiss, Edward Grant, I 20.

Curtiss, Edward Hatch, r 88.

Curtiss, Edward J., 205.

Curtiss, Edward John, I 44, 186.

Curtiss, Edward Middleton, I32.

Curtiss, Edward Shurtleff, I 75, 205 .

Curtiss, Edward Whitehead, 193, 209.

Curtiss, Edwards, 43.

Curtiss, Edwin, ı og, I 22, I 65, I 88.

Curtiss, Edwin Augustus, 89 .

Curtiss, Edwin Bennett, 199.

Curtiss, Edwin C., 92, 138 .

Curtiss, Edwin Erastus, 104.

Curtiss, Edwin Ford, 206. 
Curtiss, Edwin Mills, I 60, 197.

Curtiss, Edwin Pitts, 139.

Curtiss, Edwin R., I 48 .

Curtiss, Edwin Rodney, 131, 177.

Curtiss, Edwin Stair, 200.

Curtiss, Effie, I 43 .

Curtiss, Effie Jane, I 67 .

Curtiss, Elbert, 86, 1 16, I32, r64.

Curtiss, Elbert Everett, 198 .

Curtiss, Elbert F., I $22,170$.

Curtiss, Elbert O., 131 .

Curtiss, Elbridge, 1 1 4 .

Curtiss, Eldad, 32.

Curtiss, Eldad King, 56 .

Curtiss, Eleanor, 2 I I.

Curtiss, Eleanor Maud, r 84.

Curtiss, Eleazer, 9.

Curtiss, Electa, 57, 66, 7 I.

Curtiss, Electra, 66, 109.

Curtiss, Eli, $47,73,78,82,83$, I I6, I I 7 , I $29,164,213$.

Curtiss, Eli Elisha, ıог, 102, I 04, 151.

Curtiss, Eliadu, 15 .

Curtiss, Elias, 74, $114,214$.

Curtiss, Elidia, 34 .

Curtiss, Elihu, 21, 26, 39, 54, 72, 115 , 126 .

Curtiss, Elihu Starr, 90.

Curtiss, Elijalı, 26, 40, 53, 75, 81 .

Curtiss, Elijah Edwards, 83, 128 .

Curtiss, Eliot Robertson, 203.

Curtiss, Eliphalet, 9, I 9, 35, 64, 65, 108.

Curtiss, Elisha, xxv, 36, 62, 67, 103, I 9.

Curtiss, Elisha Mix, I 25.

Curtiss, Elisha Stevens, I 5 I.

Curtiss, Eliza, 53, 75, 98, 11 o, 117,118 , I $25,154,17 \mathrm{I}$.

Curtiss, Eliza Ann, r 05, 117.

Curtiss, Eliza Jane, r $28,137$.

Curtiss, Eliza M., 156.

Curtiss, Eliza Maria, 92.

Curtiss, Elizabeth, vii, viii, ix, xvii, xvii, xxv, xxvi, xxvii, xxviii, xxix, $x x x, 1,2$, $5,8,10,11,13,14,15,17,18,2 \mathrm{r}$, $23,25,26,28,30,33,34,39,41,43$, $47,56,58,59,64,66,71,76,83,86$, 90,94, ro1, 103, 114, $121,129,136$, 179, 193, 2r2.

Curtiss, Elizabeth Ann, 47, 81, 119,172 , 213.

Curtiss, Elizabeth Birdsey, 5 I.
Curtiss, Elizabeth Bulkley, 206.

Curtiss, Elizabeth Collins, 148 .

Curtiss, Elizabeth F., 167.

Curtiss, Elizabeth Pixlee, r 21 .

Curtiss, Elizabeth Jane, 83, r 28 .

Curtiss, Elizabeth Sophia, I 6.

Curtiss, Elizabeth Stiles, I 44.

Curtiss, Ella, 142, $169,18_{3}, 204$.

Curtiss, Ella Grosvenor, 150.

Curtiss, Ella Hewitt, $x 69$.

Curtiss, Ella Maria, 2 r 4 .

Curtiss, Elle, 41.

Curtiss, Ellen, xx, 77, 79, 129,165 .

Curtiss, Ellen C., r 44 .

Curtiss, Ellen E., 186.

Curtiss, Ellen Eliza, I 53.

Curtiss, Ellen Elizabeth, 146.

Curtiss, Ellen L., I 48 .

Curtiss, Ellen Louise, 160.

Curtiss, Ellen S., 202 .

Curtiss, Ellen T., I 30 .

Curtiss, Ellenora, r 93.

Curtiss, Elliot Plumb, r 29, r 76, $177,205$.

Curtiss, Elliott Miles, I 72.

Curtiss, Elmer, 203.

Curtiss, Elmer E., 208.

Curtiss, Elmira, r 26 .

Curtiss, Elnatlian, xxviii, I 3, r 8,4 r.

Curtiss, Elnathan Atwater, $187,200$.

Curtiss, Eloisa Ann, 53 .

Curtiss, Elsie Ann, roo.

Curtiss, Elsie Berry, ro7.

Curtiss, Elsie Catherine, 155.

Curtiss, Elvira, 98, I 9.

Curtiss, Emeline, r 05, 129.

Curtiss, Emerson Wardell, 198.

Curtiss, Emily, $8_{3}$, I 06 , I 1 I, 118 .

Curtiss, Emily Amelia, I 4 .

Curtiss, Emily Jane, 169.

Curtiss, Emily Melissa, Iо3.

Curtiss, Emily Peck, I 5 .

Curtiss, Emily Sophia, 175 .

Curtiss, Emma, r 5, 93, 1 19, 157, 162, r 64.

Curtiss, Emma A., I 48 .

Curtiss, Emma Elizabeth, 158.

Curtıss, Emma Hall, 150.

Curtiss, Emma Jane, 160.

Curtiss, Enma Louisa, I 7 .

Curtiss, Emma Lyme, r 96.

Curtiss, Emma WV., 169, 201 .

Curtiss, Emory, 142 . 
Curtiss, Enoch, I 5, 34, 77, I 22.

Curtiss, Enoch Olds, r 22.

Curtiss, Enos, 14, 32, 62 .

Curtiss, Enos Hall, r 04, 15 r.

Curtiss, Epenetus, 5 I, 89 .

Curtiss, Ephraim, xxvi, 9, r 7, r 8, 25, 34, $39,7 \mathrm{r}, 72, \mathrm{r} 5$.

Curtiss, Erastus, 49, 85,98 .

Curtiss, Erastus Ives, I 03, I 49, I 50.

Curtiss, Ernest Morton, 196.

Curtiss, Estelle Sandford, r 87 .

Curtiss, Esther, I I, I 3, 20, 28, 31,33 , $35,36,39,43,44,45,52,53,55,56$, $64,79,8 \mathrm{r}, 9 \mathrm{r}, \mathrm{roo}, \mathrm{r} 37,2 \mathrm{r} 3$.

Curtiss, Esther Alvira, r6r.

Curtiss, Esther Iouisa, y 67 .

Curtiss, Ethan, r6, 38 .

Curtiss, Ethel, r 94.

Curtiss, Eugenia, $\mathrm{r} 6 \mathrm{r}$.

Curtiss, Eula Lambert, I 87 .

Curtiss, Eunice, I 2, I3, 19, 20, 21,23 , $26,30,31,33,37,43,45,48,51,57$, $58,59,6 \mathrm{r}, 63,68,2 \times 2$.

Curtiss, Eustace, xii.

Curtiss, Eustace Sanford, r 79.

Curtiss, Eva, r 94.

Curtiss, Eva Adelaide, 135 .

Curtiss, Eva M., r 86.

Curtiss, Eva May, 2 r r.

Curtiss, Evaline, r 60 .

Curtiss, Eve, 29.

Curtiss, Everdon, xx.

Curtiss, Everett, 42, 79.

Curtiss, Ezekiel, 52, 73, 78, r r 8, r23, r 24.

Curtiss, Ezra, 22, 26, 52, 72, 76, 81, 199 .

Curtiss, Ezra Eli, y 6.

Curtiss, Fannie Edith, 167.

Curtiss, Fannie Isabella, 200.

Curtiss, Fanny, 86, 109, 1 23, I 59, r 7 I, 199.

Curtiss, Fanny C., 109.

Curtiss, Fanny H., 7o.

Curtiss, Fanny Maria, 96.

Curtiss, Fanny May, 2 I 6.

Curtiss, Fanny W., I I $\mathrm{r}$.

Curtiss, Flora Preston, 186.

Curtiss, Floyd, $147,189$.

Curtiss, Floyd Ferris, r 40.

Curtiss, Florence, $\mathbf{4} \mathbf{r}$.

Curtiss, Frances, r 36 .

Curtiss, Frances Ann, 132.

Curtiss, Frances E., 174 .
Curtiss, Frances Eddy Spencer, 168.

Curtiss, Frances J., 144, 182.

Curtiss, Frances Marian, $13 x$.

Curtiss, Francis, xxv, 39, r 50.

Curtiss, Francis E., $r 58$.

Curtiss, Francis Elbert, 164 .

Curtiss, Francis Ella, r 64.

Curtiss, Francis Eugene, r 33 .

Curtiss, Francis Ivah, r 50.

Curtiss, Francis Parker, 97.

Curtiss, Francis Randolph, r 78 .

Curtiss, Frank, r $80, r 8_{3}, \mathrm{r} 84, \mathrm{r} 89$, r 98 .

Curtiss, Frank Beal, 203.

Curtiss, Frank C., 156.

Curtiss, Frank L., 177.

Curtiss, Frank Moody, r94, 2 ro.

Curtiss, Frank Thompson, 170.

Curtiss, Franklin, 12 r, r 57, r 67, r 95.

Curtiss, Franklin A., 160 .

Curtiss, Franklin Burdette, 198 .

Curtiss, Frauklin Judson, $\mathrm{r}_{3}$.

Curtiss, Franklin Luther, $145, x_{7}$.

Curtiss, Fred D., 143.

Curtiss, Fred Hull, 159 .

Curtiss, Fred Sterling, 208.

Curtiss, Freda, 2 r r.

Curtiss, Frederic Eugene, ro6.

Curtiss, Frederic Haines, r 95, 2 ro.

Curtiss, Frederic Henry, r 75, 204.

Curtiss, Frederic J., 2 r 6.

Curtiss, Frederick, $\mathbf{r}_{32}, \mathbf{r}_{4} 8, \mathbf{r} 66, \mathbf{r}_{72}, \mathrm{r}_{89}$, $198,202$.

Curtiss, Frederick Alonzo, r 56.

Curtiss, Frederick Edgar, 154.

Curtiss, Frederick Emest, r 65 .

Curtiss, Frederick Griswold, I 25.

Curtiss, Frederick Kingsbury, I 79, 207.

Curtiss, Frederick L., I 4 I.

Curtiss, Frederick Lacey, 93.

Curtiss, Frederick Levi, 192.

Curtiss, Frederick Martin, 184 .

Curtiss, Frederick Milard, 17\%, 206.

Curtiss, Frederick Morgan, 167.

Curtiss, Frederick Stiles, 200.

Curtiss, Frederick Stoddard, r 88.

Curtiss, Frederick William, r 2 r, 135 .

Curtiss, Freeman, 77, 122.

Curtiss, Freeman G., I 70.

Curtiss, Freeman L., 122, I 7 .

Curtiss, Gabriel, xviii.

Curtiss, Gad, 65.

Curtiss, Geoffrey, 201. 
Curtiss, Goorge, xvii, xviii, xix, xx, xxv, 75, 106, I 1 8, I 28, I 50, I 72, 190 .

Curtiss, George A., I 5 I, I98, 215.

Curtiss, George Benjamin, 188.

Curtiss, George Brooks, I 54, 193.

Curtiss, George Corbin, I9o.

Curtiss, George Cowles, rog, 155.

Curtiss, George Dwight, 97.

Curtiss, George Emerson, 196.

Curtiss, George Frederick, 13 I.

Curtiss, George Graham, I 56, 194.

Curtiss, George H., I 46.

Curtiss, George Henry, I 43, 160.

Curtiss, George L., I 93.

Curtiss, George Munson, I 54, 192.

Curtiss, George Preston, 53, 213.

Curtiss, George Redfield, 106, 154.

Curtiss, George S., 143.

Curtiss, George Sample, I 80, 207.

Curtiss, George Smith, I $\$ 6$.

Curtiss, George Thomas, I 37 .

Curtiss, George IV., I 56 .

Curtiss, George Washington, I 27 , I 76.

Curtiss, George William, 196.

Curtiss, Georgia, I 65 .

Curtiss, Georgina Pell, 184.

Curtiss, Gertrude Helen, I 77 .

Curtiss, Gertrude May, I67.

Curtiss, Gideon, I $6,18,28,34,35,36,56$, 66,77 .

Curtiss, Gilbert, 35, 64 .

Curtiss, Giles, 1 7, 39, 71 .

Curtiss, Gladys Cleo, 209.

Curtiss, Gladys Hawthorne, 203.

Curtiss, Gold, $27,55$.

Curtiss, Gould, 56, 93 .

Curtiss, Grace, I 4, I 84, I 93, 208.

Curtiss, Grace Griffiths, I 65 .

Curtiss, Grace Hallam, 192.

Curtiss, Grant, I 42.

Curtiss, Grovenor IVilliam, 16 r.

Curtiss, Guy Cook, I 67 .

Curtiss, Hadden Reuben, 196.

Curtiss, Halden, 176.

Curtiss, Hamilton, 177 .

Curtiss, Hamilton Burton, I69, 201.

Curtiss, Hamson, xx.

Curtiss, Hannah, xxviii, xxx, $3,6,8$, I I, 1 3, 14, 1 7, 19, 20, 21, 22, 24, 27, 28, $35,37,39,43,44,45,46,47,50,55$, $59,61,68,71,72,74,76,79,103$, I I $1,145,2$ I 2,2 I 3.
Curtiss, Hannah Amelia, I97.

Curtiss, Hamnah C., I 71.

Curtiss, Hannah Eliza, 96.

Curtiss, Hannah Hall, I 25.

Curtiss, Hannah Lucretia, 174.

Curtiss, Hannah Maria, 96.

Curtiss, Hannah Olive, 169.

Curtiss, Hannah Susan, I 16.

Curtiss, Happalorna Katherine, ro7.

Curtiss, Harlow Clarke, I 66.

Curtiss, Harmon, 53.

Curtiss, Harmon May, I $37,18_{3}$.

Curtiss, Harold Edivard, 205.

Curtiss, Harold Marshail, I 9 I.

Curtiss, Harriet, I 3, 53, 73, 75, 87, 93, $96,98,107,108,117,120,123,127$, $162,203$.

Curtiss, Harriet Amelia, I 40.

Curtiss, Harriet Ann, I 16, I64.

Curtiss, Harriet B., I I I.

Curtiss, Harriet Louisa, 149, I9 I.

Curtiss, Harriet Louise, I 53.

Curtiss, Harriet M., I 64.

Curtiss, Harriet N., 109.

Curtiss, Harriet Stiles, 99.

Curtiss, Harrison IV., I02, I 47.

Curtiss, Harry, 208.

Curtiss, Harry Beers, I $\delta_{5}$.

Curtiss, Harry Brooks, I 30.

Curtiss, Harvey, 6I, I I I, 146.

Curtiss, Hattie, $16 z$.

Curtiss, Hawley, I о I.

Curtiss, Hazel May, 2 I I.

Curtiss, Helen, $44,73,79$, I 38 .

Curtiss, Helen Birdseye, I 24, 163 , I 73 , I 99.

Curtiss, Helen Frances, 169.

Curtiss, Helen M., 148.

Curtiss, Helen Maria, I 33.

Curtiss, Helen Marietta, 115.

Curtiss, Henrietta, 82 .

Curtiss, Henrietta Swords, I 70.

Curtiss, Henry, xix, xxv, xxvi, I 7, 26, 40, $53,57,75,85,92,94,96,100,102$, I $13,117,141,160,188,214$.

Curtiss, Henry A., Iog.

Curtiss, Henry B., 129 , I 30 , I 46.

Curtiss, Henry Barnum, I 82 .

Curtiss, Henry Burr, 97.

Curtiss, Henry Clay, I So.

Curtiss, Henry Elbert, 163 .

Curtiss, Henry Francis, 89, I 35 . 
Curtiss, Henry Gould, I4I, 185 .

Curtiss, Henry Hobart, 132.

Curtiss, Henry Holbrook, 1 78, 206, 207.

Curtiss, Henry James, $174,204$.

Curtiss, Henry I'inner, 165 .

Curtiss, Henry Sanford, 203.

Curtiss, Henry Stiles, I $\mathrm{r} 7, \mathrm{r} 85,200$.

Curtiss, Henry Thompson, 40, 750.

Curtiss, Henry Tomlinson, 53, 2 r4.

Curtiss, Henry William, I 35, r81, I 82 .

Curtiss, Hepsa, 2 I 3.

Curtiss, Hepsey, 77 .

Curtiss, Hepzibah, 45, 74, 8r, 83, 84, I 29.

Curtiss, Herbert Dewy, $9^{6}$.

Curtiss, Herbert Ellsworth, 198.

Curtiss, Herbert 'T., 209.

Curtiss, Herman, ro8, I 43.

Curtiss, Hester, 9, 29.

Curtiss, Hetty Redfield, 153 .

Curtiss, Hezekiah, 10, $21,22,46,47,56$, $8 \mathrm{r}, 94$.

Curtiss, Hila Andrews, 68.

Curtiss, Hiram, 53 , I 4.

Curtiss, Hiram Foster, I 8 I.

Curtiss, Hobart Henry, I $4 \mathrm{I}$.

Curtiss, Holbrook, $87, \mathrm{r} 33$.

Curtiss, Homer, 102, $1_{4} 8$.

Curtiss, Homer Augustus, 148,189 .

Curtiss, Horace, 63,65 , 106, 108.

Curtiss, Horace Atwater, 186.

Curtiss, Horace Daniel, 144, I86.

Curtiss, Horace Horatio, 99, I 45.

Curtiss, Horace Judington, I 75 .

Curtiss, Horatio Nelson, 90, I 36.

Curtiss, Howard, I64, I 88 .

Curtiss, Howard Everett, 198.

Curtiss, Howard J., I 7 $1,202$.

Curtiss, Howard M., 2 ro.

Curtiss, Howard Redfield, 192.

Curtiss, Howard Wheeler, 202.

Curtiss, Huldah, $37,40,47,48,61,64$, $68,69,71,74,77,83,84,88$, ror, I 14.

Curtiss, Ichabod, 8o, I 26.

Curtiss, Ichabod Francis, I 26, 175 .

Curtiss, Ida, 189, 194.

Curtiss, Ida Castella, 196.

Curtiss, Ida Fern, 2 I 2.

Curtiss, Ida May, r 98.

Curtiss, Ira, $67,74,84$, IIO, II9, I 23 , I $30,146,156,17$ I.
Curtiss, Ira B., I 34, I 80.

Curtiss, Ira Clifton, 208, 2 I I.

Curtiss, Ira Elnathan, I 7 I.

Curtiss, Ira Lamar, r8r.

Curtiss, Ira Lawrence, 90, I 36 .

Curtiss, Irah, 63.

Curtiss, Irene, I 70.

Curtiss, Ireme Augusta, 140.

Curtiss, Irene Morse, I 37.

Curtiss, Irving, 2 ro.

Curtiss, Irving G., 162 .

Curtiss, Isaac, xxviii, xxx, I I, 23, 35, 43, $47,48,54,87$, I I9, I30, I32, 2 I3.

Curtiss, Isaac B., I I 9 .

Curtiss, Isaac Judson, 50, 86.

Curtiss, Isaac Leverett, 84 .

Curtiss, Isaac R., 147.

Curtiss, Isaac W., I02, 147 .

Curtiss, Isabel, $\mathrm{xx}, \mathrm{I} 85$.

Curtiss, Isabel Sanford, 187 .

Curtiss, Isabella May, 137 .

Curtiss, Isabelle, 177 .

Curtiss, Isabelle E., I 99.

Curtiss, Isabelle Jane, I 8 I.

Curtiss, Isadora Louise, 197.

Curtiss, Israel, $1,2,5,6,10,12,14,21$, $30,31,59,64,65$.

Curtiss, Israel Josiah, I 45 .

Curtiss, Ivah, 62, 103.

Curtiss, Jabez, 40, 74, 76, 108, т 20.

Curtiss, Jacob, $15,33,63$, г 36 .

Curtiss, James, xxviii, xxx, 9, 16, 18, 20, $36,37,4 \mathrm{r}, 44,46,49,7 \mathrm{r}, 78,79,108$, I 4 , I 62 .

Curtiss, James Anson, I I 2.

Curtiss, James E., I 29.

Curtiss, James Francis, I 20.

Curtiss, James Guernsey, I43, I 85, I86.

Curtiss, James H., I I 2.

Curtiss, James Harrison, IO4.

Curtiss, James Henry, r 96.

Curtiss, James Herman, 2 I I.

Curtiss, James Herod, 68, I 12.

Curtiss, James J., I I 1, I $46,158$.

Curtiss, James McEwen, I 44, 187 .

Curtiss, James Rose Edwards, 83, 129.

Curtiss, Jane, $\mathrm{xx}, \mathrm{xxvi}, 7 \mathrm{r}, 107,134,2 \mathrm{I} 4$, 215.

Curtiss, Jane A., I 46 .

Curtiss, Jane Augusta, I 7 I.

Curtiss, Jane Eliza, I 73.

Curtiss, Jane Maria, 153. 
Curtiss, Jane Pixlee, 12 I.

Curtiss, Jane Rosilla, 168.

Curtiss, Japhet, 60, 99, 100.

Curtiss, Jason, $9 \mathrm{~S}$.

Curtiss, Jason Parker, i 5 S, 196.

Curtiss, Jauncey, 6r, 100.

Curtiss, Jeanette, 107.

Curtiss, Jemina, 8, I 7 .

Curtiss, Jennett, 105.

Curtiss, Jennette Waterbury, I 45.

Curtiss, Jennic, I 24, I 4 I, 2 I I.

Curtiss, Jemie Frances, 175 .

Curtiss, Jenny Belle, 2 I I.

Curtiss, Jeptha, 38,65 .

Curtiss, Jeremiah, xxiii, xxiv, I1, 22, 23 , $24,47,49,85$, I I $2,159$.

Curtiss, Jeremiah Judson, 19,43 .

Curtiss, Jerome, I I 2, 2 I 5.

Curtiss, Jerry, 101.

Curtiss, Jerusha, 23, 38, 43, 49, 74 .

Curtiss, Jesse, 37, 69, I 12.

Curtiss, Jesse Munson, 192.

Curtiss, Jessie Hammill, i 87 .

Curtiss, Jessie Louise, 159.

Curtiss, Joane, xviii, xx.

Curtiss, Joanna, 13,28 .

Curtiss, Job, 74 .

Curtiss, Joel, 78 , г 23.

Curtiss, Johannah, 6, 13 .

Curtiss, John, vii, viii, xii, xvii, xviii, xix, $\mathrm{xx}, \mathrm{xxi}$, xxii, xxiv, xxv, xxvi, xxvii, xxviii, xxix, $\mathrm{xxx}, \mathrm{xxxi}, \mathrm{I}, 2,3,6,7,8$, $9,13,17,19,20,24,27,28,39,41$, $44,49,52,53,57,73,76,78,79,80$, $8 \mathrm{I}, 85,88,89,90,9 \mathrm{r}, 94$, І г 7 , г 20 , $122, x 24,125,156,164,174,193$.

Curtiss, John Abner, 139.

Curtiss, John H., Ios.

Curtiss, John Beach, 95, 1 36 .

Curtiss, John Beers, 132.

Curtiss, John Burr, 206.

Curtiss, Jolun Calvin, 1 72, 202.

Curtiss, John Christlieb, 190.

Curtiss, John E., I 48 , 1 S9.

Curtiss, John Francis, 174.

Curtiss, Joln Goss, I 75 .

Curtiss, John Gould, 56, 94 .

Curtiss, John H., I 56, 214.

Curtiss, John Halsey, 14 I.

Curtiss, John Haugh, I 90.

Curtiss, John J., 1 I 4 .

Curtiss, John K., 203.
Curtiss, Jolin Leonard, 140.

Curtiss, John Lucky, I 2.

Curtiss, John Nelson, 97.

Curtiss, John P., I 7 x.

Curtiss, John Robertson, 170.

Curtiss, John Stokes, I 75, 204.

Curtiss, John IVilliam, 152.

Curtiss, Jonah, 2 I, 37 .

Curtiss, Jonathan, $1,4,5,10$, I I, I 6, 20, $2 \mathrm{I}, 36,45,67$.

Curtiss, Jonathan G., 1 I I, 159.

Curtiss, Jonas, 21 , 45, 81 .

Curtiss, Jonas Platt, 159 , I 97.

Curtiss, Jone, xviii.

Curtiss, Joseph, xii, xix, xxiv, xxvi, xxviii, xxix, $2,5,7,8,9,10,14,16,13,21$, $30,31,33,37,42,55,59,67,76,78$, 92, I 10, I 23, I 35, I 40, I 7 1, I 81 , I 94 , $2 \times 3,2 \times 4$.

Curtiss, Joseph Beebe, go.

Curtiss, Joseph Davis Beers, I 27, 176.

Curtiss, Joseph Merchant, 46 .

Curtiss, Joseph Osborn, I 84, 209.

Curtiss, Joseph Reuben, 2 I 4.

Curtiss, Joseph IV., 2 I 5, 2 I 6.

Curtiss, Josephine, 16 I.

Curtiss, Josephine May, 176.

Curtiss, Joshua, 4, 5, I о, I $7,23,3 \&, 47$, $70,113$.

Curtiss, Josiah, $4,5,6,9,12,13,21,25$, $27,39,5^{\circ}, 67,71,78,81,95,111$.

Curtiss, Josiah A., 158 .

Curtiss, Josiah Miles, 172.

Curtiss, Judson, $x 9,42,54,73,77,117$ I $8,121,123$, I $68,169,171,213$.

Curtiss, Julia, $53,69,73,88,92$, I 00, $102, x$ I I, I 19,123 , I 41 .

Curtiss, Julia A., 102, 104, 151, $171,214$.

Curtiss, Julia Ann, I 10, 1 S .

Curtiss, Julia Atwater, 149.

Curtiss, Julia Jeach, 129.

Curtiss, Julia Eliza, 186.

Curtiss, Julia Emma, 139, 141.

Curtiss, Julia M., I 46.

Curtiss, Julia W., 2 I 4 ?

Curtiss, Julian, 2 I 4.

Curtiss, Juliette, i $1 S$, 136.

Curtiss, Julius, 1 I 0 , I 57.

Curtiss, Julius A., I $7 \mathbf{I}$.

Curtiss, Julius Bolěvar, 134 , х 79 .

Curtiss, Justus, 62 .

Curtiss, Justus Burr, $8_{3}, 129$. 
Curliss, Kate, 39, 46, $115,211$.

Curtiss, Kate Butler, 195.

Curtiss, Keith, 200.

Curtiss, Keziah, 18, 4 I.

Curtiss, Kittie, I32.

Curtiss, Knells, xxiv.

Curtiss, L. Clyde, 212.

Curtiss, I. Josephine, I 55.

Curtiss, I a mbert B., Iog.

Curtiss, Lanette, $16 \mathrm{I}$.

Curtiss, lany, 136 .

Curtiss, Iaura, $64,84,91,102,113,130$, I $64, \mathrm{I} 85,2 \mathrm{I} 3$.

Curtiss, Laura A., 70.

Curtiss, Laura Jane, 149.

Curtiss, Laura L., 67.

Curtiss, Laura Maria, I6o.

Curtiss, Lauretta, ı $о 6$.

Curtiss, Lavinia, 125.

Curtiss, Lee, 91 , 138 .

Curtiss, Lee Le Count, I 7 I.

Curtiss, Lemuel, I 5 I.

Curtiss, Lemuel Johnson, In 4, I 5 I.

Curtiss, Leonard, 48, 85, I о .

Curtiss, Leonard Chester, 68.

Curtiss, Leonia, 58 .

Curtiss, Leony, 60.

Curtiss, Lepy, 76 .

Curtiss, Le Roy Gaines, I 55.

Curtiss, Le Roy Homan, 192.

Curtiss, Lester, 9 I, I 37, I $38,182$.

Curtiss, Lester Lee, I 37 .

Curtiss, Leverett, 48,84 .

Curtiss, Levi, 50, $57,61,62,63,72,80$ 86, I 01 , I 66 , I 1 $4,124,146,154,162$.

Curtiss, Levi Bradiey, I 53, I 91 .

Curtiss, Levi Johnson, 173 .

Curtiss, Levon, I 13.

Curtiss, lewis, $40,74,82$, I 19, I 22,123 , $127,128,171,213$.

Curtiss, Lewis Agur, 127 , I 76.

Curtiss, Iewis B., 120 .

Curtiss, Lewis Beers, $176,205$.

Curtiss, Lewis Benjamin, 127.

Curtiss, Lewis Charles, 203.

Curtiss, Lewis Emerson, I 58, 196.

Curtiss, Lewis Francis, 17 .

Curtiss, Lewis Frederick, 132.

Curtiss, Lewis Judson, I 16,163 , 199.

Curtiss, Lewis M., 130.

Curtiss, Lewis Martin, 2 I I.

Curtiss, Lewis Walker, 119.
Curtiss, Lidia Dot, 209.

Curtiss, Lillian Fay, 19 I.

Curtiss, Lindslay Byron, I 74.

Curtiss, Lizzie, 157, I 73, 177 .

Curtiss, Lois, $17,31,32,33,34,36,44$, 63.

Curtiss, Loly, 68.

Curtiss, Lorinda, i 58 .

Curtiss, Louis, 60.

Curtiss, Iouis Julius, I 79.

Curtiss, Louis K., $147,188$.

Curtiss, Louisa, 39, 100, 101, 103, 104, I $21,124,136,151$.

Curtiss, Louisa A., rog.

Curtiss, Louisa Amclia, 97.

Curtiss, Louisa Hall, 205.

Curtiss, Louise, I 7 I, 172.

Curtiss, Louise French, I 88.

Curtiss, Louise WVakely, I 63 .

Curtiss, Love, 30.

Curtiss, Lovie, 2 I 3.

Curtiss, Lovina, 49.

Curtiss, Lowesa Jane, 208.

Curtiss, Lucas, 49, 84 .

Curtiss, Iucella, 183 .

Curtiss, Lucetta, 2 I 5 .

Curtiss, Iucinda, 193 .

Curtiss, Lucius, $53,73,8$ I, 87,92, I 33 , 2 I 3.

Curtiss, Iucius Treat, 117.

Curtiss, Lucius Wellington, I59, I97.

Curtiss, Lucretia, 127.

Curtiss, Lucretia Ives, 103.

Curtiss, Lucy, $24,34,35,40,49,52,58$, $61,66,67,68,69,77,8_{4}, 85,90,9 \mathrm{I}$, I 00, IOI, 104,1 I 9, I $26,130,168$.

Curtiss, Lucy Ann, I 22.

Curtiss, I Jcy Maria, I05.

Curtiss, Iucy McDaniel, I 82 .

Curtiss, Lue, 35 .

Curtiss, Iulu Rose, I 96.

Curtiss, Iydia, xxviii, $35,38,43,53,62$, $63,80,81$, I 7,183 .

Curtiss, Lydia Ann, 182.

Curtiss, Lyman, I 13 .

Curtiss, Lyman Lord, i i 8, I 65.

Curtiss, Lyman Sylvestcr, 97.

Curtiss, Mabel, 27, 29, 60.

Curtiss, Mabel I Iassard, 200.

Curtiss, Mabellc Elizabeth, 209 .

Curtiss, Madlyng, xviii.

Curtiss, Major, 7o, I 13 . 
Curtiss, Malcolm, zro.

Curtiss, Marcia, 90, I 14, I25, I 36 .

Curtiss, Marcia A., 215 .

Curtiss, Marcia Maria, 9 r.

Curtiss, Marcia R., 52.

Curtiss, Marcus, 49, 69, 76, 85, 87 .

Curtiss, Margaret, xvii, xix, xxx, I6, 48, $65, \mathrm{I} 2 \mathrm{I}, \mathrm{I} 43,2 \mathrm{IO}$.

Curtiss, Margaret Ann, I 20, I 24.

Curtiss, Margaret Hoxey, 97.

Curtiss, Margaret Owen, 76, IIS, I 20.

Curtiss, Margery, 34.

Curtiss, Margett, i 70 .

Curtiss, Maria, 53, 95, 98, 100, 104, $\mathrm{II}_{3}$, Ir4, Ir 7, I20, I2I, I23, I29, I43, I62.

Curtiss, Maria E., $9 S$.

Curtiss, Maria Louise, r63.

Curtiss, Marian, ros.

Curtiss, Marian Eleanor, I 55.

Curtiss, Marie xviii, I $23,200$.

Curtiss, Marienina, i I 2.

Curtiss, Marilla, 55 .

Curtiss, Marinda, 137.

Curtiss, Marion, xviii, I 55, 194.

Curtiss, Marion B., I 77 .

Curtiss, Marjorie Allen, 207.

Curtiss, Martha, xvii, xxvii, 1 7, 18, 19, 26, $28,33,42,56,69,77,143$.

Curtiss, Martha Ann, 96 .

Curtiss, Martha B., I67.

Curtiss, Martha C., 109.

Curtiss, Martha Eliza, I9o.

Curtiss, Martha Evaline, 97.

Curtiss, Martha Judson, 2 I 3.

Curtiss, Martha L., I02, I 47 .

Curtiss, Martha Root, r I 2.

Curtiss, Martha Young, 209.

Curtiss, Martin, $7 \mathrm{r}$.

Curtiss, Martin Luther, 60.

Curtiss, Mary, xvii, xviii, xix, xx, xxvii, $\mathrm{xxx}, 1,2,7,8,10,14, \mathrm{I} 5, \mathrm{I} 6, \mathrm{I} 7, \mathrm{1} 8$, I9, 20, 21, 23, 24, 25, 27, 29, 30, 32, $35,37,39,45,50,53,54,55,57,58$, $59,60,66,67,7$ I, 73, 74, 76, 78, 8I, $8_{3}, S_{4}, 8_{5}, 87,89,92,93,95$, I о I, I 22 , I IO, I I 3 , I I4, I 2 I, I 26 , I 38, I 66, I 75, 180.

Curtiss, Mary Ada, I64.

Curtiss, Mary Alanthea, I 79 .

Curtiss, Mary Almira, I 75 .

Curtiss, Mary Ann, 48, 72, 82, 83, 98, 99, I Oo, I I I, I 44.
Curtiss, Mary A. R., Iog.

Curtiss, Mary B., I 20.

Curtiss, Mary Beers, 127.

Curtiss, Mary Caroline, 87, r 4 I.

Curtiss, Mary Cecilia, 140,184 .

Curtiss, Mary Corclelia, I 3 I.

Curtiss, Mary Cornelia, I 43, I 46.

Curtiss, Mary E., I $12,146,2$ r 5 .

Curtiss, Mary Edwards, I 70.

Curtiss, Mary Electra, I I I.

Curtiss, Mary Eliza, Io8.

Curtiss, Mary Elizabeth, I18, I 20, I 27 , I 68 .

Curtiss, Mary Ellen, 156.

Curtiss, Mary Emaline, 97.

Curtiss, Mary Emeline, 86.

Curtiss, Mary Emlen, 207.

Curtiss, Mary Eve, 56.

Curtiss, Mary Frances, I 49.

Curtiss, Mary Henrietta, I 39 .

Curtiss, Mary Jane, I 63 .

Curtiss, Mary Jennette, 145 .

Curtiss, Mary Jerusha, 173 .

Curtiss, Mary Lewis, I 23, I 7 I.

Curtiss, Mary Mallory, 99.

Curtiss, Mary Melissa, I 88.

Curtiss, Mary Pixlee, I 67.

Curtiss, Mary Rebecca, 190.

Curtiss, Mary Rice, I 3 .

Curtiss, Mary Robertson, I 70 .

Curtiss, Matilda, xx, 9r, I I6, r 29, r64.

Curtiss, Matilda Rogers, 89 .

Curtiss, Matthew, xv, I 2, 25, 27, 54, 55, 93, 2 工3.

Curtiss, Mattie Louise, 147 .

Curtiss, Mattie May, 2 r r.

Curtiss, Maud, r 99, 208.

Curtiss, May Evelyn, 177 .

Curtiss, May L., 202.

Curtiss, Naybel Edith, 196.

Curtiss, Medora C., I 75, 204.

Curtiss, Mehitabel, xxx, 10, I2, 21,22 , 26,52 .

Curtiss, Mehitabel Jane, I 5 .

Curtiss, Mehitable, 2 I 2.

Curtiss, Melissa, I $15,162$.

Curtiss, Melville Judson, I $69,201$.

Curtiss, Mercy, xxviii, 22,66 .

Curtiss, Merib, 35 .

Curtiss, Merritt, $7 x$.

Curtiss, Milbern, 208.

Curtiss, Mildred Harriet, 206. 
Curtiss, Mildred Rebecca, 205.

Curtiss, Miles, 7 I.

Curtiss, Miles L., r 30.

Curtiss, Milton, i ro.

Curtiss, Mindwell, 15 .

Curtiss, Minerva, 70.

Curtiss, Minnie, I46, I64, 18 r, 208, 2 Ir.

Curtiss, Minnie Belle, 203.

Curtiss, Minnie Jane, 216.

Curtiss, Minnie Merk, 188.

Curtiss, Mira, 76 .

Curtiss, Miriam, xix, 32, 68 .

Curtiss, Miriam Blanche, I6o.

Curtiss, Mirriam, 35 .

Curtiss, Mitchell, $23,48$.

Curtiss, Molly, 72.

Curtiss, Monson Edwards, I 29.

Curtiss, Montelle Clifton, 2 г 2.

Curtiss, Morgan, 76, i 18 , 120.

Curtiss, Morse, 9 I.

Curtiss, Moses, 14, 32, 33. .

Curtiss, Moses Osborn, 92 .

Curtiss, Myra, Ir6, rr7, I64.

Curtiss, Myrtie, 204.

Curtiss, Myrtle Almyra, i 89.

Curtiss, Nabby, 55, 60, 75 .

Curtiss, Nancy, 63, 66, 68, 69, 72, 78, ro6, I I 3 , I I 4 .

Curtiss, Nancy M., 54, I 29.

Curtiss, Nancy Maria, I I 6 .

Curtiss, Naomi, $23,74$.

Curtiss, Narcissa, 65 .

Curtiss, Nathan, 9 , I 3,15, r $8,19,28,32$, $44,56,78,79,80,98, x 23$, r 25 .

Curtiss, Nathan Bulkley, I 77, 206.

Curtiss, Nathan Selah, 13 r.

Curtiss, Nathaniel, xix, xxvi, 7, r r, $\mathrm{r}_{4}$, I $5,22,33,62$.

Curtiss, Nathaniel Bancroft, I 12.

Curtiss, Nathaniel Wheeler, 75 .

Curtiss, Nehemiah, 25, 42, 43, 50, 77,2 I 5 .

Curtiss, Nellie, 183 .

Curtiss, Nellie Elizabeth, I 87 .

Curtiss, Nellie Iona, 177.

Curtiss, Nellie Sabrina, I 96.

Curtiss, Nellie Thomson, I 88.

Curtiss, Netta A., I 83 .

Curtiss, Nettie, r 65 .

Curtiss, Nettie Barker, 197.

Curtiss, Newton, r4I.

Curtiss, Nicholas, xvii.

Curtiss, Nichols, 88, r $33, \mathrm{r} 34$.
Curtiss, Niram, 27.

Curtiss, Nirom, 89 .

Curtiss, Noah, xx.

Curtiss, Nora, I 8 I.

Curtiss, Normau, 66.

Curtiss, Norris Beach, r 73 .

Curtiss, Norris Newton, $124,173$.

Curtiss, Olive, I3, r 5, 58, 80, roo.

Curtiss, Olive Strong, 185 .

Curtiss, Oliver, 22, 30, 31 , 85, 92, 2 I 2.

Curtiss, Oliver Bronson, roz.

Curtiss, Olivia Harriet, r 86.

Curtiss, Orlando, rog.

Curtiss, Orlando F., r го.

Curtiss, Orman, 95.

Curtiss, Orren, I 10, I 56, I 57.

Curtiss, Orrin, roo, I I 3 , I 46.

Curtiss, Oscar Lee, 138 .

Curtiss, Otis Beers, I69, 201.

Curtiss, Otto E., I8o.

Curtiss, Pamelia, 67.

Curtiss, Parthenia, 5 I.

Curtiss, Patience, 64 .

Curtiss, Patty, ror.

Curtiss, Paul, I gr.

Curtiss, Paul Allen, 92, r 39, 183 .

Curtiss, Pauline, r $40, \mathrm{r} 84$, r $9 \mathrm{r}$.

Curtiss, Peter, xx, 6, 12, 13, 16, 26, 29, $35,65,108$.

Curtiss, Peter Burr, 29, 57, 95, r 42 .

Curtiss, Peter Pixlee, I 19, 167.

Curtiss, Petronill, xx.

Curtiss, Phebe, r6, r8, r9, 20, 22, 26, 27 , 30, $34,41,42,43,44,46,48,50,51$, $52,55,56,58,69,75,77,80,86,89$, $95,108,125,126$.

Curtiss, Phebe Amanda, 97.

Curtiss, Phebe Ann, 79, I05, I35.

Curtiss, Phebe Augusta, 97.

Curtiss, Phedenia, 88.

Curtiss, Phedina, 5 r.

Curtiss, Philander C., r 34, I 8 r.

Curtiss,'Philip, xvii, xviii, xx, xxx.

Curtiss, Philo, 46, 5 I, 7 r, 72, 74, 81, 88, II 3, I I 5 , I I 9, I 34, I 59 .

Curtiss, Philo Upson, 159.

Curtiss, Phineas, $37,42,68,69,77$.

Curtiss, Phobe, I 24, 20I, 2 I 2, 2 I 3 ,

Curtiss, Phœbe Eliza, I 39 .

Curtiss, Pliny, 70, I r 2, I I 3 .

Curtiss, Polly, 49, 54, 59, 6I, 62, 70, 74, $75,76,85,88, \mathrm{r}_{4}, \mathrm{x} \times 4, \mathrm{x} 23, \mathrm{x} 27,213$. 
Curtiss, Polly Ann, $S_{7}, 215$.

Curtiss, Polly I., rog.

Curtiss, Polly Samantha, I 05.

Curtiss, Prudence, 30, 36, 59, 66.

Curtiss, Rachel, I6, So, $S_{5}$, I 00, 124.

Curtiss, Rachel Jane, 84 .

Curtiss, Ralph Hamilton, 201.

Curtiss, Ray, I $\delta_{5}$.

Curtiss, Ray R., 205.

Curtiss, Raymond, 200.

Curtiss, Raymond Hammil], 200.

Curtiss, Rebecca, xxix, 2, 5, 6, S, 1 I , 13 , 14, 19, $28,39,54,55,72,73,87,93$, I39.

Curtiss, Rebecca Judson, I 64 .

Curtiss, Reginald, $\mathrm{xx}$.

Curtiss, Rejoice, 74 .

Curtiss, Reuben, $27,31,55$.

Curtiss, Reuben Bradley, 98, I 4 .

Curtiss, Reuben Judson, 55 .

Curtiss, Rhoda, 1 5, 23, 39, 60.

Curtiss, Richard, xix, $\mathrm{xx}, \mathrm{xxvii}, \mathrm{xxviii}$ xxix, 54, 2 I 3.

Curtiss, Robert, xviii, xx, $18,38,42$.

Curtiss, Robert Hallam, I 53, 192.

Curtiss, Robert Lawrence, 2 1 0.

Curtiss, Robert Meiville, 20 I.

Curtiss, Robert IV., I 66.

Curtiss, Robert Welles, 4 I, 2 I 3.

Curtiss, Robert William, I 33 .

Curtiss, Roderick Perry, 176.

Curtiss, Rodney, 79, 84, I 30.

Curtiss, Rodney Valentine, 169 .

Curtiss, Roland Anthony, i 98 .

Curtiss, Rollin William, 147 .

Curtiss, Rosa, $1+7$.

Curtiss, Rosilee, 54.

Curtiss, Roswell, 62, 71, 91, 106, I 21 , 1 36, I 68, 2 I 5.

Curtiss, Roswell Chapman, 174.

Curtiss, Rowlanel, xxvi.

Curtiss, Roxa, 57 .

Curtiss, Roxey, 7 I.

Curtiss, Roy Abijah, I 95.

Curtiss, Royal, 160, $198,21 \mathrm{I}$.

Curtiss, Russell, 58, 90.

Curtiss, Russell 'T., 93.

Curtiss, Ruth, xxv, xxviii, 6, 9, I I, I4, I 8 , $20,22,31,32,35,37,41,42,46,52$, $62,63,65,70$, So, 81, 104, 200, 20I, 208, 2 I 2.

Curtiss, Ruth A., ro3.
Curtiss, Ruth Ann, 22, 53, I 19.

Curtiss, Sabra, 67.

Curtiss, Salena, I 35.

Curtiss, Sally, 46, 49, 52, 53, 57, 64, 66, 68,76, I I $5,123,212$.

Curtiss, Sally Ann, r 07.

Curtiss, Sally M., 54 .

Curtiss, Salmon, 26, 51,87 .

Curtiss, Samantha, 95 .

Curtiss, Samuel, xxi, xxvi, xxvii, xxviii, 7 , 8 , I I, I 3, I 5, 20, 22, 24, 25, 33, 34, $40,43,46,47,48,50,62,63,75,80$, $82,94,105,114,125,141,161,164$, $174,204$.

Curtiss, Samuel Bennett, I 37,183 .

Curtiss, Samuel Brooks, I 25, I 74 .

Curtiss, Samuel C., I I 5, i 16 , I 62.

Curtiss, Samuel Edmond, 33 .

Curtiss, Samuel Elisha, 83 .

Curtiss, Samuel Ellery, 84, I 31 .

Curtiss, Samuel Henry, 175 .

Curtiss, Samuel Ives, I 03, I 49, I 50, 190.

Curtiss, Sarah, vii, xix, xxvii, xxviii, xxix, $\mathrm{xxx}, 1,5,8$, I O, I I, I 4, I 5, I 9, 20, 2 I, $22,23,24,26,30,31,32,35,36,3^{8}$, $39,40,41,44,45,49,50,51,53,58$, $59,61,63,65,66,67,72,73,74,76$, $77,78,86, S_{9}, 90,94,98$, го1, 107, I I , I I , I I $7, I_{2} 5,126,136,2$ I 2 , 213.

Curtiss, Sarah A., 147.

Curtiss, Sarah Ann, 53, 54, 81, 93, 139, 213.

Curtiss, Sarah E., 109, 172.

Curtiss, Sarah Emily, 155.

Curtiss, Sarah Fitch, I 67 .

Curtiss, Sarah G., ${ }_{5} 8$.

Curtiss, Sarah Glover, 203.

Curtiss, Sarah H., I 1 5, I $48,164,175$, 204.

Curtiss, Sarah Isabel, 141.

Curtiss, Sarali J., 79 .

Curtiss, Sarah Jane, 47, I 32 .

Curtiss, Sarah Jane B., I 50.

Curtiss, Sarah Jerusha, i 46.

Curtiss, Sarah Lena, I 79.

Curtiss, Saral Maria, 97.

Curtiss, Sarah Matida, I 2 I, 135.

Curtiss, Sarah Melvina, 97.

Curtiss, Sarah Minerva, 187.

Curtiss, Sarah Miranda, I 87 .

Curtiss, Sarah Peck, I24, I6و. 
Curtiss, Sarah Theodora, 141 .

Curtiss, Seta Ann, I87.

Curtiss, Seth, 27.

Curtiss, Seymour, 200.

Curtiss, Shelden, $9 \mathrm{I}$.

Curtiss, Sheldon, 6o, 2 I 2.

Curtiss, Sheldon Pixlee, I 18 , I 66.

Curtiss, Sherman, 57, 58, 60, 97, 100.

Curtiss, Sherman William, ${ }_{3} 8$.

Curtiss, Shuvall, 69.

Curtiss, Sidney, 2 ro.

Curtiss, Sidney F., 87, 132.

Curtiss, Sidney Ufford, 133 .

Curtiss, Silas, 26, 34, 37, 39, 42, 52, 64, $72,73,78,115,123$.

Curtiss, Silence, 55 .

Curtiss, Simeon, 35, 38, 59, 70, 94, 98, 99, II $3,145,160$.

Curtiss, Solomon, xxv, 33, 34, 36, 40, 43, $63,66,74,75,107,155$.

Curtiss, Sophia, 88, 123,157 .

Curtiss, Sophia M., i 95.

Curtiss, Sophronia, 96.

Curtiss, Spencer, r 20 .

Curtiss, Statirah, 80.

Curtiss, Stella, 193.

Curtiss, Stephen, xx, xxix, 6, I3, I4, 21 , $25,28,31,49,60,86$.

Curtiss, Stephen C., rog.

Curtiss, Stephen Edwin, I 15.

Curtiss, Stephen Fowler, 107, 155.

Curtiss, Stephen Judson, 50.

Curtiss, Stephen Stiliman, 92.

Curtiss, Stephen Welles, 72.

Curtiss, Sterling Pemberton, I 8 I, 209.

Curtiss, Stiles, $17,27,39,41,73,76$, I $6,117,122,162,170,213,215,216$.

Curtiss, Stiles Henry, $165,200$.

Curtiss, Stiles Whiting, $16_{3}, 199$.

Curtiss, Stillman H., 70 .

Curtiss, Susan, xvii, 69, 76, 86, 89, 93, 126, I $29,135,158$.

Curtiss, Susan A., I 20 .

Curtiss, Susan Caroline, I 39.

Curtiss, Susan Clarice, 2 I 2.

Curtiss, Susan J., I 72, 202.

Curtiss, Susan M., $123,124,148$.

Curtiss, Susan Rebecca, 132 .

Curtiss, Susannah, xvii, 23, 31, 56, 73, I 68 .

Curtiss, Susie, 2 I 1.

Curtiss, Sybil, $3^{8 .}$
Curtiss, Sybilla Cleora, 98.

Curtiss, Sylvester, I I $3,160$.

Curtiss, Sylvester Johnson, 105, I 52.

Curtiss, Sylva, 129.

Curtiss, Sylvia, 53, ro8.

Curtiss, Sylvia Johnsou, I 9 I.

Curtiss, Tabatha, 20, 43 .

Curtiss, Tabitha, 18 .

Curtiss, Talcott, 125, I 73 .

Curtiss, Thaddeus, $30,40,59,73,78$, 90.

Curtiss, Thaddeus Birdsey, I 16, I 63 .

Curtiss, Thankful, $x 6,33,36,37,67$.

Curtiss, Theodore, 126 .

Curtiss, Theodosia, 65,78 .

Curtiss, Theophilus, xxvi.

Curtiss, Theresa Ann, I69.

Curtiss, Theron E., I oo.

Curtiss, Theron Skeele, 99.

Curtiss, Thomas, vii, xvii, xviii, xix, xx, xxvii, xxix, $x \times x, x x x i, 1,2,6,7,8,16$, $22,35,55,114,2$ I 2 .

Curtiss, Thomas Bennett, $137, \mathbf{1} 82$.

Curtiss, Thomas E. (Hulse), \} _ { 5 3 } \text { , } 1 9 1 .

Curtiss, Thomas Emmons, is 8 , 165 .

Curtiss, Thomas H., I Io, 156 .

Curtiss, Thomas Henry, 198, 211.

Curtiss, Thomas Jefferson Barnes, I74, 203.

Curtiss, Thomas Yale, 62.

Curtiss, Timothy, $2 x, 45,8 \mathrm{x}$.

Curtiss, Titus, 32 .

Curtiss, Trephena Emily, 10\%.

Curtiss, Trueman, II

Curtiss, Truman, $58,59,158, \mathrm{x} 6 \mathrm{r}$.

Curtiss, Urania, 22.

Curtiss, Uriah Bradley, 95, 142.

Curtiss, Uriel Carlton, 107 .

Curtiss, Ury, 34, 63 .

Curtiss, Vasti, 34 .

Curtiss, Veletta, 95 .

Curtiss, Vera Cameron, 203.

Curtiss, Victor Delitzsch, 191.

Curtiss, Victor Stuart, 202.

Curtiss, Victory, 78, 124,172 .

Curtiss, Victory B., I 72 .

Curtiss, Wait, 5o, 86.

Curtiss, Waldo Camp, 199.

Curtiss, Wales Hatch, I 44, I 87.

Curtiss, Wallace Edwin, I98.

Curtiss, Walter, $x x, 126,173,183$.

Curtiss, Walter A., $18 \mathbf{r}$. 
Curtiss, Walter George, 204.

Curtiss, Walter Henry, 99.

Curtiss, Walter Judson, 201.

Curtiss, Walter Lord, 165.

Curtiss, Waldo Loren, I 6 I, 199.

Curtiss, Walter Raymond, 200.

Curtiss, Walter Shelden, 137.

Curtiss, Walter Stanley, 2 o I.

Curtiss, Walter Stiles, I 44, I 86.

Curtiss, IVatson IV., 147.

Curtiss, Welles, 2 I 3.

Curtiss, Wellington L., 199.

Curtiss, Wells, 47, 2 I 2 .

Curtiss, Wilber, 208.

Curtiss, IVilber B., r 46.

Curtiss, Wilfred Leroy, 197.

Curtiss, William, vii, viii, ix, xii, xviii, $\mathrm{xx}$, $\mathrm{xxi}$, xxiii, xxiv, xxv, xxvi, xxvii, xxviii, xxix, $\mathrm{xxx}, \mathrm{xxxi}, 1,2,3,4,5,10,12,24$, $4 \mathrm{I}, 45,46,50,54,6 \mathrm{r}, 73,76,8 \mathrm{r}, 86$,

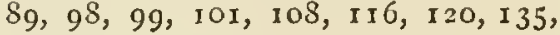
I $68,203,2 I_{3}$.

Curtiss, William Amos, 104.

Curtiss, William Anson, 164 .

Curtiss, William B., I46.

Curtiss, William Conway, I 63, 199.

Curtiss, William Day, I 30.

Curtiss, William Douglass, I 70.

Curtiss, William E., 2 I 6.

Curtiss, William Edmond, I $78,207$.

Curtiss, William Edmund, $133, \mathbf{1} 78$.

Curtiss, William H., $109,177, \mathbf{1} 83$.

Curtiss, William Hadden, 128.

Curtiss, IVilliam Henry, 133, 140, I 77 , I 84, I 96.

Curtiss, William Hubbell, 1 34, I 80.

Curtiss, William L., I $22,170,{ }_{1} \delta_{3}$.

Curtiss, William Lafayette, $\mathbf{3 2}$.

Curtiss, William Lester, 197.

Curtiss, William Lewis, 170.

Curtiss, William M., I 95.

Curtiss, William McIntire, I 84 .

Curtiss, IVilliam Munson, 104.

Curtiss, William O., 146.

Curtiss, IVilliam Osborn, 139 .

Curtiss, William Perry, I 8 r.

Curtiss, William Pixlee, 53.

Curtiss, William Preston, 58.

Curtiss, William Ray, I 38.

Curtiss, William Reuben, 187 .

Curtiss, William Robert, I 85 .

Curtiss, William Thompson, 78 .
Curtiss, William Van de Water, I40, I 84 , 209.

Curtiss, William WV., 2 I 6 .

Curtiss, Willis, 86, I 3 I, 185 .

Curtiss, Willis B., I 46 .

Curtiss, IVinfield Scott, I59, I 97.

Curtiss, IVinterton Conway, 199.

Curtiss, Zachariah, 4, 5, I I, $23,48,8_{3}$.

Curtiss, Zachariah P., 128 .

Curtiss, Zachariah Tomlinson, 54.

Curtiss, Zacheus, xxx.

Curtiss, Zebina, 64, 66, rog.

Curtiss, Zebinia, I 10.

Curtiss, Zechariah, $7 \mathrm{~S}$.

Curtiss, Zella, 147.

Cushing, Abby Bicknell, I53, I9 I.

Cushing, Alice Maria, I 53, I 9 I.

Cushing, Henry Francis, I53, I9I.

Cushing, John T., 103.

Cutler, Timothy, 24.

Daggett, Mrs. —, 70.

Danforth, John, 126 .

Davidson, Daniel, 149.

Davis, Polly, 38, 69 .

Day, Aaron, 24, 48 .

Day, Mary Ann, 24, 48 .

Day, Susannah Stanley, 24, 48.

Dayton, -, Io.

Dayton, Mary Ann, I I 3, I61.

Dealing, Orville B., I 75 .

Dean, Simeon Morton, 97.

Decker, Mary, 55.

DeForest, Benjamin, 22.

DeForrest, Alice, $47,8 z$.

DeForrest, Elihu, 47, 82 .

DeForrest, Ephraim B., 46.

DeForrest, Sarah, 47, 82 .

Demarest, Christina, 87,132 .

Deming, David, 96 .

Deming, E. S. 113 .

Deming, Selah, 49 .

Dempster, Agnes, 147.

Devine, Mary, 26, $5 \pi$.

Dill, Solomon, Jr., 65 .

Dingee, Amelia J., 137.

Disbro, Myron, $\mathrm{I} \mathbf{S}_{2}$.

Doolittle, Frances E., 130.

Doolittle, Harriet, r ro.

Dorchester, Eliza, I 65 .

Dorman, Abbie O., 199.

Doty, John, xxv.

Doty, Martha, xxv, 5 . 
Doty, Roxy Maria, I37, I82.

Doty, William, 137 .

Dowd, Almiron, I I 1 .

Dowd, Clarissa, 70, i 13.

Dowd, Elizabeth, I $13,160$.

Downs, George Newton, 160.

Downs, Monroe, 133 .

Dresser, Joseph, I5o.

Duke, Edward, xx.

Duke, Richard, xx.

Dunbar, Edward, I 7 .

Dunham, Cornelius, 63, 106.

Dunham, Jemima Andrews, 63, 106.

Dunham, Nancy, 63, 106.

Dyer, Benjamin, 64 .

Dyer, Margaret, 35, 64。

Dyer, Peggy, 48.

Eddy, Alice M., I 49.

Eddy, Malvinia R., I49.

Eddy, Zackary, 149.

Edgerton, A. J., 148.

Edmond, Elizabeth, I33.

Edmond, Elizabeth P., 87, 133 .

Edmond, William, 87 , 133 .

Edwards, Gershom, 43.

Edwards, Huldah, 48, 83, r29.

Edwards, John, 48,83 .

Edwards, Mary, 19, 43 .

Edwards, Naomi, 48, 83 .

Edwards, Rebecca Nappan, 82, I 28.

Edwards, Samuel, 2 I.

Eggleston, Samuel, 66.

Eliot, Bennett, vii.

Eliot, John, vii, viii, xxx.

Eliot, Sarah, vii, xviii.

Ellison, Charles Grant, 187 .

Ely, Elisha Miles, 53.

Ely, David, 53.

Ensign, B. Evans, I07.

Eversley, Anah, 163 .

Eversley, Eli Barnum, I63.

Eversley, Harriet, 263.

Eyre, Frances E., I 85 .

Fairchild, —, 80.

Fairchild, Ebenezer, 20.

Fairchild, Lewis, I 21.

Fairchild, Nathan, 45 .

Fairchild, Robert, 17 .

Fairchild, Sally, 8o.

Fairchild, Samuel, I 9.

Fairchild, Sarah, 40, 76 .

Farnum, Diana, Io\%.
Fay, George A., I 53.

Fayerweather, Anne, 76, I 20.

Fayerweather, John, 45.

Fickett, Eliza Abbie, I 8 I, 208.

Fickinger, James A., 175.

Filley, Willian 'T., 96.

Fitch, Edward D., 19.

Fitch, Mary Esther, I I 9, I 67.

Foot, Abigail, I5, 33 .

Foot, Amos, 57.

Foot, Comfort, 38, $7 \mathrm{I}$.

Foot, Joseph, Jr., I 5, 33 .

Foote, Robert, xxviii.

Foote, Sarah, xxviii.

Foote, Winthrop Alvin, 136.

Ford, Ann, 27.

Ford, Bathsheba, 25.

Ford, Catherine IV., I 77, 206.

Ford, Hannah, $27,54,2$ I 3.

Foster, - 1 I 8 .

Foster, Hannah, 31, 60.

Foulke, Brion, I 7 o.

Fowle, John Hunt, 139.

Fowler, Catherine, 64, I07.

Foxwell, Richard, xxix.

Foxwell, Sarah, xxix.

Francis, Emily Adelaide, 147.

Francis, Job, I о

Francis, Sarah, I or .

Fredericks, Elizabeth, 8, I 5 .

Fredericks, Mary Tuttle, I 5.

Fredericks, William, 15 .

Freeman, Darius, I 58.

French, Bennet, 58.

French, Ellen Mallory, 1 45, I 88.

French, Frances Ann, I 3 , 149.

French, George B., I 45, I 88 .

French, James, I 49.

French, Melissa, I 49.

French, Samuel, 4 .

French, Silas, 5 т.

Frister, Mary, 160.

Frost, Elizabeth, I 19.

Fulmer, Almira, 16o, I 98.

Furniss, Clara, 147 , I 89 .

Furniss, Edward, 147, I89.

Gale, Fred C., 196.

Galpin, Samuel, I 4 .

Galpin, Stephen, 28.

Gano, J. Elbridge, I 8 z.

Garbrant, Albert, I 80.

Garfield, Isaac, 66. 
Girfielel, Iucy, 36, 66.

Gates, Lafayette, 137.

Geary, IVilliam, $\mathrm{xxx}$.

Geiger, Henry C., 177.

Gerrish, Helen, I 26.

Gilbert, Abijah, 173 .

Gilbert, David Borbul, Io4.

Gilbert, Harriet Is, $177,206$.

Gilbert, Huldah, 77, 42 .

Gilbert, I. Mix, I 71 .

Gilbert, Jennette, I6S.

Gilbert, Josiah, 42, 77 .

Gilbert, Maria, I $17,164$.

Gilbert, Mary Ann, I 77, 206.

Gilbert, William T., I 77, 206.

Giles, William Ogden, 127 .

Gillet, Phebe, 1 74, 204.

Gillett, Abigail, 33, 63 .

Gillett, John, 7o, I I 3 .

Gillett, Polly, 7o, I 3 .

Gillett, Zachariah, 33, 63 .

Gilman, John, 70.

Glassburner, James, I $8 \mathbf{I}$.

Gleason, Thomas, 66.

Gleeson, Moses, I 65.

Gleeson, Polly Richardson, I 65.

Glover, Anna, 52, 90.

Glover, Eunice, 52, 90.

Glover, H. B., I 72 .

Glover, James, 52, 90.

Glover, John, 26, 88, 9o, I 36.

Glover, Marcia, 56, 94.

Glover, Marietta, 9o, 136.

Glover, Polly Curtiss, 90, 136.

Godone, Angelo, 183 .

Gold, Hezekiah, 22, 47.

Gold, Huldah, 22,47 .

Gold, Nathan, 3, 5, 8 .

Goodwin, Mary Gleeson, I I 7, 165 .

Goodwin, William, ix, 2, 3 .

Gordon, A. J., 142.

Gorham, Ann Jewett, 122 , 170.

Gorham, Anna, 40, 75.

Gorham, Hannah, 47.

Gorham, Judson, 1 I 9 .

Goss, Geraldine, 126 , 175 .

Goss, John, I 26, 175 .

Goss, Martha Matilda, I 26, 175 .

Gould, Elizabeth, 27,55 .

Gracey, —, 2 I 2.

Graham, Andrew, 28.

Grain, Alice Kneeland, I 79.
Grain, Francis H., I 79.

Granger, Gilbert, r ro.

Gray, -, 124.

Green, Estella, I 85 .

Green, Lewis S., 105.

Green, Sarah A., I I .

Green, William H., I05.

Gridley, Hannah M., 85 .

Gridley, Noah, 24 .

Gridley, Sarah L., 152.

Griffin, Francis, Io.

Griswald, Hannah, 44, 79.

Group, Agnes, 198, 2 I .

Grout, Abigail, xxvi.

Grumley, Elizabeth, 197 .

Guernsey, Anna, 98, I 43.

Guernsey, Bridgeman, 79.

Guernsey, Joseph, 23.

Guernsey, Rachel, I r, 23.

Guernsey, William, I I.

Guy, Mary, xxv.

Guy, Nicholas, xxv.

Guyon, Charles, 20.

Hadden, Ann Aspinwall, 82, 128.

Hadden, David, 82, I $2 \mathrm{~S}$.

Hadden, Laura, $8 \mathbf{2}, 128$.

Hagabone, Eliza, 137.

Hagerman, Maria, I Io.

Haines, Catherine Butler, I 57 , I94.

Haines, John, 2 I.

Haines, Mary Elizabeth, 157, 194.

Haines, Seldon, 157 , 194.

Hale, J. Montgomery, I 79.

Hale, Marian S., I 79.

Hall, Abigail, 62, 103 .

Hall, Anna, 6r, го .

Hall, Annabelle, 1 77, 205.

Hall, Benjamin, 7 .

Hall, Daniel, 7 .

Hall, Edward N., Ioz.

Hall, Elizabeth, 7 .

Hall, Enos, 62, Iо3.

Hall, Jerusha, 6 1 , 1 oo.

Hall, John, 44.

Hall, Jonas K., I I 2 .

Hall, Kirtland, $\mathbf{1} 0_{3}$.

Hall, Lucy, 6 I, y or.

Hall, Lydia, 4t, So.

Hall, Mary, Io3.

Hall, Mehitabel, $6 r$.

Hall, Nathaniel, 7, 8.

Hall, Ormiel, I 77, 205. 
Hall, Sarah, 7, I4.

Hall, Thomas, $\mathbf{5}$.

Hall, William, 85 .

Hallam, Robert A., I o5.

Hallan, Mrs. Robert A., I 54.

Hallet, Ann, xxvii.

Hallet, John, xxvii.

Halsey, Charles, 93.

Halsey, John, 93.

Halsey, William, 92.

Hamilton, Mary E., I32.

Hamlin, Willis A., I $6 \mathrm{r}$.

Hammill, Esther Sanford, 144, 187.

Hammill, Mary Frances, I44, 187 .

Hammill, William, I44, I87.

Handford, Mary, xix.

Hard, Cornelius Marcus, I87.

Hard, Ellen, 187.

Hard, Mary, 122, 1 69 .

Hare, J. Montgomery, 207.

Hare, Marian S., 207.

Harmon, William B., 158.

Harrington, E. E., 204.

Harrison, Samuel, 70.

Hart, Ida Isabel, 187 .

Harvy, - 146 .

Hasard, Anna Isabel, I 87, 209.

Haskins, Asa, 67 .

Hassard, Jane, 209.

Hassard, Thomas, 209.

Hatch, Chauncy, 88.

Hatch, Edward Walker, I47, 188.

Hatch, Keturah, 3 I, 59.

Hatch, Nancy C. Boris, I47, 188.

Hatch, Sarah Elizabeth, 147, I 88.

Havens, Elmer H., I 7 r.

Hawkins, _- 208.

Hawkins, Sylvester, 125.

Hawley, Abel, 26 .

Hawley, Abijah R., 84 .

Hawley, Clarles, 72 .

Hawley, Cyrus, 54.

Hawley, David, $5^{8 .}$

Hawley, Eli L., 54 .

Hawley, Elias Youngs, 53 .

Hawley, Elizabeth, 8.

Hawley, Frederick, 203.

Hawley, Frederick H., I 24.

Hawley, Gideon, 45.

Hawley, Helen Lonise, 203.

Hawley, Henry, I 2, 26.

Hawley, Ifenry S., I 4 r.
Hawley, Ichabod, 45.

Hawley, Joseph, viii, ix, xxiii, xxiv, 1 .

Hawley, Laura, 76 , I I 9 .

Hawley, Mary, I 2, 26 .

Hawley, Rufus, 66.

Hazen, Charles, 95 .

Healey, Effie J., 160, 198 .

Heath, Isaac, xix.

Heath, Thomas, vii.

Heaton, Harry, I 48.

Henries, Zilpha, ıоg.

Henry, Almon, 138.

Henry, Cynthia, 138 .

Henry, Howard, I 85.

Henry, Mary, 137.

Herryson, Anne, xxi.

Hewitt, Desire, 2 I 4 .

Hicock, Amos, 30.

Hicock, Bethiah, 56 .

Hicock, Curtiss, 58.

Hicock, Huldah, 14.

Hicock, Ithiel, 30 .

Hicock, Lois, 14.

Hicock, Mary, 28, 56 .

Hicock, Olive, 3 I.

Hicock, Stephen, 56 .

Higbee, Phebe Ann, 91, 138.

Higley, Adeline, 104.

Higley, Martha, 85 .

Higley, Sarah, 104.

Hill, —-, 214.

Hilson, Eleanor Johnson, 140, 184.

Hilson, Mary Elizabeth, I 40,184 .

Hilson, Thomas, 140, 184 .

Hines, Harriet, 66, I 10.

Hines, Josiah, 99.

Hines, Mary Louise, 145,187 .

Hines, Richard, 145,187 .

Hinman, —, 60 .

Hinman, David, 30, 94.

Hinman, Edward, 28.

Hinman, Elijah, 29.

Hinman, James, $5^{8}$.

Hinına, Joel, 30 .

Hinman, John, 23.

Hinman, Margeth, 99.

Hinman, Mercy, 25, 50.

Hinman, Sarah, I $4,30$.

Hinman, Sylvester, 54 .

Hinman, Titus, 30.

Hinman, Truman, 56.

Hinman, IVait, 50 . 
Hitchcock, Abigail, 68 .

Hitchcock, Augustus, 153.

Hitchcock, Betsey, 4 I, 76 .

Hitchcock, Elizabeth, 106 .

Hitchcock, Lois Atwater, I 53.

Hitchcock, Sarah Maria, ro5, I 53.

Hoffman, George, I 57 .

Holbrook, Esther, 5 1, 87 .

Holcomb, Banajah, Jr., 65 .

Holland, John, xxx.

Holland, Obedience, xxx.

Hollister, - 32 .

Holmes, George L., I 24 .

Holmes, Sarah, 9 I, ${ }_{3} 8$.

Holt, Abigail, 7 .

Holt, Joseph, 7, 8 .

Holt, Lowly, i 6.

Homan, Augusta Savage, I92.

Homan, Clara Augusta, 192.

Homan, Charles Edward, 153.

Homan, IVilliam, I92.

Hooker, Hezekiah, 12.

Hooker, Thomas, viii.

Hooper, John Jay, 137.

Hopkins, Dorothy, 55, 2 I4.

Hopkins, Stephen, 55, 214 .

Horner, Augusta Savage, I 53.

Horner, Clara Augusta, 153.

Horner, William, 153 .

Horton, Eveline, 113 .

Hosford, Elihu, r 26 .

Hoskins, Maria, I 13 , 160.

Hotchkiss, Albert, 76 .

Hotchkiss, Laura Sophia, I 53, 192.

Hotchkiss, Merriam, I6, 34 .

Hotchkiss, Olive Webb, I59, I97.

Hough, Daniel, 6 r.

Hough, Harvey, 48.

Hough, Mary A., 85 .

Hough, Mindwell, 31, 6r.

Hough, Rebecca Tuttle, I03, I 49.

Houghton, Eveline, I $6 \mathbf{r}$.

Houghton, Jared, I 6 I.

Houghton, Nancy, I6I.

How, Sarah, 7, I 4 .

How, Sarah Gilbert, 14.

How, Zachariah, 14.

Howard, Georgie, 122 , I 7 1.

Howard, Matilda A., 137, I 83 .

Howell, Ann Eliza, 162.

Hoxey, Stephen Van Renselear, 95.

Hubbard, Emily M., I O2, 148.
Hubbard, Huldah, 5 I.

Hubbard, Nellie E., I 74, 203.

Hubbell, Huldah, 88.

Hubbell, Louisa M., 93.

Hubbell, Mehitabel, 9.

Hubbell, Richard, 9 .

Hubbell, Wolcott, 59 .

Hughes, Charles H., I 46.

Huiell, John V., I 68 .

Hull, $\longrightarrow, 2$ I.

Hull, Abel, I I r, 159.

Hull, Abijah, 39 .

Hull, Charlotte Sophia, I I I, I 59.

Hull, Electa, I 59.

Hull, Jeremiah, 7 .

Hull, Martha Abbott, I 6 , I 62 .

Hulse, Ada H., I 53, I 91.

Hulse, Harriet A., I 5 , 152.

Hulse, Thomas, I 05 , I 52.

Huntington, Daniel, 58, 98 .

Huntington, Sybilla, 58,98 .

Hurd, Abiah, 5 I, 88 .

Hurd, Abigail, I 9.

Hurd, Amos, 26, 5 I, 89.

Hurd, David, I 3 .

Hurd, Esther, 9, I9.

Hurd, Graham, 30.

Hurd, John, 19.

Hurd, John, 2 d, 9.

Hurd, Nirom, 26, 5 I, 88.

Hurd, Sabrina, 5 I.

Hurd, Salina, 89 .

Hurd, Sarah, 51,88 .

Huse, Nicholas, ro.

Hussey, Mary A., I 48, I 89 .

Hutchins, Elizabeth, vii.

Hutchins, John, xxi.

Hutchins, Thomas, xxi.

Hutton, John, xx.

Hyde, Martha, I I o, I 56.

Ives, Abel, I 7, 39.

Ives, Anne, $17,39$.

Ives, Eliza, r o3, I 49.

Ives, Hannah, 62, Io3.

Ives, Jesse, Io3, 149.

Ives, John, 7.

Jacobs, Mary, 36, 67 .

Jacobs, Richard, 36,67 .

Jacobs, Thankful Kellogg, 67 .

Jandon, Elizabeth McDonald, I 40, I 84.

Jandon, Frank, 140, 184.

Jayner, Newton, III. 
Jester, Ann Eliza, I 80, 207.

Job, Herbert K., I go.

Johnson, —, I46.

Johnson, Catherine, 2 I 5.

Johnson, Ebenezer, 9, I9.

Johnsou, Hannah, 9, 19.

Johnson, John, 44 .

Johnson, John Quincy Adams, 195.

Johnson, Lambert, 8 .

Johnson, Louisa, 62, I04.

Johnson, Sarah, xx.

Johnson, Solomon, 3 I.

Jones, Emma D., I 75, 205.

Jones, Isaac, 42.

Jones, Joel, 87 .

Jones, Richard E., 1 75, 205.

Joyner, Elizabeth, I 57 .

Joyner, Frank, I 56.

Judson, Aaron, 73.

Judson, Abigail, 5, 12.

Judson, Abner, 39, 78, I 23.

Judson, Agur, 19.

Judson, Amy, I 8, 42 .

Judson, Benjamin, 39 .

Judson, Bethia, 42, 77 .

Judson, Curtiss, 40.

Judson, Daniel, 39.

Judson, David, I 2, $25,27,39$

Judson, Elizabeth, 25 .

Judson, Ephraim, 45.

Judson, Esther, 3, 9.

Judson, Ethan A., Iog.

Judson, Eunice, 9, 1 \&.

Judson, Harrjet, 78 , I 23 .

Judson, Helen, 73, I 15.

Judson, Huldah, 42, 77 .

Judson, James, I 8, 42 .

Judson, Jeremiah, 1 .

Judson, Jeremiah, Jr., 9.

Judson, John, 25.

Judson, Joseph, 3, 5, 9, I 2, I 3 .

Judson, Martha, I 8, 42.

Judson, Mary, I 2, 24, 25, 90.

Judson, Monroe, 94.

Judson, Naomi, 73, 1 I 5 , I I 6.

Judson, Phebe, I 2, $25,27,39$.

Judson, Philo, $5^{8 .}$

Judson, Prudence, I 2, 24.

Judson, Rebecca, ז 7, 39, 73, I I 6 .

Judson, Samuel, 1 2, 24, 42, 77 .

Judson, Sarah, 3, 5, 9, I 2, 25, 49.

Judson, Silas, 42 .
Judson, Stiles, 73, I I 5, I I 6 .

Judson, William, viii.

Keeler, Ransom, 85 .

Kellogg, Donald, 67 .

Kellogg, Mary, 67, I I I.

Kellogg, Timothy, 67, I I I.

Kelly, Blanche Eliza, I 62.

Kent, Abdiel, 70.

Kent, Ezekiel, 70.

Kent, Ira, 7 o.

Kent, J. V. R., 70.

Ketcham, Louisa, 90.

Keyes, Ann, xviii.

Kidder, John, $3^{8}$.

Kimball, Nancy I., I 26.

Kimberly, Abraham, 2.

Kimberly, Eleazer, xxviii.

Kimberly, Hannah, 2 .

Kimberly, Le Roy Hart, Io7.

King, Eldad, I4.

Knap, Abigail, 13, 28.

Knapp, Elizabeth, 93.

Kneeland, Alice, I 34, I 79.

Kneeland, Henry, I 34, I 79.

Kneeland, Margaret, I 34, I 79.

Kraus, Leopold, I 72.

Kummel, Henry E., 93.

Lacey, Polly, 55, 93 .

Lake, Nichols Booth, 9o.

Lamb, Loomis, I I 2.

Lambert, Emily A., I I 7, I 65 .

Lamphere, Rebecca, 97, I 43 .

Lane, Hannah L., $102,147$.

Langdon, Andrew, 106.

Langdon, John, 66.

Lapham, Amelia, 2 I 5.

Larrabee, Rollin W., I 85 .

Lash, David, 66.

Lawrence, Bigelow, 36 .

Leavenworth, Susannah, 56 .

Leavitt, Samuel, I 5 .

Le Baron, David, 63.

Le Baron, Huldah, 63 .

Le Baron, Martha Chatfield, 63 .

Lee, Lucy Camp, 85 .

Lee, Mary, 49, 85 .

Lee, Timothy, 49, 85 .

Le Sargent, John, xx.

Lewis, $\longrightarrow 53$.

Lewis, Abram C., 162.

Lewis, Agur, 54.

Lewis, Alva, 155 . 
Lewis, Benjamin, $3,47,77,82, \mathbf{I} 2 \mathbf{I}$.

Lewis, Caroline, 87,132 .

Lewis, Conway, I23.

Lewis, David, 18,76 .

Lewis, Edmund, I r.

Lewis, Eli Walker, 73.

Lewis, Elizabeth, 47, 77, 82, $121, x 68$.

I.ewis, Elizabeth A., I 2 I.

Lewis, Hannah, 156, 194.

Lewis, Hannah Wheeler, 172.

Lewis, Huldah, 47, 82 .

Lewis, Jane M., I 22, I 70.

Lewis, Job, 24.

Lewis, Joseph H., 156, 194.

Lewis, Judson, 54 .

Lewis, Latura, 94, I4 1 .

Lewis, Levi, I 23.

Lewis, Loderick Shurrell, I 13.

Lewis, Mary, 24, 49, 77, 121, 156, 194 .

Lewis, Nathan, $2.4,49$.

Lewis, Phebe, 1 $24,172$.

Lewis, Philo, 42.

Lewis, Robert, 54 .

Lewis, Sarah, 26, 42, 54, 77 .

Lewis, Sarah Catherine, 54 .

Lewis, Silah, 154 .

Lewis, Stephen, 49 .

Lewis, Stephen Curtiss, 172.

Lewis, William, 42, 77 .

Leyden, Ann E., ı 06.

Lillingston, Frederick, 20 I.

Lillingston, Grace, 20 I.

Lillingston, Isabella, 201.

Lindslay, Emily Sophia, I 25, I 74.

Lindsley, James H., 164.

Lines, Edwin L., 103.

Lingenfelter, Eva A., 131, I 77 .

Litchfield, David B., 113 .

Littlefield, Henry C., I59.

Livingston, Edward Bayard, 97.

Locke, Sarah, xxvi.

Kogan, Samuel S., 125.

Lombard, Charlotte, III, I58.

Lombard, Lemuel, i i I, I 58.

Lombard, Lucinda, I 58.

London, Ambrose, 17.

Longworthy, John, 107.

Lovejoy, $\longrightarrow, 86$.

Loveland, Ezra, 86.

Lord, Electa, 73, I 18.

Lucas, Edwin, I I 4 .

Lucas, John E., 2 I5.
Luikhart, Elizabeth K., I 76 .

Lyman, Hannah, 57, 96.

Lyman, Mary, $97, \mathbf{1} 43$.

Lyman, Rozella, 97.

Lyman, Samuel, 96.

Lyman, Sarah Maria, 97 .

Lyman, Solomon, 55 .

Lyons, Mary Elizabeth, 139, I83.

Lyons, William, I 39, 183 .

Mackay, T., Jr., I 79.

Main, Amelia Lent, I I 8, 166.

Malone, Henrietta, 102.

Mallett, Peter, 43 .

Mallory, Abigail, 3 I, 59 .

Mallory, David, 59.

Mallory, Gideon, 59 .

Mallory, John, 60.

Mallory, Mary, 6, г 2, 3 I, 6o.

Mallory, Obedient, 2 I , 45 .

Mallory, Olive, 59 .

Maltbie, Warren, rog.

Mann, Robert, 145 .

Mansfield, Catherine Hurlburt, I 54, I92.

Mansfield, Phineas, I I I.

Mansfield, Sophia Phillips, I 54, I 92.

Mansfield, Thomas Trowbridge, I54, 192.

Marcellus, George E., I 55.

March, Stephen, 7 I.

Marcy, John, Io.

Marks, Silas H., I 16.

Marshall, Anna A., I 30 .

Martin, Abigail, $3^{\circ}$.

Martin, Annie, I40, I84.

Martin, Lydia, $17,3^{8}$.

Martin, Phebe, 14, 30 .

Martin, William, 3 o.

Martingale, $\longrightarrow, 32$.

Matthews, Joseph, $\mathbf{1} 4$.

Matthews, William, Io6.

Mattoon, - 20.

Mattoon, Nathaniel, $\mathbf{7}$.

May, George, ${ }^{3}{ }^{8}$.

May, Lovina, 137.

May, Sophia, 9 I, I 37.

Mayo, Clarendon, i I 1 .

McClure, John, iso.

McClure, Mary Hawkins, I80, 208.

McClure, IVilliam, iso, 208.

McDaniel, Lany, \$9, I 35 .

McDaniel, Risdon, 89, I 35 .

McEwen, David, 98, I 44 .

McEwen, Joel, I 66. 
McEwen, John, 43.

McEwen, Minerva, 98, r 44 .

McEwen, Polly E., 77, I 22.

McEwen, Sally Booth, 98, 144.

McEwen, Sarah, I 8 , 166.

McEwen, Tryphena Gorham, x 66.

McIntire, Amy Cornell, I 40, I84.

McIntire, Ruth Lovett, 140, I 84 .

McIntire, William, I 40 , I84.

McKay, Robert Ridley, 93.

McIoughlin, Samuel, 9r.

Mead, 'Tammah, 57, 95 .

Meeks, Clotilda, 32.

Melboume, Martha, 173 .

Merriam, Nathaniel, 6I.

Merriman, Abigail, 2, 6 .

Merriman, Eliza Curtiss, 193, 209.

Merriman, Grace, 193, 209.

Merriman, Joel Bacon, 156, 193, 209.

Merriman, Josiah H., 155.

Merriman, Mary, 2,6 .

Merriman, Nathaniel, 2, 6.

Meserah, Hannah Maria, I6r.

Meymeis, Andre, 136 .

Middlebrook, —, 76 .

Miles, John, I 7 .

Miles, Philip, 148.

Miles, Susan J., 1 24, I 72.

Miller, Anna M., 1 75, 205.

Miller, Robert, 83 .

Mills, Amasa, 35 .

Mills, Elizabeth, 36, 66 .

Mills, Gideon, 36, 66 .

Mills, Joane, xx.

Mills, Treat, 24.

Mills, Washburn G., I61.

Miner, Sarah, 6, 13.

Miner, Thomas, 6.

Minor, Allen I., I 85 .

Minor, Elizabeth, 22, 47.

Minor, Ephraim, 6.

Minor, John, $1,3,13$.

Minor, Matthew, 30,58 .

Minor, Richardson, 22, 47.

Minor, Richardson, Jr., 18.

Minor, Sarah, 3o, 58.

Minor, Truman, I I 7 .

Mitchell, —, I 18.

Mitchell, John, I 3 .

Mitchell, Mary, I I, 23 .

Mix, Deborah, I6, 36 .

Mix, Thomas, 16,36 .
Modrul, Ann Elizabeth, I34, I 8 I.

Modrul, Elizabeth, I 34, I 8 I.

Modrul, Robert, I34, I 8 I.

Moffatt, Anthony, 92.

Moffatt, John L., 55 .

Monroe, Jane, 85 .

Moore, Fannie, $8 \mathrm{~g}$.

Moore, William, I 96.

Moore, IVilliam B., 195.

Morehouse, Daniel, 26.

Morris, Matthew, ix, 2, 3 .

Morris, Sarah, ix, 2, 3 .

Morse, Collins, 96.

Morse, Patty, 52, 91.

Moseley, Increase, 50.

Moseley, Olive, 50.

Moseley, Patience Hinman, 50.

Moseley, IVilliam G., I 17 .

Moses, Zebina, 65 .

Moshier, Esack, 137.

Moss, Jane, 26,53 .

Moss, John, 26, 53, 54 .

de Mott, Edward P., I 59 .

Mower, Marshall, 38 .

Munn, Gideon, 58.

Munson, Augusta, I o6, I 54.

Munson, Edgar, 105.

Munson, Ephraim, 15.

Munson, Jared, 59.

Munson, Jesse, r o6, I 54 .

Munson, Jesse, Jr., I 53.

Munson, Rebecca, 7 .

Munson, Sophia, 106, I 54 .

Munson, William, 7, 8 .

Naramore, Robert Comstock, I $2 \mathbf{x}$.

Nefus, Marcia, 90.

Nettleton, Joseph, 89 .

Newell, Catherine, 49,85 .

Newell, David, 66 .

Newell, Isaac, 49, 85 .

Newell, Rachel Pomeroy, 85 .

Newhall, Isaac, xxx.

Newman, Ferris, I 43.

Newman, Samuel, Jr., 67 .

Newman, William, xviii.

Newton, Deborah Hall Grosvenor, I 03 , I 49 , I 50 .

Newton, Larkin, 103, 149, 150.

Nichols, $\longrightarrow, 84$.

Nichols, Andrew S., 84 .

Nichols, Isaac, xxiii, xxiv.

Nichols, John, Jr., 2 I 2. 
Nichols, Joseph, 8 .

Nichols, Lewis, 54.

Nichols, Mary, 94, 141.

Nichols, Nathaniel, 26, 5 I.

Nichols, Phedina, 26, 5 I.

Nichols, Philo, 46.

Nichols, Ruth A., r $_{4}$.

Nichols, Simeon, 90.

Nichols, Theophilus, Ix.

Niles, Julia E., I 26, I 75 .

Noble, - 93.

Northrop, Thomas G., 52.

Norton, Andrew O., I 14 .

Norton, Dinah, 20, 24.

Norton, Fanny M., r63, r99.

Norton, Jedediah, $\Upsilon_{4}, 32$.

Norton, Samuel, 20,44 .

Olcott, Joseph, 65 .

Olmstead, Anne Belden, I I6, I63.

Olmstead, Fannie, I 16, 163 .

Olmstead, Jane, I I 6, I6 3 .

Osborn, Abigail, 2 I, 46.

Osborn, Jasper, 99.

Osborn, Mary, 55, 92.

Osborn, Moses, 55, 92 .

Osborn, Nancy, 2 r 2.

Outcault, Cornelius Brinkman, 140.

Overing, Elizabeth, 82, I 27.

Owen, Carolina, r io, 157 .

Owen, Ira, rio, 157 .

Owen, Sophia Standish, r ro, 157 .

Palley, Hannah, xxx.

Palley, John, xxx.

Palmer, Experience, xxix.

Palmer, John, xxix.

Palmer, Phebe, 7 .

Palmer, Solomon, 33 .

Palmer, William E., 107.

Pancost, Mary E., 109, I 55.

Pantlin, Rhoda, 69, I I 2.

Parker, — 146 .

Parker, Chestina, $x 6,35$.

Parker, Daniel, 32.

Parker, Daniel Webster, $\mathbf{3} 6$.

Parker, Eliada, 15.

Parker, Eliphalet, 35.

Parker, Esther, 33, 62.

Parker, Fannie, 94, 141 .

Parker, Hannah, 35 .

Parker, John E., I 52.

Parker, Joseph, $7,8$.

Parker, Sarah, 7 .
Parks, Joel, $3^{8}$.

Parks, Lillian Oregon, I 75 .

Parmalee, Ruth, 44, 8o.

Parsons, - 20.

Parsons, Israel M., I 25.

Parsons, Ithamar, 20.

Partridge, H. E., 147.

Patten, Robert, 33, 37 .

Pattenden, Joane, xx.

Patterson, Abel, 47, 82 .

Patterson, Amy, 47, 82 .

Patterson, Esther, 42, 79 .

Patterson, Lucy, 42, 79 .

Patterson, Samuel, 42, 79.

Patterson, Temperance Ann, 47, 82 .

Paulk, Eliakim, 150.

Paulk, Laura, I03, 150.

Paulk, Sally Craw, I 50.

Peak, Charles, xxx.

Peak, Dorcas, xxx.

Pease, Zeno K., I 48.

Pebbles, Augusta, $137,183$.

Peck, —, 124 .

Peck, Alice, 124.

Peck, Alice Birdseye, 79.

Peck, Anna, 77.

Peck, Betty, 74 .

Peck, Clemence, 72, 15.

Peck, Ephraim, 5 I.

Peck, Eugene B., r2o.

Peck, George W., I 3 o.

Peck, Hannah, 3r, r2 r, r68, r69.

Peck, Helen, r 23 , r 7 I.

Peck, Helen Birdseye, 79, I 24 .

Peck, Henry F., 132.

Peck, Joanna, 56, 93 .

Peck, Job, 40, 74, 17 r.

Peck, John, 7.

Peck, Josiah, 79, r 24.

Peck, Judson, 77, 122.

Peck, Martha, I 21, I 68.

Peck, Mary, 42, 78, x 22 .

Peck, Mary Blakeman, I 22.

Peck, Mary Helen, I 32.

Peck, Nathan, 42, 78 , 12 r.

Peck, Nathan, Jr., 23.

Peck, Russell, 86.

Peck, Samuel, 72, 93, r $_{5}$.

Peck, Sarah, 40, 74, 121 , I 68.

Peck, Simeon B., 90, 94 .

Peck, Tabitha, 42, 7 S.

Peck, Thaddeus, 77, 1 $21,168,169$. 
Peck, Thaddeus, Jr., 12 I.

Peckham, Charles, 2 I 5, 216.

Peckham, Nancy, 2 I 5, 216.

Peckham, Priscilla, 2 I 5, 216.

Peet, - $\longrightarrow 74$.

Peet, Phebe, 23, 48.

Peet, Richard, r 4.

Peet, Samuel, 79.

Peet, Thomas, 23, 48 .

Percival, Francis, 78 .

Perkins, Elisha, 68 .

Perkins, Eunice, 37, 68.

Perkins, Stephen, 31 .

Perkins, Tabatha, 32 .

Perrin, Frederick Stanton, 163 .

Perry, —, 100.

Perry, Alfred Gardner, 140.

Perry, Joseph, 26, 52 .

Perry, Roswell F., 197.

Perry, Ruth Ann, z6, 52 .

Phelps, - 212.

Phelps, Alonzo, $15^{8 .}$

Phelps, Asaph, I 53.

Phelps, Jannette, 158.

Phelps, Julia Hannah, 105, 153.

Phelps, Juliette Bradley, 15 S.

Phelps, Polly, $6_{3}$.

Phelps, Sophia Pearl, 153.

Philleo, Emily, 142.

Philleo, Mary, 142, 185 .

Pickett, David, 9.

Pickett, Margaret, 9 .

Pickitt, Daniel, 19.

Pickitt, Margaret, 19.

Pickitt, Mary, 19.

Pierce, E. A., I 9 I.

Pierson, Abraham, 44.

Pinkham, Hezekiah, xxv.

Pinkham, Mary, xxr.

Pinneo, Henry Osmond, r 40.

Pitcher, Eugene, 185 .

Pilts, Henrietta, 92, 138 .

Pitts, John, 92, 138 .

Pixlee, Elizabeth, 73, I $8,121$.

I'ixlee, Peter, 73, I 18.

Pixley, Peter, 47.

Plum, Rachel, $15,34$.

Plum, Waitstill John, I5.

Plumb, Benjamin, 79.

Poggiasuasa, Emanuel, Prince of, 176.

Poison, Chloe, 65.

Porter, David, 6o.
Porter, Elizabeth, 5.

Porter, Elizabeth A., 87.

Porter, Hannah, 5, 1 I.

Porter, Nathaniel, 5, I r.

Porter, Ruth, 5, 11 .

Post, Frederick, 165.

Potwin, Benjamin, 62.

Powell, $\longrightarrow 34$.

Pratt, Carrie A., 133 .

Preston, Betterus, 56.

Preston, Jehiel, 28, 56.

Preston, Martha, 1 3, $28,56$.

Preston, IVilliam, 28.

Prince, James, 74.

Pugeley, Mary, 107, I 55.

Puiner, Rosa, 165 .

Purion, Helen, 200.

Putney, Lucy, $17,3^{S}$.

Pynchon, William, viii.

Quintord, Anson, I 16, 164.

Quintord, Mary Frances, I 16, 164.

Quintord, Polly, I I6, 164 .

Race, Nicholas, I I 1 .

Randall, Richard, I 35 .

Ranger, George F., 193.

Rathbun, James Sylvester, 97.

Raymond, Cornelia I., I $63,200$.

Read, Charles Henry, 97.

Redfield, Ann, 105.

Redfield, Augustus, 62, I05.

Redfield, Horace, I 04.

Redfield, Mehitable, 62, I 05.

Reed, John, xviii.

Reed, Newton, 125.

Reeve, Emma L., I 3 I.

Ressique, Jacob, 22 .

Rice, Moses, 48 .

Rice, Silas, 62.

Richards, - 32 .

Rider, Abigail Terry, xxvii.

Rider, Elizabeth, xxvii.

Rider, Mary Harriet, 141.

Rider, Thomas, xxvii.

Robbins, Appleton, 66.

Roberts, Samuel, 102.

Robertson, Catherine Mumford, I 2 2, 170.

Robertson, John, 122, 170.

Robinson, - - 20.

Robinson, Abigail M., 9 I, I 38 .

Robinson, Anna, 69.

Robinson, Maria, 214.

Robinson, Sabrina, I I I, 157. 
Robinson, Sanuuel, 69.

Robinson, Timothy, Ir I, 157.

Rogers, J. Augustus, 170.

Rogers, Matilda, 89 .

Roice, Ebenezer, 15.

Root, Elisha, 24.

Root, Jonathan, 24, 48.

Root, Josiah, 69.

Root, J. T., 160.

Root, Kczial, 69.

Root, Margaret, 24, 48 .

Root, Martha, 37, 60 .

Root, Nettie, i6r, 199.

Root, Ruth, 24, 48 .

Rose, —, ix, 5 .

Rose, Betsey, 9 r, r 37 .

Rose, Elizabeth, 4.

Rosecrans, Hannah, 1 34, I8o.

Rosecrans, Jacob, r 34, iso.

Ross, Alva, rog.

Rowell, Thomas, ro.

Roys, Abner, 67, I 10, 157 , r 95 .

Roys, Ella, $\mathrm{r}_{57}, \mathrm{x}_{95}$.

Roys, Harvey, I I r.

Roys, Isaac, 67.

Roys, Jane Spurr, 157 , r 95.

Roys, Levi, 67.

Roys, Ruth, 67, i ro.

Ruffin, Corinna B., $146, \mathrm{r} 88$.

Ruffin, Robert J., r 46, 188.

Rugg, Charles, 85 .

Ruggles, John, xxi.

Ruggles, Thomas, xviii.

Ruspoli, Emanuel, Prince of, $\mathbf{r} 76$.

Russell, Benjamin, r64.

Russell, William, r 9 .

Rutley, Ezra, roz.

Sage, Simon, 157 , r 95.

Sage, Susan, $157,195$.

Salmon, Richard, 23 .

Salter, Rebccca, 16,37 .

Salter, Sarah, I 7, 38 .

Sample, Polly, 88, 34.

Sanborn, Mary A., I 74 .

Sanford, Elizabeth, I44, I 86.

Sanford, Isaac R., 195.

Sanger, $\stackrel{4}{2}$, I 13 .

Sansome, Ann, xviii.

Sawyer, George, 97 .

Scarlet, Robert, xx.

Schmitz, Margaret, 121,167 .

Schmitz, Peter, r21, 167 .
Schmitz, Susan, r2 r, 167.

Scott, Eunice, 62, ro6.

Scoville, Eunice, I 33, 178 .

Scoville, Mary Anne, 133, r 78.

Scoville, IVilliam Henry, $133,178$.

Scranton, - 80 .

Scranton, David, 44.

Scribner, James, 1 i 6.

Seaman, Samuel Edward, 168.

Searle, Elizabeth, xxi.

Searle, William, xxi.

Secor, Moses, I 20.

Seeley, Davikl, 23.

Seeright, William, $\mathrm{I}_{3}$.

Selleck, James K., 163 .

Sessions, Jared D., 150, 190.

Sessions, Latra IV., I 50, I go.

Seymour, Bevil, so.

Seymour, Lizzie, r 48, r 89 .

Seymour, Matthew, 22.

Sharp, Ira, I 42 .

Sheldon, Emeline, r6o, 197.

Sheldon, Emily Louise, r 98.

Sheldon, Orlando, I 60.

Shelly, Mary, xviii.

Shelton, Ambrose, x 55.

Shelton, Avis Newton, 73, I I 5 .

Shelton, Daniel, 39, 73, 15.

Shelton, David, I 29 .

Shelton, Edwin, 1 r6.

Shelton, Jeannette, 56, 94 .

Shelton, Mary, 39, 73 .

Shelton, Mehitable, 73, r 15.

Shepherd, Fred, 203.

Shepherd, Jerre, 9 I.

Sherman, Aaron, 58.

Sherman, Alonzo, r 20.

Sherman, Benjamin, 45 .

Shernan, Ebenezer, 40, 75 .

Sherman, George L., 8 I.

Sherman, Job, 29, 57.

Sherman, Phebe, 29, 40, 57, 75.

Sherman, Samuel, Jr., ro.

Sherwood, —, 53 .

Sherwood, Aaron Banks, I 29.

Sherwood, Lucile, 126 .

Shipman, Benjamin, 95 .

Short, Helen, $x 8_{3}$.

Shumwag, Sophia, 95, 142 .

Sicor, Theodosius F., 120.

Silliman, - 173 .

Skelton, —, 86. 
Slater, Benjamin, 6o, 99.

Slater, Esther Preston, 60, 99.

Slater, Jennet, 60, 99.

Sledge, Elizabeth, 38, 70.

Slocum, Frances E., I5I.

Smith, - 2 I .

Smith, Anson, 8g.

Smith, Carrie, 95 .

Smith, Elijah, 62.

Smith, Frances M., I 5 I.

Smith, Haunah, xxv.

Smith, Jane B., I 20.

Smith, John, xxv, 36 .

Smith, Joseph, I 60.

Smith, Julius B., I61.

Smith, Lucretia, 132.

Smith, Lucy, 35, 64 .

Smith, Mary, I98.

Smith, Samuel, 117 .

Smith, Samuel C., I 25.

Smith, Timothy, 16.

Smith, Walter D., I 26.

Smith, William, 57 .

Sniffen, Deborah, I 2 I, 168.

Southworth, Mary, I 18 , I65.

Southworth, William, I 9.

Spelman, John Adams, 150.

Spencer, Charles, $121,167$.

Spencer, Frances Catherine, I $21,167$.

Spencer, Lucretia Marsylva, I 67 .

Spencer, Martha, 6 .

Sprague, John, 96.

Sprague, Lucy, 57, 96.

Squire, Allan B., I 54 .

Squire, Francis Orvis, I 95, 2 I o.

Squire, Helen Lawrence, 195, 210.

Squires, Mercy J., I 25, 173 .

St. John, Adonijah, 86 .

Stagg, Joseph H., 24.

Stair, Edwin, I 65, 200.

Stair, Lucia Marcia, I 65, 200.

Stair, Marcia Lucia Cobb, 165, 200.

Standing, Eliza Anu, 64, 107.

Stansbury, Catherine, 92, 139.

Stansbury, Jonas, 92, I 39.

Stansbury, Mary Cccilia, 92, I 39.

Staples, —, 74 .

Stebbins, Alice, $148,189$.

Stebbins, Ellen, 132 .

Stebbins, IVilliam, 86.

Steele, Deborah Lacey, 58.

Steele, Delia Augusta Perry, I 27, r 76.
Steele, Emma Sophia, 1 27, r 76.

Steele, Lucy J., 160, I 98.

Steele, Michael, $5^{8}$.

Steele, Naucy, I I I, I 58.

Steele, Roderick Cadwell, $127,176$.

Stephens, Mary Ann, I 12 , 159.

Stevens, - 129.

Stevens, Helen, I 42.

Stevens, Laura Ann, r 59, 197.

Stevens, Maria, I42.

Stevens, Sally L., $107,155$.

Stevenson, Betsey, 67, I 10.

Stevenson, Hannah, 67, I 10.

Stevenson, John, 122.

Stevenson, 'Thomas, 67, I 10.

Steward, George Thomas, 209.

Stillman, Charles, 193.

Stiles, Bathsheba, 9 .

Stiles, Elizabeth, 9, I 7 .

Stiles, Ephraim, xxiii, xxiv, 3, 9, 17.

Stiles, Eunice, 29.

Stiles, Frances, $1 S_{5}$.

Stiles, Francis, 29, 143.

Stiles, Henry B., $143, \times 85$.

Stiles, Mary, 29.

Stiles, Mary Jennette, I 43 , I 85 .

Stiles, Roderick, 98 .

Stiles, Rufus, 98.

Stoddard, - 30.

Stoddard, Abijah, I 3 .

Stoddard, Anthony, 1 4, 30.

Stoddard, Eliakim, I 3.

Stoddard, Elisha, I 4, 59.

Stoddard, Gideon, I 3 .

Stoddard, Jessie, 204 .

Stoddard, Mary, I 4, 30, 59 .

Stodder, John, Jr., xxix.

Stone, Eleanor, xxi.

Stone, Ellen, I 72 .

Stone, Hannah, I 38.

Stork, Francis, $180,208$.

Strange, Estella, I 8 I.

Stratton, - 123.

Stratton, Althea J., 173.

Stratton, Thomas, 2 I.

Stridwell, Charles A., I 64.

Strong, Anthony, 58.

Strong, Benjamin, 60, 100.

Strong, Elizabeth McDonald, I 40, 184 .

Strong, Esther (Mead), 50, 86.

Strong, Flora (Preston), 98, 143.

Strong, Jennette Mallory, 99, I 45 . 
Strong, John, 98, I 43.

Strong, Julia, 6o, roo.

Strong, Julia Flora, 98, 143.

Strong, Lucy, 6o, 99.

Strong, Martha Jennette, 99, 145.

Strong, Olive, 50, 86.

Strong, Oliver, I 40,184 .

Strong, Selah, 50, 86.

Strong, Stoddard, 99, 145.

Strong, 'Thomas, $3 \mathrm{I}$.

Stuart, Adela, I 72, 202.

Stukeley, Dr., xx.

Sullivan, Harriet Eliza, 104, 152.

Summers, Eunice, 2 I, 45 .

Summers, Luke, $4 \mathrm{I}$.

Summers, Maria, 98, 143.

Summers, Mary, 10, 20.

Sutliff, Samuel, 20.

Sylvester, Joseph, xxix.

Sylvester, Mary, xxix.

Symons, Michael, $x x$.

Sypert, Mary, 99, 145 .

Sypert, Stephen, 99, 145.

Taber, Susan A., 181, 209.

Tabor, L. H., 7o.

Tailor, Mary, xviii.

Talbot, Ellen V., 1 71, 202.

Talleyrand-Perigord, Maurice, Marquis de, I 76.

Teasdale, Belle, i 82.

Telfair, - 2 I 4 .

Terrell, Julantia E., Iог, 146.

Thomas, Isaiah, 2 I 5 .

Thompson, - $93,212$.

Thompson, Abigail, 5, 10, 1 2, 27.

Thompson, Abigail Judson, 2 I 5.

Thompson, Ambrose, I $7,40$.

Thompson, Anne, 1 7, 40.

Thompson, David, Jr., 40.

Thompson, George, I 22.

Thompson, Hezekiah, 46.

Thompson, John, 5, 9, 10, 39, 42, 77 .

Thompson, Joseph, 73.

Thompson, Josephine, 194.

Thompson, Mary, 42, 77 .

Thompson, Mehitabel, 42.

Thompson, Mirabel, 5, jo.

Thompson, Nancy, 213, 215 .

Thompson, Nehemiah, 215.

Thompson, Ruth, 78.

Thompson, Samuel, 16.

Thompson, Sarah, 22, 46 .
Thompson, Thankful, 20,44 .

Thompson Timothy, 3 r.

Thompsorr, William, I 70.

Tibbals, Dorothy, 40, 75 .

Tillotson, Curtiss, 96.

Todd, Charles H., 70.

Todd, Theodore Wallace, 140.

Tomlinson, — 60 .

Tomlinson, Agur, 41.

Tomlinson, Amy, 26, 53, 54 .

Tomlinson, Anne, 26,53 .

Tomlinson, Bathsheba, 3, 9.

Tomlinson, Henry, 3, 9.

Tomlinson, Homer S., 144.

Tomlinson, Isaac, 87 .

Tomlinson, Joseph, 73.

Tomlinso1, Sarah, 26,54 .

Tomlinson, Zachariah, 26, 53, 54 .

Tomson, William, xviii.

Towne, Phebe, 26, $5 \mathrm{r}$.

Towner, —, roo.

Towner, Eunice, 14, 30.

Towner, Martha, I4, 30.

Traver, —, 198.

Treat, Robert, xxiv.

Trubee, David, 77.

Trubee, Samuel Comfort, 77.

Trumbull, Benjamin, viii.

Tryon, Emma L., I 47.

Tryon, Thomas, $6 \mathrm{I}$.

Tucke, Ebenezer Franklin, 194, 2 ro.

Tucke, Florence Irene, r 94, 2 I o.

Tucke, Mary Robinson, 194, 2 Io.

Tuller, Omas, II 1 .

Turney, Aaron, 77.

Tuttle, Lucinda, I 10.

Tuttle, Manderville, I 37.

Twaight, Joane, $x x$.

Tyler, Alonzo, 96.

Tyler, Jane Eliza, 135, $18 \mathrm{I}$.

Ufford, Abigail, 72 .

Ufford, Anna, 72.

Ufford, Benjamin, 72.

Ufford, Frances, 87,133 .

Ufford, Phebe, 4 I.

Ufford, Samuel, 72.

Ufford, Sarah, 18, 4 I.

Ufford, Thomas, 41 .

Uffort, Samuel, 8.

Uffort, Thomas, vii, viii.

Underwood, Henry, I Io.

Upson, Julia Ann, I02, 148. 
Upson, Philo, III.

Upton, Helen, I I 4, I 6.2.

Utley, Origen, I 48.

Van der Burgh, Lydia, 86, I $3 \mathbf{x}$.

Vanderslip, George, I 96,21 I.

Vanderslip, Jane, i 96,2 I 1 .

Vanderslip, Lucinda, r 96, 2 I 1 .

Van de Water, Mary, 92, I 39 .

Van de Water, Phebe, 92, I 39, I 40.

Van de Water, Sarah Anne, 92, 140.

Van de Water, William, 92, I39, 140.

Vincent, Robert, $\mathrm{xx}$.

Wadsworth, Nancy, 80, r25.

Wadsworth, S. J., Iog.

Wainwright, William, 20.

Wakeley, —, I 29.

Wakelyn, Henry, ix, I.

Walcott, Gov. Oliver, 105.

Walker, Hepzebah, 40, 74 .

Walker, James, 40, 74 .

Walker, Jerusha, 40, 74 .

Walker, John, Iо \%.

Wallace, F. A., 147.

Walsh, Emma, i 80.

Walsh, Jerusha, I80, 207.

Walsh, Joseph, 180, 207, 2 I 1.

Walsh, Lena, 207, 2 I I.

Ward, Dr., 29.

Warren, Jane, I I 2.

Washburn, - 45 .

Washburn, John, r.

Washington, Anyy, xx.

Washington, John, xx.

Weakley, Dinah, 9, x 9 .

Webb, Elizabeth, I 2.

Webster, Amy Ann, 102, 147.

Weeks, Charles H., I67.

Weller, Oliver S., 98.

Weller, Robert, xxvi.

Weller, Rose, xxviii.

Welles, ——, ix, 5, 86.

Welles, Abigail, 2 I 2.

Welies, Anna, 76, I I 9 .

Welles, Benjamin, 43, 124 .

Welles, David, I 15.

Welles, Elbert, I 24.

Welles; Eli, I 19.

Welles, Elizabeth, 2, 9, 18, 25 .

Welles, George, I 15.

Welles, Hannah, I I, 22.

Welles, Isaac, 86.

Welles, James, 40.
Welles, John, ix, xxiii, xxiv, 25.

Welles, John, Jr., 9, I 8.

Welles, Joseph, 42, 77 .

Welles, Joseph, Jr., I 8.

Welles, Lonisa, i 76.

Welles, Margaret, xviii.

Welles, Martha, 42, 77 .

WVelles, Mary, 25.

Welles, Mary R., I3I.

Welles, Phebe, 42, 77 .

Welles, Prudence, 2 I 2.

Welles, Robert, I 2, 40.

Welles, Roswell, I 15.

Welles, Sarah, 4, 18, 40.

Welles, Samuel, i r, 22.

Welles, Samuel, Jr., 2 I 2.

Welles, Thomas, ix, 2.

Welles, Walter le Curteis de, xx.

Wentworth, Lois, xxviii.

Wentworth, Sarah Jane, 172.

Westover, Hannah, 39, 7 r.

Wetmore, Gibert, I60.

Wheatley, Jane, 85 .

Wheeler, Cyrus, i 5.

Wheeler, Ezra, 83 .

Wheeler, Ezra C., 52 .

Wheeler, Jerusha, 74 .

Wheeler, Mary E. H., 214.

Wheeler, Nathaniel, 74.

Wheeler, Rachel, 74.

Wheeler, Rebecca, xxx.

Wheeler, Sarah A., 1 $24,173$.

Wheeler, Thomas, xxx.

Wheeler, Wilmot C., I 67 .

White, Amanda, $137,182$.

White, George C., I 44 .

White, O. F., I 75 .

White, Thomas, I37.

Whitehead, Grace Knott, 156, 193.

Whitehead, John Campbell, 156, 193.

Whitehead, Susan, 156 , I93.

Whitfield, Rebecca, 92.

Whitfield, Thomas, 92.

Whiting, Hannah, I 16, I 62 .

Whiting, Hannah Curtiss, 162 .

Whiting, Jane I., I 16,162 .

Whiting, Mary, 16, 37.

Whiting, Nathan, 162.

Whiting, Seymour Conway, 72, 162 .

Whitlow, Ida, r 35, I 8 I.

Whitlow, Josiah, r 35, r8r.

Whitney, Joseph, 43. 
Wickham, Isaac, 31, 60 .

Wickham, Patience, 31, 60.

Wilbur, Willian, 91.

Wilcockson, Timothy, xxiii, xxiv.

Wilcox, Abigail, 1 06, I 5 I, I 53.

Wilcox, Amos, 65.

Wilcox, Annie E., I o6, I53.

Wilcox, Cherissa, 65 .

II'ilcox, Daniel, 9 I.

Wilcox, George, $\mathrm{I}_{3}$.

Wilcox, George Horace, 197.

Wilcox, Harriet R., I 5 I.

Wilcox, Henry L., 193.

IVilcox, Jerusha, 45, $\mathrm{S}_{\mathbf{I}}$.

Wilcox, Oliver S., 49.

Wilcox, Silas, I o6, I 5 I, 153.

Wilcoxson, John, Jr., I 3 .

Wilcoxson, Silvanus, 36 .

Wilcoxson, William, viii, jx.

Wildman, John Rockwell, 57.

William, Michael, xv.

Williams, ——, I 18.

IVilliams, George, 158 , $19^{6 .}$

IVilliams, Harriet, r 58 , ig6.

Williams, Harriet Monervia, I 58, 196.

Villiams, John T., I 37 .

Williams, Sherman, 91 .

Willoughby, Artimesia, I r o, 156.

Willoughby, Salmon, I ro, i 56 .

Willoughby, Salome Jeffers, I 56.

Wilmot, Asahel, 35.
Wilson, Charles, I 58 , 195.

Wilson, James A., I 35 .

Wilson, Jannette, 158 , 195.

Wilson, Jarvis, 93.

Wilson, Olive, 167, 201 .

Winslow, Myron, I 41.

IVinthrop, —, vii.

Wood, Simon, 97.

Woodhul, Curtiss, 123.

Woodon, Orrin, 1 I 4 .

Woodruff, Joshua, 36 .

Woodruff, Phebe, 173 .

Woodruff, Solomon, 65 .

Woodruff, Sylvanus, 35 .

Woodruff, Wyllys, 84 .

Woodward, Eunice, $3^{\circ}, 5^{8}$.

Woodward, John, 32 .

Woolsey, Electa A., I I 9 .

Wooster, Anna, 73, I I 8.

Wooster, Joseph, 4 I.

Wright, Frederick L., I $3^{2}$.

Wright, Maria, 97, I43.

Yale, Captain, 6.

Yale, Eunice, I 4.

Yale, Juliette, I 5 I.

Yale, Mary, 32.

Yale, Theophilus, 7 .

Yale, Thomas, 32.

Yale, Waitstill, 17 .

Young, Ida F., I 84 .

Young, John, 29. 




, 


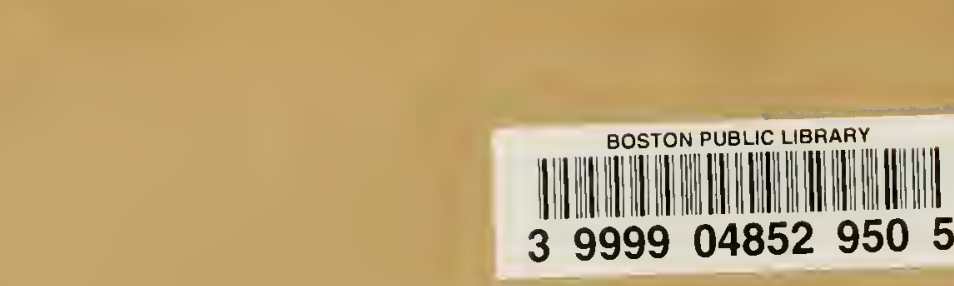

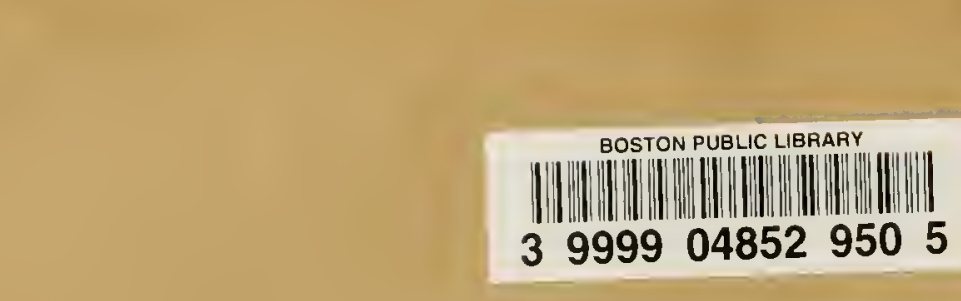

.

.

.

.

.

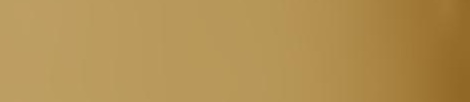

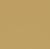

.

.

.

.

.

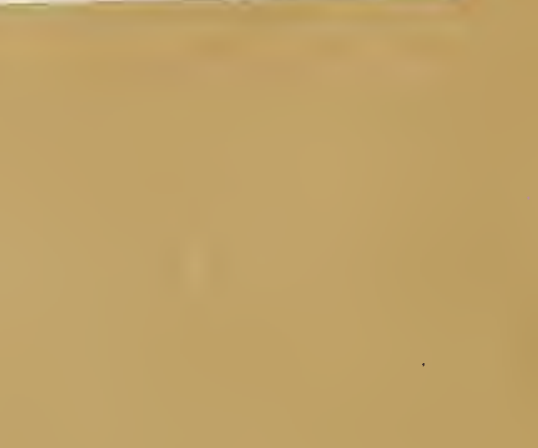

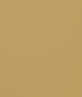

.

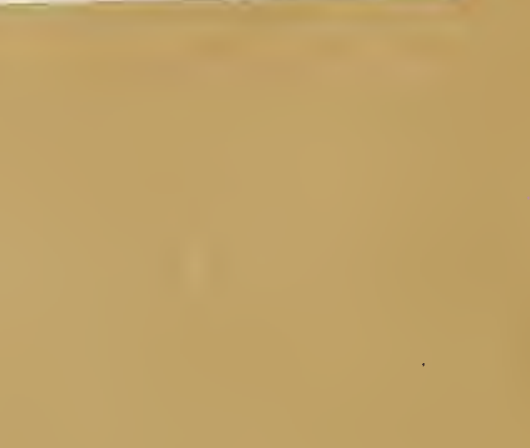

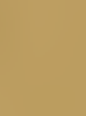

(

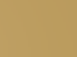

(n)

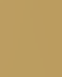

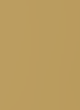

(1)

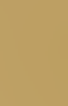

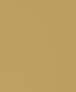

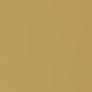

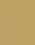

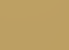

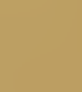

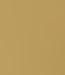

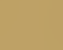

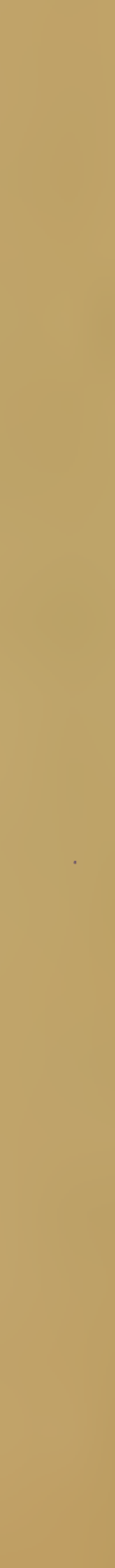

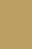

Ad 

80. a 11,15

(1) $i=9,9=\quad$ in 